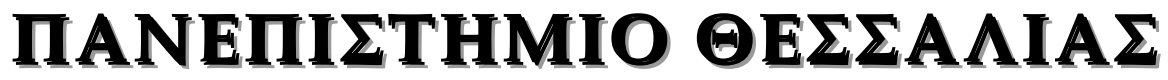

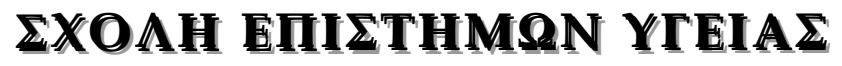 \\ TMHMA IATPIKH
}

NEYPOАОГIКН КАINIKH

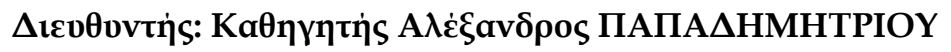

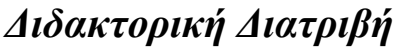

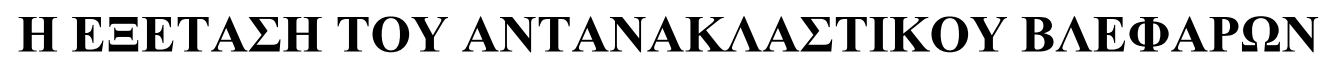

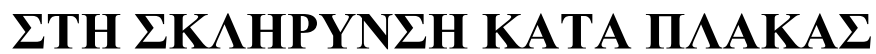

vлó

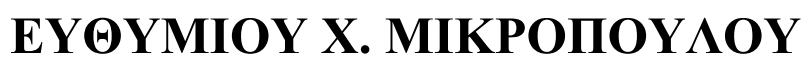

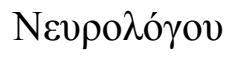

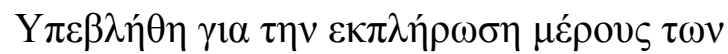

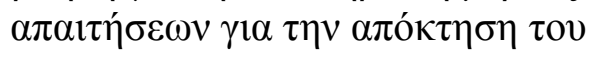

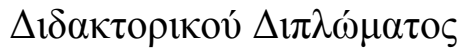

$\Lambda \alpha ́ \rho ı \sigma \alpha, 2011$ 


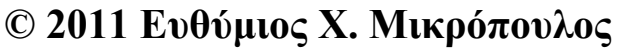

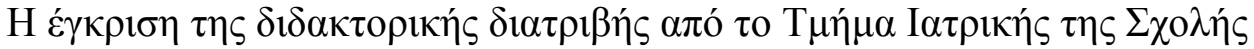

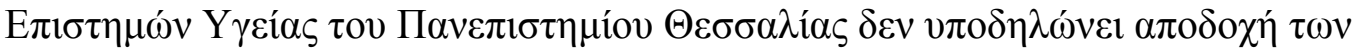

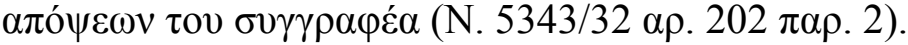




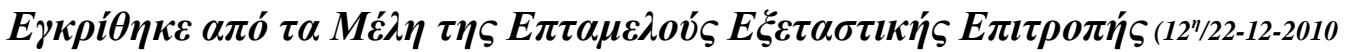

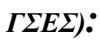

$1^{o \varsigma} E \xi \varepsilon \tau \alpha \sigma \tau \eta \dot{\varsigma}$

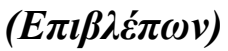

$2^{o \varsigma} E \xi \varepsilon \tau \alpha \sigma \tau \eta \dot{\varsigma} \varsigma$

$3^{o \varsigma} E \xi \varepsilon \tau \alpha \sigma \tau \eta \dot{~}$

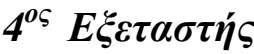

$5^{o \varsigma} E \xi \varepsilon \tau \alpha \sigma \tau \eta \dot{\zeta}$

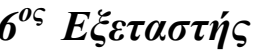

$7^{o \varsigma} E \xi \varepsilon \tau \alpha \sigma \tau \eta \dot{\zeta}$

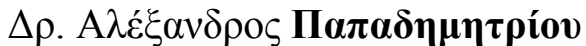

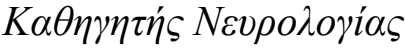

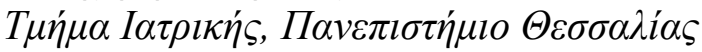

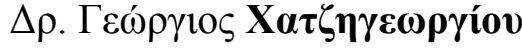

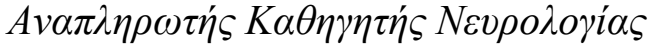

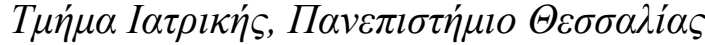

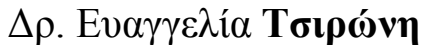

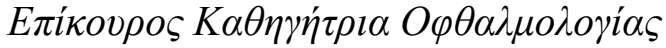

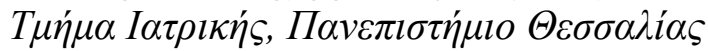

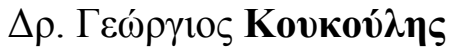

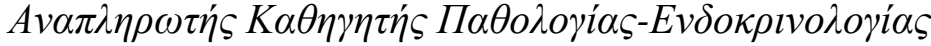

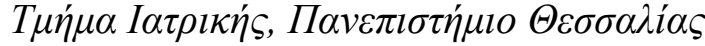

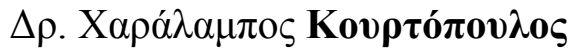

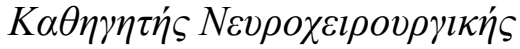

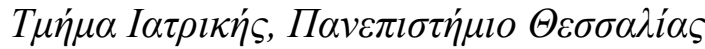

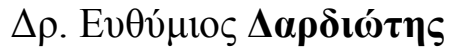

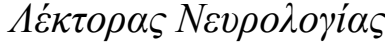

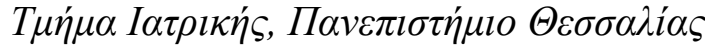

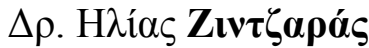

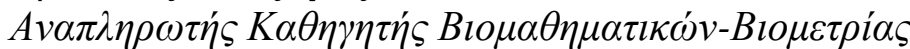

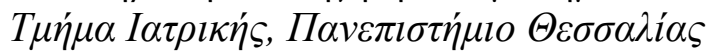




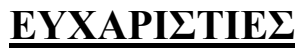

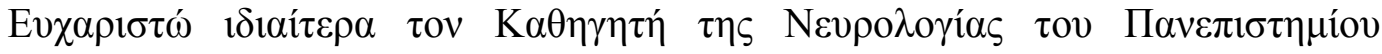

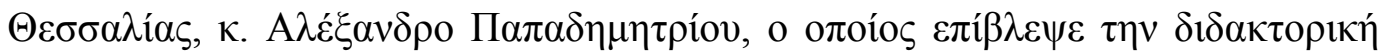

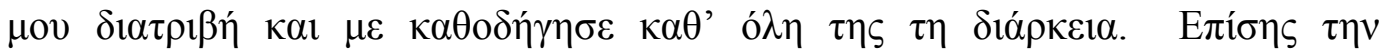

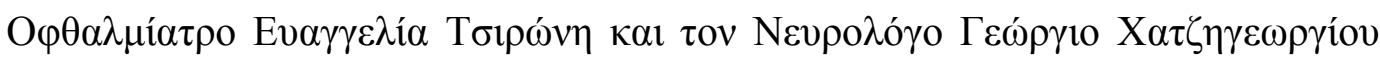

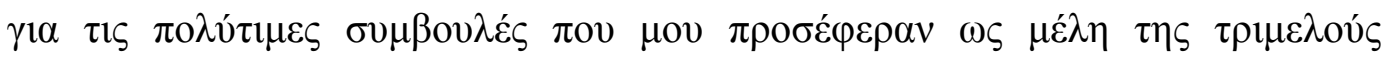

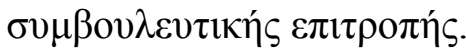

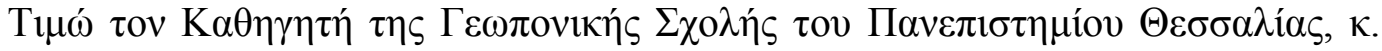

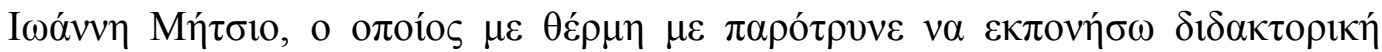

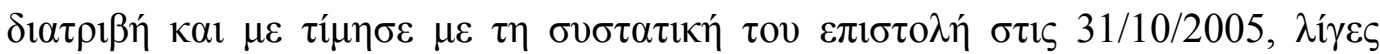

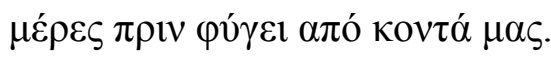

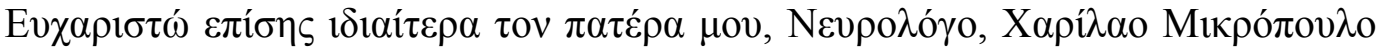

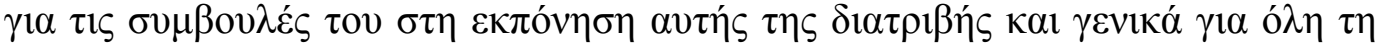

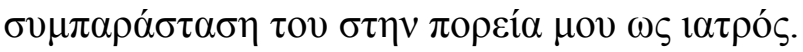

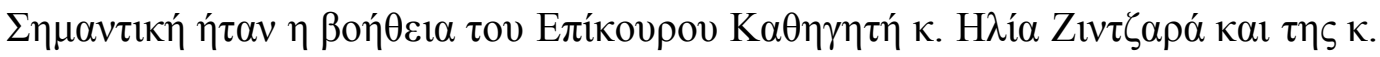

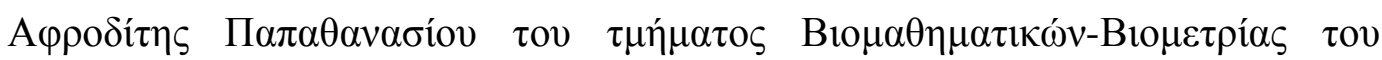

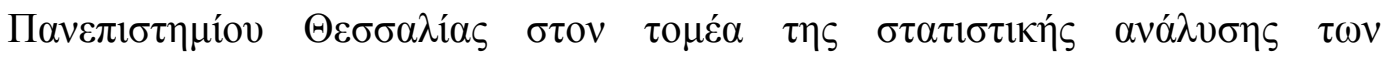

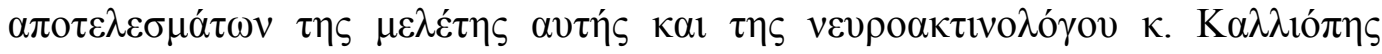

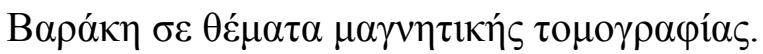

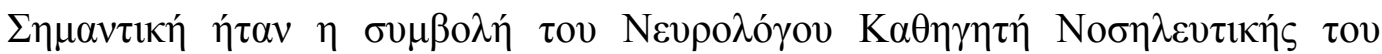

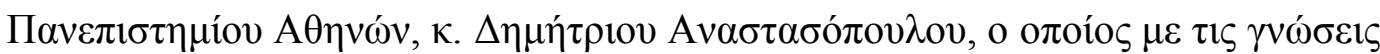

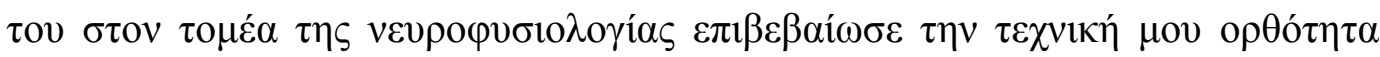

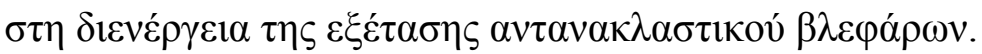

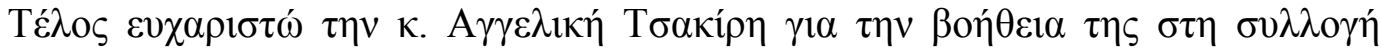

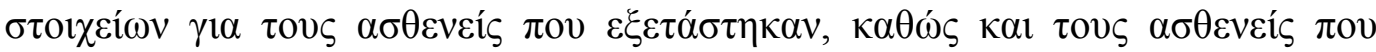

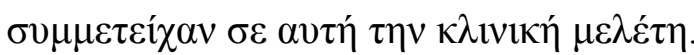




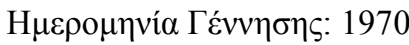

\section{EYNTOMO BIOГPAФIKO}

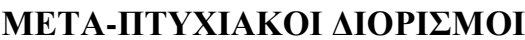

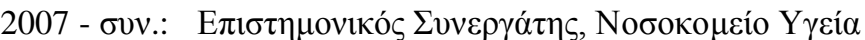

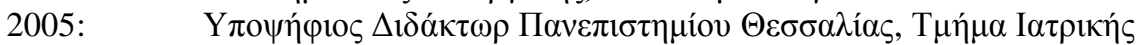

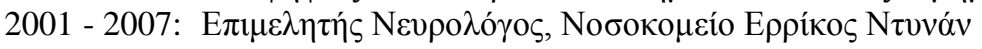

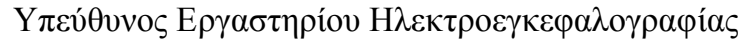

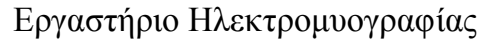

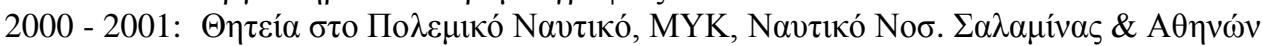

\section{EKПАI $\Delta E Y \Sigma H / T I T \Lambda O I ~ \Sigma \Pi O Y \Delta \Omega N:$

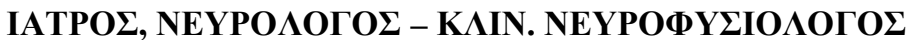

2000: $\Delta \imath \pi \lambda \omega \mu \alpha \tau o v ́ \chi o \varsigma$, American Board of Psychiatry and Neurology

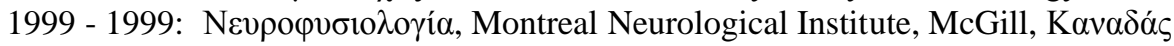

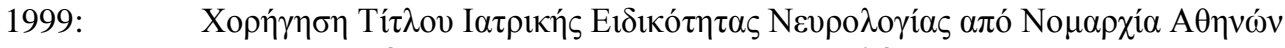

1998: Хори́ $\quad$ Х

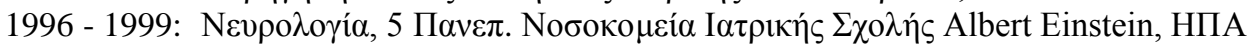

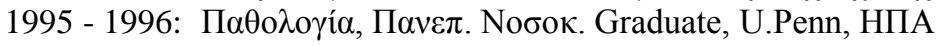

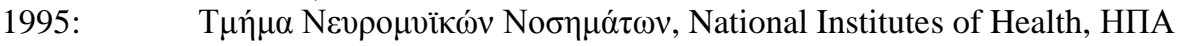

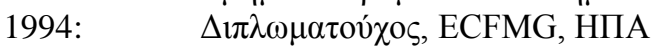

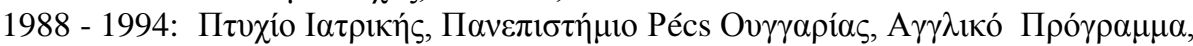

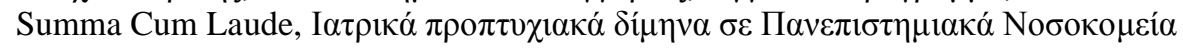

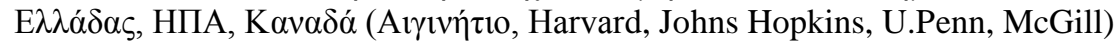

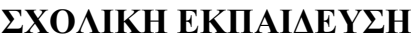

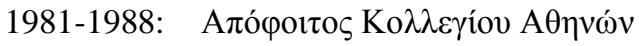

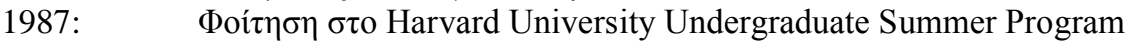

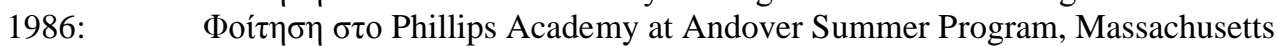

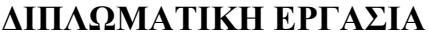

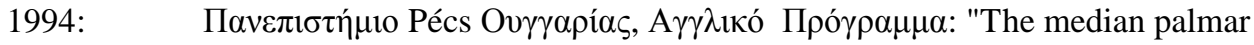
orthodromic sensory conduction study within the framework of the carpal tunnel syndrome and its electroneurographic diagnostic techniques".

\section{$\triangle H M O \Sigma I E Y \Sigma E I \Sigma$}

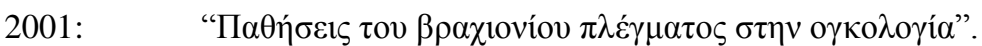

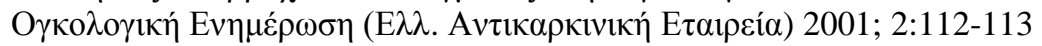

2010: "Supratentorial Multiple Sclerosis lesions affect the Blink Reflex Test". E.H.Mikropoulos, A.Papathanasiou, G.Hadjigeorgiou, E.Tsironi, A.Papadimitriou. The Open Neurology Journal 2010;4: 92-99

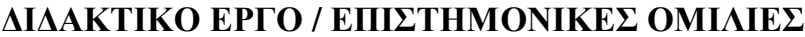

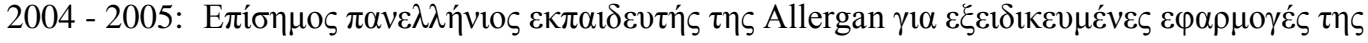

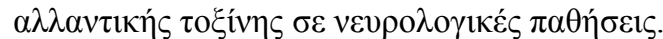

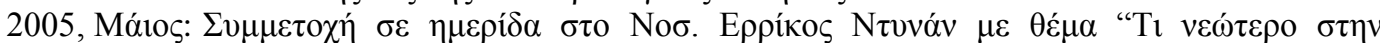

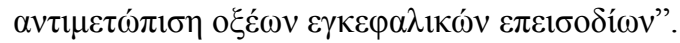

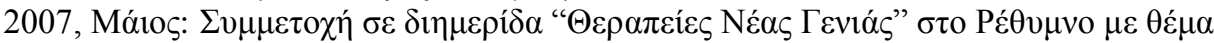

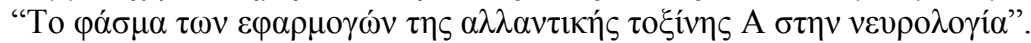

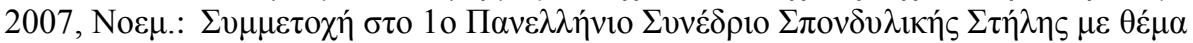

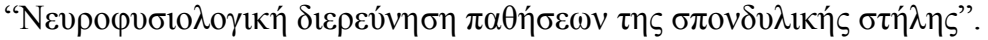

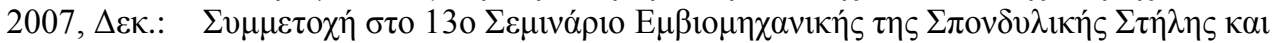

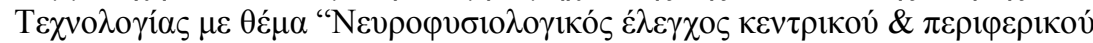

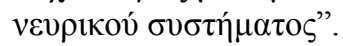

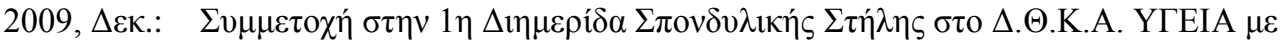

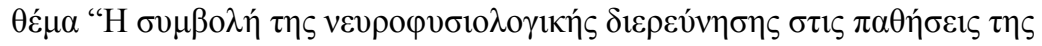
$\sigma \pi \mathrm{ov \delta v \lambda \iota \kappa ท́} \sigma \tau \eta \dot{\lambda} \eta \varsigma^{\prime}$. 


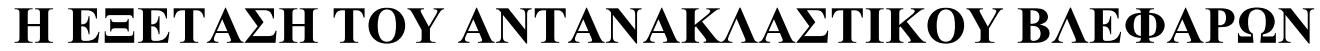

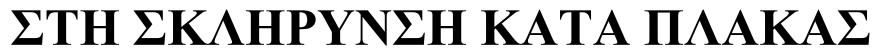

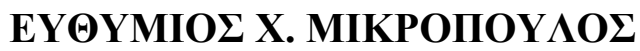

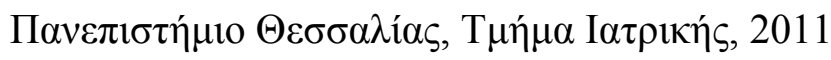

\section{ТРIMЕАН Г ГМВВУАЕYТIКН ЕПIТРОПН}

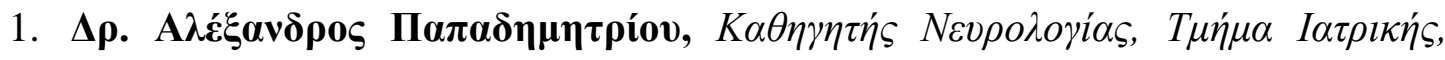

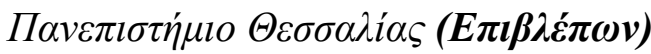

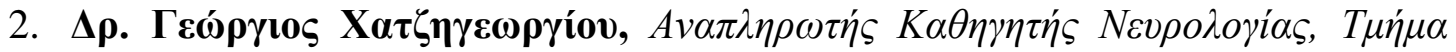

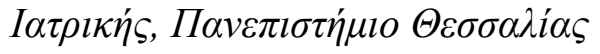

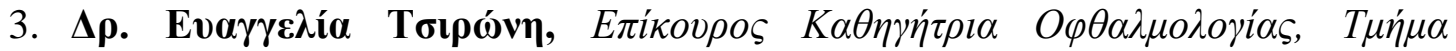

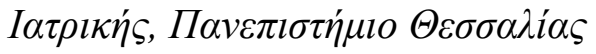




\section{ПЕРІАНЧН}

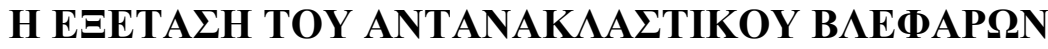

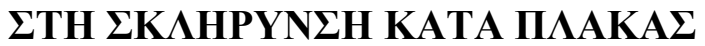

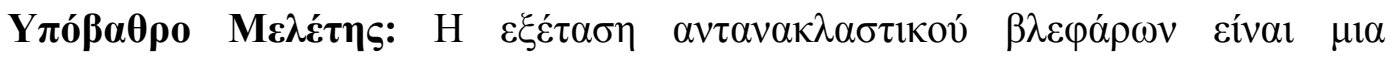

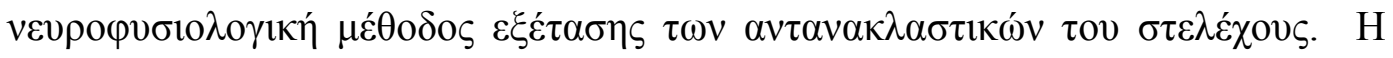

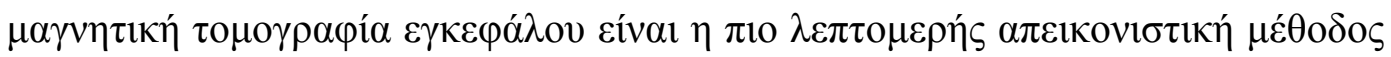

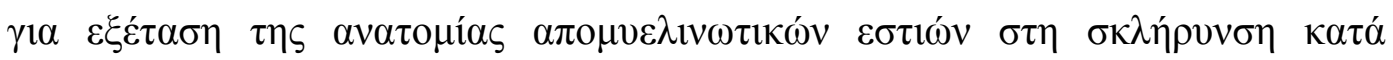
$\pi \lambda \alpha ́ \kappa \alpha \varsigma$.

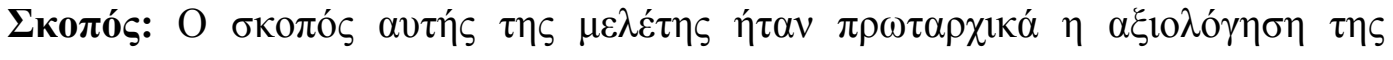

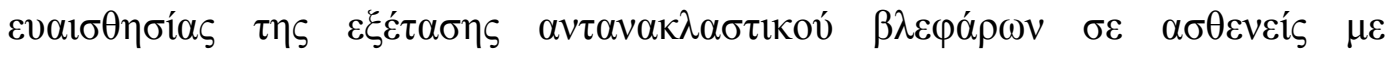

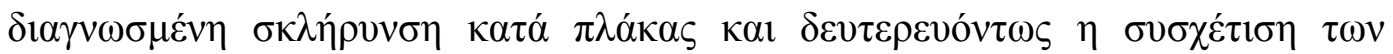

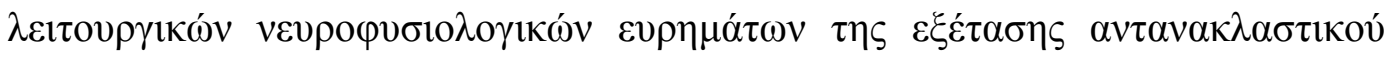

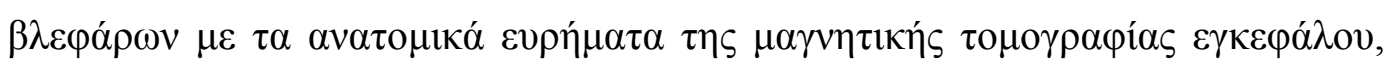

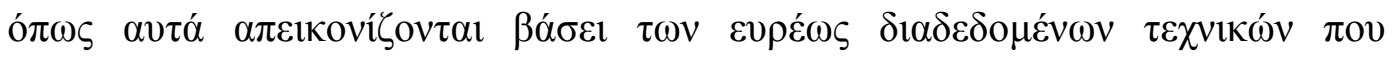

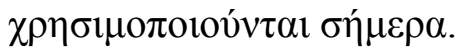

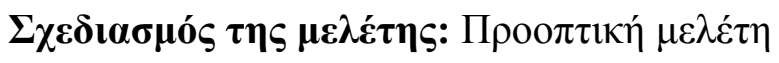

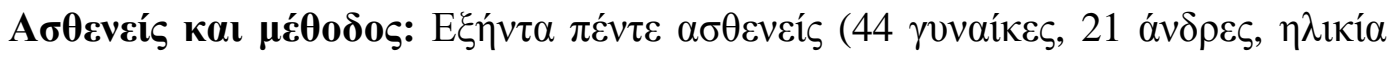

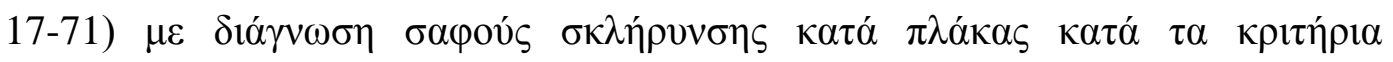

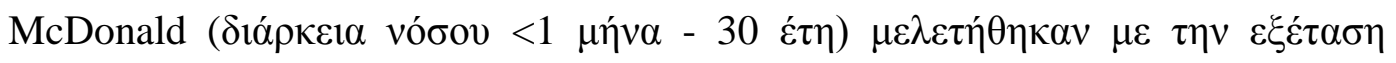

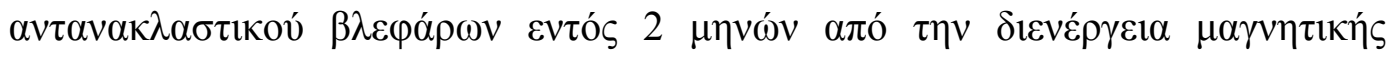

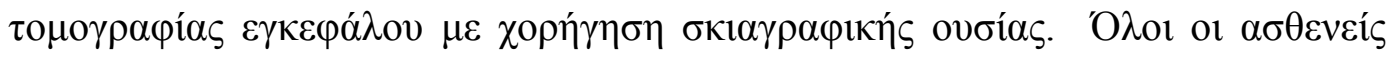

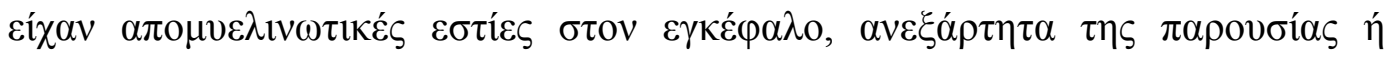

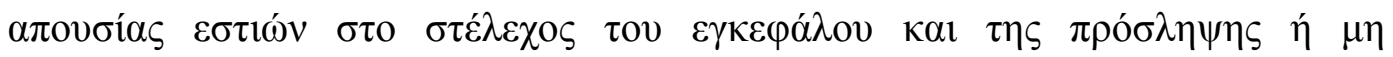

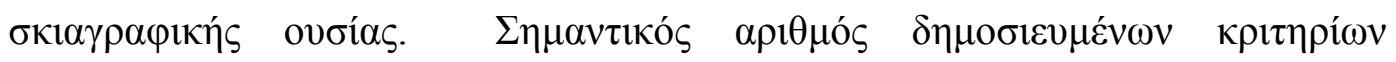

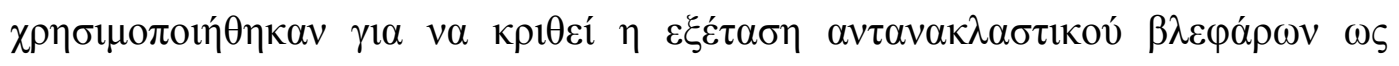
$\pi \alpha \theta 0 \lambda$ о

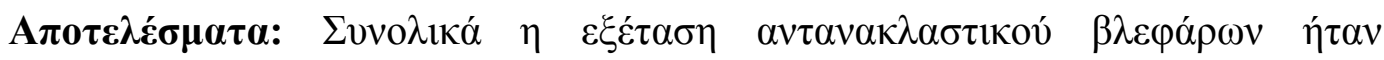

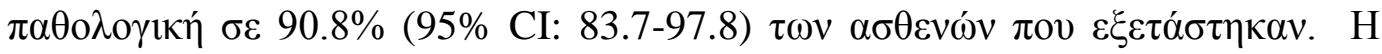




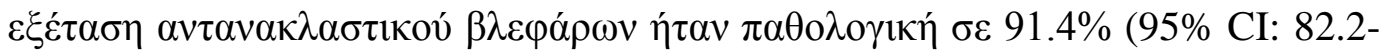

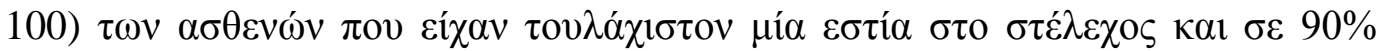

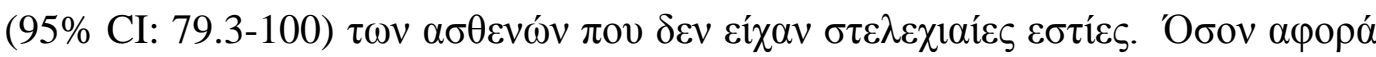

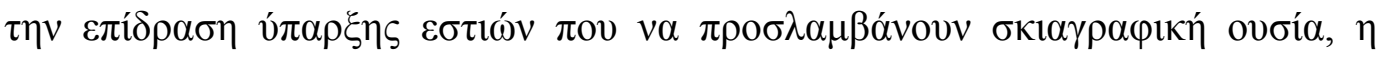

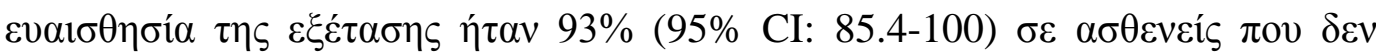

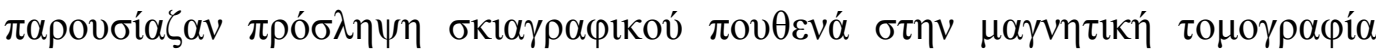

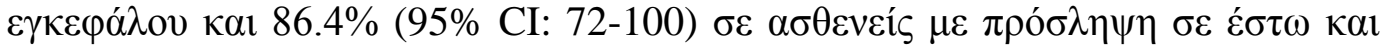

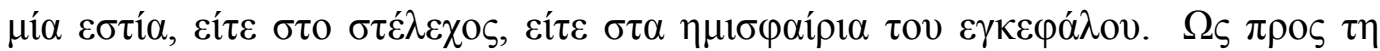

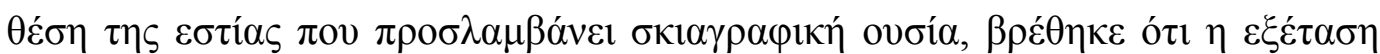

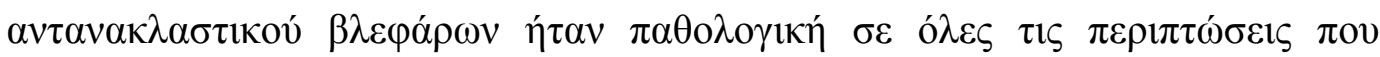

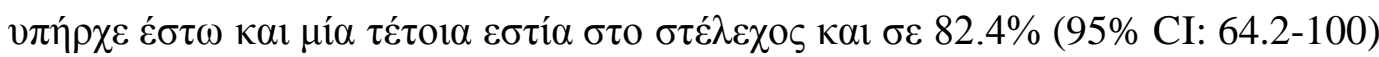

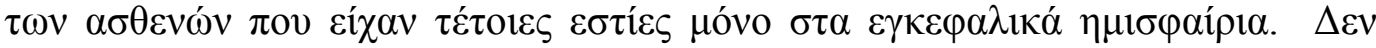

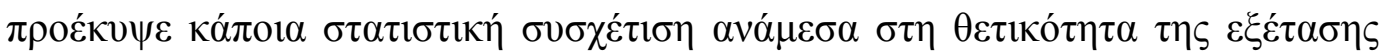

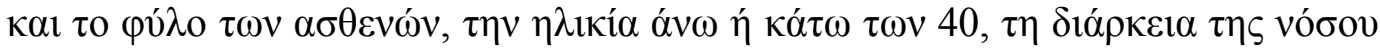

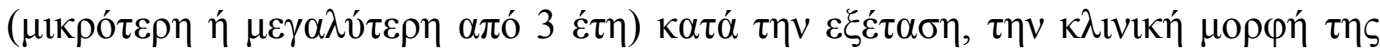

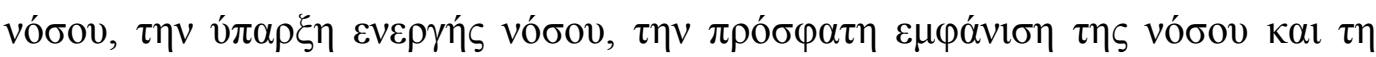

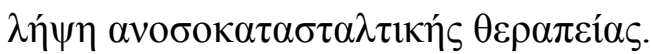

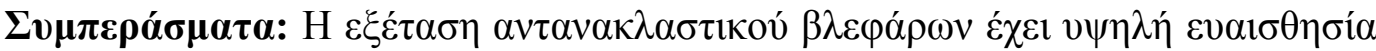

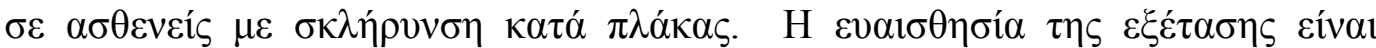

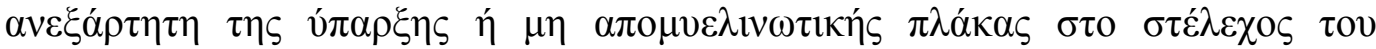

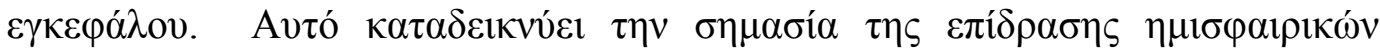

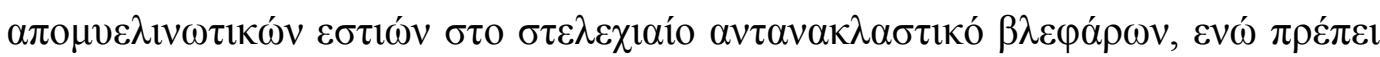

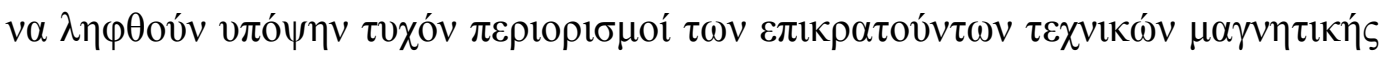

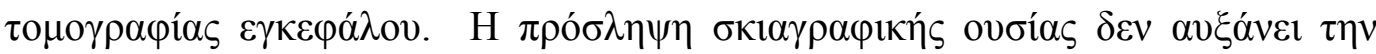

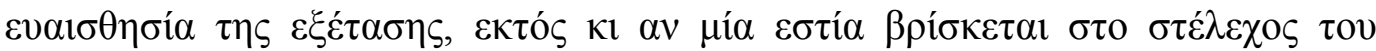

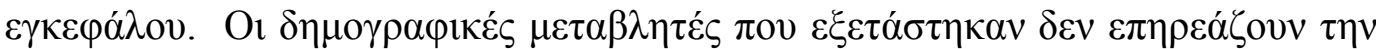

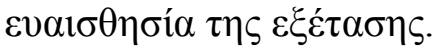




\section{SUMMARY}

\section{A STUDY OF THE BLINK REFLEX TEST IN MULTIPLE SCLEROSIS}

Objective: The purpose of this study was to evaluate the sensitivity of the blink reflex test in patients diagnosed with multiple sclerosis and to identify whether the presence of demyelinating plaques in the brainstem or contrast enhancement in the magnetic resonance imaging (MRI) study of the patients' brain affects the results.

Research Design: Prospective cohort study

Patients and Methods: Sixty five patients (44 women, 21 men, age 17-71) who meet the McDonald criteria for diagnosis of definite multiple sclerosis (disease duration $<1$ month -30 years) underwent the blink reflex test within 2 months of an MRI of the brain that was performed using gadolinium. All patients had demyelinating plaques in the brain, regardless of the presence or absence of brainstem lesions or gadolinium enhancement. Several published criteria were used to classify the blink reflex test as positive or negative.

Results: Overall, the test was positive in $90.8 \%$ (95\% CI: 83.7-97.8) of the patients examined. The test was positive in 91.4\% (95\% CI: 82.2-100) of examined patients with at least one brainstem lesion and in 90\% (95\% CI: $79.3-$ 100) of patients who had no brainstem lesions in their MRI. As to the presence of gadolinium-enhancing lesions anywhere on MRI, the test was positive in $93.3 \%$ (95\% CI: 85.4-100) of patients with no enhancement anywhere in the brain and in $86.4 \%$ (95\% CI: 72-100) of patients with at least one enhancing lesion, whether in the cerebral hemispheres or the brainstem. Finally, as to the location of gadolinium enhancement, where present, it was found that the test was positive in $100 \%$ of patients with at least one enhancing lesion in the brainstem and in $82.4 \%$ (95\% CI: 64.2-100) of patients whose only enhancing lesions were in the cerebral hemispheres. No statistical correlation was found between positivity of results and gender, age above or below 40, disease duration (more or less than 3 years), 
type of MS, acuteness of an MS event, whether MS diagnosis was recent or not, immunosuppressive treatment or not.

Conclusions: The blink reflex test has a high sensitivity in patients with multiple sclerosis, regardless of the presence or absence of demyelinating plaques in the brainstem. This points to the influence of supratentorial MS lesions on the BRT, while limitations of commonly used imaging techniques must be considered. Gadolinium enhancement in the brain MRI does not increase the sensitivity of the test, unless it occurs in a brainstem plaque. Gender, age, disease duration, type of MS, acuteness of an MS event and whether MS diagnosis was recent or not were not variables affecting the results. 


\section{ПEPIEXOMENA}

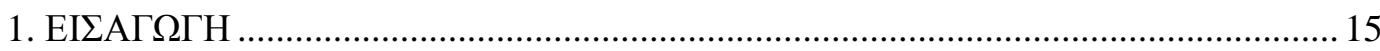

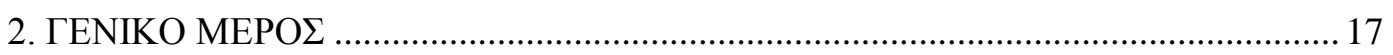

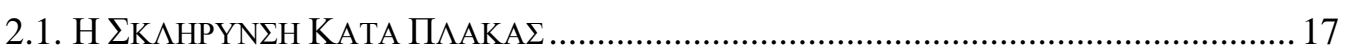

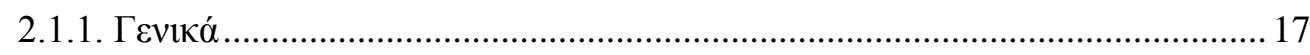

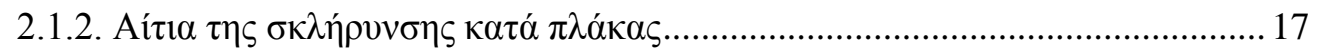

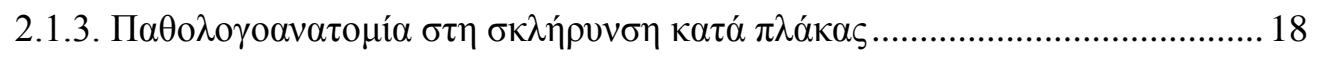

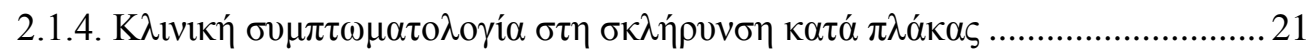

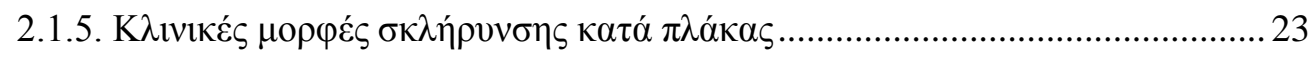

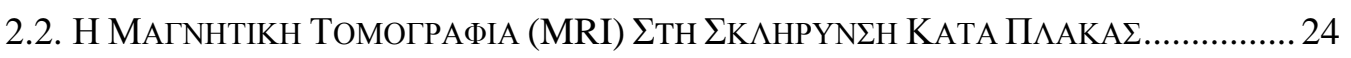

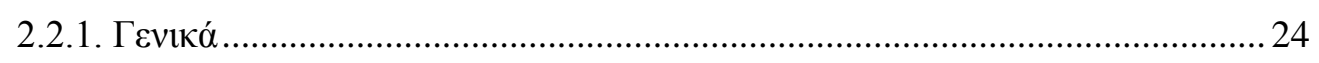

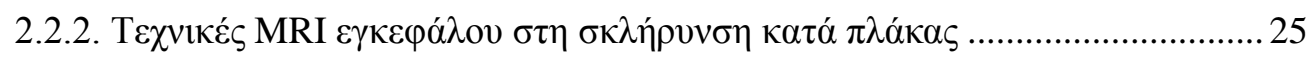

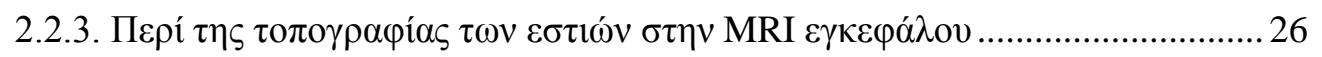

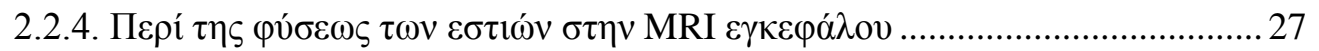

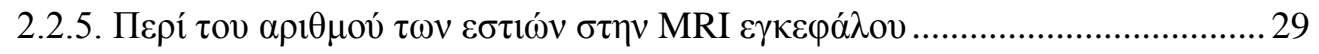

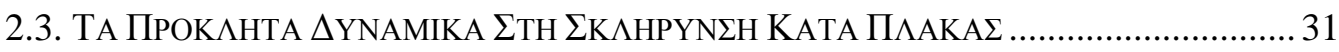

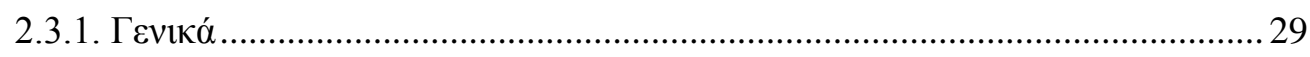

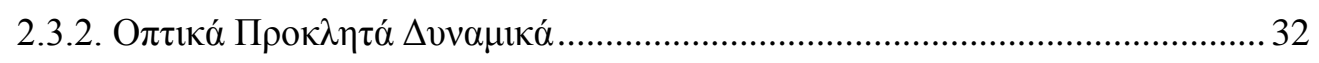

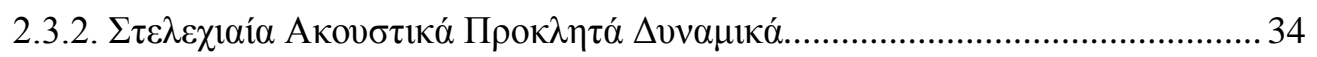

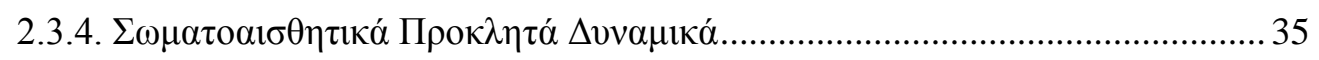

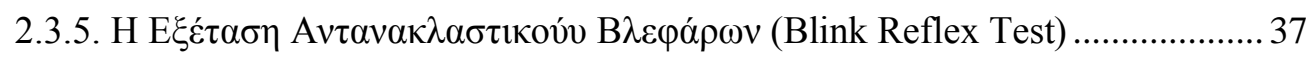

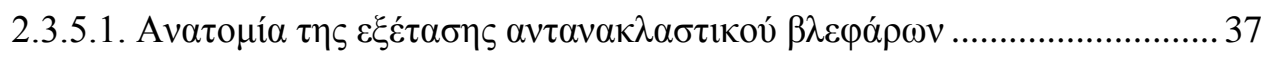

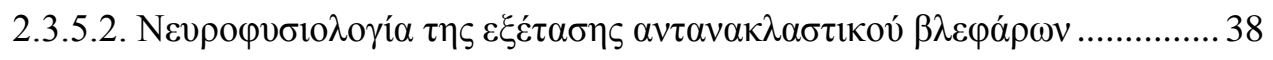

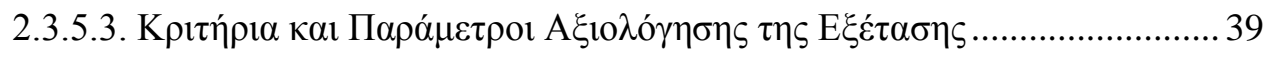

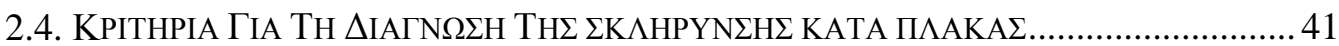




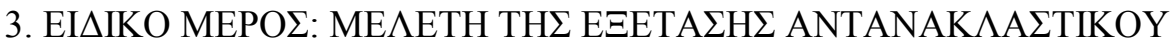

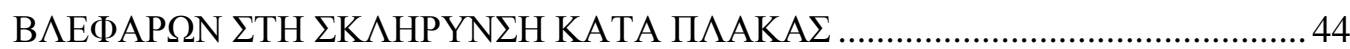

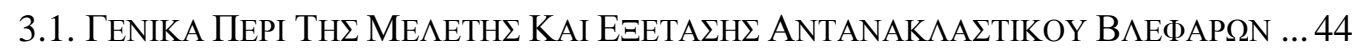

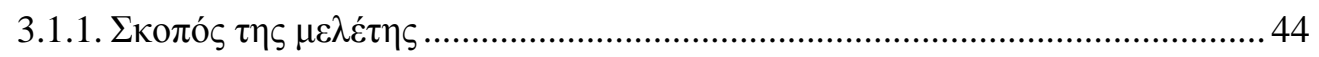

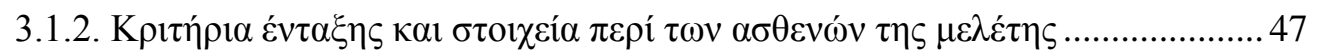

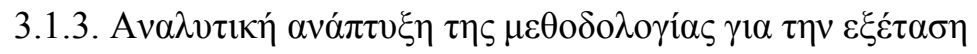

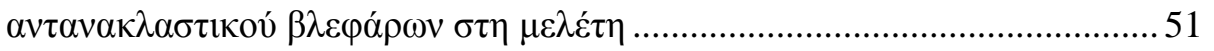

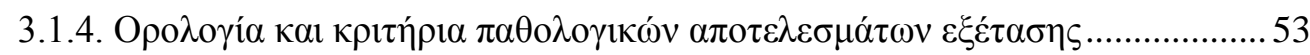

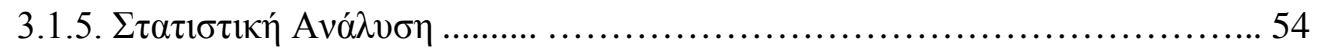

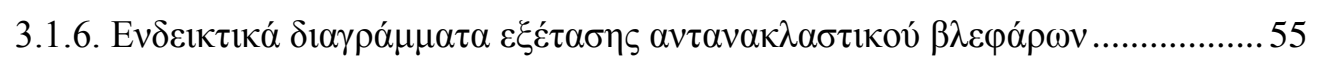

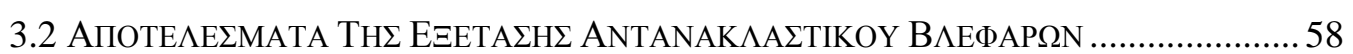

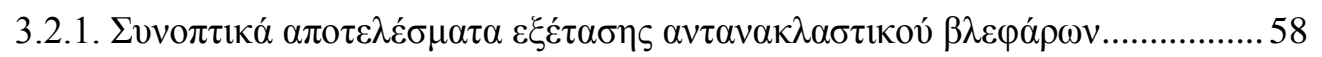

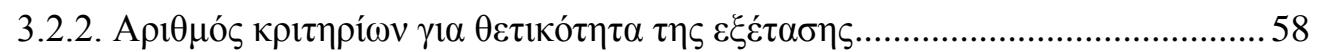

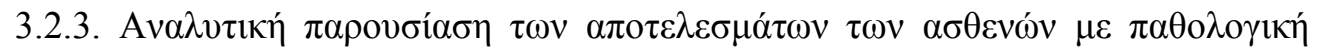

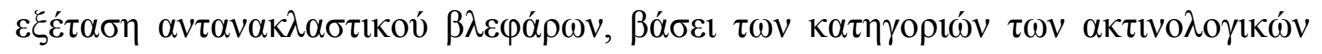

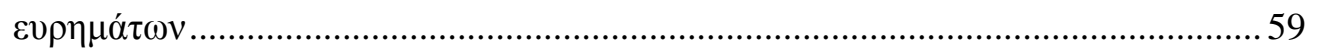

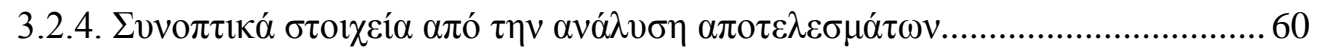

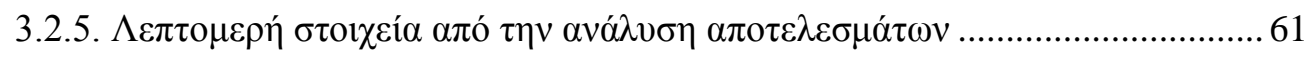

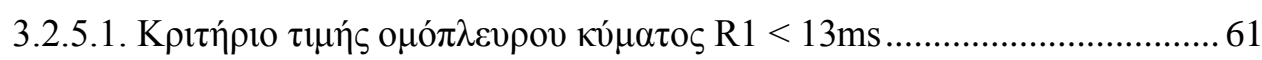

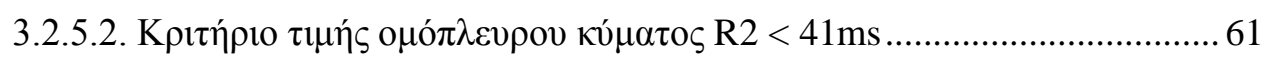

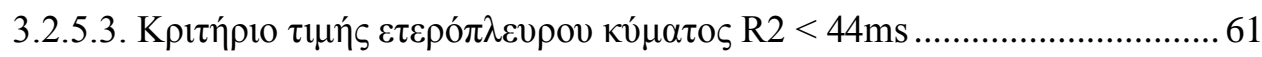

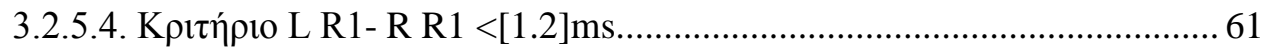

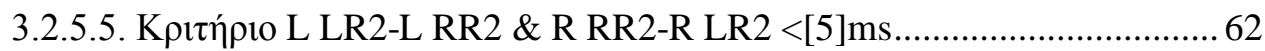

3.2.5.6. Kpıтท́рı L LR2-R LR2 \& L RR2-R RR2 <[7]ms.................................... 62

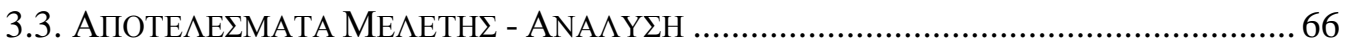

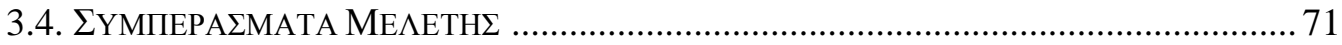

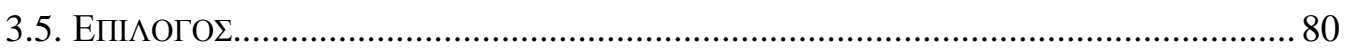

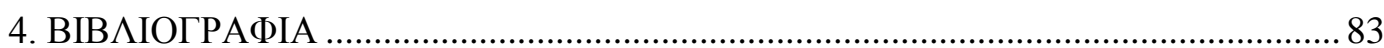




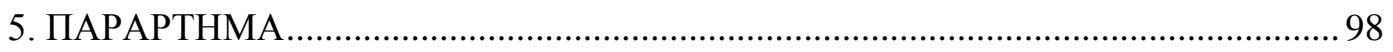

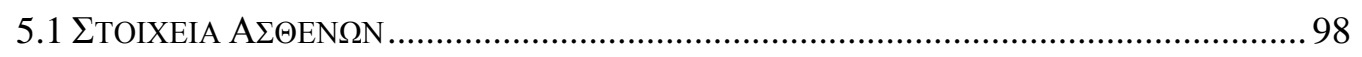

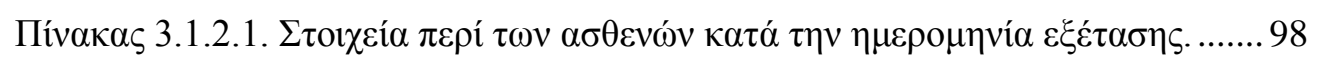

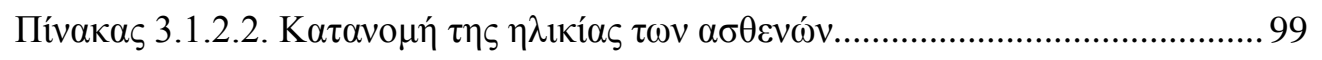

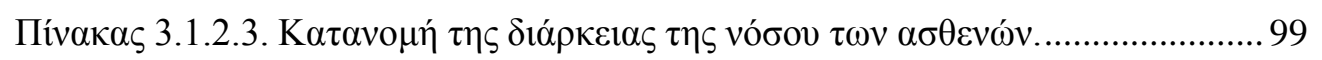

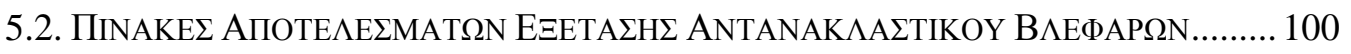

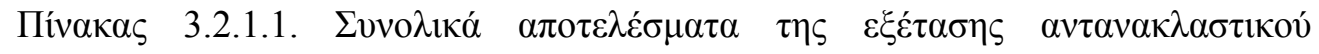

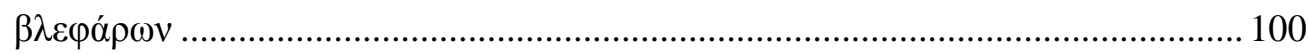

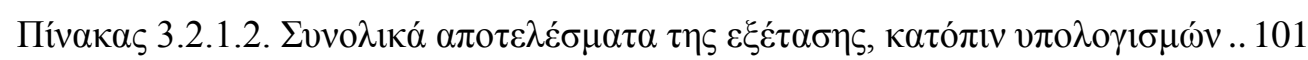

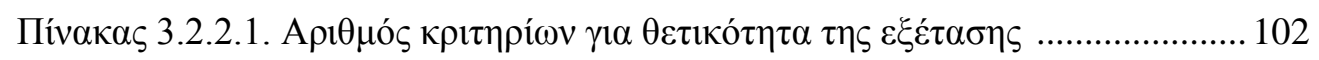

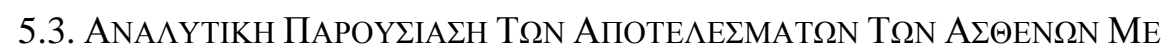

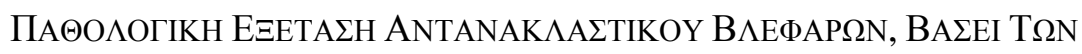

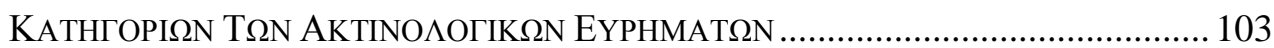

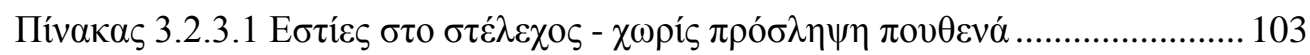

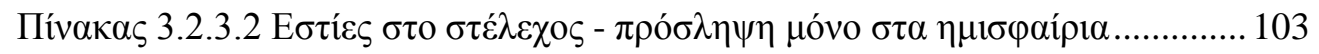

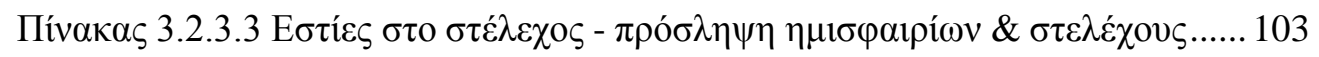

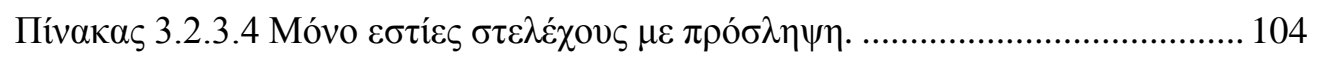

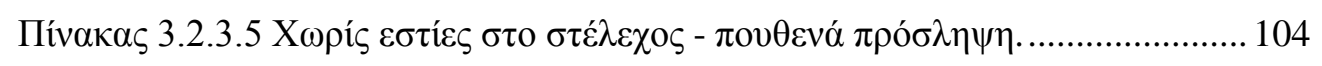

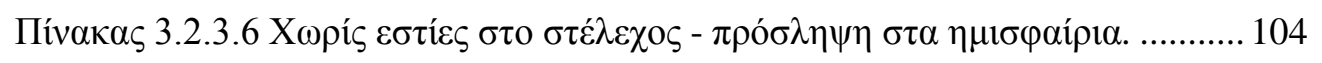

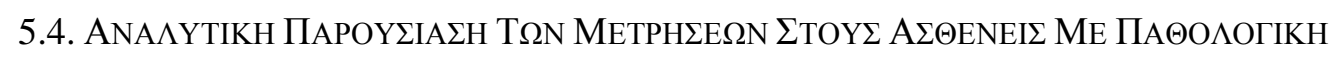

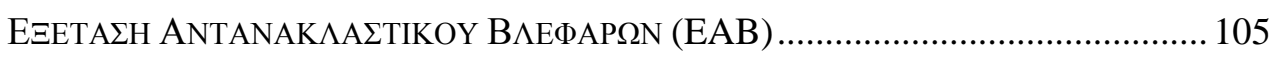

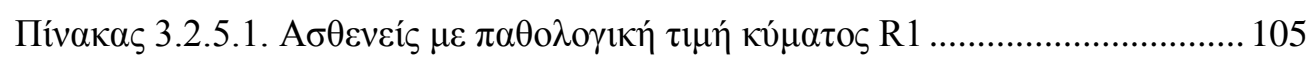

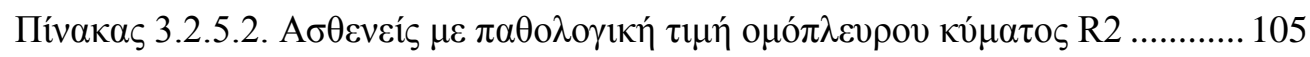

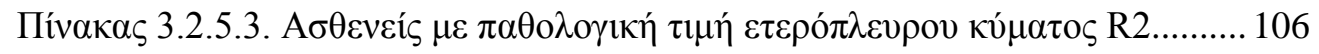

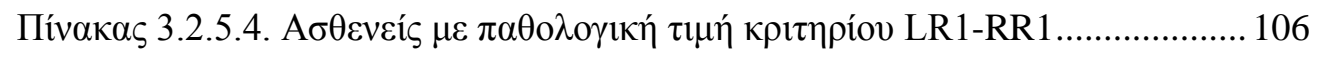

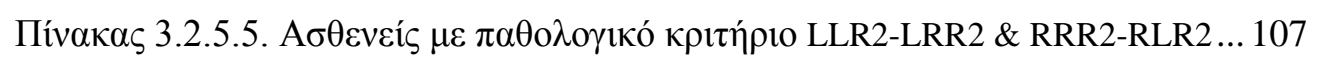

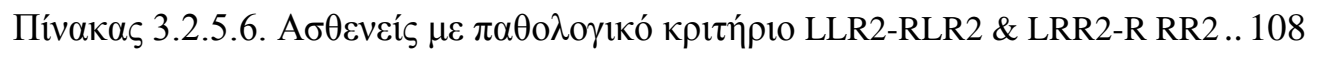

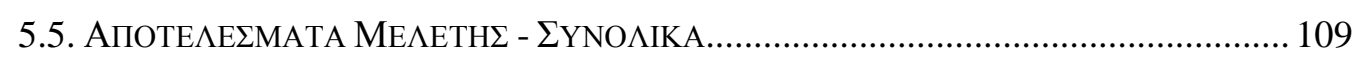

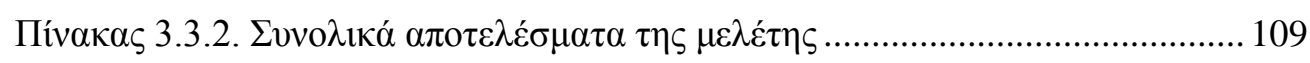

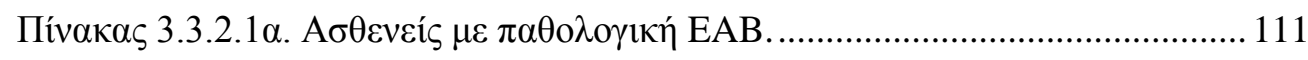

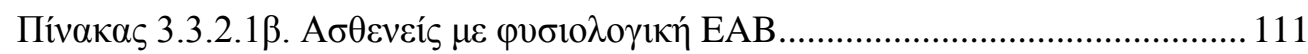

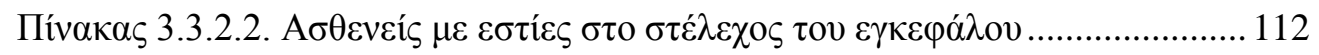

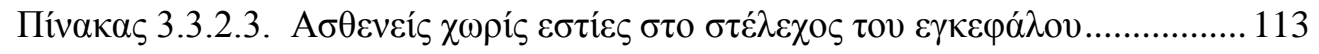

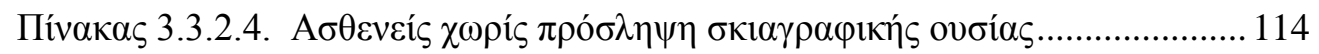

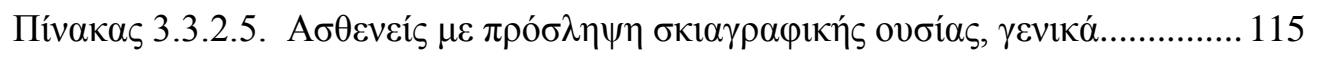




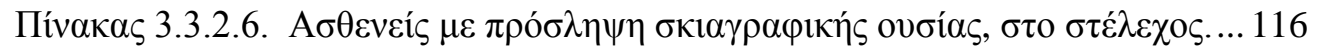

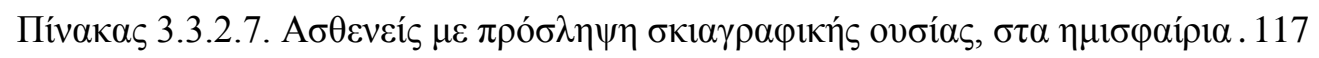

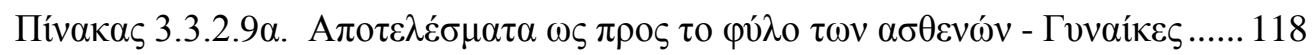

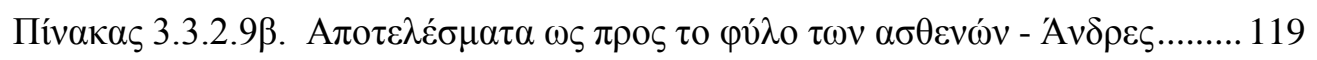

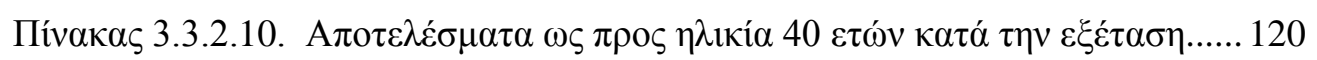

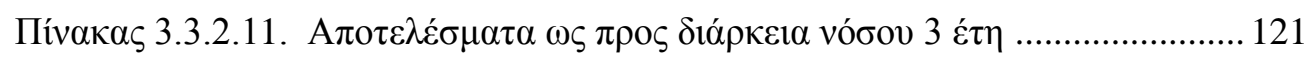

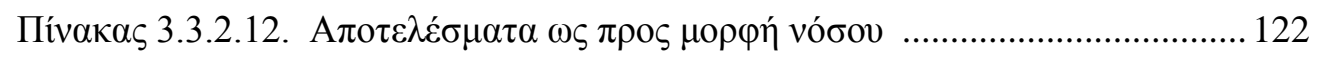

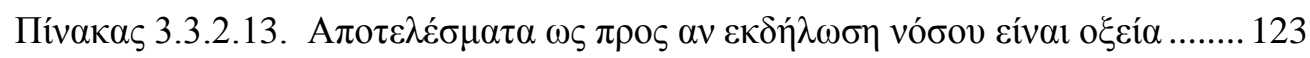

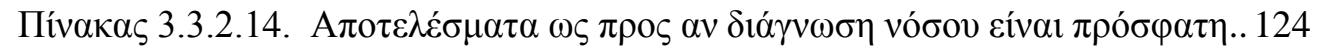




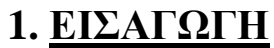

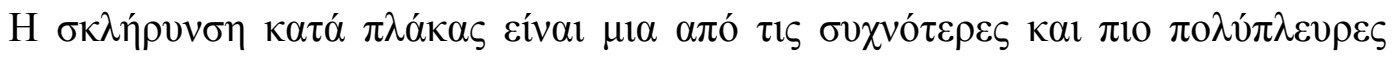

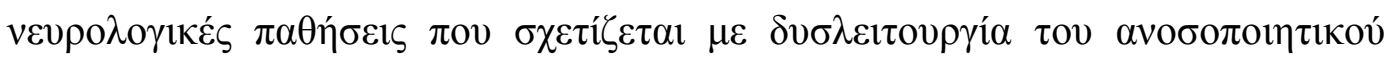

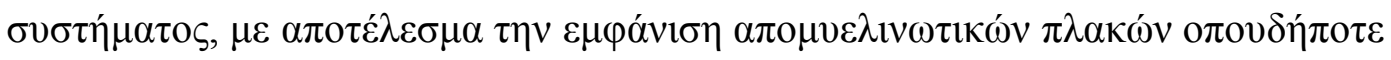

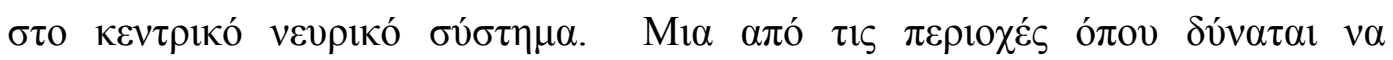

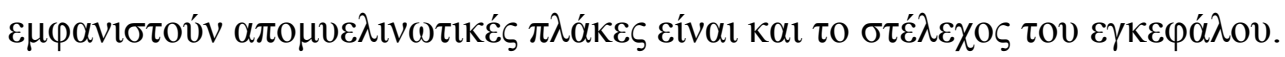

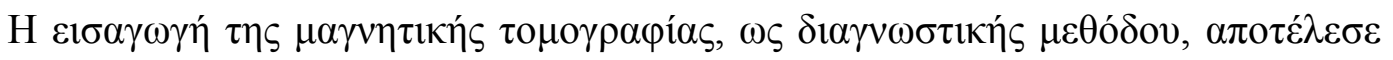

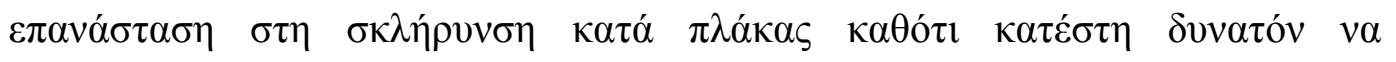

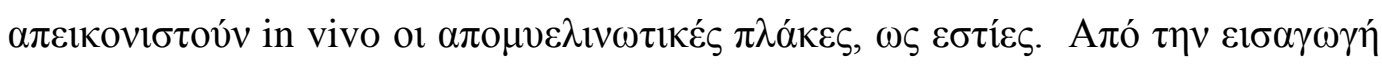

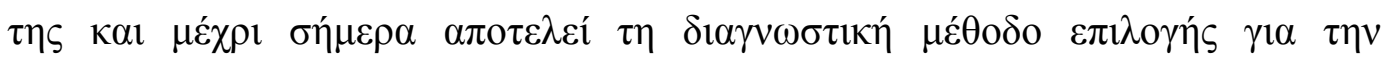

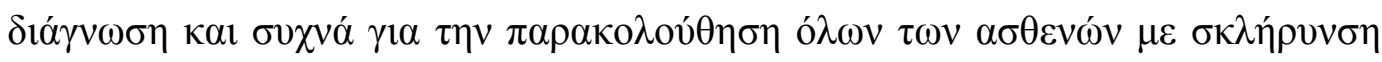

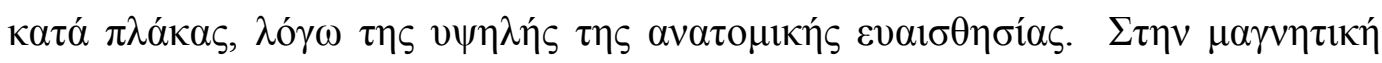

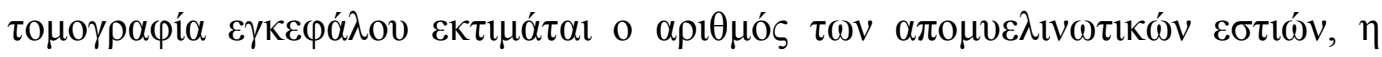

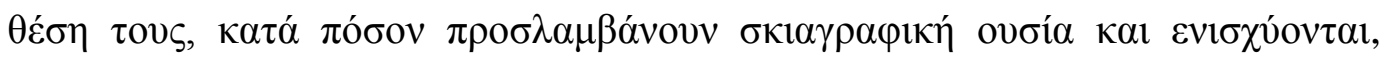

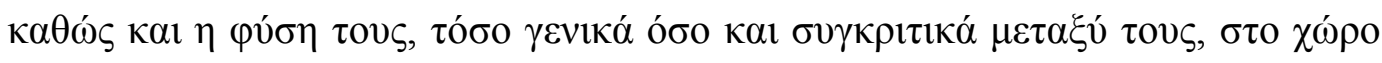

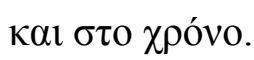

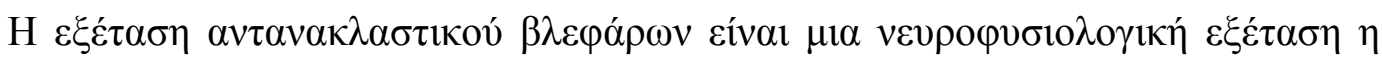

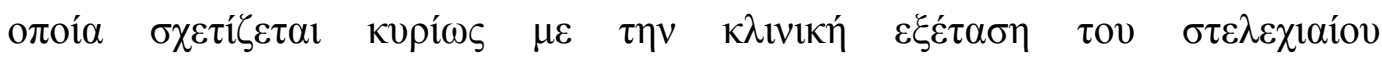

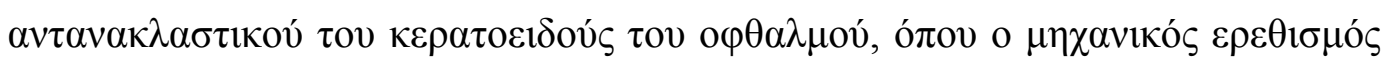

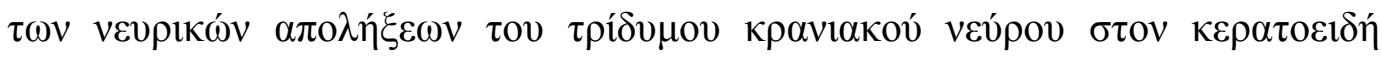

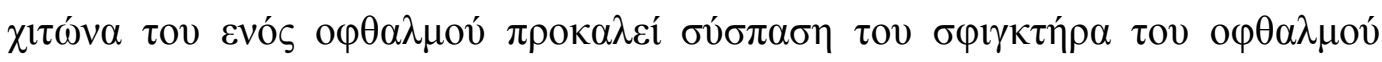

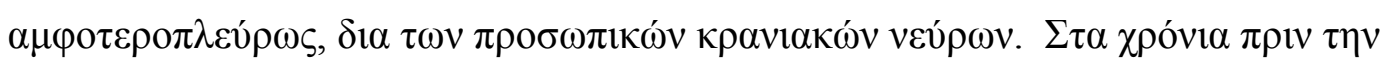

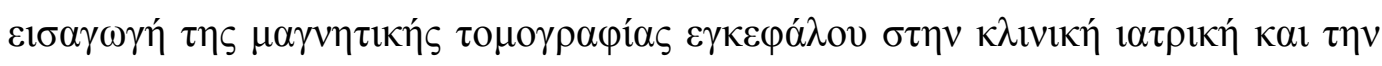

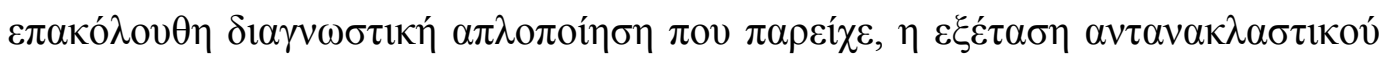

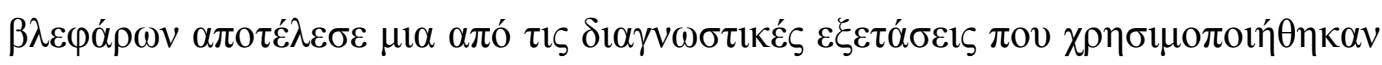

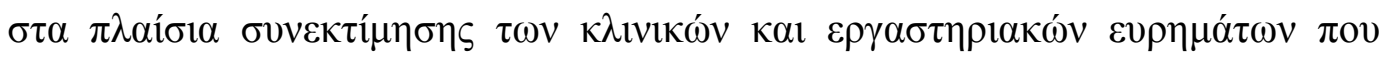

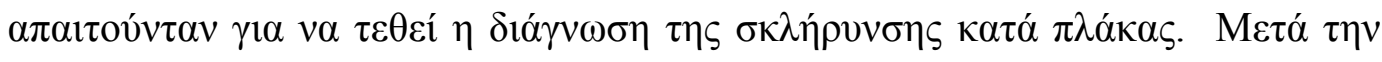

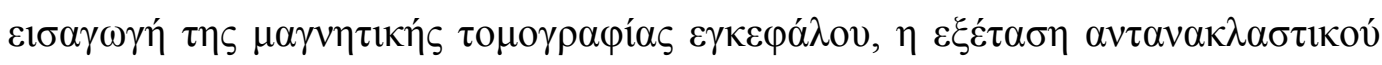

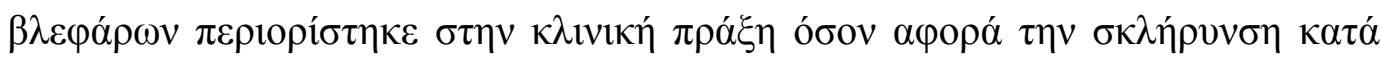

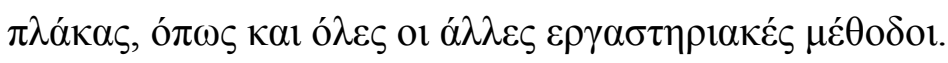




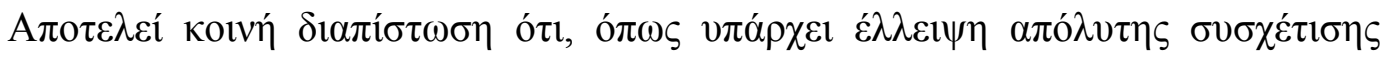

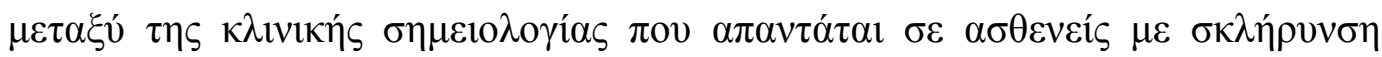

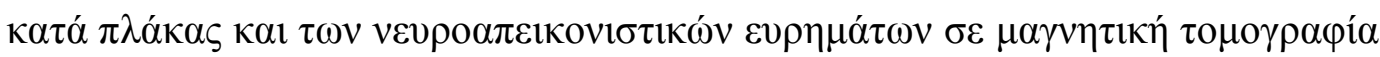

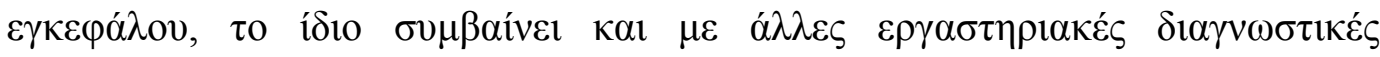

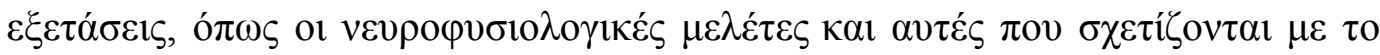

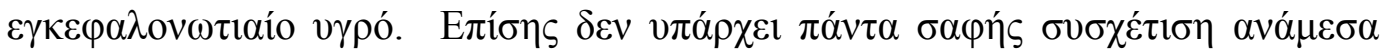

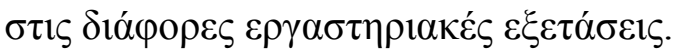

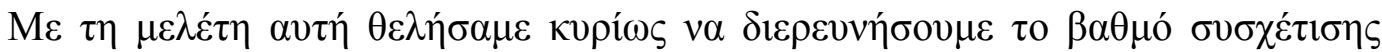

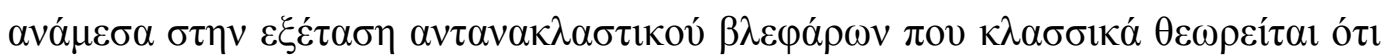

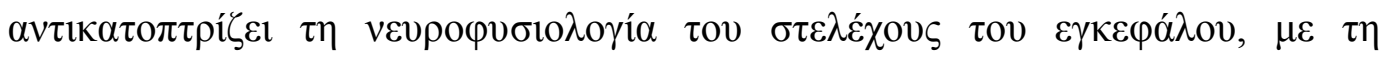

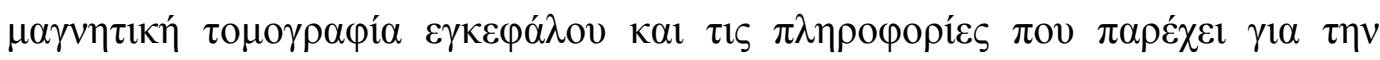

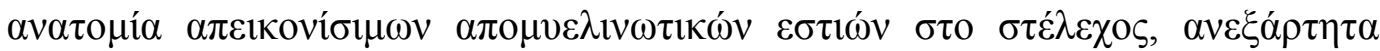

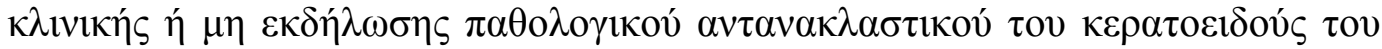

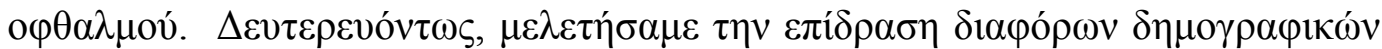

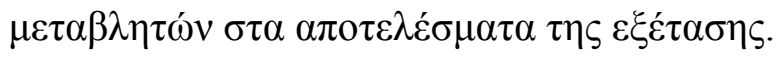




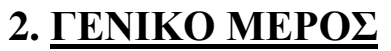

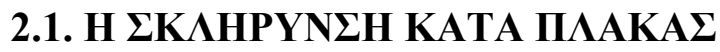

\subsection{1. ГENIKA}

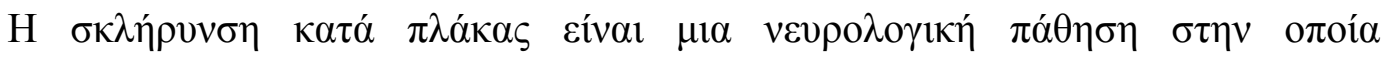

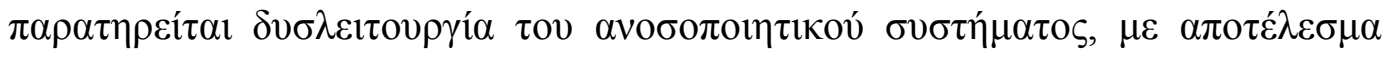

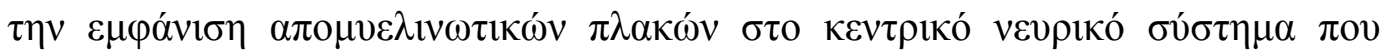

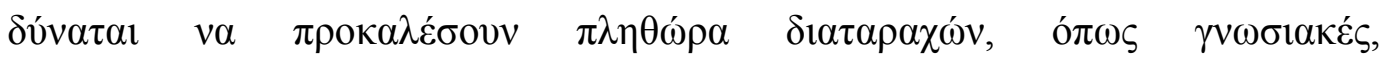

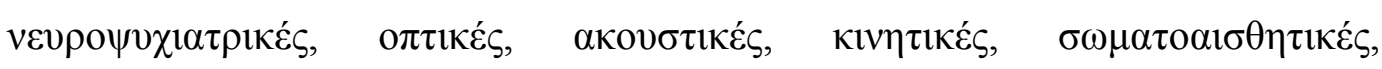

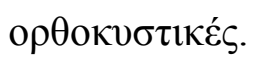

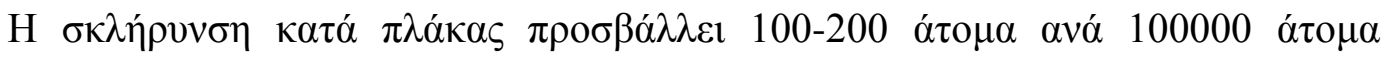

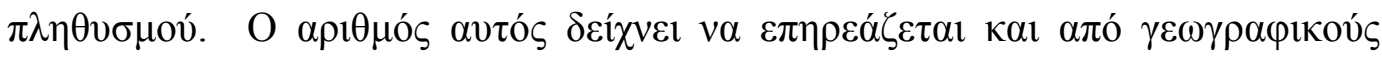

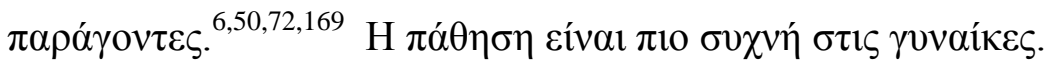

\subsubsection{AITIA TH $\Sigma$ $\Sigma \Lambda H P Y N \Sigma H \Sigma$ KATA П $\Lambda A K A \Sigma$}

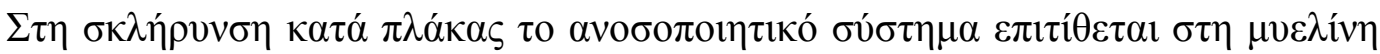

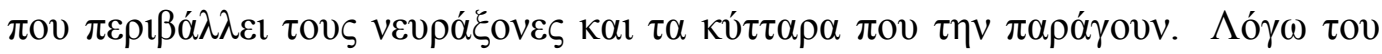

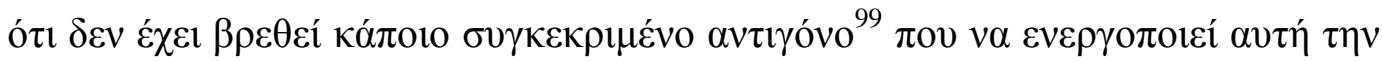

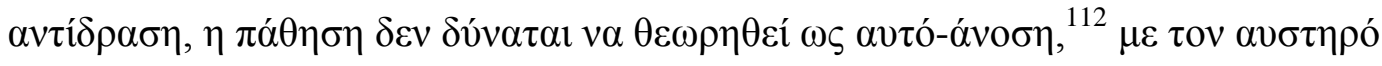

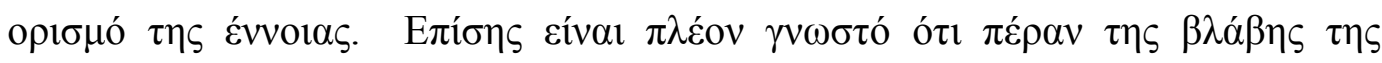

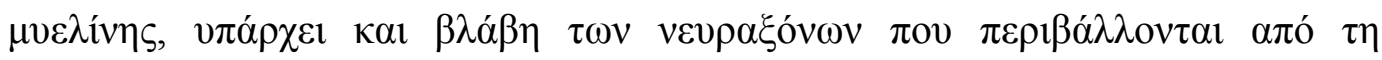

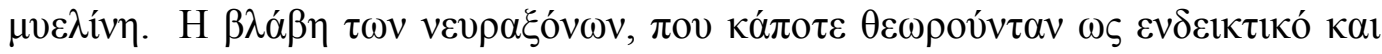

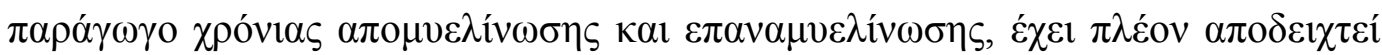

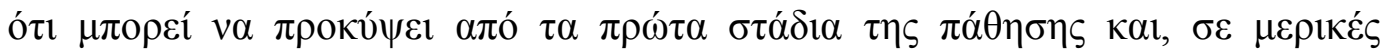

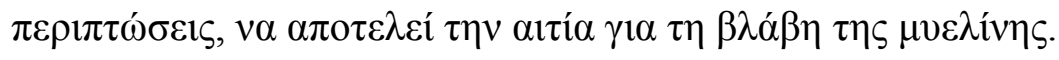

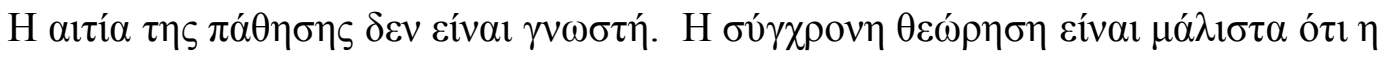

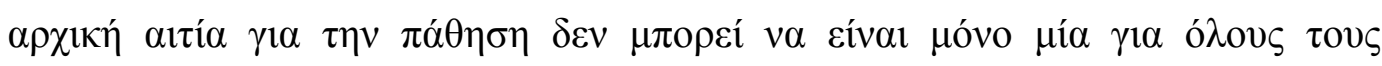

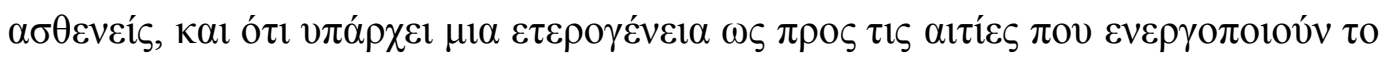

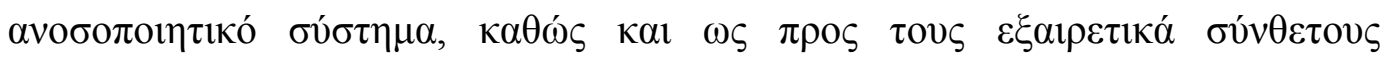




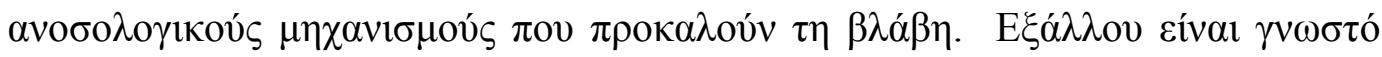

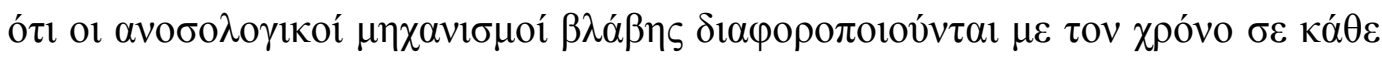

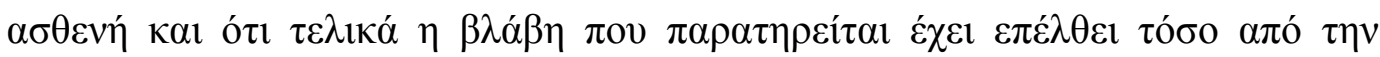

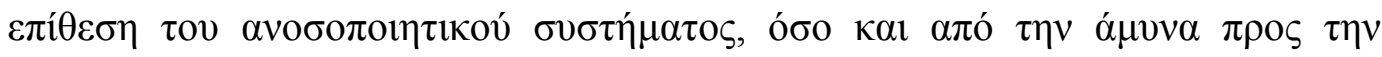

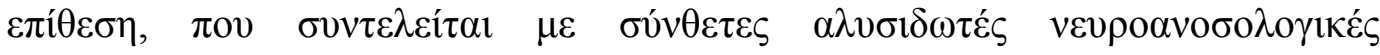
$\alpha \nu \tau \imath \delta \rho \alpha ́ \sigma \varepsilon \imath \varsigma$.

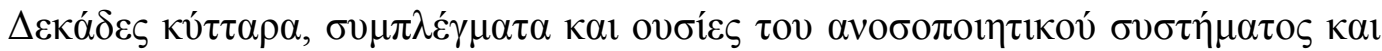

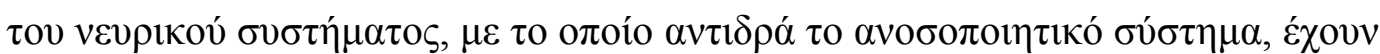

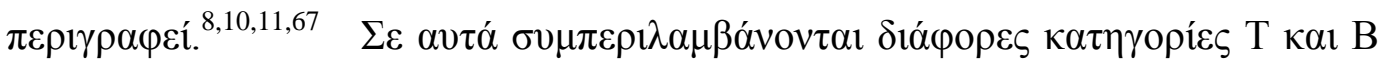

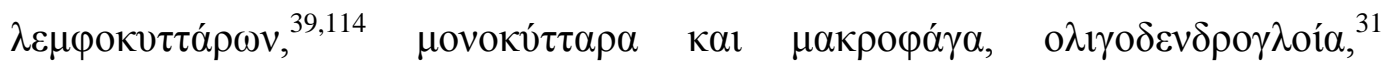

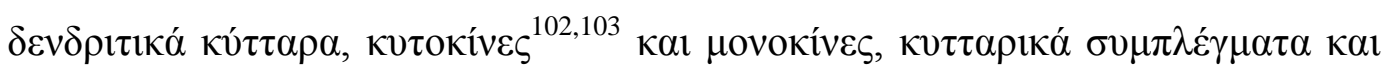

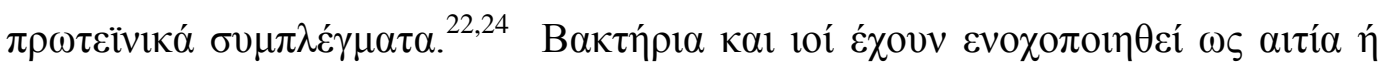

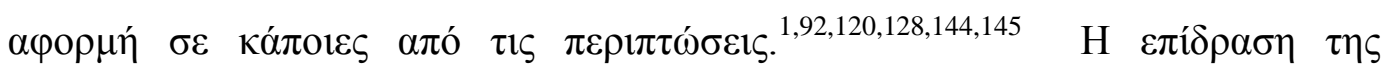

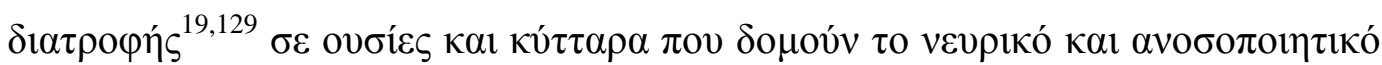

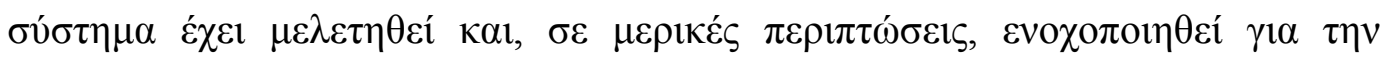

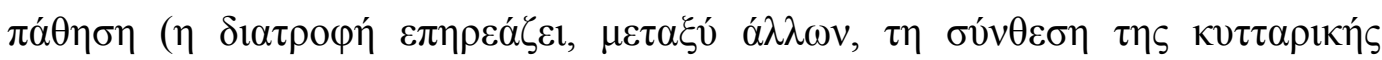

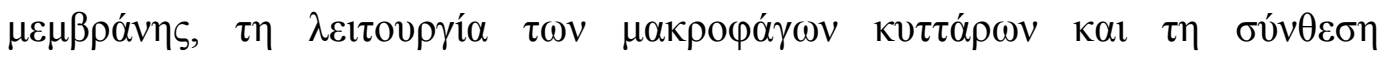

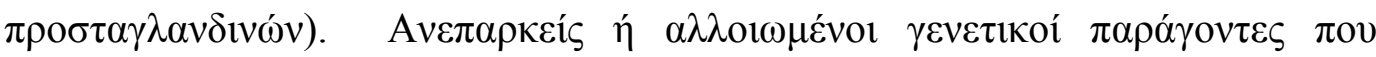

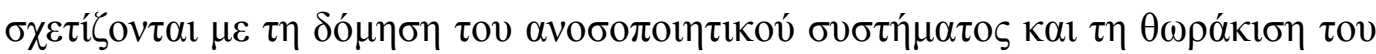

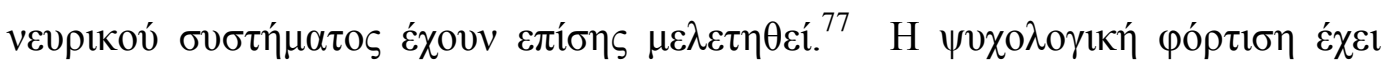

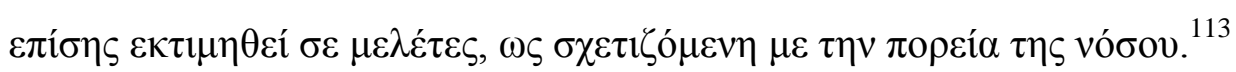

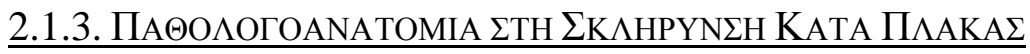

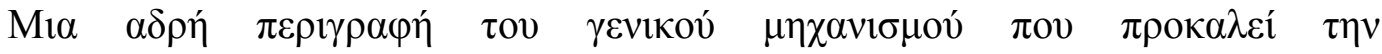

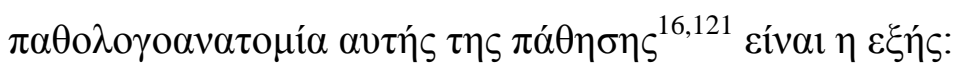

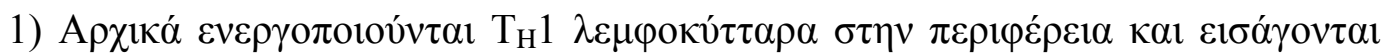

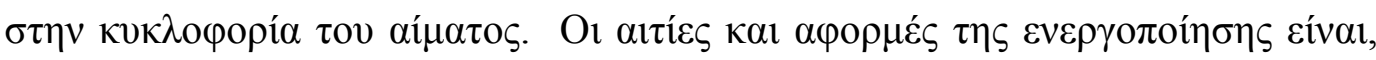
о́ $\pi \omega \varsigma \pi \rho о \alpha v \alpha \varphi \varepsilon ́ \rho \theta \eta \kappa \varepsilon, \pi \circ \lambda \lambda \varepsilon \dot{\varepsilon}$.

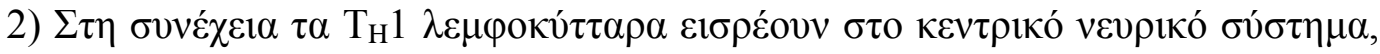

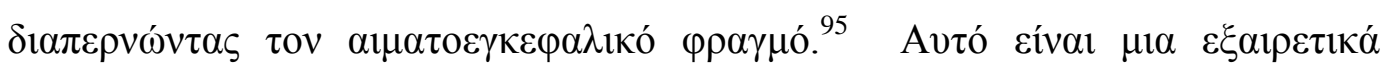




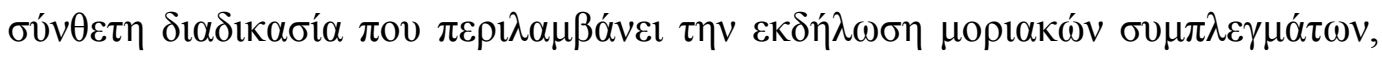

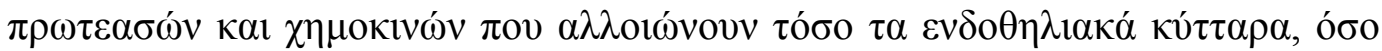

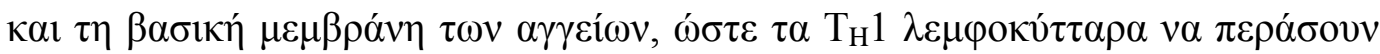

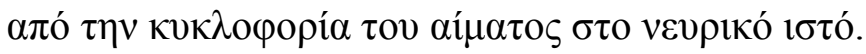

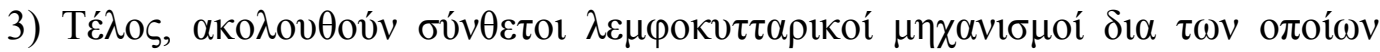

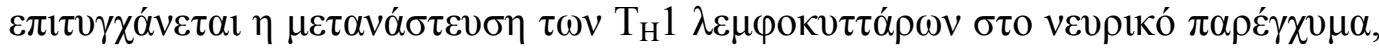

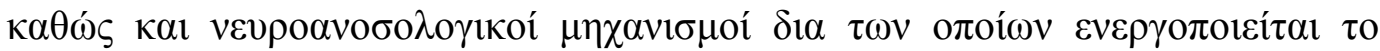

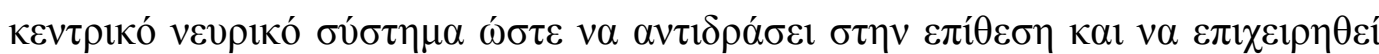
$\varepsilon \pi \alpha v \alpha \mu v \varepsilon \lambda i v \omega \sigma \eta .^{124}$

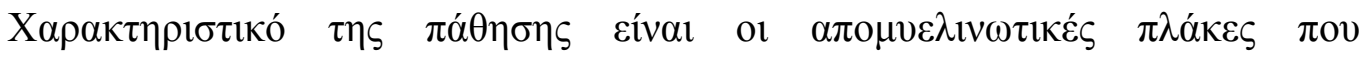

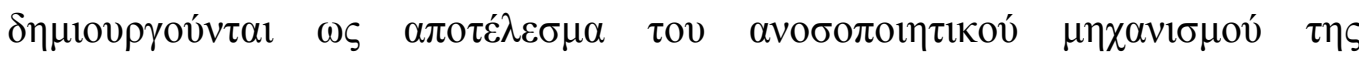

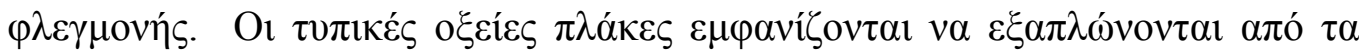

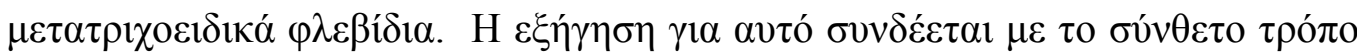

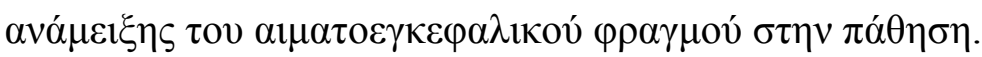

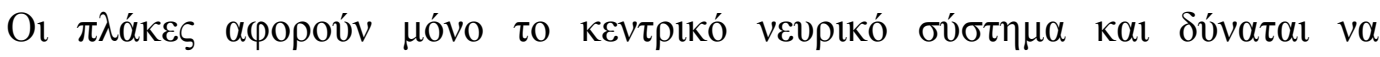

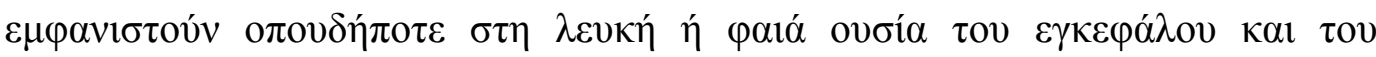

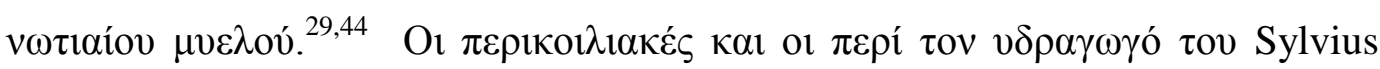

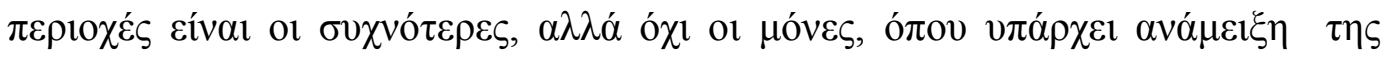

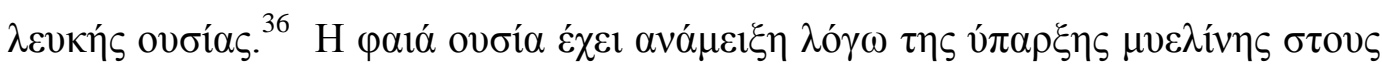

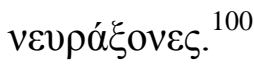

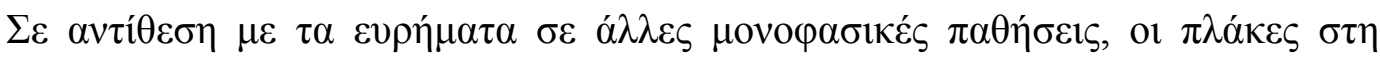

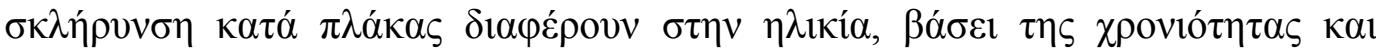

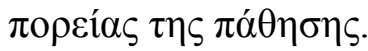

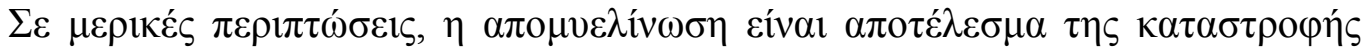

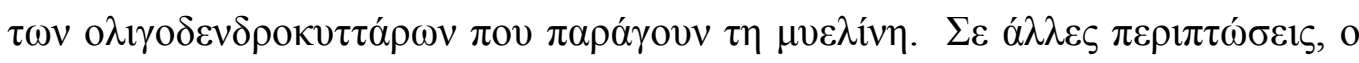

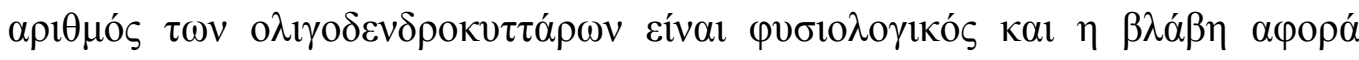

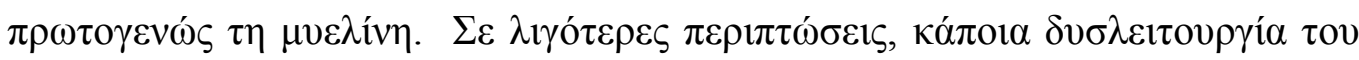

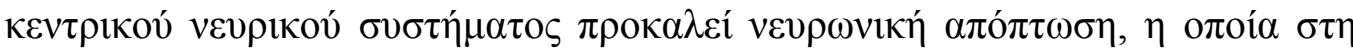

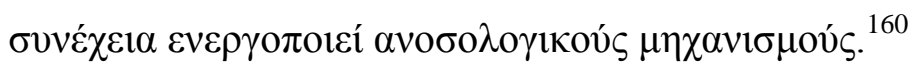




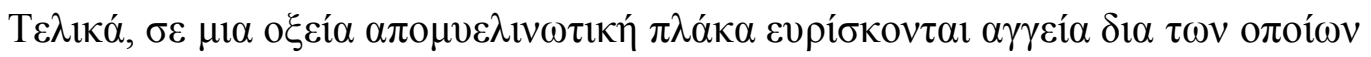

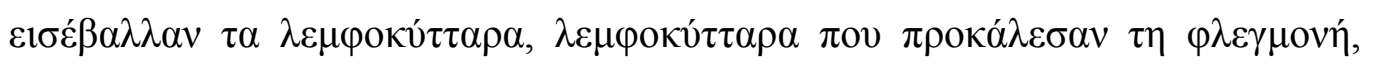

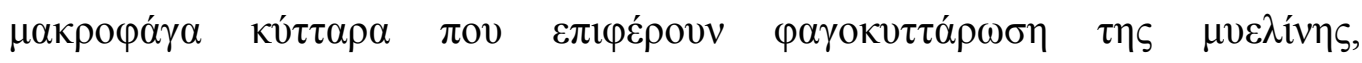

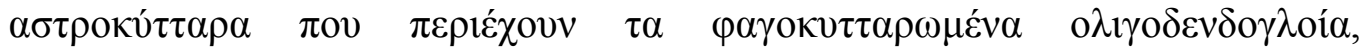

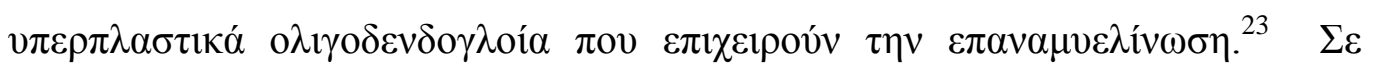

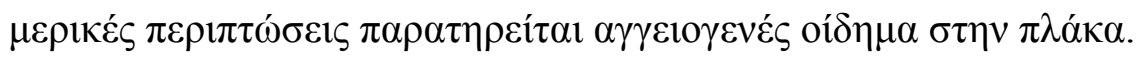

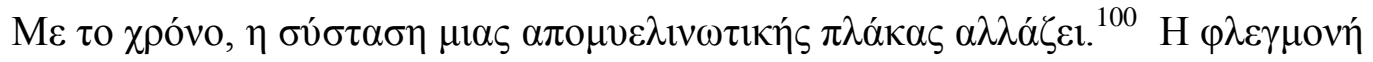

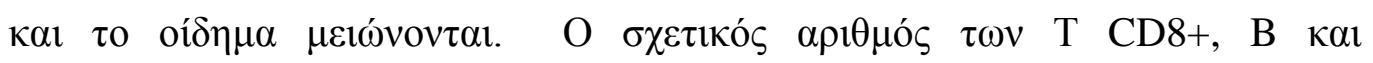

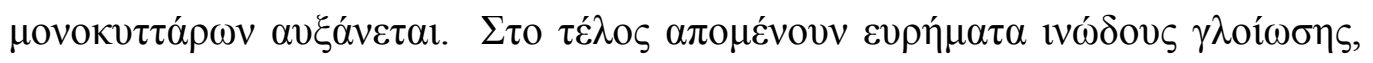

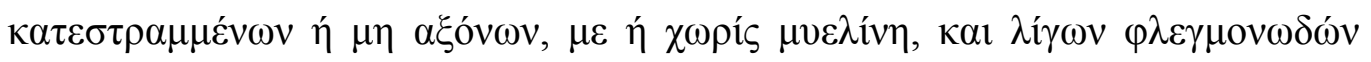

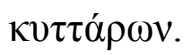

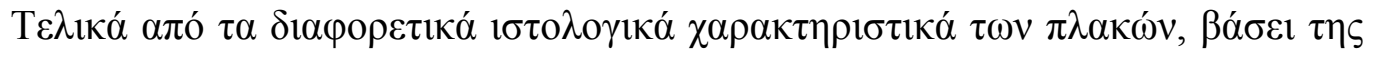

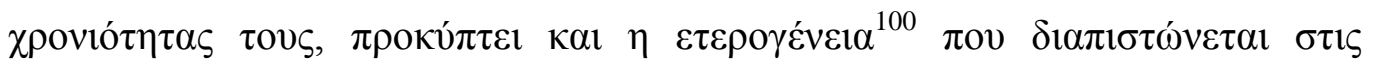

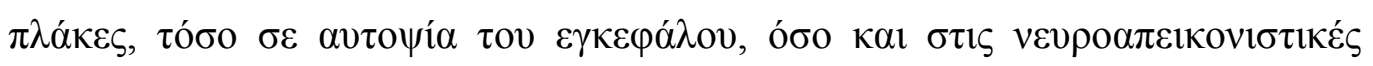

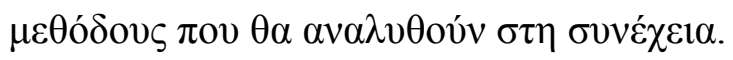

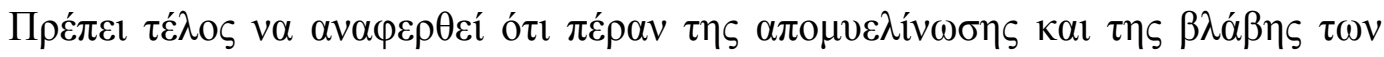

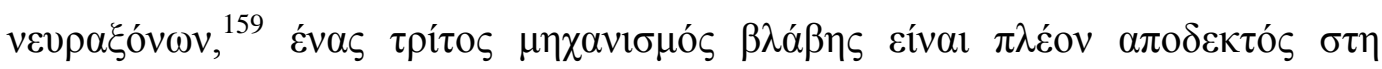

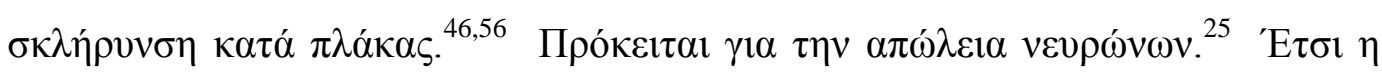

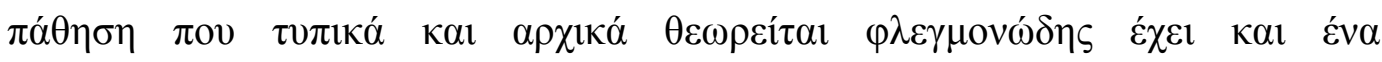

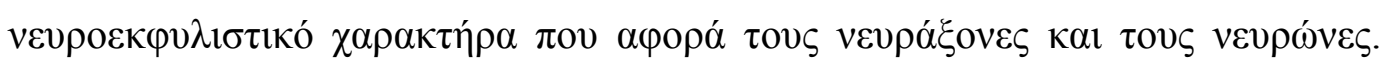

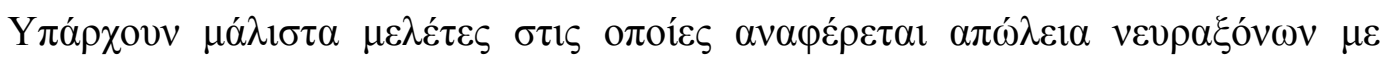

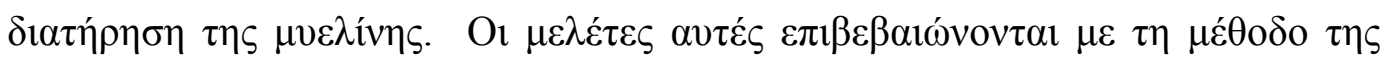

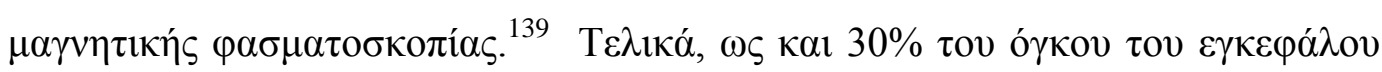

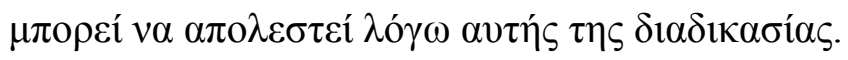




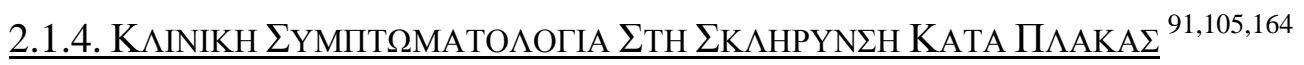

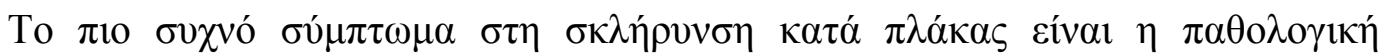

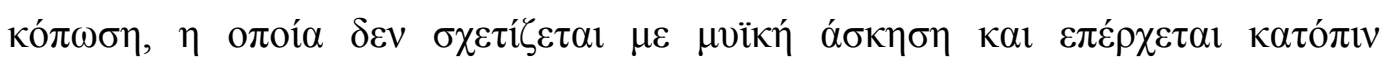

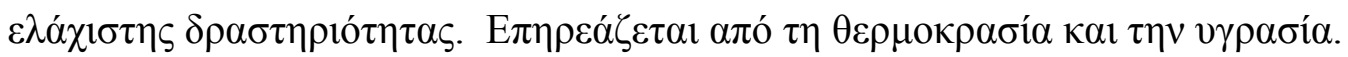

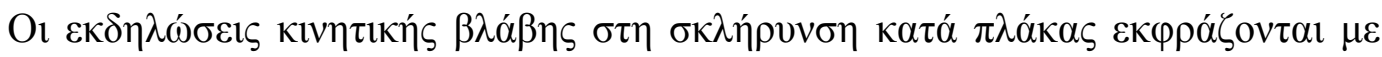

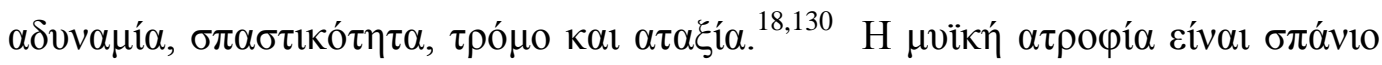

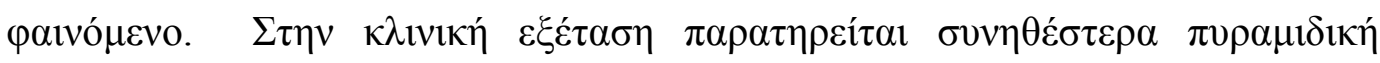

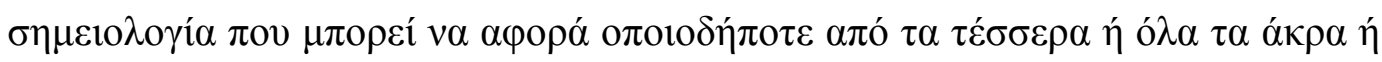

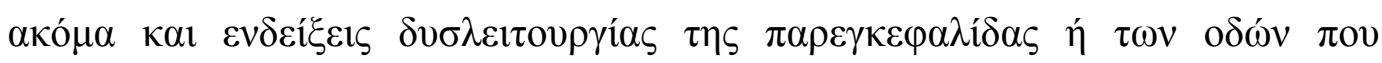

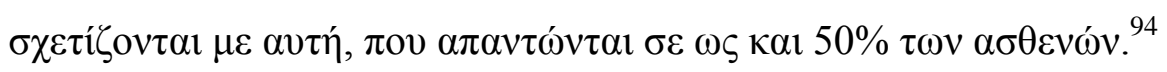

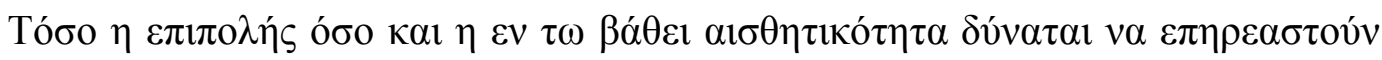

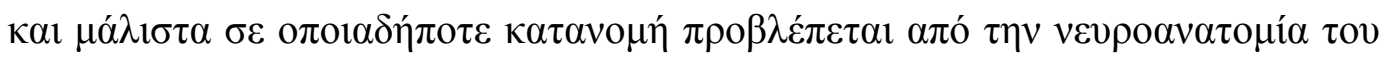

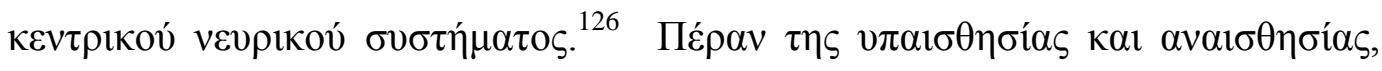

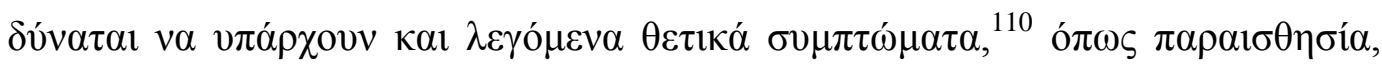

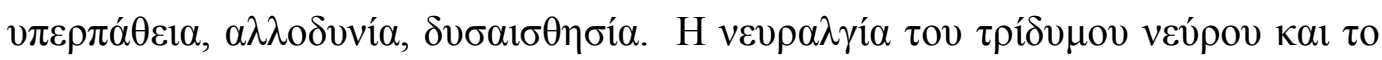

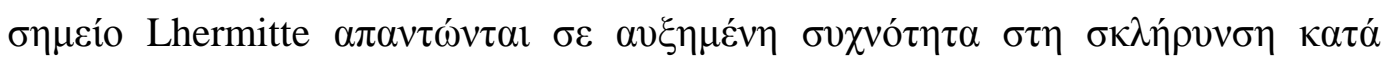

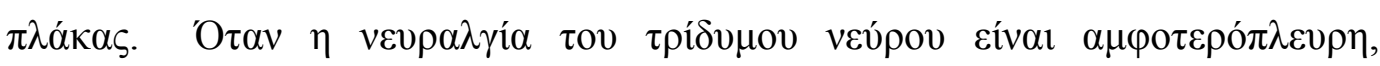

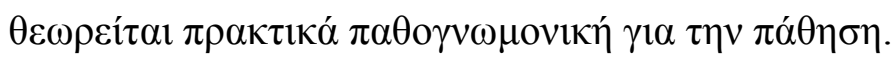

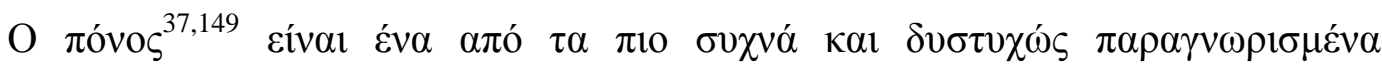

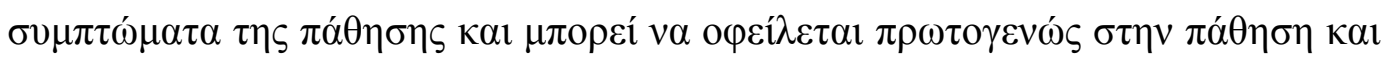

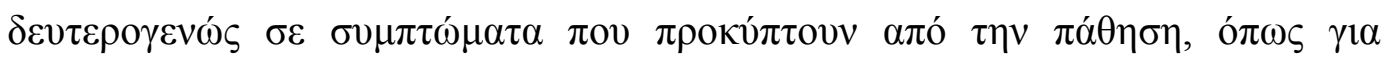

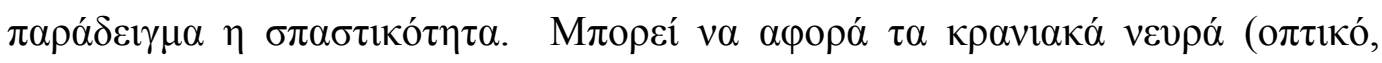

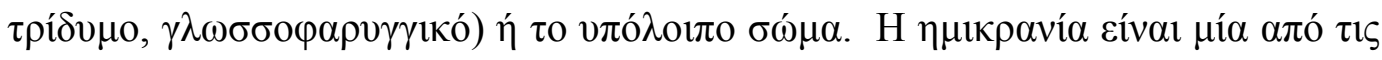

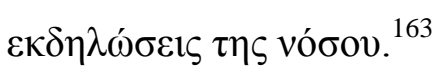

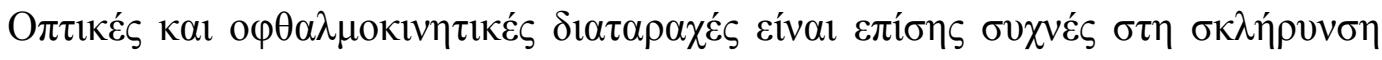

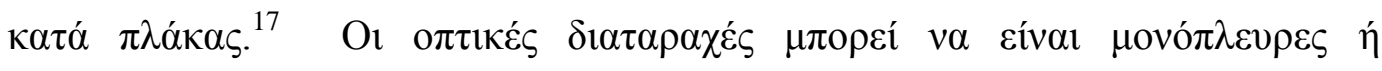

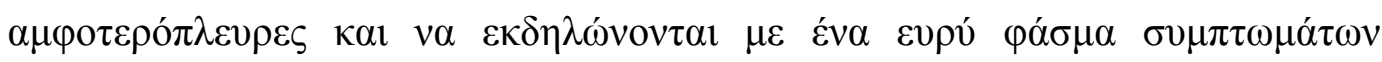

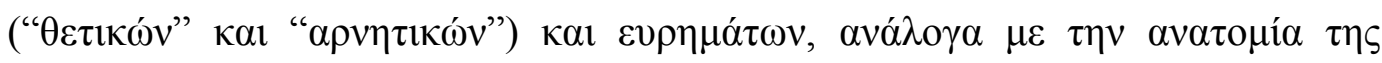

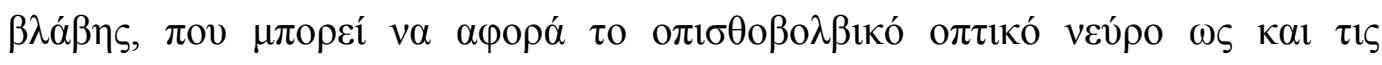

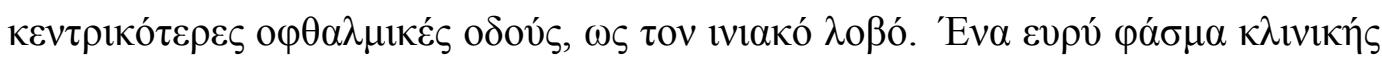




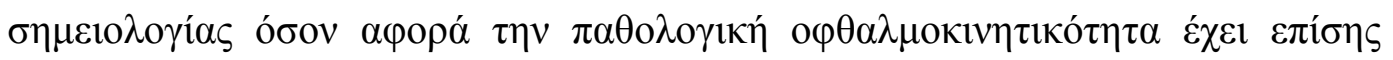

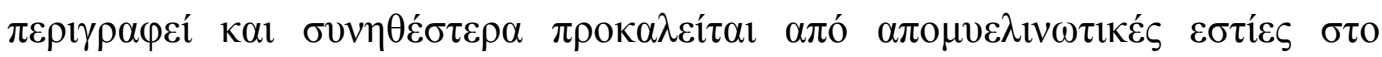

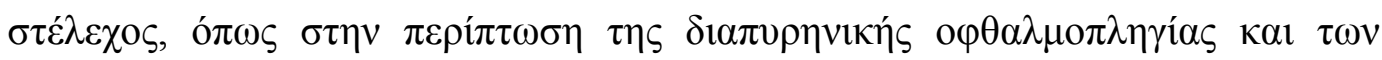

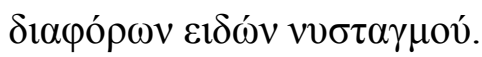

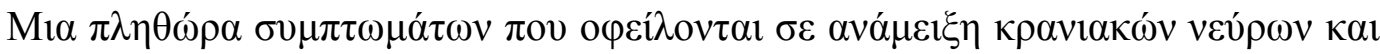

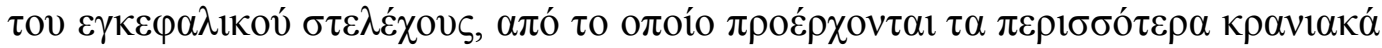

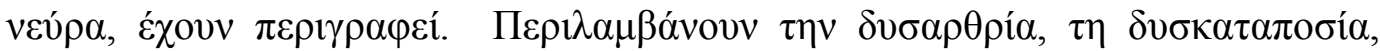

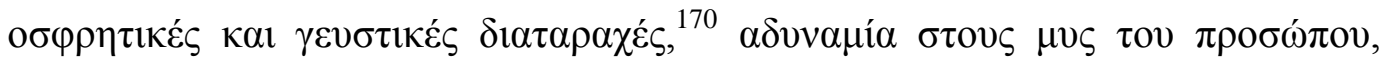

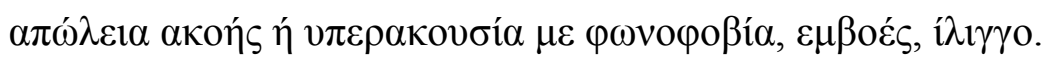

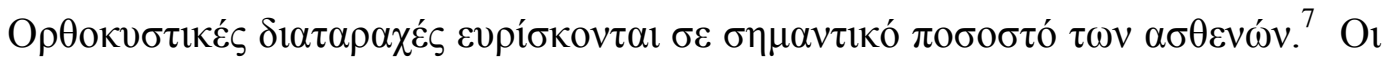

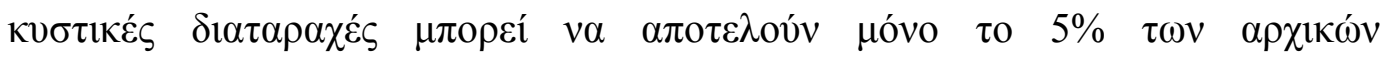

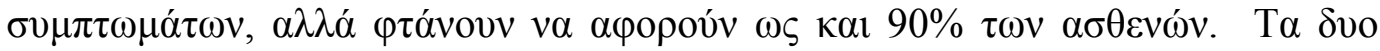

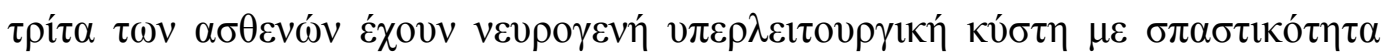

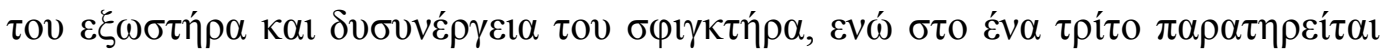

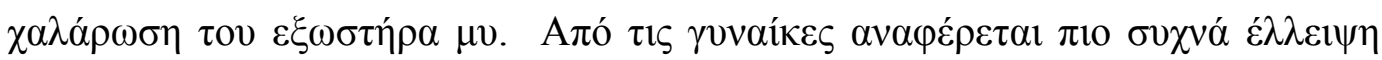

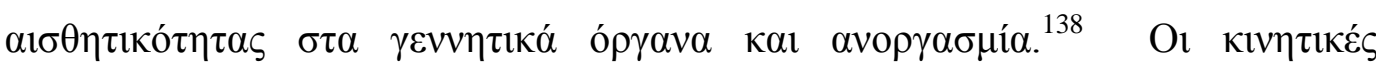

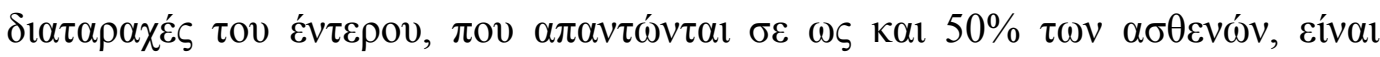

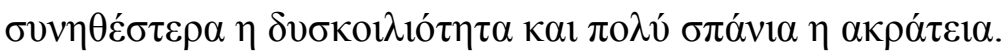

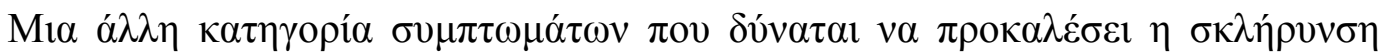

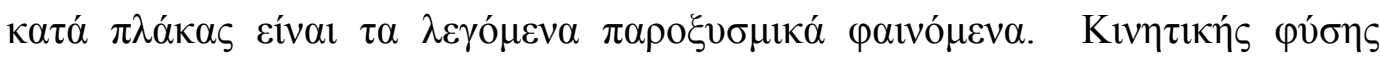

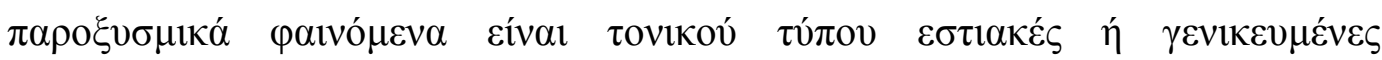

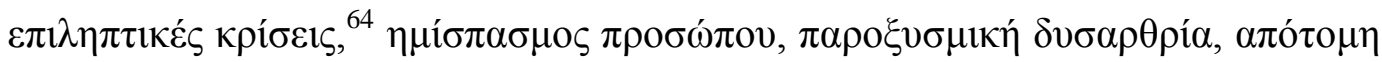

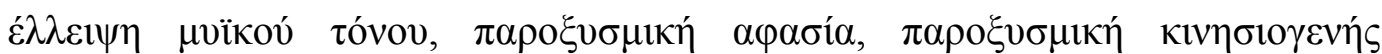

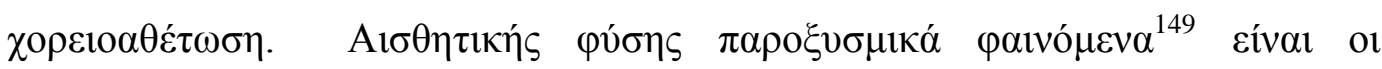

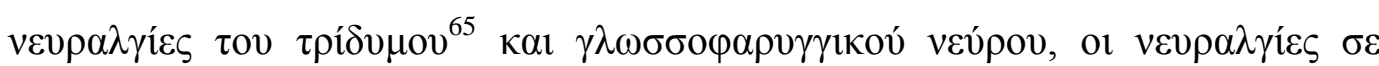

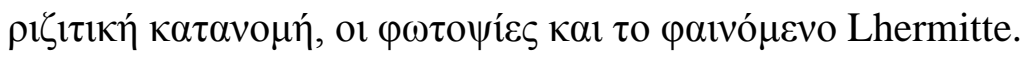

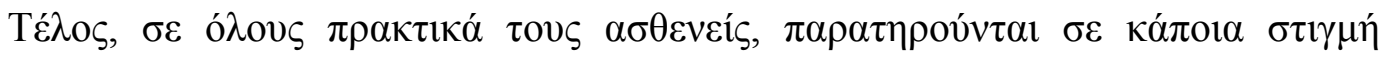

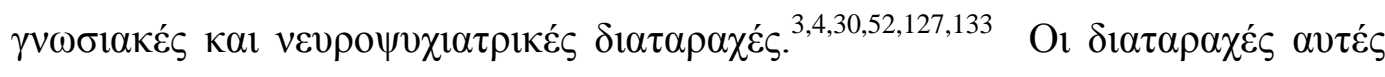

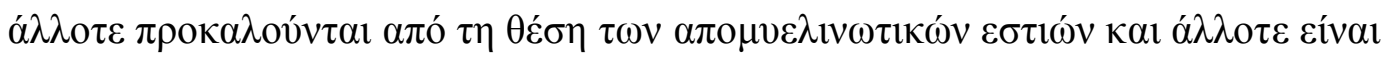

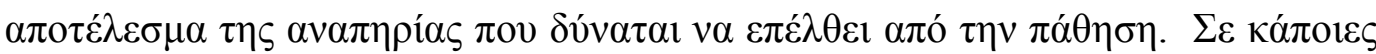




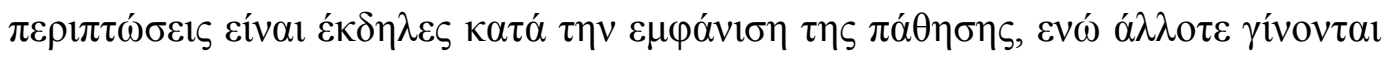

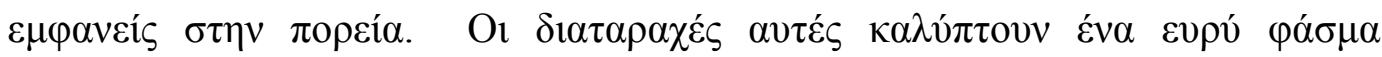

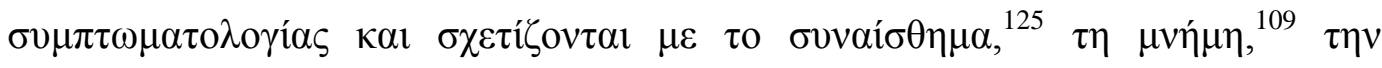

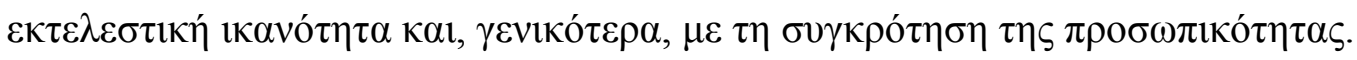

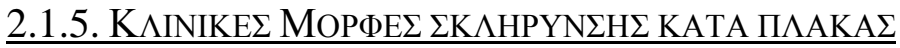

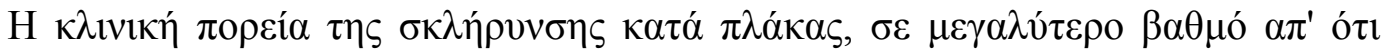

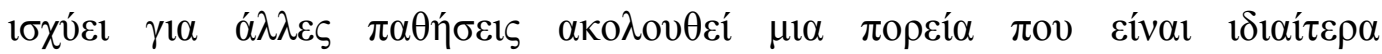

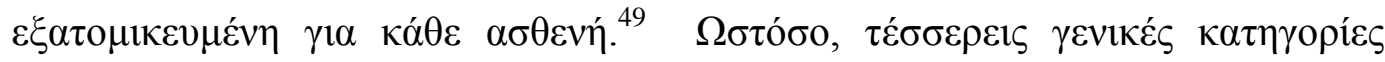

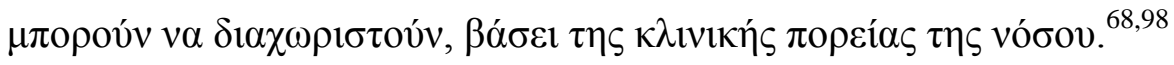

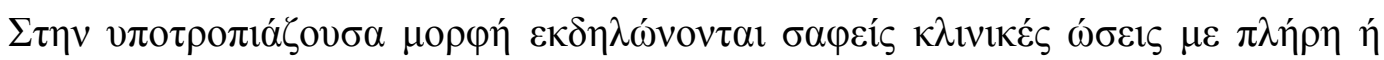

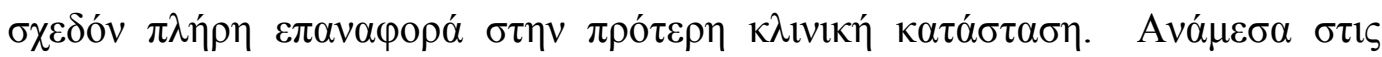

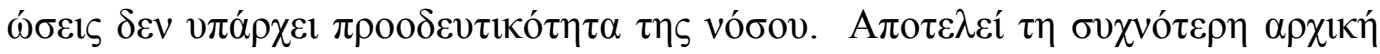

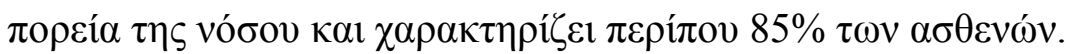

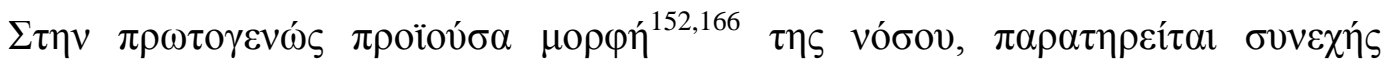

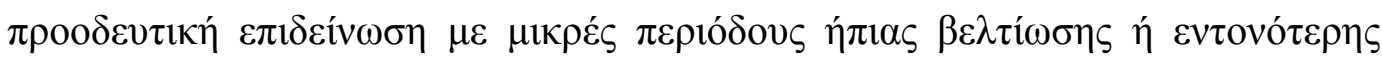

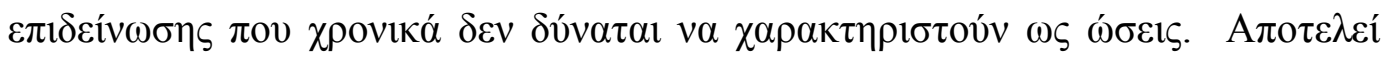

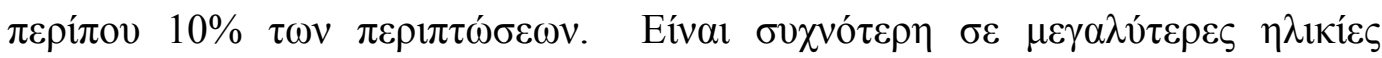

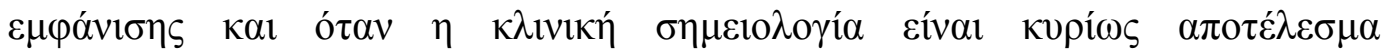

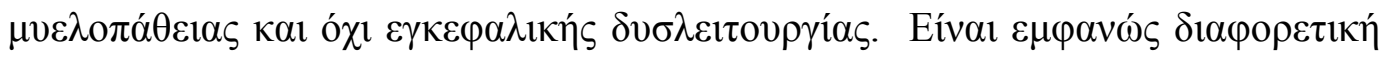

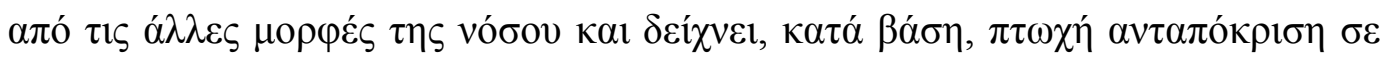

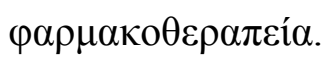

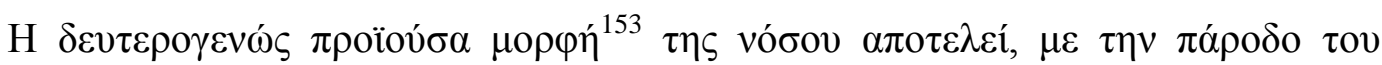

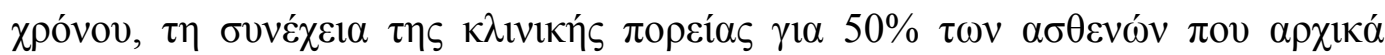

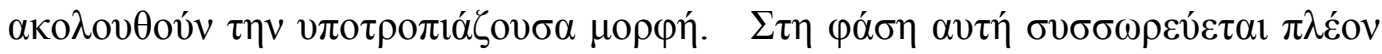
$\varepsilon \mu \varphi \alpha \nu \omega ́ \varsigma \alpha \nu \alpha \pi \eta \rho i ́ \alpha .{ }^{40}$

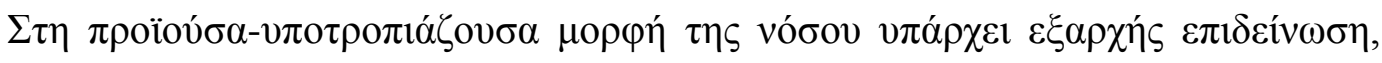
$\pi \mathrm{ov} \varepsilon \kappa \delta \eta \lambda \omega ́ v \varepsilon \tau \alpha$

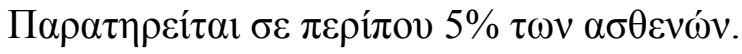




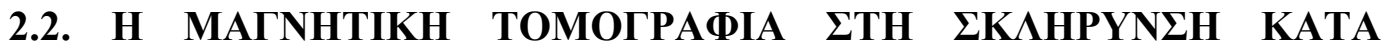

\section{П $\Lambda \mathbf{A K A \Sigma}$}

\subsection{1. ГENIKA}

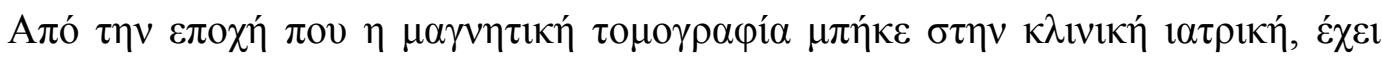

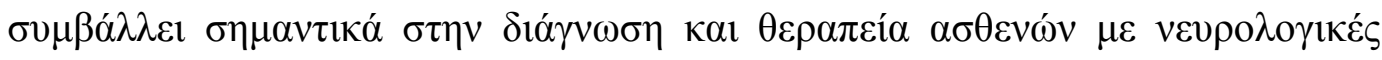

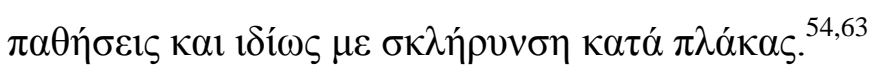

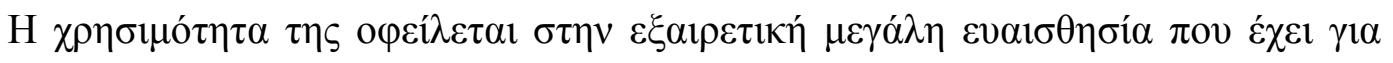

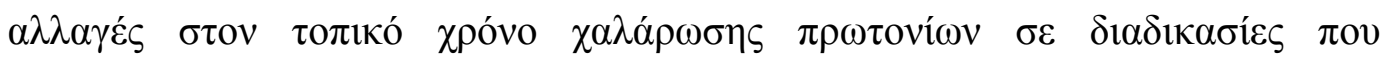

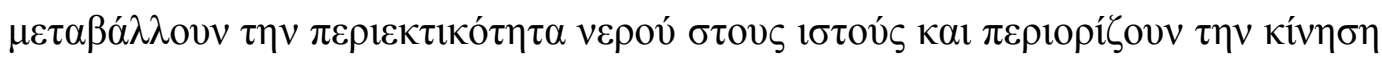

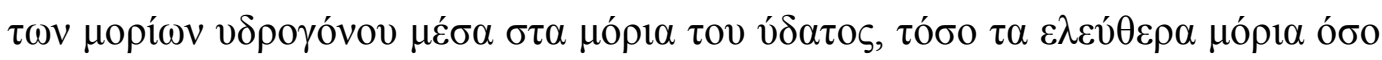

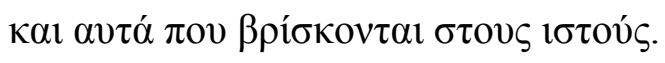

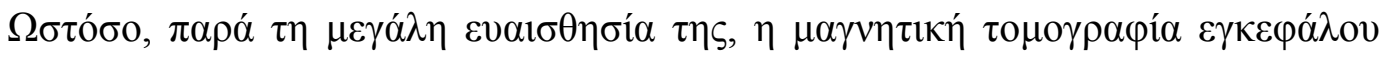

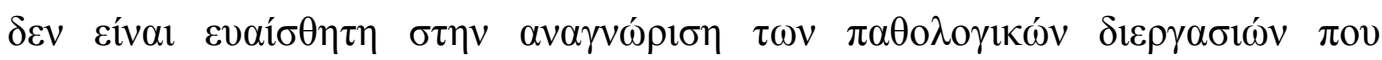

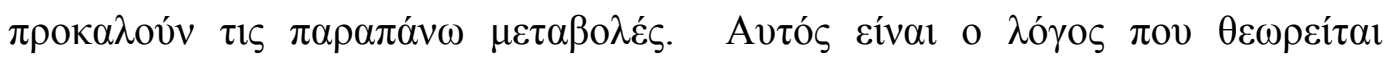

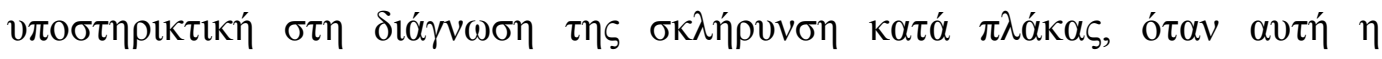

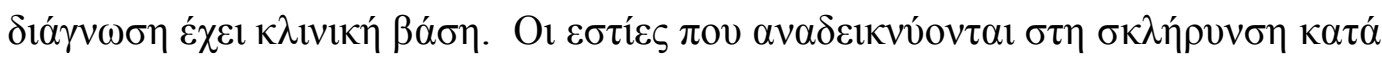

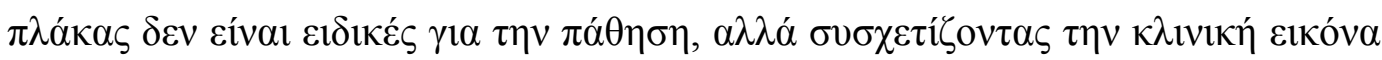

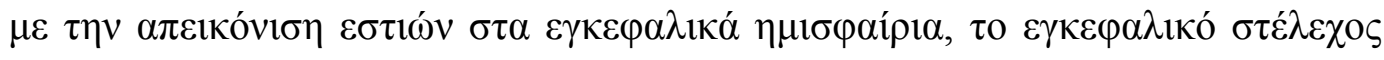

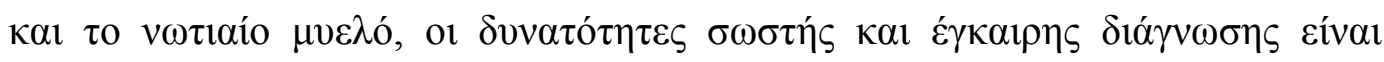
$\alpha v \xi \eta \mu \varepsilon \dot{v \varepsilon \varsigma . ~}$

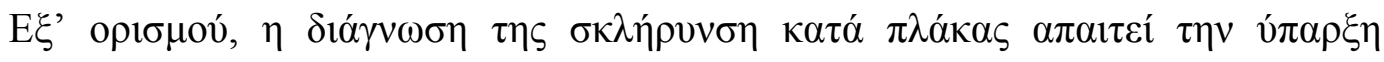

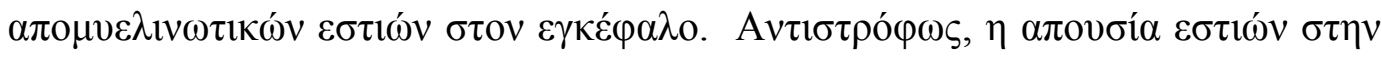

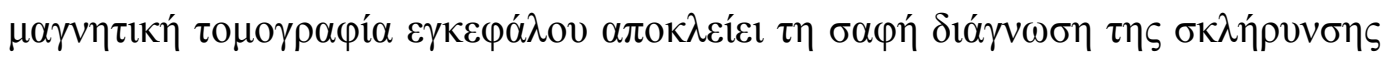

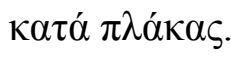

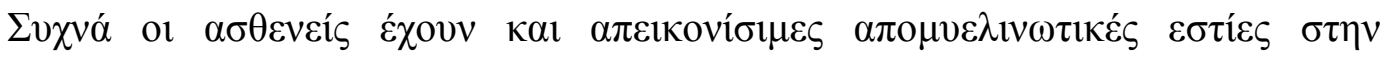

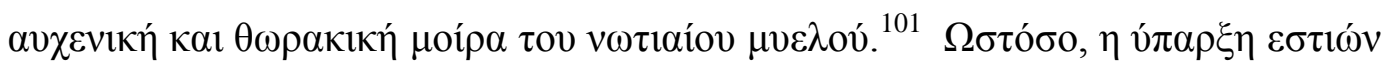

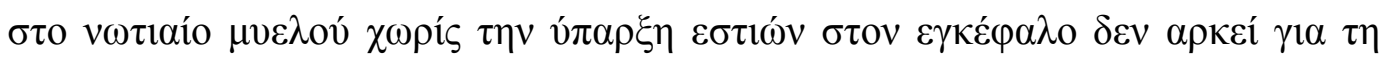

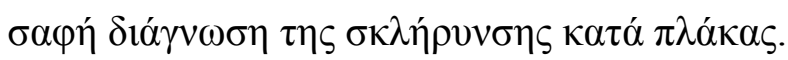




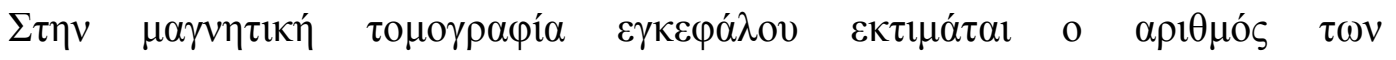

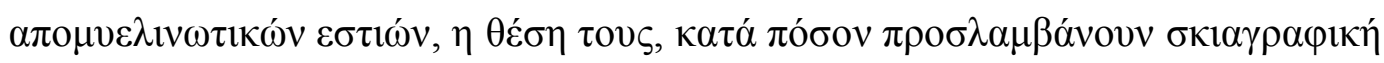

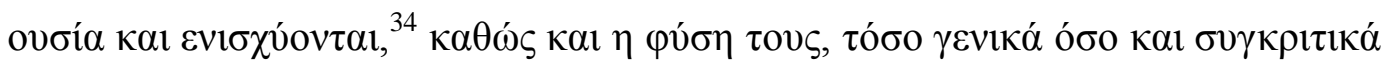

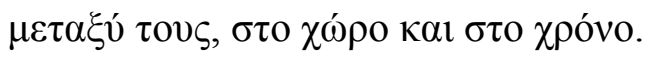

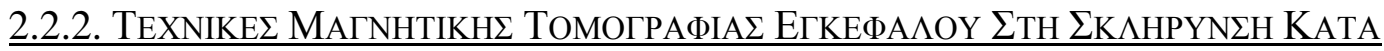

\section{$\underline{\Pi \Lambda \mathrm{AKA \Sigma}}$}

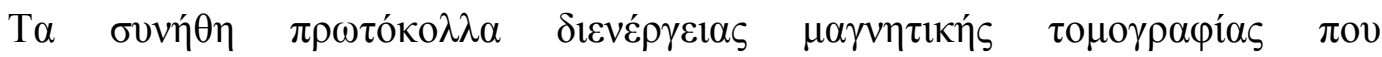

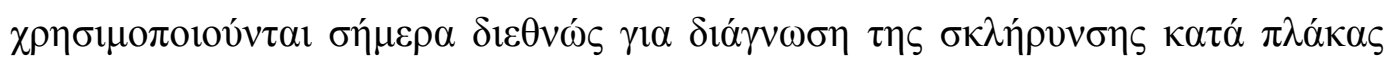

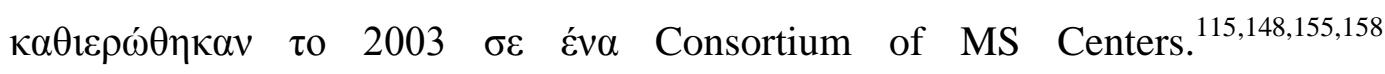

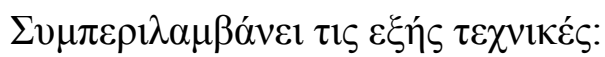

Sagittal FLAIR (Fluid-Attenuating Inversion Recovery)

Axial dual echo proton density

T2-weighted axial FLAIR

Axial Gadolinium T1 series

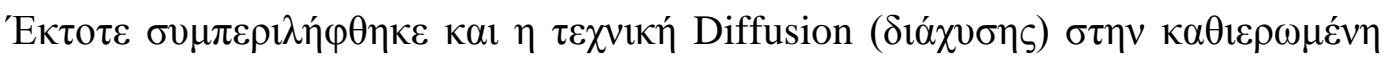

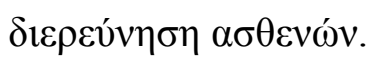

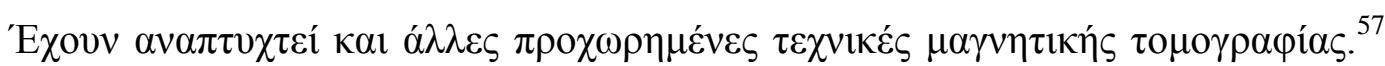
$\Sigma v \mu \pi \varepsilon \rho i \lambda \alpha \mu \beta \alpha ́ v o v v \tau \eta$ magnetization transfer ratio (MTR) imaging, ${ }^{157} \tau \eta$ dual

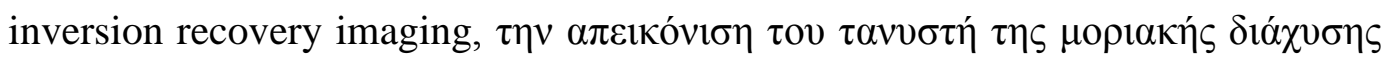
(diffusion tensor imaging, fractional anisotropy, tractography), ${ }^{35,70} \tau \eta \mu \alpha \gamma v \eta \tau \iota \kappa \eta ́$ фабнатобколі́ dimensional chemical shift imaging), $\tau \eta \lambda \varepsilon\left\llcorner\tau o v \rho \gamma ı \eta^{\prime}\right.$ (functional) MRI $^{59} \kappa \alpha \iota$

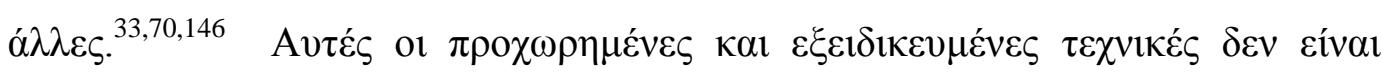

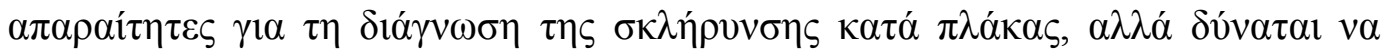

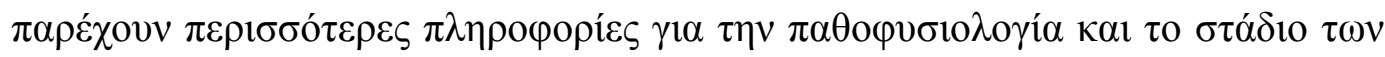

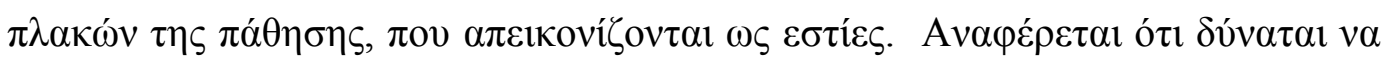

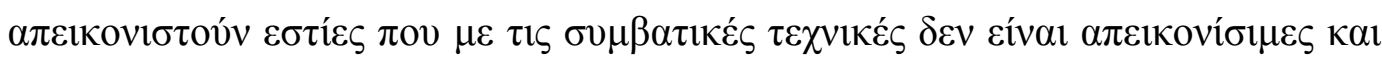

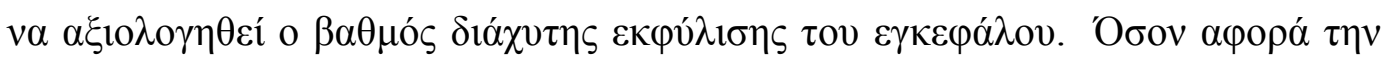

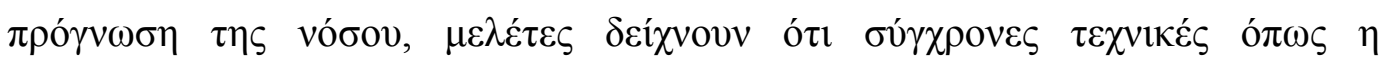

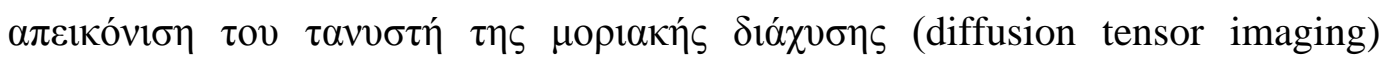




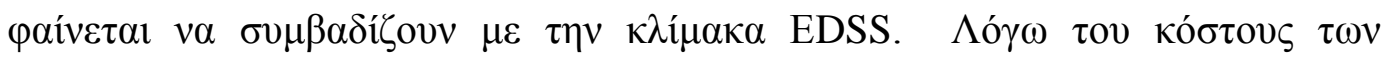

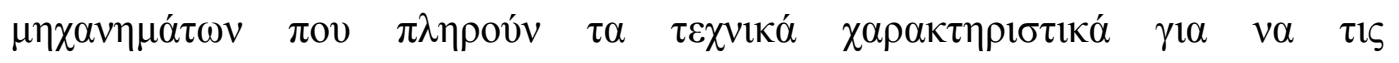

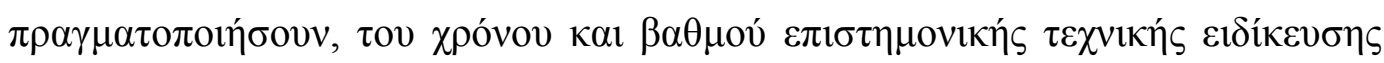

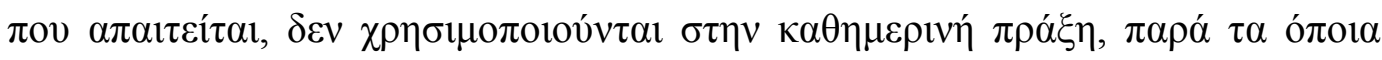

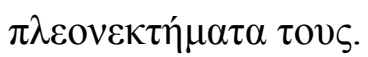

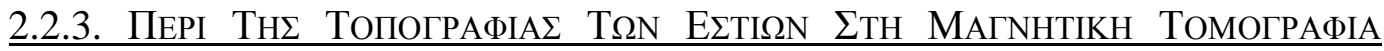

\section{$\underline{\text { ЕГКЕФА } \Lambda \mathrm{OY}}$}

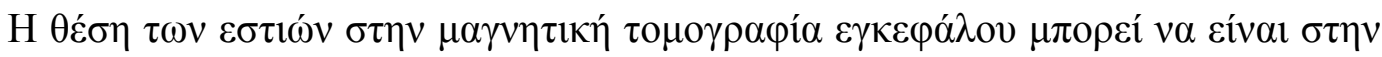

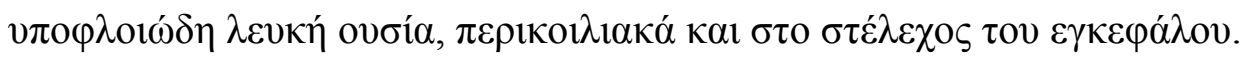

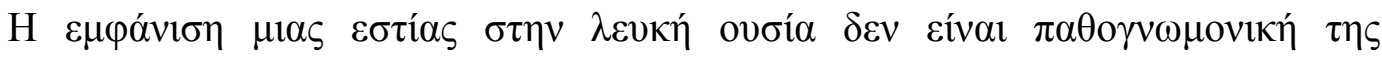

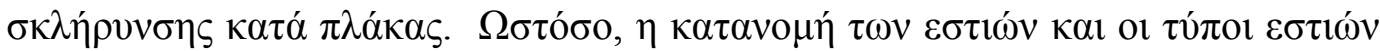

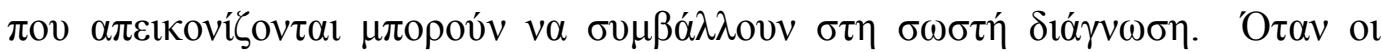

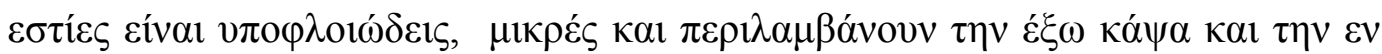

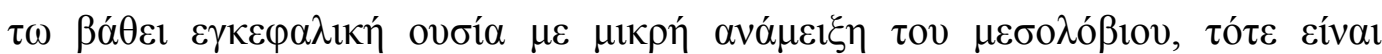

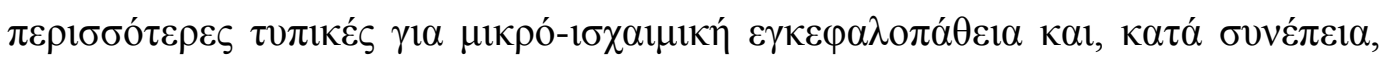

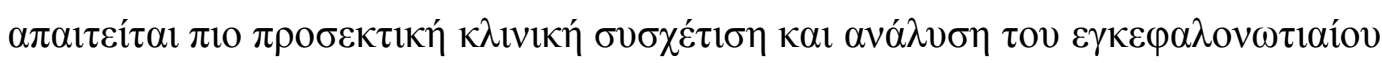
vүроv́.

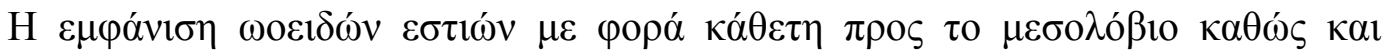

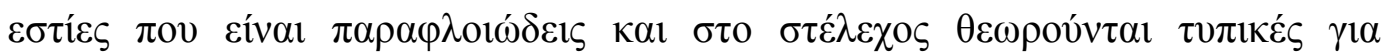

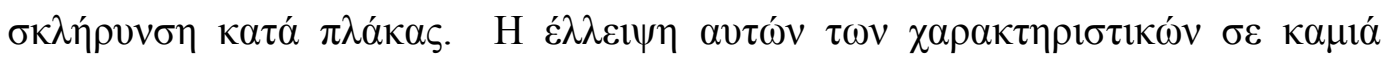

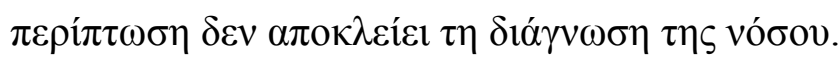

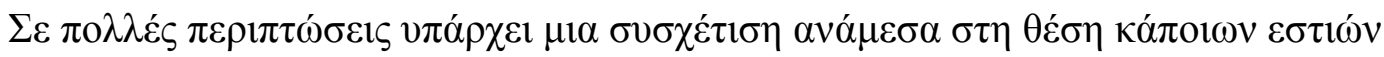

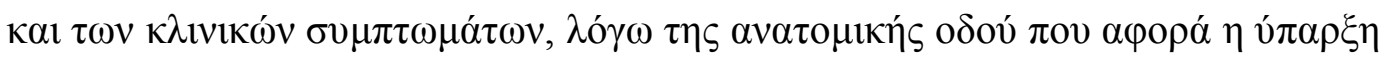

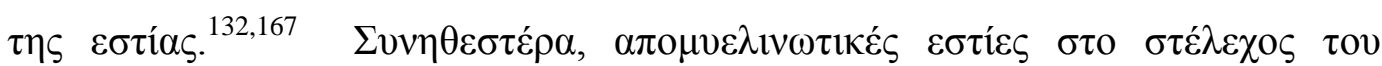

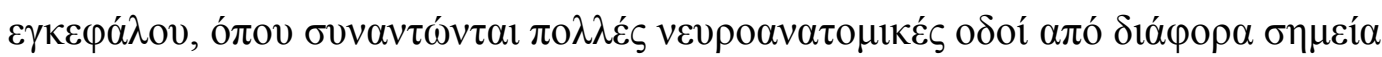

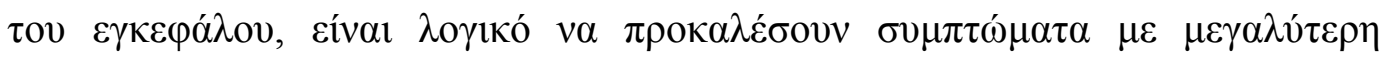

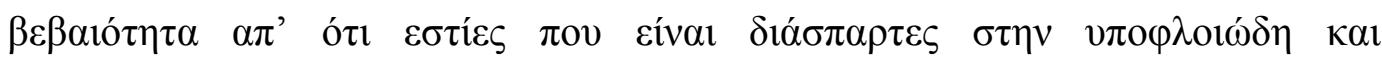

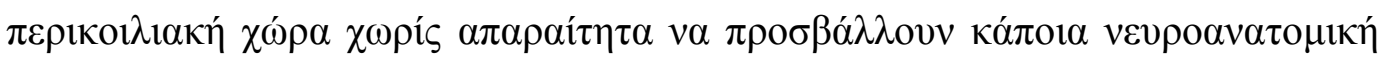

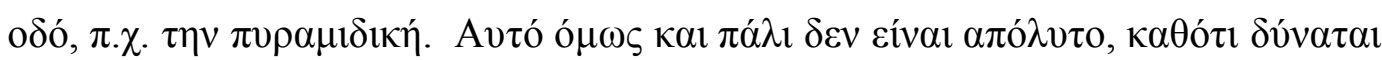

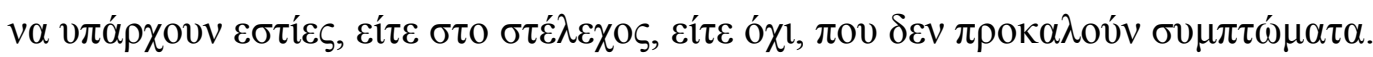




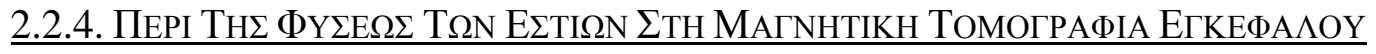

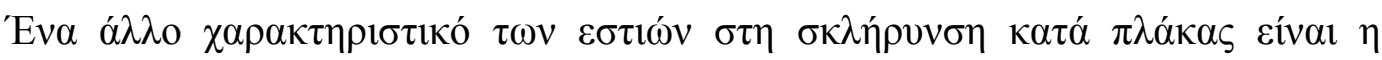

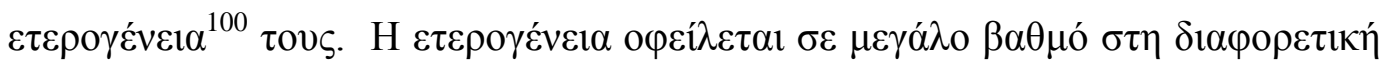

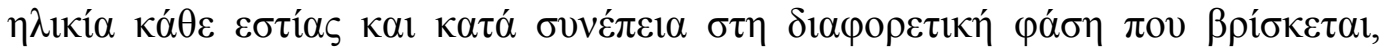

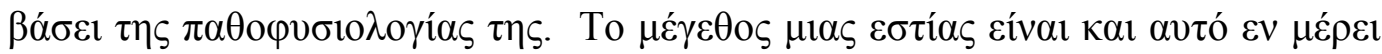

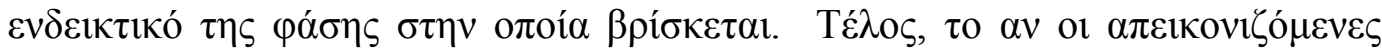

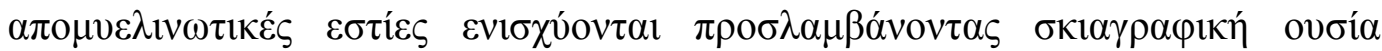

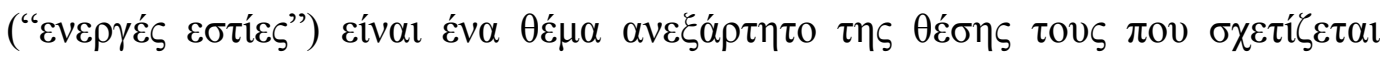

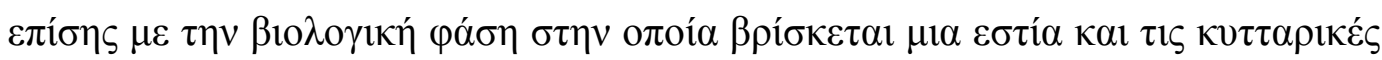

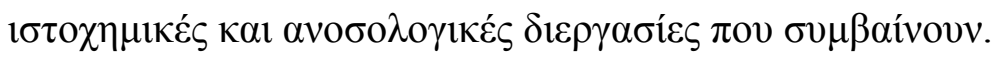

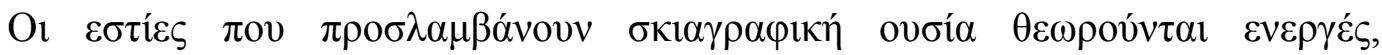

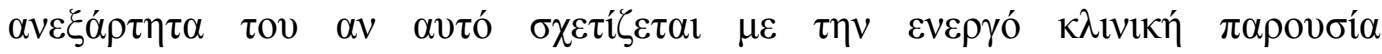

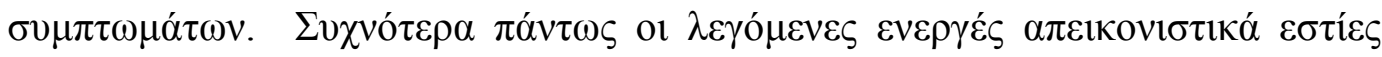

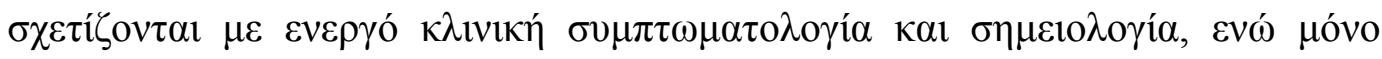

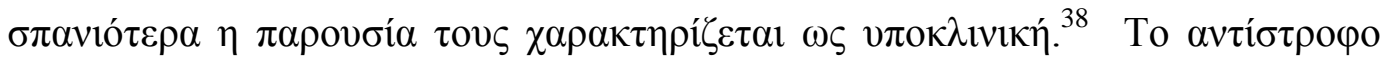

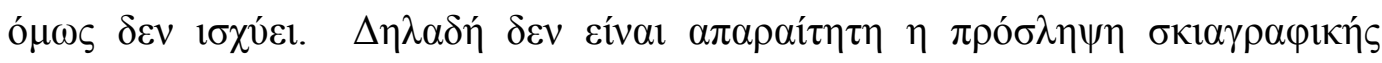

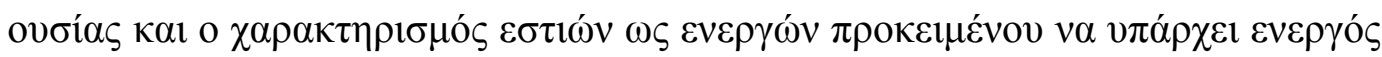

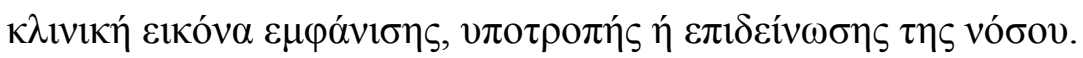

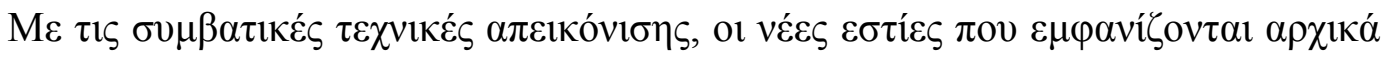

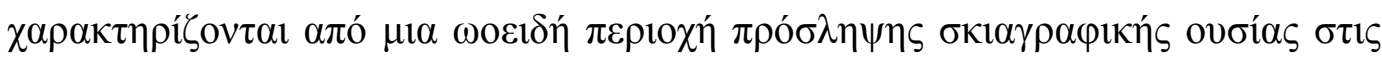

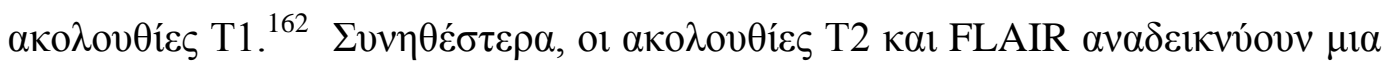

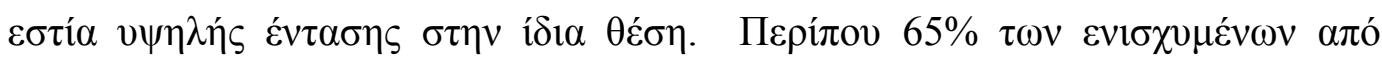

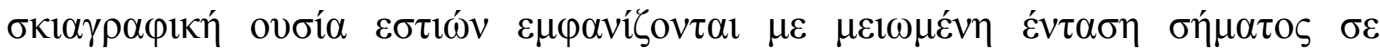

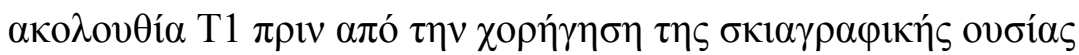

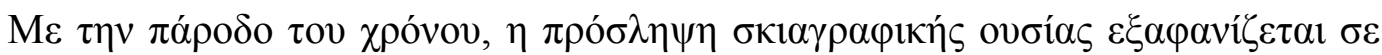

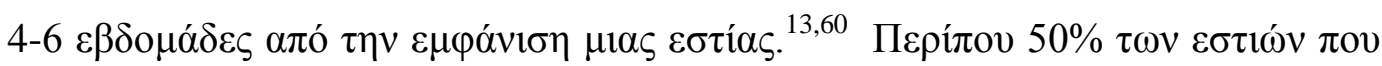

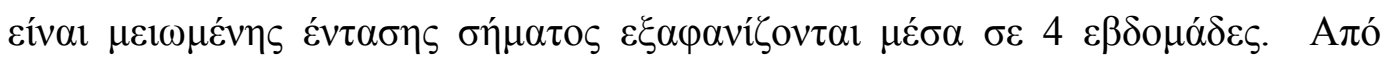

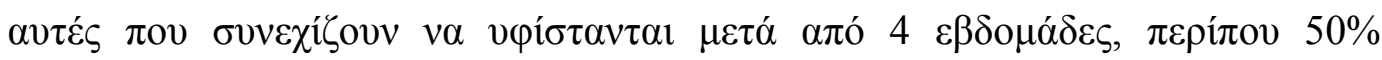

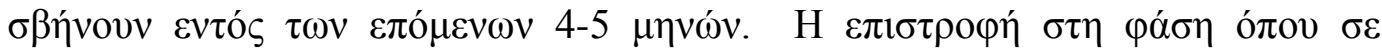

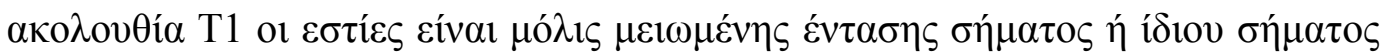




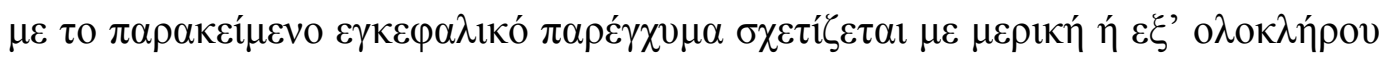
$\varepsilon \pi \alpha v \alpha \mu v \varepsilon \lambda i v \omega \sigma \eta{ }^{12}$

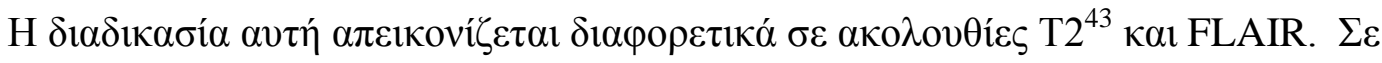

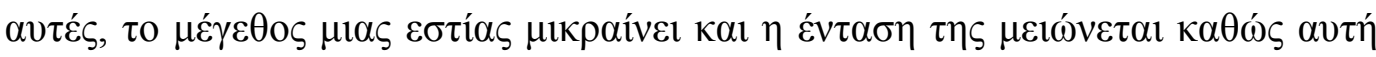

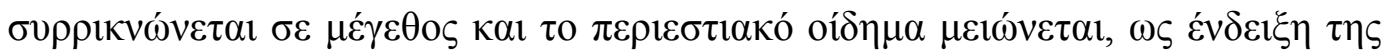

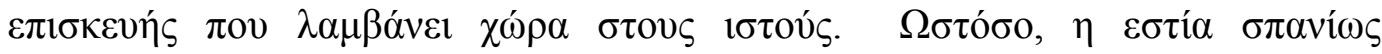

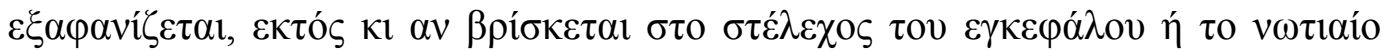
$\mu v \varepsilon \lambda$ ó $^{-}$

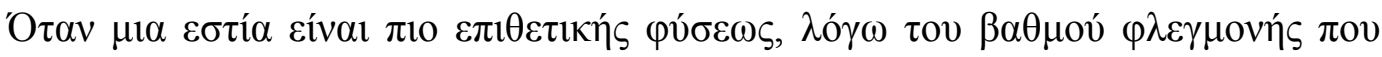

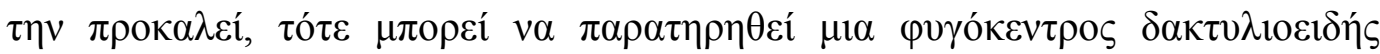

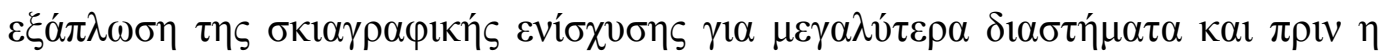

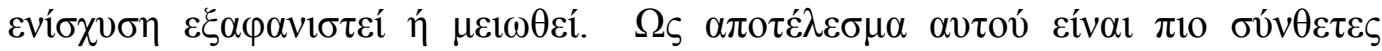

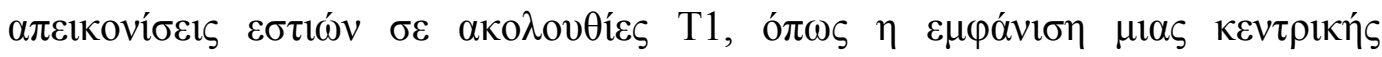

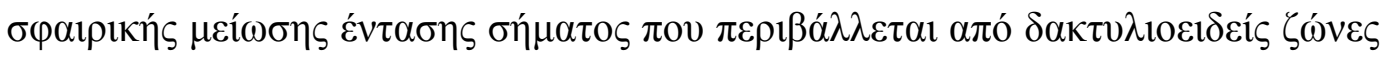

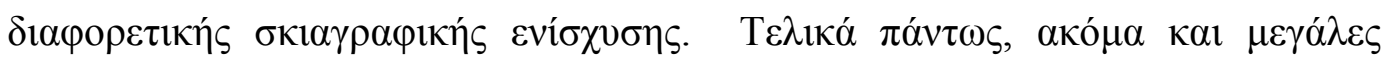

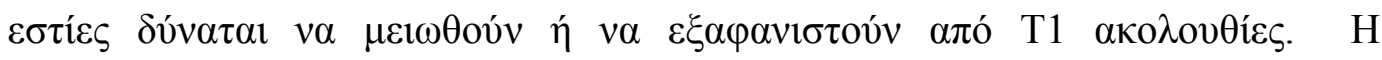

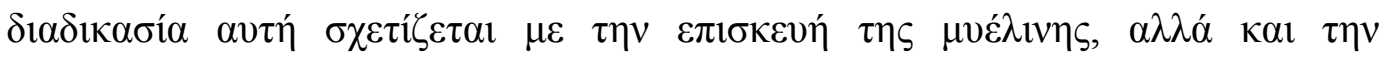

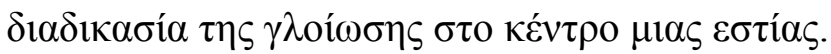

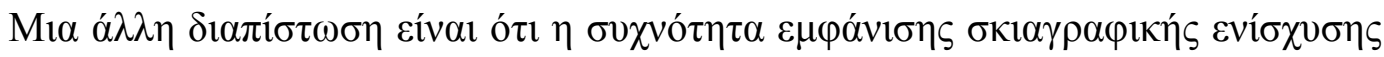

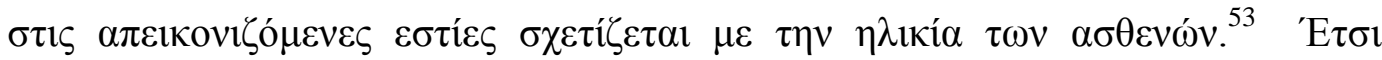

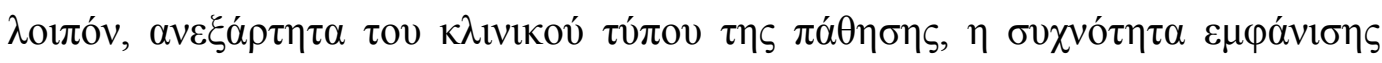

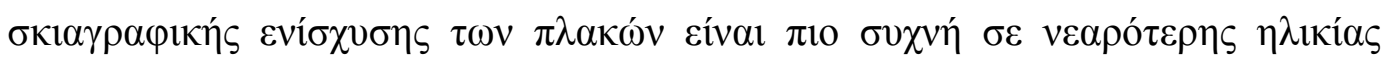

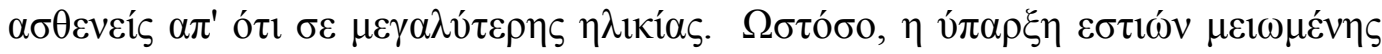

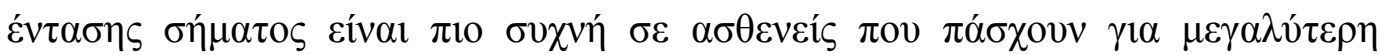

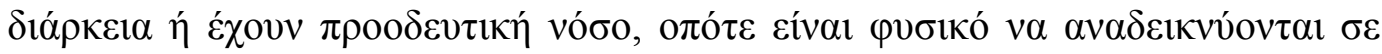

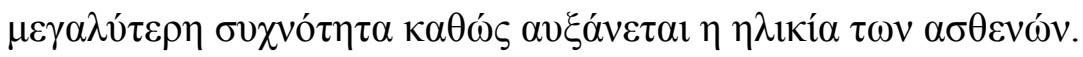

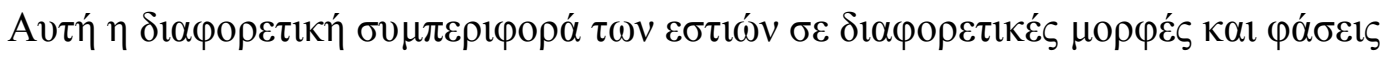

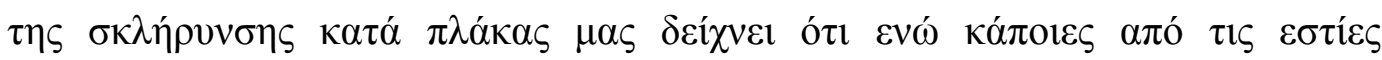

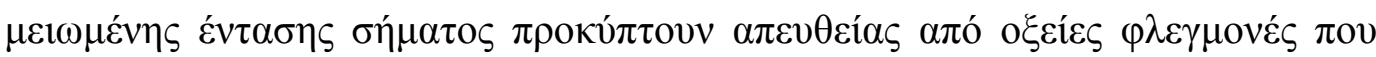

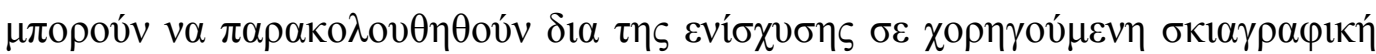




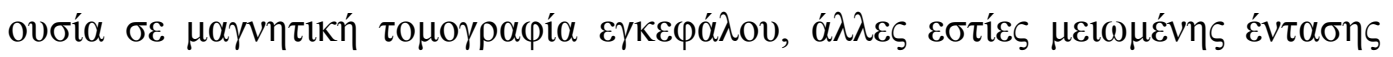

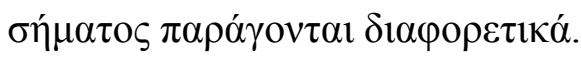

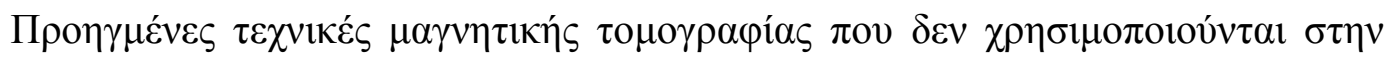

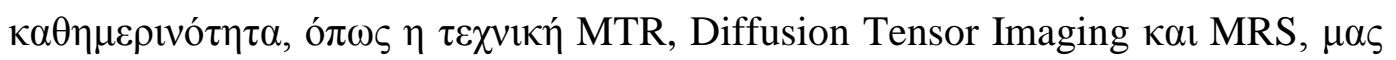

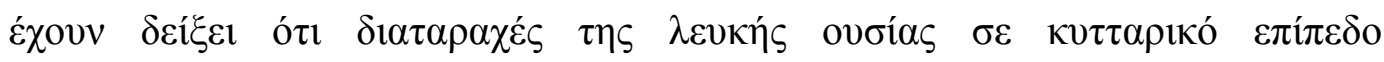

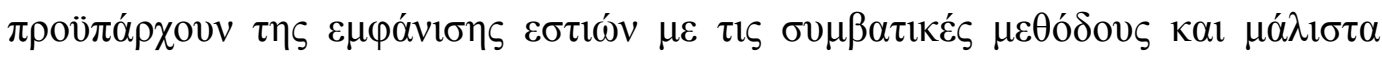

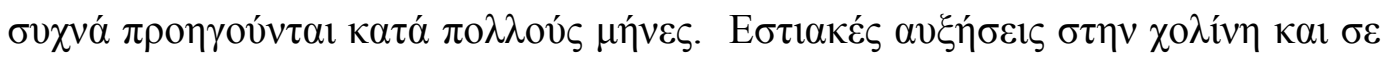

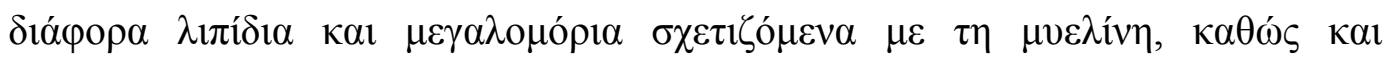

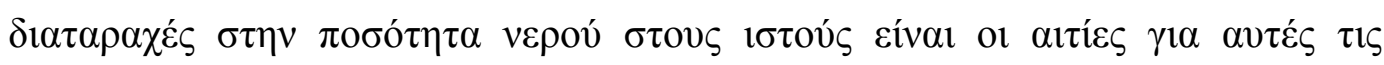

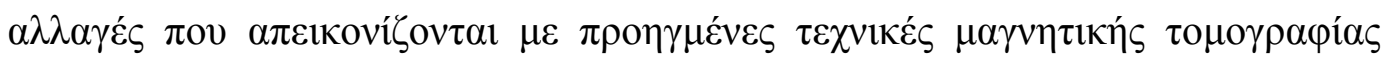

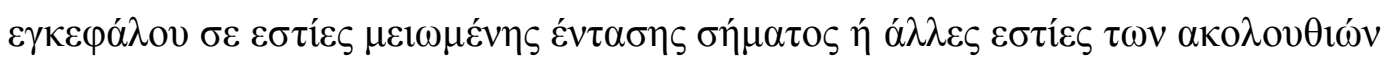
T1, T2, FLAIR.

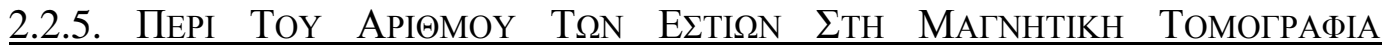
ЕГКЕФА ОY

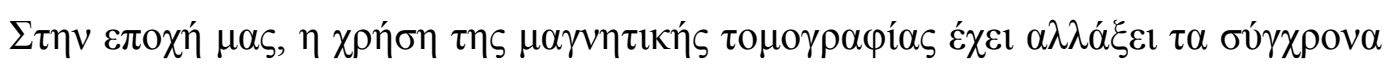

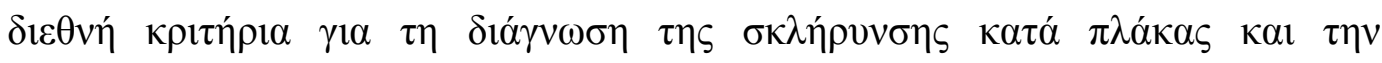

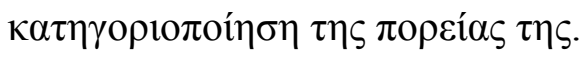

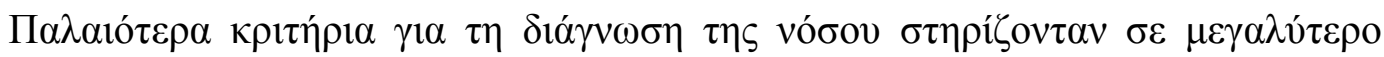

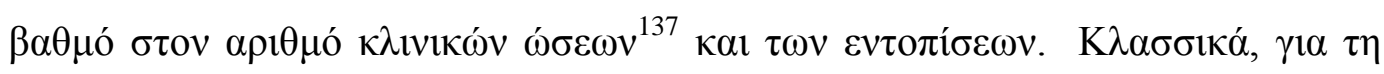

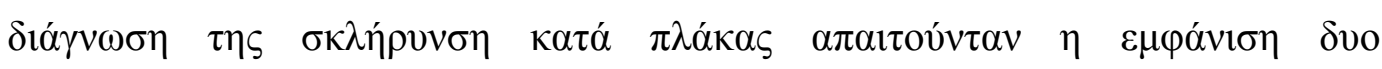

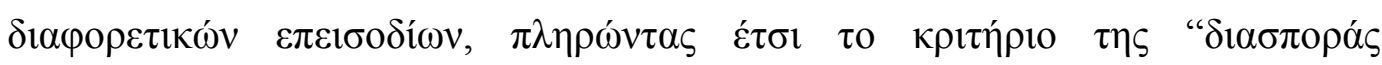

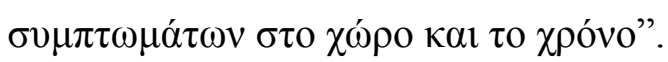

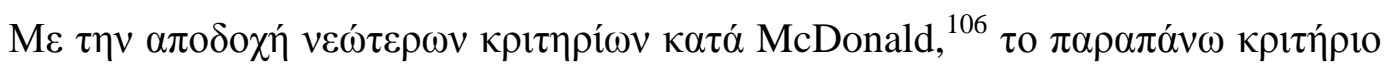

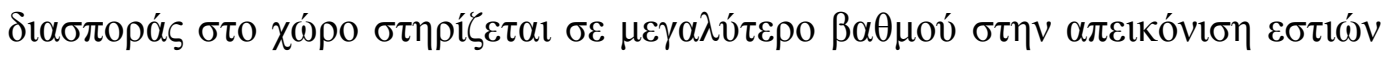

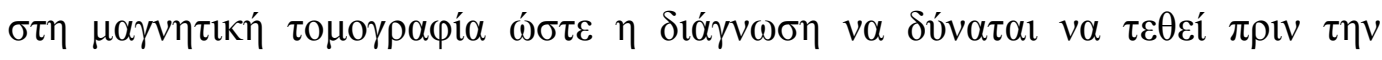

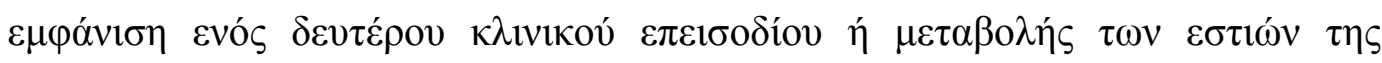

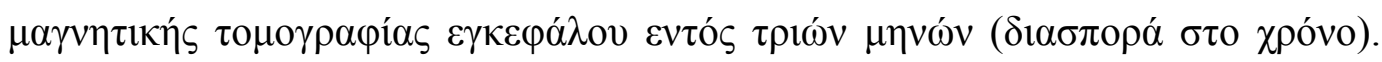

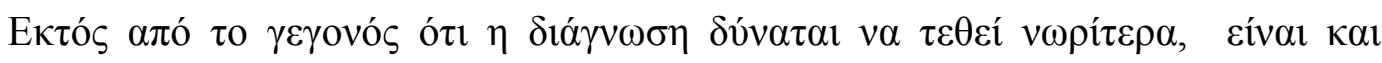

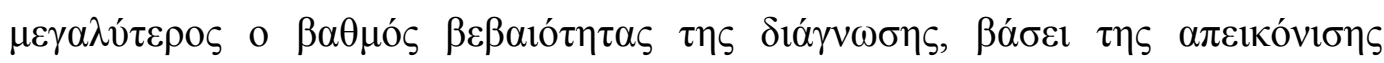




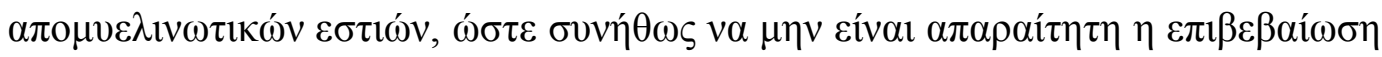

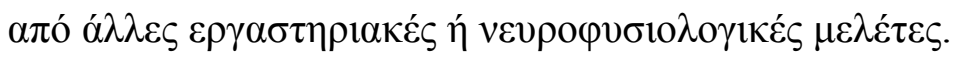

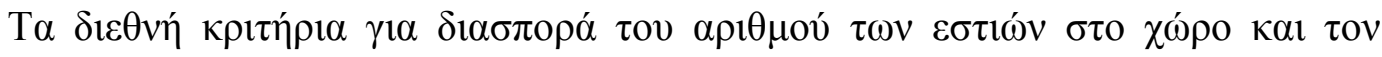

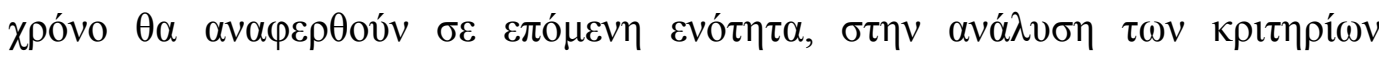

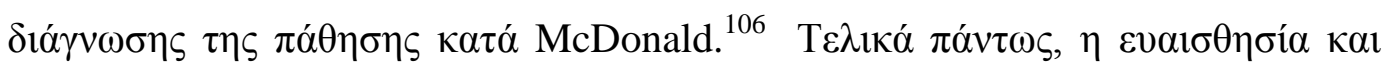

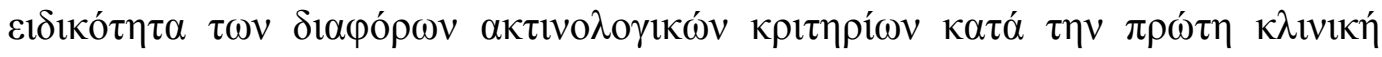

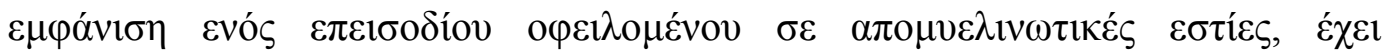

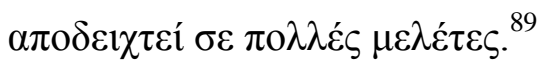

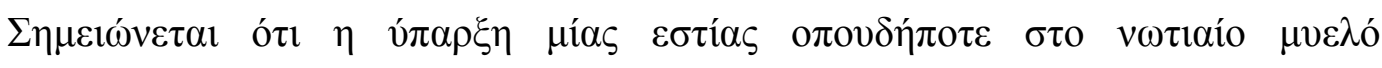

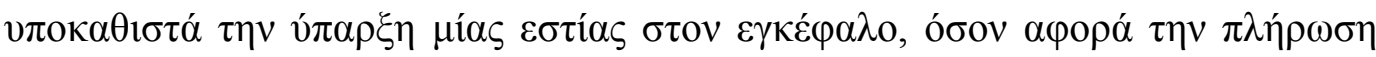

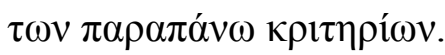

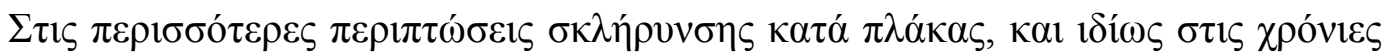

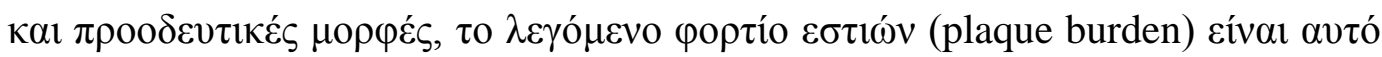

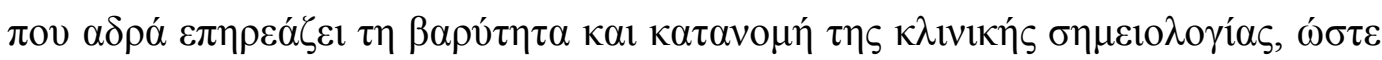

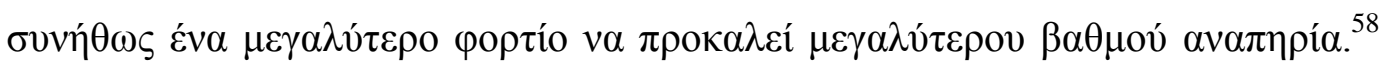

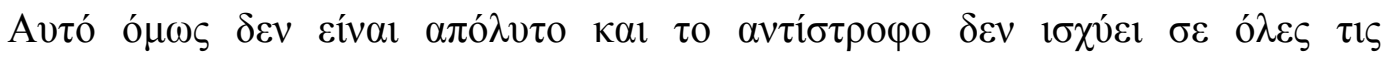
$\pi \varepsilon \rho ı \tau \tau \omega ́ \sigma \varepsilon 1 \varsigma$.

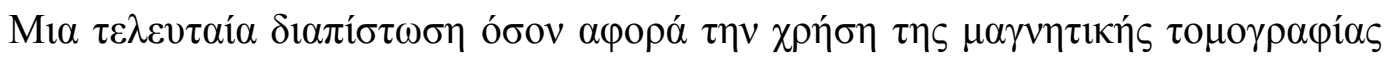

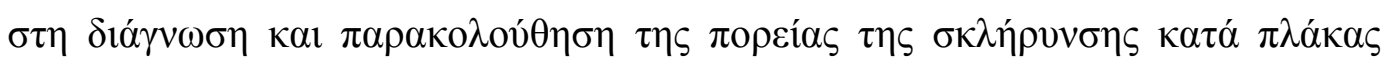

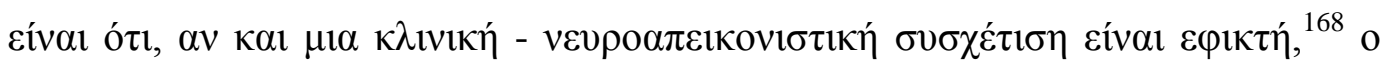

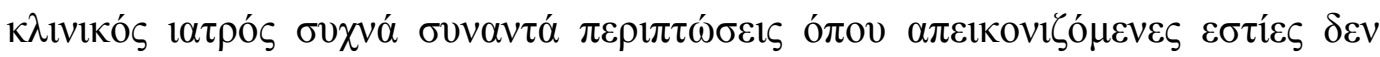

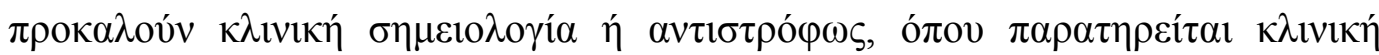

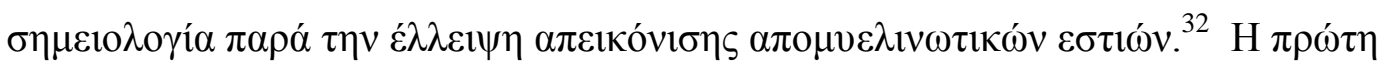

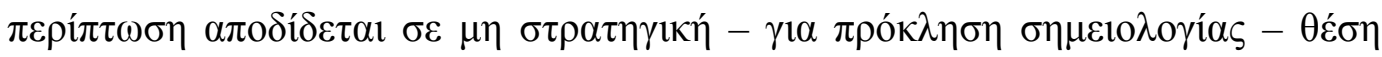

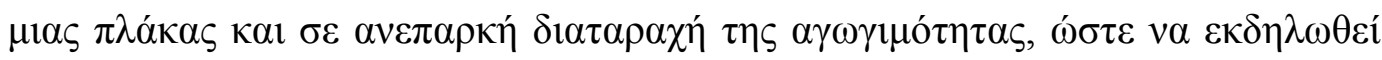

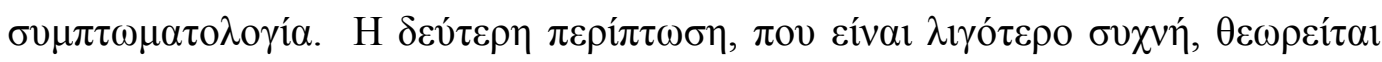

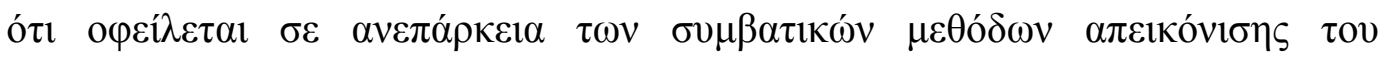
$\varepsilon \gamma \kappa \varepsilon \varphi \alpha ́ \lambda o v$. 


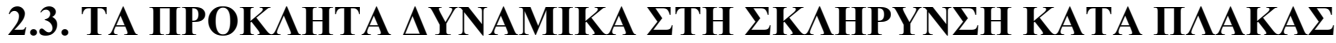

\subsection{1. ГENIKA}

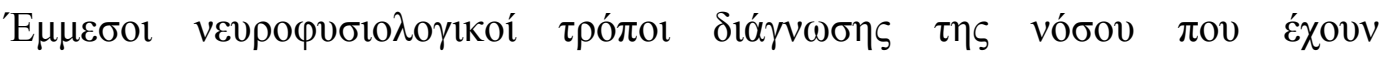

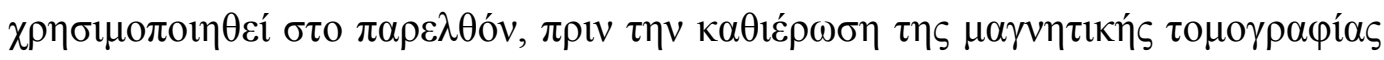

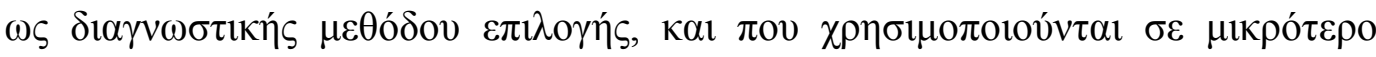

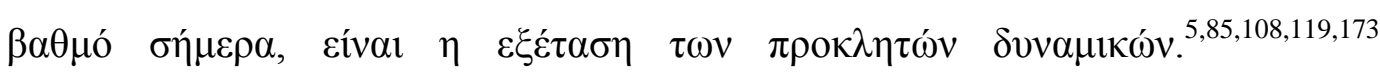

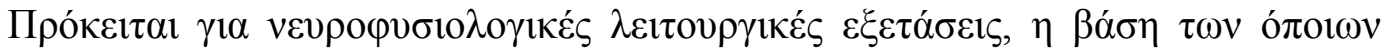

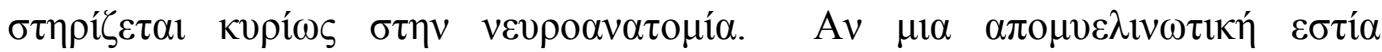

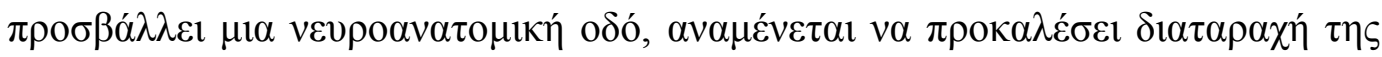

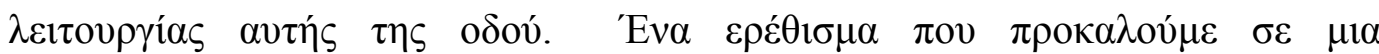

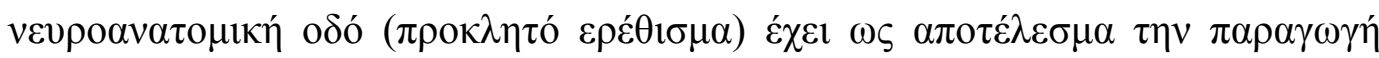

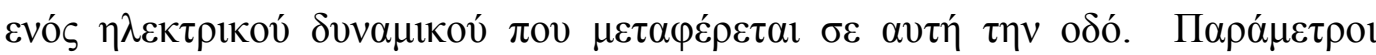

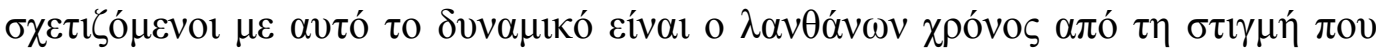

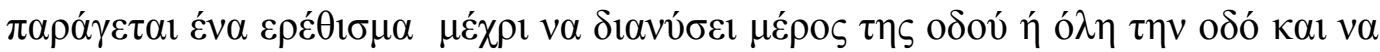

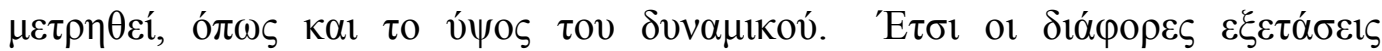

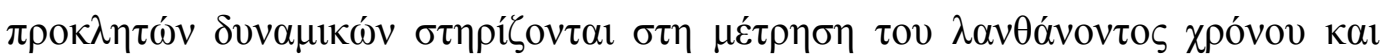

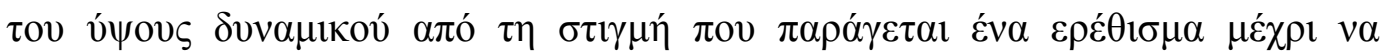

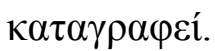

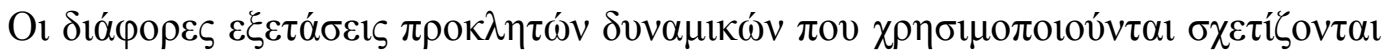

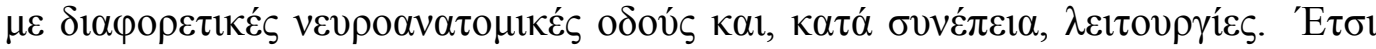

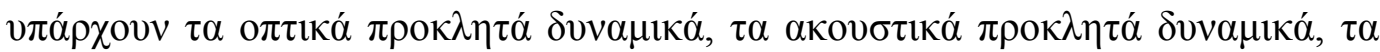

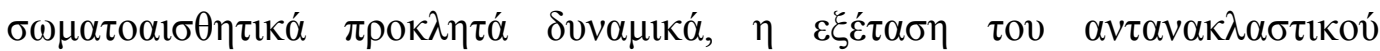

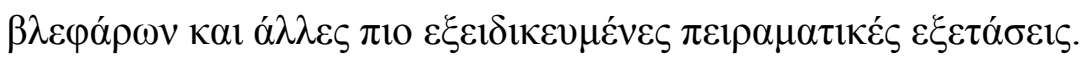

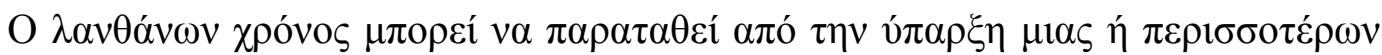

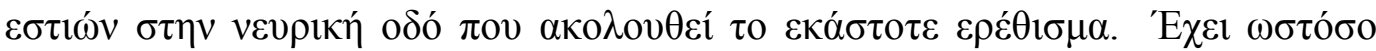

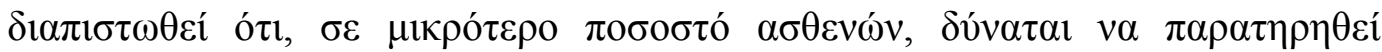

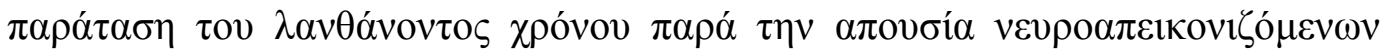

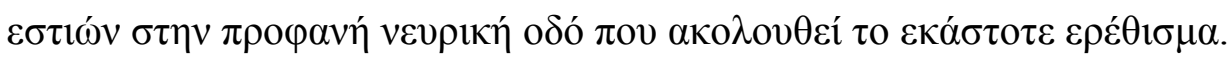

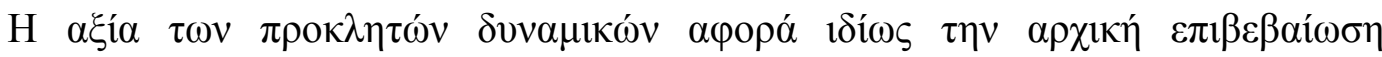

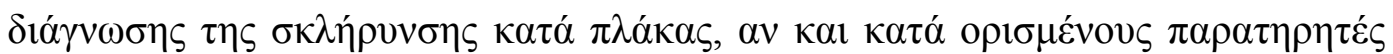

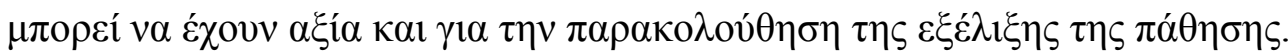




\subsection{2. ОПТТКА ПРОК $\Lambda$ HТА $\triangle$ YNAMIKA}

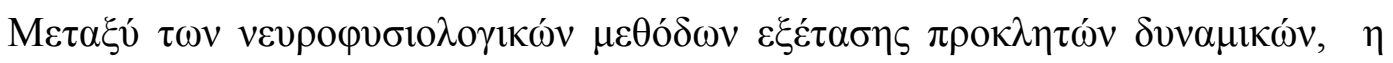

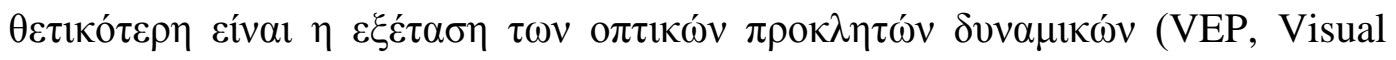
Evoked Potentials). H $\mu \varepsilon \lambda \varepsilon \dot{\tau} \eta \tau \omega v$ о $\tau \tau$

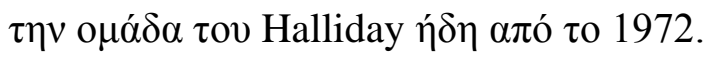

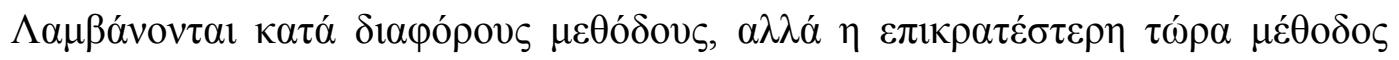

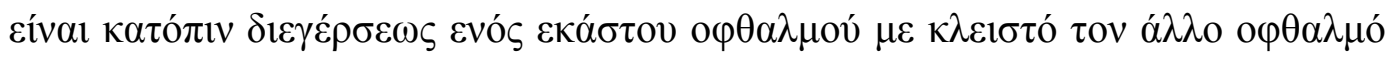

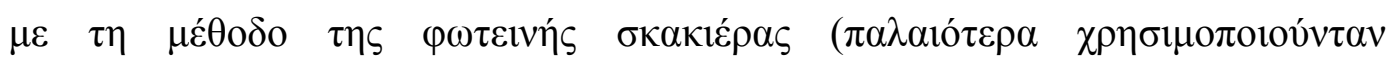

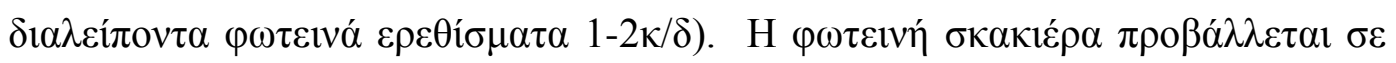

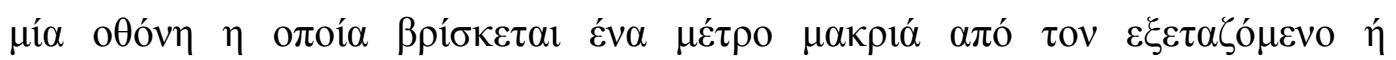

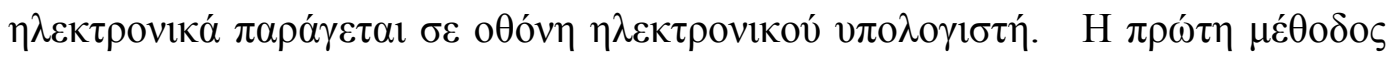

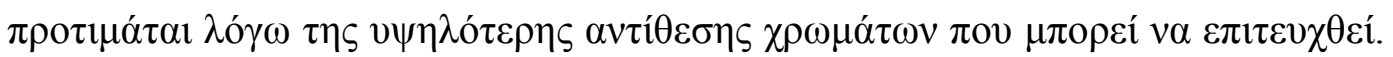

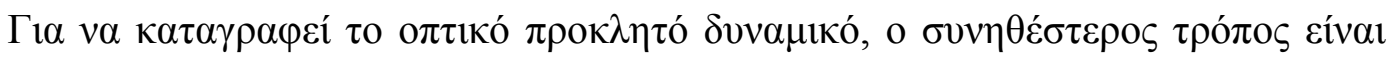

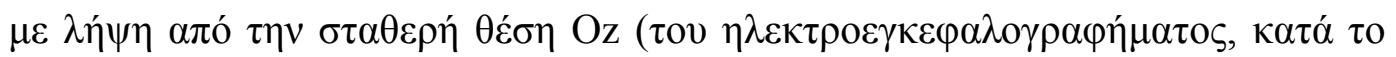

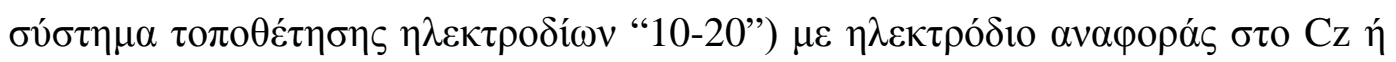

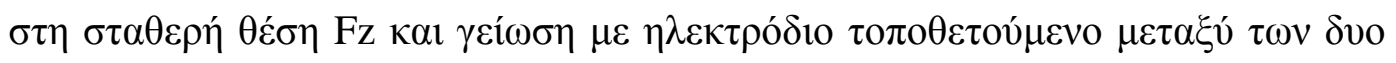

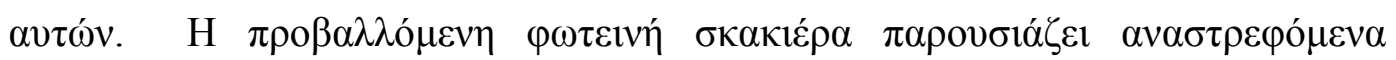

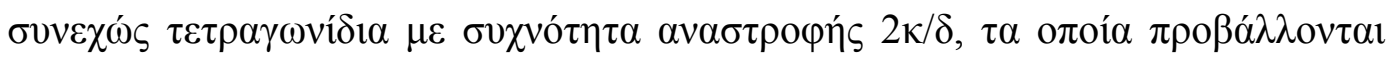

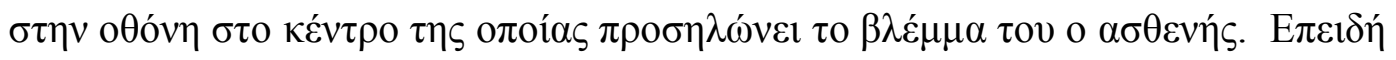

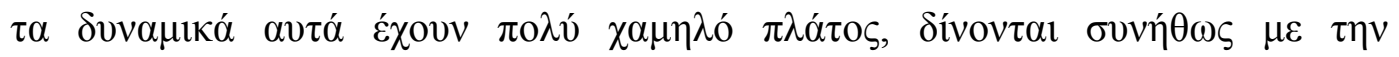

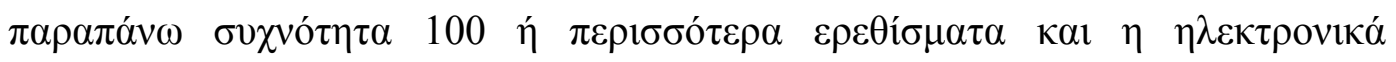

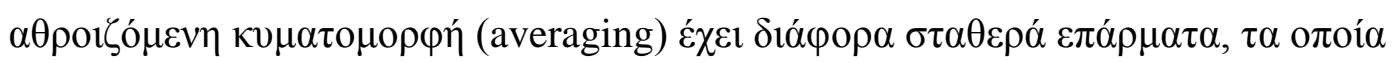

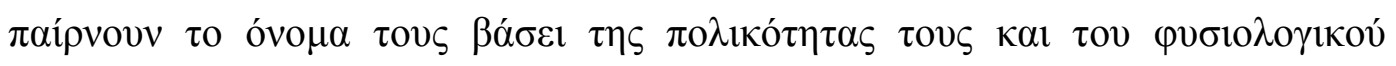
$\lambda \alpha v \theta \alpha \dot{v o v \tau o \zeta ~ \chi \rho o ́ v o v ~ \kappa \alpha \tau \alpha \gamma \rho \alpha \varphi ท ́ \varsigma ~ \tau o v \varsigma . ~}$

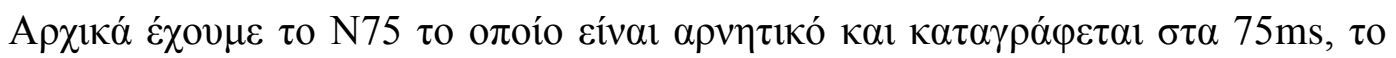

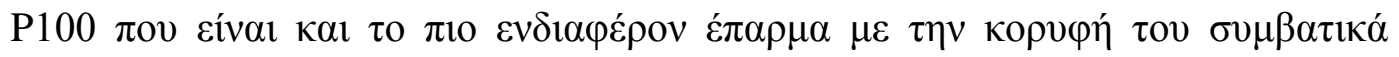

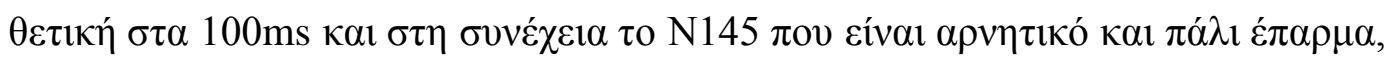

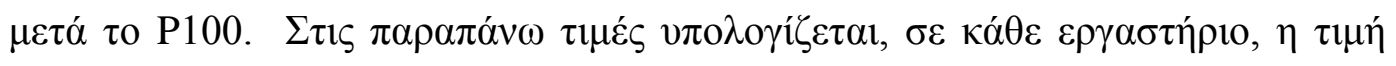

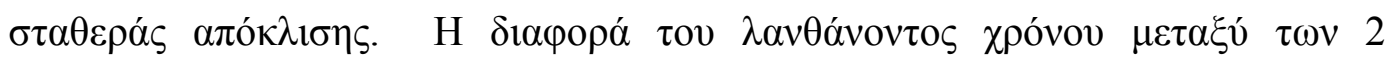

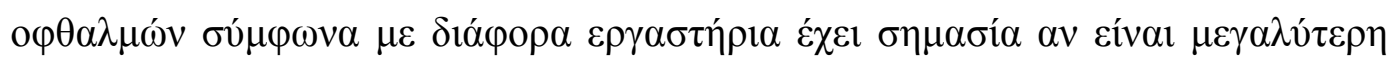

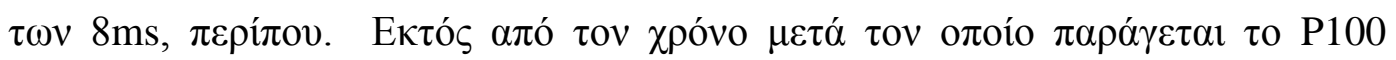




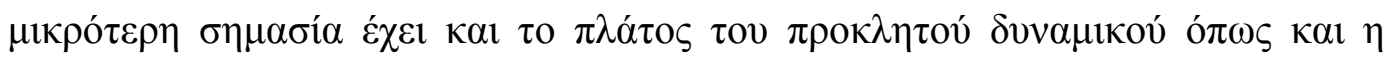

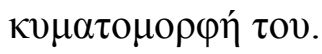

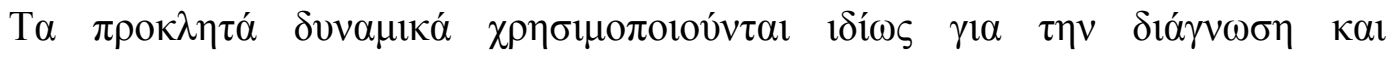

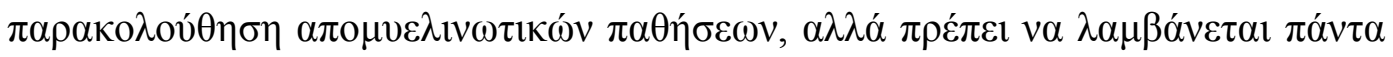

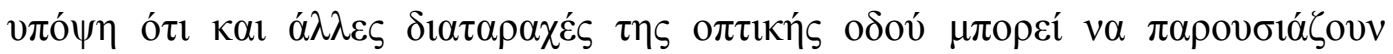

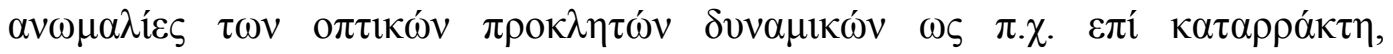

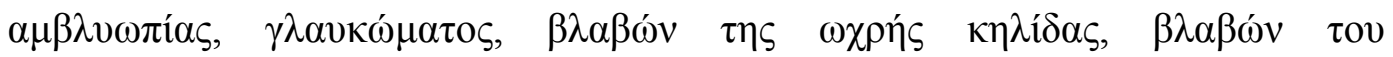

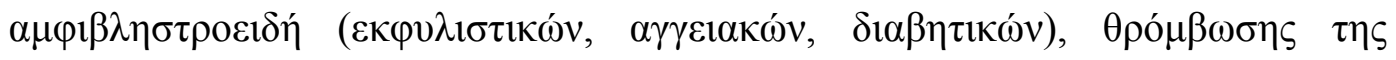

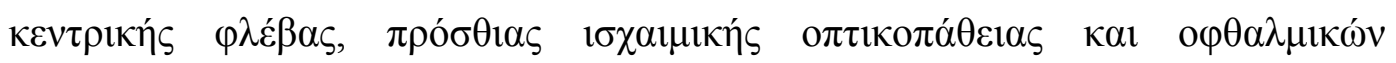

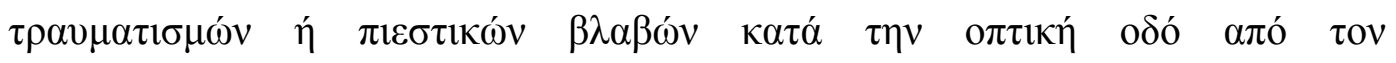

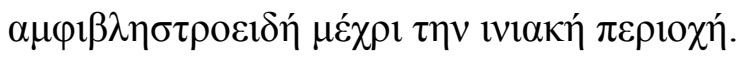

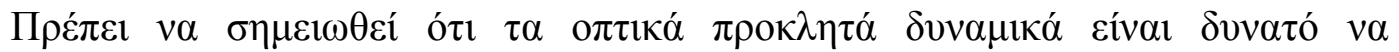

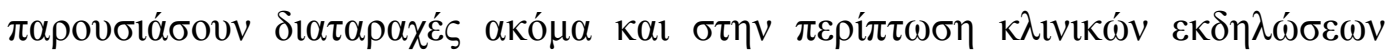

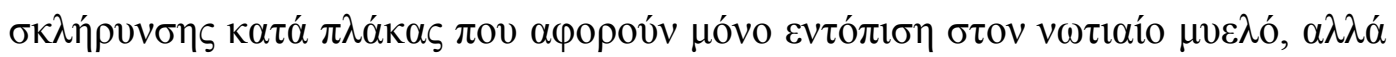

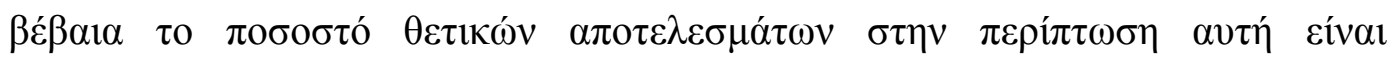

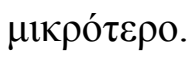

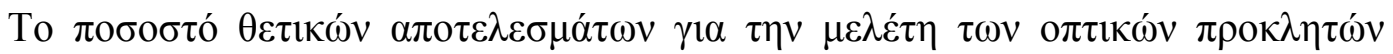

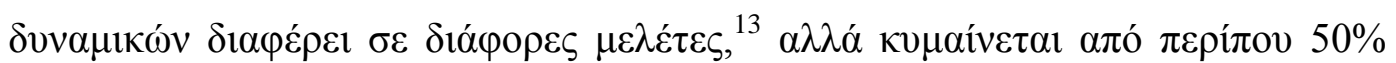

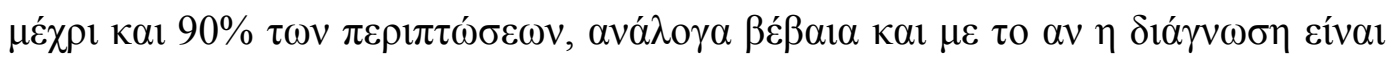

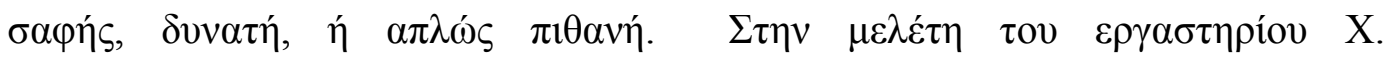

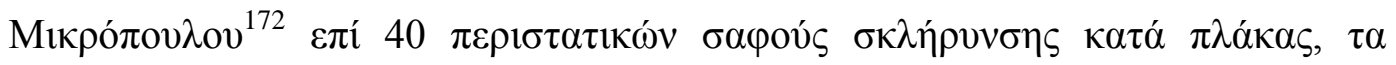

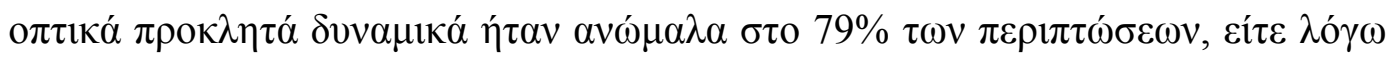

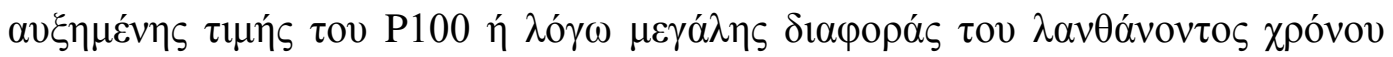

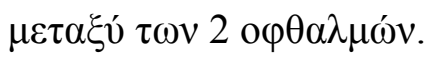

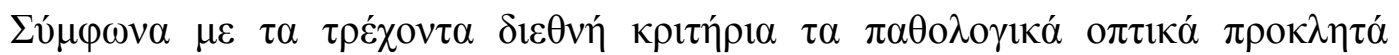

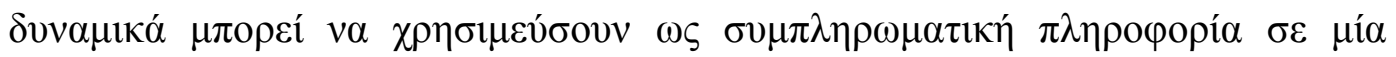

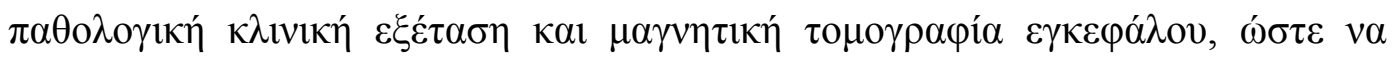

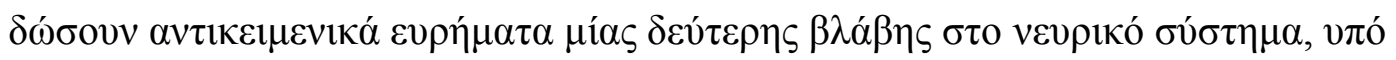

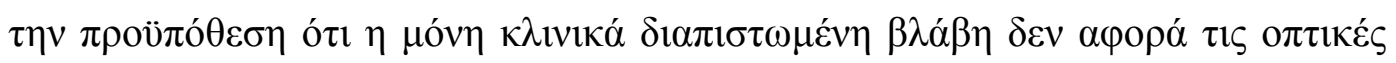

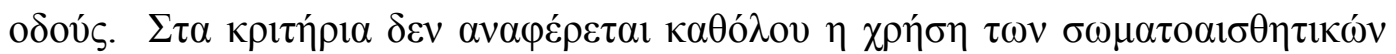




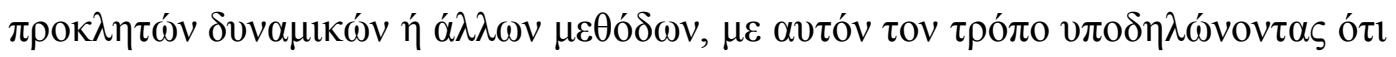

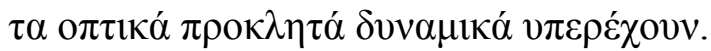

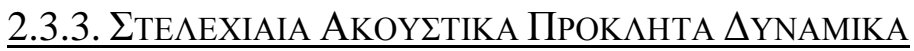

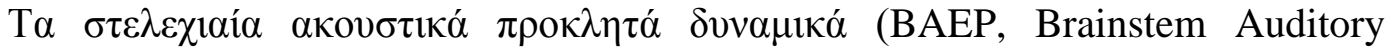

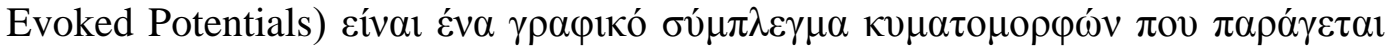

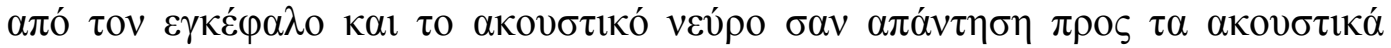

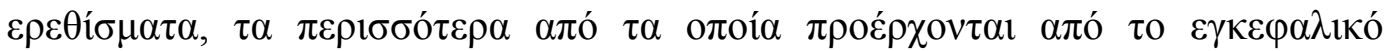
$\sigma \tau \varepsilon ́ \lambda \varepsilon \chi \circ \varsigma$.

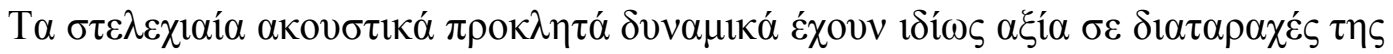

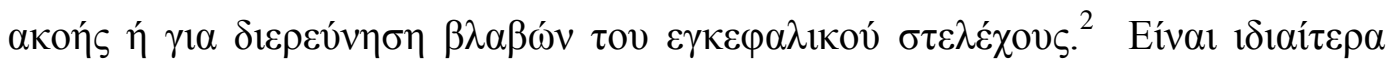

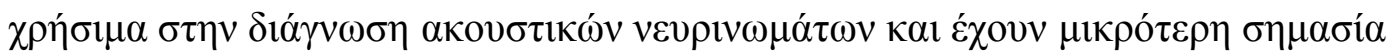

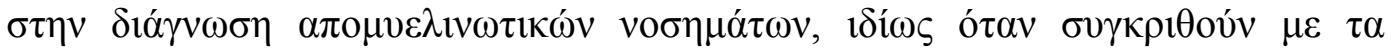

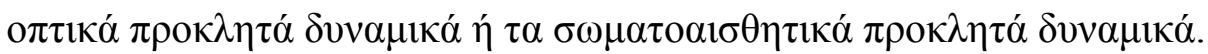

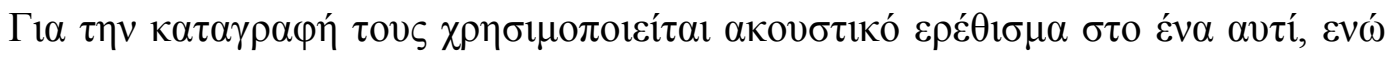

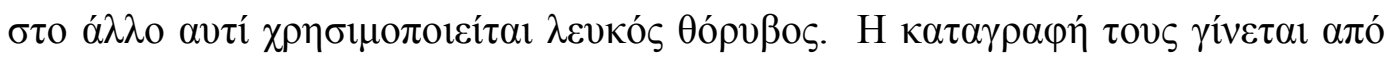

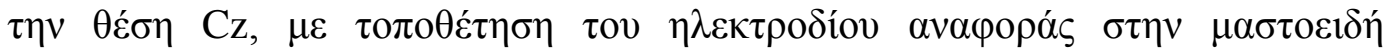

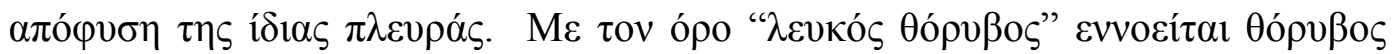

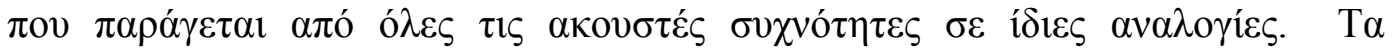

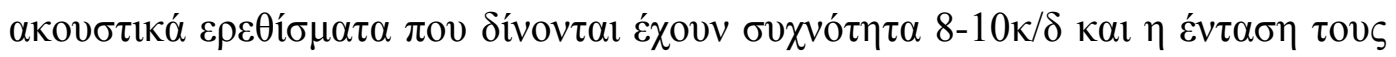

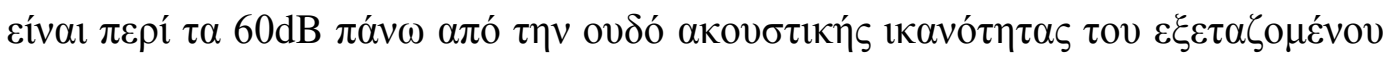

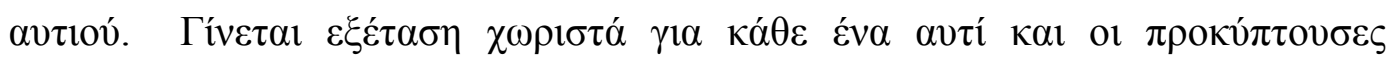

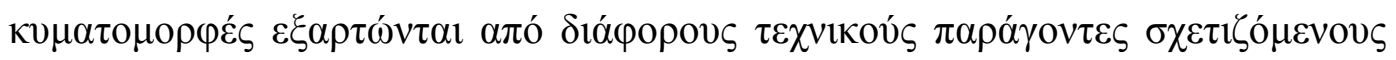

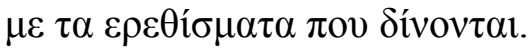

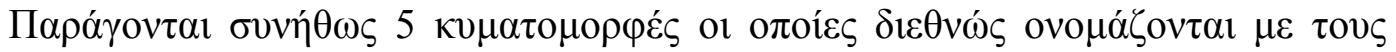

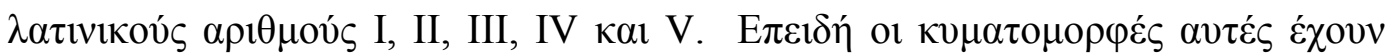

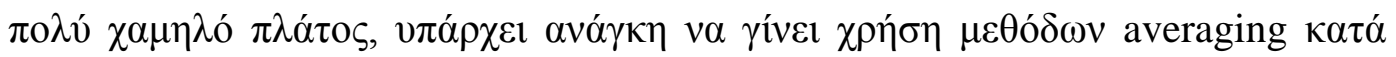

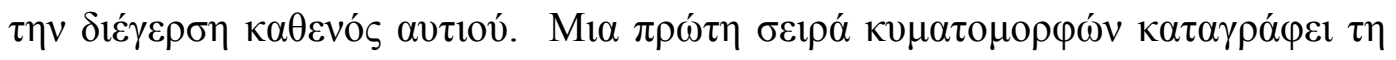

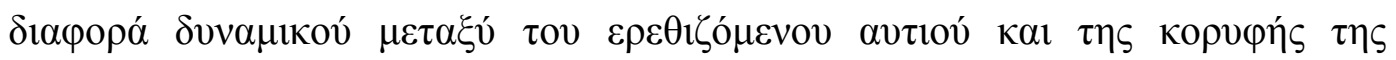

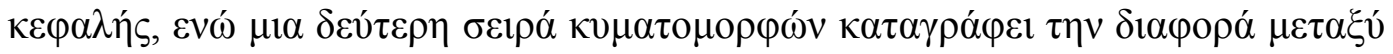

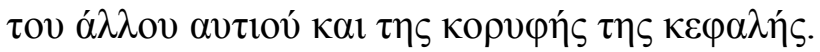




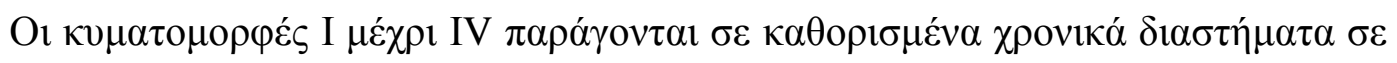

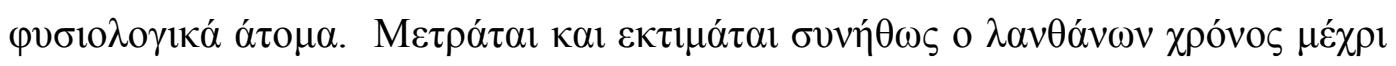

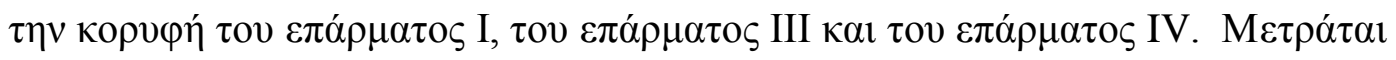

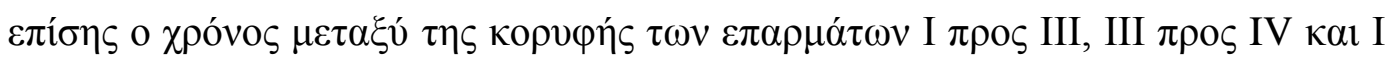

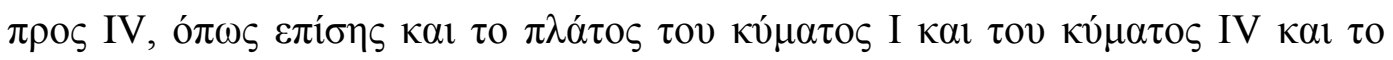

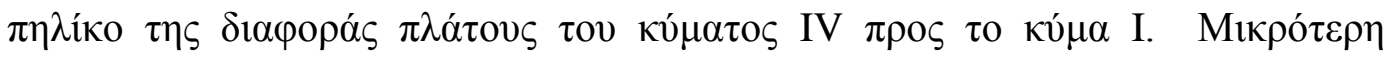

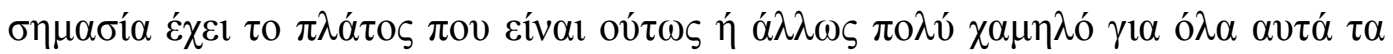

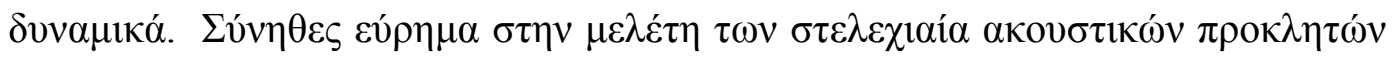

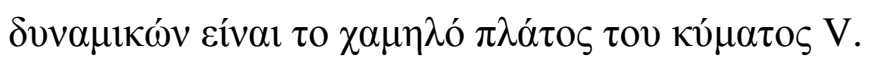

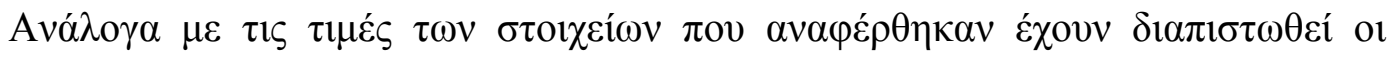

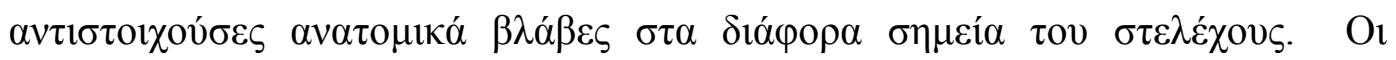

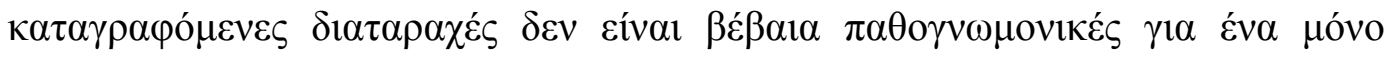

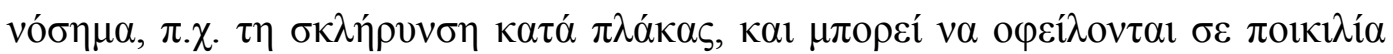

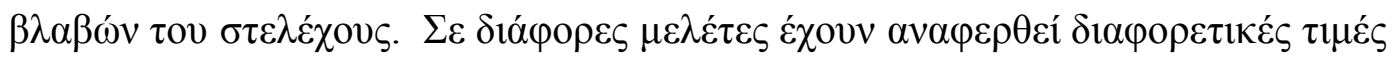

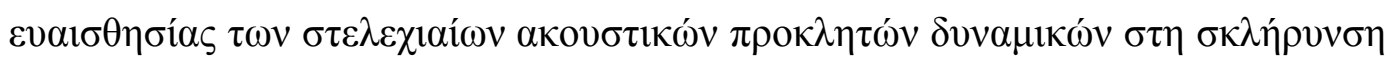

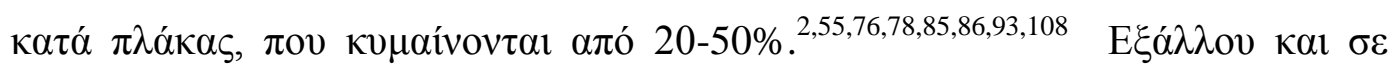

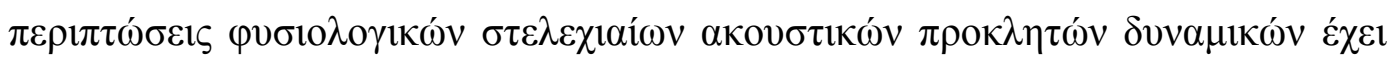

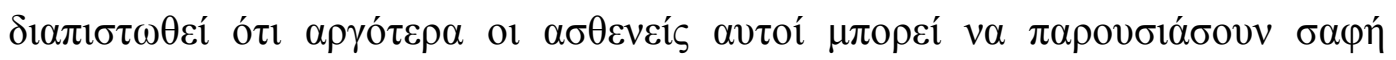

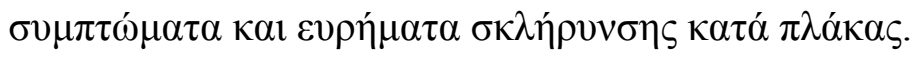

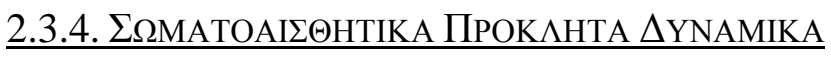

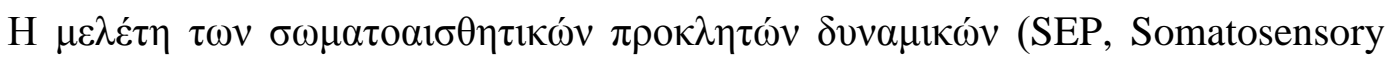

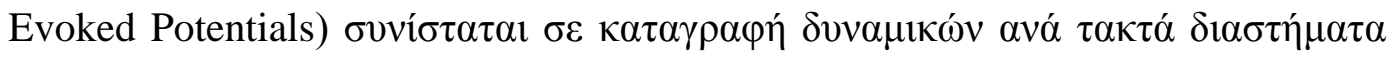

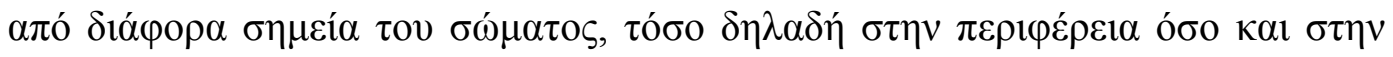

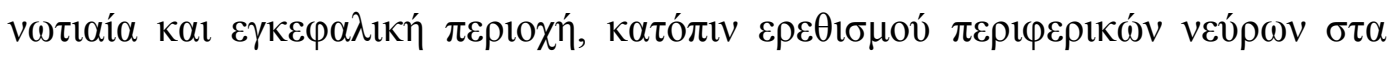

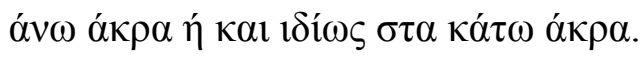

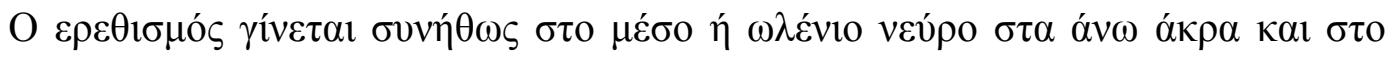

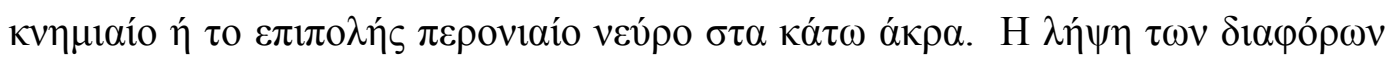

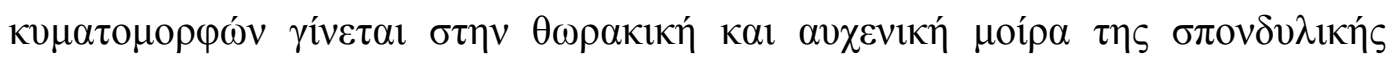

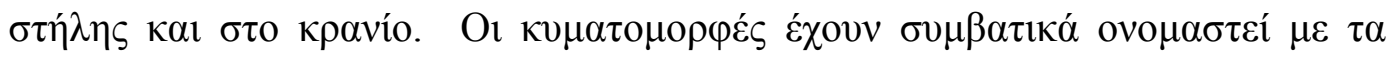




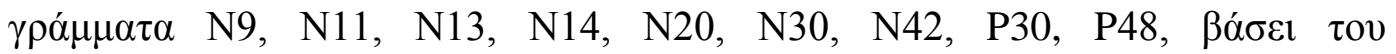

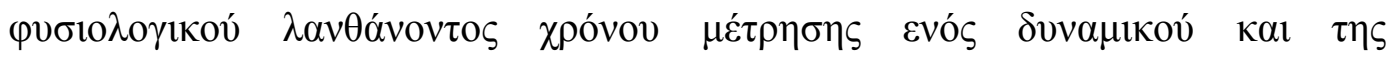

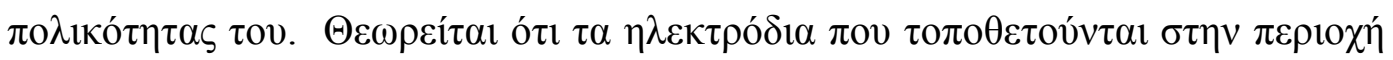

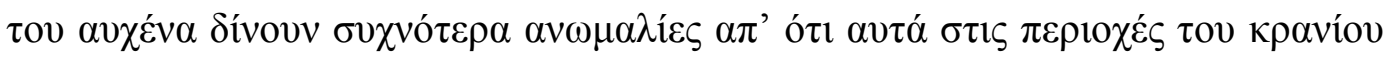

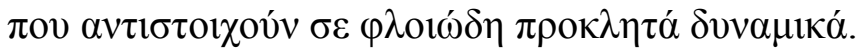

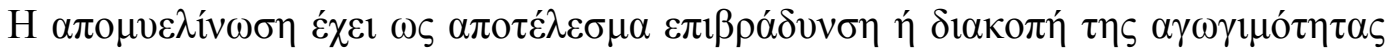

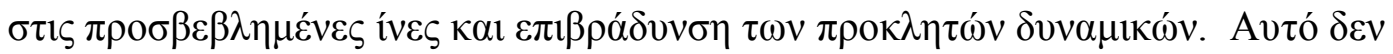

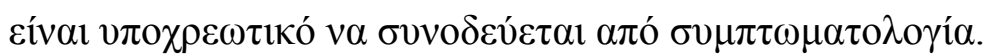

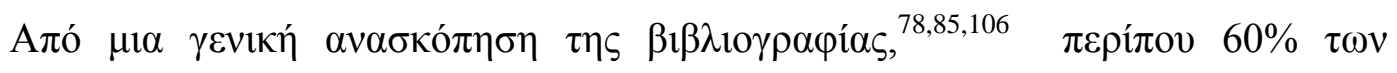

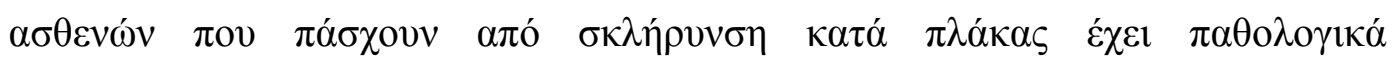

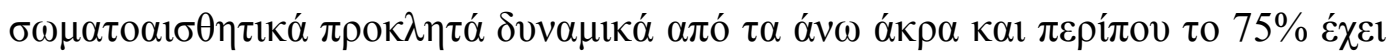

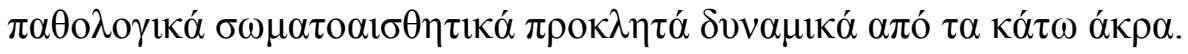




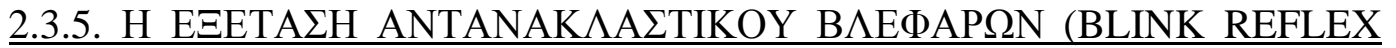 TEST) $\Sigma$ TH $\Sigma$ K $\Lambda$ HPYN $\Sigma H$ KATA П $\Lambda$ AKA $\Sigma{ }^{55,81,140,141,142}$}

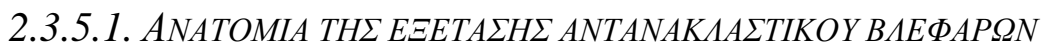

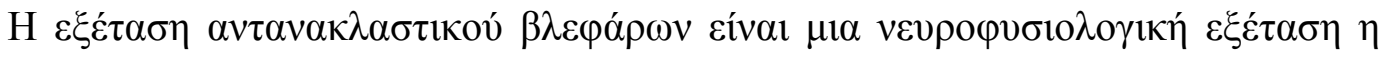

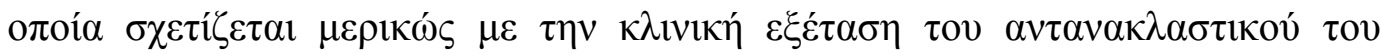

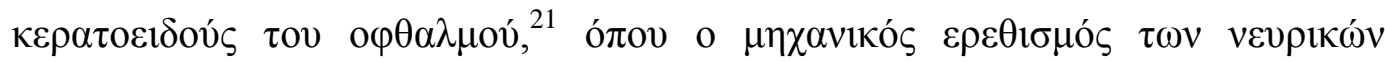

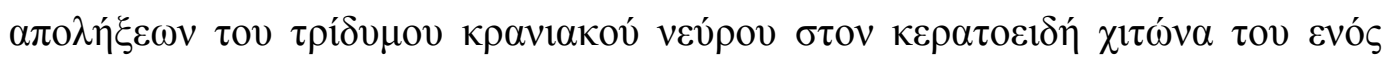

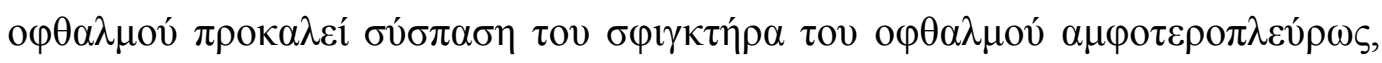

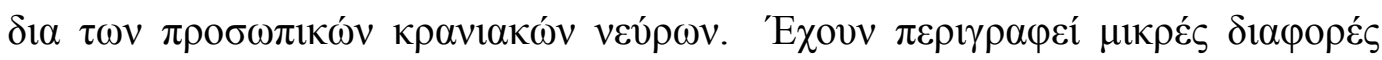

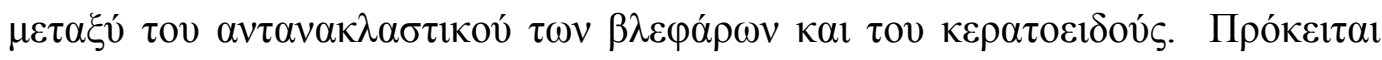

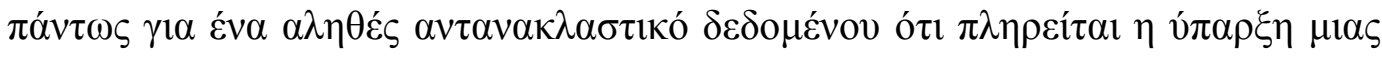

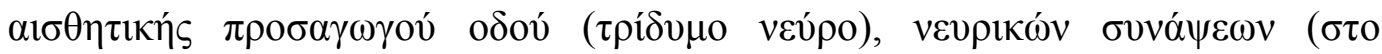

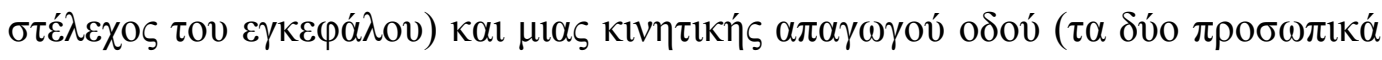

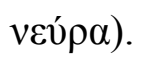

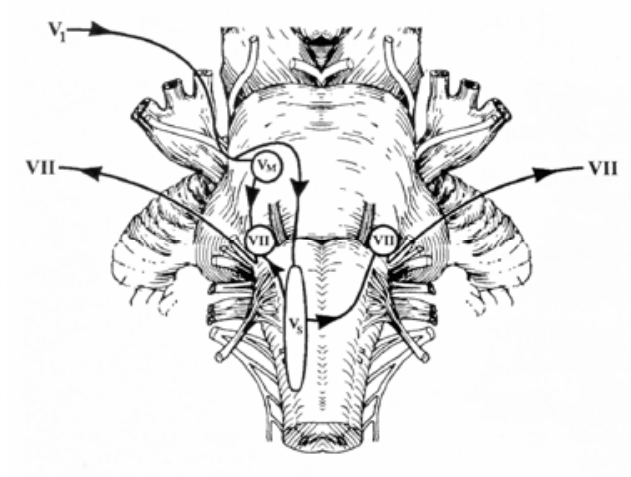

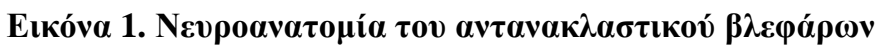

A $\pi$ ó D.Preston, B.Shapiro, Electromyography and Neuromuscular Disorders, $1^{\eta}$ Eк $\delta ., ~ 1998$, Butterworth-Heinemann, $\sigma .58$

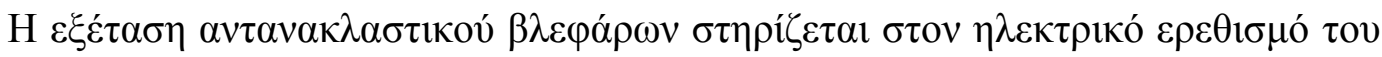

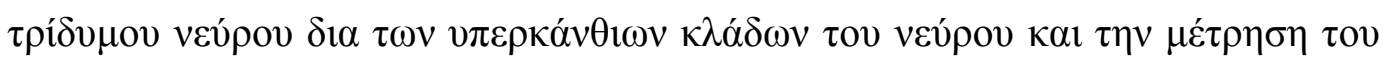

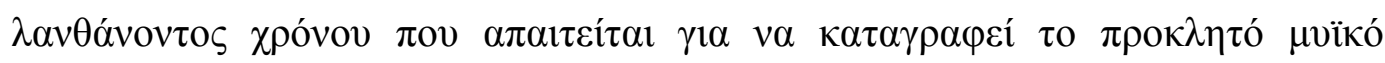

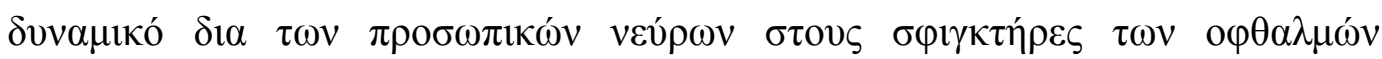

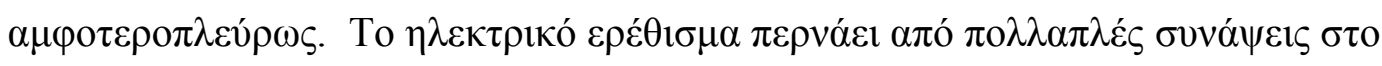

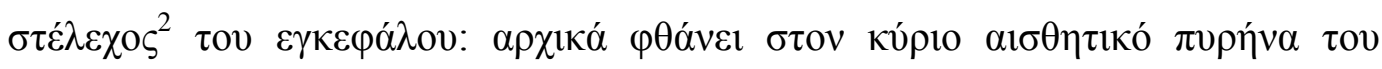

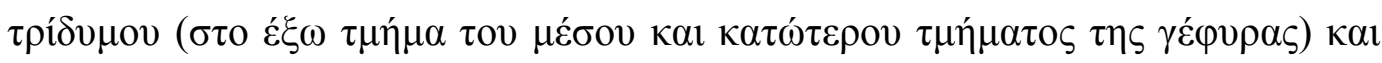

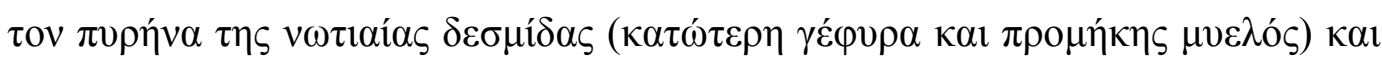




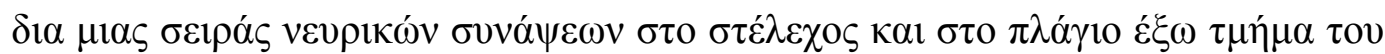

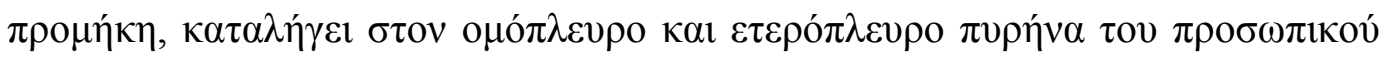
vev́pov (Eıкóva 1).

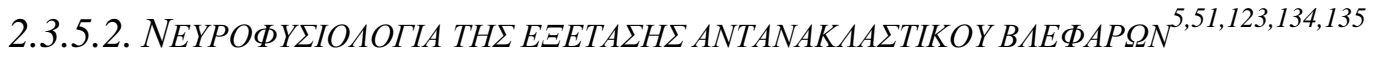

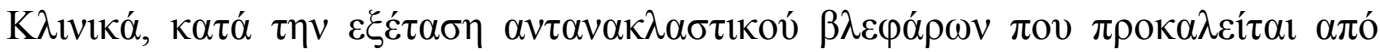

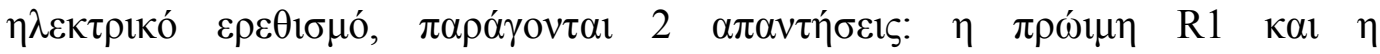

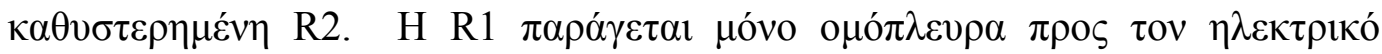

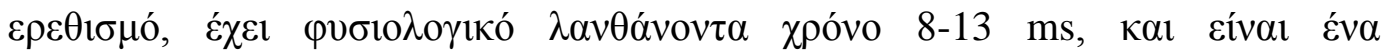

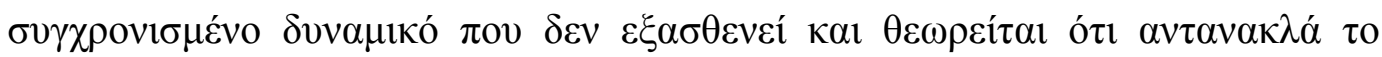

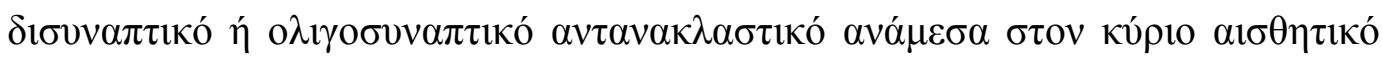

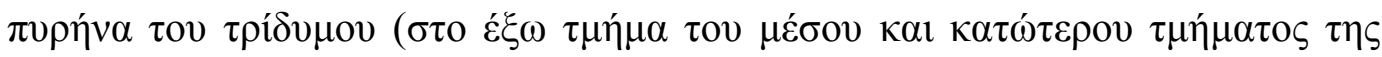

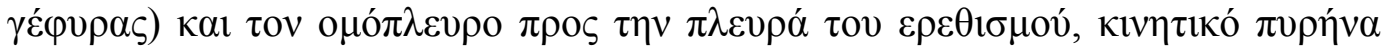

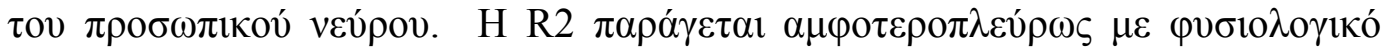

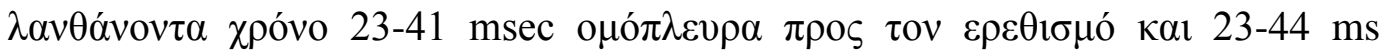

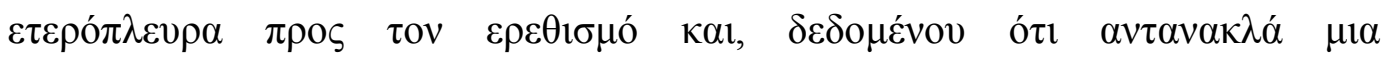

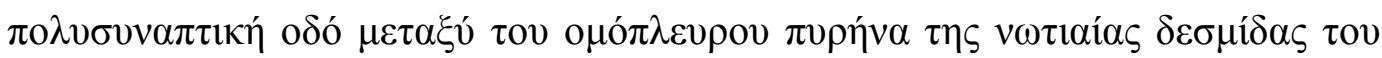

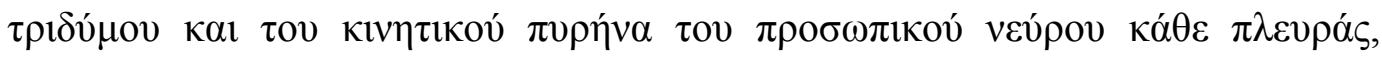

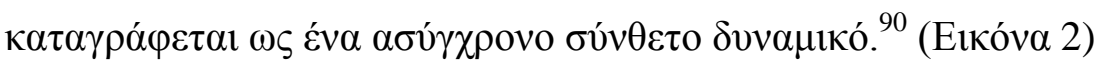

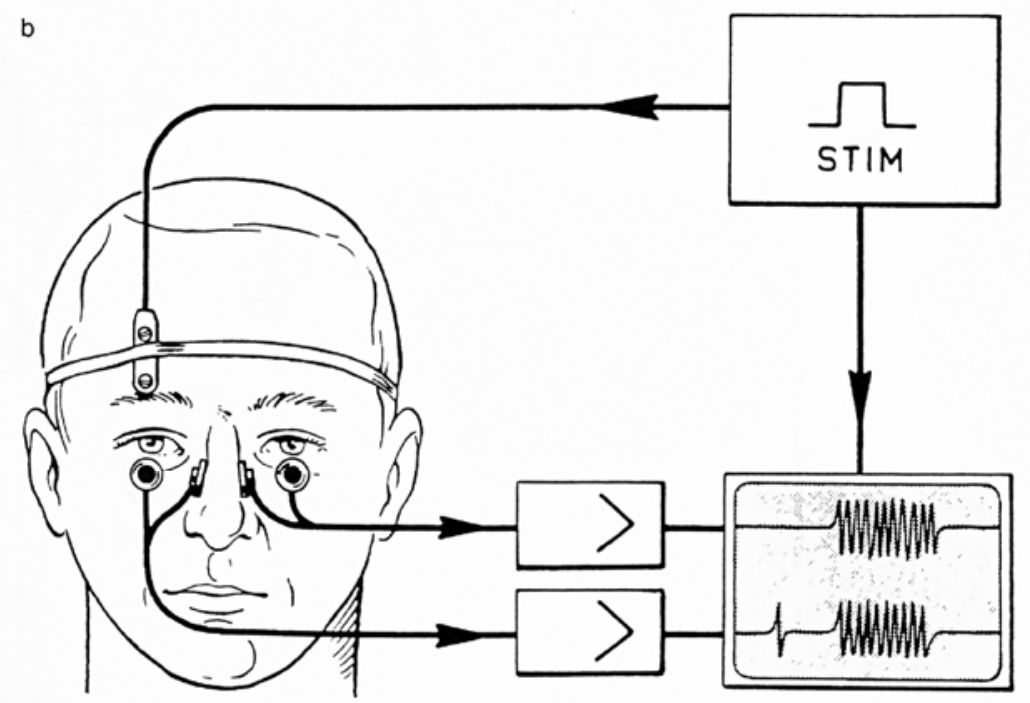

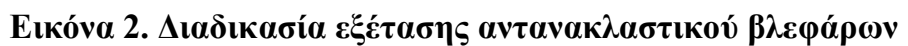

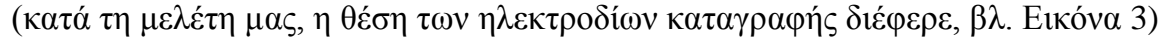
A $\pi$ ó Stöhr M., Iatrogene Nerveläsionen. G. Thieme, Stuttgart-New York, 1980 


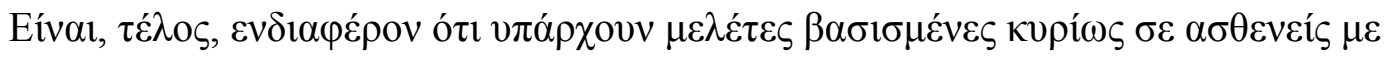

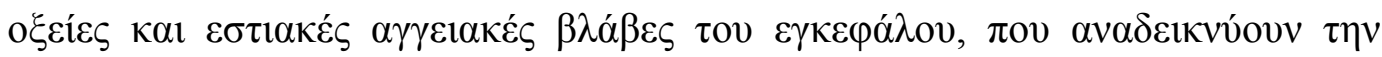

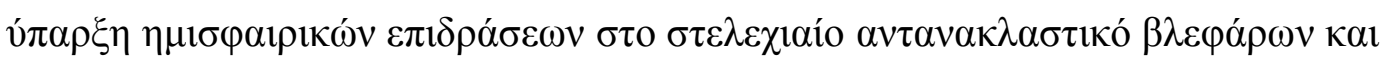

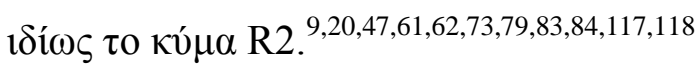

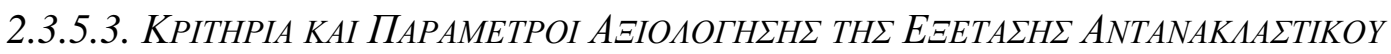
$B \Lambda E \Phi A P \Omega N$

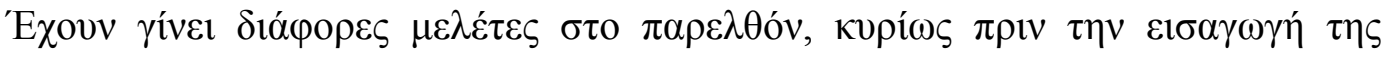

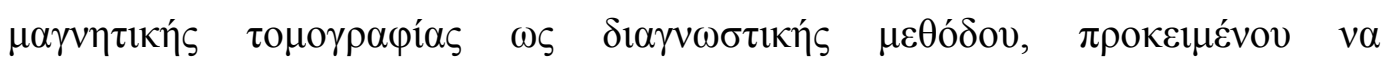

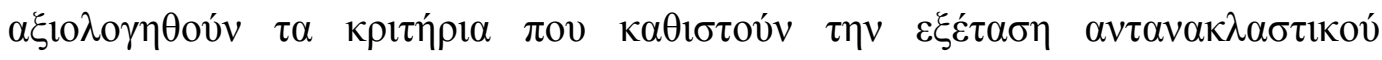

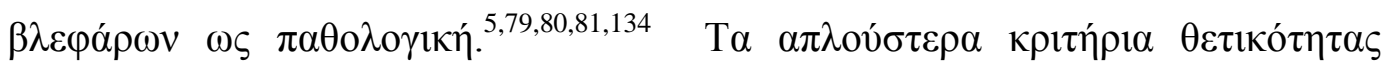

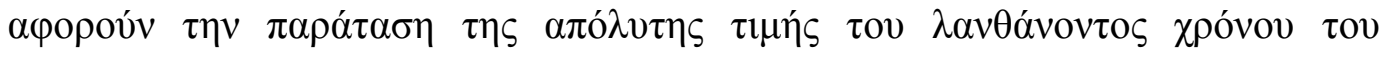

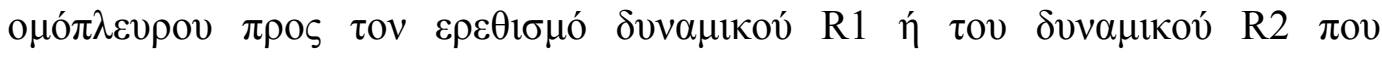

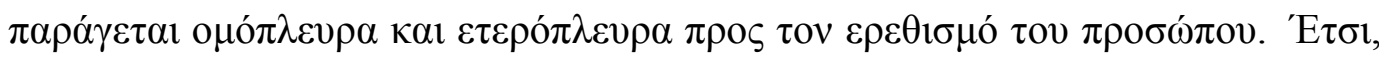

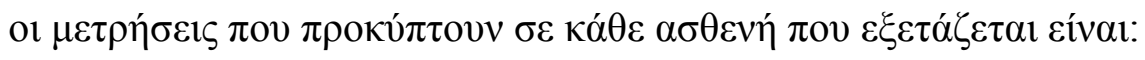

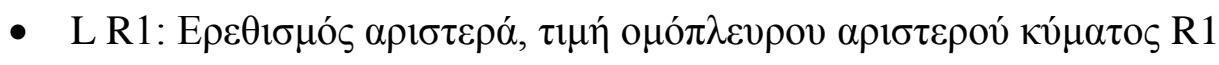

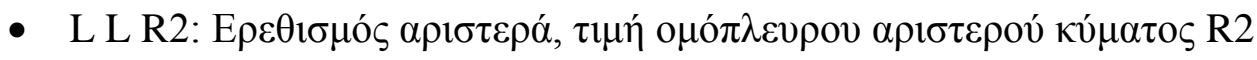

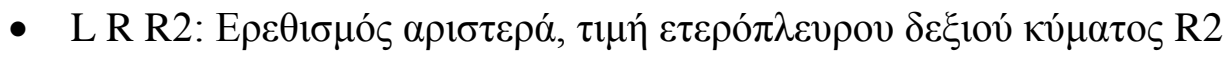

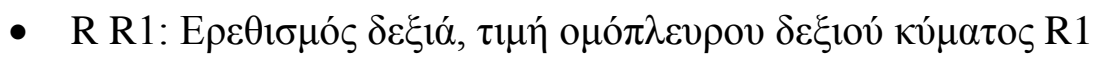

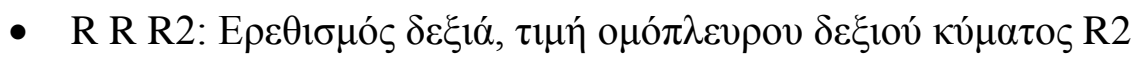

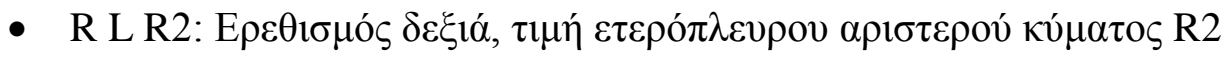

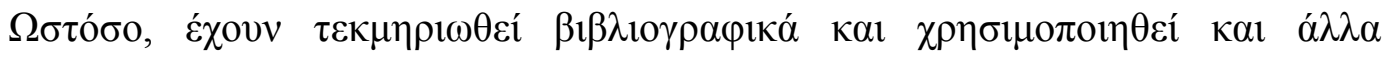

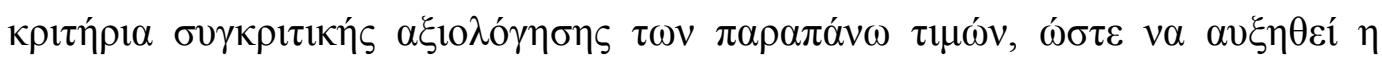

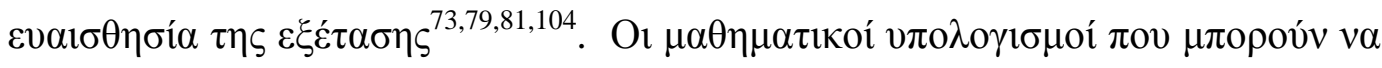

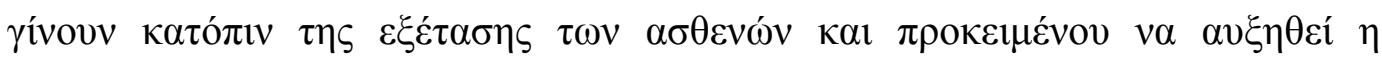

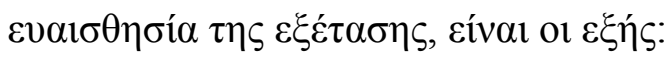

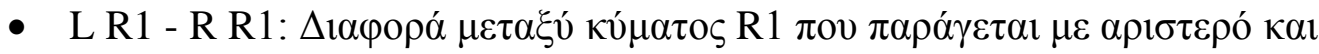
$\delta \varepsilon \xi$ ıó $\varepsilon \rho \varepsilon \theta \imath \sigma \mu o ́$

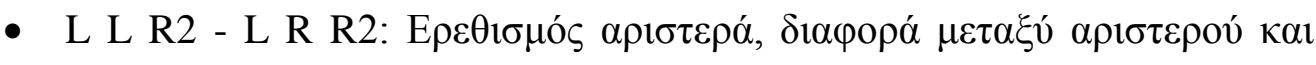

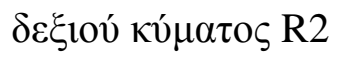




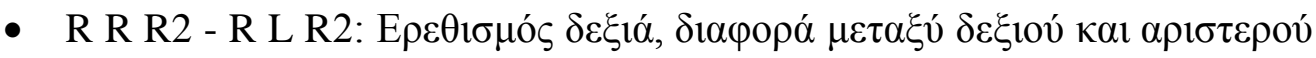
$\kappa v ́ \mu \alpha \tau$ ○ R2

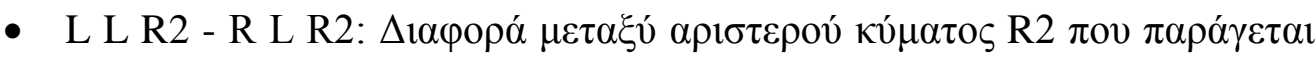
$\alpha \pi$ ó $\alpha \rho \imath \sigma \tau \varepsilon \rho o ́ ~ \kappa \alpha \imath ~ \delta \varepsilon \xi$ ló $\varepsilon \rho \varepsilon \theta 1 \sigma \mu o ́$

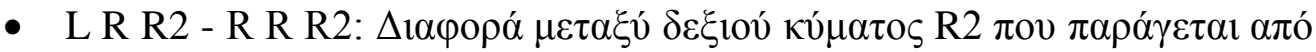
$\alpha \rho \imath \sigma \tau \varepsilon \rho o ́ ~ \kappa \alpha \imath ~ \delta \varepsilon \xi$ ló $\varepsilon \rho \varepsilon \theta \imath \_\mu o ́$ 


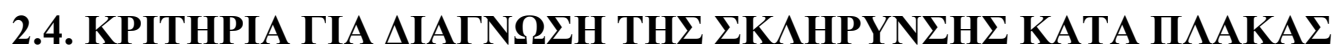

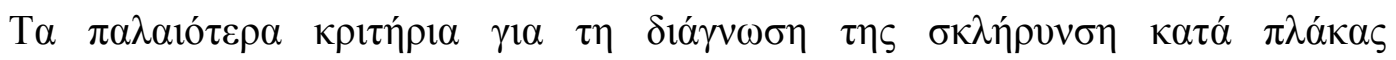

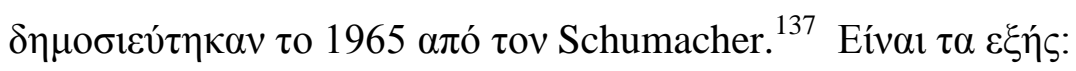

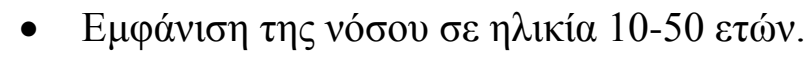

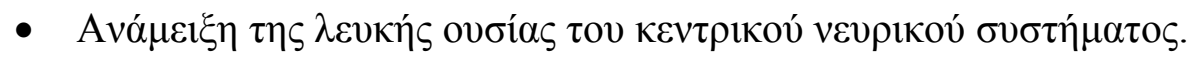

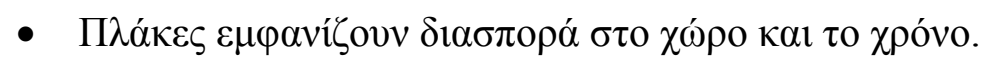

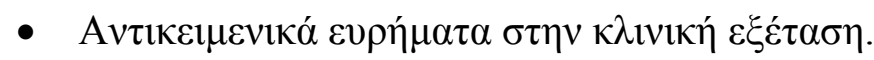

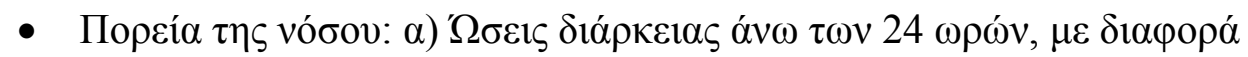

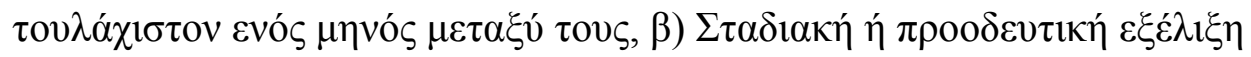

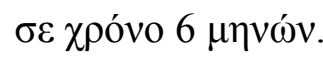

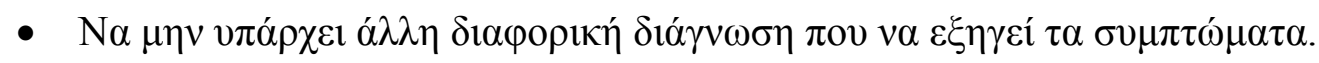

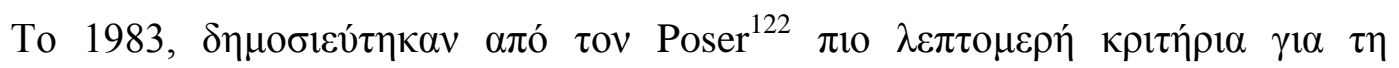

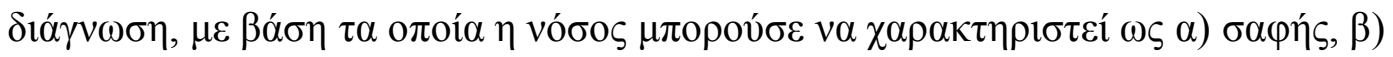

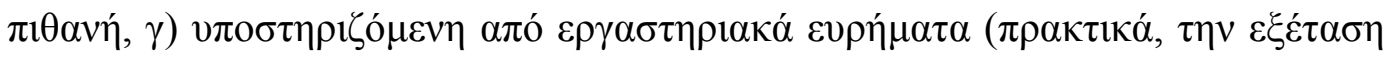

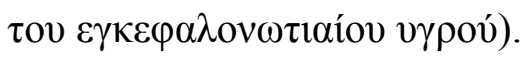

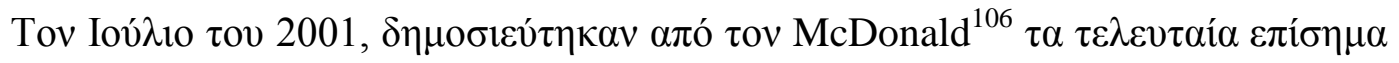

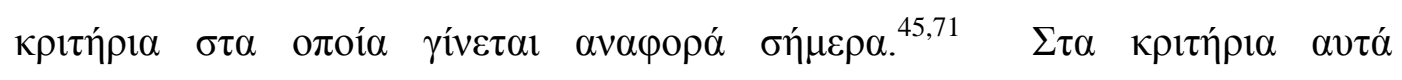

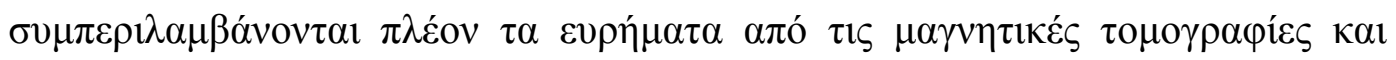

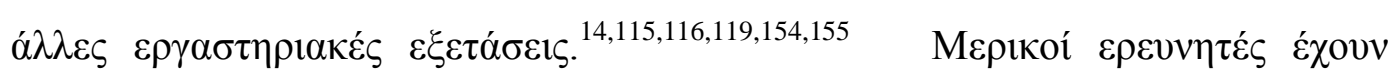

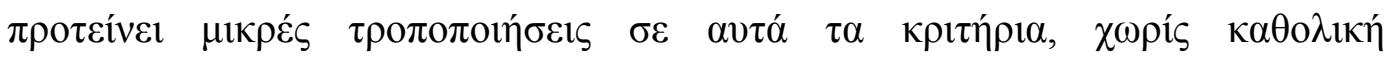
$\alpha \pi \mathrm{o \delta o} \chi \eta^{15,156}$

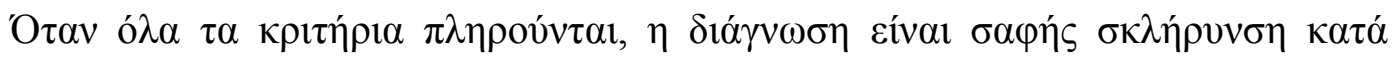

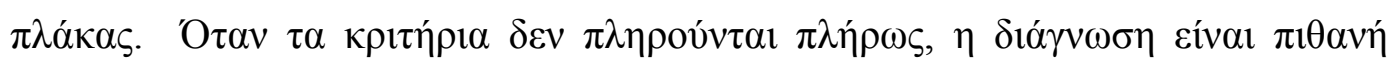

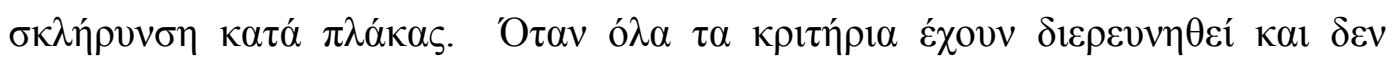

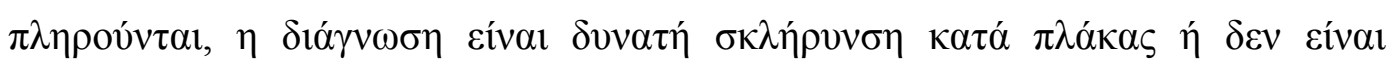

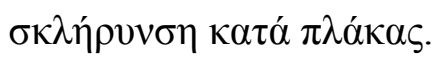




\section{KАТНГОРIA A}

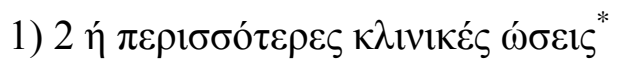

2) 2 ฑ́ $\pi \varepsilon \rho ı \sigma \sigma o ́ \tau \varepsilon \rho \alpha \varepsilon v \rho \eta ́ \mu \alpha \tau \alpha \sigma \tau \eta v \varepsilon \xi \xi \dot{\varepsilon} \tau \alpha \sigma \eta^{* *}$

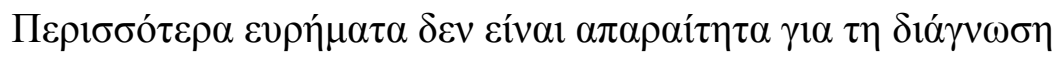

\section{КАТНГОРІА B}

1) 2 ท́ $\pi \varepsilon \rho 1 \sigma \sigma o ́ \tau \varepsilon \rho \varepsilon \varsigma \kappa \lambda \imath v ı \kappa \varepsilon ́ \varsigma \omega ́ \sigma \varepsilon ı \varsigma^{*}$

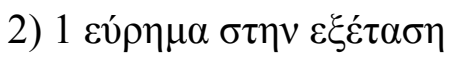

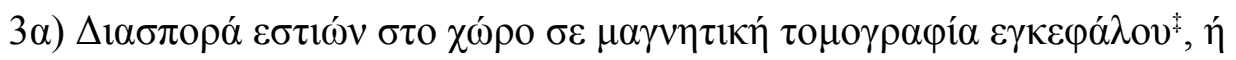

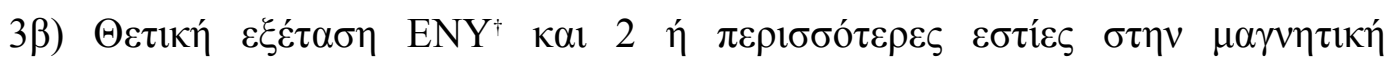

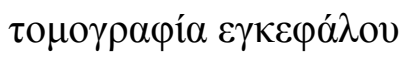

\section{$\underline{\text { KАТНГОРІА } \Gamma}$}

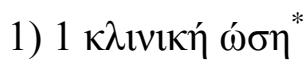

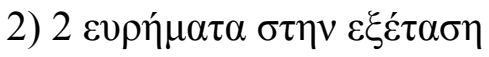

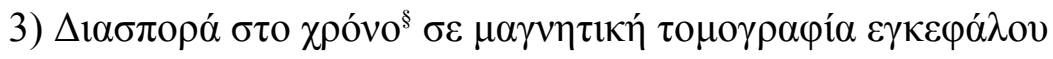

\section{$\underline{\text { KATНГОРIA } \Delta}$}

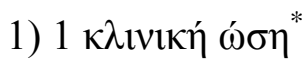

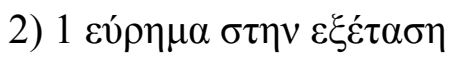

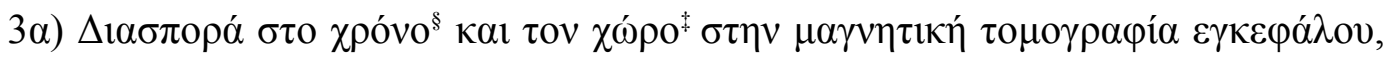
ท́

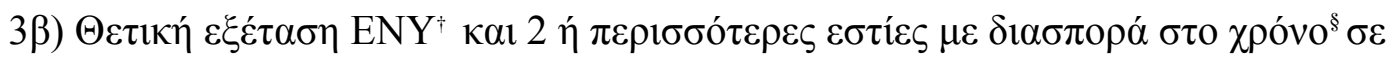

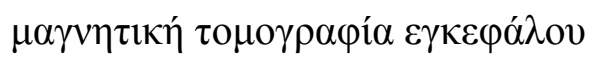

\section{$\underline{\text { KАTHГОРIA E }}$}

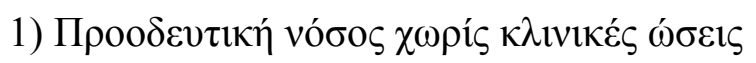

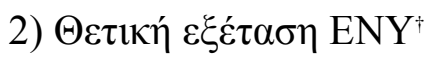

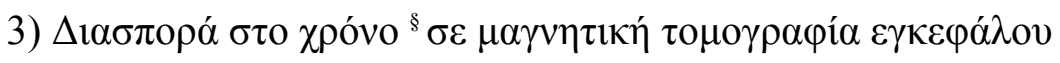

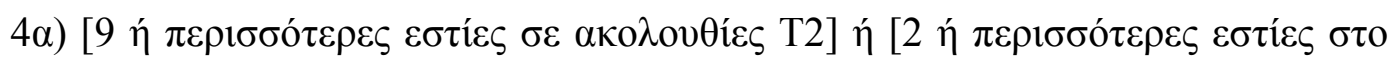

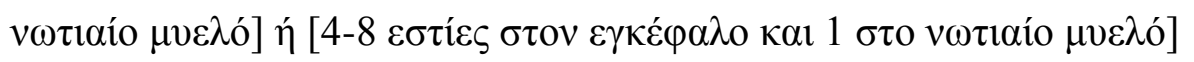

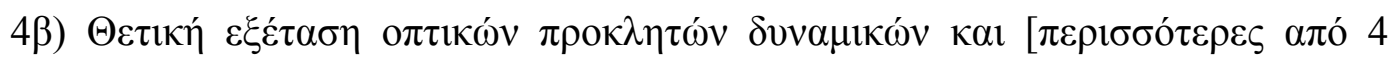

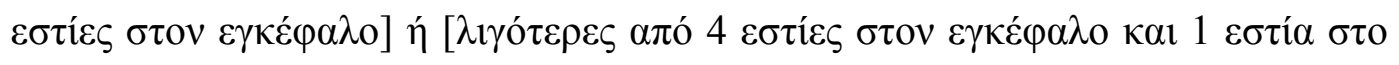

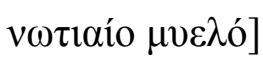




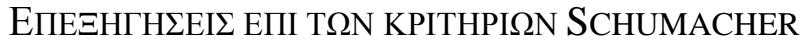

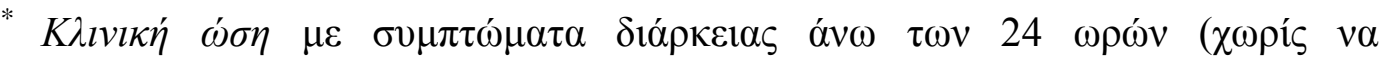

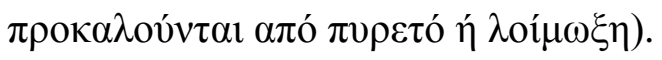

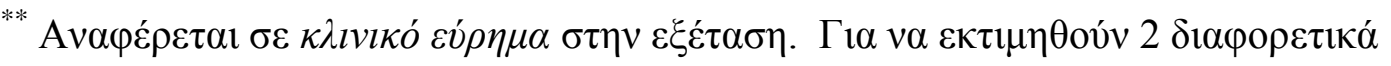

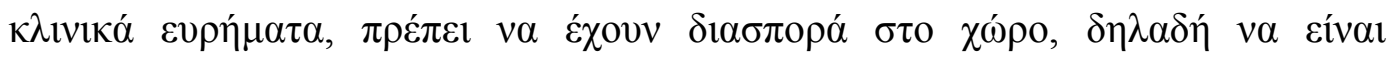

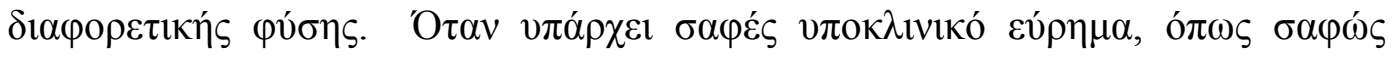

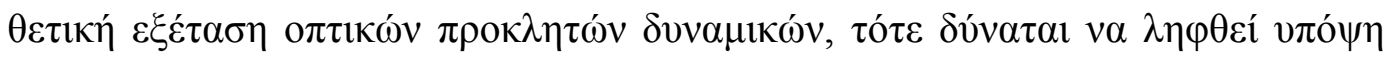

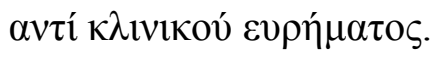

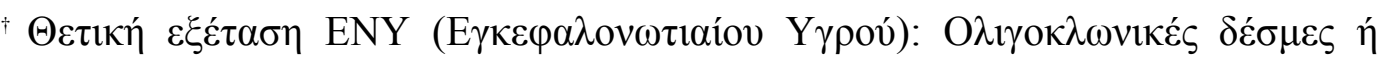

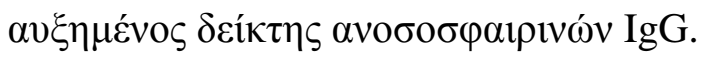

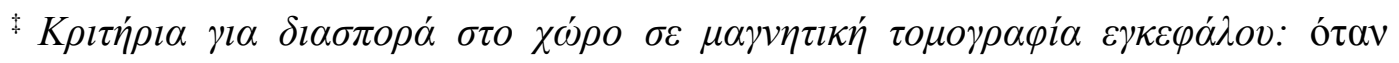

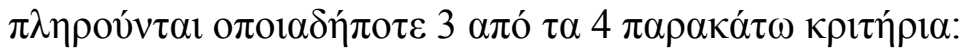

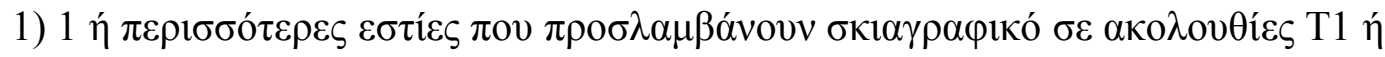

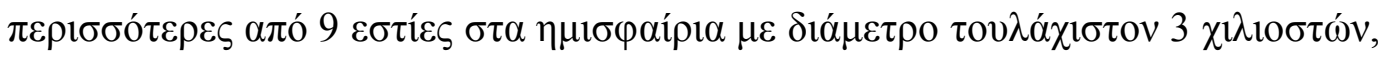
$\sigma \varepsilon \alpha \kappa о \lambda о v \theta i ́ \varepsilon \varsigma \mathrm{T} 2$.

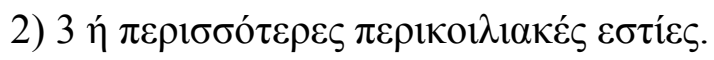

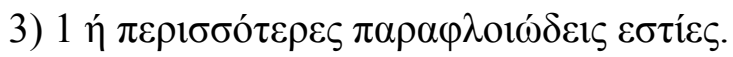

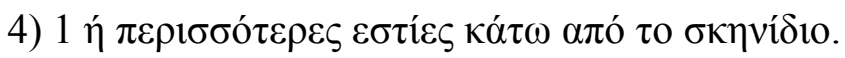

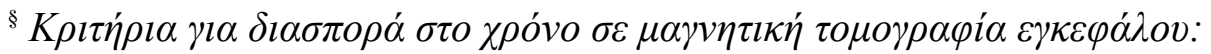

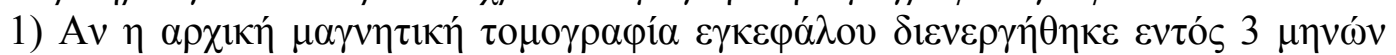

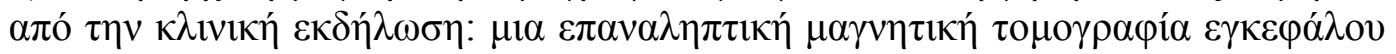

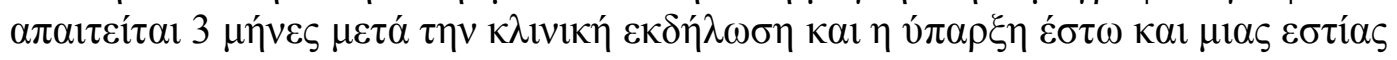

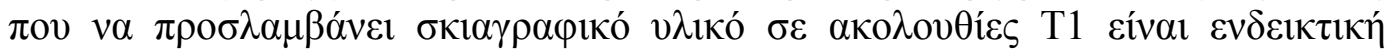

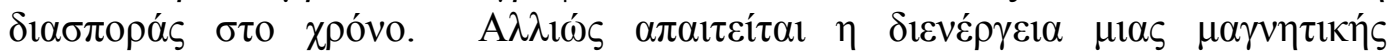

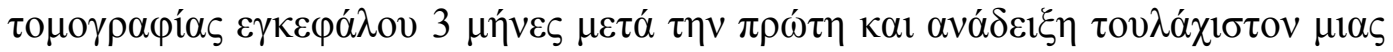

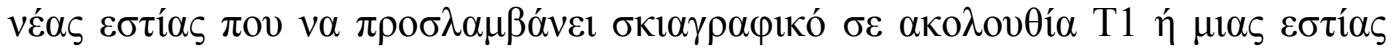

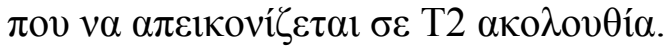

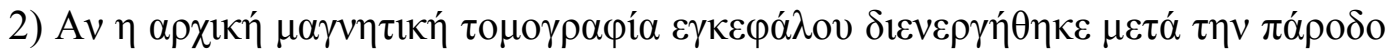

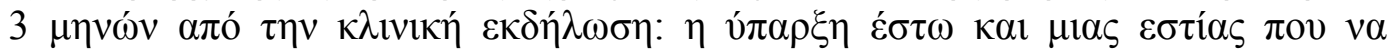

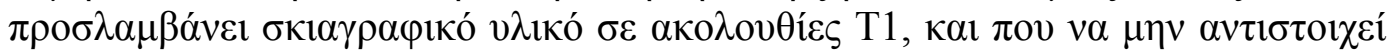

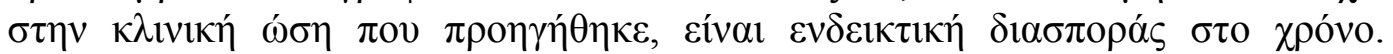

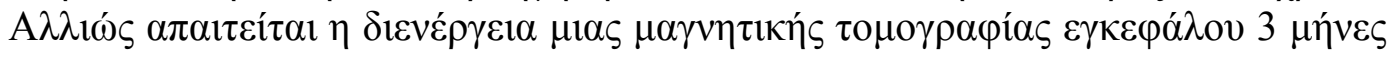

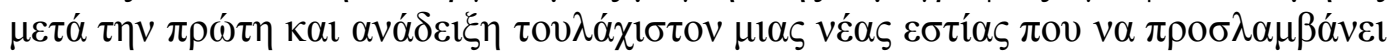

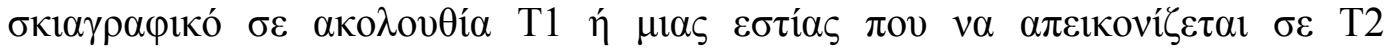

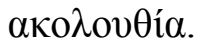




\section{ME}

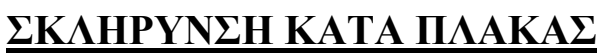

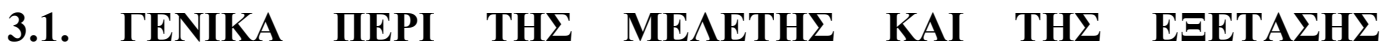

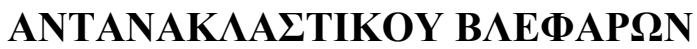

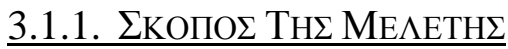

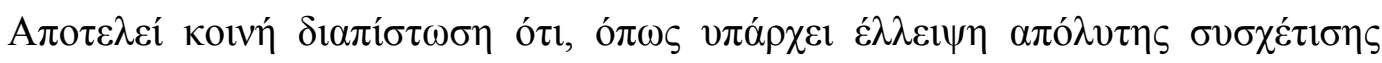

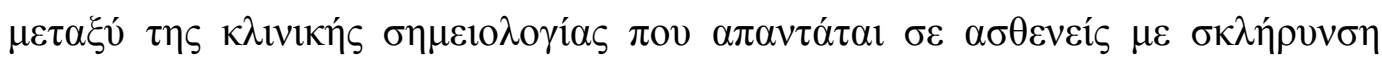

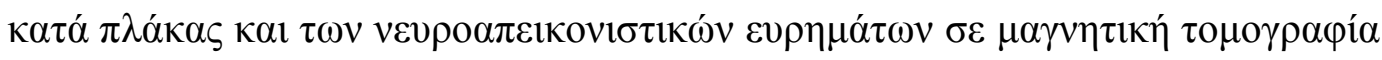

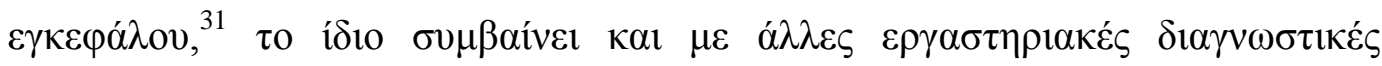

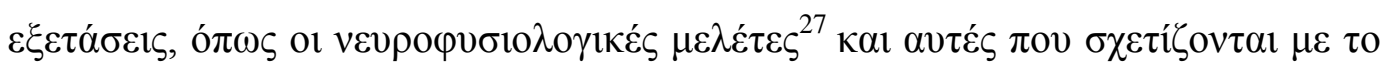

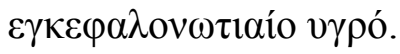

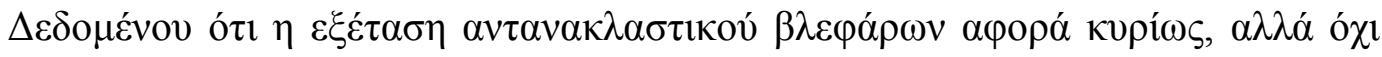

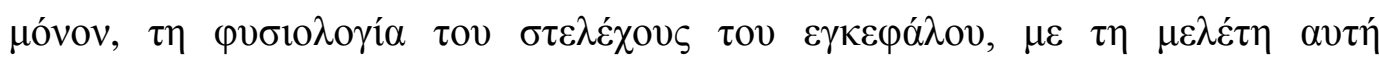

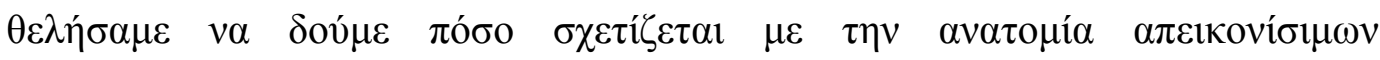

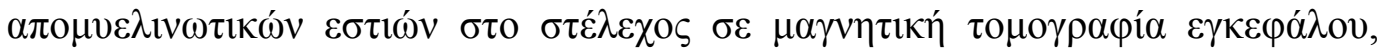

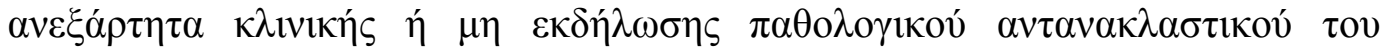

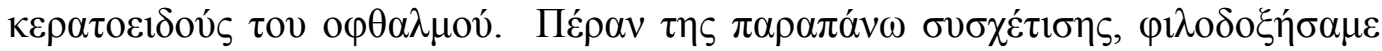

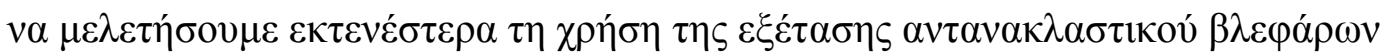

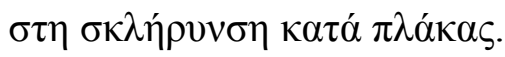

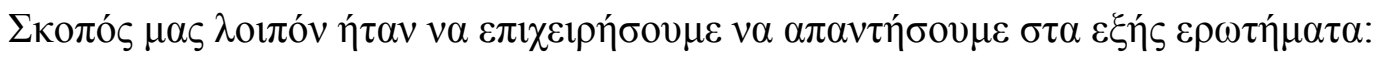

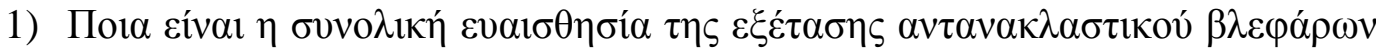

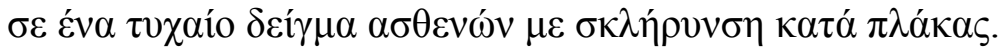

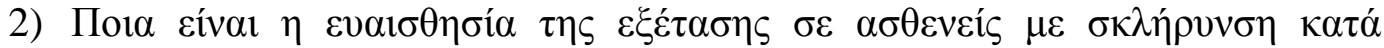

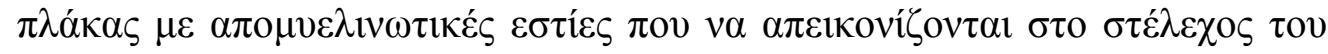
$\varepsilon \gamma \kappa \varepsilon \varphi \alpha ́ \lambda o v$.

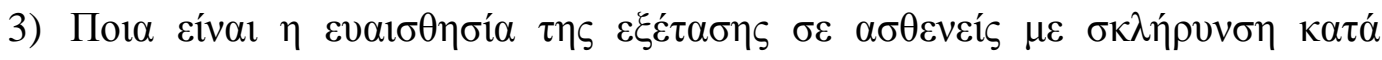

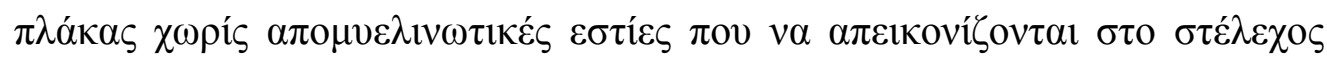

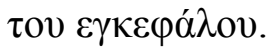




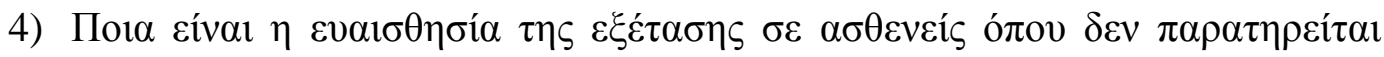

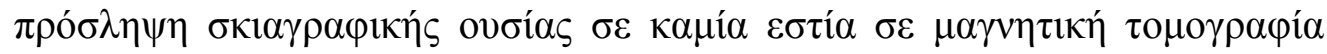
$\varepsilon \gamma \kappa \varepsilon \varphi \alpha ́ \lambda o v$.

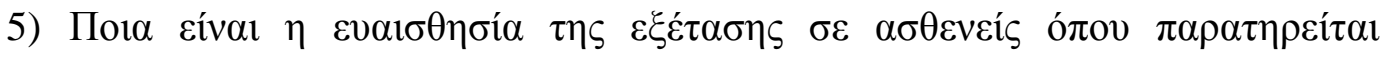

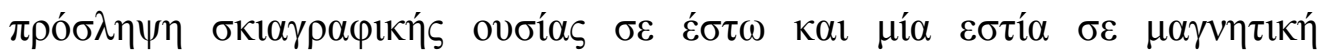

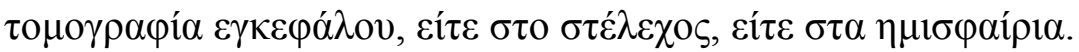

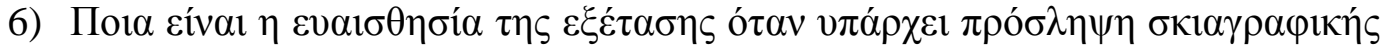

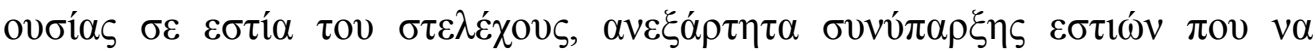

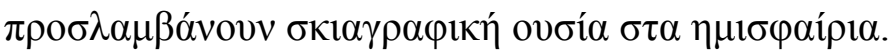

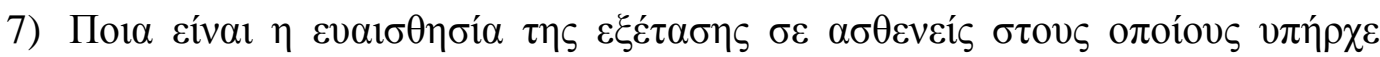

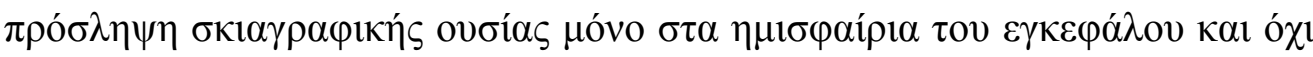

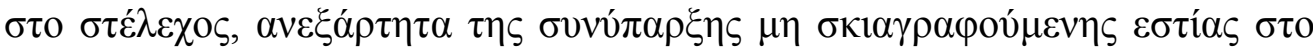
$\sigma \tau \varepsilon \dot{\lambda} \varepsilon \chi \chi \circ \varsigma$.

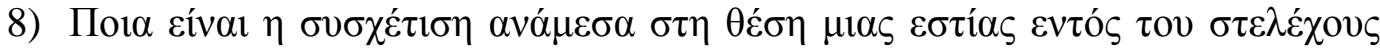

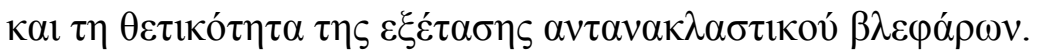

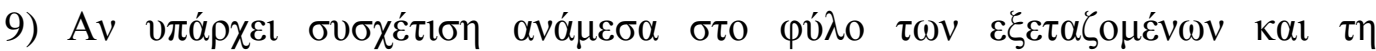

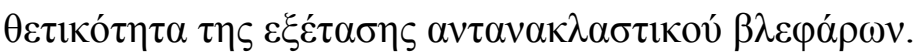

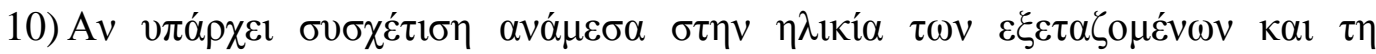

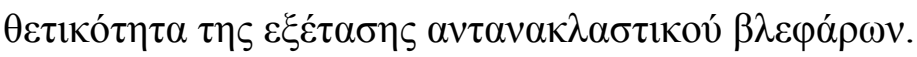

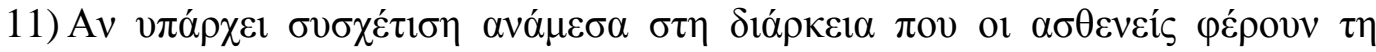

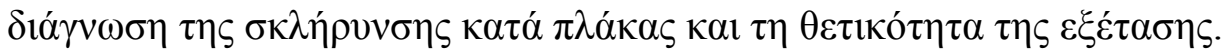

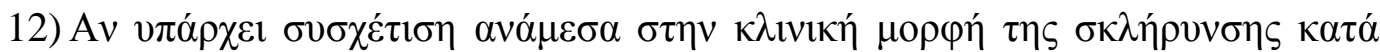

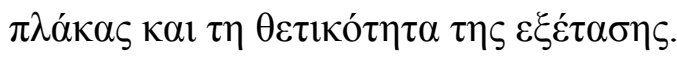

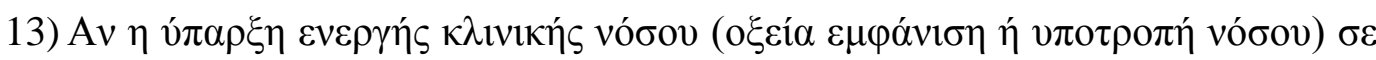

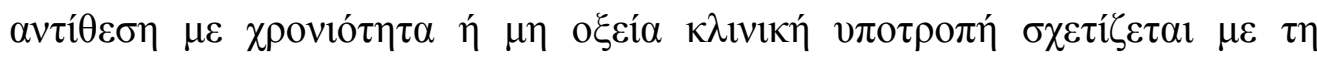

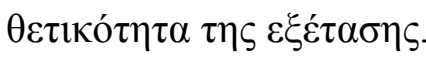

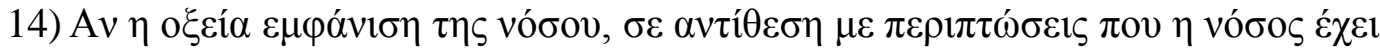

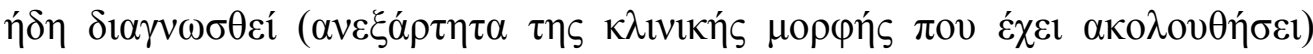
$\sigma \chi \varepsilon \tau i \zeta \zeta \varepsilon \tau \alpha 1 \mu \varepsilon \tau \eta \theta \varepsilon \tau 1 \kappa o ́ \tau \eta \tau \alpha \tau \eta \varsigma \varepsilon \xi \dot{\varepsilon} \tau \alpha \sigma \eta \zeta$.

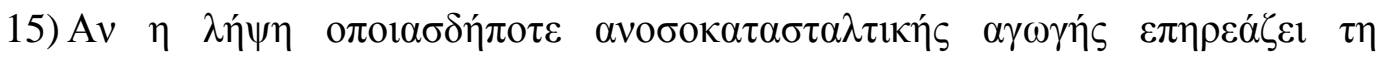

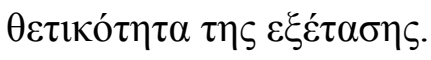




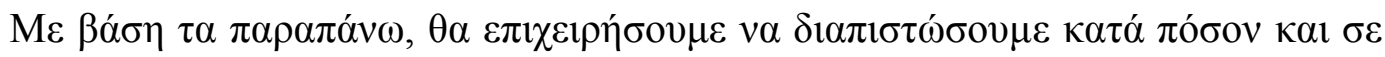

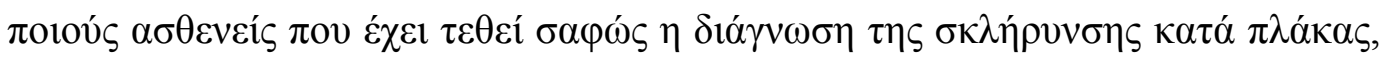

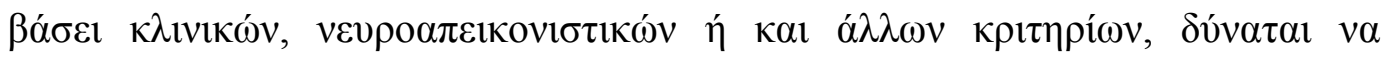

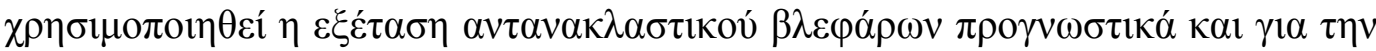

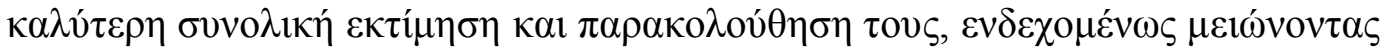

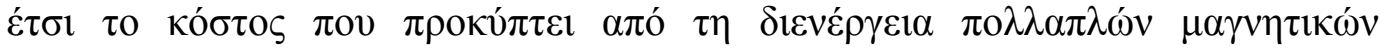

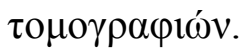




\subsubsection{KPITHPIA ENTA $\Xi H \Sigma$ KAI $\Sigma$ TOIXEIA ПEPI T $\Omega$ N A $\Sigma \Theta E N \Omega N$ TH $\Sigma$ ME $\Lambda$ ETH $\Sigma$}

\subsubsection{KAINIKA KPITHPIA}

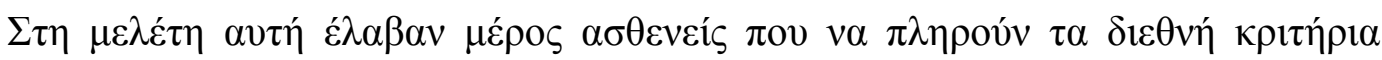

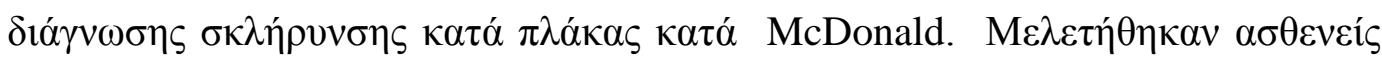

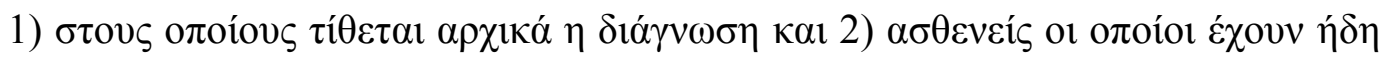

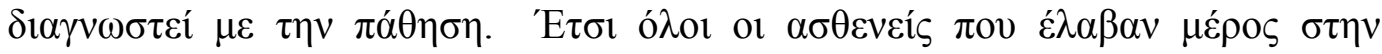

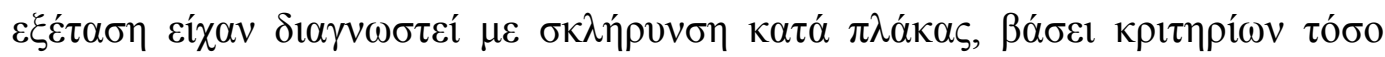

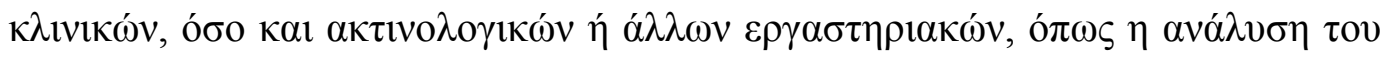

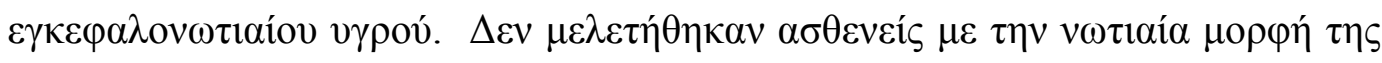

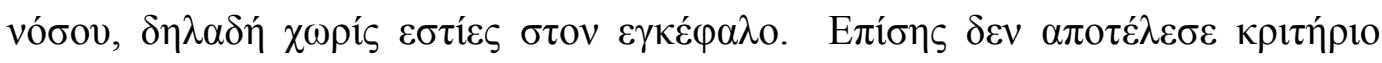

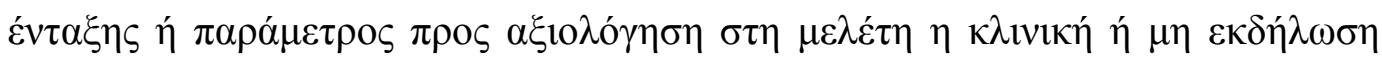

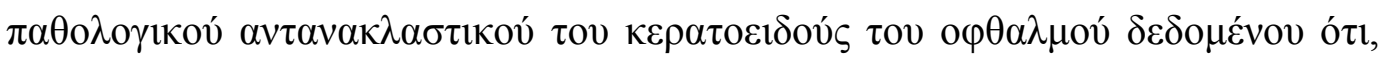

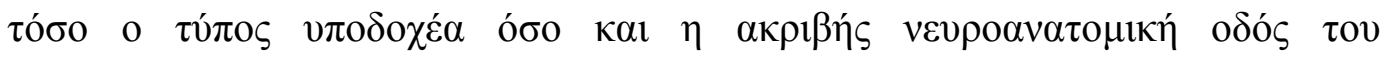

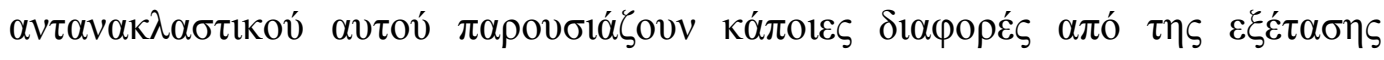

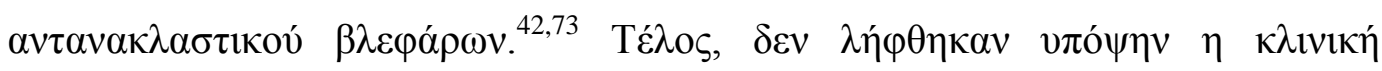

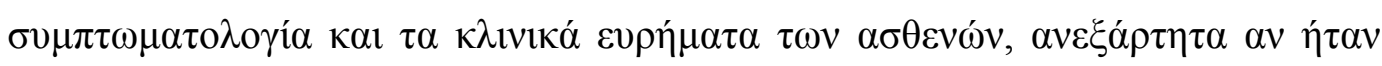

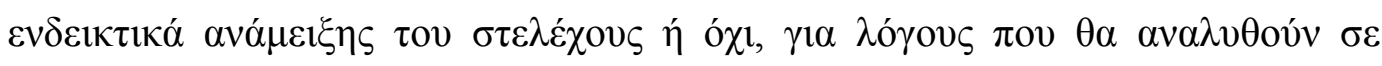
$\varepsilon \pi$ ó $\mu \varepsilon v \eta \varepsilon v o ́ \tau \eta \tau \alpha(\sigma .75)$.

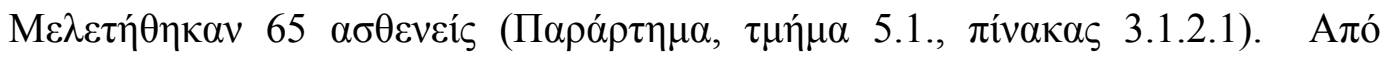

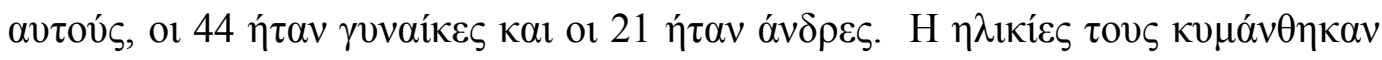

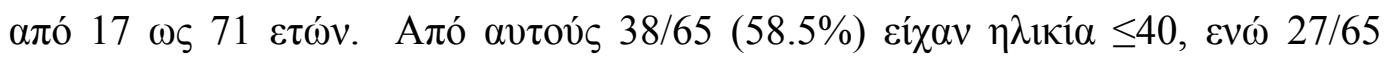

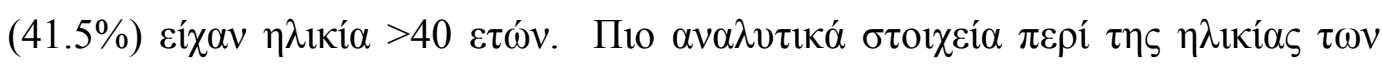

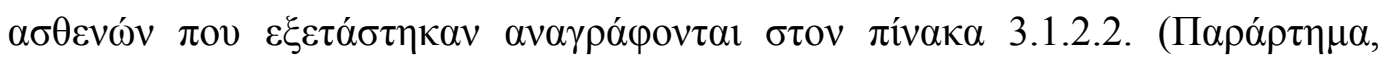
$\tau \mu \eta ́ \mu \alpha$ 5.1.).

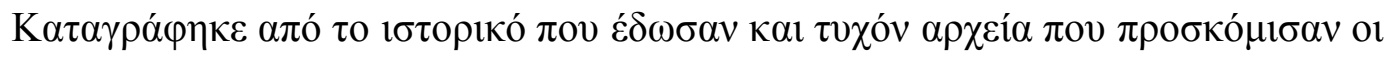

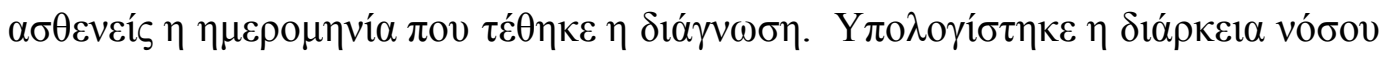

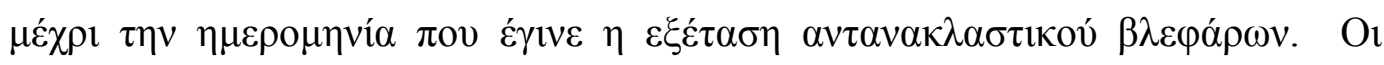

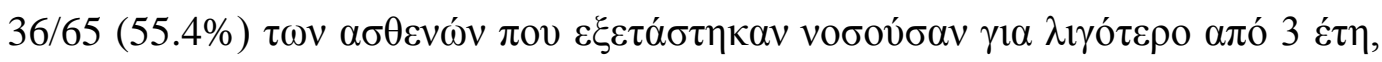

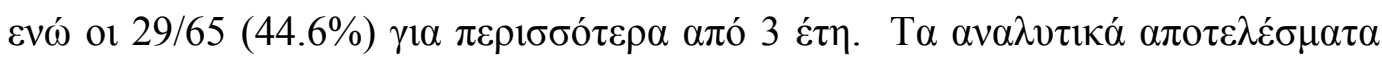

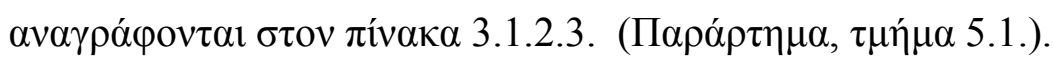




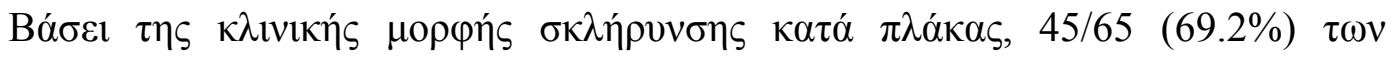

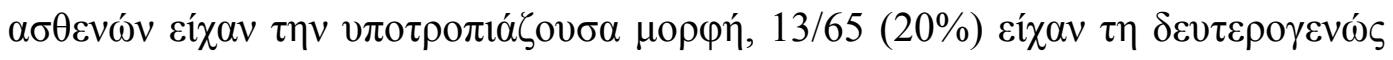

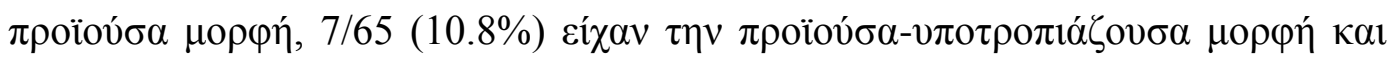

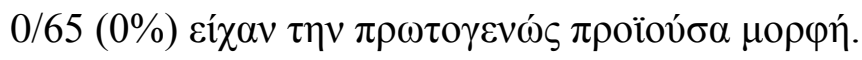

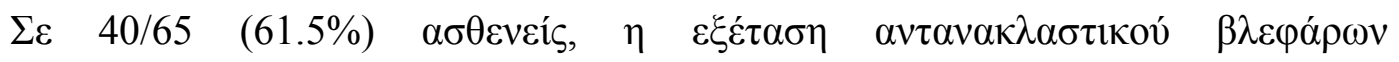

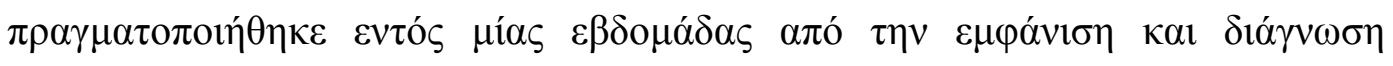

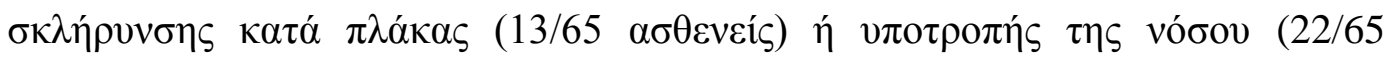

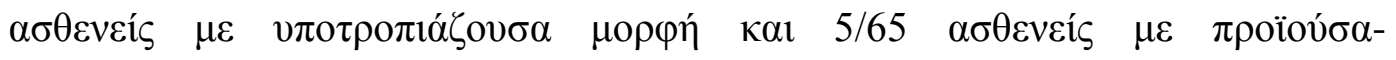

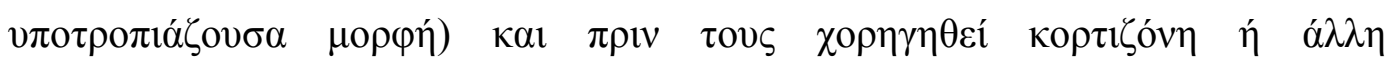

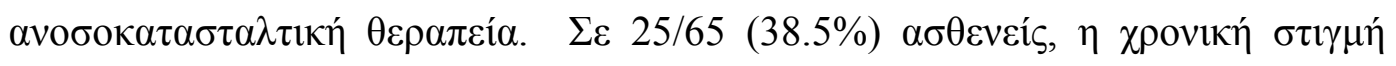

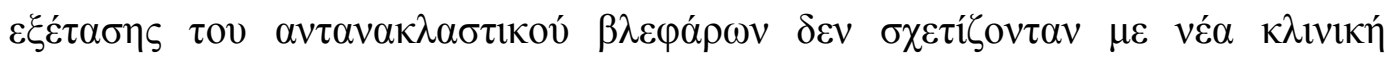

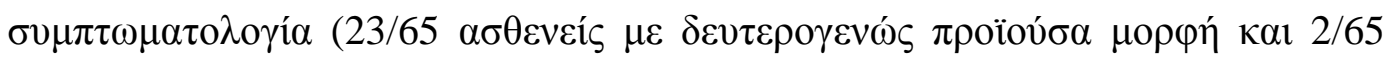

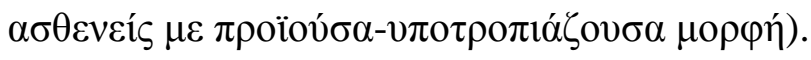

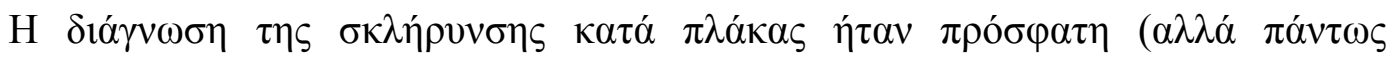

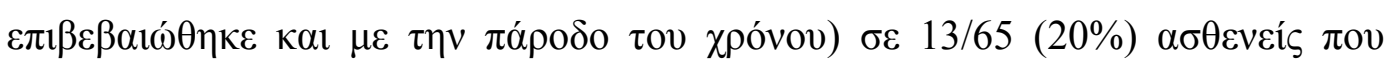

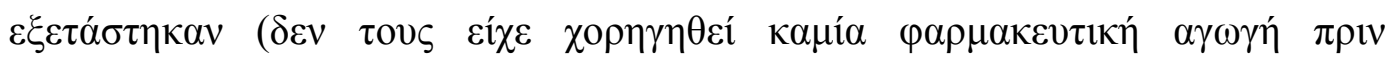

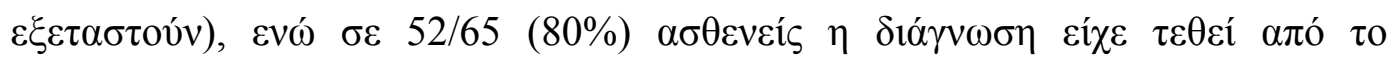
$\pi \alpha \rho \varepsilon \lambda \theta$ óv.

\subsubsection{2. АКТINOАОГIКА КРІТНРIA}

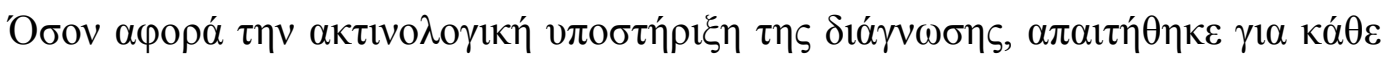

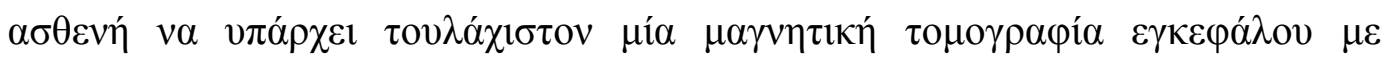

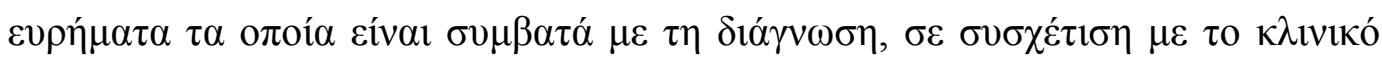

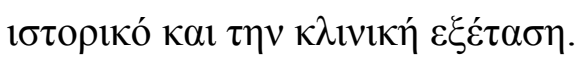

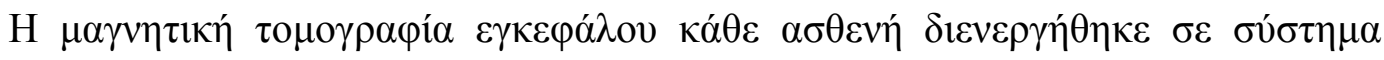

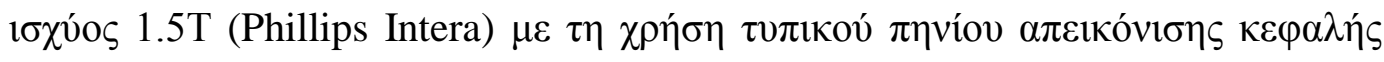

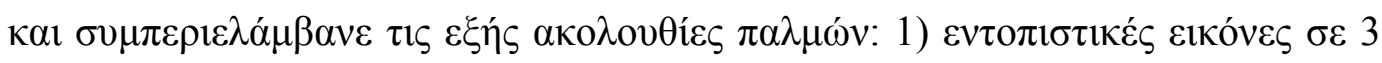

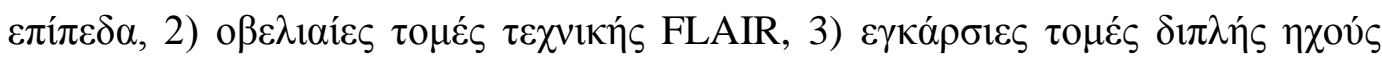

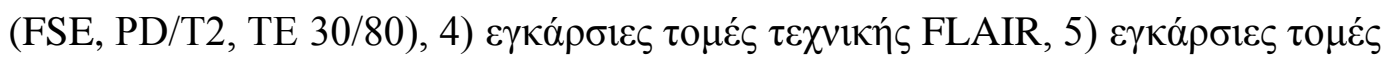

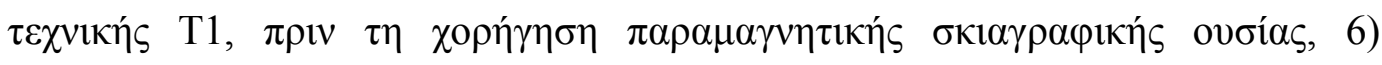

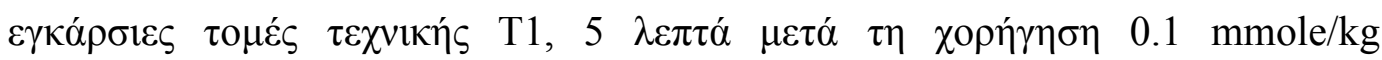




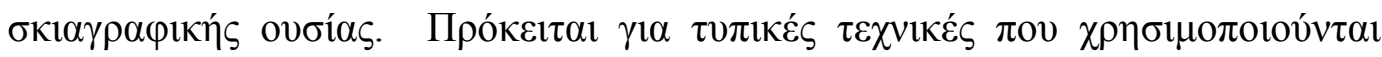
$\delta ı \theta v \omega ́ \varsigma^{148}$

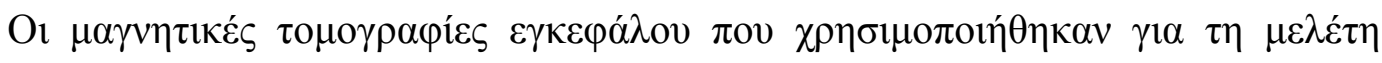

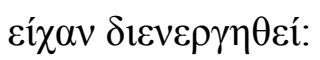

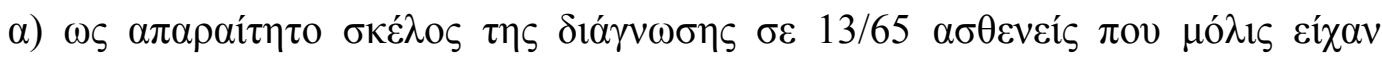

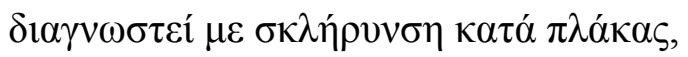

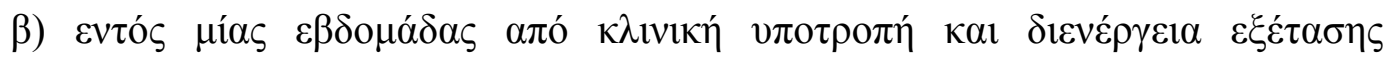

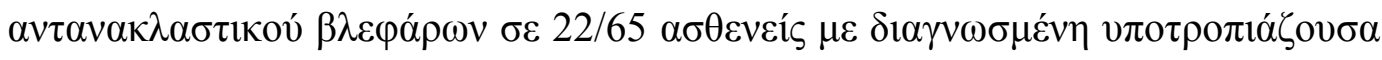

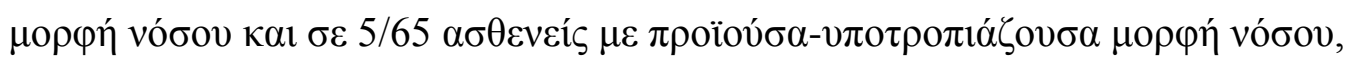

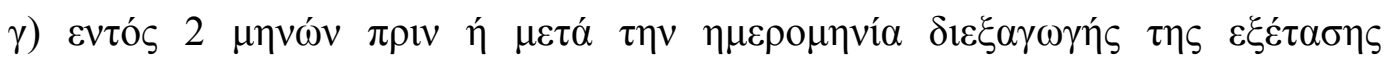

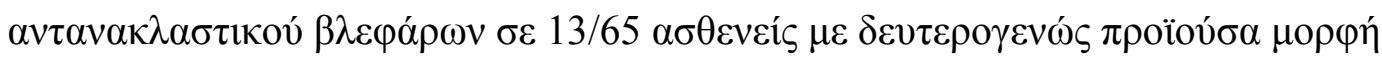

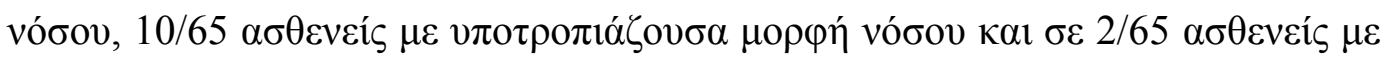

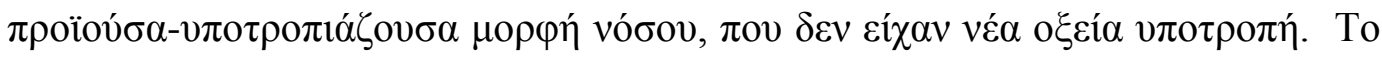

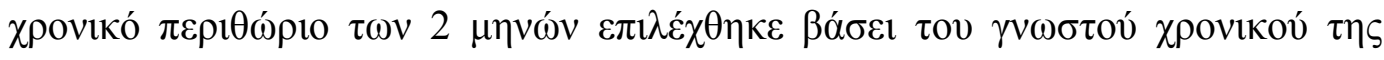

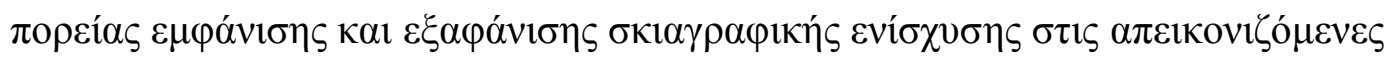
$\varepsilon \sigma \tau i ́ \varepsilon \varsigma$.

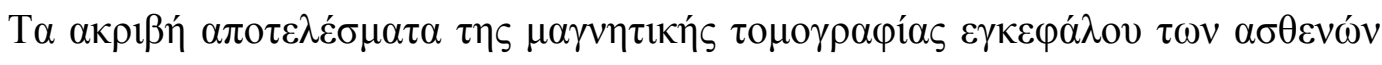

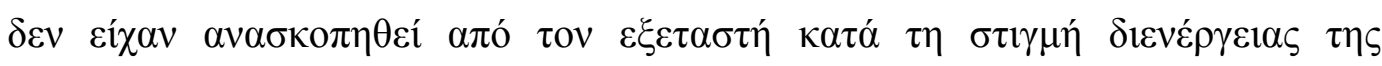
$\varepsilon \xi \dot{\varepsilon} \tau \alpha \sigma \eta \varsigma \alpha v \tau \alpha \nu \alpha \kappa \lambda \alpha \sigma \tau \imath \kappa o v ́ ~ \beta \lambda \varepsilon \varphi \alpha ́ \rho \omega v$.

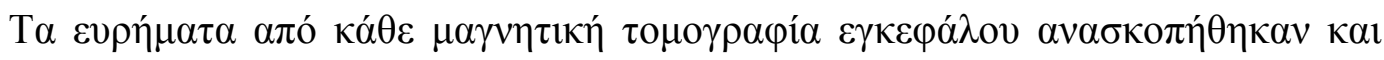

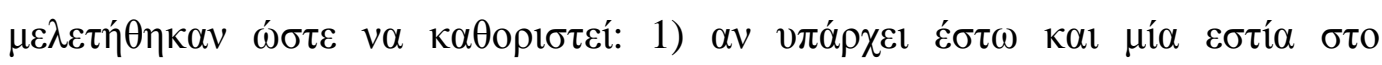

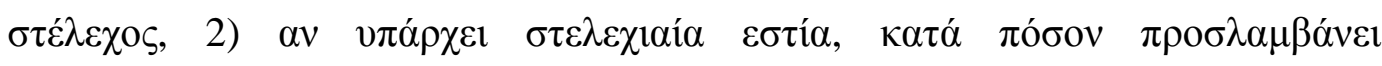

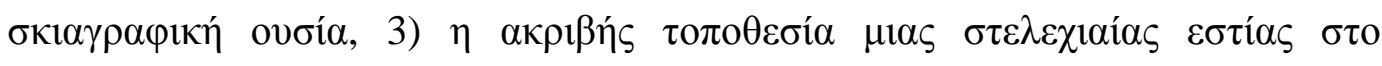

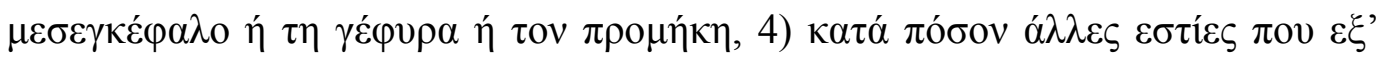

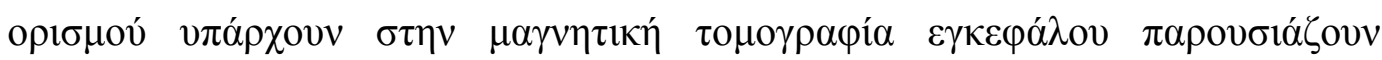

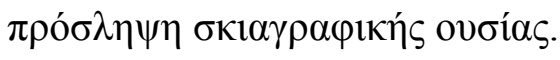

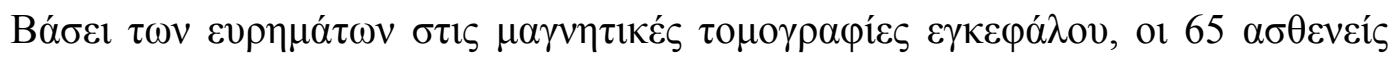

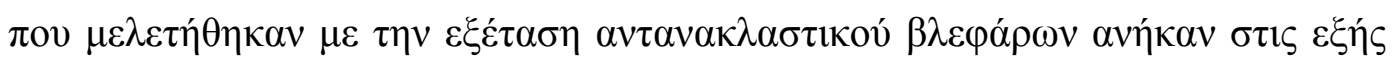

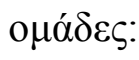




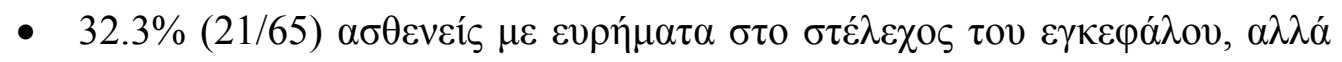

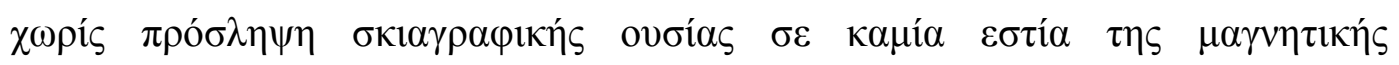

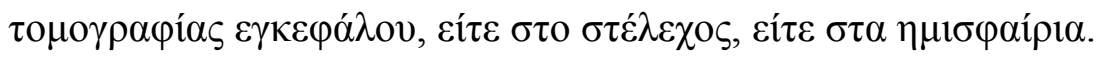

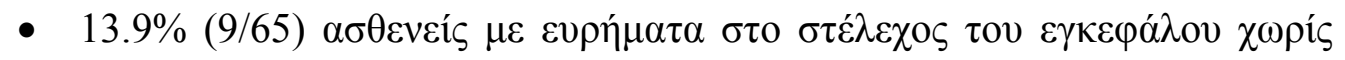

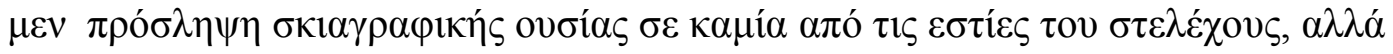

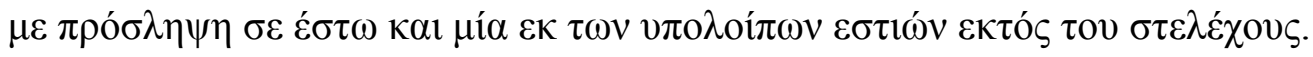

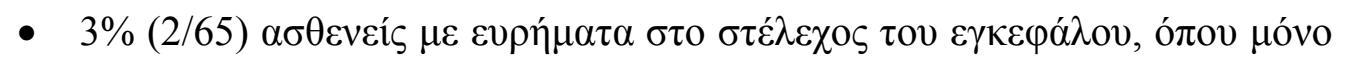

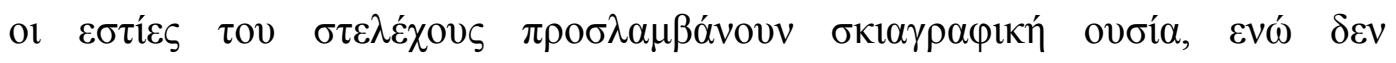

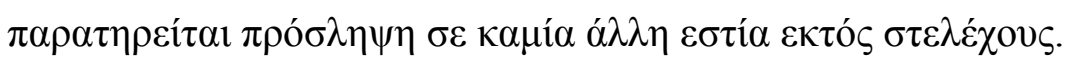

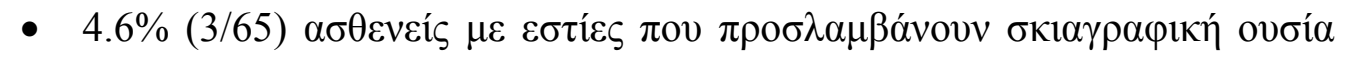

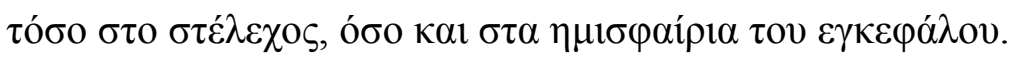

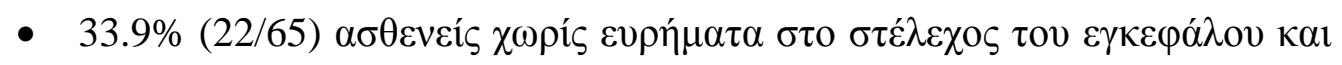

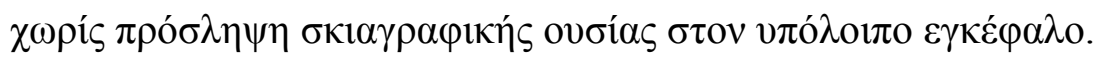

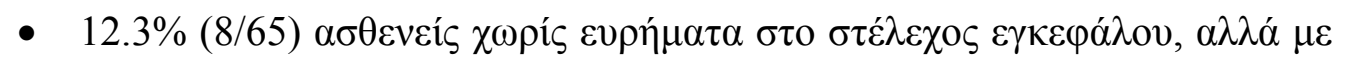

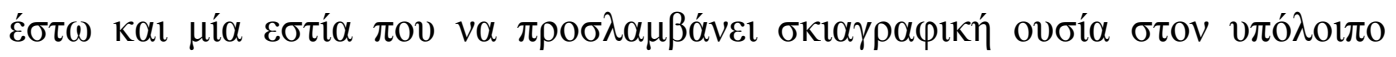

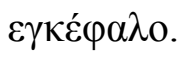




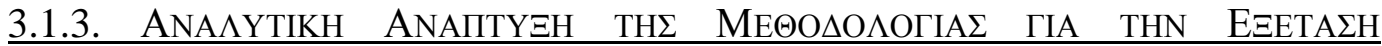

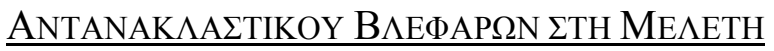

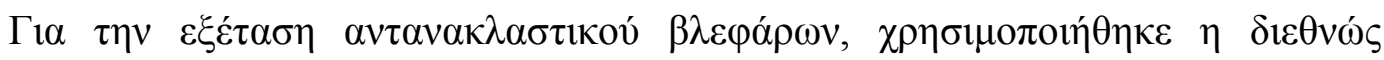

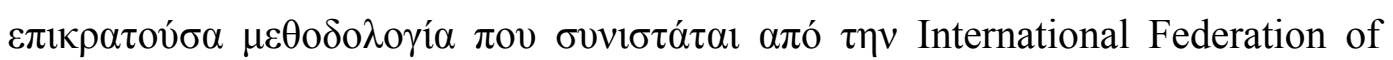

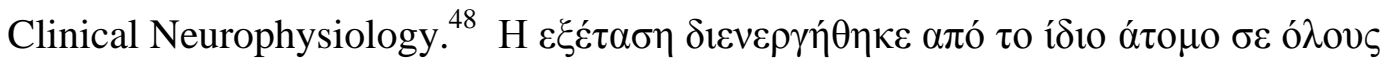

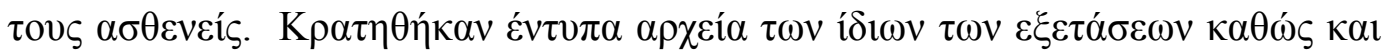

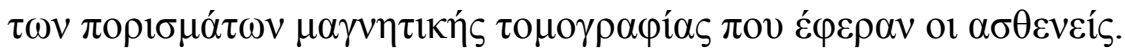

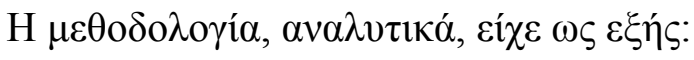

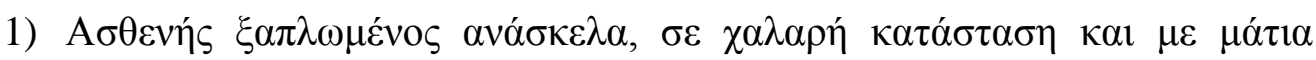

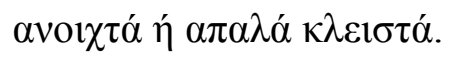

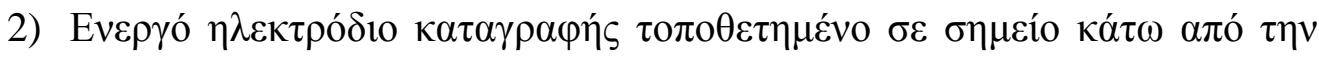

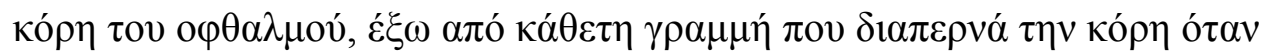

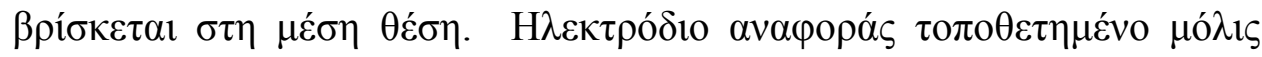

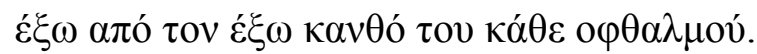

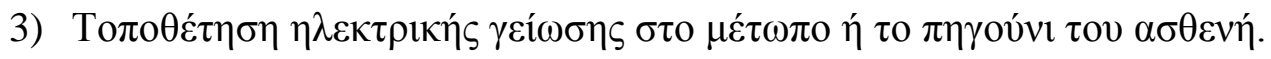

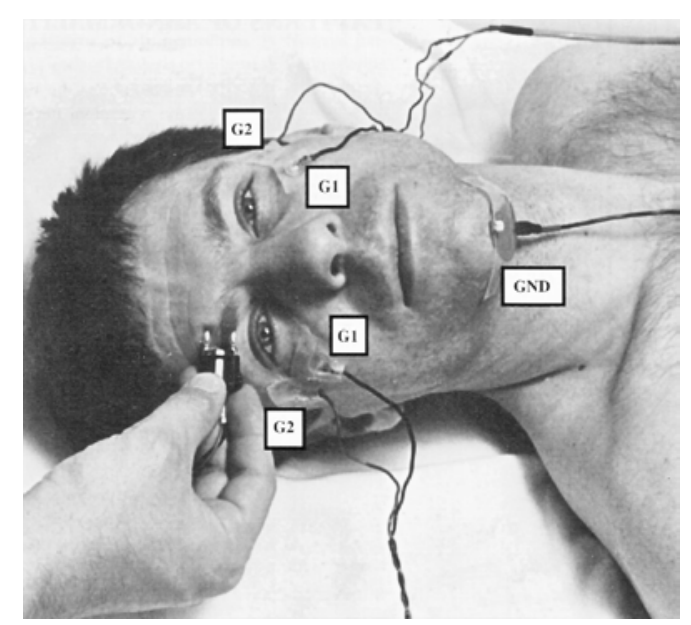

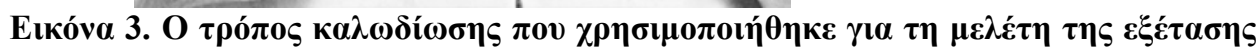

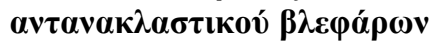

A $\pi$ ó D.Preston, B.Shapiro, Electromyography and Neuromuscular Disorders, $1^{\eta}$ Eк $\delta$., 1998, Butterworth-Heinemann, $\sigma .59$

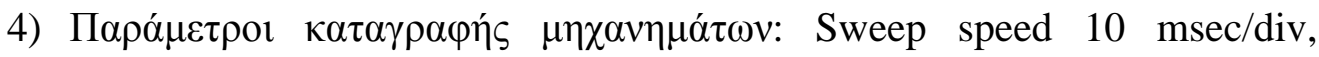
Sensitivity $100-200 \mu$ V/div, Motor Filter settings $10 \mathrm{~Hz} \& 10 \mathrm{kHz}$.

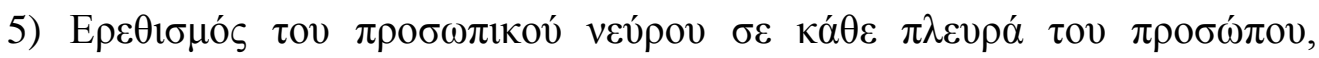

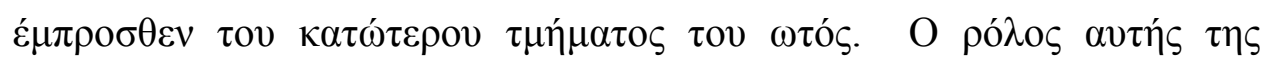

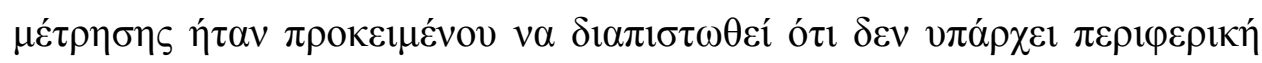




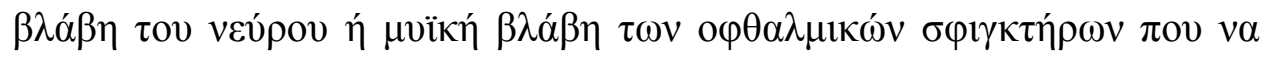

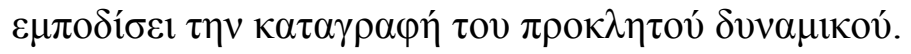

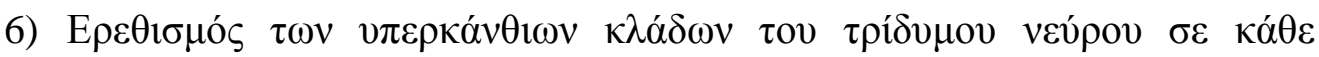

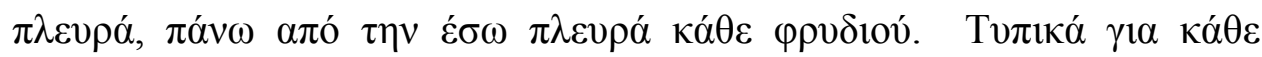

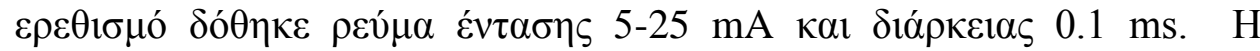

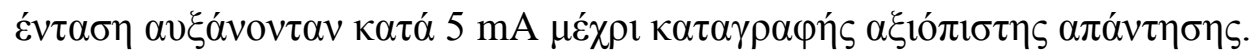

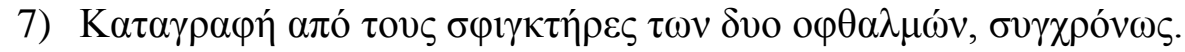

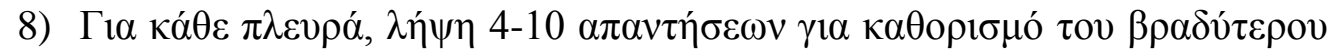

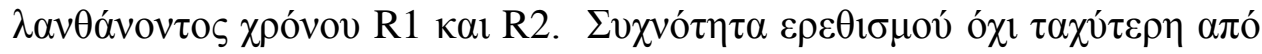

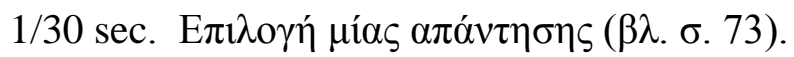

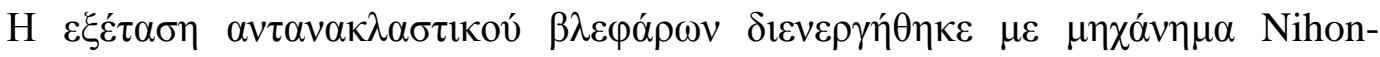

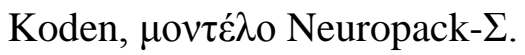




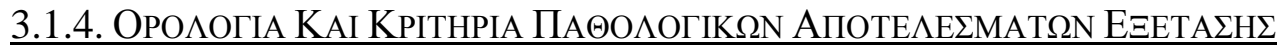

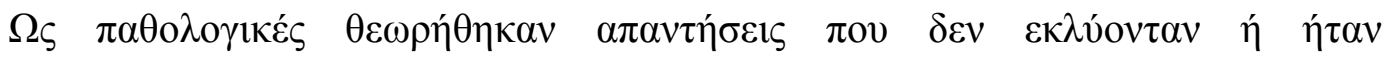

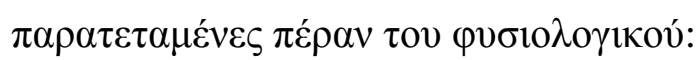

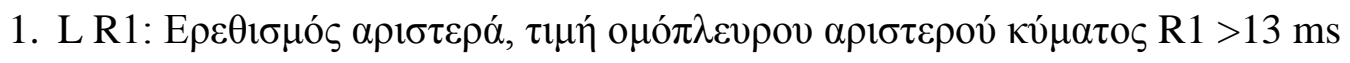

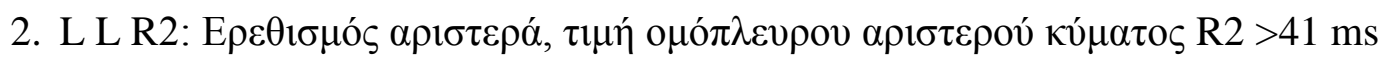

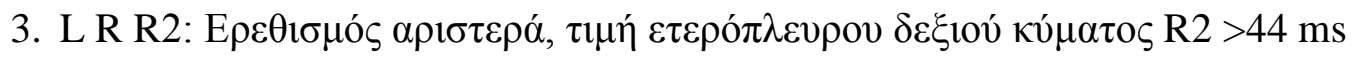

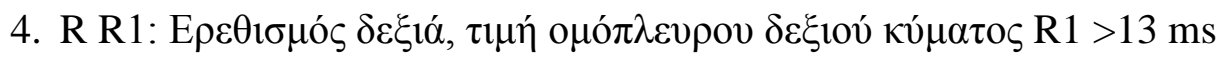

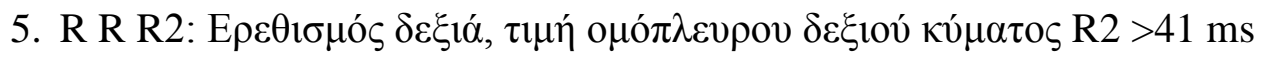

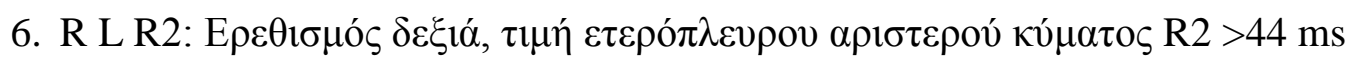

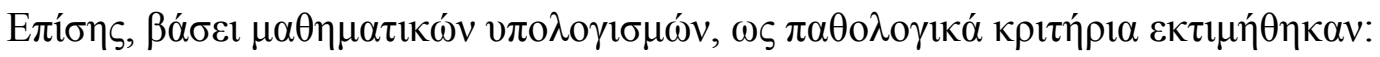

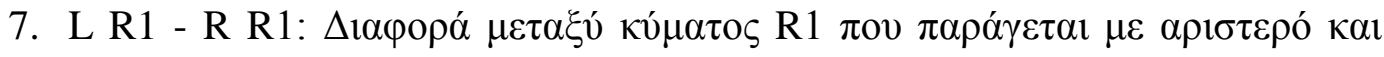
$\delta \varepsilon \xi$ ıó $\varepsilon \rho \varepsilon \theta \imath \sigma \mu o ́ ~>1.2 \mathrm{~ms}$

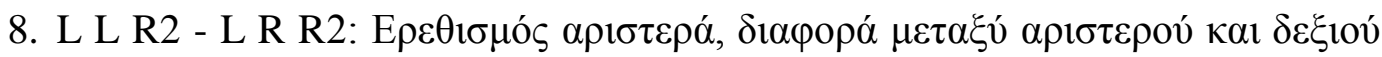
$\kappa v ́ \mu \alpha \tau \circ \varsigma \mathrm{R} 2>5 \mathrm{~ms}$

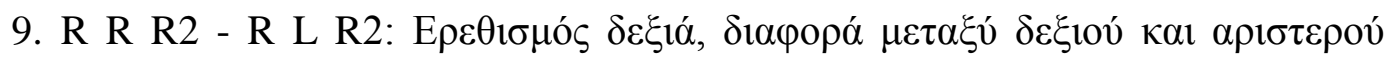
$\kappa v ́ \mu \alpha \tau \circ \varsigma \mathrm{R} 2>5 \mathrm{~ms}$

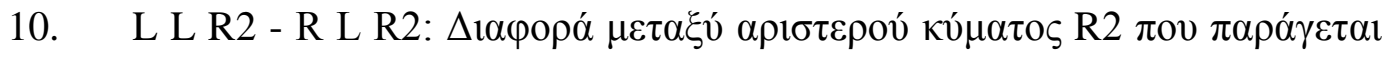
$\alpha \pi$ ó $\alpha \rho \imath \sigma \tau \varepsilon \rho$ ó $\kappa \alpha \imath \delta \varepsilon \xi$ ió $\varepsilon \rho \varepsilon \theta 1 \sigma \mu$ ó $>7$ ms

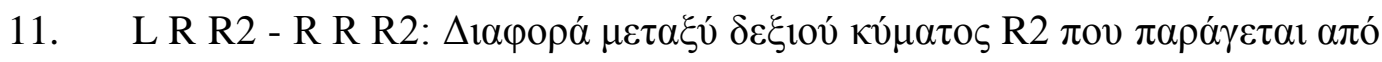

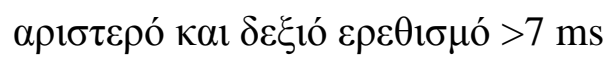

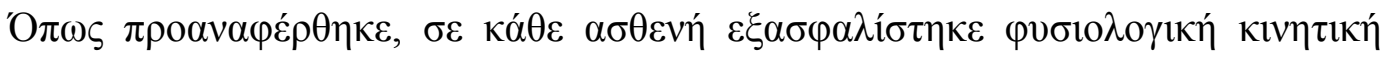

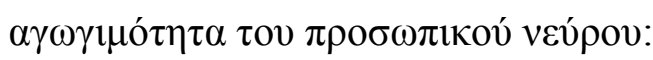

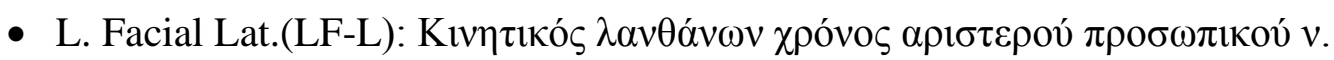

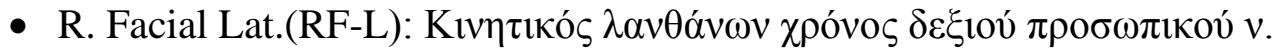
Фvбıһо

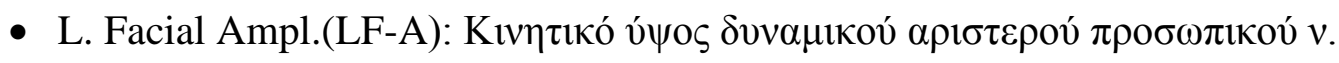

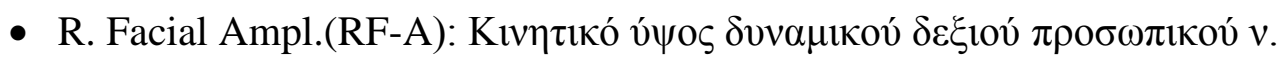

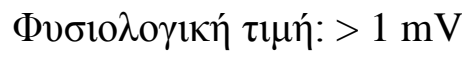




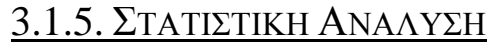

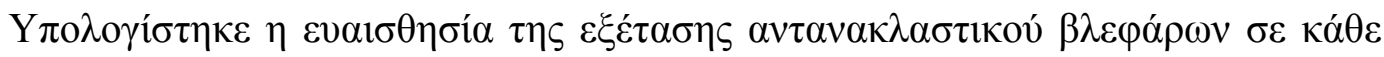

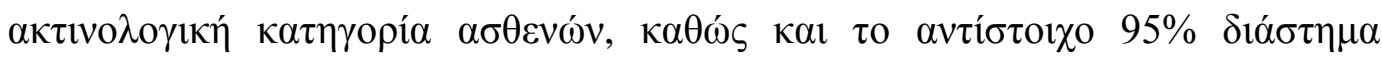
$\varepsilon \mu \pi \imath \sigma \tau о \sigma u ́ v \eta \varsigma$.

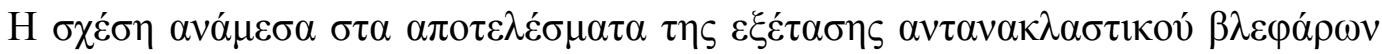

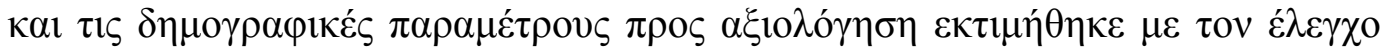
$\chi^{2}$ 
3.1.6. EN $\triangle$ EIKTIKA $\triangle$ IAГPAMMATA TH $\Sigma$ E $\Xi E T A \Sigma H \Sigma$ ANTANAK $\Lambda$ ALTIKOY B $\Lambda$ EФAP $\Omega N$

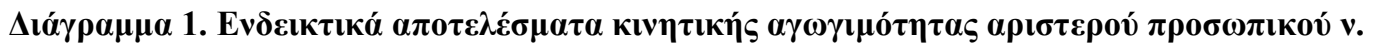

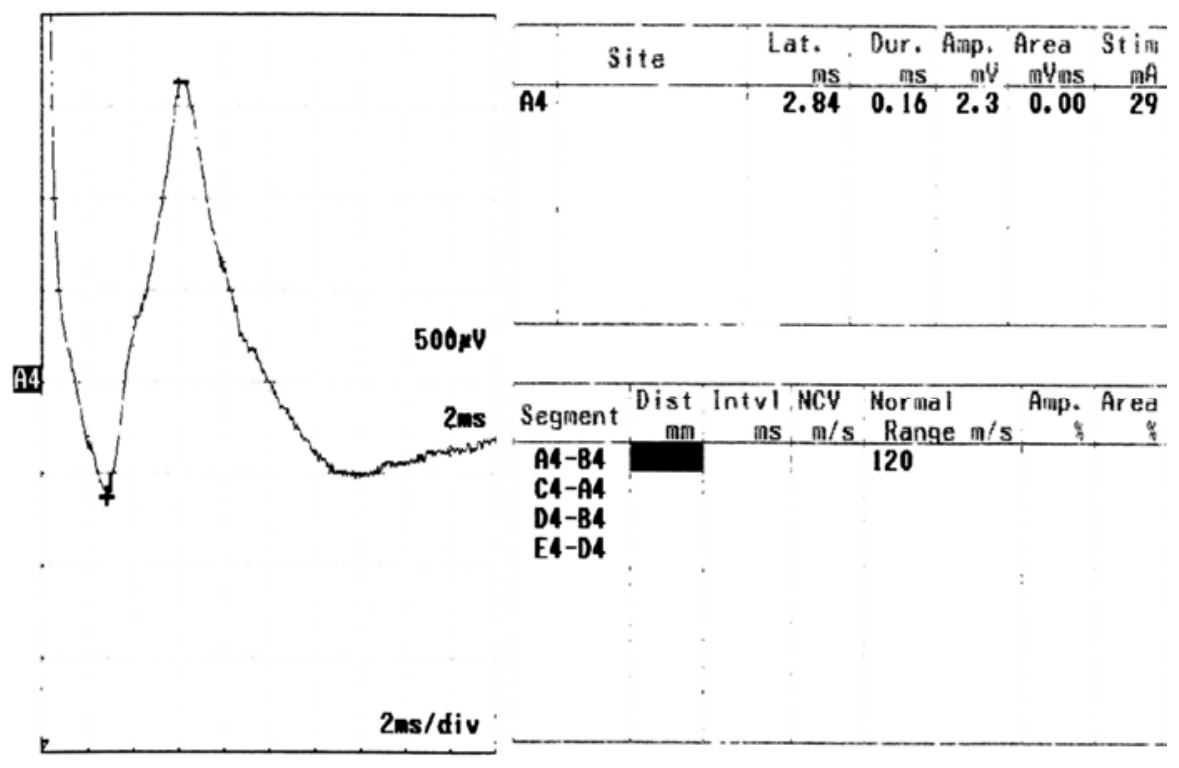

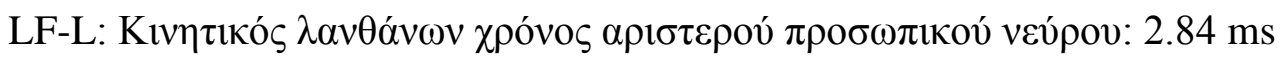

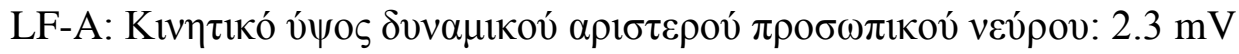

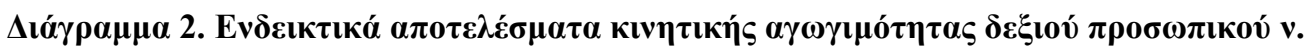

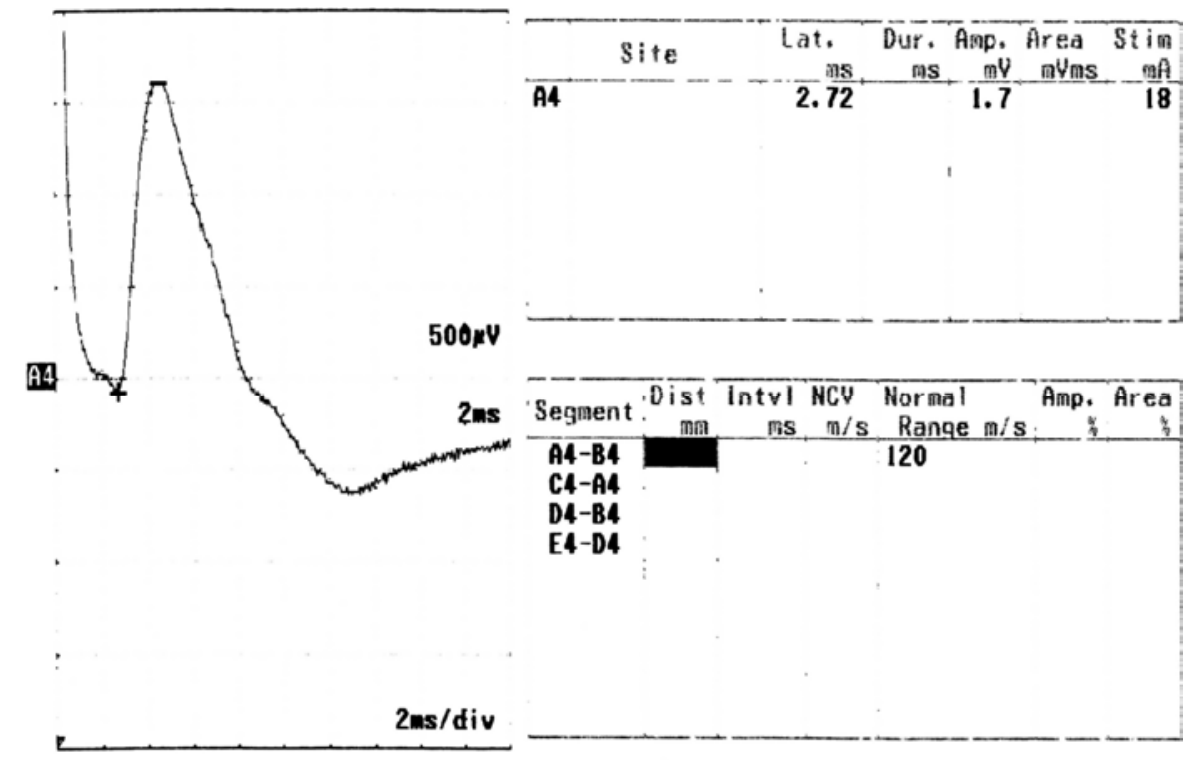

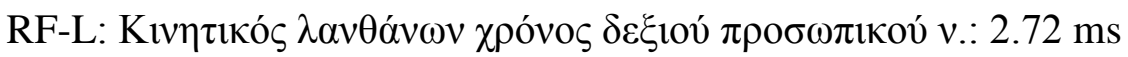

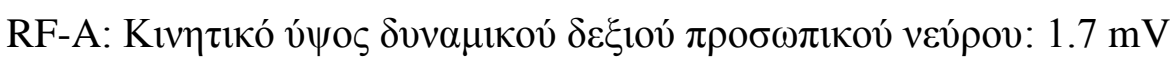




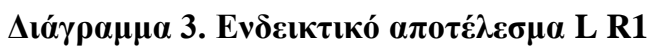

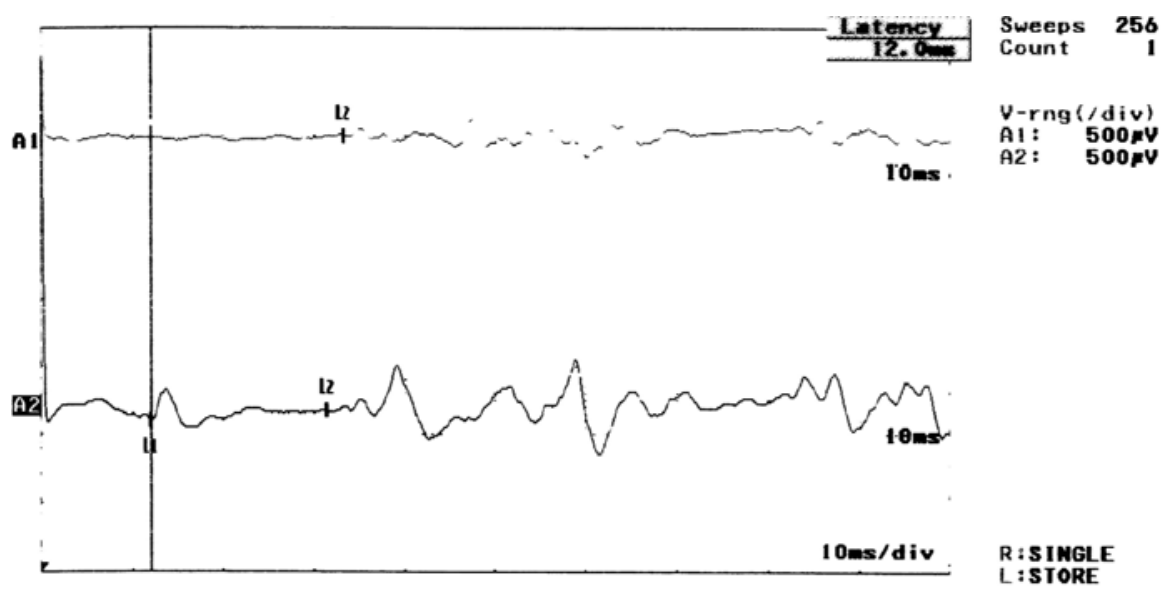

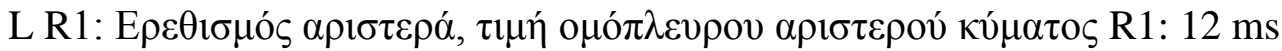

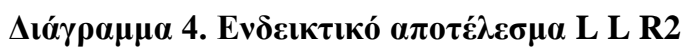

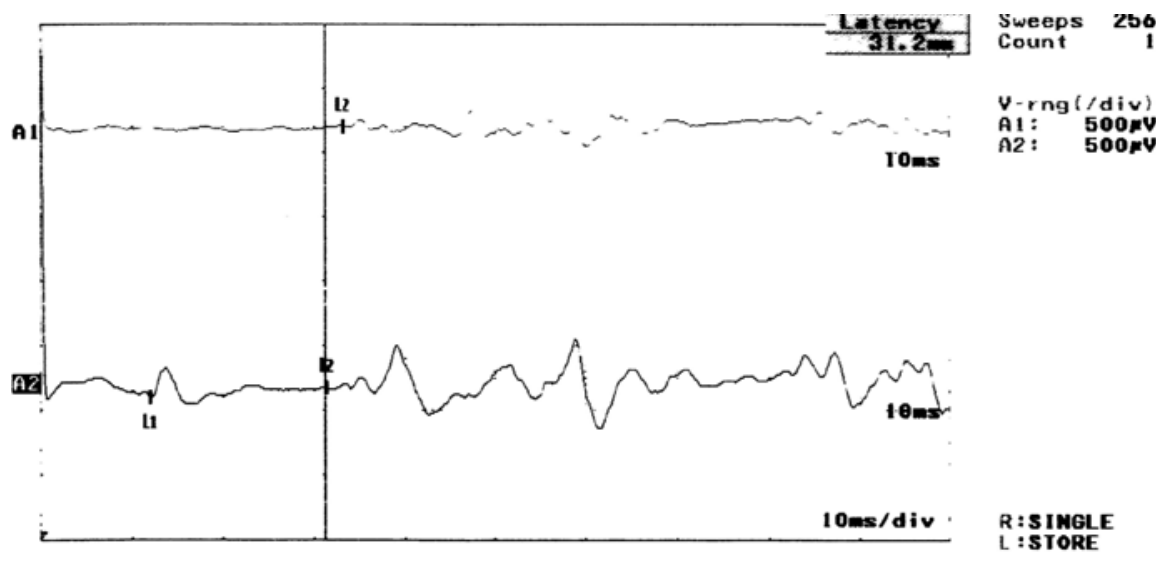

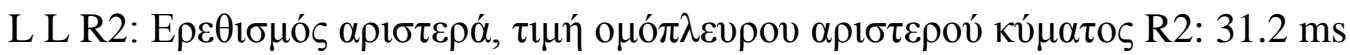

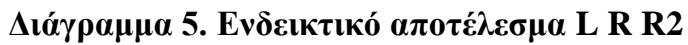

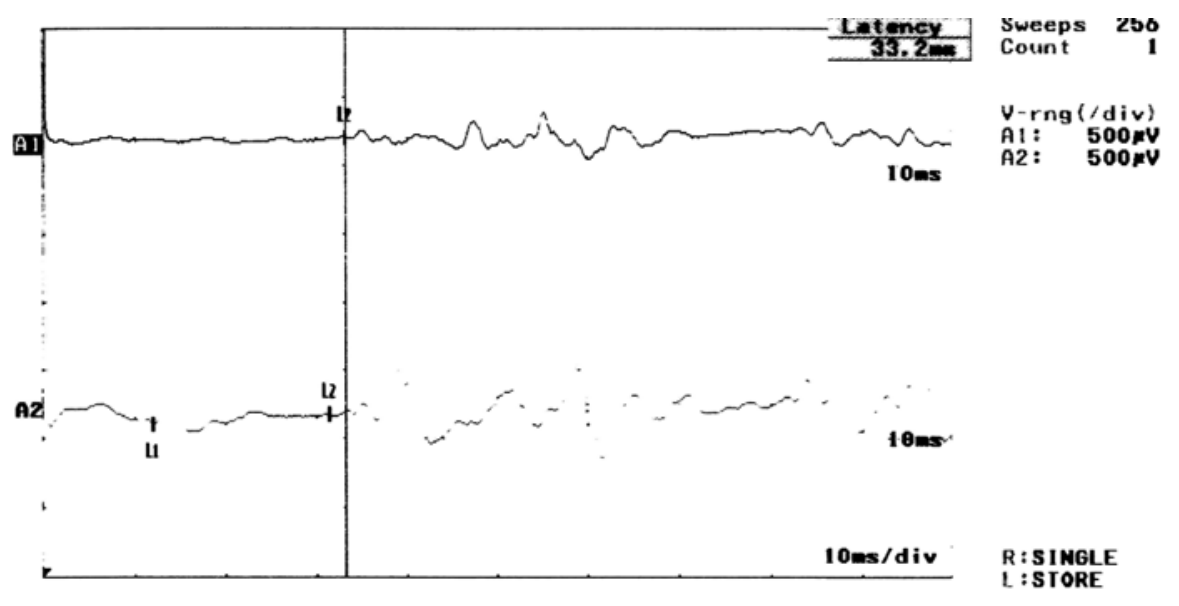

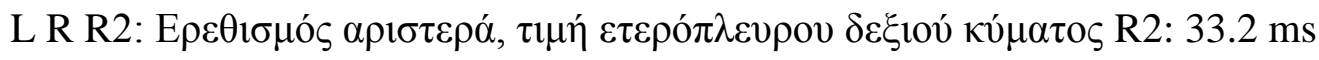




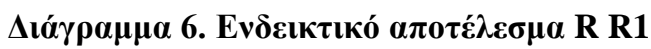

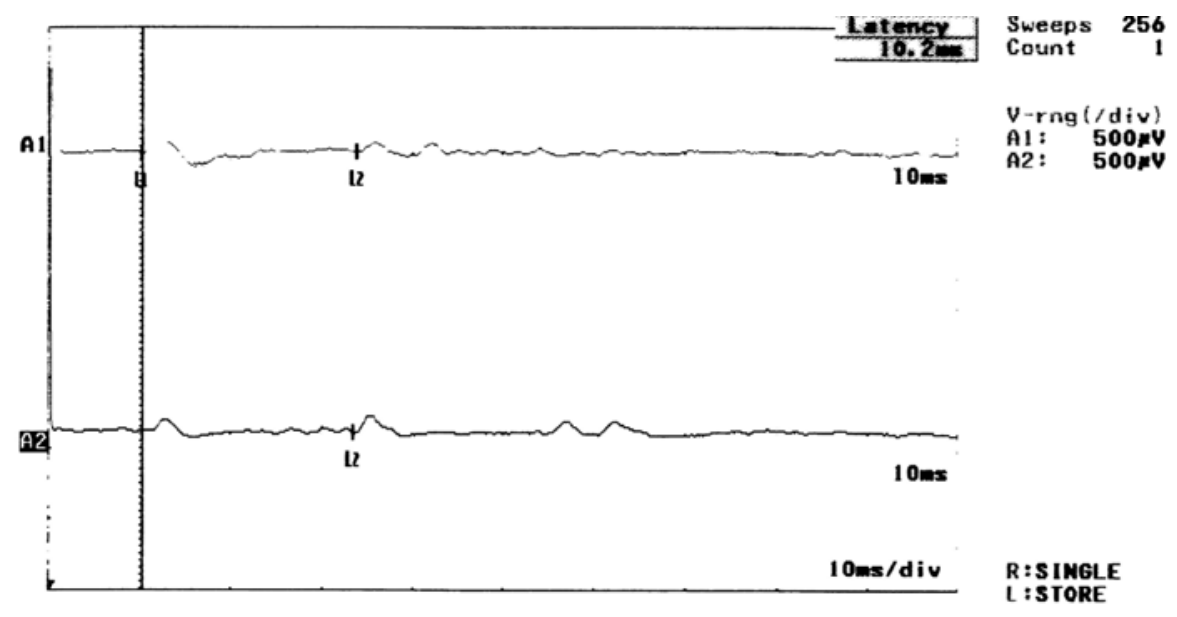

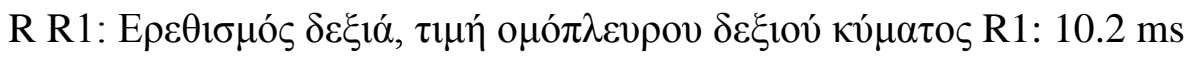

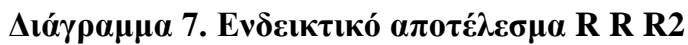

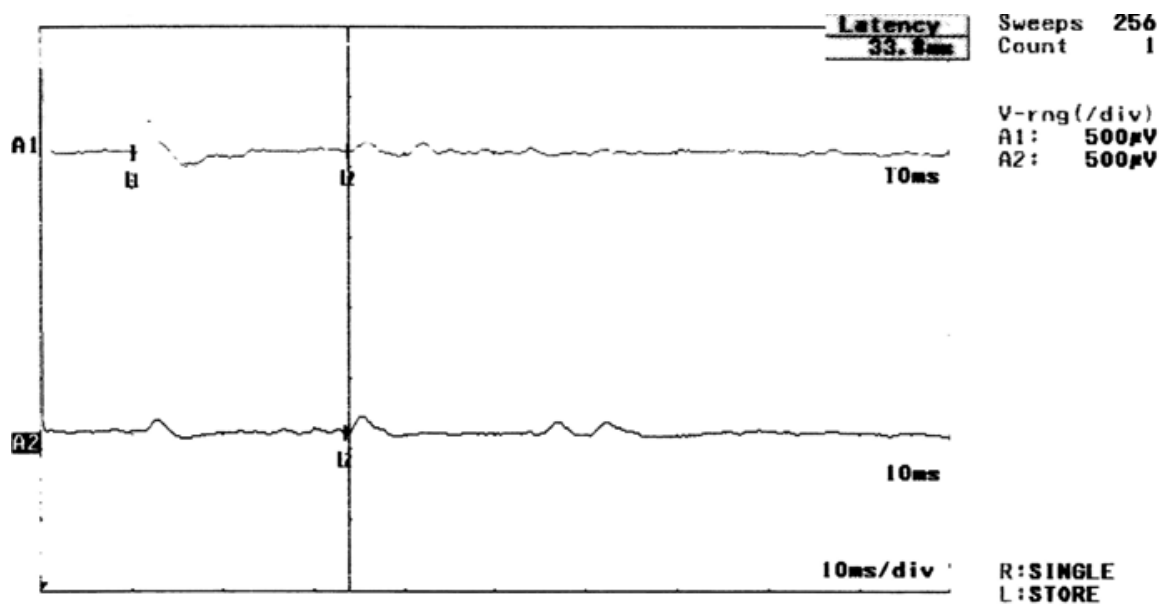

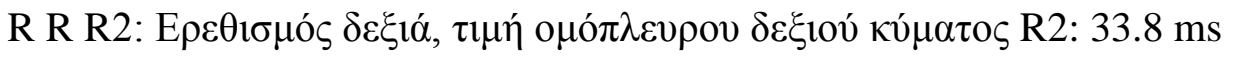

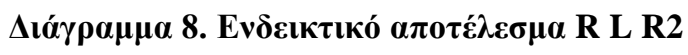

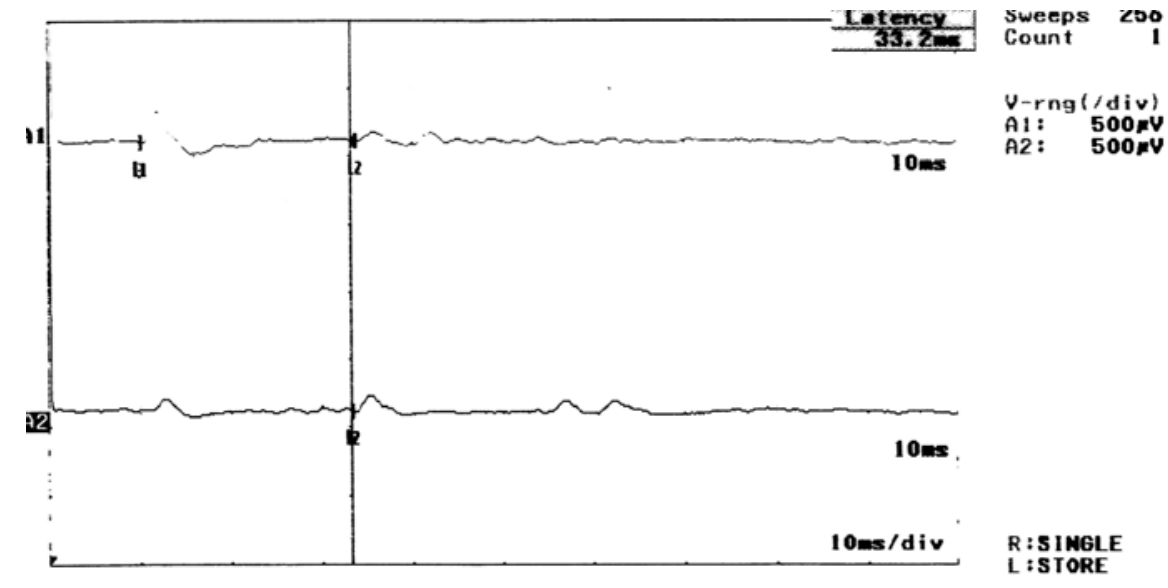

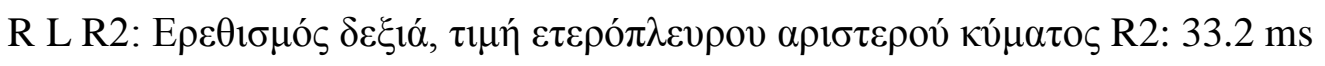




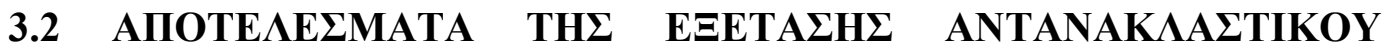

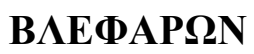

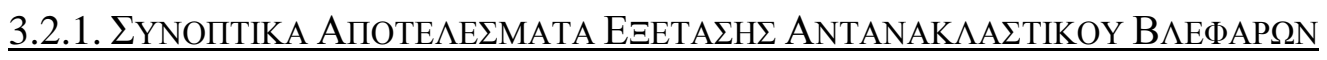

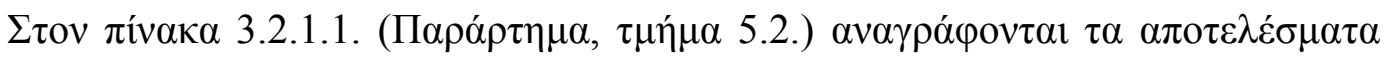

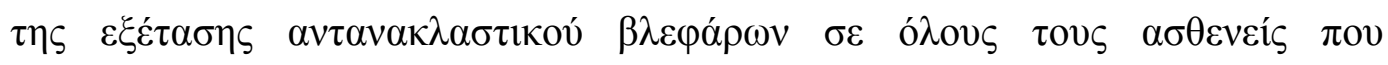

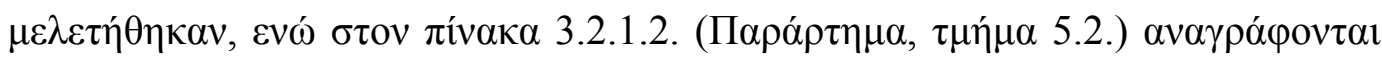
$\tau \alpha$ a

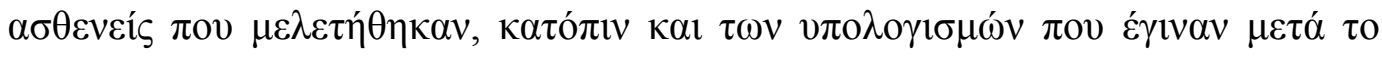

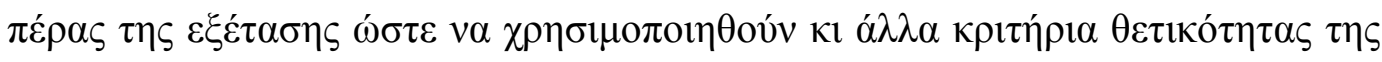
$\varepsilon \xi \dot{\varepsilon} \tau \alpha \sigma \eta \zeta$.

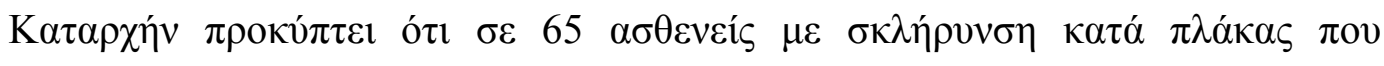

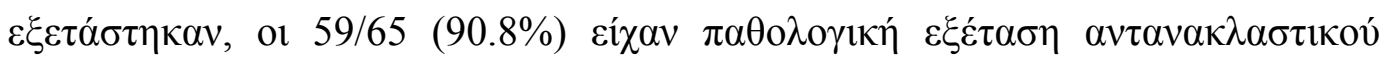

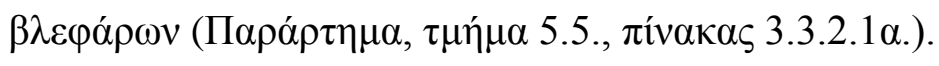

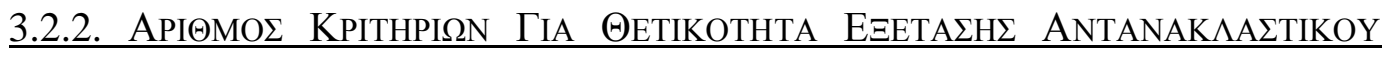

\section{$\underline{\mathrm{B} \Lambda \mathrm{E} \Phi \mathrm{AP} \Omega \mathrm{N}}$}

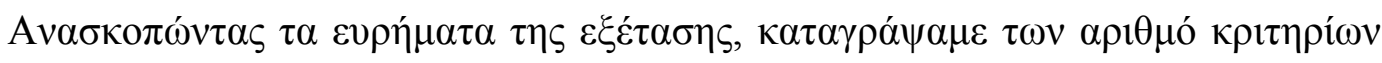

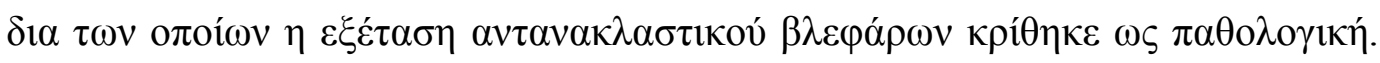

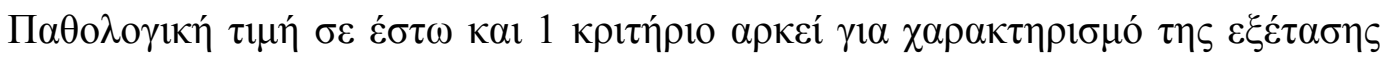

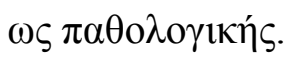

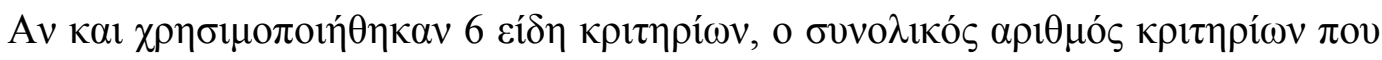

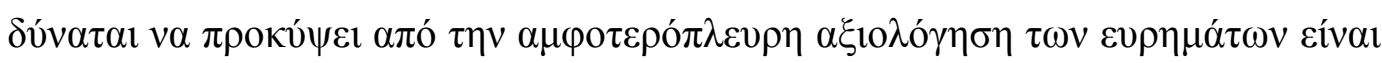

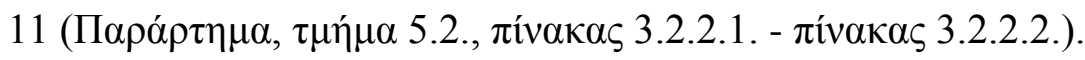

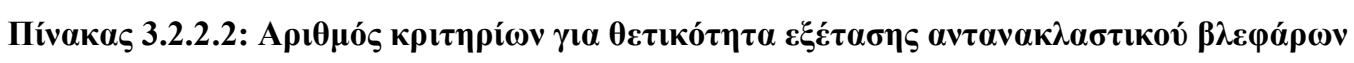

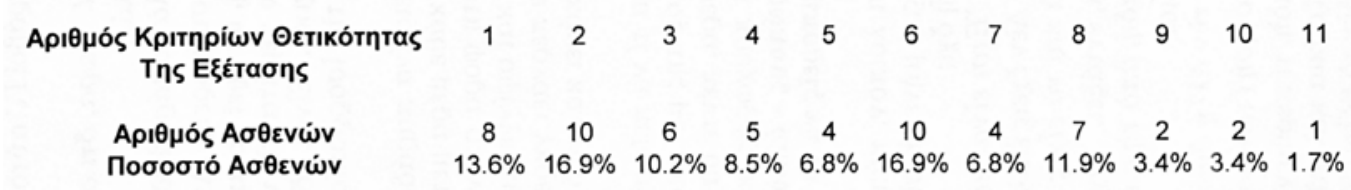

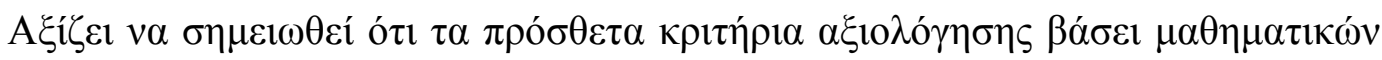

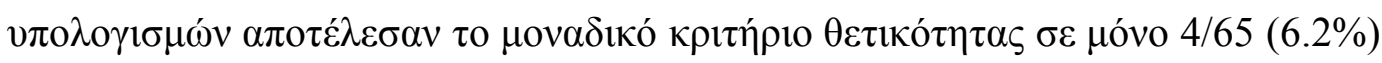

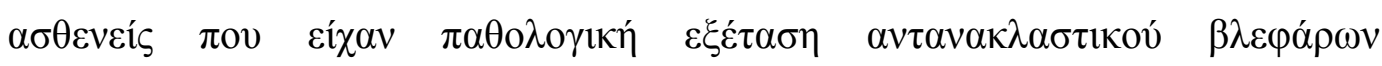

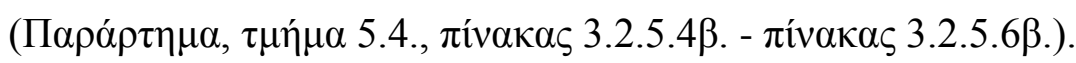




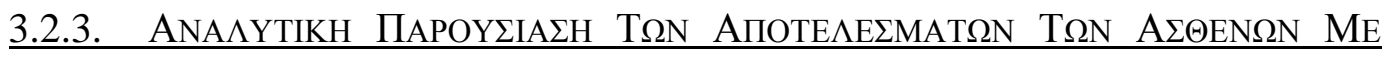

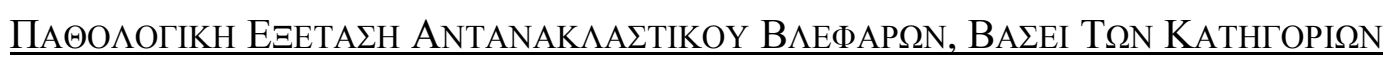
T $\Omega$ N AKTINO $\Lambda$ OГIK $\Omega$ N EYPHMAT $\Omega N$

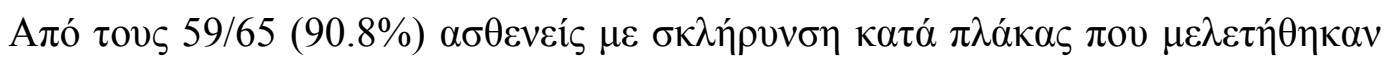

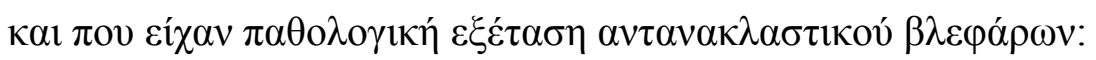

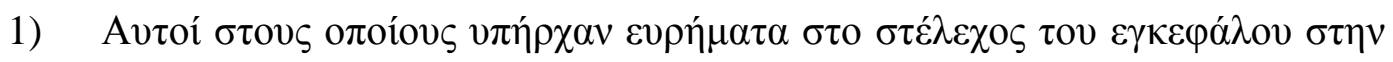

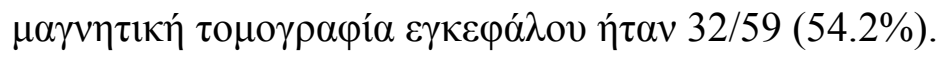

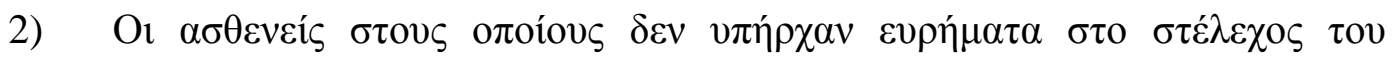

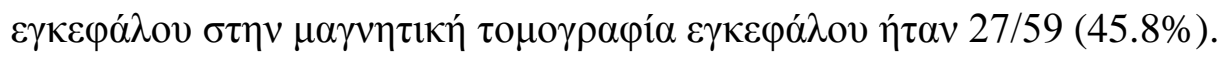

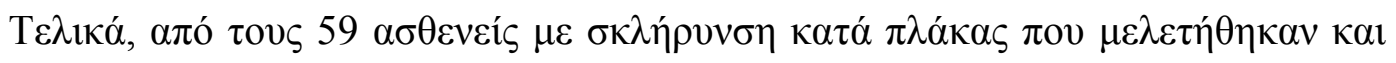

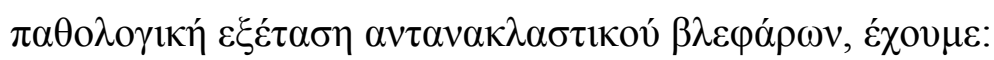

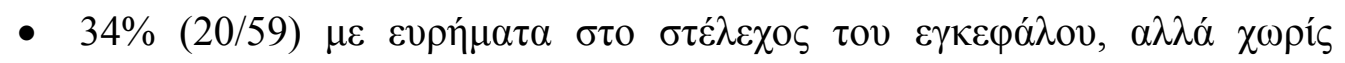

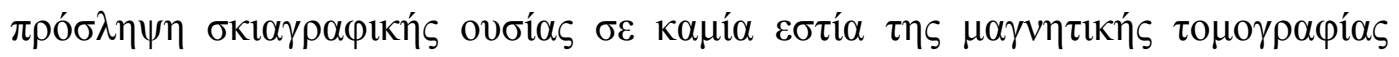

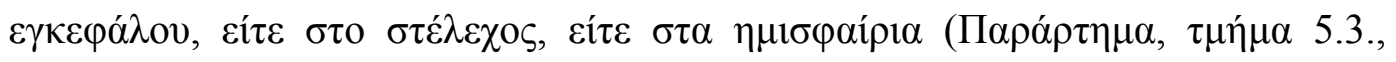

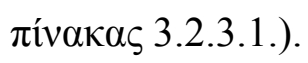

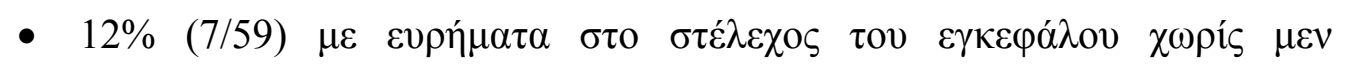

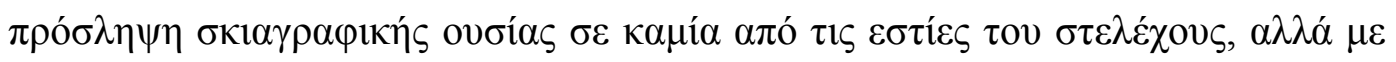

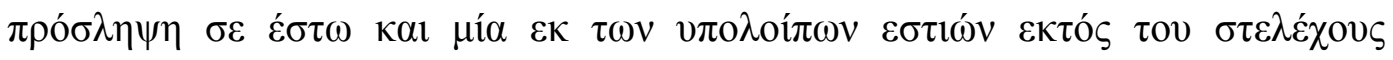

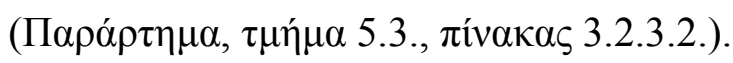

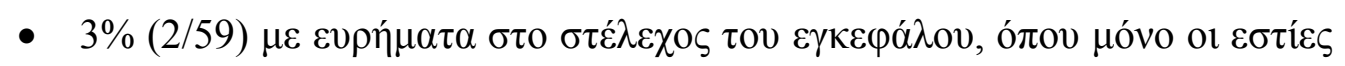

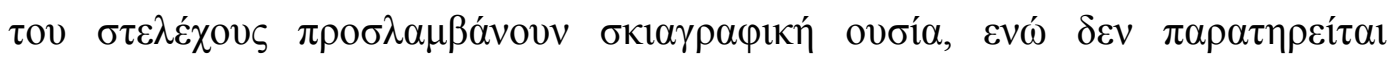

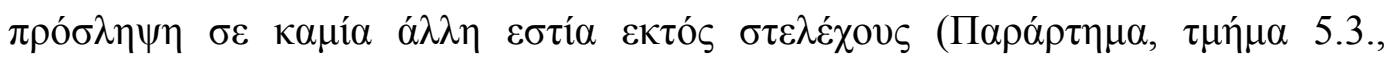

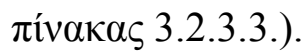

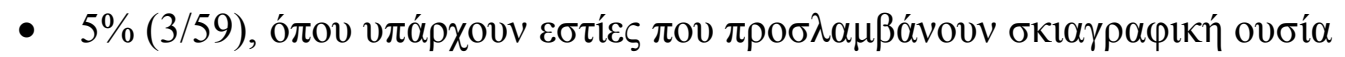

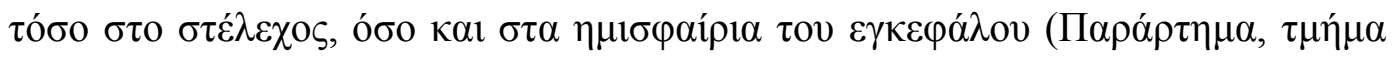

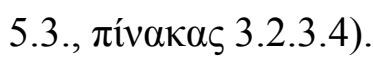

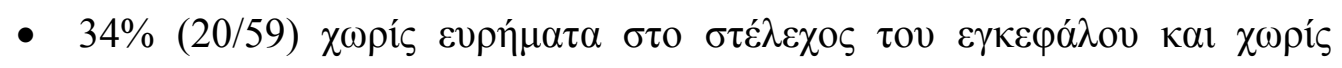

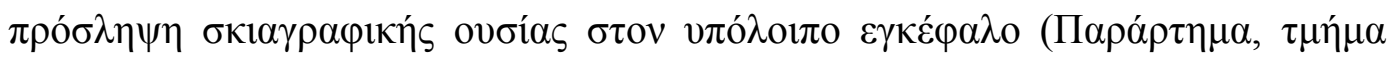

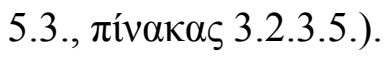

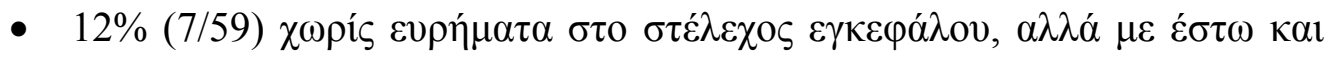

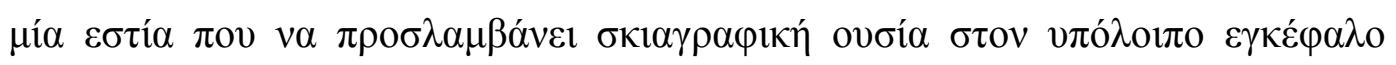

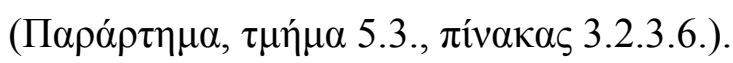




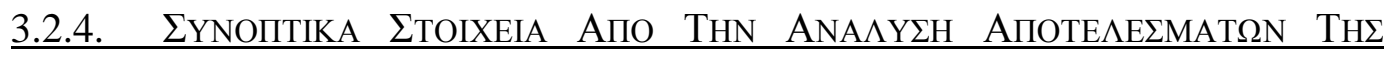

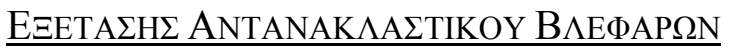

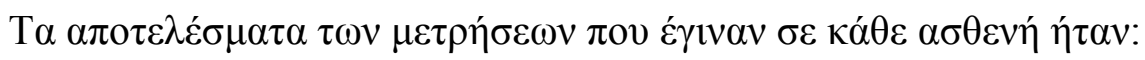

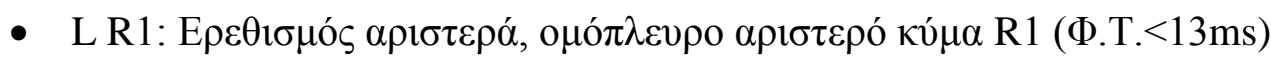

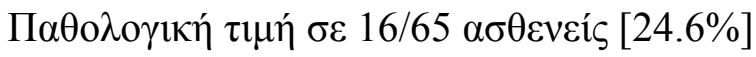

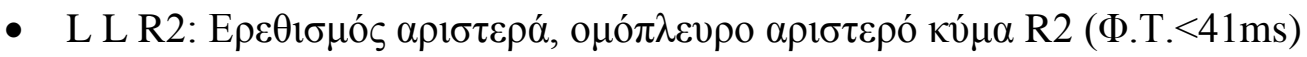

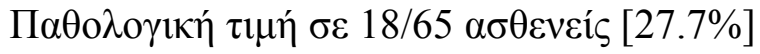

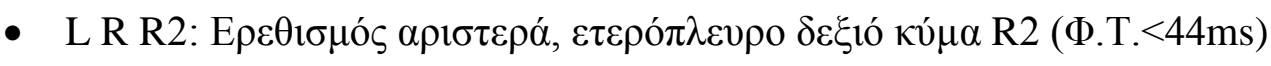

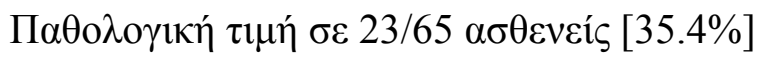

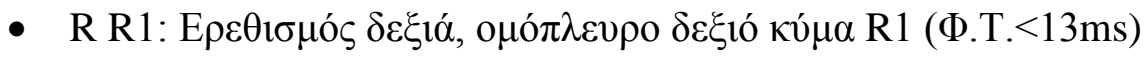

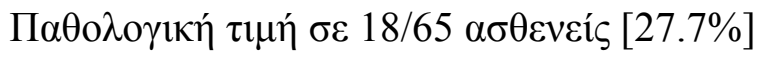

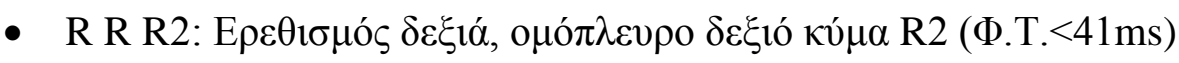

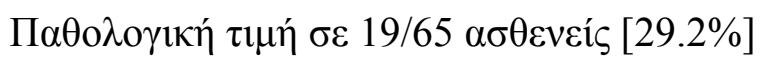

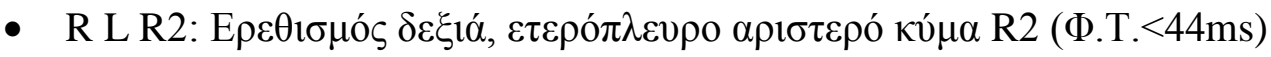

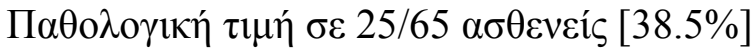

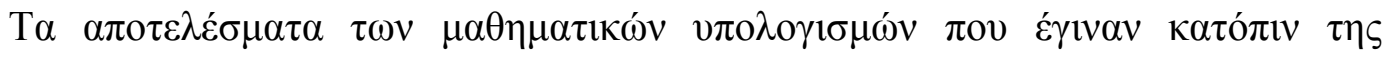
$\varepsilon \xi \dot{\varepsilon} \tau \alpha \sigma \eta \zeta:$

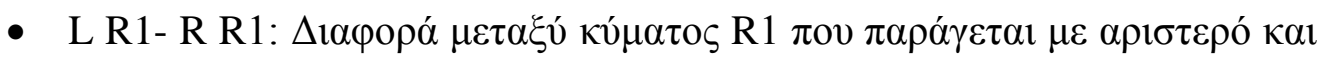
$\delta \varepsilon \xi$ ió $\varepsilon \rho \varepsilon \theta 1 \sigma \mu o ́$ (Ф.T. <[1.2]ms)

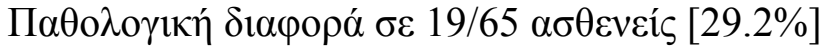

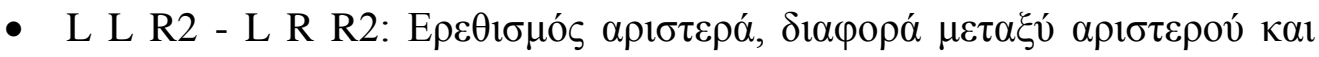

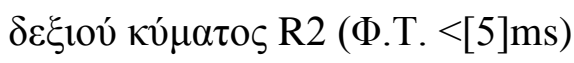

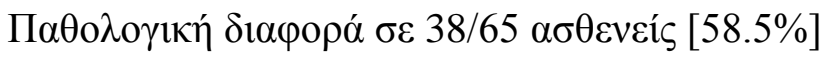

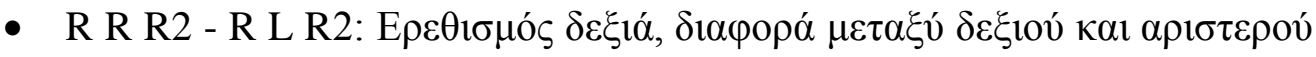

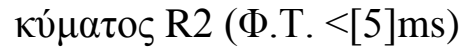

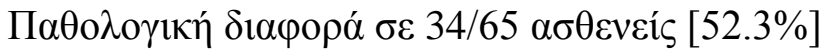

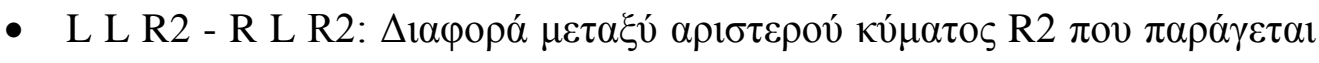
$\alpha \pi$ ó $\alpha \rho \imath \sigma \tau \varepsilon \rho o ́ ~ \kappa \alpha \imath ~ \delta \varepsilon \xi$ ló $\varepsilon \rho \varepsilon \theta \imath \sigma \mu o ́ ~(\Phi . T . ~<[7] \mathrm{ms})$

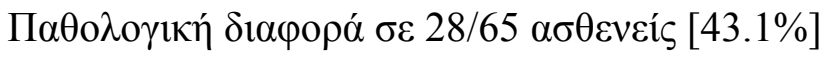

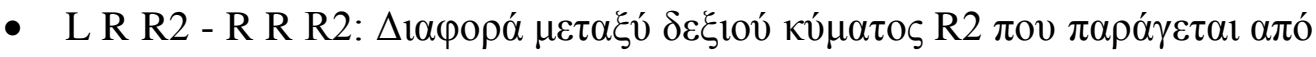
$\alpha \rho \imath \sigma \tau \varepsilon \rho o ́ ~ \kappa \alpha \imath ~ \delta \varepsilon \xi$ ló $\varepsilon \rho \varepsilon \theta 1 \sigma \mu o ́ ~(Ф . T . ~<[7] \mathrm{ms})$

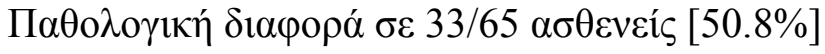




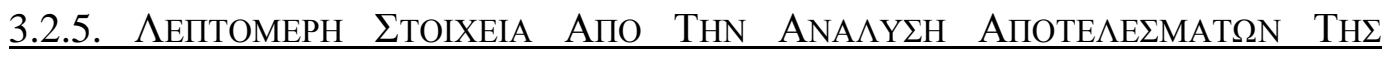

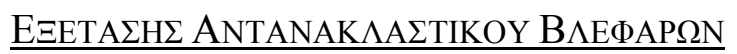

\subsubsection{1. КРІТНРІО ТІМНГ ОМОПАЕҮРОУ КУМАТОЕ $R 1<13 \mathrm{~ms}$}

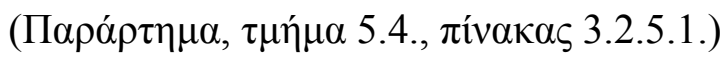

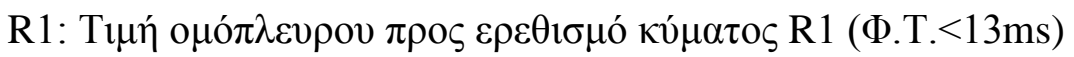

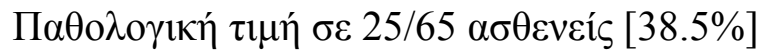

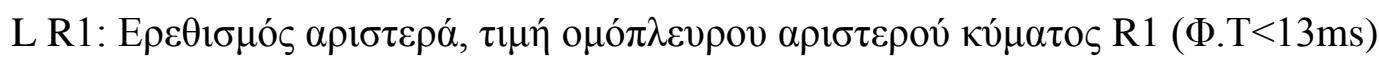

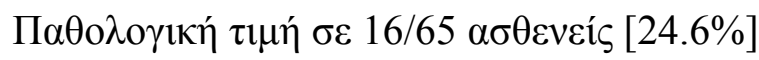

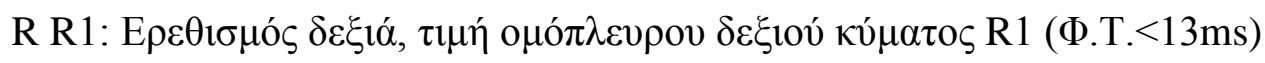

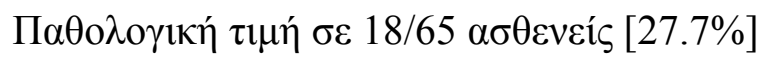

\subsubsection{2. КРІТНРІО ТІМНГ ОМОПАЕҮРОУ КУМАТОЕ $R 2<41 \mathrm{~ms}$}

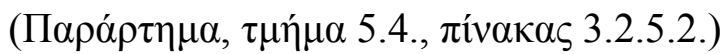

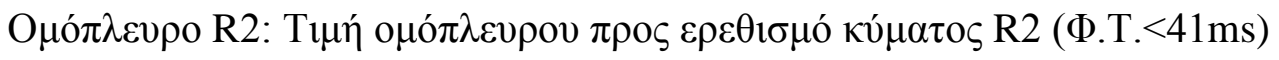

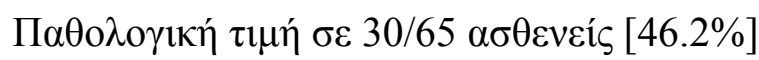

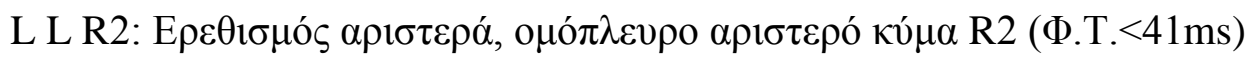

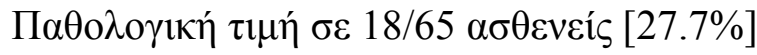

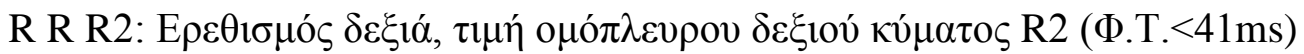

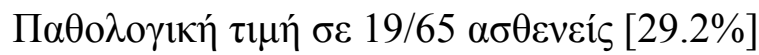

\subsubsection{3. КРІТНРІО ТIMНЕ ЕТЕРОПИЕҮРОY КУМАТОГ $R 2<44 \mathrm{~ms}$}

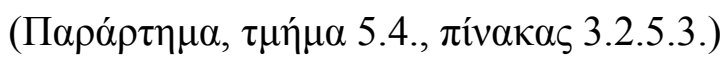

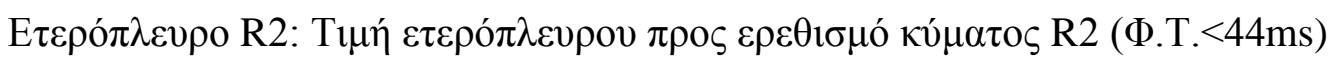

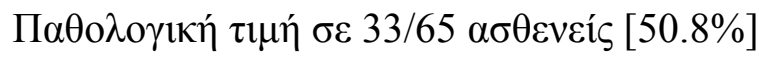

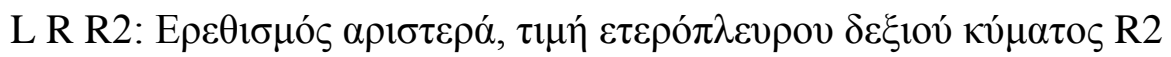

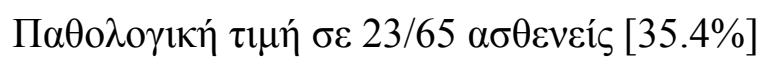

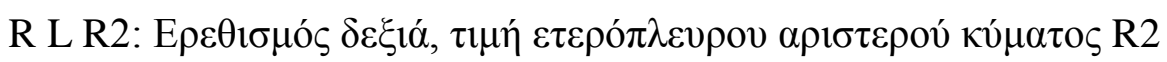

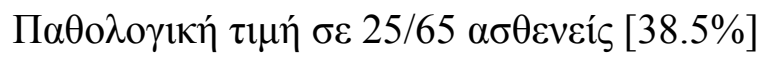

\subsubsection{KPITHPIO L R1 - $R R 1<[1.2] \mathrm{ms}$}

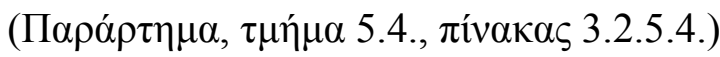

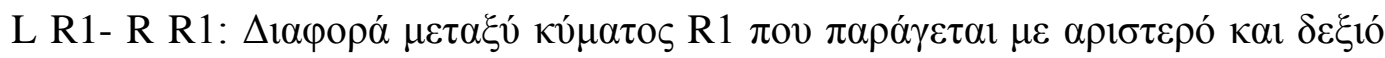
$\varepsilon \rho \varepsilon \theta 1 \sigma \mu$ ó $\left.^{(\Phi . T . ~}<[1.2] \mathrm{ms}\right)$

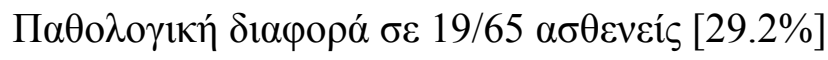




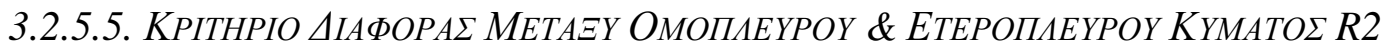
АПО ЕРЕАІГМО ГТН МІА ПАЕҮРА (Ф.T. < [5] $\mathrm{ms})$

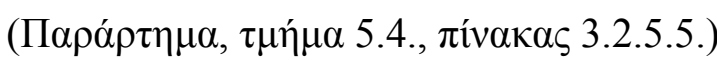

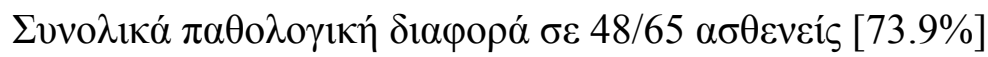

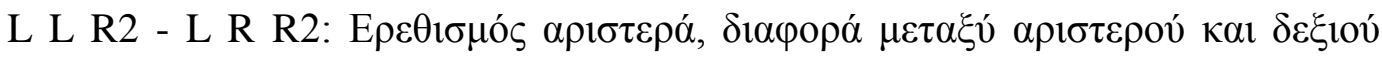

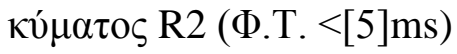

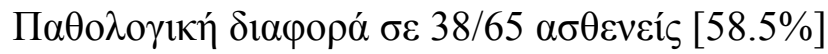

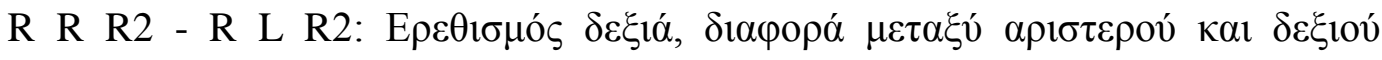
$\kappa v ́ \mu \alpha \tau o \varsigma$ R2 (Ф.T. <[5]ms)

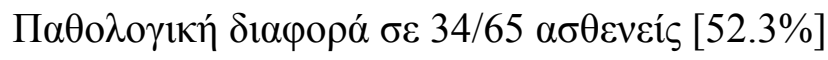

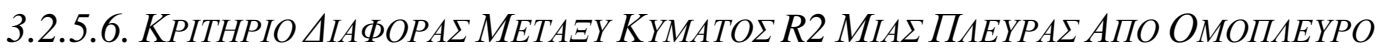
\& ЕТЕРОПИЕҮРО ЕРЕОIГМО (Ф.T. < [7] $\mathrm{ms})$

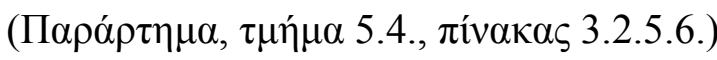

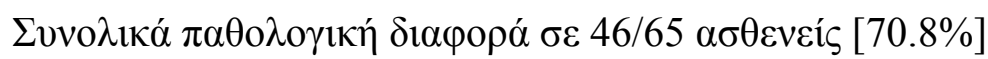

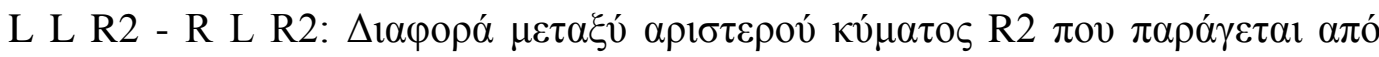

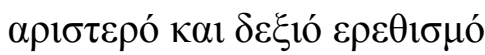

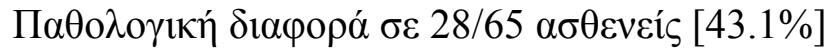

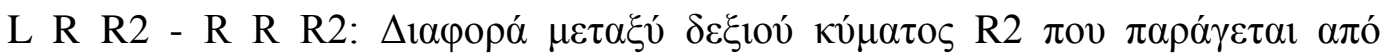

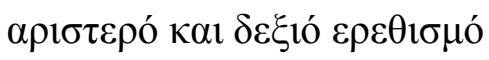

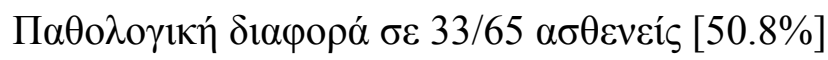

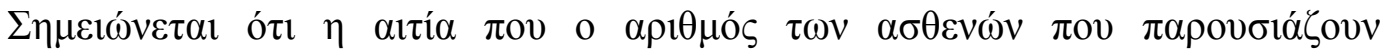

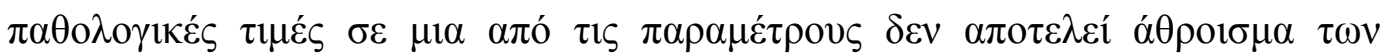

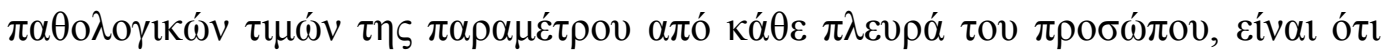

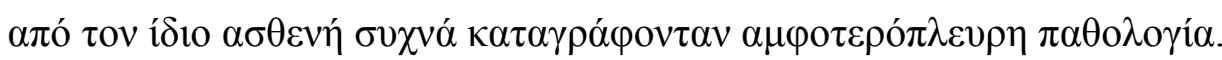

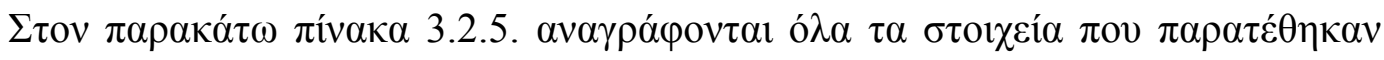

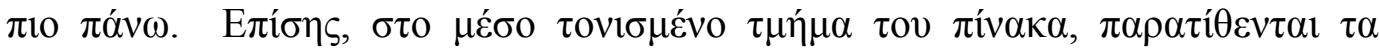

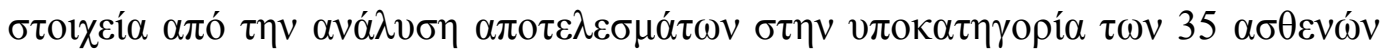
$\mu \varepsilon \sigma \tau \varepsilon \lambda \varepsilon \chi 1 \alpha i ́ \varepsilon \zeta \varepsilon \sigma \tau i ́ \varepsilon \varsigma$. 


\begin{tabular}{|c|c|c|c|}
\hline 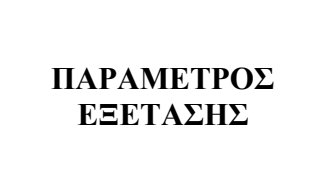 & & 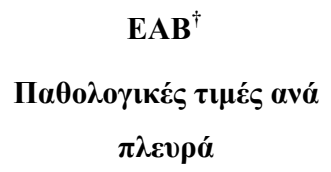 & 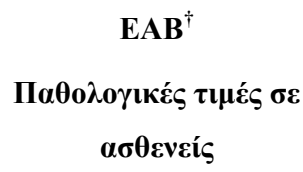 \\
\hline \multirow{2}{*}{ 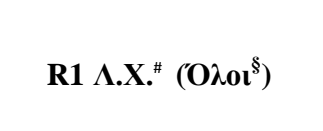 } & 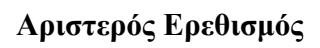 & $17 / 65(26.2 \%)$ & \multirow{2}{*}{$26 / 65(40 \%)$} \\
\hline & 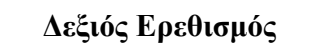 & $18 / 65(27.7 \%)$ & \\
\hline \multirow{2}{*}{ 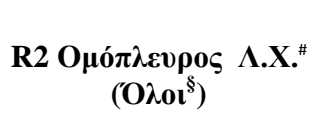 } & 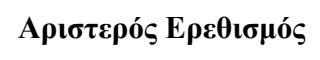 & $20 / 65(30.8 \%)$ & \multirow{2}{*}{$35 / 65(53.8 \%)$} \\
\hline & 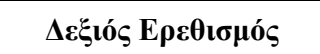 & $22 / 65(33.8 \%)$ & \\
\hline \multirow{2}{*}{ 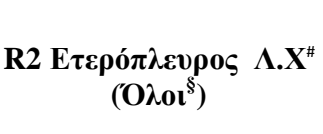 } & 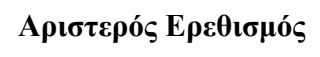 & $24 / 65(36.9 \%)$ & \multirow{2}{*}{$34 / 65(52.3 \%)$} \\
\hline & $\Delta \varepsilon \xi ı$ ló $\mathrm{E \rho \varepsilon \theta \imath \sigma \mu ó \varsigma}$ & $25 / 65(38.5 \%)$ & \\
\hline \multirow{2}{*}{ R1 $\Lambda . X . .^{\#}\left(\Sigma \tau \varepsilon \lambda^{*}\right)$} & 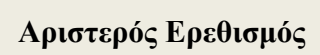 & $12 / 35(34.3 \%)^{\frac{1}{t}}$ & \multirow{2}{*}{$16 / 35(45.7 \%)^{\ddagger}$} \\
\hline & 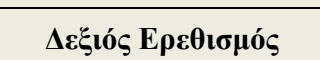 & $12 / 35(34.3 \%)^{\frac{1}{7}}$ & \\
\hline \multirow{2}{*}{ 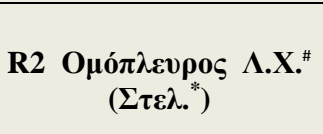 } & 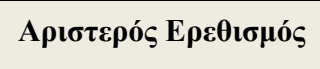 & $12 / 35(34.3 \%)^{\ddagger}$ & \multirow{2}{*}{$16 / 35(45.7 \%)^{\ddagger}$} \\
\hline & 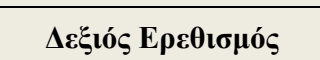 & $12 / 35(34.3 \%)^{\ddagger}$ & \\
\hline \multirow{2}{*}{ 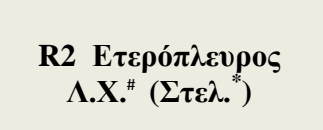 } & 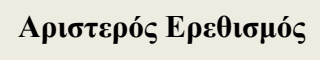 & $15 / 35(45.7 \%)$ & \multirow{2}{*}{$22 / 35(62.9 \%)$} \\
\hline & 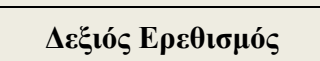 & $17 / 35(48.6 \%)$ & \\
\hline \multicolumn{4}{|l|}{ 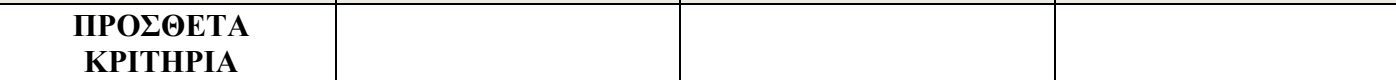 } \\
\hline 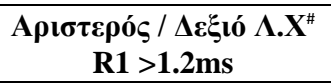 & & & $19 / 65(29.2 \%)$ \\
\hline \multirow{2}{*}{ 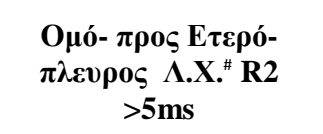 } & 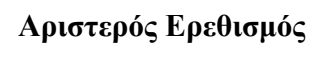 & $38 / 65(58.5 \%)$ & \multirow{2}{*}{$48 / 65(73.9 \%)$} \\
\hline & 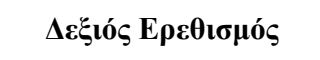 & $34 / 65(52.3 \%)$ & \\
\hline \multirow{2}{*}{ 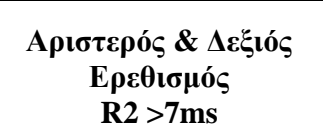 } & 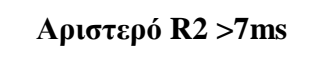 & $28 / 65(43.1 \%)$ & \multirow{2}{*}{$46 / 65(70.8 \%)$} \\
\hline & $\Delta \varepsilon \xi$ ıó $\mathrm{R} 2>7 \mathrm{~ms}$ & $33 / 65(50.8 \%)$ & \\
\hline
\end{tabular}

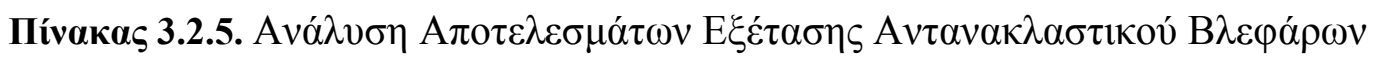

${ }^{\dagger} \mathrm{EAB}: \mathrm{E} \xi \dot{\varepsilon} \tau \alpha \sigma \eta \mathrm{A} v \tau \alpha v \alpha \kappa \lambda \alpha \sigma \tau 1 \kappa o v ́ \mathrm{~B} \lambda \varepsilon \varphi \alpha ́ \rho \omega \nu$

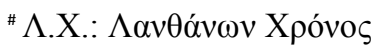

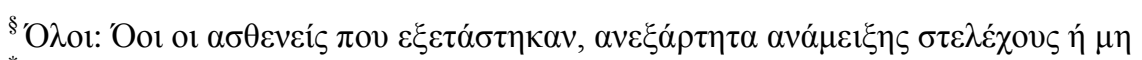

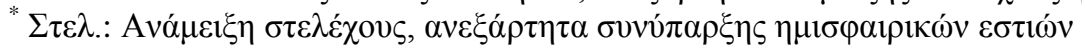

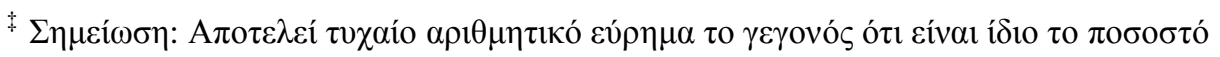

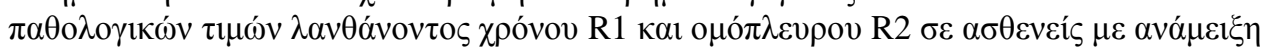

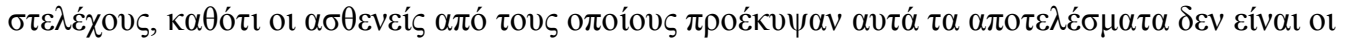
ísıo. 


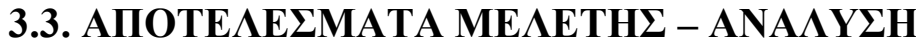

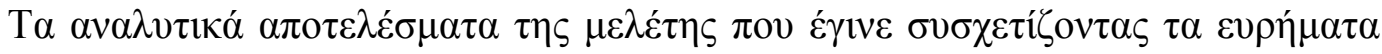

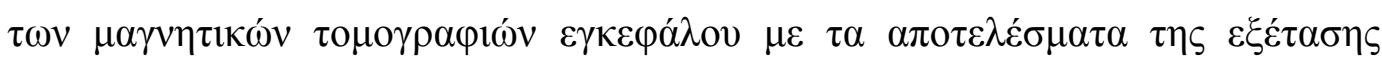

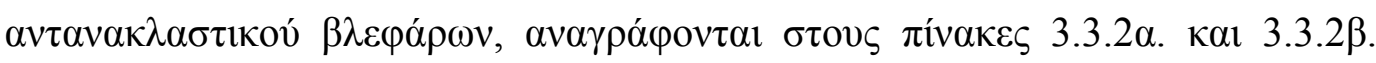

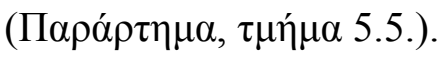

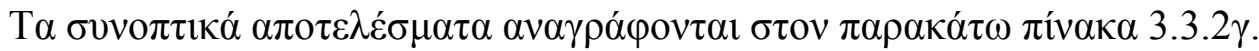

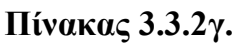

\begin{tabular}{|c|c|c|}
\hline \multicolumn{2}{|c|}{ MRI* $\varepsilon \gamma \kappa \varepsilon \varphi \alpha ́ \lambda o v$} & 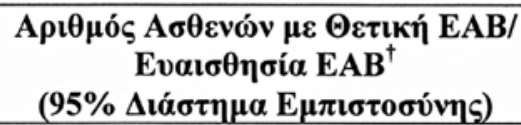 \\
\hline \multicolumn{2}{|c|}{ 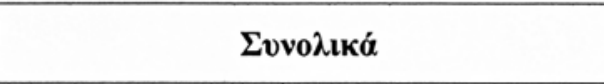 } & $\begin{array}{c}59 / 65 \\
90.8 \%(83.7-97.8 \%)\end{array}$ \\
\hline \multirow{2}{*}{ 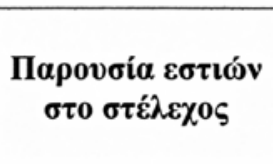 } & 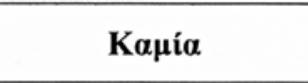 & $\begin{array}{c}27 / 30 \\
90.0 \%(79.3-100 \%)\end{array}$ \\
\hline & $\geq 1$ & $\begin{array}{c}32 / 35 \\
91.4 \%(82.2-100 \%)\end{array}$ \\
\hline \multirow{2}{*}{ 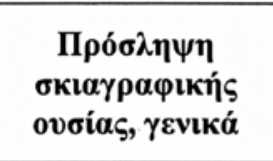 } & 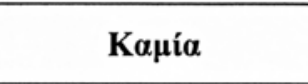 & $\begin{array}{c}40 / 43 \\
93 \%(85.4-100 \%)\end{array}$ \\
\hline & $\sigma \varepsilon \geq 1 \varepsilon \sigma \tau i ́ \varepsilon \varsigma$ & $\begin{array}{c}19 / 22 \\
86.4 \%(72-100 \%)\end{array}$ \\
\hline \multirow{2}{*}{ 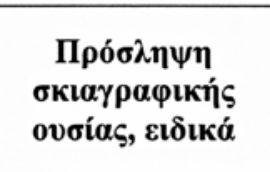 } & $\begin{array}{c}\Sigma \varepsilon \varepsilon \sigma \tau i \varepsilon \varsigma \sigma \tau \varepsilon \lambda \varepsilon ́ \chi 0 v \varsigma \\
(\kappa \alpha \iota \eta \mu \iota \sigma \varphi \alpha \iota \rho i ́ \omega v)\end{array}$ & $\begin{array}{c}5 / 5 \\
100 \%\end{array}$ \\
\hline & 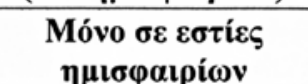 & $\begin{array}{c}14 / 17 \\
82.4 \%(64.2-100 \%)\end{array}$ \\
\hline
\end{tabular}

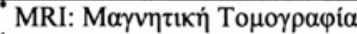

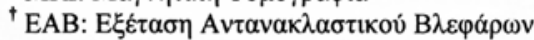

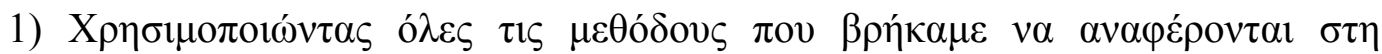

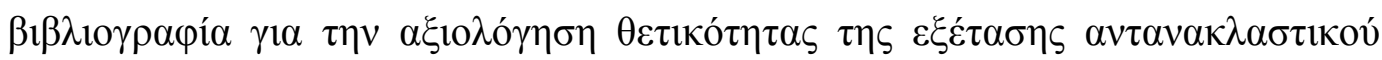

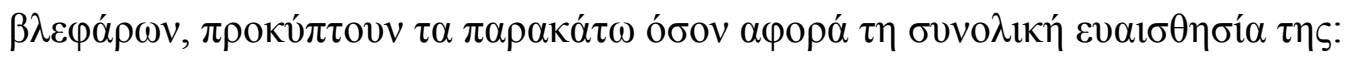

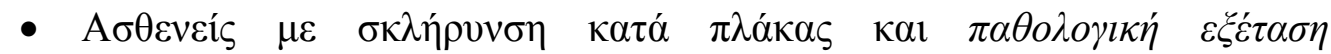

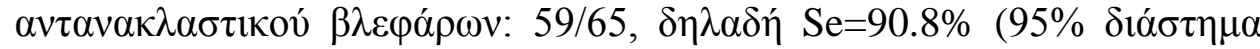

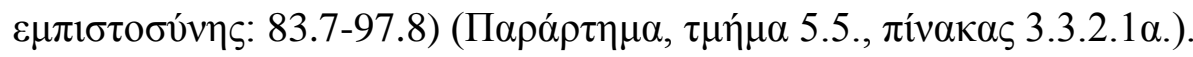

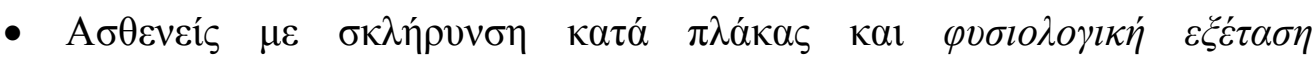
$\alpha \nu \tau \alpha v \alpha \kappa \lambda \alpha \sigma \tau 1 \kappa o v ́ \quad \beta \lambda \varepsilon \varphi \alpha ́ \rho \omega v: \quad 6 / 65, \quad \delta \eta \lambda \alpha \delta \dot{\eta} \quad 9.2 \% \quad(95 \% \quad \delta i \alpha ́ \sigma \tau \eta \mu \alpha$

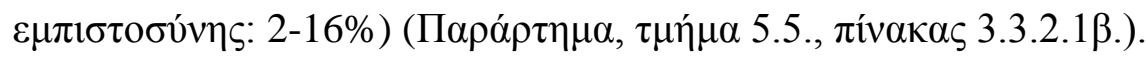

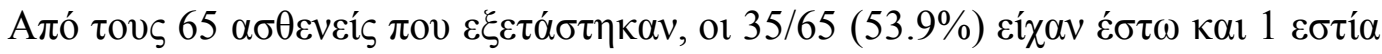

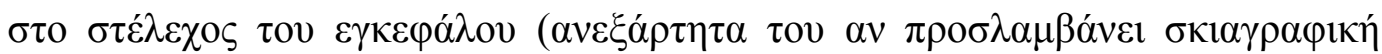

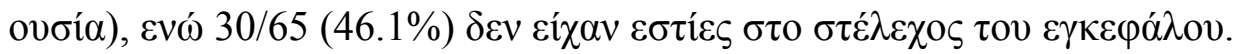




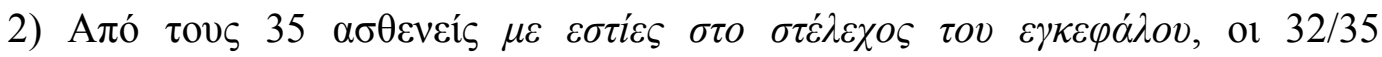

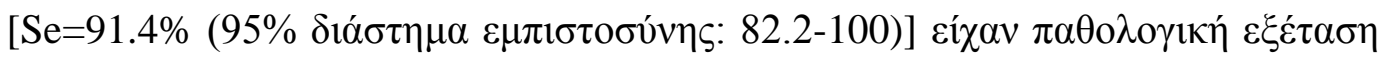

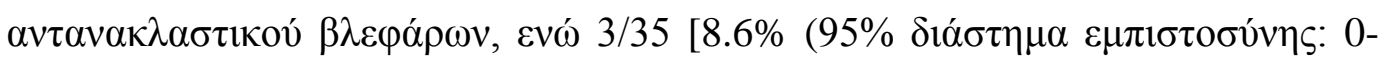

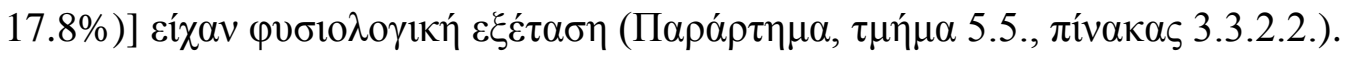

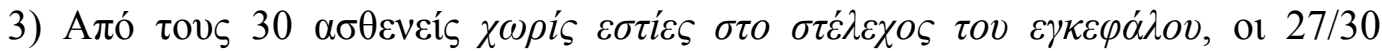

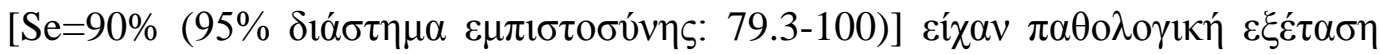

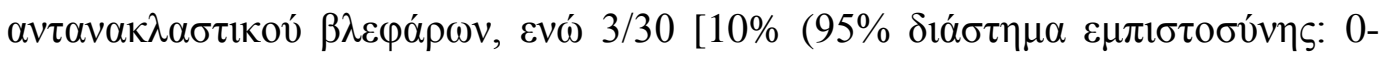

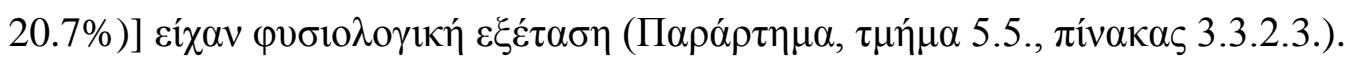

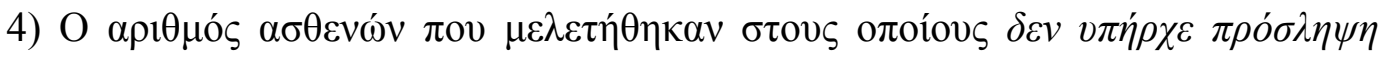

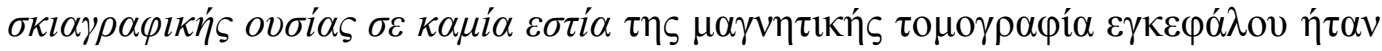

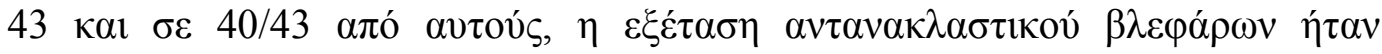

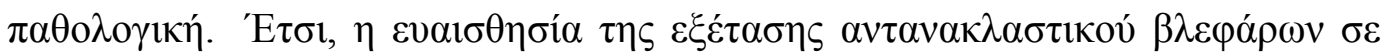

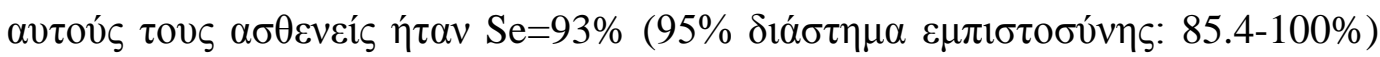

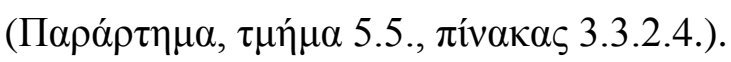

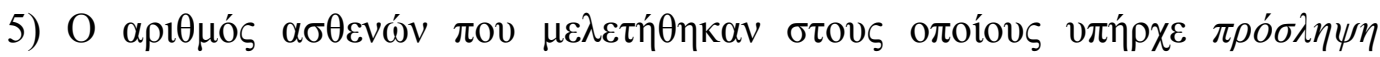

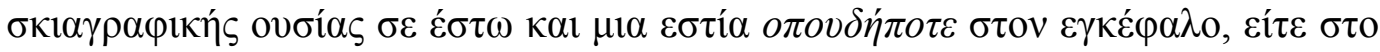

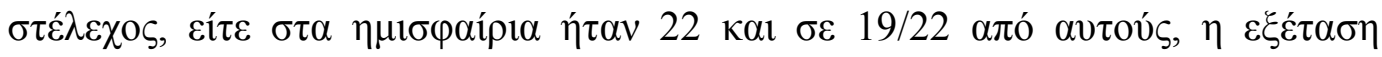

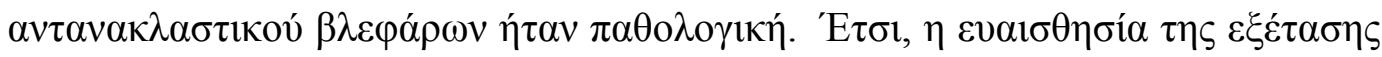

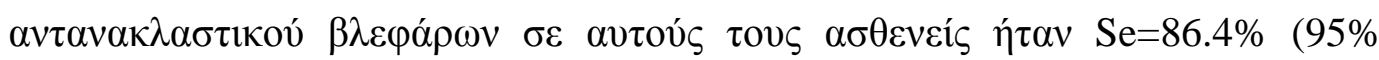

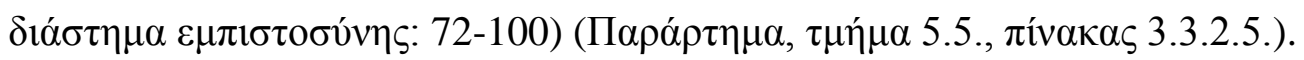

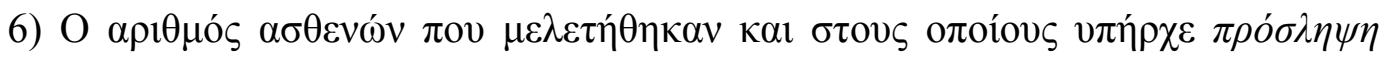

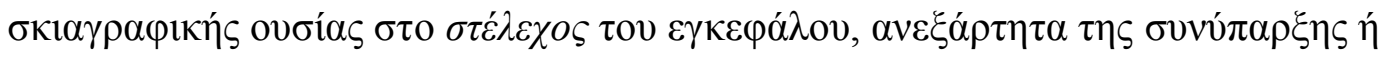

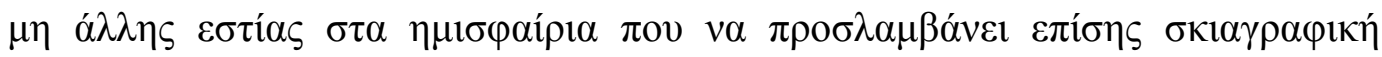

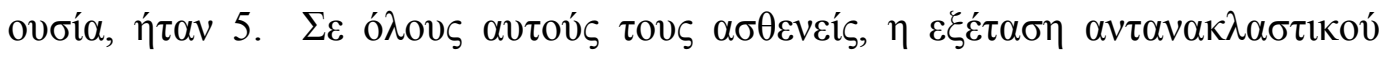

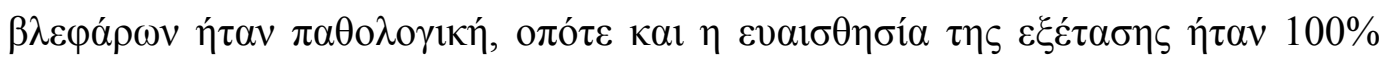

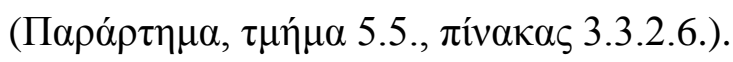

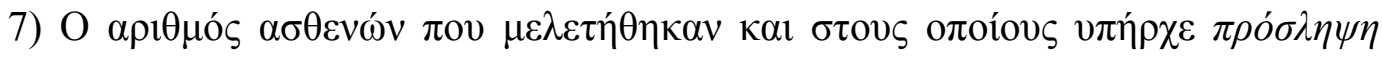

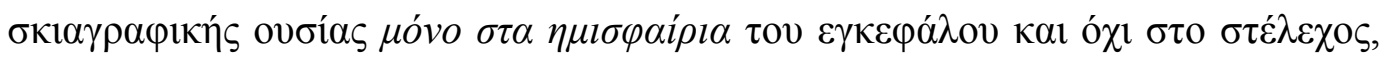




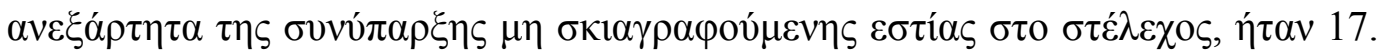

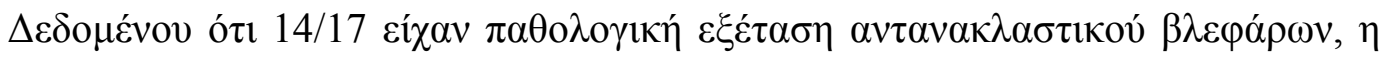

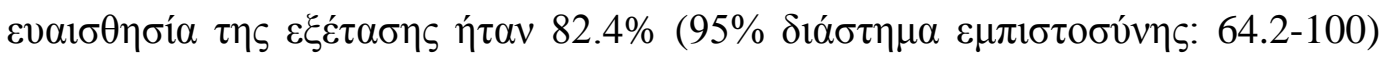

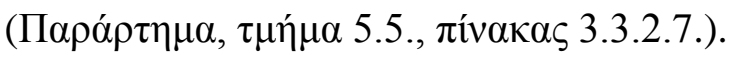

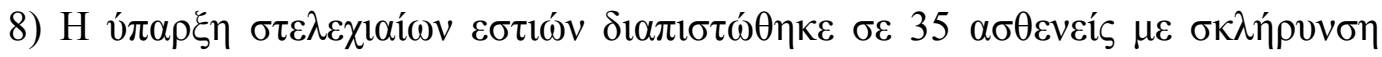

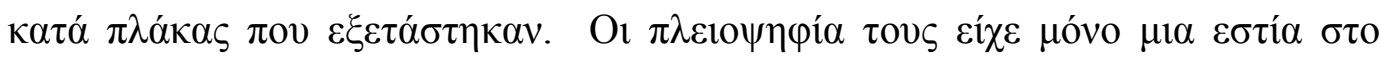

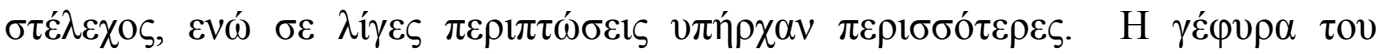

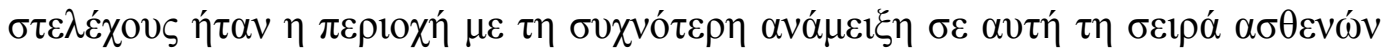

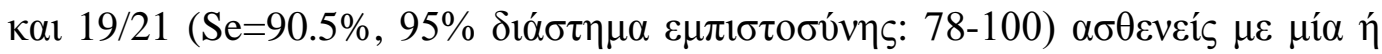

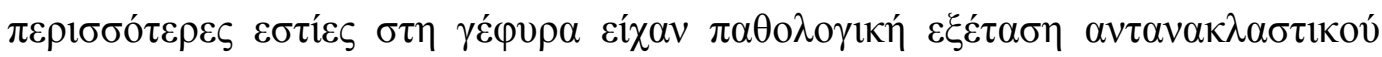

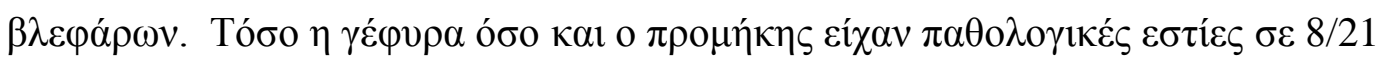

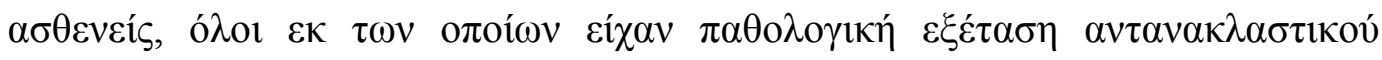

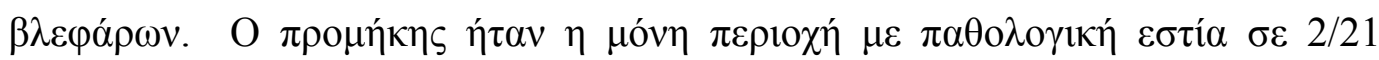

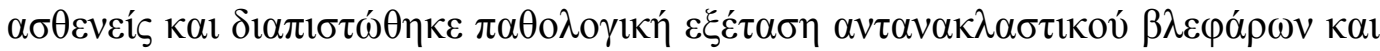

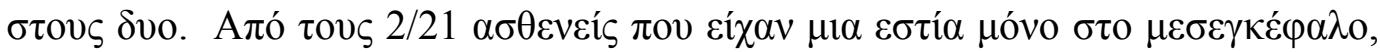

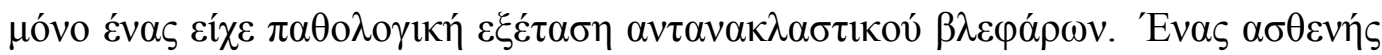

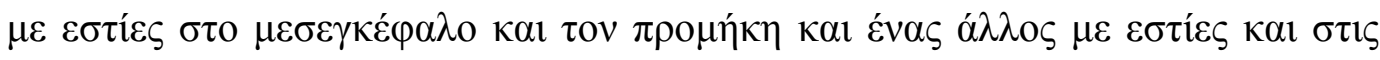

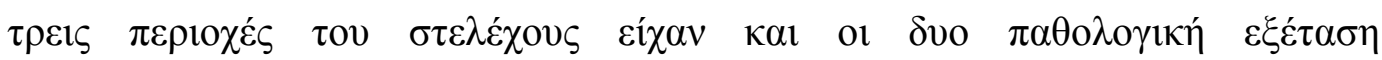

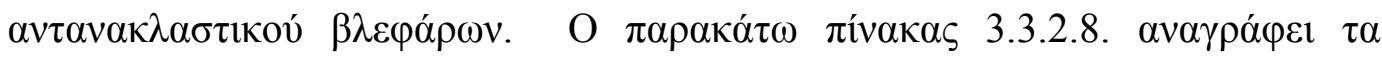

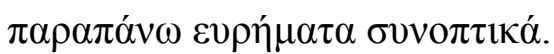

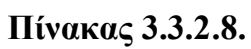

\begin{tabular}{|c|c|c|}
\hline \multirow[t]{2}{*}{ 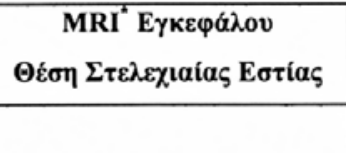 } & \multicolumn{2}{|c|}{$\begin{array}{c}\mathrm{A} \pi 0 \tau \varepsilon \lambda \varepsilon \sigma \mu \alpha \tau \alpha \mathrm{EAB}^{\dagger} \\
\mathrm{A \rho} \theta \mu \delta \varsigma \varsigma \mathrm{A} \sigma \theta \varepsilon v \omega \mathrm{v}\end{array}$} \\
\hline & 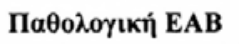 & 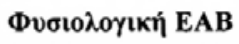 \\
\hline 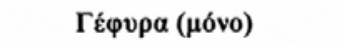 & $19 / 21$ & $2 / 21$ \\
\hline 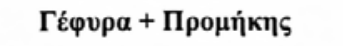 & $8 / 8$ & $0 / 8$ \\
\hline 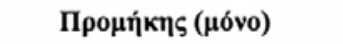 & $2 / 2$ & $0 / 2$ \\
\hline 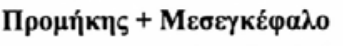 & $1 / 1$ & $0 / 1$ \\
\hline 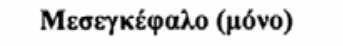 & $1 / 2$ & $1 / 2$ \\
\hline 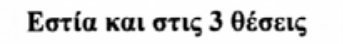 & $1 / 1$ & $0 / 1$ \\
\hline EYNOAO & $32 / 35(91.4 \%)$ & $3 / 35(8.6 \%)$ \\
\hline
\end{tabular}

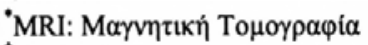

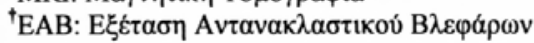




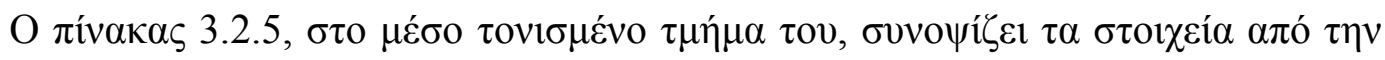

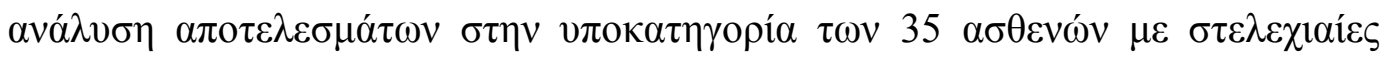
$\varepsilon \sigma \tau i ́ \varepsilon \varsigma . \Sigma v v o \pi \tau 1 \kappa \alpha ́:$

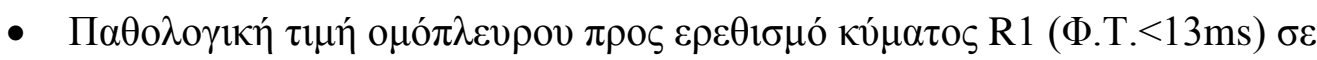
$16 / 35 \alpha \sigma \theta \varepsilon v \varepsilon i ́ s$ [45.7\%]

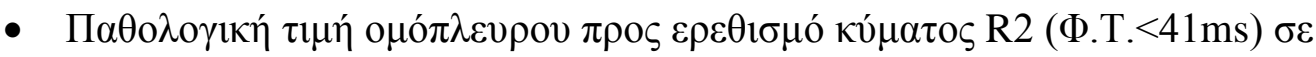
$16 / 35 \alpha \sigma \theta \varepsilon v \varepsilon i ́ \varsigma$ [45.7\%]

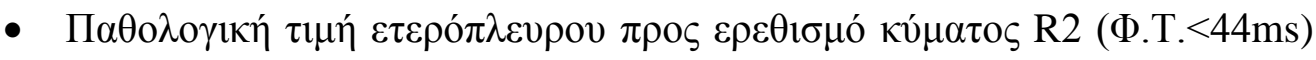
$\sigma \varepsilon 22 / 35 \alpha \sigma \theta \varepsilon v \varepsilon i ́ s[62.9 \%]$

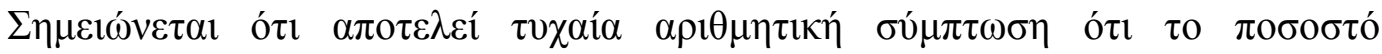

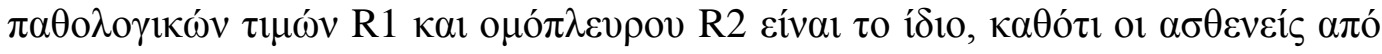

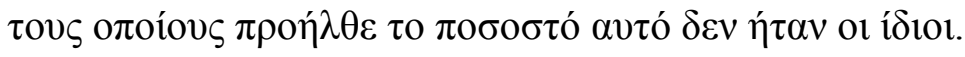

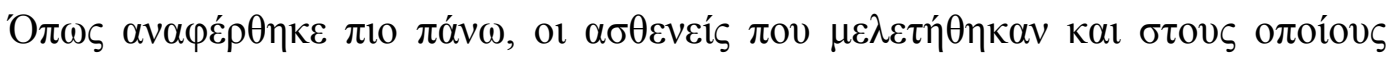

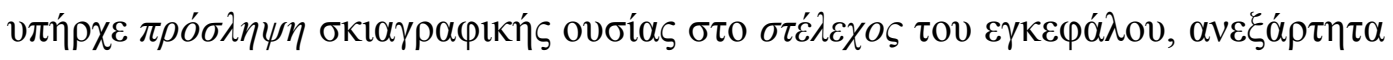

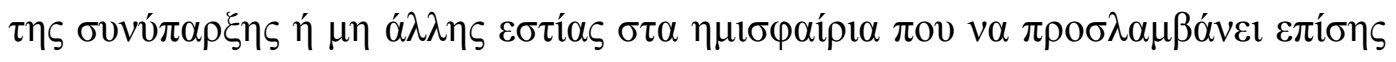

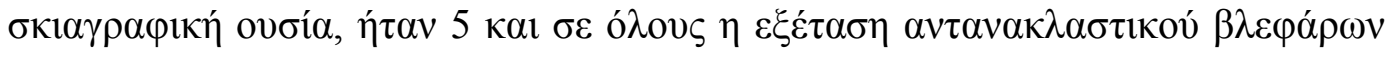

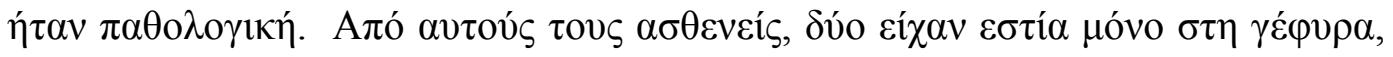

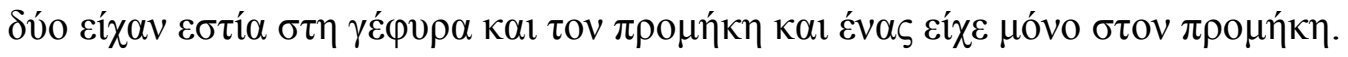

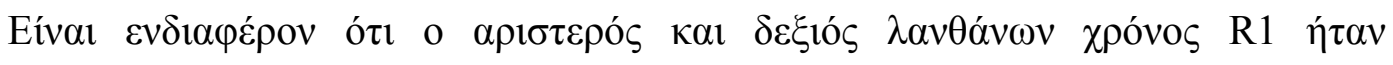

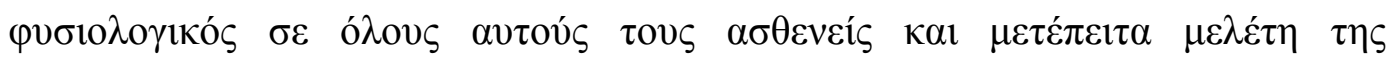

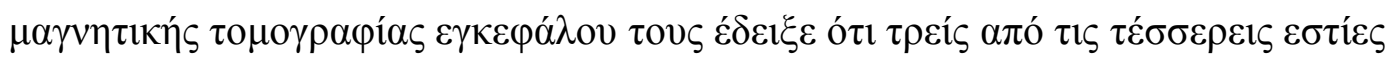

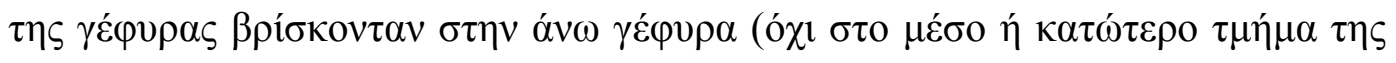

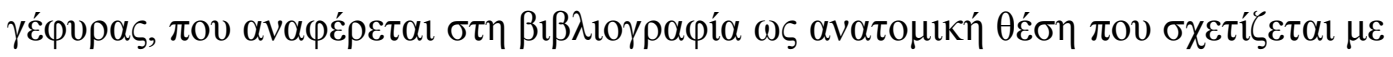

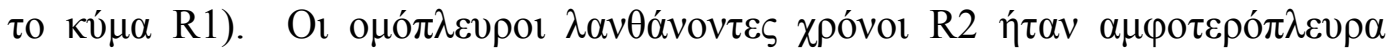

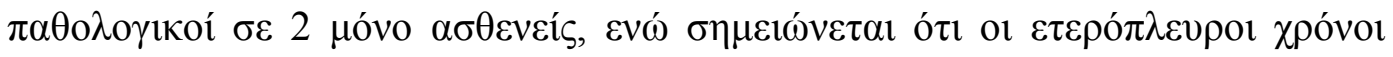

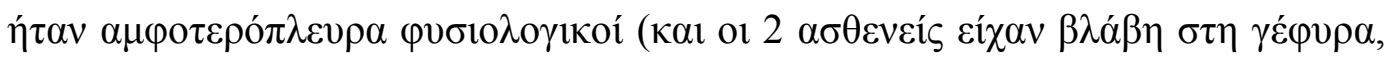

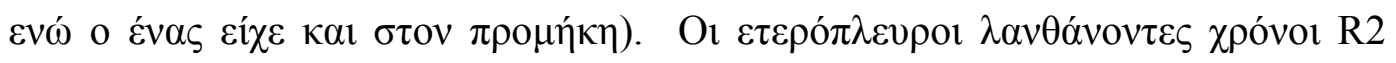

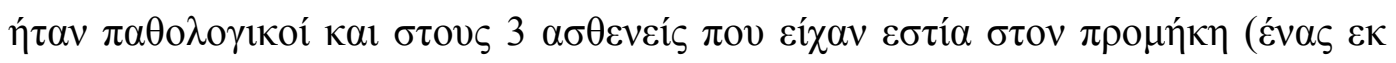

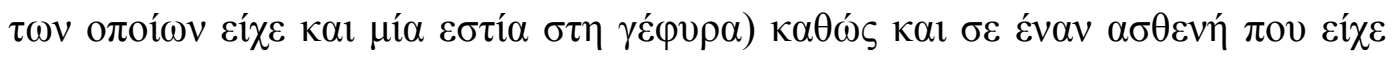

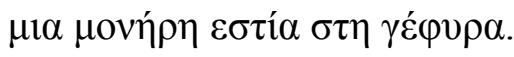


A

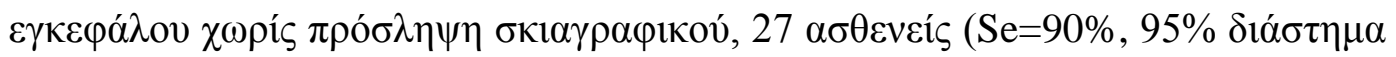

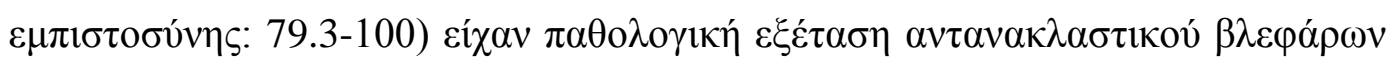

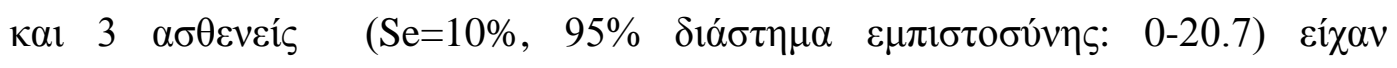

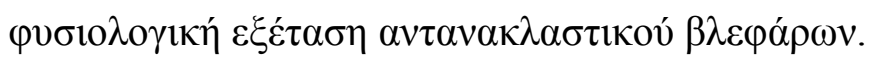

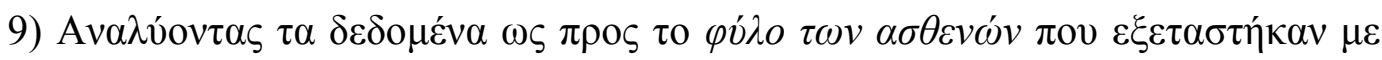

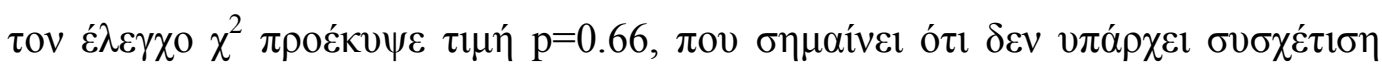

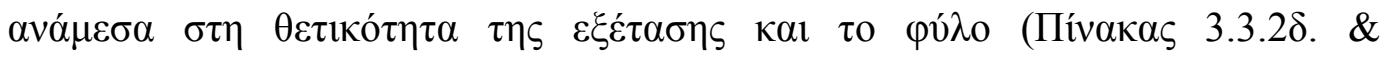

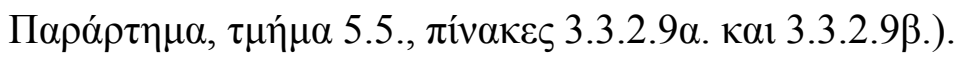

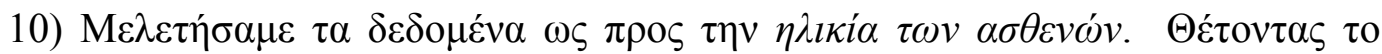

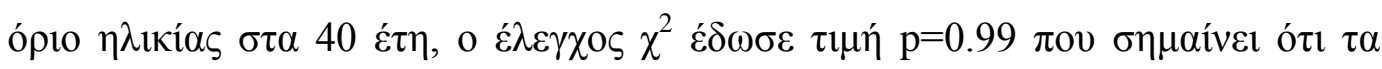

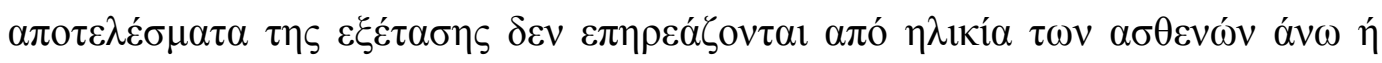

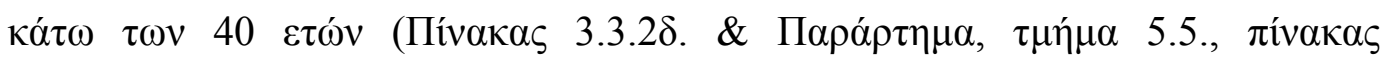
3.3.2.10.).

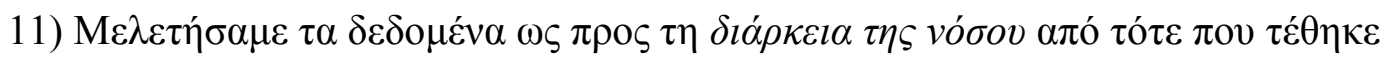

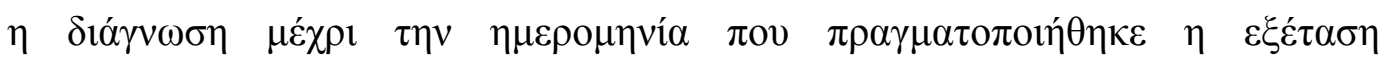

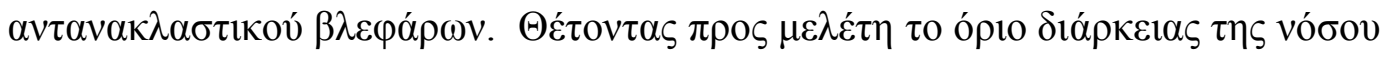

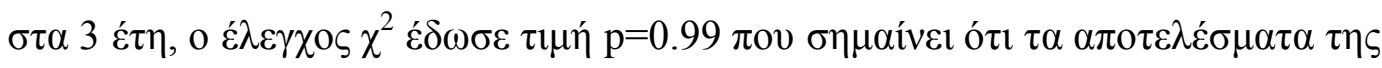

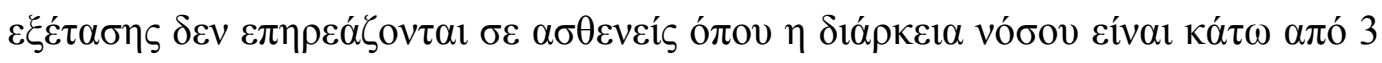

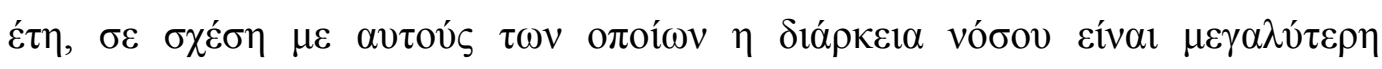

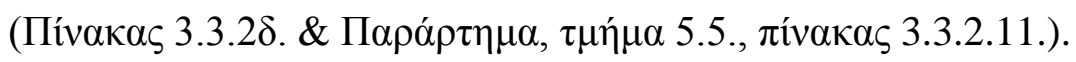

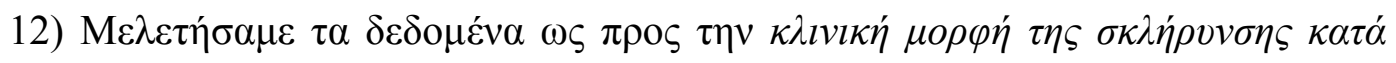

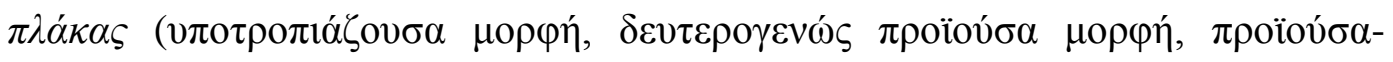

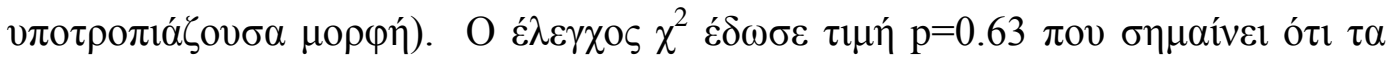

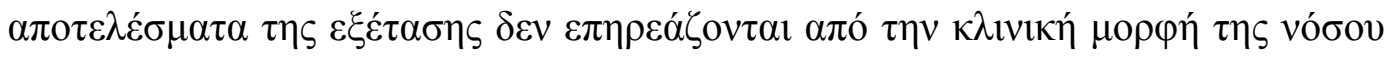

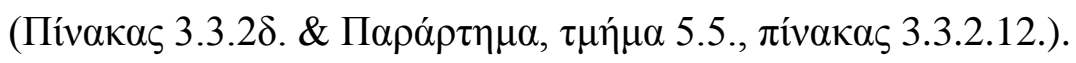

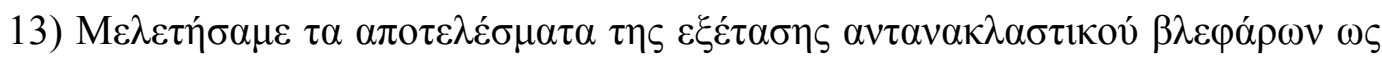

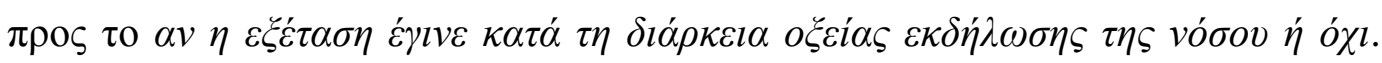

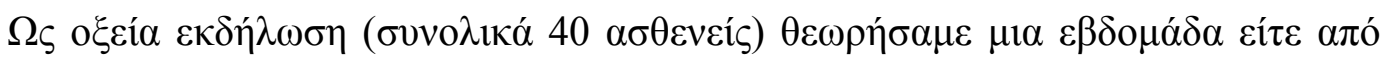

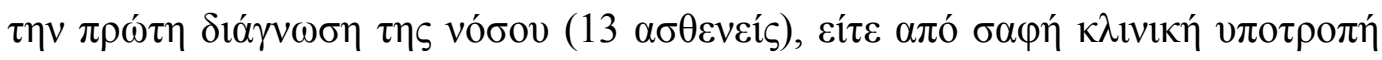




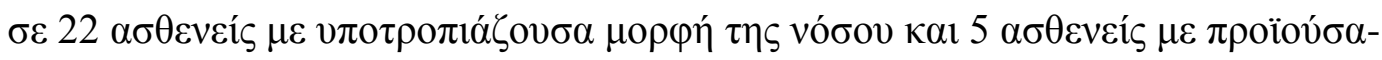

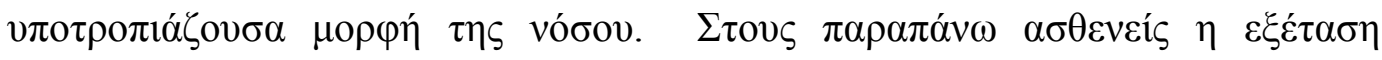

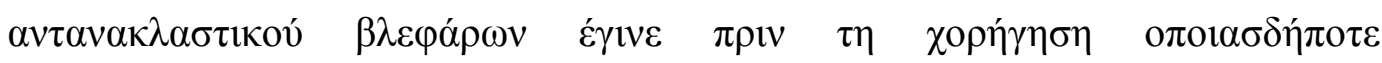

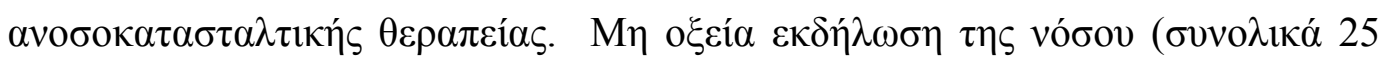

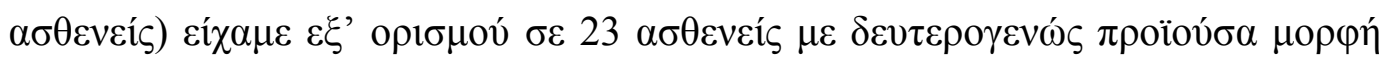

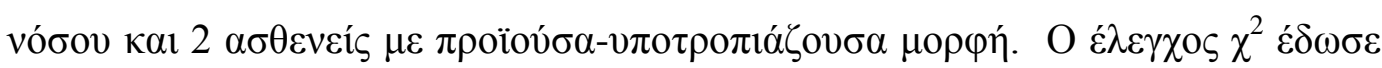

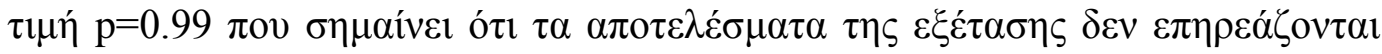

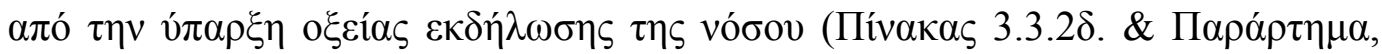
$\tau \mu \eta ́ \mu \alpha$ 5.5., $\pi i ́ v \alpha \kappa \alpha \varsigma$ 3.3.2.13.).

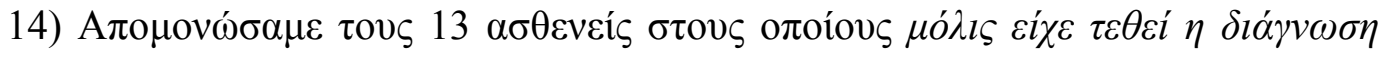

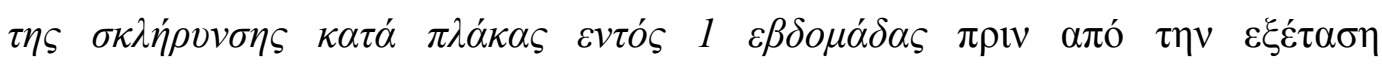

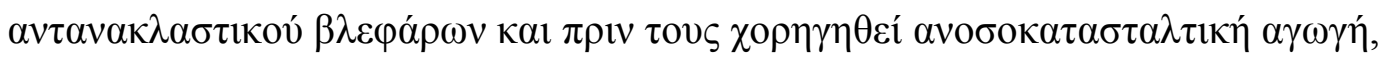

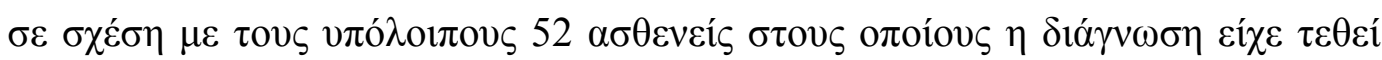

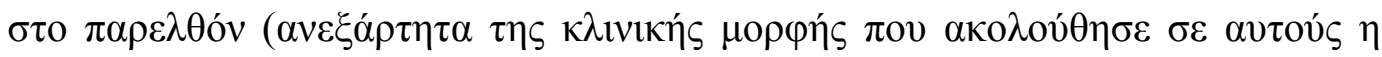

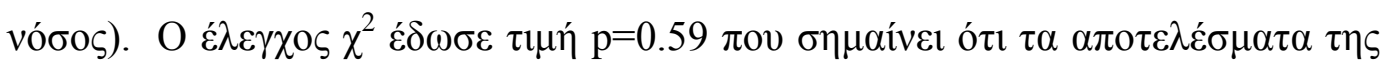

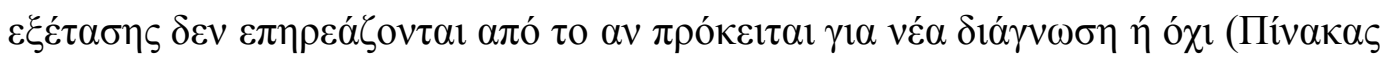

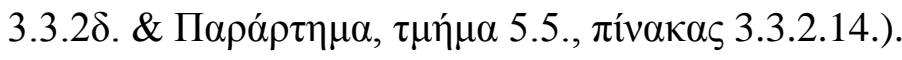

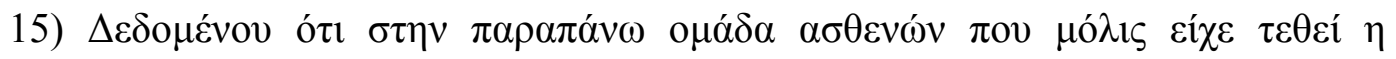

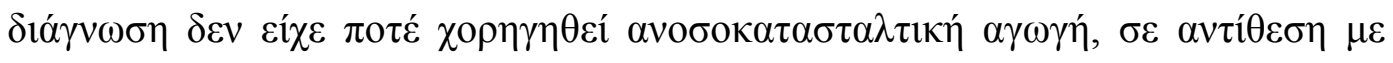

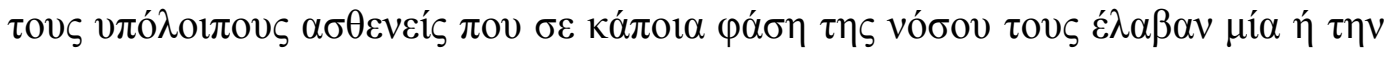

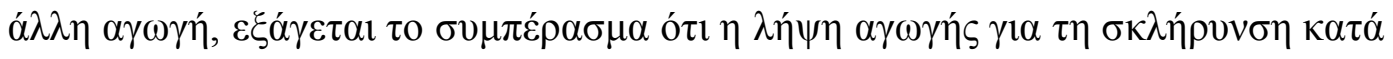

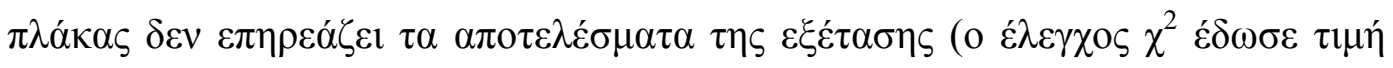
$\mathrm{p}=0.59)$.

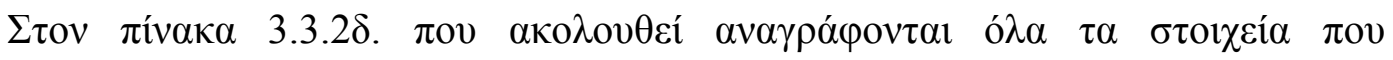

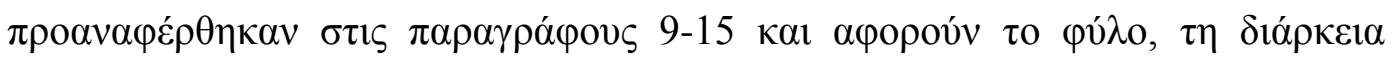

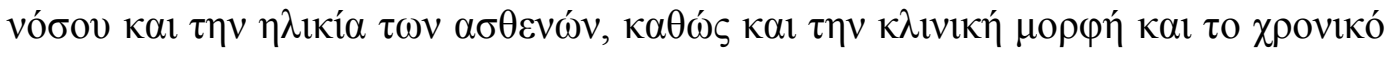

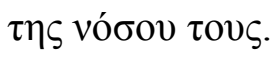


б. 70

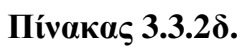

\begin{tabular}{|c|c|c|c|c|}
\hline & & \multicolumn{2}{|c|}{$\mathbf{E A B}^{\dagger}$} & \multirow{2}{*}{ P-value* } \\
\hline & & Өєтเкท́ & 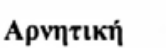 & \\
\hline \multirow[b]{2}{*}{ Фúdo } & 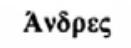 & $95.2 \%(20 / 21)$ & $4.8 \%$ & \multirow{2}{*}{0.66} \\
\hline & Гvvаíкє૬ & $88.6 \%(39 / 44)$ & $11.4 \%$ & \\
\hline \multirow{2}{*}{ 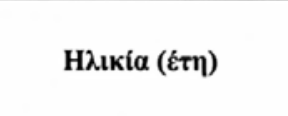 } & $>40$ & $92.6 \%(25 / 27)$ & $7.4 \%$ & \multirow{2}{*}{0.99} \\
\hline & $\leq 40$ & $89.5 \%(34 / 38)$ & $10.5 \%$ & \\
\hline \multirow{2}{*}{ 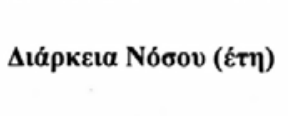 } & $>3$ & $89.7 \%(26 / 29)$ & $10.3 \%$ & \multirow{2}{*}{0.99} \\
\hline & $\leq 3$ & $91.7 \%(33 / 36)$ & $8.3 \%$ & \\
\hline \multirow{3}{*}{ 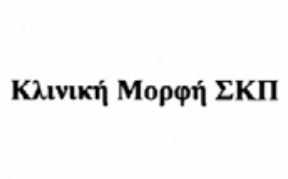 } & RRMS & $88.9 \%(40 / 45)$ & $11.1 \%$ & \multirow{3}{*}{0.63} \\
\hline & RPMS & $100 \%(7 / 7)$ & $0 \%$ & \\
\hline & SPMS & $92.3 \%(12 / 13)$ & $7.7 \%$ & \\
\hline \multirow{2}{*}{ 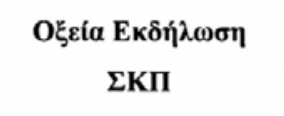 } & Naí & $90 \%(36 / 40)$ & $10 \%$ & \multirow{2}{*}{0.99} \\
\hline & $O_{\chi 1}$ & $92 \%(23 / 25)$ & $8 \%$ & \\
\hline \multirow{2}{*}{ 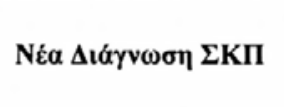 } & Naí & $84.6 \%(11 / 13)$ & $15.4 \%$ & \multirow{2}{*}{0.59} \\
\hline & $\mathrm{O} \chi \mathrm{l}$ & $92.3 \%(48 / 52)$ & $7.7 \%$ & \\
\hline
\end{tabular}

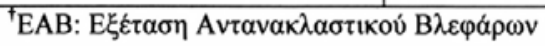

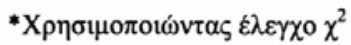

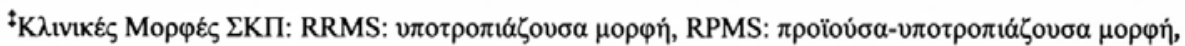

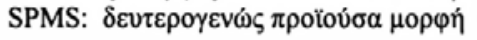




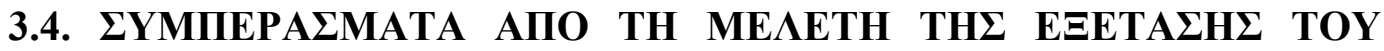

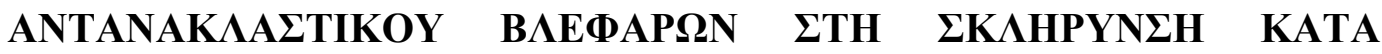

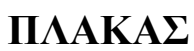

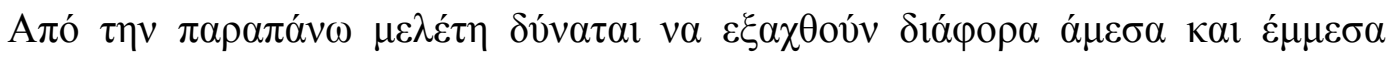

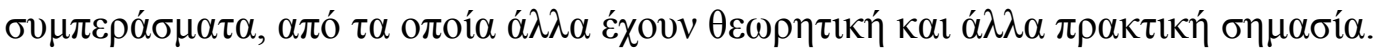

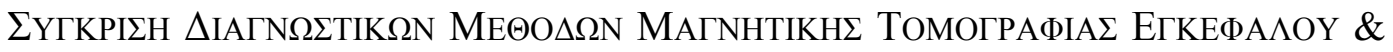
E $\Xi E T A \Sigma H \Sigma$ ANTANAK $\Lambda$ A $\Sigma T I K O Y$ B $\Lambda$ EФAP $\Omega N$

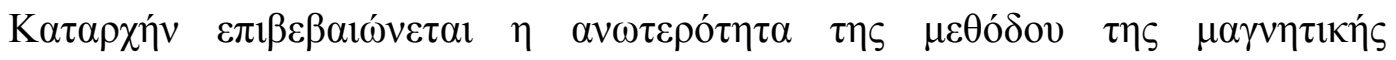

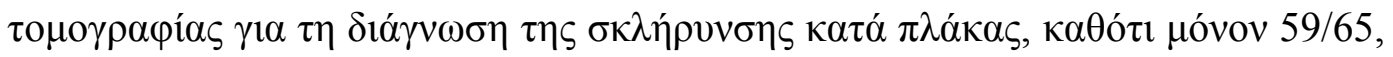

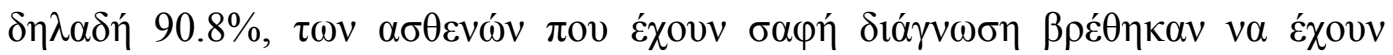
$\pi \alpha \theta 0 \lambda$ о

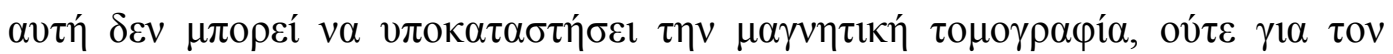

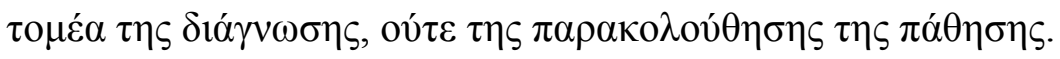

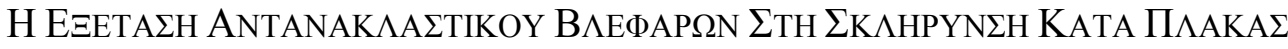

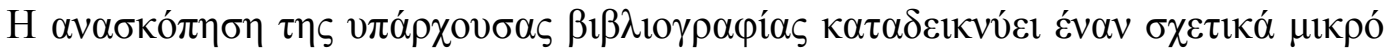

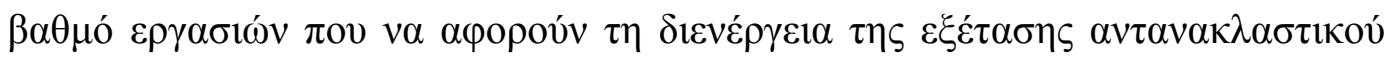

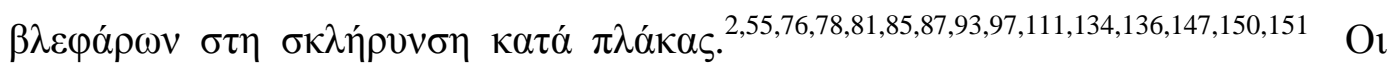

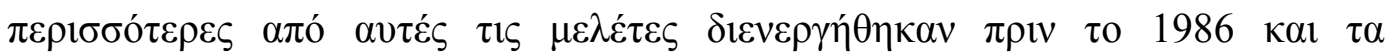

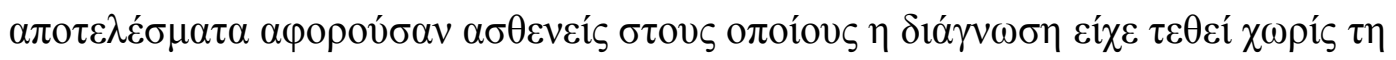

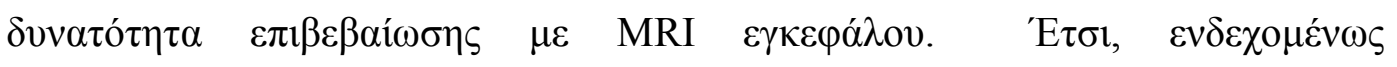

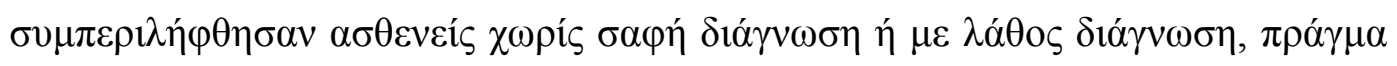

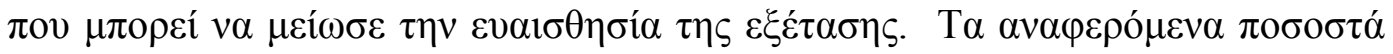

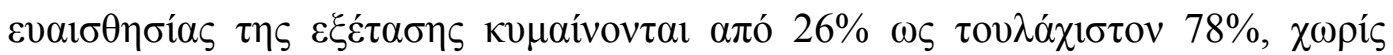

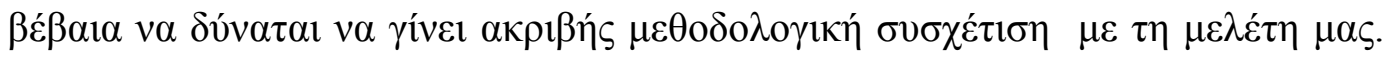

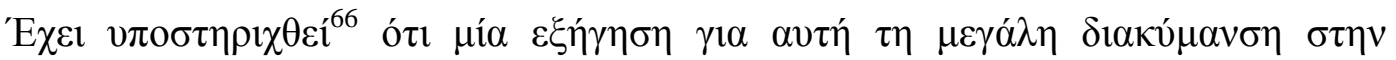

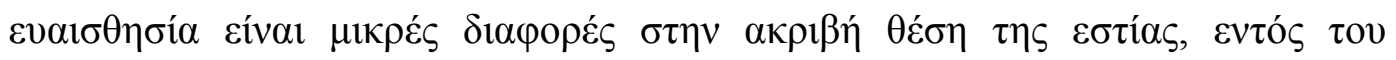

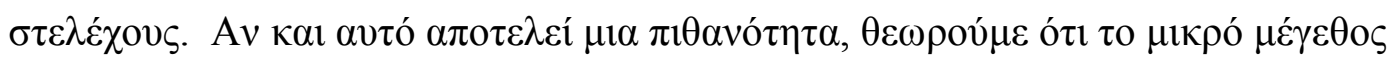

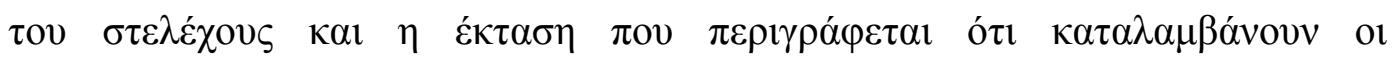

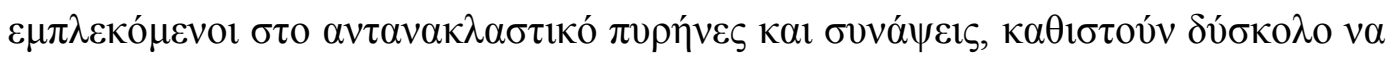

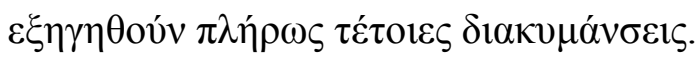




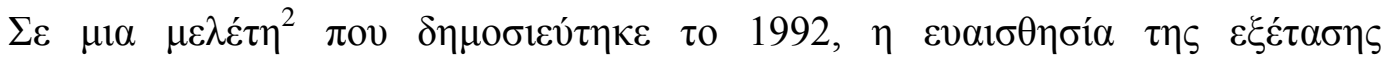

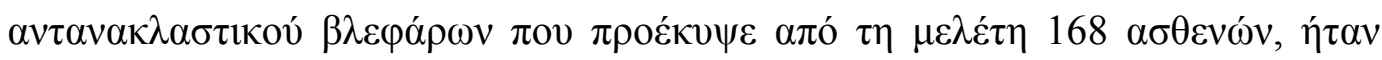

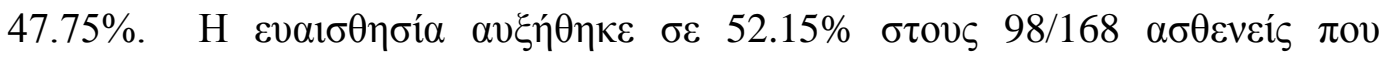

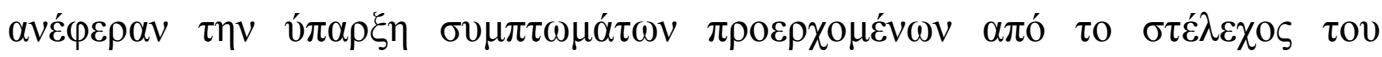

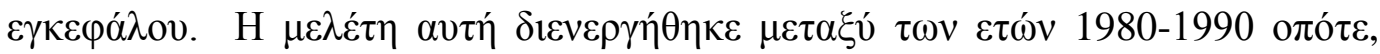

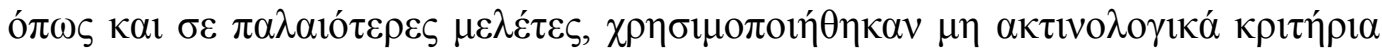

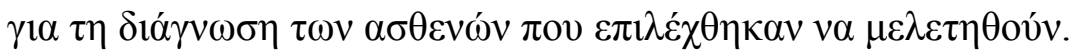

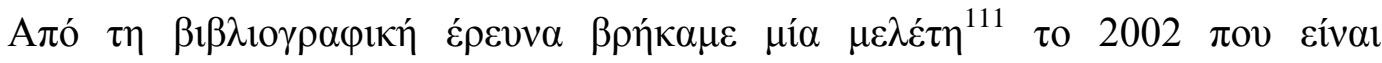

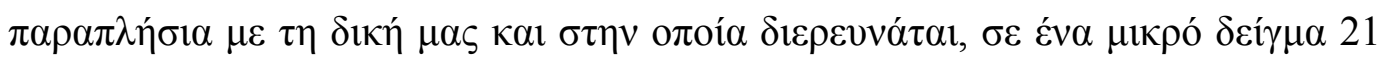

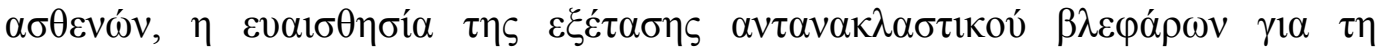

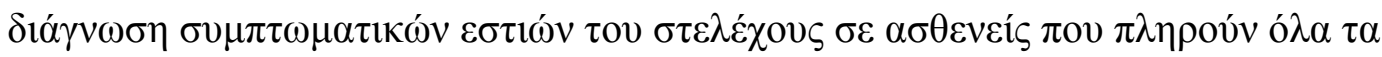

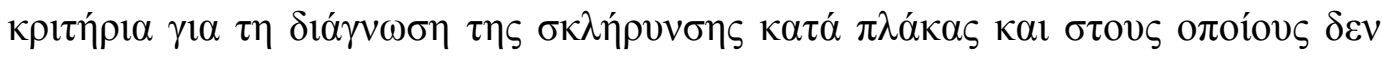

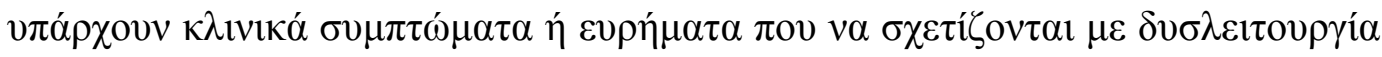

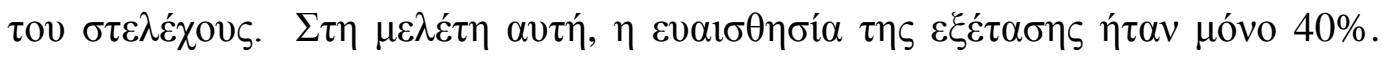

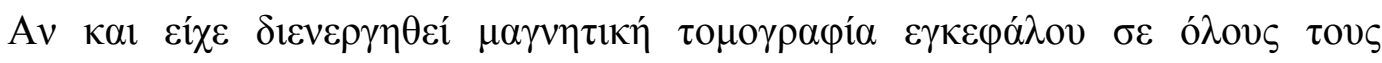

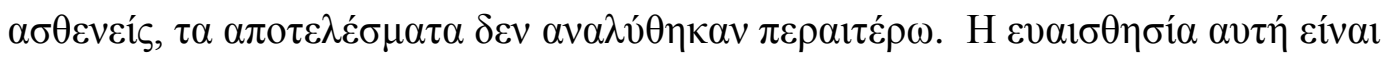

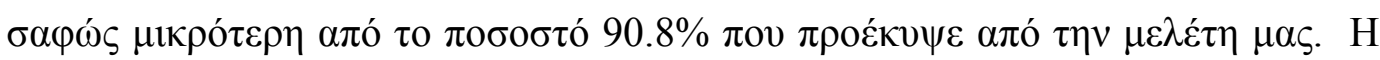

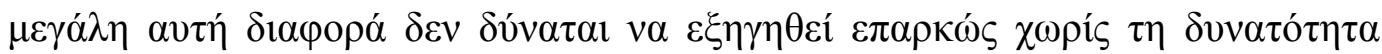

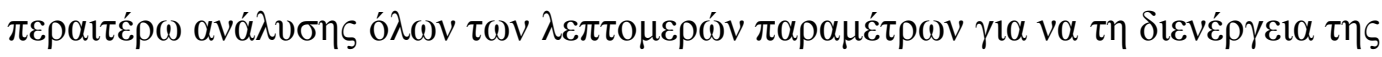

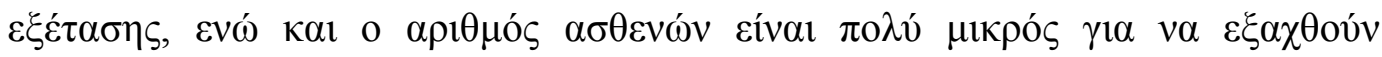

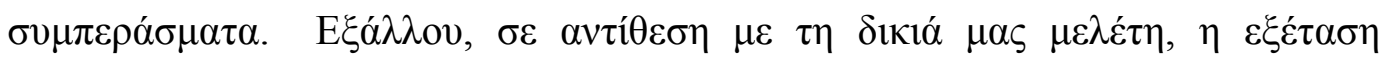

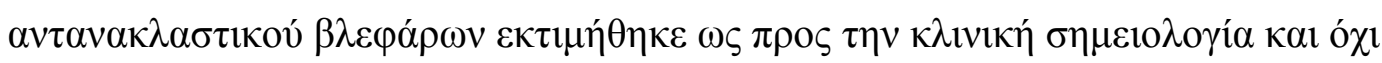

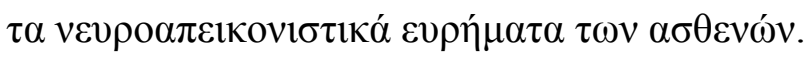

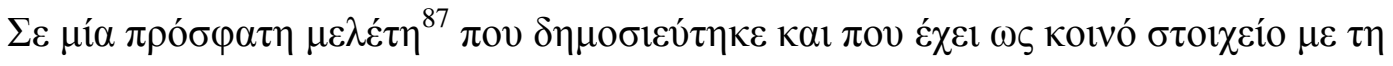

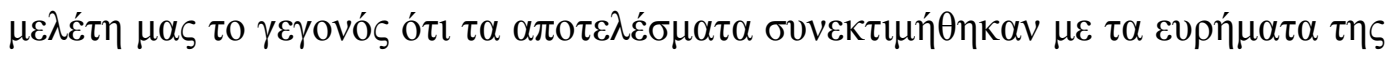

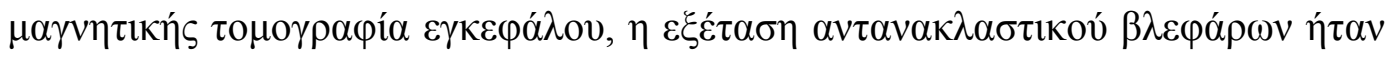

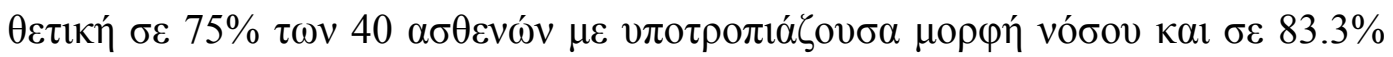

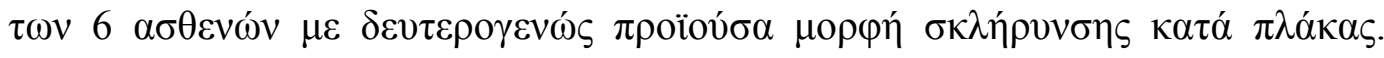

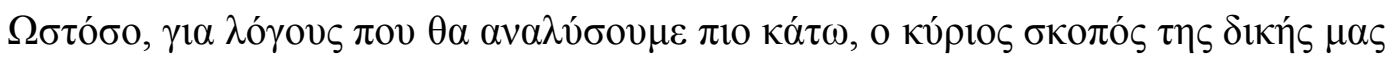

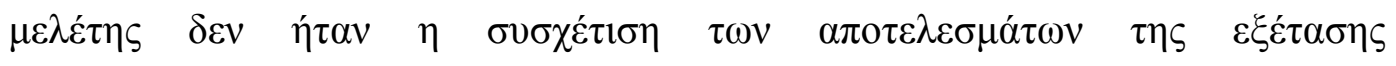




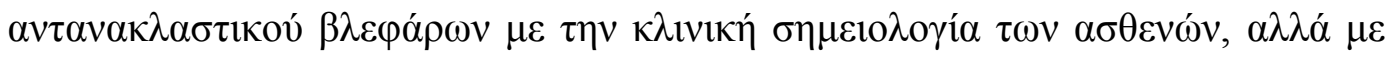

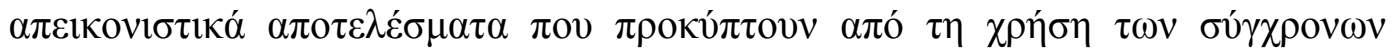

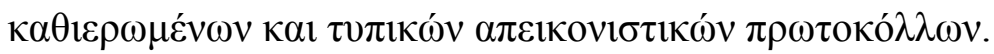

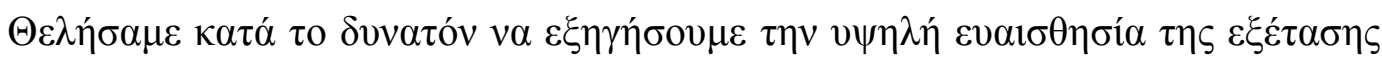

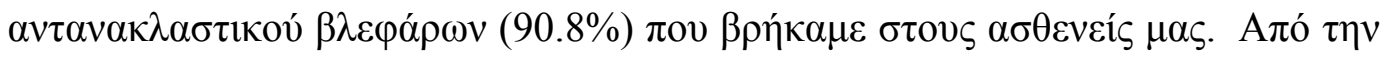

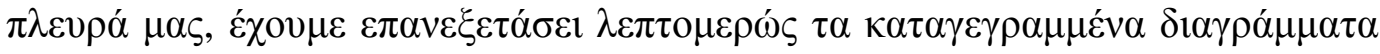

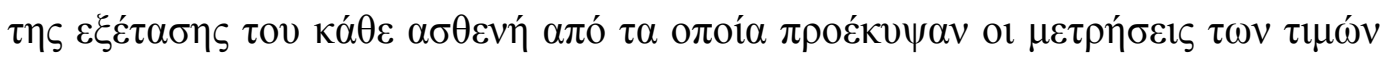

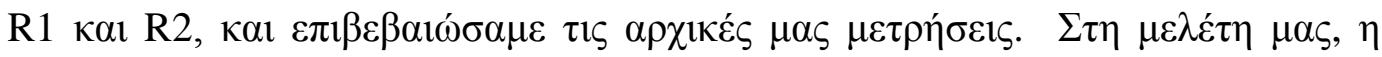

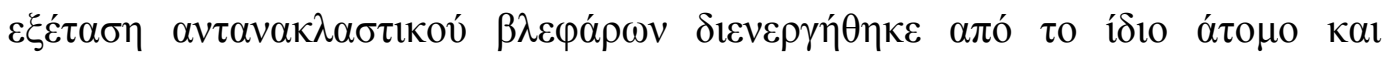

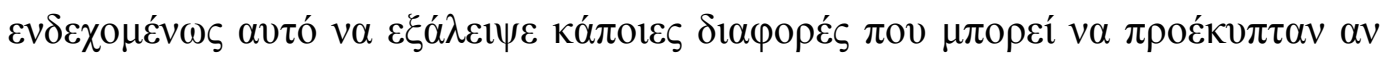

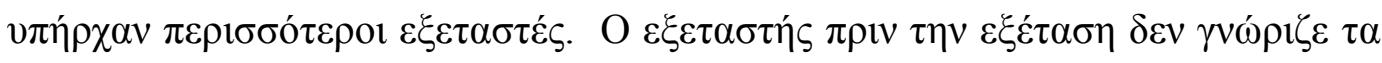

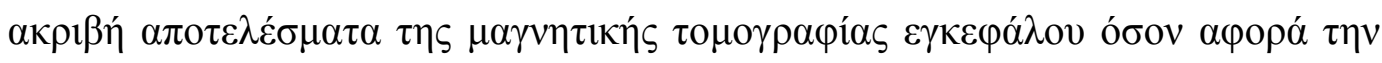

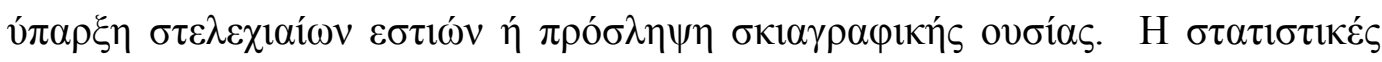

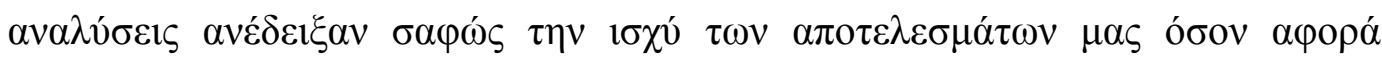

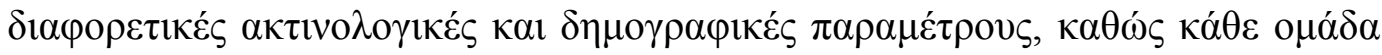

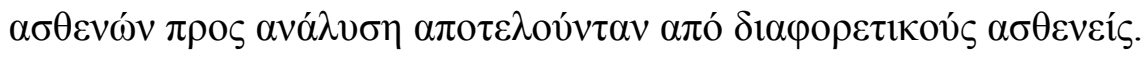

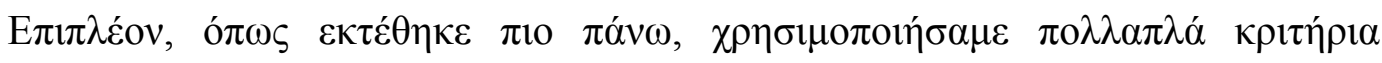

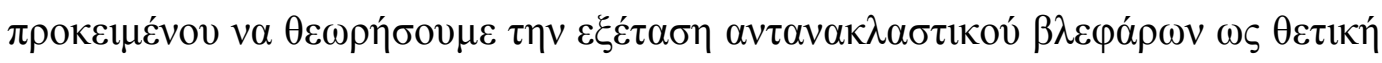

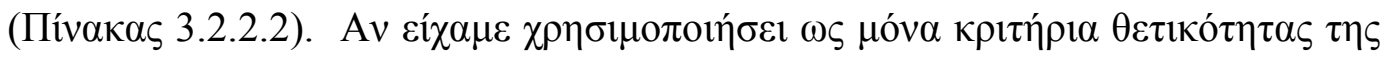

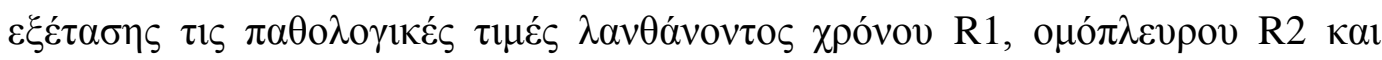

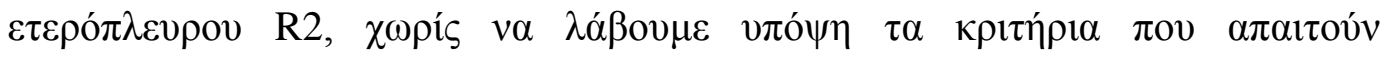

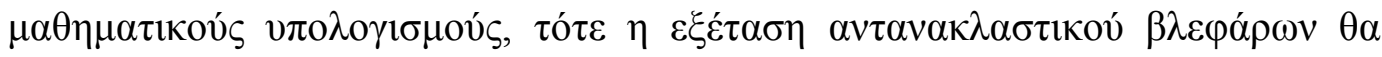

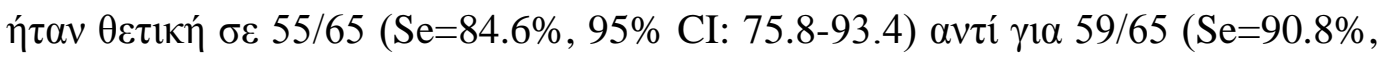

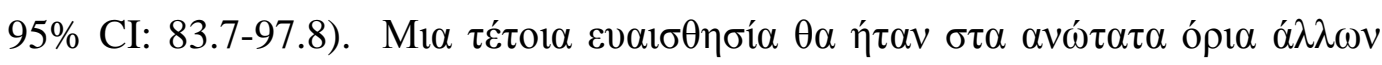

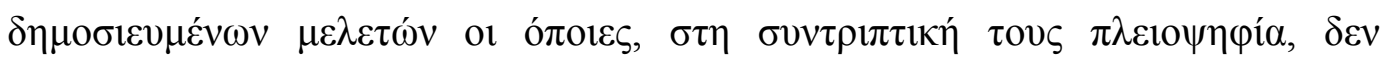

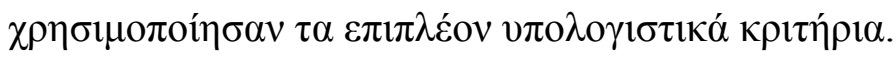

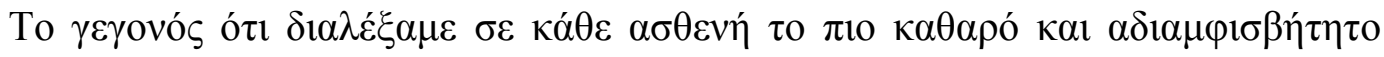

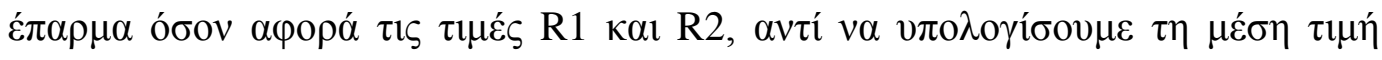

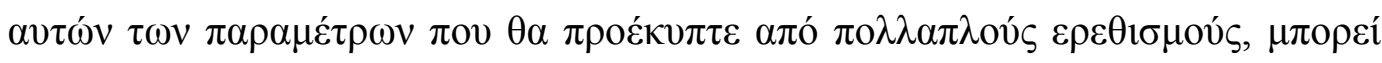

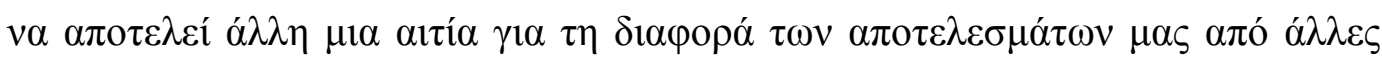




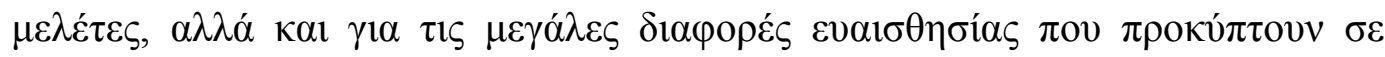

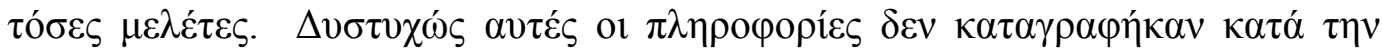

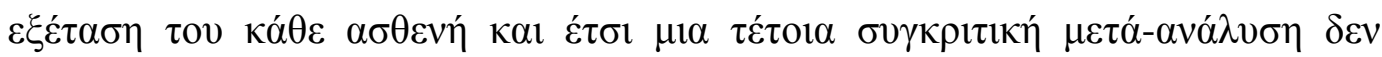

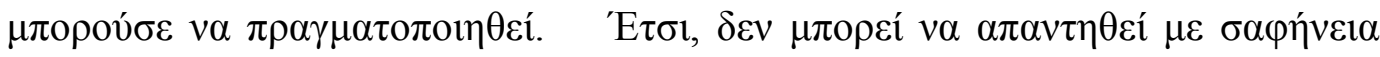

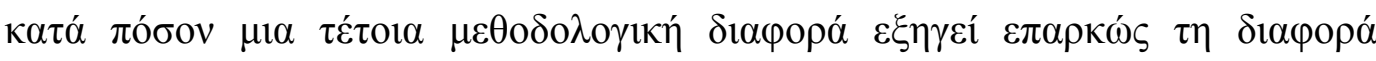

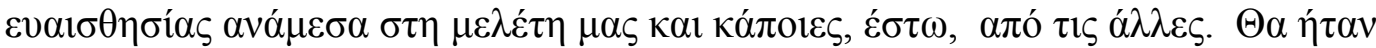

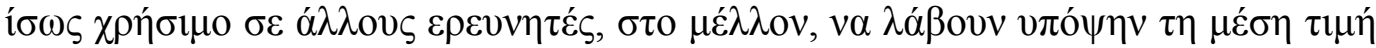

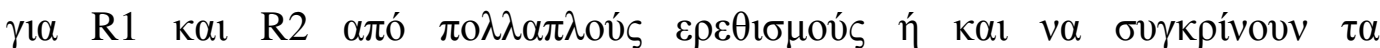

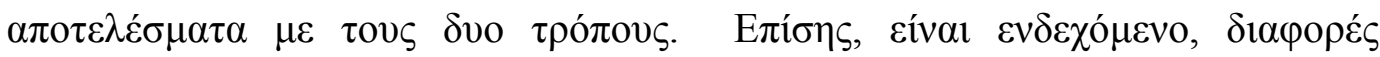

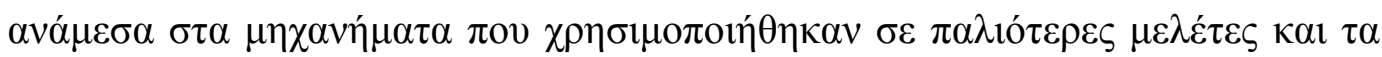

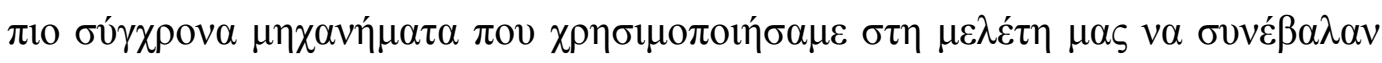

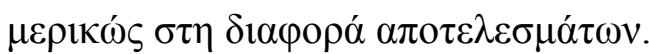

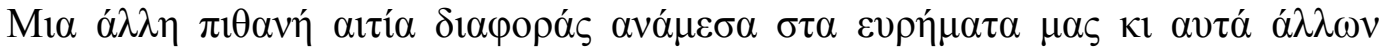

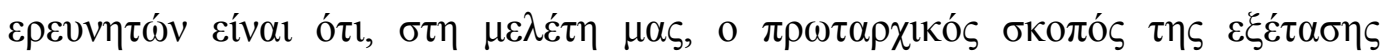

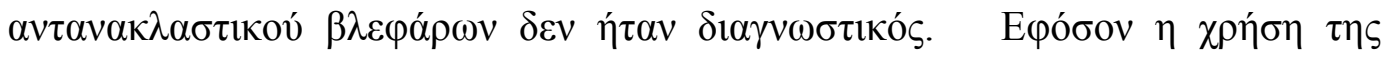

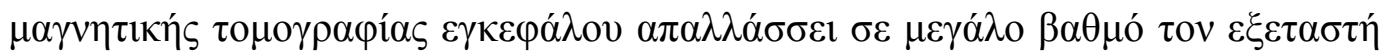

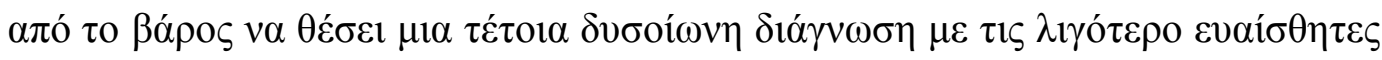

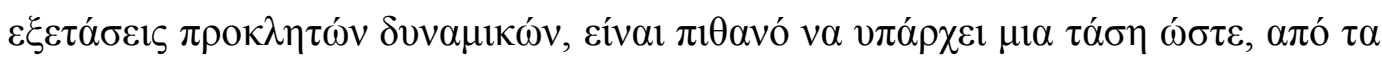

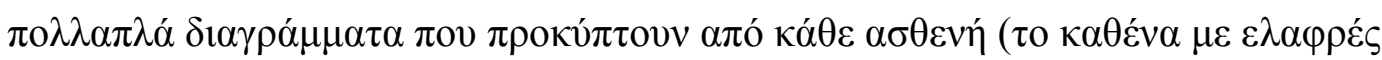

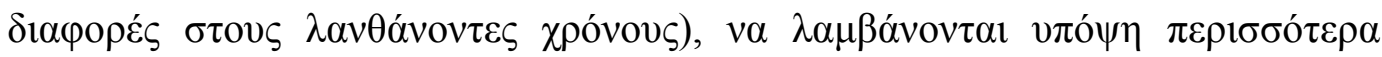

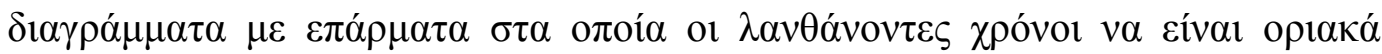

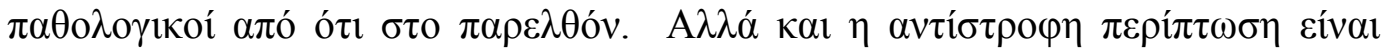

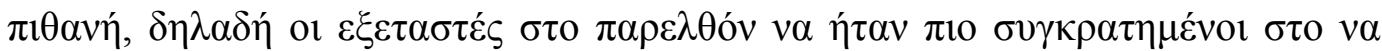

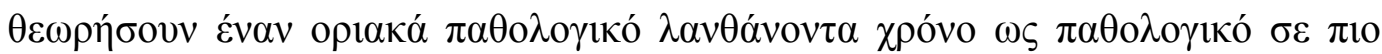

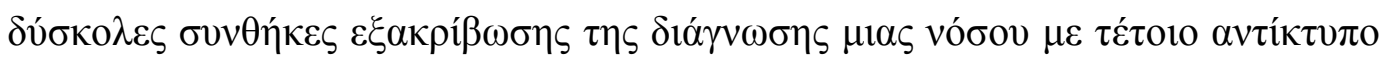
$\sigma \varepsilon \dot{\varepsilon} v \alpha \nu \alpha \sigma \theta \varepsilon v \eta \dot{~}$

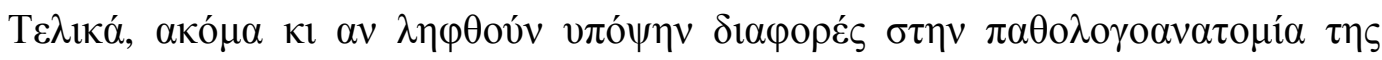

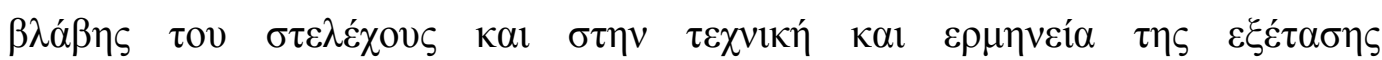

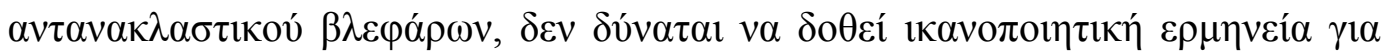

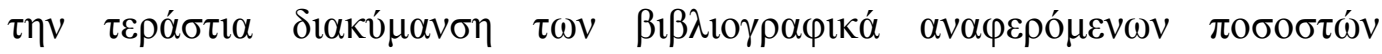

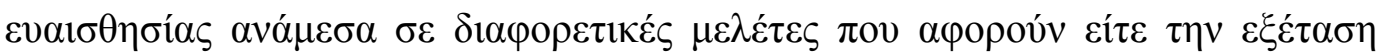




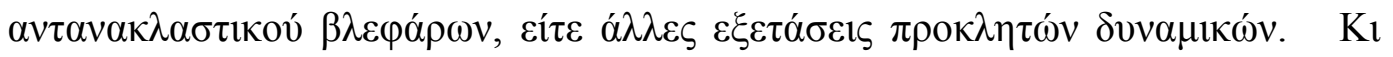

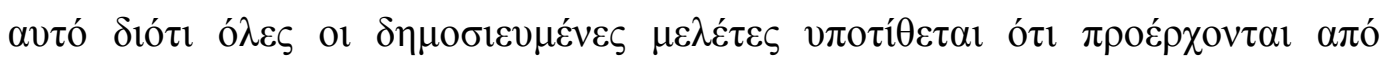

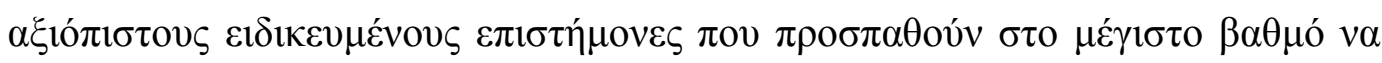

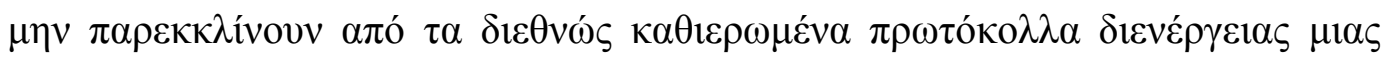
$\varepsilon \xi \dot{\varepsilon} \tau \alpha \sigma \eta \varsigma$.

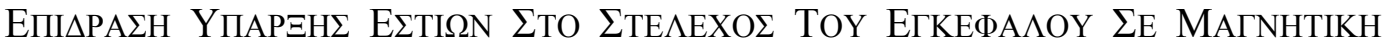
ТОМОГРАФІА ЕГКЕФА ОО

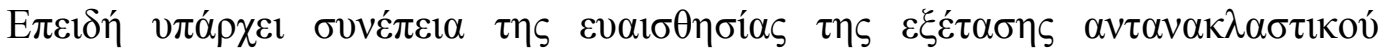

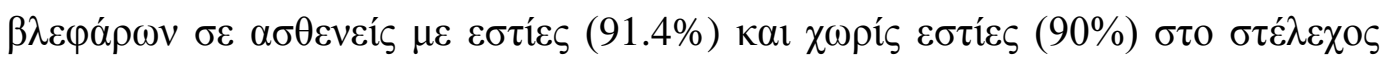

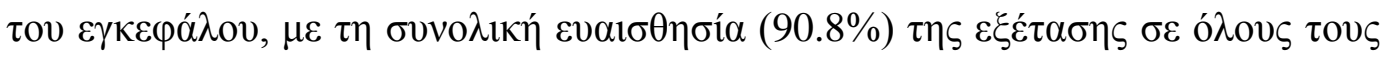

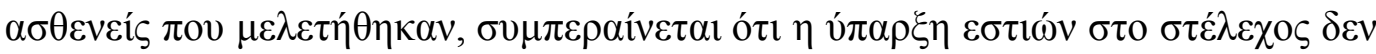

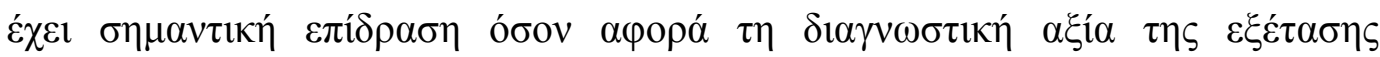
$\alpha v \tau \alpha v \alpha \kappa \lambda \alpha \sigma \tau 1 \kappa o v ́ ~ \beta \lambda \varepsilon \varphi \alpha ́ \rho \omega v$.

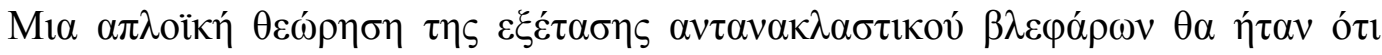

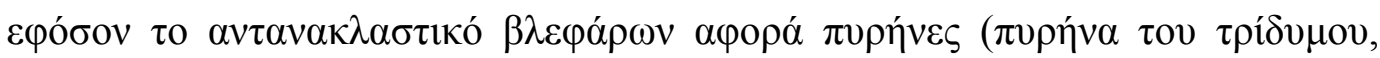

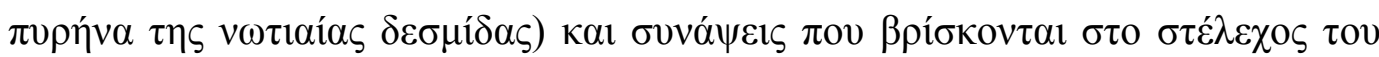

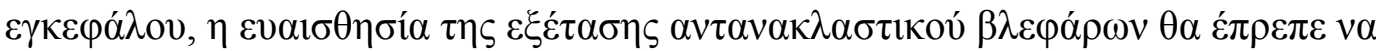

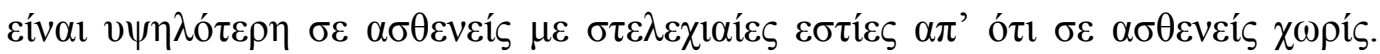

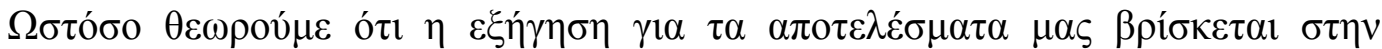

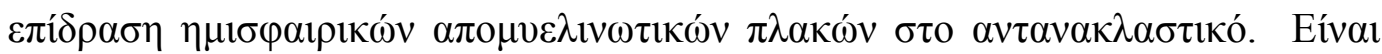

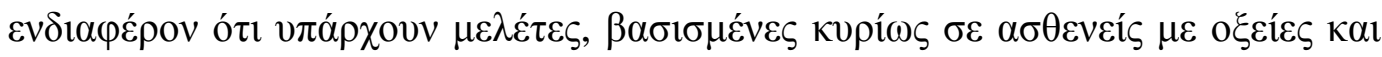

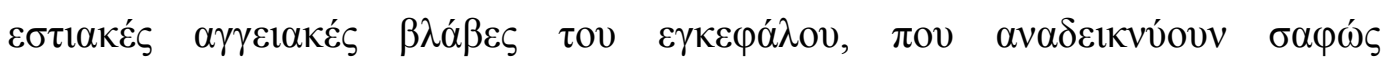

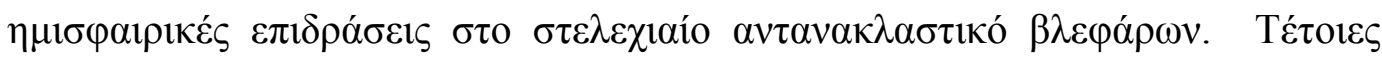

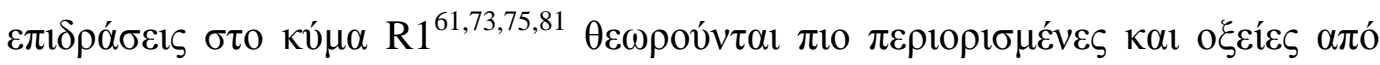

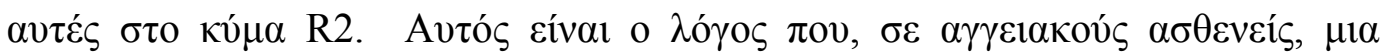

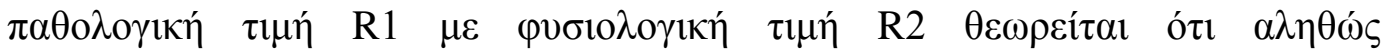

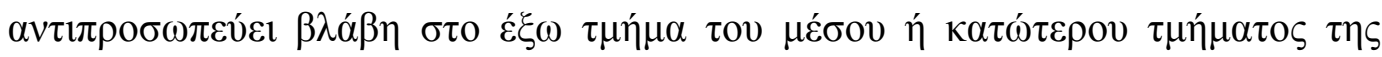

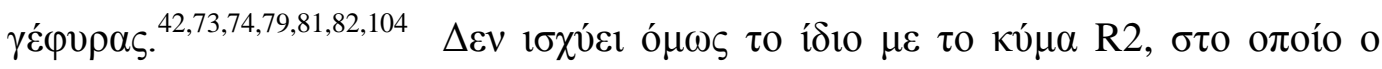

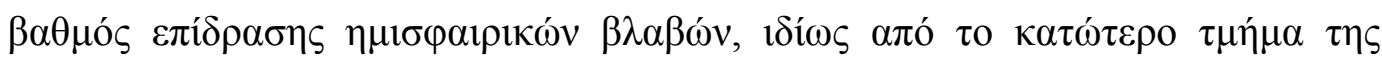
o

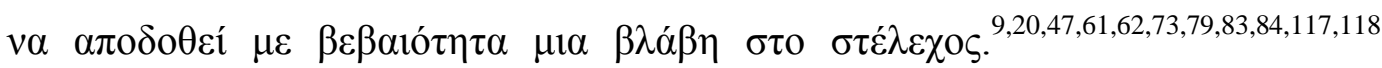

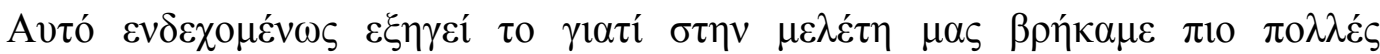




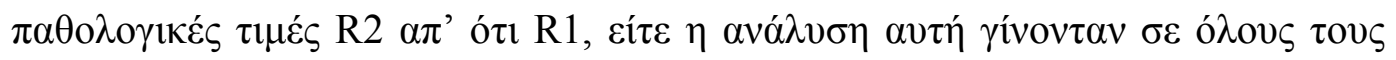

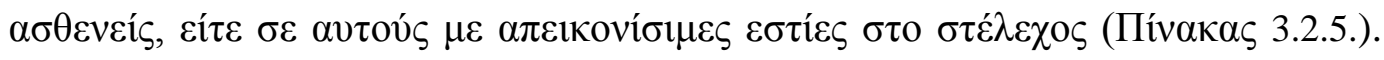

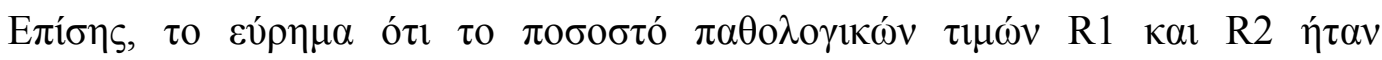

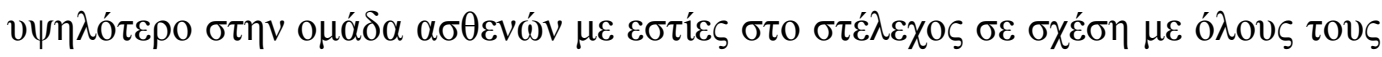

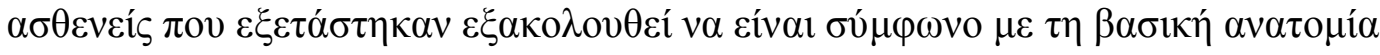

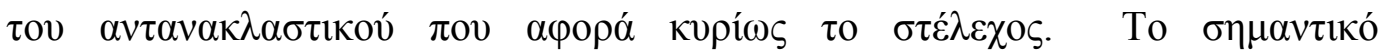

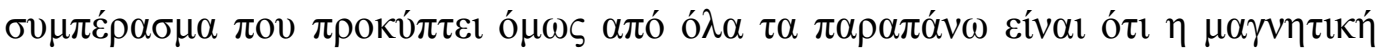

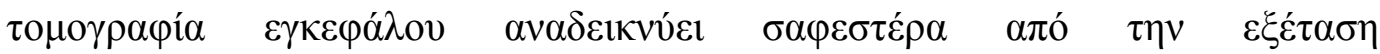

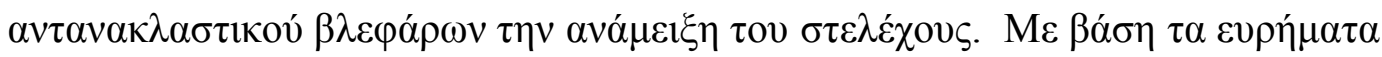

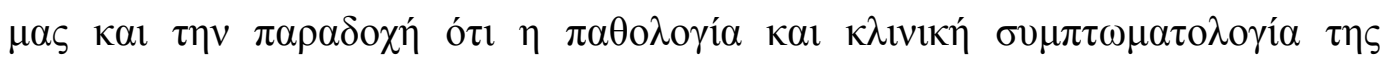

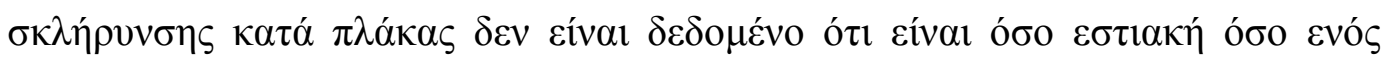

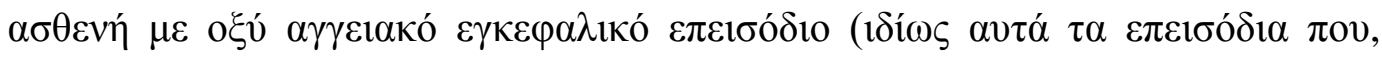

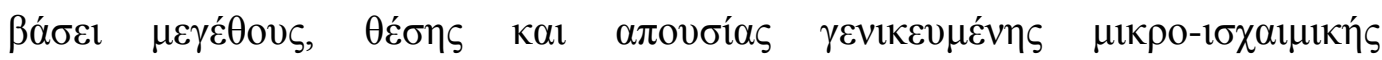

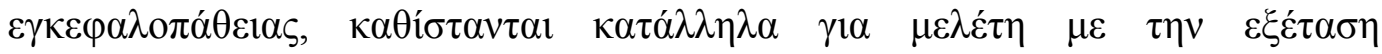

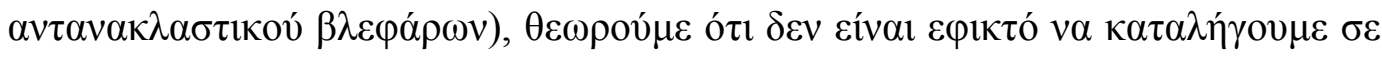

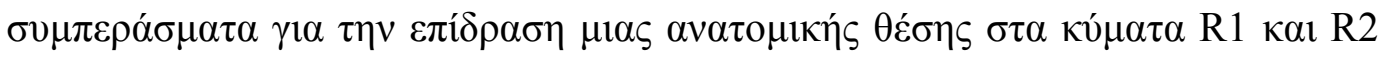

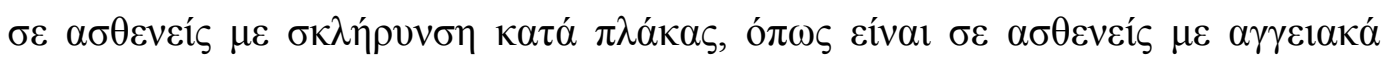

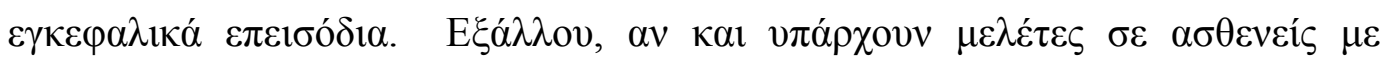

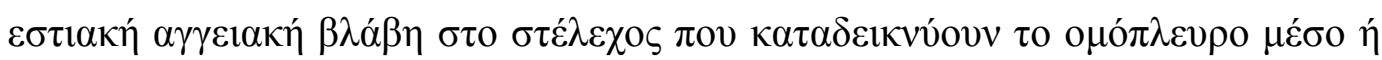

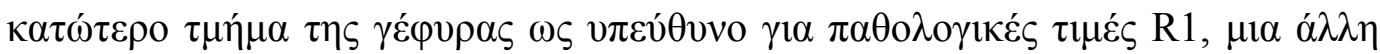

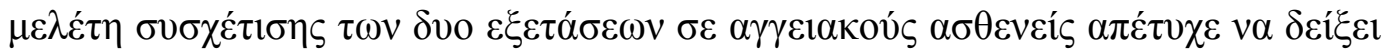

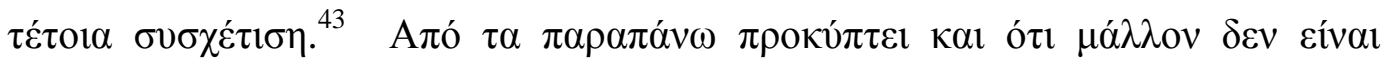

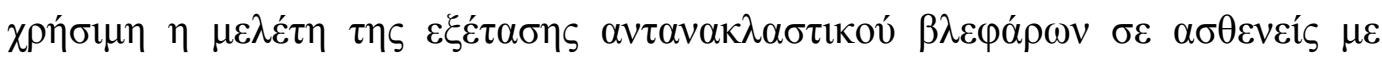

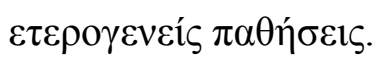

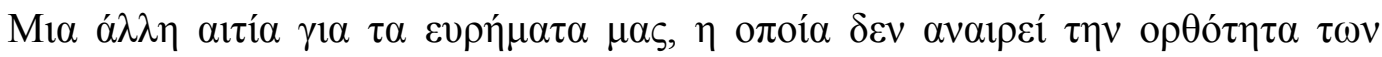

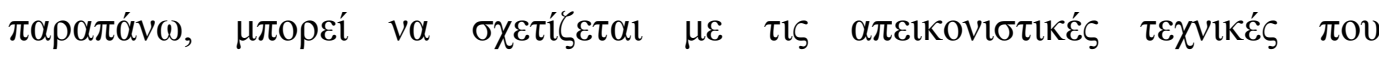

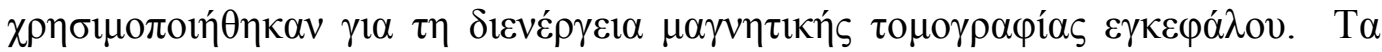

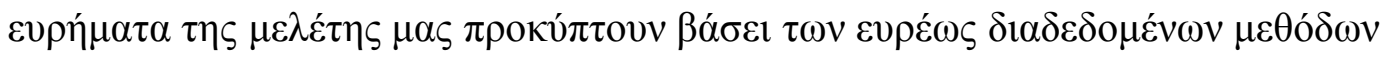

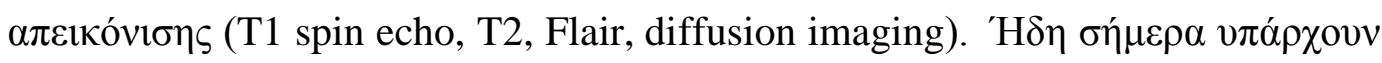

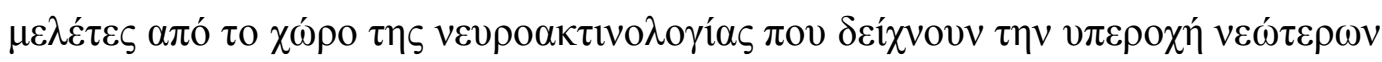

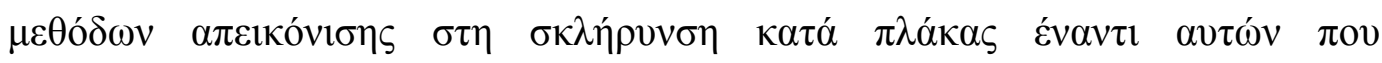

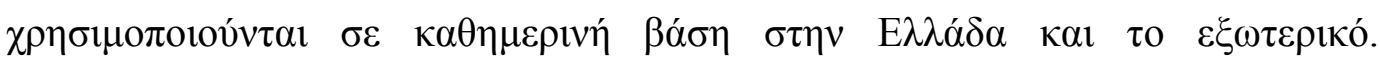
$\Sigma v \mu \pi \varepsilon \rho \iota \lambda \alpha \mu \beta \alpha ́ v o v v \tau \eta v$ magnetization transfer ratio (MTR) imaging, ${ }^{157}$ dual 
inversion recovery imaging, diffusion tensor imaging ${ }^{35,70}$ (fractional anisotropy,

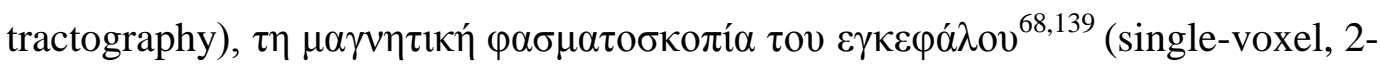

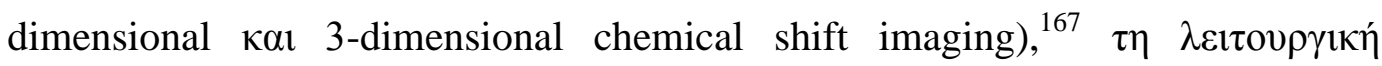

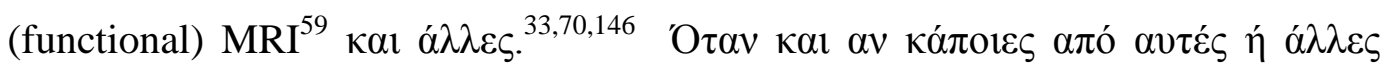

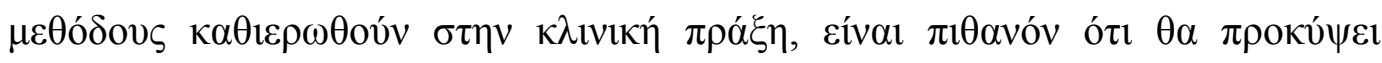

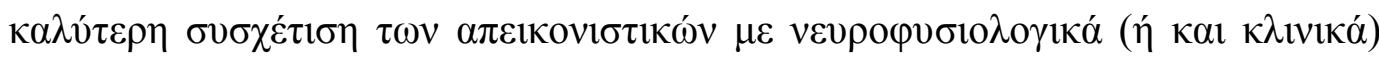

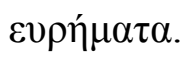

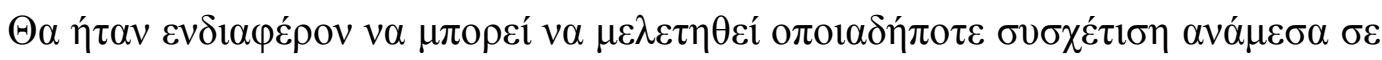

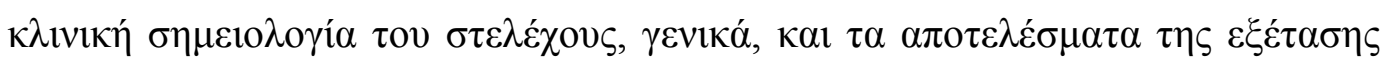

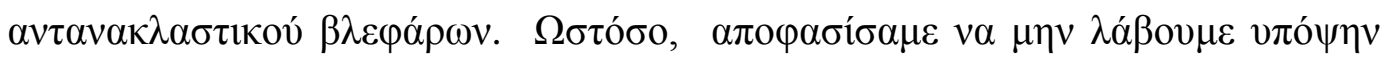

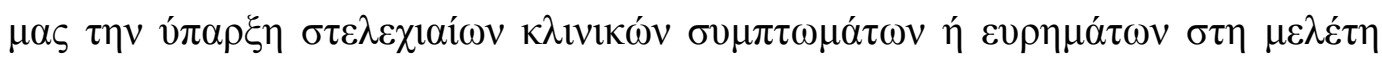

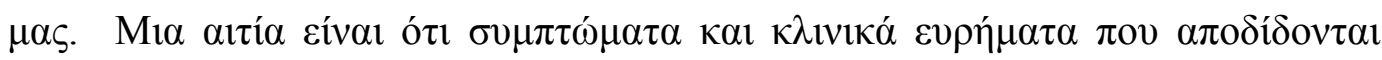

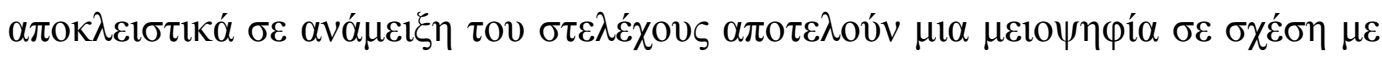

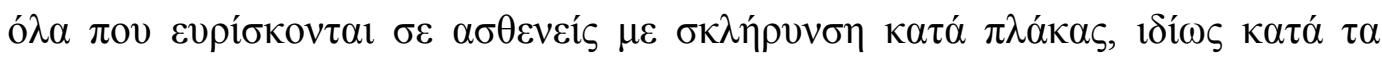

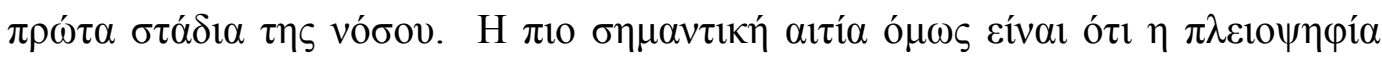

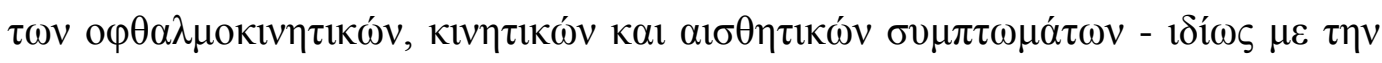

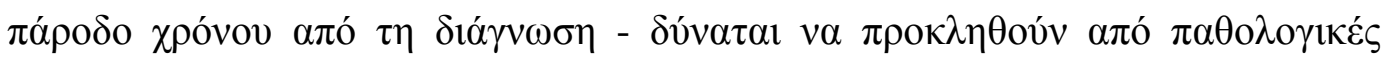

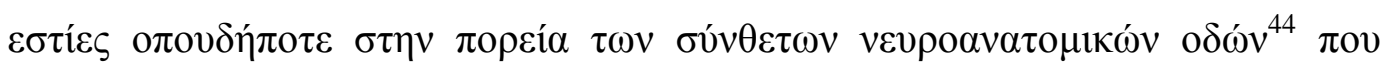

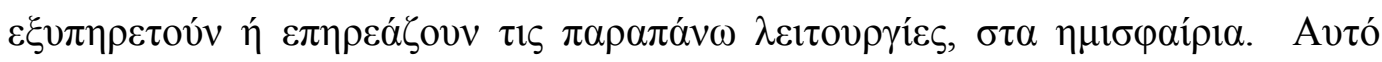

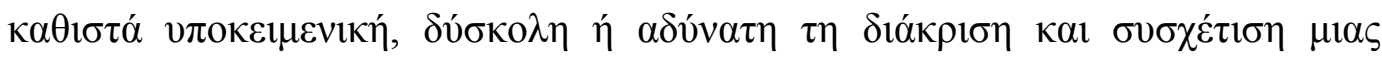

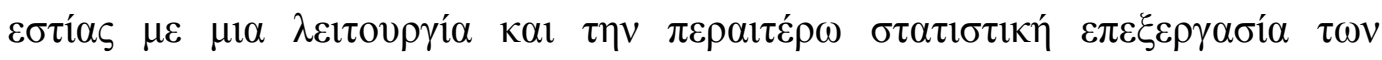

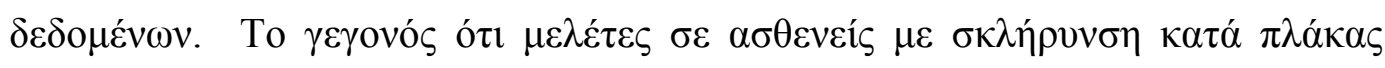

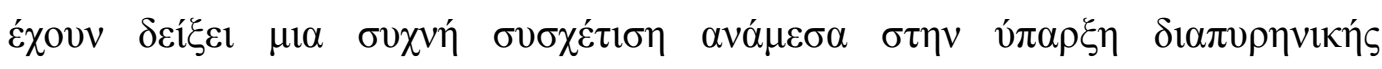

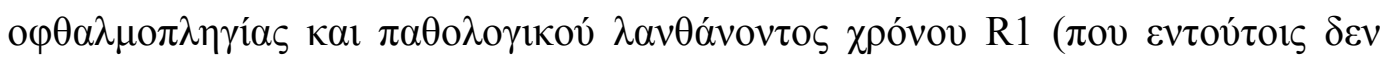

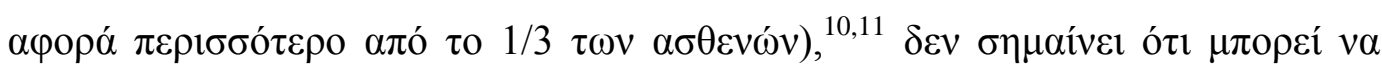

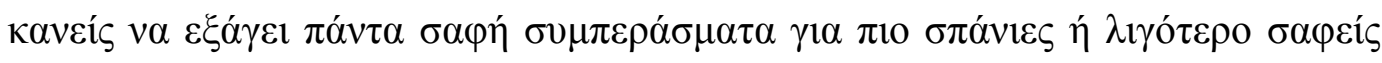

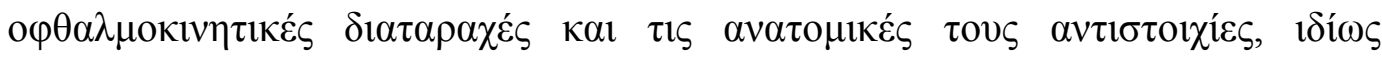

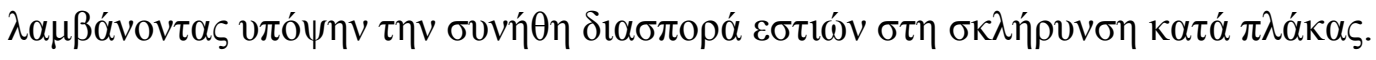

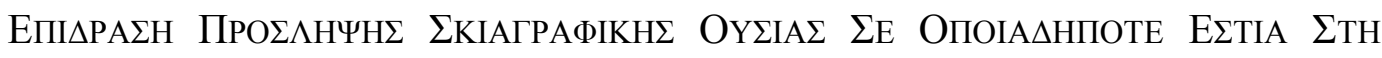

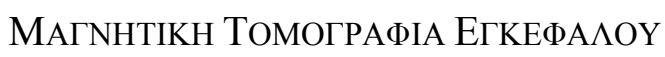

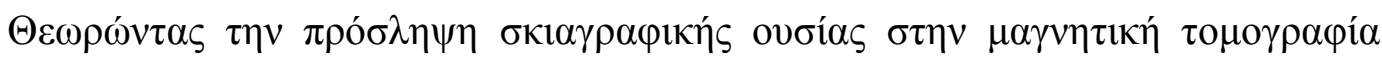

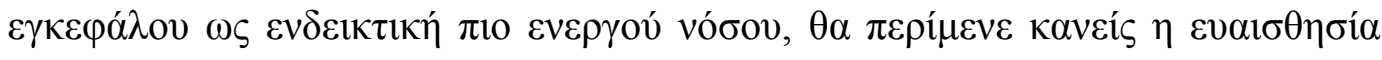




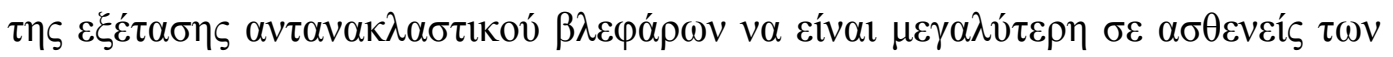

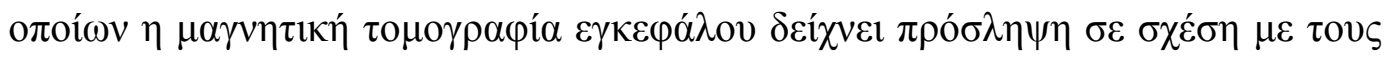

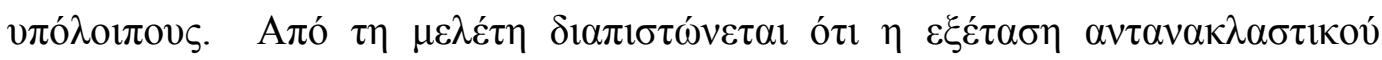

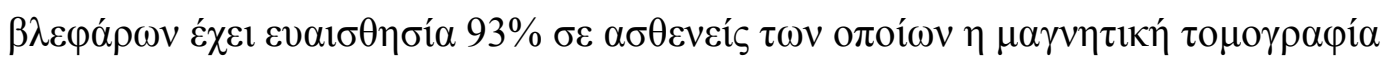

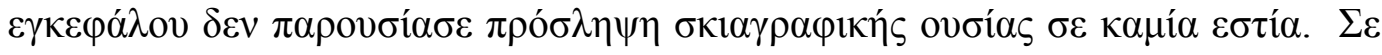

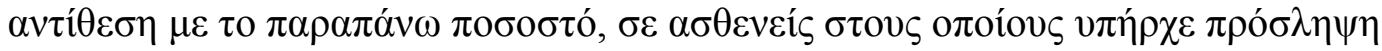

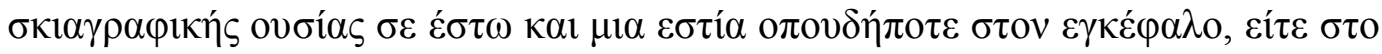

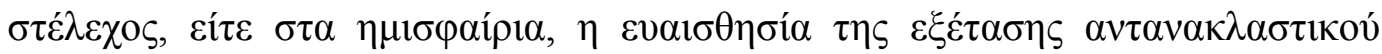

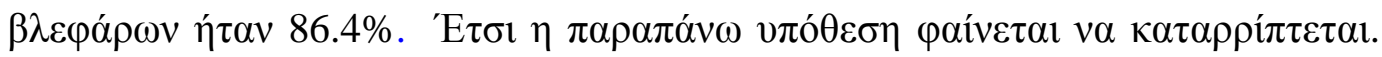

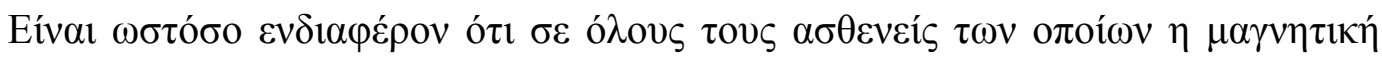

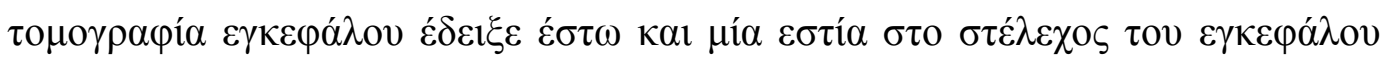

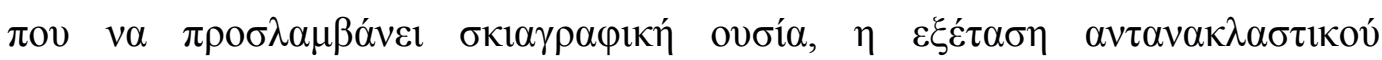

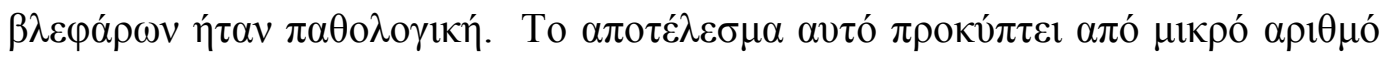

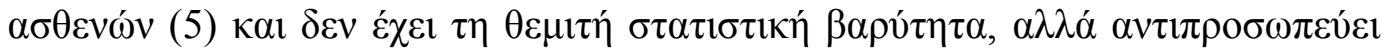

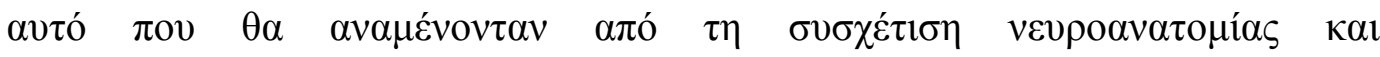

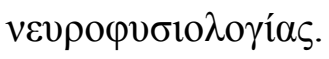

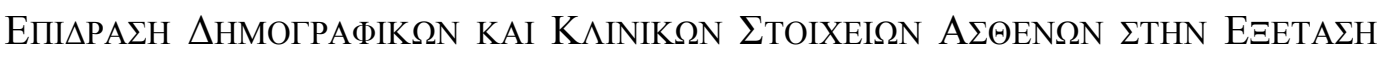
ANTANAK $\Lambda$ ALTIKOY B $\Lambda$ EФAP $\Omega N$

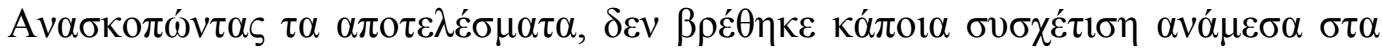

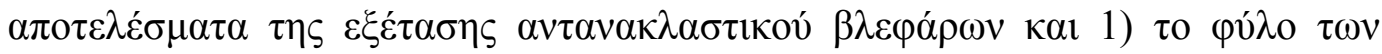

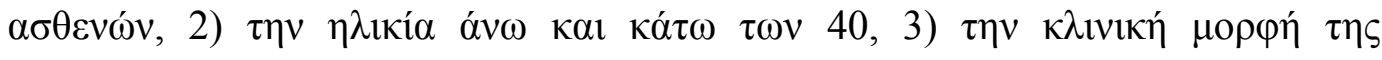

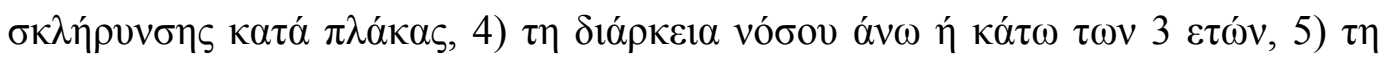

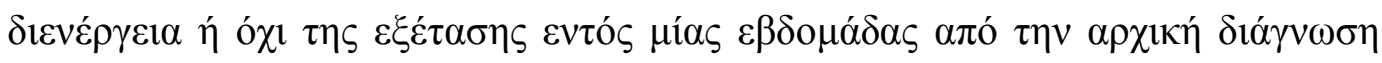

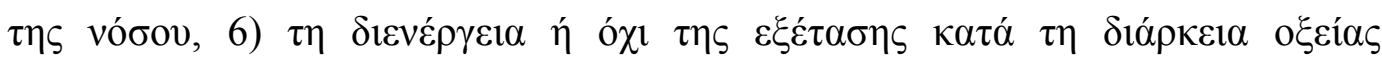

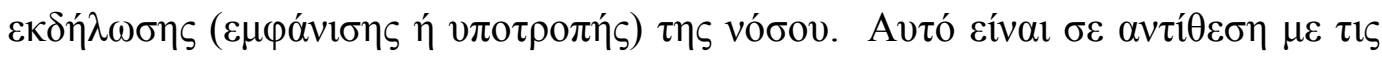

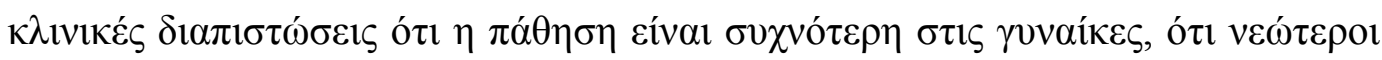

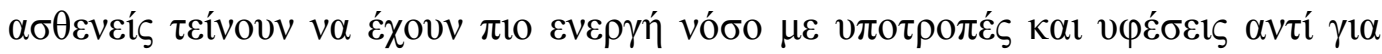

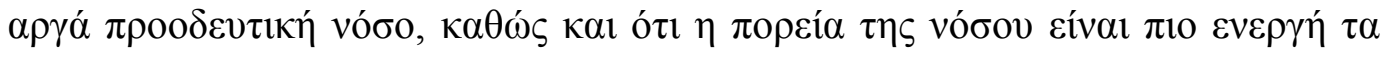

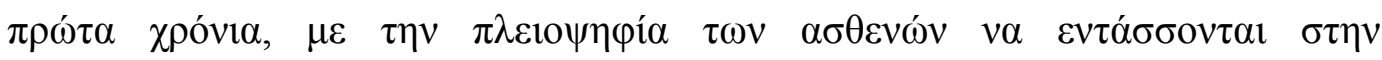

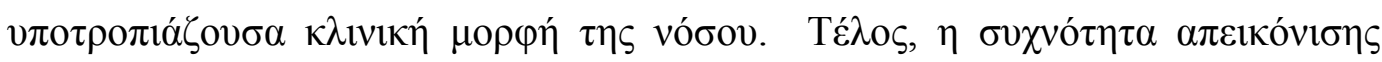

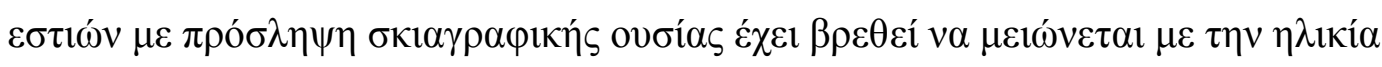

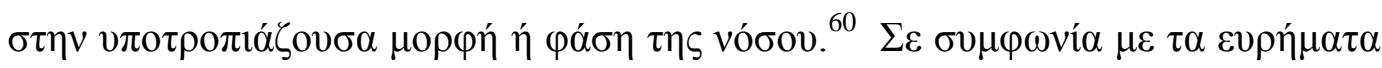

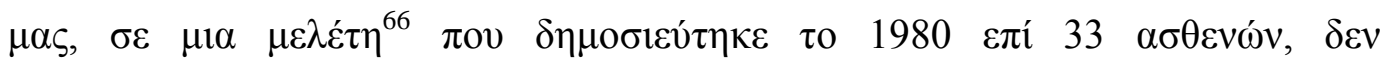




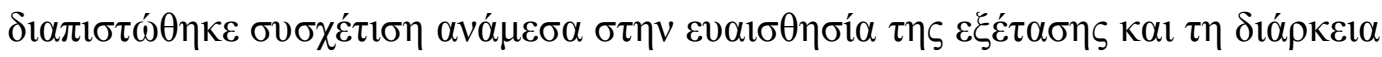
vóбov.

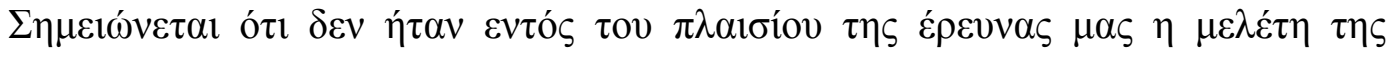

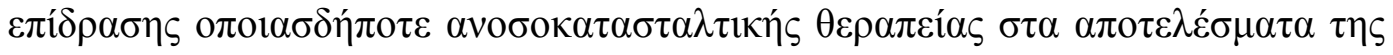

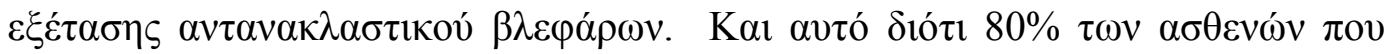

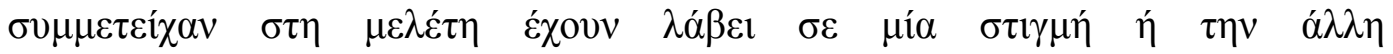

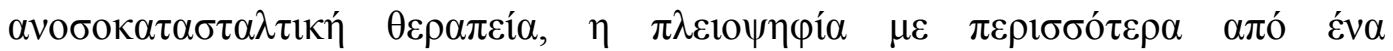

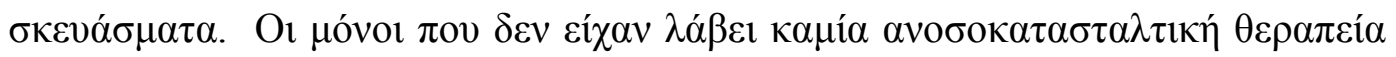

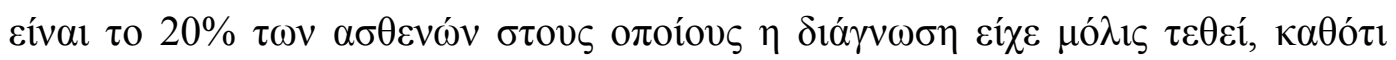

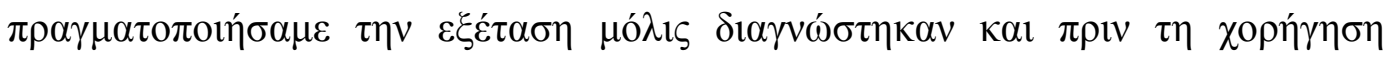

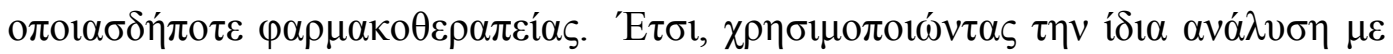

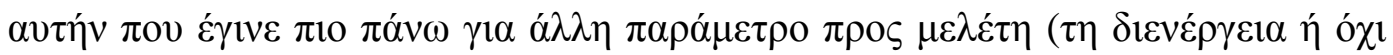

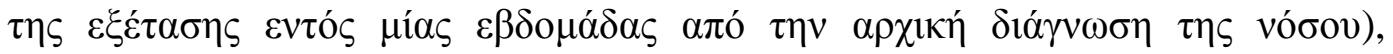

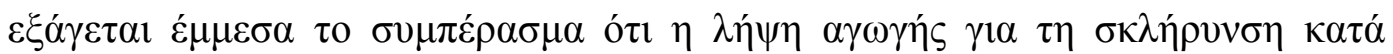
$\pi \lambda \alpha ́ \kappa \alpha \varsigma \delta \varepsilon v \varepsilon \pi \eta \rho \varepsilon \alpha ́ \zeta \varepsilon 1 \tau \alpha \alpha \pi 0 \tau \varepsilon \lambda \varepsilon ́ \sigma \mu \alpha \tau \alpha \tau \eta \varsigma \varepsilon \xi \dot{\varepsilon} \tau \alpha \sigma \eta \varsigma$.

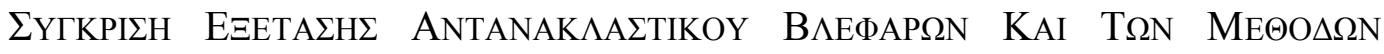

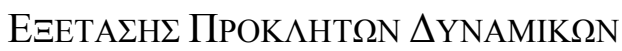

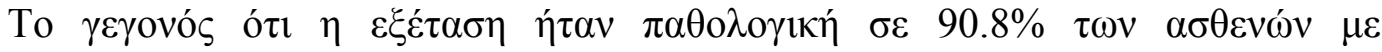

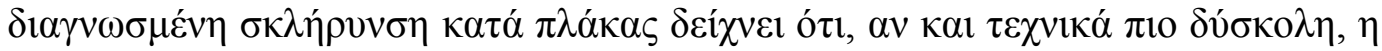

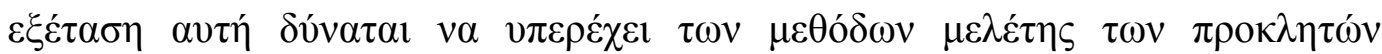

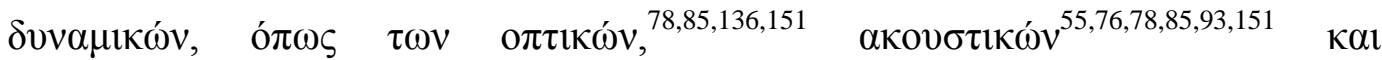

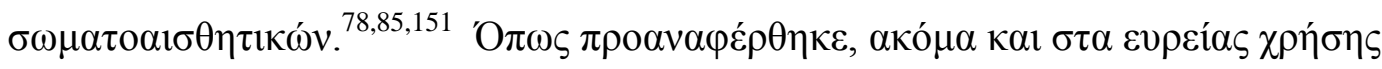

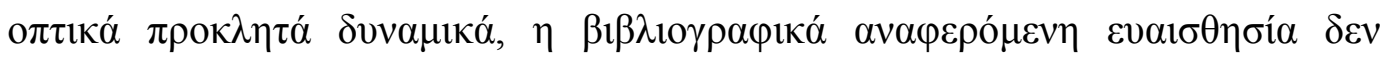

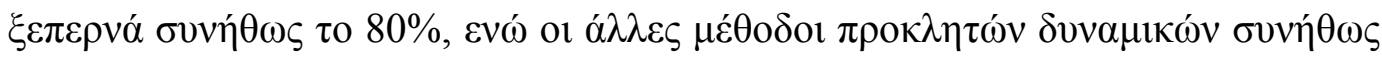

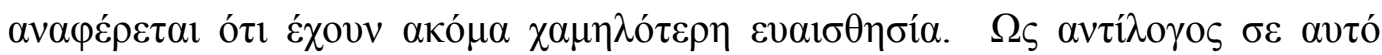

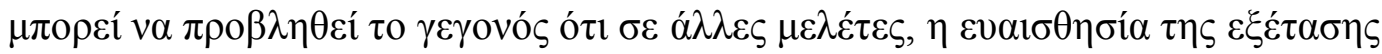

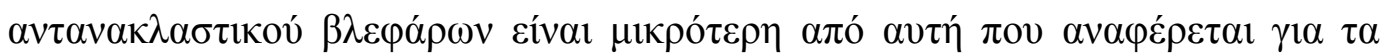

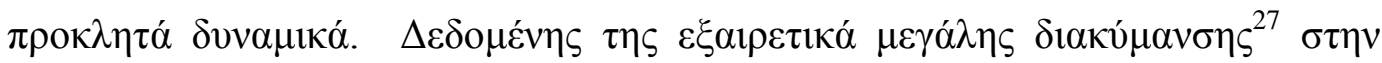

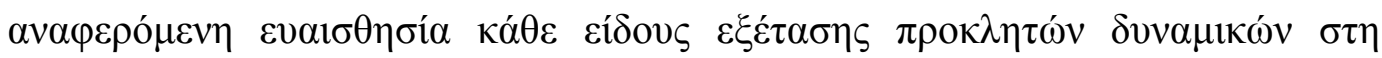

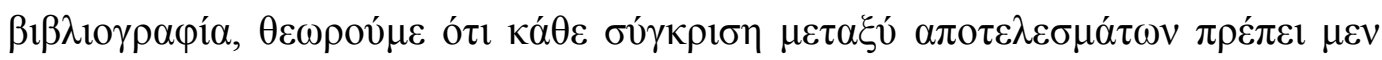

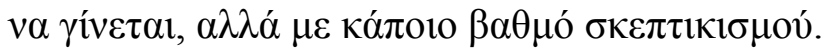




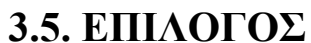

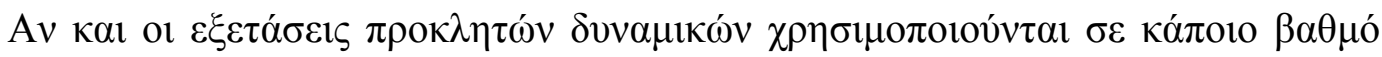

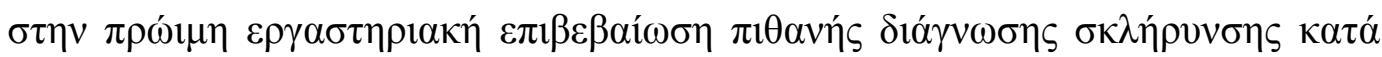

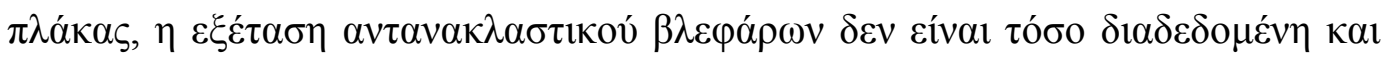

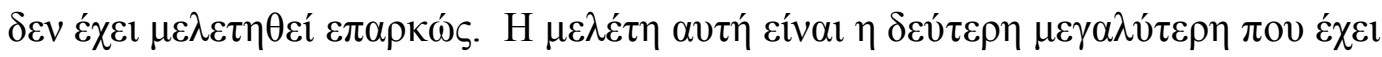

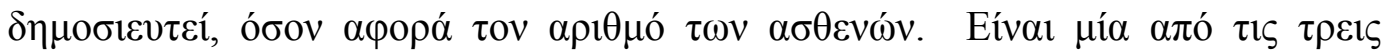

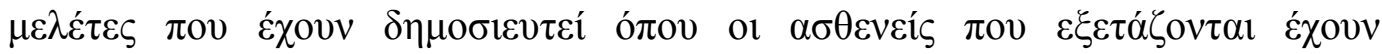

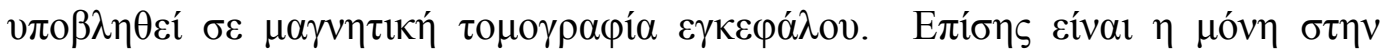

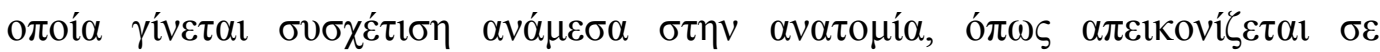

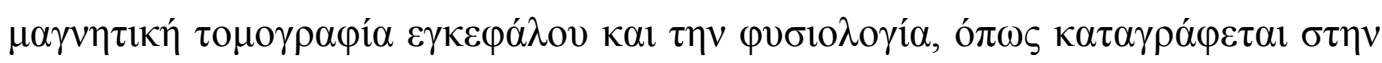

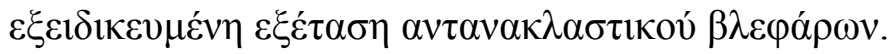

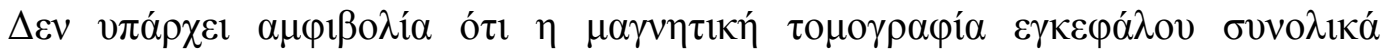

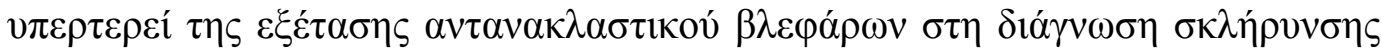

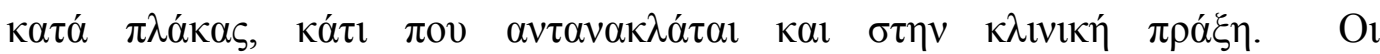

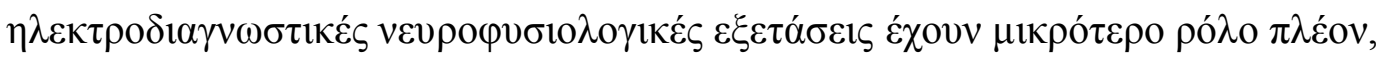

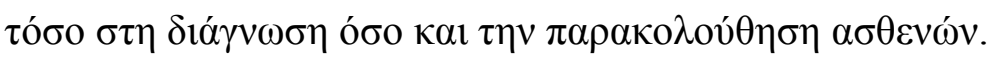

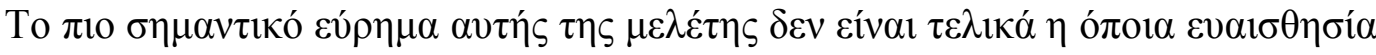

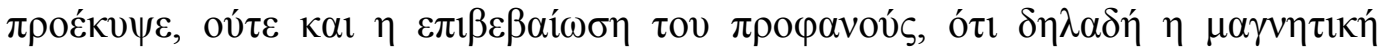

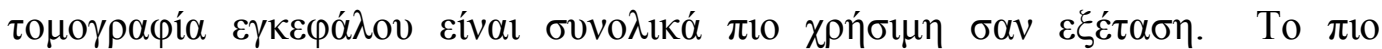

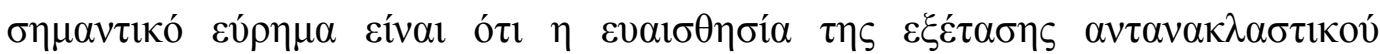

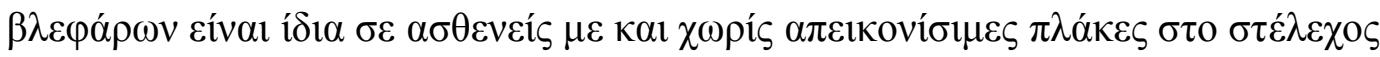

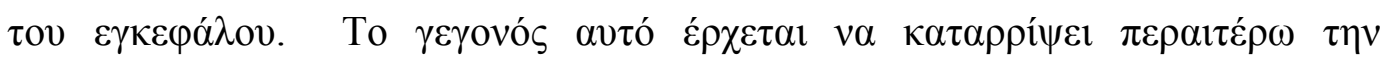

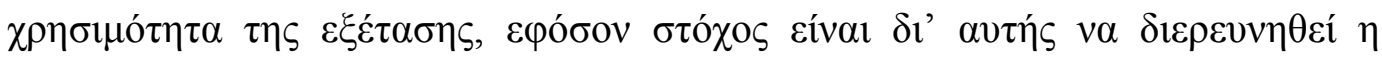

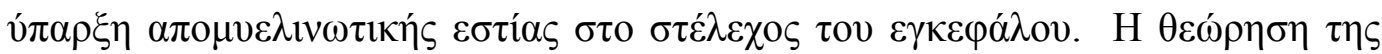

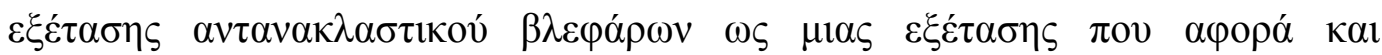

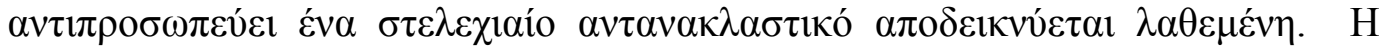

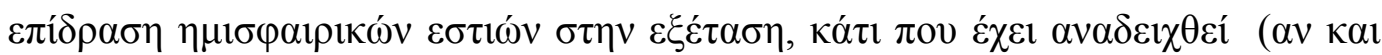

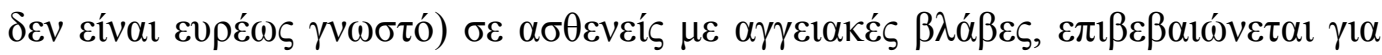

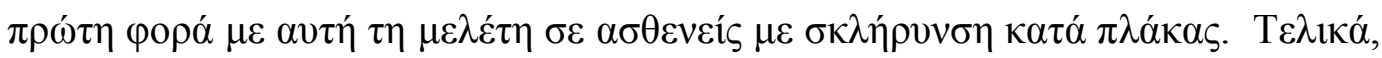

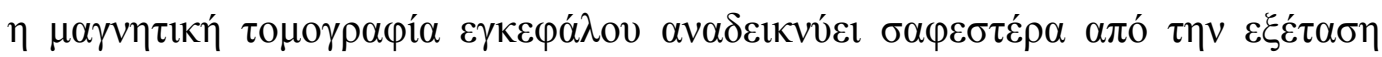

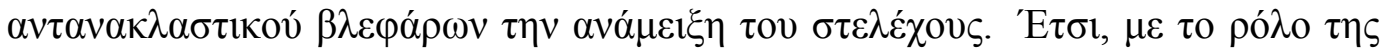

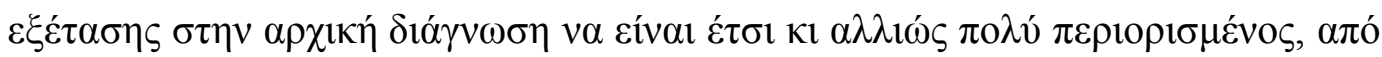




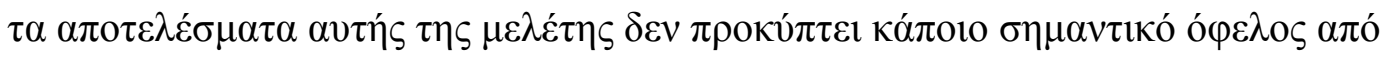

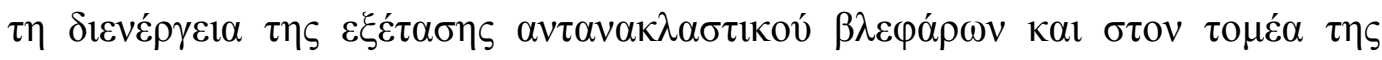

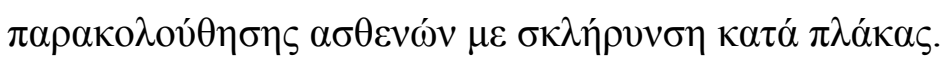

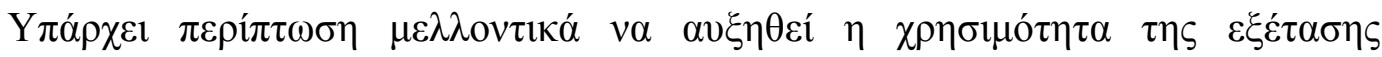

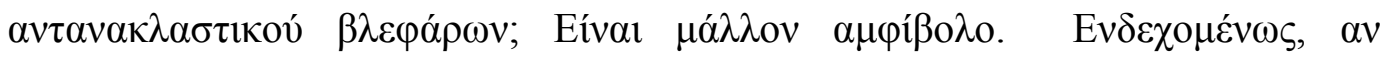

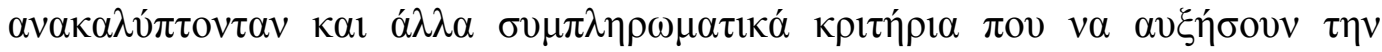

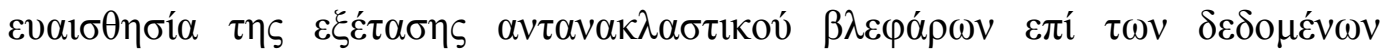

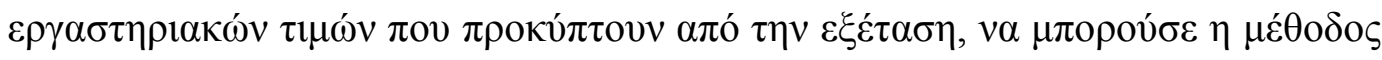

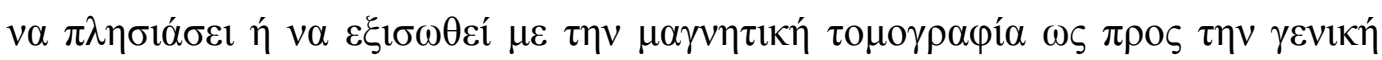

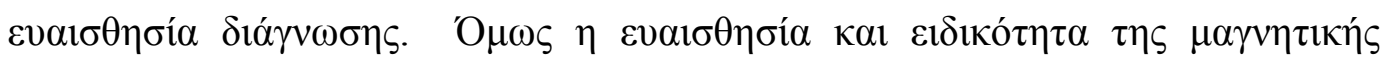

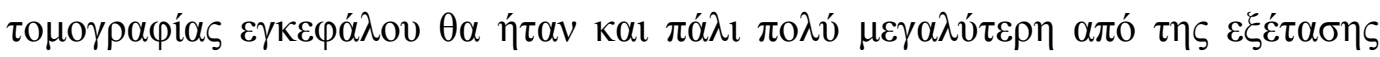

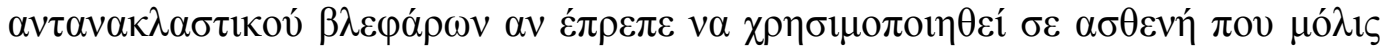

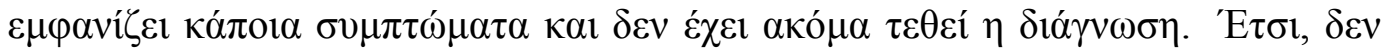

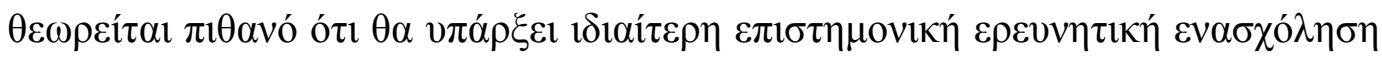

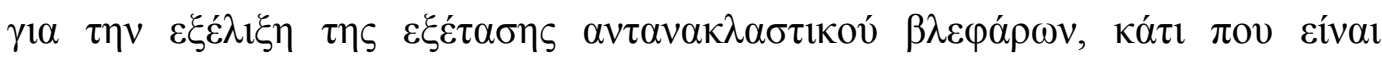

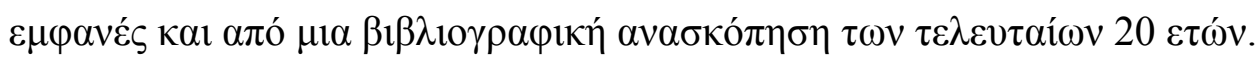

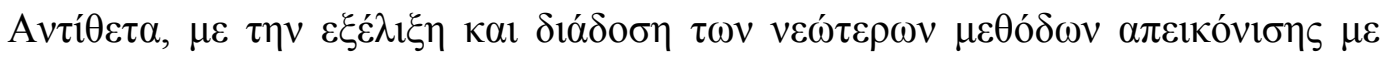

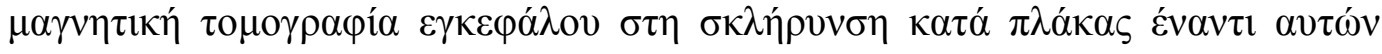

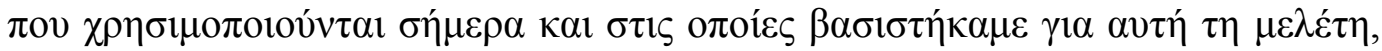

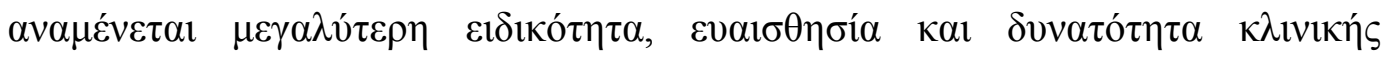

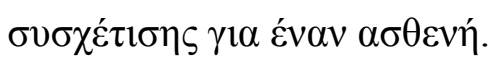

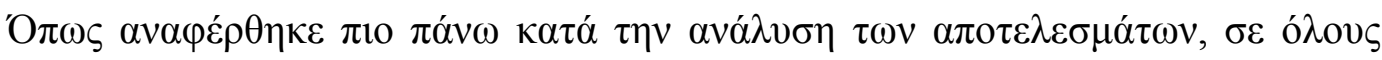

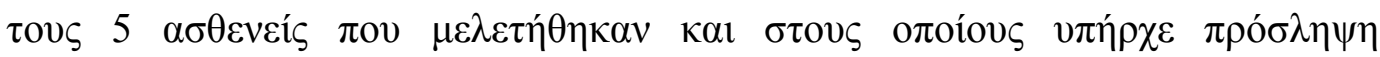

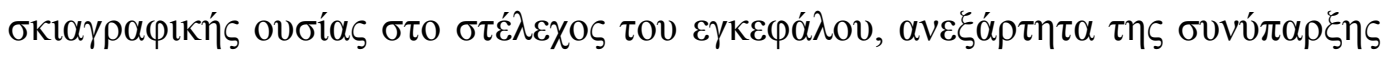

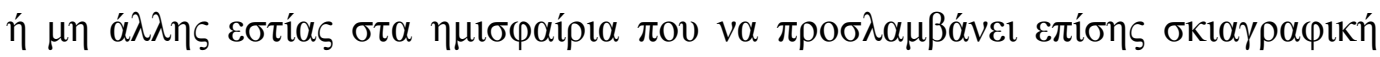

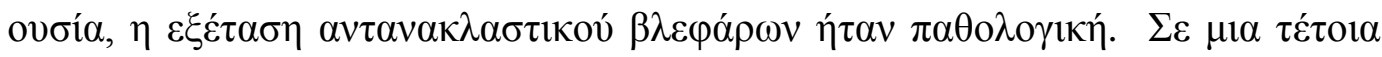

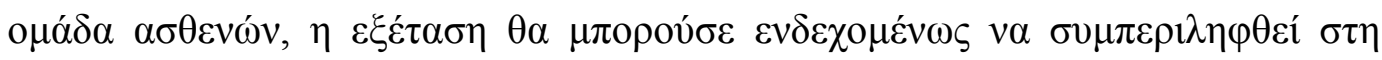

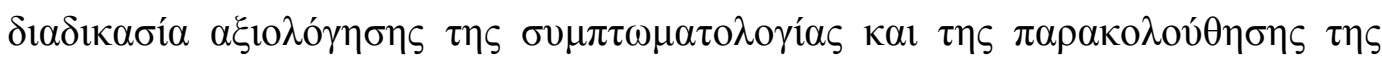

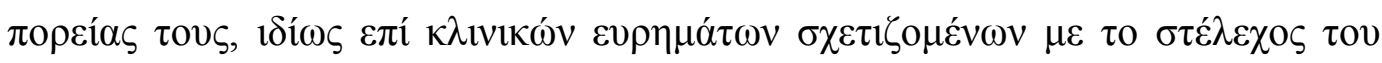
$\varepsilon \gamma \kappa \varepsilon \varphi \alpha ́ \lambda$ ov $\alpha \pi \varepsilon \imath \kappa o ́ v ı\rceil \eta ~ \varepsilon \sigma \tau \imath \omega ́ v ~ \sigma \tau \alpha ~ \eta \mu \imath \sigma \varphi \alpha i ́ p ı$. 


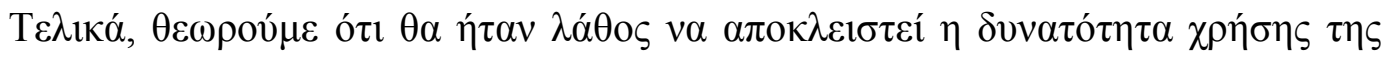

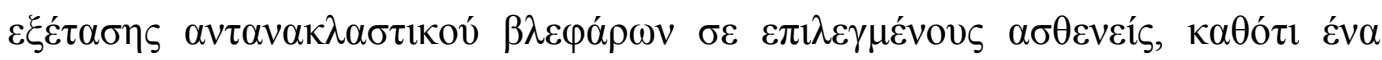

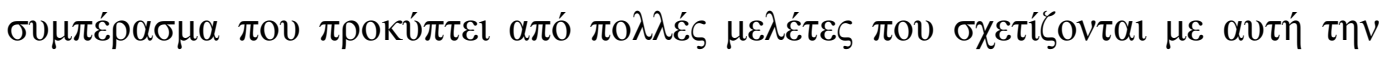

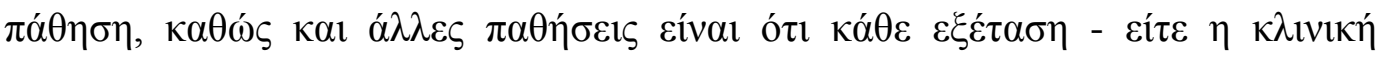

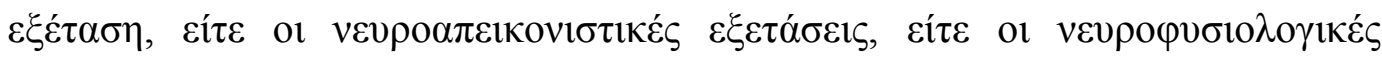

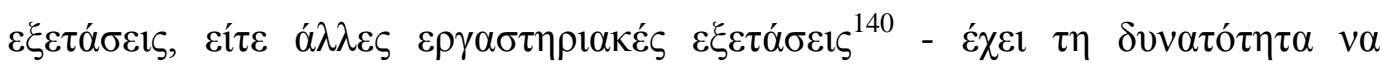

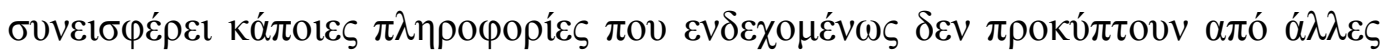

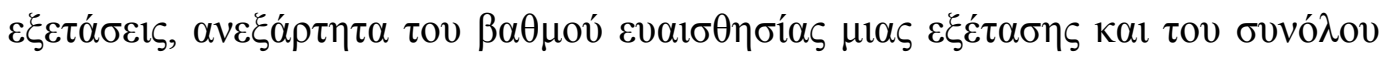

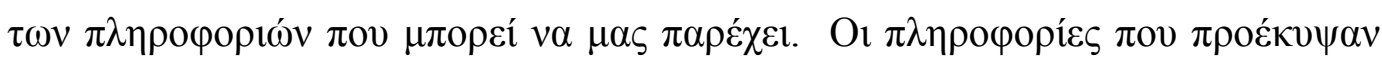

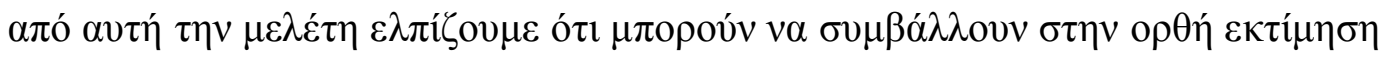

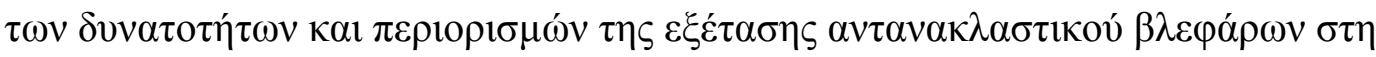

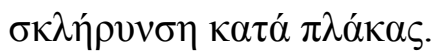




\section{4. ВIВАIOГРАФIA}

1) Alter M, Zhang ZX, Davanipour Z, Sobel E, Zibulewski J, Schwartz G, Friday G. Multiple sclerosis and childhood infections. Neurology 1986; 36: 1386-9.

2) Alonso F, Traba A, Roldan R, Esteban A. Brainstem lesion in multiple sclerosis, blink reflex, and brainstem auditory evoked potentials. Arch Neurobiol (Madr) 1992; 55(3): 89-98.

3) Amato MP, Ponziani G, Siracusa G, Sorbi S. Cognitive dysfunction in early-onset multiple sclerosis: a reappraisal after 10 years. Arch Neurol 2001; 58: 1602-1606.

4) Amato MP, Troiano M, Goretti B et al. Benign multiple sclerosis beyond expanded disability status scale: a thorough investigation on cognitive, psychological and social aspects. Neurology 2004; 62 (suppl. 5): A228.

5) Aminoff M. Electrodiagnosis in Clinical Neurology, first edition. Churchill-Livingstone, 1980: 299.

6) Anderson DW, Ellenberg JH, Leventhal CM, Reingold SC, Rodriguez M, Silberberg DH. Revised estimate of the prevalence of multiple sclerosis in the United States. Ann Neurol 1992; 31: 333-6.

7) Andrews KL, Husmann DA. Bladder dysfunction and management in multiple sclerosis. Mayo Clin Proc 1997; 72: 1176-83.

8) Antel JP, Bania MB, Reder AT, Cashman N. Activated suppressor cell dysfunction in chronic progressive multiple sclerosis. J Immunol 1986; 137: $137-41$.

9) Aramideh M, Ongerboer de Visser BW, Koelman JH, Majoie CB, Holstege G. The late blink reflex response abnormality due to lesion of the lateral tegmental field. Brain 1997; 120: 1685-1692.

10) Arnett HA, Mason J, Marino M et al. TNF alpha promotes proliferation of oligodendrocyte progenitors and remyelination. Nat Neurosci 2001; 4: 1116-1122.

11) Balashov KE, Smith DR, Khoury SJ, Hafler DA, Weiner HL. Increased interleukin 12 production in progressive multiple sclerosis: 
Induction by activated CD4+ T cells via CD40 ligand. Proc Natl Acad Sci USA 1997; 94: 599-603.

12) Bagnato F, Jeffries N, Richert ND et al. Evolution of T1 black holes in patients with multiple sclerosis imaged monthly for 4 years. Brain 2003; 126: $1782-1789$.

13) Barkhof F, Bruck W, De Groot CJ et al. Remyelinated lesions in multiple sclerosis: magnetic resonance image appearance. Arch Neurol 2003a; 60: 1073-1081.

14) Barkhof F, Rocca M, Francis G et al. Validation of diagnostic magnetic resonance imaging criteria for multiple sclerosis and response to interferon beta1a. Ann Neurol 2003b; 53: 718-724.

15) Barkhof F, Filippi M, Miller DH et al. Comparison of MRI criteria at first presentation to predict conversion to clinically definite multiple sclerosis. Brain 1997; 120: 2059-2069.

16) Barnett MH, Prineas JW. Relapsing and remitting multiple sclerosis: pathology of the newly forming lesion. Ann Neurol 2004; 55: 458-468.

17) Beck RW, Trobe JD, Moke PS et al. High- and low-risk profiles for the development of multiple sclerosis within 10 years after optic neuritis: experience of the optic neuritis treatment trial. Arch Ophthalmol 2003; 121: 944-949.

18) Benedetti MG, Piperno R, Simoncini L et al. Gait abnormalities in minimally impaired multiple sclerosis patients. Mult Scler 1999; 5: 363368.

19) Ben-Shlomo Y. Dietary fat in the epidemiology of multiple sclerosis: Has the situation been adequately assessed? Neuroepidemiology 1992; 11: $214-25$.

20) Berardelli A. Accornero N, Cruccu G, Fabiano F, Guerrisi V, Manfredi M. The orbicularis oculi response after hemispheral damage. $J$ Neurol Neurosurg Psychiatry 1983; 46: 837-843.

21) Berardelli A et al. The corneal reflex and the R2 component of the blink reflex. Neurology 1985; 35(6): 797-801.

22) Berger $\mathbf{T}$, Rubner $\mathbf{P}$, Schautzer $\mathbf{F}$ et al. Antimyelin antibodies as a predictor of clinically definite multiple sclerosis after a first demyelinating event. N Engl J Med 2003; 349: 139-145. 
23) Bieber AJ, Kerr S, Rodriguez M. Efficient central nervous system remyelination requires T cells. Ann Neurol 2003; 53: 680-684.

24) Bielekova B, Goodwin B, Richert $\mathbf{N}$ et al. Encephalitogenic potential of the myelin basic protein peptide (amino acids 83-99) in multiple sclerosis: results of a phase II clinical trial with an altered peptide ligand. Nat Med 2000; 6: 1167-1175.

25) Bjartmar C, Kinkel RP, Kidd $\mathbf{G}$ et al. Axonal loss in normal-appearing white matter in a patient with acute MS. Neurology 2001; 57: 1248-1252.

26) Brazis P, Masdeu J, Biller J. Localization in Clinical Neurology, third edition. Little, Brown and Company (Inc), 1996: 155-250, 449-534.

27) Buggenhout E. Success and failure of evoked potentials in detecting clinical and subclinical lesions in multiple sclerosis. Clin Neurol Neurosurg 1982; 84(1): 3-14.

28) Buntinx $\mathbf{M}$, Stinissen $\mathbf{P}$, Steels $\mathbf{P}$ et al. Immune-mediated oligodendrocyte injury in multiple sclerosis: molecular mechanisms and therapeutic interventions. Critical Rev Immunol 2002; 22: 391-424.

29) Bo L, Vedeler CA, Nyland $\mathbf{H}$ et al. Subpial demyelination in the cerebral cortex of multiple sclerosis patients. J Neuropathol Exp Neurol 2003; 62: 723-732.

30) Bobholz JA, Rao SM. Cognitive dysfunction in multiple sclerosis: a review of recent developments. Curr Opin Neurol 2003; 16: 283-288.

31) Brex PA, Ciccarelli 0, O'Riordan A et al. A longitudinal study of abnormalities on MRI and disability from multiple sclerosis. $N$ Engl J Med 2002; 346: 158-164.

32) Brex PA, Miszkiel KA, O'Riordan JI et al. Assessing the risk of early multiple sclerosis in patients with clinically isolated syndromes: the role of a follow up MRI. J Neurol Neurosurg Psychiatry 2001; 70: 390-393.

33) Castriota-Scanderbeg A, Fasano F, Hagberg G et al. Coefficient D(av) is more sensitive than fractional anisotropy in monitoring progression of irreversible tissue damage in focal nonactive multiple sclerosis lesions. Am J Neuroradiol 2003; 24: 663-670.

34) Charil A, Zijdenbos AP, Taylor J et al. Statistical mapping analysis of lesion location and neurological disability in multiple sclerosis: application to 452 patient data sets. Neuroimage $2003 ; \mathbf{1 9}$ : 532-544. 
35) Ciccarelli O, Werring DJ, Barker GJ et al. A study of the mechanisms of normal-appearing white matter damage in multiple sclerosis using diffusion tensor imaging-evidence of Wallerian degeneration. J Neurol 2003; 250: 287-292.

36) Cifelli A. Arridge M, Jezzard $\mathbf{P}$ et al. Thalamic neurodegeneration in multiple sclerosis. Ann Neurol 2002; 52: 650-653.

37) Clifford DB, Trotter JL. Pain in multiple sclerosis. Arch Neurol 1984; 41: $1270-2$.

38) CHAMPS Study Group. MRI predictors of early conversion to clinically definite MS in the CHAMPS placebo group. Neurology 2002; 59: 9981005.

39) Cross Ali, Trotter JL, Lyons J. B cells and antibodies in CNS demyelinating ease. J Neuroimmunol 2001; 112: 1-14.

40) Confavreux C, Vukusic S, Moreau T, Adeleine P. Relapses and progression of disability in multiple sclerosis. N Engl J Med 2000; 343: 1430-1438.

41) Courville $\mathbf{J}$. The nucleus of the facial nerve. The relation between cellular groups and peripheral branches of the nerve. Brain Res 1966; 1: 338-354.

42) Cruccu G, Iannetti G, Marx J, Thoemke F, Truini A, Fitzek S, Galeotti F et al. Brainstem reflex circuits revisited. Brain 2007; 128: 386394.

43) Dalton CM, Brex PA, Miszkiel KA et al. Application of the new McDonald criteria to patients with clinically isolated syndromes suggestive of multiple sclerosis. Ann Neurol 2002; 52: 47-53.

44) Dalton CM, Chard DT, Davies GR et al. Early development of multiple sclerosis is associated with progressive grey matter atrophy in patients resenting with clinically isolated syndromes. Brain 2004; 127: 1101-1107.

45) Dalton CM, Brex PA, Miszkiel KA et al. New T2 lesions enable an earlier diagnosis of multiple sclerosis in clinically isolated syndromes. Ann Neurol 2003; 53: 673-676.

46) De Stefano N, Narayanan S, Francis SJ et al. Diffuse axonal and tissue injury in patients with multiple sclerosis with low cerebral lesion load and no disability. Arch Neurol 2002; 59: 1565-1571. 
47) Dengler R, Kossev A, Gippner C, Struppler A. Quantitative analysis of blink reflexes in patients with hemiplegic disorders. Electroencephalogr Clin Neurophysiol 1982; 53: 513-524.

48) Deuschl G, Eisen A, editors. Recommendations for the practice of clinical neurophysiologic. IFCN Guidelines. Electroencephalogr Clin Neurophys 1999; Suppl. 52

49) Devonshire V, Sayao AL, McDonnell G et al. Benign multiple sclerosis: an assessment of disability and disease progression in patients with this diagnosis. Mult Scler 2003; 9 (suppl 1): 5128-5129.

50) Ebers GC, Sadovnick AD. The geographic distribution of multiple sclerosis: a review. Neuroepidemiology 1993; 12: 1-5

51) Eisen A, Ross, M. Studies of Late Responses. In: Electromyography and Nerve Conduction Studies. AAN Annual Meeting 1993; 150: 40-41

52) Fassbender K, Schmidt R, Mößner R, Kischka U, Kühnen J, Schwartz A, Hennerici M. Mood disorders and dysfunction of the hypothalamic-pituitary-adrenal axis in multiple sclerosis. Arch Neurol 1998; 55: 66-72.

53) Fazekas F, Offenbacher $\mathbf{H}$, Fuchs $\mathbf{S}$ et al. Criteria for an increased specificity of MRI interpretation in elderly subjects with suspected multiple sclerosis. Neurology 1988; 38: 1822-1825.

54) Fazekas F, Barkhof F, Filippi $\mathbf{M}$ et al. The contribution of magnetic resonance imaging to the diagnosis of multiple sclerosis. Neurology 1999; 53: $448-456$.

55) Ferguson IT, Ramsden RT, Lythgoe M. Brain stem auditory evoked potentials and blink reflexes in multiple sclerosis. J Larygol Otol 1985; 99: 677-683.

56) Filippi M, Bozzali M, Rovaris $\mathbf{M}$ et al. Evidence for widespread axonal damage at the earliest clinical stage of multiple sclerosis. Brain 2003; 126: 433-437.

57) Filippi M, Grossman RI. MRI techniques to monitor MS evolution: the present and the future. Neurology 2002; 58: 1147-1153.

58) Filippi M, Horsfield MA, Morrissey SP et al. Quantitative brain MRI lesion load predicts the course of clinically isolated syndromes suggestive of multiple sclerosis. Neurology 1994; 44: 635-641. 
59) Filippi M, Rocca MA, Colombo B et al. Functional magnetic resonance imaging correlates of fatigue in multiple sclerosis. Neuroimage 2002; 14: 559-567.

60) Filippi M, Wolinsky JS, Sormani MP et al. Enhancement frequency decreases with increasing age in relapsing-remitting multiple sclerosis. Neurology 2001b; 56: 422-423.

61) Fisher MA, Shahani BT, Young RR. Assessing segmental excitability after acute rostral lesions: the blink reflex. Neurology 1979; 29: 45-50.

62) Fitzek S, Fitzek C, Marx J, Speckter H, Urban PP, Thömke F, Stoeter P, Hopf HC. Blink reflex R2 changes in lower brainstem - an electrophysiological and MR-imaging study. J Neurol Neurosurg Psychiatry 1999; 67: 630-636.

63) Frohman EM, Goodin DS, Calabrese PA et al. The utility of MRI in suspected MS: report of the Therapeutics and Technology Assessment Subcommittee of the American Academy of Neurology. Neurology 2003; 61: 602-611.

64) Gambardella A, Valentino P, Labate A et al. Temporal lobe epilepsy as a unique manifestation of multiple sclerosis. Can J Neurol Sci 2003; 30: 228-232.

65) Gass A, Kitchen N, MacManus DG, Moseley IF, Hennerici MG, Miller DH. Trigeminal neuralgia in patients with multiple sclerosis: lesion localization with magnetic resonance imaging. Neurology 1997; 49: $1142-4$.

66) Gil R, Lefèvre JP. Electrologic syndromes of alteration of elicited blink reflex in multiple sclerosis. Study of a group of 33 patients (author's transl). Sem Hop 1980; 56(5-6): 214-20.

67) Giuliani F, Goodyer CG, Antel JP, Yong VW. Vulnerability of human neurons to T cell-mediated cytotoxicity. J Immunol 2003; 171: 368-379.

68) Hawkins SA, McDonnell GV. Benign multiple sclerosis? Clinical course, long term follow up, and assessment of prognostic factors. J Neurol Neurosurg Psychiatry 1999; 67: 148-152.

69) Helms G. Volume correction for edema in single-volume proton MR spectroscopy of contrast-enhancing multiple sclerosis lesions. Magn Reson Med 2001; 46: 256-263. 
70) Henry RG, Oh J, Nelson SJ, Pelletier D. Directional diffusion in relapsing-remitting multiple sclerosis: a possible in vivo signature of Wallerian degeneration. J Magn Reson Imaging 2003; 18: 420-426.

71) Herndon RM, Coyle PK, Murray TJ et al. Report of the consensus panel on the New International Panel Guidelines for diagnosis of MS. Int J MS Care 2002; 4: 170-173.

72) Hogancamp WE, Rodriguez M, Weinshenker BG. The epidemiology of multiple sclerosis. Mayo Clin Proc 1997; 72: 871-8.

73) Hopf HC. Topodiagnostic value of brain stem reflexes. Muscle Nerve 1994; 17: 475-484.

74) Hopf HC, Thömke F, Gutmann L. Midbrain vs. pontine medial longitudinal fasciculus lesions: the utilization of masseter and blink reflexes. Muscle Nerve 1991; 14: 326-330.

75) Kaplan PE, Kaplan C. Blink reflex: review of methodology and its application to patients with stroke syndrome. Arch Phys Med Rehabil 1980; 61: 30-33.

76) Kayamori R, Dickins QS, Yamada T, Kimura J. Brainstem auditory evoked potential and blink reflex in multiple sclerosis. Neurology 1984; 34(10): 1318-23.

77) Kerschensteiner M, Stadelmann C, Dechant G et al. Neurotrophic cross-talk between the nervous and immune systems: implications for neurological diseases. Ann Neurol 2003; 53: 292-304.

78) Khoshbin S, Hallett M. Multimodality evoked potentials and blink reflex in multiple sclerosis. Neurology 1981; 31(2): 138-44.

79) Kimura J. Electrodiagnosis in Diseases of Nerve and Muscle: principles and practice, third edition. New York: Oxford University Press, Inc., 2001: 409-438.

80) Kimura J. In: Common Problems in Electrodiagnostic Medicine, AAEM Course A, 1990: 10.

81) Kimura, J. Electrically elicited blink reflex in diagnosis of Multiple Sclerosis - review of 260 patients over a seven year period. Brain 1975; 98(3): 413-426.

82) Kimura J. Alteration of the orbicularis oculi reflex by pontine lesions: study in multiple sclerosis. Arch Neurol 1970; 22: 156-161. 
83) Kimura J, Lyon LW. Orbicularis oculi reflex in the Wallenberg syndrome: alteration of the late reflex by lesion of the spinal tract and nucleus of the trigeminal nerve. J Neurol Neurosurg Psychiatry 1972; 35: 228-233.

84) Kimura J, Wilkinson JT, Damasio H, Adam HR jr, Shivapour E, Yamada T. Blink reflex in patients with hemispheric cerebrovascular accident (CVA). J Neurol Sci 1985; 67: 15-28.

85) Kjaer M. The value of brain stem auditory, visual and somatosensory evoked potentials and blink reflexes in the diagnosis of multiple sclerosis. Acta Neurol Scand 1980; 62(4): 220-36.

86) Kjaer M. The value of brain stem evoked potentials and the blink reflex in multiple sclerosis. Acta Neurol Scand 1981; 65(8): 113-114.

87) Klissourski M, Novachkova S, Tzvetanov P, Alexiev F. Orbicularis oculi reflex abnormalities in patients in patients with multiple sclerosis: a clinical, EMG, and MRI investigation. Electromyogr Clin Neurophysiol 2009; 49(1): 59-63.

88) Kossev A, Dengler R, Struppler A. Quantitative assessment of the blink reflex in normals. Electromyogr Clin Neurophysiol 1983; 23: 501-511.

89) Koziol J, Wagner S, Sobel F, Lloyd S et al. Predictive Value of Lesions for Relapses in Relapsing-remitting Multiple Sclerosis. Am J Neuroradiology 2001; 22: 284-291.

90) Kumaran MS et al. Wavelet decomposition of the Blink Reflex R2 component enables improved discrimination of Multiple Sclerosis. Clin Neurophysiol 2000; 111(5): 810-20.

91) Kurtzke JF. Clinical manifestations of multiple sclerosis. In: Vinken PJ, Bruyn, eds. Handbook of clinical neurology. Amsterdam: Elsevier Science, 1970: 161-216.

92) Kurtzke JF. Epidemiologic evidence for multiple sclerosis as an infection. Clin Microbiol Rev 1993; 6: 382-427.

93) Lacquaniti F, Benna P, Gilli M, Troni W, Bergamasco B. Brain stem auditory evoked potentials and blink reflexes in quiescent multiple sclerosis. Electroencephalogr Clin Neurophysiol 1979; 47(5): 607-10.

94) Larson EB, Burnison DS, Brown WS. Callosal function in multiple sclerosis: bimanual motor coordination. Cortex 2002; 38: 201-214. 
95) Lassmann H, Suchanek G, Ozawa K. Histopathology and the bloodcerebrospinal fluid barrier in multiple sclerosis. Ann Neurol 1994; 36: S42-6.

96) Lowitzsch K, Lüder G. Habituation of the blink reflex. Electroencephalogr Clin Neurophysiol 1985; 60: 525-531.

97) Lowitzsch K, Kuhnt U, Sackmann C, Maurer K, Hopf HC, Schott D et $a l$. Visual pattern evoked responses and blink reflexes in assessment of MS diagnosis. J Neurol 1976; 213: 17-32.

98) Lublin FD, Reingold SC. Defining the clinical course of multiple sclerosis: results of an international survey. National Multiple Sclerosis Society (USA) Advisory Committee on Clinical Trials of New Agents in Multiple Sclerosis. Neurology 1996; 46: 907-911.

99) Lucchinetti CF, Brück W, Rodriguez M, Lassmann H. Distinct patterns of multiple sclerosis pathology indicates heterogeneity in pathogenesis. Brain Pathol 1996; 6: 259-74.

100) Lucchinetti C, Bruck W, Parisi J et al. Heterogeneity of multiple sclerosis lesions: implications for the pathogenesis of demyelination. Ann Neurol 2000; 47: 707-717.

101) Lycklama G, Thompson A, Filippi M et al. Spinal-cord MRI in multiple sclerosis. Lancet Neurol 2003; 2: 555-562.

102) Maimone D, Gregory S, Arnason BGW, Reder AT. Cytokine levels in the cerebrospinal fluid and sera of patients with multiple sclerosis. $J$ Neuroimmunol 1991a; 32: 67-74.

103) Maimone D, Reder AT. Soluble CD8 levels in the cerebrospinal fluid and serum of patients with multiple sclerosis. Neurology 1991; 41: 8514.

104) Marx J, Thoemke F, Fitzek S, Vucurevic G, Fitzek C, Mika-Gruettner A et al. Topodiagnostic value of blink reflex R1 changes: A digital postprocessing MRI correlation study. Muscle Nerve 2001; 24: 13271331.

105) Matthews WB. Clinical aspects. In: Compston A ed. McAlpine’s Multiple Sclerosis. New York: Churchill Livingstone, 1985: 49-278.

106) McDonald WI, Compston A, Edan G, Goodkin D, Hartung HP, Lublin FB et al. Recommended diagnostic criteria for multiple sclerosis: 
guidelines from the International Panel on the diagnosis of multiple sclerosis. Ann Neurol 2001; 50: 121-127.

107) Meinke U, Ferbert A. Blink reflex in patients with an ischemic lesion of the brain-stem verified by MRI. J Neurol 1993; 241: 37-44.

108) Misulis K. In: Spehlmann's Evoked Potential Primer, Second Edition. Butterworth-Heinemann, 1994:285.

109) Moriarty DM, Blackshaw AJ, Talbot PR et al. Memory dysfunction in multiple sclerosis corresponds to juxtacortical lesion load on fast fluidattenuated inversion-recovery MR images. Am J Neuroradiol 1999; 20: 1956-1962.

110) Moulin DE, Foley KM, Ebers GC. Pain syndromes in multiple sclerosis. Neurology 1988; 38: 1830-4.

111) Nazliel B, Irkeç C, Koçer B. The roles of Blink Reflex and Sympathetic Skin Response in Multiple Sclerosis diagnosis. Mult Scler 2002 Dec; 8(6): $500-4$.

112) Nevo U, Kipnis J, Golding I et al. Autoimmunity as a special case of immunity: removing threats from within. Trends Mol Med 2003; 9: 88-93.

113) Nisipeanu P, Korczyn AD. Psychological stress as risk factor for exacerbations in multiple sclerosis. Neurology 1993; 43: 1311-2.

114) O' Connor KC, Bar-Or A, Hafler DA. The neuroimmunology of multiple sclerosis: possible roles of $\mathrm{T}$ and $\mathrm{B}$ lymphocytes in immunopathogenesis. J Clin Immunol 2001; 21: 81-92.

115) Offenbacher H, Fazekas F, Schmidt R, Freidl W, Flooh E, Payer F, Lechner H. Assessment of MRI criteria for a diagnosis of MS. Neurology 1993; 43: 905-9.

116) O' Riordan JI, Thompson AJ, Kingsley DP et al. The prognostic value of brain MRI in clinically isolated syndromes of the CNS. A 10-year follow-up. Brain 1998; 121: 495-503.

117) Ongerboer de Visser BW, Kuypers HGJM. Late blink reflex changes in lateral medullary lesions. Brain 1978; 101: 281-294.

118) Ongerboer de Visser BW. Comparative study of corneal and blink reflex latencies in patients with segmental or with cerebral lesions. In Desmedt JE (ed) Motor Control Mechanisms in Health and Disease. New York: Raven Press, 1983: 757-772. 
119) Paty DW, Oger JJ, Kastrukoff LF et al. MRI in the diagnosis of MS: a prospective study with comparison of clinical evaluation, evoked potentials, oligoclonal banding, and CT. Neurology 1988; 38: 180-185.

120) Perry VH, Newman TA, Cunningham C. The impact of systemic infection on the progression of neurodegenerative disease. Nature Rev Neurosci 2003; 4: 103-112.

121) Poser CM. Pathogenesis of multiple sclerosis. Acta Neuropathol 1986; 71: 1-10.

122) Poser CM, Paty DW, Scheinberg L. New diagnostic criteria for multiple sclerosis: guidelines for research protocols. Ann Neurol 1983; 13: $227-31$.

123) Preston D, Shapiro B. Electromyography and Neuromuscular Disorders, first edition. Butterworth-Heinemann, 1998: 57-62.

124) Prineas JW, Barnard RO, Kwon EE, Sharer LR, Cho E-S. Multiple sclerosis: remyelination of nascent lesions. Ann Neurol 1993; 33: 137-51.

125) Pujol J, Bello J, Deus J, Martí-Vilalta JL, Capdevila A. Lesions in the left arcuate fasciculus region and depressive symptoms in multiple sclerosis. Neurology 1997; 49: 1105-10.

126) Rae-Grant AD, Eckert NJ, Bartz 5, Reed JF. Sensory symptoms of multiple sclerosis: a hidden reservoir of morbidity. Mult Scler 1999; 5: 179-183.

127) Rao SM, Leo GJ, Bernardin L, Unverzagt F. Cognitive dysfunction in multiple sclerosis. I. Frequency, patterns, and prediction. Neurology 1991; 41: 685-91.

128) Rapp NS, Gilroy J, Lerner AM. Role of bacterial infection in exacerbation of multiple sclerosis. Am J Phys Med Rehabil 1995; 4: 4158.

129) Reynolds EH. Multiple sclerosis and vitamin B12 metabolism. $J$ Neuroimmunol 1992; 40: 225-30.

130) Riechert T, Hassler R, Mundinger F, Bronisch F, Schmidt K. Pathologic-anatomical findings and cerebral localization in stereotactic treatment of extrapyramidal motor disturbances in multiple sclerosis. Confinia Neurol 1975; 37: 24-40. 
131) Rossi A, Scarpini C. Gating of trigemino-facial reflex from lowthreshold trigeminal and extratrigeminal cutaneous fibers in humans. $J$ Neurol Neurosurg Psychiatry 1992; 55: 774-780.

132) Rovaris M, Comi G, Ladkani D, Wolinsky J, Filippi M. Short-Term Correlations between Clinical and MR Imaging Findings in RelapsingRemitting Multiple Sclerosis. Am J Neuroradiol 2003; 24: 75-81.

133) Ruggieri RM, Palermo R, Vitello G et al. Cognitive impairment in patients suffering from relapsing-remitting multiple sclerosis with EDSS scores < or 3.5. Acta Neurol Scand 2003; 108: 323-328.

134) Sanders EA, Ongerboer de Visser BW, Barendswaard EC, Arts RJ. Jaw, blink and corneal reflex latencies in multiple sclerosis. J Neurol Neurosurg Psychiatry 1985; 48(12): 1284-9.

135) Sanders E Reulen JP, Van der Velde EA, Hogenhuis LA. The diagnosis of multiple sclerosis. Contribution of non-clinical tests. J Neurol Sci 1986; 72(2-3): 273-85.

136) Scarpalezos S, Stamboulis E, Eudokimidis J, Siafakas A, Monastiriotis N. Contribution of visual evoked potentials, electronystagmography and the blink reflex to the diagnosis of multiple sclerosis. Rev Neurol (Paris) 1986; 142(10): 753-8.

137) Schumacher GA, Beebe GW, Kibler RF et al. Problems of experimental trials of therapy in multiple sclerosis: report by the panel on the evaluation of experimental trials of therapy in multiple sclerosis. Ann NY Acad Sci 1965; 122: 552-568.

138) Schoenberg H. Bladder and sexual dysfunction in multiple sclerosis. In: Antel JP. Neurologic clinics. Philadelphia, 1983: 601-13.

139) Sharma R, Narayana PA, Wolinsky JS. Grey matter abnormalities in multiple sclerosis: proton magnetic resonance spectroscopic imaging. Mult Scler 2001; 7: 221-226.

140) Shahani BT, Young RR. Human Orbicularis Oculi Reflexes. Neurology 1972; 22: 149

141) Shahani BT. The Human Blink Reflex. J Neurol Neurosurg Psychiatry 1970; 33: 792.

142) Shahani BT, Young RR. A note on Blink Reflexes. J Physiol (Lond) 1968; 198: 103. 
143) Shahani BT, Young RR. The Blink Reflex. In Goodgold J and Eberstein A ed. Electrodiagnosis of Neuromuscular Diseases. Xxxxxx, 1977: 240250.

144) Sibley WA. Risk factors in multiple sclerosis-implications for pathogenesis. In Serlupi CG, ed. A multidisciplinary approach to myelin diseases. New York, 1988: 227-32.

145) Sibley WA, Bamford CR, Clark K. Clinical viral infections and multiple sclerosis. Lancet 1985; 1: 1313-5.

146) Silver NC, Tofts PS, Symms MR et al. Quantitative contrast-enhanced magnetic resonance imaging to evaluate blood-brain barrier integrity in multiple sclerosis: a preliminary study. Mult Scler 2001; 7: 75-82.

147) Simmat G, Gil R, Boissonnot L, Lefevre JP. The blink reflex and the diagnosis of multiple sclerosis. Clinical correlations in 66 patients. Rev Electroencephalogr Neurophysiol Clin 1985; 15(1): 107-10.

148) Simon J, Li D, Traboulsee A, Coyle P, Arnold D et al. Standardized MR Imaging Protocol for Multiple Sclerosis: Consortium of MS Centers Consensus Guidelines. Am J Neuroradiol 2006; 27: 455-461.

149) Stenager E, Knudsen L, Jensen K. Acute and chronic pain syndromes in multiple sclerosis. Acta Neurol Scand 1991; 84: 197-200.

150) Tackmann W, Ettlin T. Blink reflexes elicited by electrical, acoustic and visual stimuli. II. Their relation to visual-evoked potentials and auditory brain stem evoked potentials in the diagnosis of multiple sclerosis. Eur Neurol 1982; 21(4): 264-9.

151) Tackmann W, Strenge H, Barth R, Sojka-Raytscheff A. Evaluation of various brain structures in multiple sclerosis with multimodality evoked potentials, blink reflex and nystagmography. J Neurol 1980; 224(1): 3346.

152) Thompson AJ, Montalban X, Barkhof $\mathbf{F}$ et al. Diagnostic criteria for primary progressive multiple sclerosis: a position paper. Ann Neurol 2000; 47: 831-835.

153) Thompson AJ, Kermode AG, Wicks D, MacManus DG, Kendall BE, Kingsley DPE, McDonald WI. Major differences in the dynamics of primary and secondary progressive multiple sclerosis. Ann Neurol 1991; 29: 53-62. 
154) Tintoré M, Rovira A, Martinez $\mathbf{M}$ et al. Isolated demyelinating syndromes: comparison of different MR imaging criteria to predict conversion to clinically definite multiple sclerosis. Am J Neuroradiol 2000; 21: 702-706.

155) Tintoré M, Rovira A, Rio J et al. New diagnostic criteria for multiple sclerosis: application in first demyelinating episode. Neurology 2003; 60: 27-30.

156) Tintoré M, Rovira A, Brieva $\mathbf{L}$ et al. Isolated demyelinating syndromes: comparison of CSF oligocional bands and different MR imaging criteria to predict conversion to CDMS. Mult Scler 2001; 7: 359-363.

157) Traboulsee A, Dehmeshki J, Peters KR et al. Disability in multiple sclerosis is related to normal appearing brain tissue MTR histogram abnormalities. Mult Scler 2003; 9: 566-573.

158) Traboulsee A, Li D, Frank J et al. Consortium of MS Centers MRI protocol for the diagnosis and follow-up of MS. June 2003.

159) Trapp BD, Peterson J, Ransohoff RM, Rudick R, Mörk S, Bö L. Axonal transection in the lesions of multiple sclerosis. $N$ Engl $\mathrm{J} \mathrm{Med}$ 1998; 338: 278-285.

160) Trapp BD, Ransohoff RM, Fisher E, Rudick RA. Neurodegeneration in multiple sclerosis: relationship to neurological disability. Neuroscientist 1999; 5: 48-57.

161) Trontelj MA, Trontelj JV. Reflex arc of the first component of the human blink reflex: a single motoneuron study. J Neurol Neurosurg Psychiatry 1978; 41: 538-547.

162) van Walderveen MA, Lycklama H, Nijeholt GJ, Ader Hi et al. Hypointense lesions on T1-weighted spin-echo magnetic resonance imaging: relation to clinical characteristics in subgroups of patients with multiple sclerosis. Neurology 2001; 58: 76-81.

163) Watkins SM, Espir MLE. Migraine and multiple sclerosis. J Neurol Neurosurg Psychiatry 1969; 32: 35-7.

164) Weinshenker BG, Ebers GC. The natural history of multiple sclerosis. Can J Neurol Sci 1987; 14: 255-61. 
165) Whitaker JN, Wolinsky JS, Narayana PA et al. Relationship of urinary myelin basic protein-like material with cranial magnetic resonance imaging in advanced multiple sclerosis. Arch Neurol 2001; 58: 49-54.

166) Wolinsky JS; PROMiSe Study Group. The diagnosis of primary progressive multiple sclerosis. J Neurol Sci 2003; 206: 145-152.

167) Wolinsky JS, Narayana PA. Magnetic resonance spectroscopy in multiple sclerosis: window into the diseased brain. Curr Opin Neurol 2002; 15: 247-251.

168) Wolinsky JS, Narayana PA, Johnson KP; Multiple Sclerosis Study Group and the MRI Analysis Center. United States open-label glatiramer acetate extension trial for relapsing multiple sclerosis: MRI and clinical correlates. Mult Scler 2001; 7: 33-41.

169) Wynn DR, Rodriguez M, O'Fallon WM, Kurland LT. A reappraisal of the epidemiology of multiple sclerosis in Olmsted County, Minnesota. Neurology 1990; 40: 780-786.

170) Zorzon M, Ukmar M, Bragadin LM et al. Olfactory dysfunction and extent of white matter abnormalities in multiple sclerosis: a clinical and MR study. Mult Scler 2000; 6: 386-390.

171) Xu T, Cui LY, Li BH, DU H. Clinical value of blink reflex: analysis of 548 patients. Zhonghua Yi Xue Za Zhi 2007; 87(19): 1348-50.

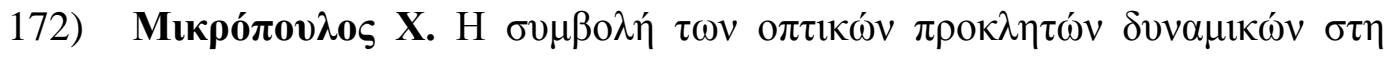

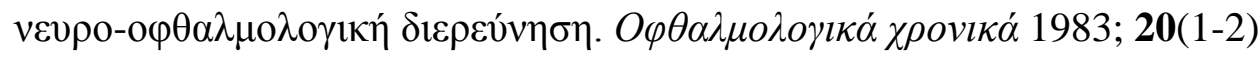

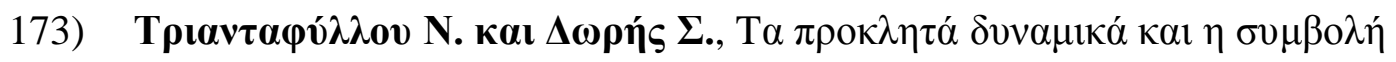

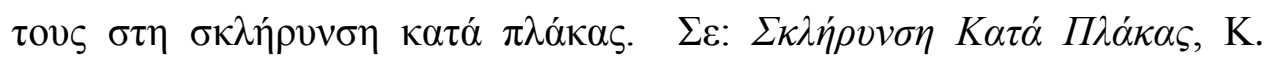

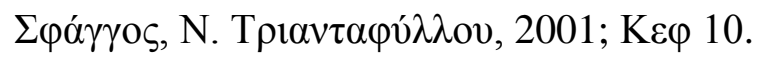




\section{5. ПАРАРТНМА}

$5.1 \Sigma$ TOIXEIA A $\Sigma \Theta E N \Omega N$

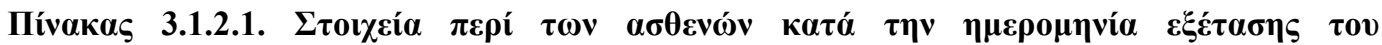

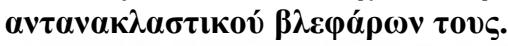

\begin{tabular}{|c|c|c|c|c|c|c|c|c|c|c|}
\hline $\mathbf{A} / \mathbf{A}$ & Ф010 & Ннер. Геv. & 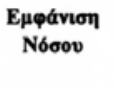 & Ннер. Blink & H人uxia & $\begin{array}{c}\text { Aldepueva } \\
\text { Nórov }\end{array}$ & H $\mu$. MRI & $\begin{array}{c}\text { Eted. Eotís } \\
\text { ГEvıќ́ }\end{array}$ & 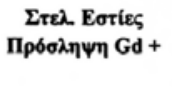 & 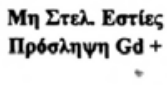 \\
\hline AA01 & $\Theta$ & 15/12/1977 & Aup-06 & $6 / 10 / 2006$ & 29 & 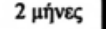 & 25/9/2006 & $\mathrm{Nai}$ & $O x$ & $\mathrm{Nai}$ \\
\hline $\mathrm{AA} 02$ & $\Theta$ & $18 / 7 / 1966$ & Iovv-06 & $22 / 6 / 2006$ & 40 & $1 \mu \eta \dot{v a \zeta}$ & $20 / 6 / 2006$ & $O x$ & $O_{x}$ & $O_{x}$ \\
\hline $\mathrm{AA03}$ & $\Theta$ & $30 / 10 / 1971$ & 1995 & $24 / 2 / 2006$ & 34 & $11 \mathrm{ktm}$ & $21 / 2 / 2006$ & $\mathrm{Nai}$ & $O x$ & $O x^{n}$ \\
\hline AA04 & A & $7 / 1 / 1961$ & $\Phi \varepsilon \beta-04$ & $10 / 5 / 2006$ & 45 & $2 \mathrm{\varepsilon tm}$ & $11 / 5 / 2006$ & $\mathrm{Nai}$ & $\mathrm{Ox}_{1}$ & $O x$ \\
\hline AA05 & A & $14 / 12 / 1962$ & 1998 & $28 / 12 / 2006$ & 44 & $8 \mathrm{Etm}$ & $18 / 12 / 2006$ & $O x$ & $O x$ & $O x$ \\
\hline AA06 & $\Theta$ & $14 / 6 / 1957$ & 2002 & $25 / 1 / 2007$ & 49 & 5 हाग & $24 / 1 / 2007$ & $\mathrm{Nai}$ & $O x$ & $O x$ \\
\hline AA07 & $\Theta$ & 16/7/1982 & Iovג- 05 & $24 / 11 / 2006$ & 24 & $16 \mu$ กர์ & $24 / 11 / 2006$ & $\mathrm{Nai}$ & $\mathrm{O} x a$ & $O_{x}$ \\
\hline AA08 & $\Theta$ & 15/6/1960 & $\Delta \varepsilon K-02$ & $28 / 12 / 2006$ & 47 & 4 हाग & $28 / 12 / 2006$ & Naí & $N \alpha i$ & $O_{x}$ \\
\hline AA09 & $\Theta$ & $1 / 1 / 1947$ & 1998 & $6 / 2 / 2006$ & 59 & 8 हाँ & $2 / 2 / 2006$ & $\mathrm{Nai}$ & $\mathrm{N} \alpha i$ & $\mathrm{~N} \alpha i$ \\
\hline AA10 & A & $6 / 3 / 1936$ & 1976 & $2 / 11 / 2006$ & 71 & $30 \mathrm{Etm}$ & $16 / 11 / 2006$ & $\mathrm{Nai}$ & $O x$ & $O_{x i}$ \\
\hline AA11 & $\Theta$ & 18/8/1965 & Iav-06 & $3 / 11 / 2006$ & 41 & $11 \mu$ กர์ & $18 / 10 / 2006$ & $O_{x}$ & $O_{x}$ & $O_{x}$ \\
\hline AA12 & $\Theta$ & 4/6/1966 & 2003 & $11 / 1 / 2007$ & 41 & 4 हाग & $28 / 12 / 2006$ & $\mathrm{Nai}$ & $0 x$ & $\mathrm{~N} \alpha i$ \\
\hline AA13 & $\Theta$ & $28 / 11 / 1980$ & Avy-04 & $27 / 11 / 2006$ & 26 & $2 \mathrm{\varepsilon tग}$ & $27 / 11 / 2006$ & Naí & $O_{x}$ & $O_{x}$ \\
\hline AA14 & $\Theta$ & 14/1/1961 & Okt-06 & $7 / 11 / 2006$ & 46 & $1 \mu$ mivas & $27 / 10 / 2006$ & $O_{x a}$ & $O_{x}$ & $\mathrm{Nai}$ \\
\hline AA15 & A & $3 / 1 / 1956$ & 1993 & $22 / 11 / 2006$ & 51 & $13 \mathrm{ktm}$ & $21 / 11 / 2006$ & $\mathrm{Nai}$ & $0 x$ & $0 x$ \\
\hline AA16 & $\Theta$ & $1 / 4 / 1972$ & 1998 & $30 / 3 / 2006$ & 34 & $8 \mathrm{~km}$ & 22/032006 & $\mathrm{Nai}$ & $0 x$ & $0 x$ \\
\hline AA17 & $\Theta$ & $1 / 5 / 1978$ & lovג-05 & 21/7/2006 & 28 & $12 \mu \eta \dot{v} \varepsilon \varepsilon$ & $23 / 6 / 2006$ & $O_{x y}$ & $O x$ & $O_{x i}$ \\
\hline AA18 & A & $22 / 1 / 1963$ & 1993 & $3 / 11 / 2006$ & 44 & $13 \mathrm{Em}$ & $30 / 10 / 2006$ & $\mathrm{Naí}$ & $0 x$ & $0 x$ \\
\hline AA19 & $\Theta$ & 23/1/1955 & 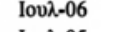 & 21/7/2006 & 51 & $1 \mu \eta$ vas & 18/7/2006 & 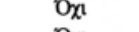 & $0 x^{n}$ & $0 x$ \\
\hline AA20 & $\Theta$ & $6 / 2 / 1965$ & Iovג- -05 & $21 / 6 / 2006$ & 41 & $11 \mu \eta \dot{v \varepsilon \varepsilon}$ & 13/6/2006 & $0 x$ & $0 x$ & $\mathrm{Nai}$ \\
\hline AA21 & A & 20/5/1972 & lovג-04 & $6 / 4 / 2006$ & 34 & $21 \mu \eta \dot{v} \mathrm{E}$ & $5 / 4 / 2006$ & $0 x$ & $0 x$ & $0 x$ \\
\hline AA22 & $\Theta$ & $18 / 2 / 1948$ & I $\alpha v=03$ & $30 / 3 / 2006$ & 58 & $3 \mathrm{~km}$ & $31 / 3 / 2006$ & $0 x$ & $0 x$ & $0 x$ \\
\hline AA23 & A & 12/8/1981 & 2000 & $8 / 6 / 2006$ & 25 & 6 हाँा & $5 / 6 / 2006$ & $\mathrm{Nai}$ & $0 x$ & $0 x$ \\
\hline AA24 & A & 12/7/1938 & Map-04 & $16 / 3 / 2006$ & 68 & $24 \mu \eta \dot{V} \mathrm{VEs}$ & $30 / 3 / 2006$ & $0 x$ & $0 x$ & $0 x$ \\
\hline AA25 & A & 29/9/1986 & Фєß-06 & $31 / 3 / 2006$ & 20 & $2 \mu \eta \dot{v} v \varepsilon s$ & 23/3/2006 & $\mathrm{Nai}$ & $0 x$ & Naí \\
\hline AA26 & A & $8 / 3 / 1977$ & A $\pi \rho-05$ & $24 / 2 / 2006$ & 29 & $10 \mu \eta \dot{V} \mathrm{VE}$ & $3 / 3 / 2006$ & $0 x$ & $0 x$ & $0 x$ \\
\hline AA27 & A & $1 / 1 / 1958$ & 1981 & $17 / 3 / 2006$ & 48 & $15 \mathrm{ktm}$ & $17 / 2 / 2006$ & $O x$ & $0 x$ & $\mathrm{Nai}$ \\
\hline AA28 & $\Theta$ & $11 / 8 / 1973$ & Okt-02 & $12 / 1 / 2007$ & 33 & $4 \varepsilon \pi \eta$ & $14 / 12 / 2006$ & $0 x$ & $0 x$ & $0 x$ \\
\hline AA29 & $\Theta$ & 4/2/1969 & 1995 & $29 / 3 / 2006$ & 37 & $11 \mathrm{~km}$ & $21 / 3 / 2006$ & $0 x$ & $0 x$ & $0 x$ \\
\hline AA30 & $\Theta$ & 4/10/1989 & Avy-06 & 26/9/2006 & 17 & 2 нก์ve६ & 20/9/2006 & $0 x$ & $O x$ & $\mathrm{Nai}$ \\
\hline AA31 & A & $18 / 4 / 1962$ & I $\alpha v-02$ & $28 / 2 / 2006$ & 44 & 4 होग & 23/2/2006 & $\mathrm{Nai}$ & $o_{x}$ & $0 x$ \\
\hline AA32 & A & $18 / 4 / 1962$ & I $\alpha v-02$ & $5 / 12 / 2006$ & 45 & $4 \mathrm{Em}$ & $4 / 12 / 2006$ & $\mathrm{Naf}$ & $0 \times 2$ & $0 x$ \\
\hline AA33 & $\Theta$ & $26 / 6 / 1970$ & $\Phi \varepsilon \beta-02$ & $17 / 3 / 2006$ & 36 & 4 हाग & $16 / 3 / 2006$ & Naí & $0 x$ & $0 x$ \\
\hline AA34 & A & $2 / 10 / 1967$ & 1989 & $23 / 8 / 2006$ & 39 & $17 \mathrm{\varepsilon tm}$ & 9/8/2006 & $\mathrm{Nai}$ & $0 x$ & $\mathrm{Nai}$ \\
\hline AA35 & $\Theta$ & $5 / 5 / 1960$ & Mat-04 & $27 / 1 / 2006$ & 46 & $20 \mu \eta \dot{v} \varepsilon \varepsilon$ & 23/1/2006 & $0 x$ & $0 x$ & $0 x a$ \\
\hline AA36 & $\Theta$ & 7/10/1958 & 21-Iav & $24 / 2 / 2006$ & 47 & 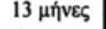 & $23 / 2 / 2006$ & $O x$ & $O x$ & $O x$ \\
\hline AA37 & $\theta$ & $23 / 12 / 1975$ & Mati-06 & 19/5/2006 & 30 & 1 нńvas & $16 / 5 / 2006$ & $\mathrm{Nal}$ & $O_{x}$ & $\mathrm{Nai}$ \\
\hline AA38 & $\theta$ & $8 / 4 / 1977$ & Map-06 & $28 / 3 / 2006$ & 29 & $1 \mu$ ñvas & 17/3/2006 & $\mathrm{Nai}$ & $0 x^{1}$ & $O x^{2}$ \\
\hline AA39 & $\Theta$ & $21 / 12 / 1967$ & Iovv-82 & $14 / 12 / 2006$ & 39 & 24 होர & $9 / 12 / 2006$ & $\mathrm{Nai}$ & $O x$ & $0 x$ \\
\hline AA40 & $\Theta$ & 8/1/1977 & 2006 & $14 / 2 / 2006$ & 29 & $1 \mu$ Invas & 9/2/2006 & $\mathrm{Naí}$ & $O_{x}$ & $\mathrm{~N} \alpha \dot{i}$ \\
\hline AA41 & $\Theta$ & $12 / 11 / 1960$ & I $\alpha v=07$ & $31 / 7 / 2007$ & 47 & $1 \mu$ ñvas & $24 / 1 / 2007$ & $O_{x y}$ & $O x$ & $O_{x x}$ \\
\hline AA42 & $\Theta$ & $7 / 3 / 1982$ & Iovv-06 & $22 / 6 / 2006$ & 24 & $1 \mu$ ñvas & 19/6/2006 & $\mathrm{N} \alpha i$ & $O x$ & $\mathrm{~N} \alpha \mathfrak{l}^{\prime}$ \\
\hline AA43 & $\Theta$ & 28/5/1958 & 1998 & $25 / 1 / 2007$ & 49 & $9 \mathrm{\varepsilon tm}$ & $24 / 1 / 2007$ & $O x$ & $\mathrm{Ox}$ & $\mathrm{Ox}$ \\
\hline AA44 & A & $21 / 8 / 1970$ & Noe-06 & $15 / 11 / 2006$ & 36 & 1 нñvas & $14 / 11 / 2006$ & $\mathrm{N \alpha i}$ & Naí & $O x$ \\
\hline AA45 & $\Theta$ & $18 / 8 / 1978$ & 1998 & $12 / 5 / 2006$ & 28 & $8 \mathrm{EtT}$ & $10 / 5 / 2006$ & $\mathrm{Nai}$ & $O x$ & $O_{x}$ \\
\hline AA46 & A & 25/12/1959 & 1998 & $15 / 5 / 2006$ & 46 & $8 \mathrm{~km}$ & $12 / 5 / 2006$ & $\mathrm{Nai}$ & $O_{x}$ & $O_{x}$ \\
\hline AA47 & A & $8 / 12 / 1977$ & Avy-04 & $16 / 11 / 2006$ & 29 & 2 हाग & $14 / 11 / 2006$ & $O x$ & $O x$ & $O x$ \\
\hline AA48 & $\Theta$ & $8 / 10 / 1963$ & Mât-05 & $29 / 1 / 2007$ & 43 & 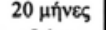 & $17 / 1 / 2007$ & $O_{x 1}$ & $O_{x}$ & $O_{x}$ \\
\hline AA49 & $\Theta$ & $25 / 6 / 1972$ & 2001 & $28 / 2 / 2006$ & 34 & 5 हٔाท & $27 / 2 / 2006$ & $O_{x i}$ & $O_{x i}$ & $O x$ \\
\hline AA50 & $\Theta$ & 4/1/1979 & Iovג -05 & $4 / 7 / 2006$ & 27 & $12 \mu \tilde{I} \mathrm{~V} \varepsilon \varsigma$ & $3 / 7 / 2006$ & $\mathrm{~N} \alpha \hat{i}$ & $N \alpha \tilde{i}$ & $\mathrm{~N} \alpha \hat{i}$ \\
\hline AA51 & $\Theta$ & $22 / 10 / 1976$ & 1997 & $21 / 7 / 2006$ & 30 & $9 \mathrm{Eth}$ & 20/7/2006 & $O x$ & $O x$ & Naí \\
\hline AA52 & $\Theta$ & $7 / 4 / 1971$ & 2003 & $22 / 6 / 2006$ & 35 & 3 हाग & 19/6/2006 & $O x$ & $O x$ & $O x$ \\
\hline AA53 & $\Theta$ & $7 / 3 / 1987$ & A $\pi \rho-05$ & $27 / 3 / 2006$ & 19 & $11 \mu \eta \tilde{v} v \varepsilon \varsigma$ & $22 / 3 / 2006$ & $\mathrm{Nai}$ & $O_{x h}$ & $O x$ \\
\hline AA54 & $\Theta$ & $24 / 7 / 1959$ & $A \pi \rho-06$ & $7 / 4 / 2006$ & 47 & 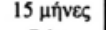 & 6/4/2006 & $O_{x 1}$ & $O x$ & $\mathrm{~N} \alpha \dot{i}$ \\
\hline AA55 & A & 17/4/1978 & 1999 & $30 / 3 / 2006$ & 28 & 7 हाग & 20/3/2006 & $\mathrm{Nai}$ & $\mathrm{Ox}_{x}$ & $O x$ \\
\hline AA56 & $\Theta$ & $6 / 10 / 1980$ & Map-05 & $22 / 6 / 2006$ & 26 & 1 દ́os & $21 / 6 / 2006$ & Naí & $O_{x x}$ & $\mathrm{Nai}$ \\
\hline AA57 & $\Theta$ & 7/2/1977 & 2001 & $5 / 5 / 2006$ & 29 & 5 होग & 13/4/2006 & $O_{x n}$ & $O x$ & $\mathrm{~N} \alpha i$ \\
\hline AA58 & $\Theta$ & 14/9/1956 & Iouv- 06 & 9/6/2006 & 50 & $1 \mu$ rivas & $6 / 6 / 2006$ & $\mathrm{~N} \alpha \dot{i}$ & $O_{x y}$ & $\mathrm{Ox}_{\mathrm{x}}$ \\
\hline AA59 & $\Theta$ & 13/10/1977 & 2002 & $10 / 5 / 2006$ & 29 & $4 \mathrm{Etm}$ & $10 / 5 / 2006$ & $\mathrm{~N} a f$ & $O_{x a}$ & $O_{x}$ \\
\hline AA60 & $\Theta$ & $26 / 12 / 1976$ & $\Sigma \varepsilon \pi-02$ & $15 / 9 / 2006$ & 30 & $4 \mathrm{E} \pi \mathrm{I}$ & 11/9/2006 & $O_{x n}$ & $O_{x n}$ & $0 x$ \\
\hline AA61 & A & 3/8/1989 & Map-06 & $12 / 4 / 2006$ & 17 & $1 \mu$ İvas & $4 / 5 / 2006$ & $\mathrm{Nai}$ & $\mathrm{Nai}$ & $\mathrm{Nai}$ \\
\hline AA62 & $\Theta$ & 3/4/1970 & Avy-06 & $12 / 9 / 2006$ & 36 & 1 ні́vas & 4/9/2006 & $\mathrm{Naí}$ & $O_{x}$ & $\mathrm{Naf}$ \\
\hline AA63 & $\theta$ & 17/10/1977 & Iav-05 & $30 / 10 / 2006$ & 29 & $22 \mu \tilde{n} \mathbf{v \varepsilon \varsigma}$ & 26/9/2006 & $O x$ & $O x$ & Naí \\
\hline AA64 & A & $11 / 12 / 1953$ & 1980 & 27/9/2006 & 53 & $26 \mathrm{ktm}$ & 25/9/2006 & $\mathrm{O} x \mathrm{I}$ & $\mathrm{O} x \mathrm{I}$ & $\mathrm{OxI}$ \\
\hline AA65 & A & 1/10/1979 & $\Sigma \varepsilon \pi-06$ & 14/9/2006 & 27 & $1 \mu \eta \dot{v}$ vas & 13/9/2006 & $\mathrm{O} x \mathrm{I}$ & $O_{x}$ & $O_{x}$ \\
\hline
\end{tabular}




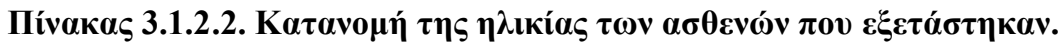

\begin{tabular}{|c|c|c|c|}
\hline 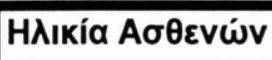 & 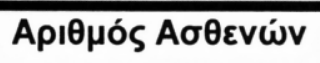 & 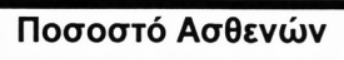 & 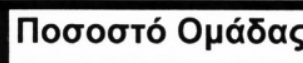 \\
\hline$<21 \varepsilon \tau \omega ́ v$ & 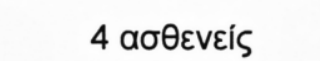 & $6.2 \%$ & \\
\hline 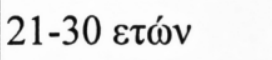 & 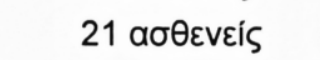 & $32.3 \%$ & \\
\hline 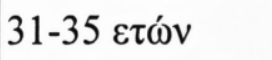 & 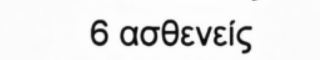 & $9.2 \%$ & \\
\hline 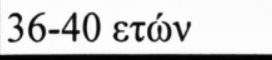 & 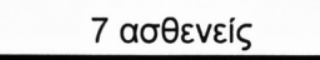 & $10.8 \%$ & $58.5 \%$ \\
\hline 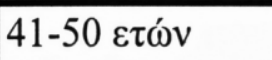 & 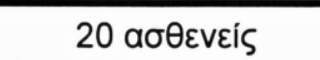 & $30.8 \%$ & \\
\hline 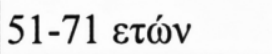 & 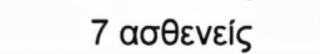 & $10.8 \%$ & $41.5 \%$ \\
\hline
\end{tabular}

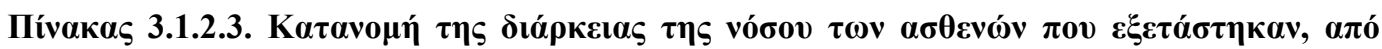

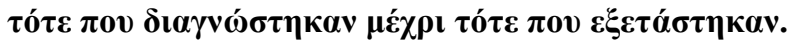

\begin{tabular}{|c|c|c|c|}
\hline 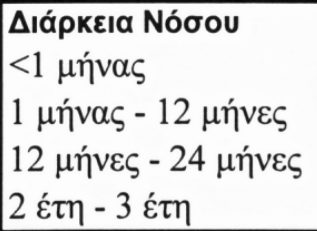 & 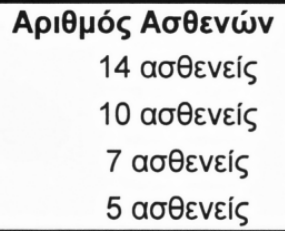 & 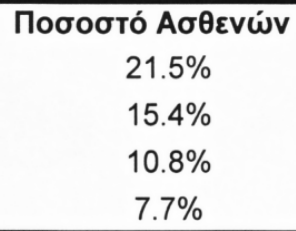 & 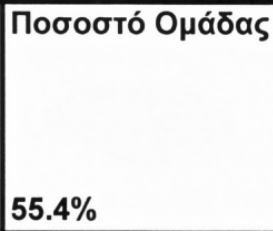 \\
\hline 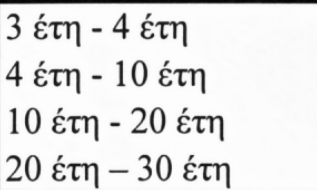 & 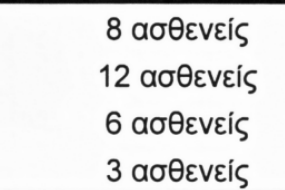 & $\begin{array}{c}12.3 \% \\
18.5 \% \\
9.2 \% \\
4.6 \%\end{array}$ & $44.6 \%$ \\
\hline
\end{tabular}


5.2. ПINAKE $\Sigma$ AПOTE $\Lambda$ E $\Sigma$ MAT $\Omega$ N E $\Xi E T A \Sigma H \Sigma$ ANTANAK $\Lambda$ A $\Sigma T I K O Y$ B $\Lambda$ EФAP $\Omega N$

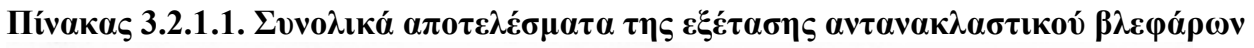

\begin{tabular}{|c|c|c|c|c|c|c|c|c|c|c|c|}
\hline $\mathbf{A} / \mathbf{A}$ & 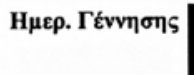 & $\begin{array}{c}\text { LR1 } \\
<13 \mathrm{~ms}\end{array}$ & $\begin{array}{l}\text { L LR2 } \\
<41 \mathrm{~ms}\end{array}$ & $\begin{array}{l}\text { L RR2 } \\
<44 \mathrm{~ms}\end{array}$ & $\begin{array}{c}\mathrm{RR} 1 \\
<13 \mathrm{~ms}\end{array}$ & $\begin{array}{l}\text { R RR2 } \\
<41 \mathrm{~ms}\end{array}$ & $\begin{array}{l}\text { R LR2 } \\
<44 \mathrm{~ms}\end{array}$ & $\begin{array}{c}\text { LF-L } \\
\text { ms }\end{array}$ & $\begin{array}{c}\text { LF-A } \\
\mathrm{mV}\end{array}$ & $\begin{array}{c}\text { RF-L } \\
\text { ms }\end{array}$ & $\begin{array}{c}\text { RF-A } \\
\mathrm{mV}\end{array}$ \\
\hline $\mathrm{AA} 01$ & $15 / 12 / 1977$ & 12,60 & 34,40 & 29,80 & 13,00 & 27,20 & 29,20 & 3,40 & 4,30 & 2,80 & 3,60 \\
\hline $\mathrm{AA} 02$ & $18 / 7 / 1966$ & 13,00 & 30,80 & 34,60 & 11,60 & 43,20 & 42,60 & 3,28 & 2,40 & 3,16 & 2,70 \\
\hline AA03 & $30 / 10 / 1971$ & 12,20 & 27,40 & 60,40 & 10,00 & 0,00 & 23,80 & 2,96 & 2,10 & 2,68 & 1,80 \\
\hline AA04 & $7 / 1 / 1961$ & 14,20 & 30,00 & 26,60 & 13,40 & 38,60 & 0,00 & 2,00 & 2,50 & 2,00 & 2,40 \\
\hline AA05 & $14 / 12 / 1962$ & 11,80 & 38,40 & 39,60 & 12,20 & 37,40 & 38,20 & 3,36 & 4,50 & 3,44 & 4,00 \\
\hline AA06 & $14 / 6 / 1957$ & 13,00 & 41,00 & 0,00 & 13,00 & 64,00 & 48,00 & 3,16 & 3,00 & 3,48 & 2,80 \\
\hline AA07 & $16 / 7 / 1982$ & 13,80 & 26,20 & 24,20 & 14,00 & 25,20 & 33,20 & 3,20 & 2,80 & 2,96 & 3,00 \\
\hline AA08 & $15 / 6 / 1960$ & 11,60 & 29,40 & 31,40 & 12,00 & 39,80 & 28,80 & 3,36 & 3,40 & 3,20 & 3,70 \\
\hline AA09 & $1 / 1 / 1947$ & 10,60 & 0,00 & 0,00 & 11,20 & 0,00 & 0,00 & 3,12 & 2,10 & 3,15 & 1,50 \\
\hline AA10 & $6 / 3 / 1936$ & 12,40 & 44,20 & 0,00 & 10,80 & 40,60 & 46,60 & 2,44 & 0,90 & 2,36 & 0,70 \\
\hline AA11 & $18 / 8 / 1965$ & 11,20 & 34,60 & 29,00 & 11,40 & 32,00 & 49,00 & 2,52 & 3,10 & 2,48 & 3,10 \\
\hline $\mathrm{AA} 12$ & $4 / 6 / 1966$ & 13,40 & 33,60 & 36,60 & 12,60 & 43,60 & 38,60 & 2,92 & 3,30 & 2,80 & 3,80 \\
\hline $\mathrm{AA} 13$ & $28 / 11 / 1980$ & 12,40 & 31,00 & 0,00 & 13,00 & 41,00 & 51,60 & 2,76 & 3,10 & 2,96 & 2,60 \\
\hline AA14 & $14 / 1 / 1961$ & 11,60 & 29,40 & 0,00 & 12,20 & 31,80 & 30,40 & 3,48 & 3,00 & 3,48 & 3,30 \\
\hline AA15 & $3 / 1 / 1956$ & 13,00 & 47,00 & 31,60 & 12,20 & 30,80 & 0,00 & 2,32 & 2,50 & 2,44 & 2,00 \\
\hline AA16 & $1 / 4 / 1972$ & 11,40 & 36,20 & 30,00 & 11,80 & 38,60 & 0,00 & 2,36 & 3,60 & 2,60 & 3,40 \\
\hline AA17 & $1 / 5 / 1978$ & 11,40 & 37,00 & 32,00 & 9,20 & 33,20 & 37,60 & 2,96 & 3,80 & 2,48 & 3,30 \\
\hline AA18 & $22 / 1 / 1963$ & 13,80 & 45,00 & 41,60 & 13,80 & 55,00 & 0,00 & 2,64 & 2,50 & 3,08 & 2,70 \\
\hline AA19 & $23 / 1 / 1955$ & 14,00 & 45,80 & 0,00 & 13,40 & 43,80 & 45,40 & 3,48 & 4,60 & 3,76 & 4,00 \\
\hline AA20 & $6 / 2 / 1965$ & 11,00 & 45,60 & 0,00 & 9,80 & 38,00 & 49,60 & 2,40 & 1,80 & 2,84 & 2,20 \\
\hline AA21 & $20 / 5 / 1972$ & 12,20 & 0,00 & 28,60 & 12,00 & 41,00 & 0,00 & 2,68 & 1,80 & 2,16 & 1,90 \\
\hline AA22 & $18 / 2 / 1948$ & 13,40 & 33,60 & 34,60 & 13,20 & 32,20 & 36,20 & 3,40 & 2,30 & 3,04 & 2,30 \\
\hline AA23 & $12 / 8 / 1981$ & 13,40 & 38,00 & 0,00 & 13,60 & 34,00 & 30,00 & 3,08 & 3,50 & 2,96 & 3,20 \\
\hline AA24 & $12 / 7 / 1938$ & 10,25 & 26,25 & 20,95 & 10,20 & 19,90 & 20,00 & 3,35 & 0,70 & 2,45 & 0,70 \\
\hline AA25 & $29 / 9 / 1986$ & 13,60 & 0,00 & 0,00 & 11,40 & 36,80 & 49,90 & 2,60 & 2,80 & 2,60 & 2,80 \\
\hline AA26 & $8 / 3 / 1977$ & 11,00 & 30,00 & 31,60 & 13,80 & 59,60 & 63,20 & 3,52 & 3,20 & 2,96 & 2,80 \\
\hline AA27 & $1 / 1 / 1958$ & 12,40 & 30,40 & 37,80 & 13,20 & 34,00 & 39,00 & 2,56 & 2,20 & 1,76 & 1,50 \\
\hline AA28 & $11 / 8 / 1973$ & 13,40 & 40,20 & 39,60 & 12,80 & 30,60 & 35,60 & 3,08 & 2,70 & 2,80 & 3,10 \\
\hline AA29 & $4 / 2 / 1969$ & 12,20 & 43,00 & 58,20 & 12,40 & 40,20 & 55,80 & 2,12 & 1,60 & 2,32 & 0,90 \\
\hline $\mathrm{AA} 30$ & $4 / 10 / 1989$ & 11,40 & 30,00 & 36,60 & 11,20 & 33,80 & 35,60 & 2,80 & 4,90 & 2,80 & 5,30 \\
\hline $\mathrm{AA} 31$ & $18 / 4 / 1962$ & 9,20 & 37,80 & 42,40 & 10,20 & 26,80 & 30,40 & 2,00 & 1,50 & 2,68 & 1,30 \\
\hline $\mathrm{AA} 32$ & $18 / 4 / 1962$ & 12,00 & 37,20 & 38,20 & 12,80 & 36,80 & 48,20 & 3,04 & 1,90 & 3,12 & 2,70 \\
\hline AA33 & $26 / 6 / 1970$ & 12,00 & 31,20 & 33,20 & 10,20 & 33,80 & 33,20 & 2,84 & 2,30 & 2,72 & 1,70 \\
\hline AA34 & $2 / 10 / 1967$ & 13,00 & 33,20 & 37,60 & 12,20 & 37,20 & 31,20 & 3,28 & 1,40 & 3,08 & 1,10 \\
\hline AA35 & $5 / 5 / 1960$ & 12,80 & 0,00 & 0,00 & 11,00 & 46,40 & 53,80 & 2,70 & 2,41 & 2,60 & 2,28 \\
\hline AA36 & $7 / 10 / 1958$ & 12,00 & 30,80 & 28,60 & 12,80 & 34,20 & 33,00 & 2,52 & 1,90 & 3,28 & 1,60 \\
\hline $\mathrm{AA} 37$ & $23 / 12 / 1975$ & 12,80 & 34,00 & 34,00 & 12,60 & 38,20 & 38,00 & 2,56 & 2,30 & 2,68 & 2,60 \\
\hline $\mathrm{AA} 38$ & $8 / 4 / 1977$ & 12,60 & 0,00 & 71,20 & 9,80 & 39,80 & 0,00 & 1,92 & 2,40 & 2,00 & 2,50 \\
\hline AA39 & $21 / 12 / 1967$ & 12,20 & 43,40 & 0,00 & 12,40 & 41,20 & 0,00 & 3,20 & 3,20 & 3,12 & 3,30 \\
\hline AA40 & $8 / 1 / 1977$ & 13,40 & 38,40 & 0,00 & 13,20 & 0,00 & 0,00 & 1,80 & 2,50 & 2,00 & 2,00 \\
\hline AA41 & $12 / 11 / 1960$ & 12,20 & 31,40 & 39,40 & 12,40 & 38,00 & 35,40 & 1,84 & 1,90 & 2,12 & 2,00 \\
\hline AA42 & $7 / 3 / 1982$ & 12,40 & 29,00 & 28,00 & 12,20 & 33,20 & 29,00 & 2,84 & 2,90 & 3,08 & 2,20 \\
\hline AA43 & $28 / 5 / 1958$ & 11,80 & 29,60 & 0,00 & 12,00 & 31,80 & 37,00 & 2,32 & 1,80 & 2,20 & 1,20 \\
\hline AA44 & $21 / 8 / 1970$ & 12,40 & 50,00 & 41,20 & 12,00 & 48,20 & 40,60 & 3,40 & 2,00 & 3,28 & 2,50 \\
\hline AA45 & $18 / 8 / 1978$ & 11,60 & 37,80 & 39,20 & 11,40 & 38,00 & 37,00 & 3,48 & 2,00 & 3,16 & 2,80 \\
\hline AA46 & $25 / 12 / 1959$ & 12,00 & 48,40 & 62,80 & 12,20 & 46,40 & 41,00 & 2,12 & 1,60 & 2,36 & 2,00 \\
\hline $\mathrm{AA} 47$ & $8 / 12 / 1977$ & 11,40 & 36,80 & 38,40 & 11,80 & 38,40 & 30,20 & 2,80 & 3,80 & 2,48 & 3,70 \\
\hline AA48 & $8 / 10 / 1963$ & 12,00 & 34,80 & 39,80 & 13,40 & 33,40 & 35,40 & 2,36 & 2,50 & 2,40 & 2,60 \\
\hline AA49 & $25 / 6 / 1972$ & 12,80 & 24,00 & 29,20 & 13,00 & 36,80 & 0,00 & 2,16 & 1,00 & 2,12 & 1,50 \\
\hline AA50 & $4 / 1 / 1979$ & 11,20 & 37,00 & 28,20 & 11,60 & 30,60 & 43,40 & 2,96 & 4,20 & 3,24 & 4,00 \\
\hline AA51 & $22 / 10 / 1976$ & 11,00 & 38,80 & 38,00 & 11,60 & 37,40 & 42,00 & 3,20 & 3,10 & 3,08 & 3,50 \\
\hline AA52 & $7 / 4 / 1971$ & 12,40 & 28,80 & 39,80 & 12,60 & 32,20 & 30,40 & 2,90 & 1,30 & 2,64 & 1,50 \\
\hline AA53 & $7 / 3 / 1987$ & 12,00 & 0,00 & 0,00 & 13,80 & 0,00 & 0,00 & 1,92 & 3,20 & 2,08 & 2,70 \\
\hline AA54 & $24 / 7 / 1959$ & 12,80 & 34,40 & 32,60 & 9,80 & 30,80 & 30,40 & 2,88 & 3,40 & 2,96 & 3,00 \\
\hline AA55 & $17 / 4 / 1978$ & 13,80 & 40,00 & 43,40 & 12,60 & 35,60 & 31,60 & 2,32 & 2,20 & 2,44 & 2,40 \\
\hline AA56 & $6 / 10 / 1980$ & 13,00 & 39,80 & 48,00 & 12,80 & 38,80 & 39,60 & 1,84 & 1,60 & 2,12 & 2,00 \\
\hline AA57 & $7 / 2 / 1977$ & 10,80 & 38,20 & 36,60 & 12,40 & 34,80 & 30,80 & 2,56 & 2,10 & 2,96 & 3,00 \\
\hline AA58 & $14 / 9 / 1956$ & 14,00 & 0,00 & 0,00 & 14,00 & 0,00 & 0,00 & 3,16 & 1,30 & 3,36 & 1,00 \\
\hline AA59 & $13 / 10 / 1977$ & 11,40 & 33,80 & 34,60 & 13,20 & 29,60 & 28,80 & 2,44 & 0,90 & 2,12 & 0,90 \\
\hline AA60 & $26 / 12 / 1976$ & 12,60 & 42,00 & 30,00 & 13,20 & 49,60 & 47,00 & 3,96 & 3,30 & 3,88 & 2,80 \\
\hline AA61 & $3 / 8 / 1989$ & 12,60 & 39,60 & 44,60 & 10,80 & 31,00 & 28,80 & 2,52 & 3,10 & 2,68 & 3,20 \\
\hline AA62 & $3 / 4 / 1970$ & 12,80 & 40,40 & 39,40 & 12,00 & 39,80 & 63,60 & 2,72 & 4,20 & 2,76 & 3,50 \\
\hline AA63 & $17 / 10 / 1977$ & 10,40 & 33,20 & 27,60 & 12,20 & 31,80 & 33,40 & 3,24 & 2,10 & 3,32 & 2,30 \\
\hline AA64 & $11 / 12 / 1953$ & 11,20 & 39,40 & 36,40 & 12,60 & 38,60 & 40,20 & 2,16 & 2,80 & 1,96 & 2,50 \\
\hline AA65 & $1 / 10 / 1979$ & 11,20 & 40,40 & 0,00 & 12,00 & 47,40 & 0,00 & 2,24 & 2,30 & 2,20 & 2,70 \\
\hline
\end{tabular}




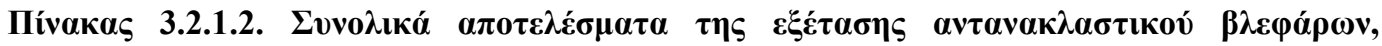

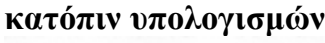

\begin{tabular}{|c|c|c|c|c|c|c|c|c|c|c|c|}
\hline $\begin{array}{c}\mathbf{A} / \mathbf{A} \\
\mathbf{K} \rho t \tau .\end{array}$ & $\begin{array}{c}\text { LR1 } \\
<13 \mathrm{~ms}\end{array}$ & $\begin{array}{l}\text { L LR2 } \\
<41 \mathrm{~ms}\end{array}$ & $\begin{array}{l}\text { L RR2 } \\
<44 \mathrm{~ms}\end{array}$ & $\begin{array}{c}\text { LLR2-LRR2 } \\
<[5] \mathrm{ms}\end{array}$ & $\begin{array}{c}\text { RR1 } \\
<13 \mathrm{~ms}\end{array}$ & $\begin{array}{l}\text { R RR2 } \\
<41 \mathrm{~ms}\end{array}$ & $\begin{array}{l}\text { R LR2 } \\
<44 \mathrm{~ms}\end{array}$ & $\begin{array}{c}\text { RRR2-RLR2 } \\
<[5] \mathrm{ms}\end{array}$ & $\begin{array}{l}\text { LR1-RR1 } \\
<[1.2] \mathrm{ms}\end{array}$ & $\begin{array}{c}\text { LLR2-RLR2 } \\
<[7] \mathrm{ms}\end{array}$ & $\begin{array}{c}\text { LRR2-RRR2 } \\
<[7] \mathrm{ms}\end{array}$ \\
\hline $\mathrm{AA} 01$ & 12,60 & 34,40 & 29,80 & 4,60 & 13,00 & 27,20 & 29,20 & $-2,00$ & $-0,40$ & 5,20 & 2,60 \\
\hline $\mathrm{AA} 02$ & 13,00 & 30,80 & 34,60 & $-3,80$ & 11,60 & 43,20 & 42,60 & 0,60 & 1,40 & $-11,80$ & $-8,60$ \\
\hline $\mathrm{AA} 03$ & 12,20 & 27,40 & 60,40 & $-33,00$ & 10,00 & 0,00 & 23,80 & $-23,80$ & 2,20 & 3,60 & 60,40 \\
\hline $\mathrm{AA} 04$ & 14,20 & 30,00 & 26,60 & 3,40 & 13,40 & 38,60 & 0,00 & 38,60 & 0,80 & 30,00 & $-12,00$ \\
\hline $\mathrm{AA} 05$ & 11,80 & 38,40 & 39,60 & $-1,20$ & 12,20 & 37,40 & 38,20 & $-0,80$ & $-0,40$ & 0,20 & 2,20 \\
\hline AA06 & 13,00 & 41,00 & 0,00 & 41,00 & 13,00 & 64,00 & 48,00 & 16,00 & 0,00 & $-7,00$ & $-64,00$ \\
\hline AA07 & 13,80 & 26,20 & 24,20 & 2,00 & 14,00 & 25,20 & 33,20 & $-8,00$ & $-0,20$ & $-7,00$ & $-1,00$ \\
\hline AA08 & 11,60 & 29,40 & 31,40 & $-2,00$ & 12,00 & 39,80 & 28,80 & 11,00 & $-0,40$ & 0,60 & $-8,40$ \\
\hline AA09 & 10,60 & 0,00 & 0,00 & - & 11,20 & 0,00 & 0,00 & - & $-0,60$ & - & - \\
\hline AA 10 & 12,40 & 44,20 & 0,00 & 44,20 & 10,80 & 40,60 & 46,60 & $-6,00$ & 1,60 & $-2,40$ & $-40,60$ \\
\hline AA11 & 11,20 & 34,60 & 29,00 & 5,60 & 11,40 & 32,00 & 49,00 & $-17,00$ & $-0,20$ & $-14,40$ & $-3,00$ \\
\hline $\mathrm{AA} 12$ & 13,40 & 33,60 & 36,60 & $-3,00$ & 12,60 & 43,60 & 38,60 & 5,00 & 0,80 & $-5,00$ & $-7,00$ \\
\hline $\mathrm{AA} 13$ & 12,40 & 31,00 & 0,00 & 31,00 & 13,00 & 41,00 & 51,60 & $-10,60$ & $-0,60$ & $-20,60$ & $-41,00$ \\
\hline AA14 & 11,60 & 29,40 & 0,00 & 29,40 & 12,20 & 31,80 & 30,40 & 1,40 & $-0,60$ & $-1,00$ & $-31,80$ \\
\hline AA15 & 13,00 & 47,00 & 31,60 & 15,40 & 12,20 & 30,80 & 0,00 & 30,80 & 0,80 & 47,00 & 0,80 \\
\hline AA16 & 11,40 & 36,20 & 30,00 & 6,20 & 11,80 & 38,60 & 0,00 & 38,60 & $-0,40$ & 36,20 & $-8,60$ \\
\hline $\mathrm{AA} 17$ & 11,40 & 37,00 & 32,00 & 5,00 & 9,20 & 33,20 & 37,60 & $-4,40$ & 2,20 & $-0,60$ & $-1,20$ \\
\hline AA18 & 13,80 & 45,00 & 41,60 & 3,40 & 13,80 & 55,00 & 0,00 & 55,00 & 0,00 & 45,00 & $-13,40$ \\
\hline AA19 & 14,00 & 45,80 & 0,00 & 45,80 & 13,40 & 43,80 & 45,40 & $-1,60$ & 0,60 & 0,40 & $-43,80$ \\
\hline $\mathrm{AA} 20$ & 11,00 & 45,60 & 0,00 & 45,60 & 9,80 & 38,00 & 49,60 & $-11,60$ & 1,20 & $-4,00$ & $-38,00$ \\
\hline $\mathrm{AA} 21$ & 12,20 & 0,00 & 28,60 & $-28,60$ & 12,00 & 41,00 & 0,00 & 41,00 & 0,20 & - & $-12,40$ \\
\hline $\mathrm{AA} 22$ & 13,40 & 33,60 & 34,60 & $-1,00$ & 13,20 & 32,20 & 36,20 & $-4,00$ & 0,20 & $-2,60$ & 2,40 \\
\hline AA23 & 13,40 & 38,00 & 0,00 & 38,00 & 13,60 & 34,00 & 30,00 & 4,00 & $-0,20$ & 8,00 & $-34,00$ \\
\hline AA24 & 10,25 & 26,25 & 20,95 & 5,30 & 10,20 & 19,90 & 20,00 & $-0,10$ & 0,05 & 6,25 & 1,05 \\
\hline AA25 & 13,60 & 0,00 & 0,00 & - & 11,40 & 36,80 & 49,90 & $-13,10$ & 2,20 & $-49,90$ & $-36,80$ \\
\hline AA26 & 11,00 & 30,00 & 31,60 & $-1,60$ & 13,80 & 59,60 & 63,20 & $-3,60$ & $-2,80$ & $-33,20$ & $-28,00$ \\
\hline AA27 & 12,40 & 30,40 & 37,80 & $-7,40$ & 13,20 & 34,00 & 39,00 & $-5,00$ & $-0,80$ & $-8,60$ & 3,80 \\
\hline AA28 & 13,40 & 40,20 & 39,60 & 0,60 & 12,80 & 30,60 & 35,60 & $-5,00$ & 0,60 & 4,60 & 9,00 \\
\hline AA29 & 12,20 & 43,00 & 58,20 & $-15,20$ & 12,40 & 40,20 & 55,80 & $-15,60$ & $-0,20$ & $-12,80$ & 18,00 \\
\hline $\mathrm{AA} 30$ & 11,40 & 30,00 & 36,60 & $-6,60$ & 11,20 & 33,80 & 35,60 & $-1,80$ & 0,20 & $-5,60$ & 2,80 \\
\hline $\mathrm{AA} 31$ & 9,20 & 37,80 & 42,40 & $-4,60$ & 10,20 & 26,80 & 30,40 & $-3,60$ & $-1,00$ & 7,40 & 15,60 \\
\hline $\mathrm{AA} 32$ & 12,00 & 37,20 & 38,20 & $-1,00$ & 12,80 & 36,80 & 48,20 & $-11,40$ & $-0,80$ & $-11,00$ & 1,40 \\
\hline $\mathrm{AA} 33$ & 12,00 & 31,20 & 33,20 & $-2,00$ & 10,20 & 33,80 & 33,20 & 0,60 & 1,80 & $-2,00$ & $-0,60$ \\
\hline AA34 & 13,00 & 33,20 & 37,60 & $-4,40$ & 12,20 & 37,20 & 31,20 & 6,00 & 0,80 & 2,00 & 0,40 \\
\hline AA35 & 12,80 & 0,00 & 0,00 & - & 11,00 & 46,40 & 53,80 & $-7,40$ & 1,80 & $-53,80$ & $-46,40$ \\
\hline AA36 & 12,00 & 30,80 & 28,60 & 2,20 & 12,80 & 34,20 & 33,00 & 1,20 & $-0,80$ & $-2,20$ & $-5,60$ \\
\hline $\mathrm{AA} 37$ & 12,80 & 34,00 & 34,00 & - & 12,60 & 38,20 & 38,00 & 0,20 & 0,20 & $-4,00$ & $-4,20$ \\
\hline AA38 & 12,60 & 0,00 & 71,20 & $-71,20$ & 9,80 & 39,80 & 0,00 & 39,80 & 2,80 & - & 31,40 \\
\hline AA 39 & 12,20 & 43,40 & 0,00 & 43,40 & 12,40 & 41,20 & 0,00 & 41,20 & $-0,20$ & 43,40 & $-41,20$ \\
\hline $\mathrm{AA} 40$ & 13,40 & 38,40 & 0,00 & 38,40 & 13,20 & 0,00 & 0,00 & - & 0,20 & 38,40 & - \\
\hline AA41 & 12,20 & 31,40 & 39,40 & $-8,00$ & 12,40 & 38,00 & 35,40 & 2,60 & $-0,20$ & $-4,00$ & 1,40 \\
\hline $\mathrm{AA} 42$ & 12,40 & 29,00 & 28,00 & 1,00 & 12,20 & 33,20 & 29,00 & 4,20 & 0,20 & 0,00 & $-5,20$ \\
\hline AA43 & 11,80 & 29,60 & 0,00 & 29,60 & 12,00 & 31,80 & 37,00 & $-5,20$ & $-0,20$ & $-7,40$ & $-31,80$ \\
\hline AA44 & 12,40 & 50,00 & 41,20 & 8,80 & 12,00 & 48,20 & 40,60 & 7,60 & 0,40 & 9,40 & $-7,00$ \\
\hline AA45 & 11,60 & 37,80 & 39,20 & $-1,40$ & 11,40 & 38,00 & 37,00 & 1,00 & 0,20 & 0,80 & 1,20 \\
\hline AA46 & 12,00 & 48,40 & 62,80 & $-14,40$ & 12,20 & 46,40 & 41,00 & 5,40 & $-0,20$ & 7,40 & 16,40 \\
\hline AA47 & 11,40 & 36,80 & 38,40 & $-1,60$ & 11,80 & 38,40 & 30,20 & 8,20 & $-0,40$ & 6,60 & 0,00 \\
\hline AA48 & 12,00 & 34,80 & 39,80 & $-5,00$ & 13,40 & 33,40 & 35,40 & $-2,00$ & $-1,40$ & $-0,60$ & 6,40 \\
\hline AA49 & 12,80 & 24,00 & 29,20 & $-5,20$ & 13,00 & 36,80 & 0,00 & 36,80 & $-0,20$ & 24,00 & $-7,60$ \\
\hline AA50 & 11,20 & 37,00 & 28,20 & 8,80 & 11,60 & 30,60 & 43,40 & $-12,80$ & $-0,40$ & $-6,40$ & $-2,40$ \\
\hline AA51 & 11,00 & 38,80 & 38,00 & 0,80 & 11,60 & 37,40 & 42,00 & $-4,60$ & $-0,60$ & $-3,20$ & 0,60 \\
\hline AA52 & 12,40 & 28,80 & 39,80 & $-11,00$ & 12,60 & 32,20 & 30,40 & 1,80 & $-0,20$ & $-1,60$ & 7,60 \\
\hline AA 53 & 12,00 & 0,00 & 0,00 & - & 13,80 & 0,00 & 0,00 & - & $-1,80$ & - & - \\
\hline AA54 & 12,80 & 34,40 & 32,60 & 1,80 & 9,80 & 30,80 & 30,40 & 0,40 & 3,00 & 4,00 & 1,80 \\
\hline AA55 & 13,80 & 40,00 & 43,40 & $-3,40$ & 12,60 & 35,60 & 31,60 & 4,00 & 1,20 & 8,40 & 7,80 \\
\hline AA56 & 13,00 & 39,80 & 48,00 & $-8,20$ & 12,80 & 38,80 & 39,60 & $-0,80$ & 0,20 & 0,20 & 9,20 \\
\hline AA57 & 10,80 & 38,20 & 36,60 & 1,60 & 12,40 & 34,80 & 30,80 & 4,00 & $-1,60$ & 7,40 & 1,80 \\
\hline AA58 & 14,00 & 0,00 & $\mathbf{0 , 0 0}$ & - & 14,00 & 0,00 & 0,00 & - & 0,00 & - & - \\
\hline AA59 & 11,40 & 33,80 & 34,60 & $-0,80$ & 13,20 & 29,60 & 28,80 & 0,80 & $-1,80$ & 5,00 & 5,00 \\
\hline AA60 & 12,60 & 42,00 & 30,00 & 12,00 & 13,20 & 49,60 & 47,00 & 2,60 & $-0,60$ & $-5,00$ & $-19,60$ \\
\hline AA61 & 12,60 & 39,60 & 44,60 & $-5,00$ & 10,80 & 31,00 & 28,80 & 2,20 & 1,80 & 10,80 & 13,60 \\
\hline AA62 & 12,80 & 40,40 & 39,40 & 1,00 & 12,00 & 39,80 & 63,60 & $-23,80$ & 0,80 & $-23,20$ & $-0,40$ \\
\hline AA63 & 10,40 & 33,20 & 27,60 & 5,60 & 12,20 & 31,80 & 33,40 & $-1,60$ & $-1,80$ & $-0,20$ & $-4,20$ \\
\hline AA64 & 11,20 & 39,40 & 36,40 & 3,00 & 12,60 & 38,60 & 40,20 & $-1,60$ & $-1,40$ & $-0,80$ & $-2,20$ \\
\hline AA65 & 11,20 & 40,40 & 0,00 & 40,40 & 12,00 & 47,40 & 0,00 & 47,40 & $-0,80$ & 40,40 & $-47,40$ \\
\hline
\end{tabular}




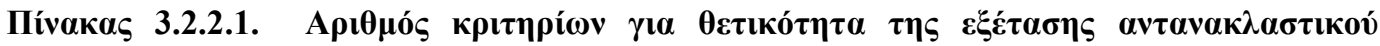

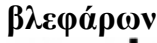

\begin{tabular}{|c|c|c|c|c|c|c|c|c|c|c|c|c|c|}
\hline & $\mathrm{A} / \mathrm{A}$ & 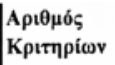 & $\begin{array}{l}\text { LR1 } \\
<13 \mathrm{~ms}\end{array}$ & $\begin{array}{l}\text { L LR2 } \\
<41 \mathrm{~ms}\end{array}$ & $\begin{array}{l}\text { L RR2 } \\
<44 \mathrm{~ms}\end{array}$ & $\begin{array}{l}\text { LLR2-LRR2 } \\
<[5] \mathrm{msec}\end{array}$ & $\begin{array}{l}\text { RR1 } \\
<13 \mathrm{~ms}\end{array}$ & $\begin{array}{l}\text { R RR2 } \\
<41 \mathrm{~ms}\end{array}$ & $\begin{array}{l}\text { R LR2 } \\
<44 \mathrm{~ms}\end{array}$ & $\begin{array}{l}\text { RRR2-RLR2 } \\
<[5] \mathrm{msec}\end{array}$ & $\begin{array}{l}\text { LR1-RR1 } \\
<|1.2| \mathrm{msec}\end{array}$ & $\begin{array}{l}\text { LLR2-RLR2 } \\
<[7] \mathrm{msec}\end{array}$ & $\begin{array}{l}\text { LRR2-RRR2 } \\
<[7] \mathrm{msec}\end{array}$ \\
\hline 1) & AA05 & 0 & 11,80 & 38,40 & 39,60 & $-1,20$ & 12,20 & 37,40 & 38,20 & $-0,80$ & $-0,40$ & 0,20 & 2,20 \\
\hline 2) & AA36 & 0 & 12,00 & 30,80 & 28,60 & 2,20 & 12,80 & 34,20 & 33,00 & 1,20 & $-0,80$ & $-2,20$ & $-5,60$ \\
\hline 3) & AA 37 & 0 & 12,80 & 34,00 & 34,00 & 0,00 & 12,60 & 38,20 & 38,00 & 0,20 & 0,20 & $-4,00$ & $-4,20$ \\
\hline 4) & $\mathrm{AA} 42$ & 0 & 12,40 & 29,00 & 28,00 & 1,00 & 12,20 & 33,20 & 29,00 & 4,20 & 0,20 & 0,00 & $-5,20$ \\
\hline 5) & AA45 & o & 11,60 & 37,80 & 39,20 & $-1,40$ & 11,40 & 38,00 & 37,00 & 1,00 & 0,20 & 0,80 & 1,20 \\
\hline 6) & AA51 & 0 & 11,00 & 38,80 & 38,00 & 0,80 & 11,60 & 37,40 & 42,00 & $-4,60$ & $-0,60$ & $-3,20$ & 0,60 \\
\hline 1) & $\mathrm{AA} 01$ & 1 & 12,60 & 34,40 & 29,80 & 4,60 & 13,00 & 27,20 & 29,20 & $-2,00$ & $-0,40$ & 5,20 & 2,60 \\
\hline 2) & AA24 & 1 & 10,25 & 26,25 & 20,95 & 5,30 & 10,20 & 19,90 & 20,00 & $-0,10$ & 0,05 & 6,25 & 1,05 \\
\hline 3) & $\mathrm{AA} 30$ & 1 & 11,40 & 30,00 & 36,60 & $-6,60$ & 11,20 & 33,80 & 35,60 & $-1,80$ & 0,20 & $-5,60$ & 2,80 \\
\hline 4) & $\mathrm{AA} 33$ & 1 & 12,00 & 31,20 & 33,20 & $-2,00$ & 10,20 & 33,80 & 33,20 & 0,60 & 1,80 & $-2,00$ & $-0,60$ \\
\hline 5) & AA41 & 1 & 12,20 & 31,40 & 39,40 & $-8,00$ & 12,40 & 38,00 & 35,40 & 2,60 & $-0,20$ & $-4,00$ & 1,40 \\
\hline 6) & AA54 & 1 & 12,80 & 34,40 & 32,60 & 1,80 & 9,80 & 30,80 & 30,40 & 0,40 & 3,00 & 4,00 & 1,80 \\
\hline 7) & $\mathrm{AA} 47$ & 1 & 11,40 & 36,80 & 38,40 & $-1,60$ & 11,80 & 38,40 & 30,20 & 8,20 & $-0,40$ & 6,60 & 0,00 \\
\hline 8) & AA64 & 1 & 11,20 & 39,40 & 36,40 & 3,00 & 12,60 & 38,60 & 40,20 & $-1,60$ & $-1,40$ & $-0,80$ & $-2,20$ \\
\hline 1) & AA08 & 2 & 11,60 & 29,40 & 31,40 & $-2,00$ & 12,00 & 39,80 & 28,80 & 11,00 & $-0,40$ & 0,60 & $-8,40$ \\
\hline 2) & AA17 & 2 & 11,40 & 37,00 & 32,00 & 5,00 & 9,20 & 33,20 & 37,60 & $-4,40$ & 2,20 & $-0,60$ & $-1,20$ \\
\hline 3) & $\mathrm{AA} 22$ & 2 & 13,40 & 33,60 & 34,60 & $-1,00$ & 13,20 & 32,20 & 36,20 & $-4,00$ & 0,20 & $-2,60$ & 2,40 \\
\hline 4) & $\mathrm{AA} 31$ & 2 & 9,20 & 37,80 & 42,40 & $-4,60$ & 10,20 & 26,80 & 30,40 & $-3,60$ & $-1,00$ & 7,40 & 15,60 \\
\hline 5) & AA 34 & 2 & 13,00 & 33,20 & 37,60 & $-4,40$ & 12,20 & 37,20 & 31,20 & 6,00 & 0,80 & 2,00 & 0,40 \\
\hline 6) & AA50 & 2 & 11,20 & 37,00 & 28,20 & 8,80 & 11,60 & 30,60 & 43,40 & $-12,80$ & $-0,40$ & $-6,40$ & $-2,40$ \\
\hline 7) & AA52 & 2 & 12,40 & 28,80 & 39,80 & $-11,00$ & 12,60 & 32,20 & 30,40 & 1,80 & $-0,20$ & $-1,60$ & $\mathbf{7 , 6 0}$ \\
\hline 8) & AA57 & 2 & 10,80 & 38,20 & 36,60 & 1,60 & 12,40 & 34,80 & 30,80 & 4,00 & $-1,60$ & 7,40 & 1,80 \\
\hline 9) & AA59 & 2 & 11,40 & 33,80 & 34,60 & $-0,80$ & 13,20 & 29,60 & 28,80 & 0,80 & $-1,80$ & 5,00 & 5,00 \\
\hline 10) & AA63 & 2 & 10,40 & 33,20 & 27,60 & 5,60 & 12,20 & 31,80 & 33,40 & $-1,60$ & $-1,80$ & $-0,20$ & $-4,20$ \\
\hline 1) & AA14 & 3 & 11,60 & 29,40 & 0,00 & 29,40 & 12,20 & 31,80 & 30,40 & 1,40 & $-0,60$ & $-1,00$ & $-31,80$ \\
\hline 2) & AA28 & 3 & 13,40 & 40,20 & 39,60 & 0,60 & 12,80 & 30,60 & 35,60 & $-5,00$ & 0,60 & 4,60 & 9,00 \\
\hline 3) & AA 32 & 3 & 12,00 & 37,20 & 38,20 & $-1,00$ & 12,80 & 36,80 & 48,20 & $-11,40$ & $-0,80$ & $-11,00$ & 1,40 \\
\hline 4) & AA48 & 3 & 12,00 & 34,80 & 39,80 & $-5,00$ & 13,40 & 33,40 & 35,40 & $-2,00$ & $-1,40$ & $-0,60$ & 6,40 \\
\hline 5) & AA56 & 3 & 13,00 & 39,80 & 48,00 & $-8,20$ & 12,80 & 38,80 & 39,60 & $-0,80$ & 0,20 & 0,20 & 9,20 \\
\hline 6) & AA62 & 3 & 12,80 & 40,40 & 39,40 & 1,00 & 12,00 & 39,80 & 63,60 & $-23,80$ & 0,80 & $-23,20$ & $-0,40$ \\
\hline 1) & $\mathrm{AA} 07$ & 4 & 13,80 & 26,20 & 24,20 & 2,00 & 14,00 & 25,20 & 33,20 & $-8,00$ & $-0,20$ & $-7,00$ & $-1,00$ \\
\hline 2) & AA11 & 4 & 11,20 & 34,60 & 29,00 & 5,60 & 11,40 & 32,00 & 49,00 & $-17,00$ & $-0,20$ & $-14,40$ & $-3,00$ \\
\hline 3) & $\mathrm{AA} 12$ & 4 & 13,40 & 33,60 & 36,60 & $-3,00$ & 12,60 & 43,60 & 38,60 & 5,00 & 0,80 & $-5,00$ & $-7,00$ \\
\hline 4) & AA27 & 4 & 12,40 & 30,40 & 37,80 & $-7,40$ & 13,20 & 34,00 & 39,00 & $-5,00$ & $-0,80$ & $-8,60$ & 3,80 \\
\hline 5) & AA55 & 4 & 13,80 & 40,00 & 43,40 & $-3,40$ & 12,60 & 35,60 & 31,60 & 4,00 & 1,20 & 8,40 & 7,80 \\
\hline 1) & $\mathrm{AA} 02$ & 5 & 13,00 & 30,80 & 34,60 & $-3,80$ & 11,60 & 43,20 & 42,60 & 0,60 & 1,40 & $-11,80$ & $-8,60$ \\
\hline 2) & $\mathrm{AA} 16$ & 5 & 11,40 & 36,20 & 30,00 & 6,20 & 11,80 & 38,60 & 0,00 & 38,60 & $-0,40$ & 36,20 & $-8,60$ \\
\hline 3) & $\mathrm{AA} 43$ & 5 & 11,80 & 29,60 & 0,00 & 29,60 & 12,00 & 31,80 & 37,00 & $-5,20$ & $-0,20$ & $-7,40$ & $-31,80$ \\
\hline 4) & AA61 & 5 & 12,60 & 39,60 & 44,60 & $-5,00$ & 10,80 & 31,00 & 28,80 & 2,20 & 1,80 & 10,80 & 13,60 \\
\hline 1) & $\mathrm{AA} 03$ & 6 & 12,20 & 27,40 & 60,40 & $-33,00$ & 10,00 & 0,00 & 23,80 & $-23,80$ & 2,20 & 3,60 & 60,40 \\
\hline 2) & $\mathrm{AA} 04$ & 6 & 14,20 & 30,00 & 26,60 & 3,40 & 13,40 & 38,60 & 0,00 & 38,60 & 0,80 & 30,00 & $-12,00$ \\
\hline 3) & AA15 & 6 & 13,00 & 47,00 & 31,60 & 15,40 & 12,20 & 30,80 & 0,00 & 30,80 & 0,80 & 47,00 & 0,80 \\
\hline 4) & $\mathrm{AA} 21$ & 6 & 12,20 & 0,00 & 28,60 & $-28,60$ & 12,00 & 41,00 & 0,00 & 41,00 & 0,20 & 0,00 & $-12,40$ \\
\hline 5) & AA23 & 6 & 13,40 & 38,00 & 0,00 & 38,00 & 13,60 & 34,00 & 30,00 & 4,00 & $-0,20$ & 8,00 & $-34,00$ \\
\hline 6) & AA26 & 6 & 11,00 & 30,00 & 31,60 & $-1,60$ & 13,80 & 59,60 & 63,20 & $-3,60$ & $-2,80$ & $-33,20$ & $-28,00$ \\
\hline 7) & AA29 & 6 & 12,20 & 43,00 & 58,20 & $-15,20$ & 12,40 & 40,20 & 55,80 & $-15,60$ & $-0,20$ & $-12,80$ & 18,00 \\
\hline 8) & $\mathrm{AA} 44$ & 6 & 12,40 & 50,00 & 41,20 & 8,80 & 12,00 & 48,20 & 40,60 & 7,60 & 0,40 & 9,40 & $-7,00$ \\
\hline 9) & AA49 & 6 & 12,80 & 24,00 & 29,20 & $-5,20$ & 13,00 & 36,80 & 0,00 & 36,80 & $-0,20$ & 24,00 & $-7,60$ \\
\hline 10) & AA60 & 6 & 12,60 & 42,00 & 30,00 & 12,00 & 13,20 & 49,60 & 47,00 & 2,60 & $-0,60$ & $-5,00$ & $-19,60$ \\
\hline 1) & $\mathrm{AA} 10$ & 7 & 12,40 & 44,20 & 0,00 & 44,20 & 10,80 & 40,60 & 46,60 & $-6,00$ & 1,60 & $-2,40$ & $-40,60$ \\
\hline 2) & $\mathrm{A} A 20$ & 7 & 11,00 & 45,60 & 0,00 & 45,60 & 9,80 & 38,00 & 49,60 & $-11,60$ & 1,20 & $-4,00$ & $-38,00$ \\
\hline 3) & AA46 & 7 & 12,00 & 48,40 & 62,80 & $-14,40$ & 12,20 & 46,40 & 41,00 & 5,40 & $-0,20$ & 7,40 & 16,40 \\
\hline 4) & AA65 & 7 & 11,20 & 40,40 & 0,00 & 40,40 & 12,00 & 47,40 & 0,00 & 47,40 & $-0,80$ & 40,40 & $-47,40$ \\
\hline 1) & AA09 & 8 & 10,60 & 0,00 & 0,00 & 0,00 & 11,20 & 0,00 & 0,00 & 0,00 & $-0,60$ & 0,00 & 0,00 \\
\hline 2) & $\mathrm{AA} 13$ & 8 & 12,40 & 31,00 & 0,00 & 31,00 & 13,00 & 41,00 & 51,60 & $-10,60$ & $-0,60$ & $-20,60$ & $-41,00$ \\
\hline 3) & AA19 & 8 & 14,00 & 45,80 & 0,00 & 45,80 & 13,40 & 43,80 & 45,40 & $-1,60$ & 0,60 & 0,40 & $-43,80$ \\
\hline 4) & AA35 & 8 & 12,80 & 0,00 & 0,00 & 0,00 & 11,00 & 46,40 & 53,80 & $-7,40$ & 1,80 & $-53,80$ & $-46,40$ \\
\hline 5) & AA38 & 8 & 12,60 & 0,00 & 71,20 & $-71,20$ & 9,80 & 39,80 & 0,00 & 39,80 & 2,80 & 0,00 & 31,40 \\
\hline 6) & $\mathrm{AA} 39$ & 8 & 12,20 & 43,40 & 0,00 & 43,40 & 12,40 & 41,20 & 0,00 & 41,20 & $-0,20$ & 43,40 & $-41,20$ \\
\hline 7) & $\mathrm{AA} 40$ & 8 & 13,40 & 38,40 & 0,00 & 38,40 & 13,20 & 0,00 & 0,00 & 0,00 & 0,20 & 38,40 & 0,00 \\
\hline 1) & AA18 & 9 & 13,80 & 45,00 & 41,60 & 3,40 & 13,80 & 55,00 & 0,00 & 55,00 & 0,00 & 45,00 & $-13,40$ \\
\hline 2) & AA25 & 9 & 13,60 & 0,00 & 0,00 & 0,00 & 11,40 & 36,80 & 49,90 & $-13,10$ & 2,20 & $-49,90$ & $-36,80$ \\
\hline 1) & AA06 & 10 & 13,00 & 41,00 & 0,00 & 41,00 & 13,00 & 64,00 & 48,00 & 16,00 & 0,00 & $-7,00$ & $-64,00$ \\
\hline 2) & AA53 & 10 & 12,00 & 0,00 & 0,00 & 0,00 & 13,80 & 0,00 & 0,00 & 0,00 & $-1,80$ & 0,00 & 0,00 \\
\hline 1) & AA58 & 11 & 14,00 & 0,00 & 0,00 & 0,00 & 14,00 & 0,00 & 0,00 & 0,00 & 0,00 & 0,00 & 0,00 \\
\hline
\end{tabular}




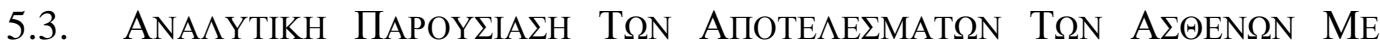

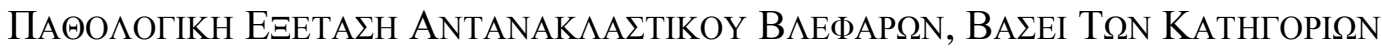
T $\Omega$ N AKTINO $\Lambda$ OГIK $\Omega$ N EYPHMAT $\Omega N$

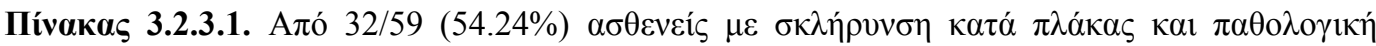

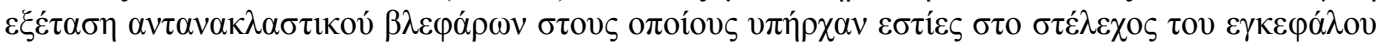

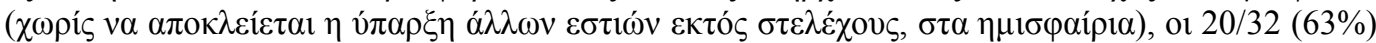

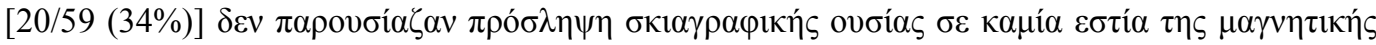

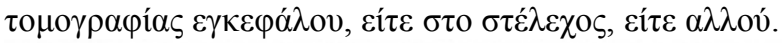

\begin{tabular}{|c|c|c|c|c|c|c|c|c|c|c|c|}
\hline $\begin{array}{c}\text { A/A } \\
\text { Kpır. } \\
\text { AA03 }\end{array}$ & \begin{tabular}{|c|} 
LR1 \\
$<13 \mathrm{~ms}$ \\
12,20
\end{tabular} & $\begin{array}{l}\text { L LR2 } \\
<41 \mathrm{~ms} \\
27,40\end{array}$ & $\begin{array}{l}\text { L RR2 } \\
<44 \mathrm{~ms} \\
60,40\end{array}$ & $\begin{array}{c}\text { LLR2-LRR2 } \\
<[5] \mathrm{ms} \\
-33,00\end{array}$ & \begin{tabular}{|c} 
RR1 \\
$<13 \mathrm{~ms}$ \\
10,00
\end{tabular} & $\begin{array}{c}\text { R RR2 } \\
<41 \mathrm{~ms} \\
0,00\end{array}$ & $\begin{array}{l}\text { R LR2 } \\
<44 \mathrm{~ms} \\
23,80\end{array}$ & $\begin{array}{c}\text { RRR2-RLR2 } \\
<[5] \mathrm{ms} \\
-23,80\end{array}$ & $\begin{array}{c}\text { LR1-RR1 } \\
<[1.2] \mathrm{ms} \\
2,20\end{array}$ & $\begin{array}{c}\text { LLR2-RLR2 } \\
<[7] \mathrm{ms} \\
3,60\end{array}$ & $\begin{array}{c}\text { LRR2-RRR2 } \\
<[7] \mathrm{ms} \\
60,40\end{array}$ \\
\hline AA04 & 14,20 & 30,00 & 26,60 & 3,40 & 13,40 & 38,60 & 0,00 & 38,60 & 0,80 & 30,00 & $-12,00$ \\
\hline AA06 & 13,00 & 41,00 & 0,00 & 41,00 & 13,00 & 64,00 & 48,00 & 16,00 & 0,00 & $-7,00$ & $-64,00$ \\
\hline AA07 & 13,80 & 26,20 & 24,20 & 2,00 & 14,00 & 25,20 & 33,20 & $-8,00$ & $-0,20$ & $-7,00$ & $-1,00$ \\
\hline AA 10 & 12,40 & 44,20 & 0,00 & 44,20 & 10,80 & 40,60 & 46,60 & $-6,00$ & 1,60 & $-2,40$ & $-40,60$ \\
\hline AA13 & 12,40 & 31,00 & 0,00 & 31,00 & 13,00 & 41,00 & 51,60 & $-10,60$ & $-0,60$ & $-20,60$ & $-41,00$ \\
\hline AA15 & 13,00 & 47,00 & 31,60 & 15,40 & 12,20 & 30,80 & 0,00 & 30,80 & 0,80 & 47,00 & 0,80 \\
\hline AA16 & 11,40 & 36,20 & 30,00 & 6,20 & 11,80 & 38,60 & 0,00 & 38,60 & $-0,40$ & 36,20 & $-8,60$ \\
\hline AA18 & 13,80 & 45,00 & 41,60 & 3,40 & 13,80 & 55,00 & 0,00 & 55,00 & 0,00 & 45,00 & $-13,40$ \\
\hline AA23 & 13,40 & 38,00 & 0,00 & 38,00 & 13,60 & 34,00 & 30,00 & 4,00 & $-0,20$ & 8,00 & $-34,00$ \\
\hline AA31 & 9,20 & 37,80 & 42,40 & $-4,60$ & 10,20 & 26,80 & 30,40 & $-3,60$ & $-1,00$ & 7,40 & 15,60 \\
\hline AA32 & 12,00 & 37,20 & 38,20 & $-1,00$ & 12,80 & 36,80 & 48,20 & $-11,40$ & $-0,80$ & $-11,00$ & 1,40 \\
\hline AA33 & 12,00 & 31,20 & 33,20 & $-2,00$ & 10,20 & 33,80 & 33,20 & 0,60 & 1,80 & $-2,00$ & $-0,60$ \\
\hline AA38 & 12,60 & 0,00 & 71,20 & $-71,20$ & 9,80 & 39,80 & 0,00 & 39,80 & 2,80 & - & 31,40 \\
\hline AA39 & 12,20 & 43,40 & 0,00 & 43,40 & 12,40 & 41,20 & 0,00 & 41,20 & $-0,20$ & 43,40 & $-41,20$ \\
\hline AA46 & 12,00 & 48,40 & 62,80 & $-14,40$ & 12,20 & 46,40 & 41,00 & 5,40 & $-0,20$ & 7,40 & 16,40 \\
\hline AA53 & 12,00 & 0,00 & 0,00 & - & 13,80 & 0,00 & 0,00 & - & $-1,80$ & - & - \\
\hline AA55 & 13,80 & 40,00 & 43,40 & $-3,40$ & 12,60 & 35,60 & 31,60 & 4,00 & 1,20 & 8,40 & 7,80 \\
\hline AA58 & 14,00 & 0,00 & 0,00 & - & 14,00 & 0,00 & 0,00 & - & 0,00 & - & - \\
\hline AA59 & 11,40 & 33,80 & 34,60 & $-0,80$ & 13,20 & 29,60 & 28,80 & 0,80 & $-1,80$ & 5,00 & 5,00 \\
\hline
\end{tabular}

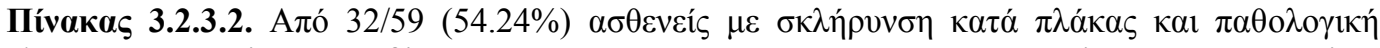

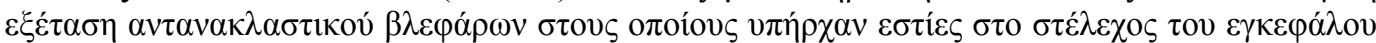

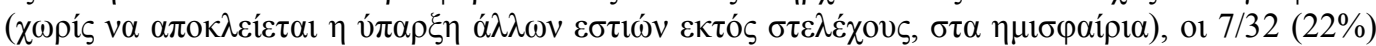

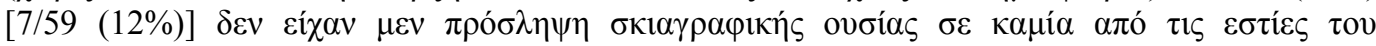

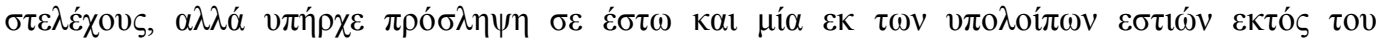
$\sigma \tau \varepsilon \lambda \varepsilon ́ \chi 0 \nu \varsigma$.

\begin{tabular}{|c|c|c|c|c|c|c|c|c|c|c|c|}
\hline $\begin{array}{c}\mathbf{A} / \mathbf{A} \\
\mathbf{K} \rho \mathbf{t \tau} .\end{array}$ & $\begin{array}{c}\text { LR1 } \\
<13 \mathrm{~ms}\end{array}$ & $\begin{array}{l}\text { L LR2 } \\
<41 \mathrm{~ms}\end{array}$ & $\begin{array}{l}\text { L RR2 } \\
<44 \mathrm{~ms}\end{array}$ & $\begin{array}{c}\text { LLR2-LRR2 } \\
<[5] \mathrm{ms}\end{array}$ & $\begin{array}{c}\text { RR1 } \\
<13 \mathrm{~ms}\end{array}$ & $\begin{array}{l}\text { R RR2 } \\
<41 \mathrm{~ms}\end{array}$ & $\begin{array}{l}\text { R LR2 } \\
<44 \mathrm{~ms}\end{array}$ & $\begin{array}{c}\text { RRR2-RLR2 } \\
<[5] \mathrm{ms}\end{array}$ & $\begin{array}{c}\text { LR1-RR1 } \\
<[1.2] \mathrm{ms}\end{array}$ & $\begin{array}{c}\text { LLR2-RLR2 } \\
<[7] \mathrm{ms}\end{array}$ & $\begin{array}{c}\text { LRR2-RRR2 } \\
<[7] \mathrm{ms}\end{array}$ \\
\hline AA01 & 12,60 & 34,40 & 29,80 & 4,60 & 13,00 & 27,20 & 29,20 & $-2,00$ & $-0,40$ & 5,20 & 2,60 \\
\hline AA12 & 13,40 & 33,60 & 36,60 & $-3,00$ & 12,60 & 43,60 & 38,60 & 5,00 & 0,80 & $-5,00$ & $-7,00$ \\
\hline AA25 & 13,60 & 0,00 & 0,00 & 0,00 & 11,40 & 36,80 & 49,90 & $-13,10$ & 2,20 & $-49,90$ & $-36,80$ \\
\hline AA34 & 13,00 & 33,20 & 37,60 & $-4,40$ & 12,20 & 37,20 & 31,20 & 6,00 & 0,80 & 2,00 & 0,40 \\
\hline AA40 & 13,40 & 38,40 & 0,00 & 38,40 & 13,20 & 0,00 & 0,00 & - & 0,20 & 38,40 & - \\
\hline AA56 & 13,00 & 39,80 & 48,00 & $-8,20$ & 12,80 & 38,80 & 39,60 & $-0,80$ & 0,20 & 0,20 & 9,20 \\
\hline AA62 & 12,80 & 40,40 & 39,40 & 1,00 & 12,00 & 39,80 & 63,60 & $-23,80$ & 0,80 & $-23,20$ & $-0,40$ \\
\hline
\end{tabular}

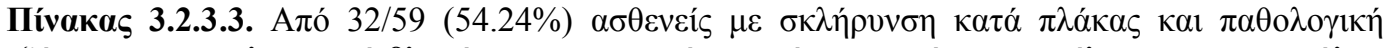

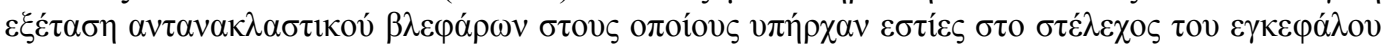

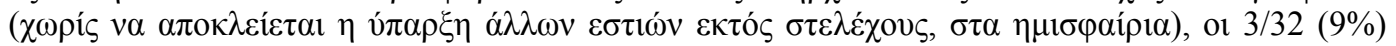

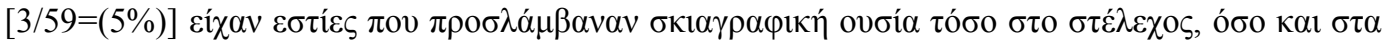

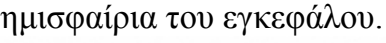

\begin{tabular}{|c|c|c|c|c|c|c|c|c|c|c|c|}
\hline $\begin{array}{c}\mathbf{A} / \mathbf{A} \\
\mathbf{K} \rho \mathbf{t} .\end{array}$ & $\begin{array}{c}\text { LR1 } \\
<13 \mathrm{~ms}\end{array}$ & $\begin{array}{l}\text { L LR2 } \\
<41 \mathrm{~ms}\end{array}$ & $\begin{array}{l}\text { L RR2 } \\
<44 \mathrm{~ms}\end{array}$ & $\begin{array}{c}\text { LLR2-LRR2 } \\
<[5] \mathrm{ms}\end{array}$ & $\begin{array}{c}\text { RR1 } \\
<13 \mathrm{~ms}\end{array}$ & $\begin{array}{l}\text { R RR2 } \\
<41 \mathrm{~ms}\end{array}$ & $\begin{array}{l}\text { R LR2 } \\
<44 \mathrm{~ms}\end{array}$ & $\begin{array}{c}\text { RRR2-RLR2 } \\
<[5] \mathrm{ms}\end{array}$ & $\begin{array}{c}\text { LR1-RR1 } \\
<[1.2] \mathrm{ms}\end{array}$ & $\begin{array}{c}\text { LLR2-RLR2 } \\
<[7] \mathrm{ms}\end{array}$ & $\begin{array}{c}\text { LRR2-RRR2 } \\
<[7] \mathrm{ms}\end{array}$ \\
\hline AA09 & 10,60 & 0,00 & 0,00 & - & 11,20 & 0,00 & 0,00 & - & $-0,60$ & - & - \\
\hline AA5C & 11,20 & 37,00 & 28,20 & 8,80 & 11,60 & 30,60 & 43,40 & $-12,80$ & $-0,40$ & $-6,40$ & $-2,40$ \\
\hline AA6 & 12,60 & 39,60 & 44,60 & $-5,00$ & 10,80 & 31,00 & 28,80 & 2,20 & 1,80 & 10,80 & 13,60 \\
\hline
\end{tabular}




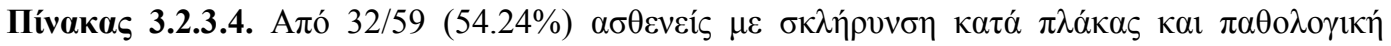

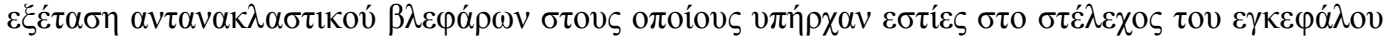

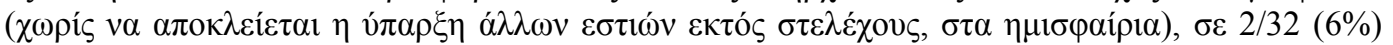

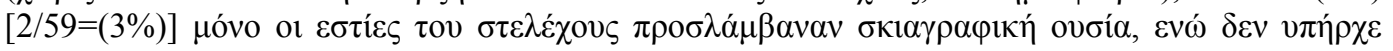

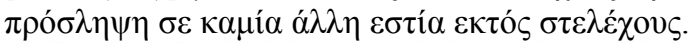

\begin{tabular}{|c|c|c|c|c|c|c|c|c|c|c|c|}
\hline 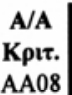 & $\begin{array}{c}\text { LR1 } \\
<13 \mathrm{~ms} \\
11,60\end{array}$ & $\begin{array}{l}\text { L LR2 } \\
<41 \mathrm{~ms} \\
29,40\end{array}$ & $\begin{array}{l}\text { L RR2 } \\
<\mathbf{4 4 m s} \\
31,40\end{array}$ & $\begin{array}{c}\text { LLR2-LRR2 } \\
<[5] \mathrm{ms} \\
-2,00\end{array}$ & \begin{tabular}{|c} 
RR1 \\
$<13 \mathrm{~ms}$ \\
12,00
\end{tabular} & $\begin{array}{l}\text { R RR2 } \\
<41 \mathrm{~ms} \\
39,80\end{array}$ & $\begin{array}{l}\text { R LR2 } \\
<44 \mathrm{~ms} \\
28,80\end{array}$ & $\begin{array}{c}\text { RRR2-RLR2 } \\
<[5] \mathrm{ms} \\
11,00\end{array}$ & $\begin{array}{c}\text { LR1-RR1 } \\
<[1.2] \mathrm{ms} \\
-0,40\end{array}$ & $\begin{array}{c}\text { LLR2-RLR2 } \\
<[7] \mathrm{ms} \\
0,60\end{array}$ & $\begin{array}{c}\text { LRR2-RRR2 } \\
<[7] \mathrm{ms} \\
-8,40\end{array}$ \\
\hline AA44 & 12,40 & 50,00 & 41,20 & 8,80 & 12,00 & 48,20 & 40,60 & 7,60 & 0,40 & 9,40 & $-7,00$ \\
\hline
\end{tabular}

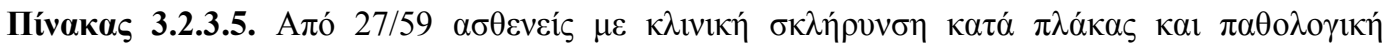

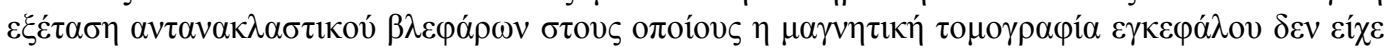

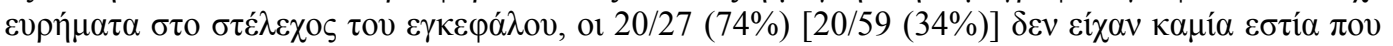

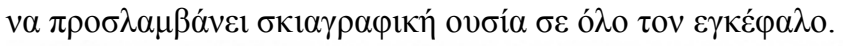

\begin{tabular}{|c|c|c|c|c|c|c|c|c|c|c|c|}
\hline $\begin{array}{c}\text { A/A } \\
\text { Kpıt. } \\
\text { AA02 }\end{array}$ & $\begin{array}{c}\text { LR1 } \\
<13 \mathrm{~ms} \\
13,00\end{array}$ & $\begin{array}{c}\text { L LR2 } \\
<41 \mathrm{~ms} \\
30,80\end{array}$ & $\begin{array}{c}\text { L RR2 } \\
<44 \mathrm{~ms} \\
34,60\end{array}$ & $\begin{array}{c}\text { LLR2-LRR2 } \\
<\text { [5] ms } \\
-3,80\end{array}$ & $\begin{array}{c}\text { RR1 } \\
<13 \mathrm{~ms} \\
11,60\end{array}$ & $\begin{array}{c}\text { R RR2 } \\
<41 \mathrm{~ms} \\
43,20\end{array}$ & $\begin{array}{c}\text { R LR2 } \\
<44 \mathrm{~ms} \\
42,60\end{array}$ & $\begin{array}{c}\text { RRR2-RLR2 } \\
<\text { [5] ms } \\
0,60\end{array}$ & $\begin{array}{c}\text { LR1-RR1 } \\
<[1.2] \mathrm{ms} \\
1,40\end{array}$ & $\begin{array}{c}\text { LLR2-RLR2 } \\
<[7] \mathrm{ms} \\
-11,80\end{array}$ & $\begin{array}{c}\text { LRR2-RRR2 } \\
<[7] \mathrm{ms} \\
-\mathbf{8 , 6 0}\end{array}$ \\
\hline AA11 & 11,20 & 34,60 & 29,00 & 5,60 & 11,40 & 32,00 & 49,00 & $-17,00$ & $-0,20$ & $-14,40$ & $-3,00$ \\
\hline AA17 & 11,40 & 37,00 & 32,00 & 5,00 & 9,20 & 33,20 & 37,60 & $-4,40$ & 2,20 & $-0,60$ & $-1,20$ \\
\hline AA19 & 14,00 & 45,80 & 0,00 & 45,80 & 13,40 & 43,80 & 45,40 & $-1,60$ & 0,60 & 0,40 & $-43,80$ \\
\hline AA21 & 12,20 & 0,00 & 28,60 & $-28,60$ & 12,00 & 41,00 & 0,00 & 41,00 & 0,20 & 0,00 & $-12,40$ \\
\hline AA22 & 13,40 & 33,60 & 34,60 & $-1,00$ & 13,20 & 32,20 & 36,20 & $-4,00$ & 0,20 & $-2,60$ & 2,40 \\
\hline AA24 & 10,25 & 26,25 & 20,95 & 5,30 & 10,20 & 19,90 & 20,00 & $-0,10$ & 0,05 & 6,25 & 1,05 \\
\hline AA26 & 11,00 & 30,00 & 31,60 & $-1,60$ & 13,80 & 59,60 & 63,20 & $-3,60$ & $-2,80$ & $-33,20$ & $-28,00$ \\
\hline AA28 & 13,40 & 40,20 & 39,60 & 0,60 & 12,80 & 30,60 & 35,60 & $-5,00$ & 0,60 & 4,60 & 9,00 \\
\hline AA29 & 12,20 & 43,00 & 58,20 & $-15,20$ & 12,40 & 40,20 & 55,80 & $-15,60$ & $-0,20$ & $-12,80$ & 18,00 \\
\hline AA35 & 12,80 & 0,00 & 0,00 & - & 11,00 & 46,40 & 53,80 & $-7,40$ & 1,80 & $-53,80$ & $-46,40$ \\
\hline AA41 & 12,20 & 31,40 & 39,40 & $-8,00$ & 12,40 & 38,00 & 35,40 & 2,60 & $-0,20$ & $-4,00$ & 1,40 \\
\hline AA43 & 11,80 & 29,60 & 0,00 & 29,60 & 12,00 & 31,80 & 37,00 & $-5,20$ & $-0,20$ & $-7,40$ & $-31,80$ \\
\hline AA47 & 11,40 & 36,80 & 38,40 & $-1,60$ & 11,80 & 38,40 & 30,20 & 8,20 & $-0,40$ & 6,60 & 0,00 \\
\hline AA48 & 12,00 & 34,80 & 39,80 & $-5,00$ & 13,40 & 33,40 & 35,40 & $-2,00$ & $-1,40$ & $-0,60$ & 6,40 \\
\hline AA49 & 12,80 & 24,00 & 29,20 & $-5,20$ & 13,00 & 36,80 & 0,00 & 36,80 & $-0,20$ & 24,00 & $-7,60$ \\
\hline AA52 & 12,40 & 28,80 & 39,80 & $-11,00$ & 12,60 & 32,20 & 30,40 & 1,80 & $-0,20$ & $-1,60$ & 7,60 \\
\hline AA60 & 12,60 & 42,00 & 30,00 & 12,00 & 13,20 & 49,60 & 47,00 & 2,60 & $-0,60$ & $-5,00$ & $-19,60$ \\
\hline AA64 & 11,20 & 39,40 & 36,40 & 3,00 & 12,60 & 38,60 & 40,20 & $-1,60$ & $-1,40$ & $-0,80$ & $-2,20$ \\
\hline AA65 & 11,20 & 40,40 & 0,00 & 40,40 & 12,00 & 47,40 & 0,00 & 47,40 & $-0,80$ & 40,40 & $-47,40$ \\
\hline
\end{tabular}

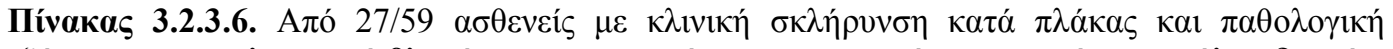

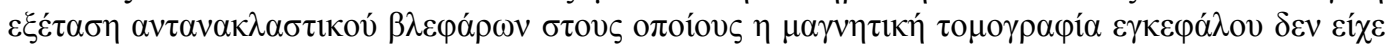

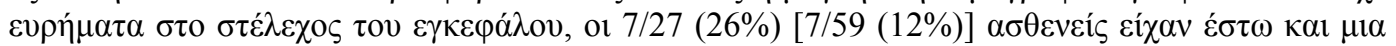

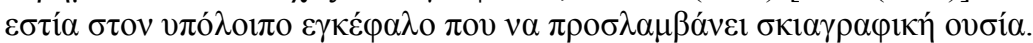

\begin{tabular}{|c|c|c|c|c|c|c|c|c|c|c|c|}
\hline $\begin{array}{r}\mathbf{A} / \mathbf{A} \\
\mathbf{K} \rho \iota \tau .\end{array}$ & $\begin{array}{c}\text { LR1 } \\
<13 \mathrm{~ms}\end{array}$ & $\begin{array}{l}\text { L LR2 } \\
<41 \mathrm{~ms}\end{array}$ & $\begin{array}{l}\text { L RR2 } \\
<44 \mathrm{~ms}\end{array}$ & $\begin{array}{c}\text { LLR2-LRR2 } \\
<[5] \mathrm{ms}\end{array}$ & $\begin{array}{c}\text { RR1 } \\
<13 \mathrm{~ms}\end{array}$ & $\begin{array}{l}\text { R RR2 } \\
<41 \mathrm{~ms}\end{array}$ & $\begin{array}{l}\text { R LR2 } \\
<44 \mathrm{~ms}\end{array}$ & $\begin{array}{c}\text { RRR2-RLR2 } \\
<[5] \mathrm{ms}\end{array}$ & $\begin{array}{c}\text { LR1-RR1 } \\
<[1.2] \mathrm{ms}\end{array}$ & $\begin{array}{c}\text { LLR2-RLR2 } \\
<[7] \mathrm{ms}\end{array}$ & $\begin{array}{c}\text { LRR2-RRR2 } \\
<[7] \mathrm{ms}\end{array}$ \\
\hline AA14 & 11,60 & 29,40 & 0,00 & 29,40 & 12,20 & 31,80 & 30,40 & 1,40 & $-0,60$ & $-1,00$ & $-31,80$ \\
\hline AA20 & 11,00 & 45,60 & 0,00 & 45,60 & 9,80 & 38,00 & 49,60 & $-11,60$ & 1,20 & $-4,00$ & $-38,00$ \\
\hline AA27 & 12,40 & 30,40 & 37,80 & $-7,40$ & 13,20 & 34,00 & 39,00 & $-5,00$ & $-0,80$ & $-8,60$ & 3,80 \\
\hline AA30 & 11,40 & 30,00 & 36,60 & $-6,60$ & 11,20 & 33,80 & 35,60 & $-1,80$ & 0,20 & $-5,60$ & 2,80 \\
\hline AA54 & 12,80 & 34,40 & 32,60 & 1,80 & 9,80 & 30,80 & 30,40 & 0,40 & 3,00 & 4,00 & 1,80 \\
\hline AA57 & 10,80 & 38,20 & 36,60 & 1,60 & 12,40 & 34,80 & 30,80 & 4,00 & $-1,60$ & 7,40 & 1,80 \\
\hline AA63 & 10,40 & 33,20 & 27,60 & 5,60 & 12,20 & 31,80 & 33,40 & $-1,60$ & $-1,80$ & $-0,20$ & $-4,20$ \\
\hline
\end{tabular}




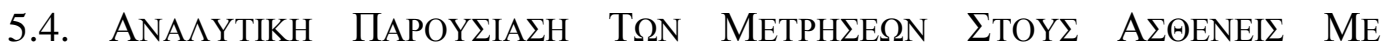

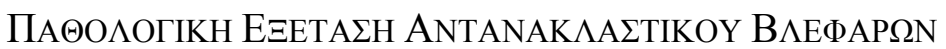

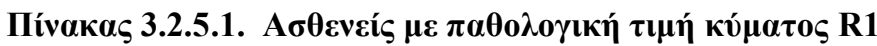

\begin{tabular}{|c|c|c|c|c|c|c|c|c|c|c|c|}
\hline $\begin{array}{c}\mathbf{A} / \mathbf{A} \\
\mathbf{K} \rho \mathbf{t} .\end{array}$ & $\begin{array}{c}\begin{array}{c}\text { LR1 } \\
<13 \mathrm{~ms}\end{array} \\
\end{array}$ & $\begin{array}{l}\text { L LR2 } \\
<41 \mathrm{~ms}\end{array}$ & $\begin{array}{l}\text { L RR2 } \\
<44 \mathrm{~ms}\end{array}$ & $\begin{array}{c}\text { LLR2-LRR2 } \\
<[5] \mathrm{ms}\end{array}$ & $\begin{array}{c}\text { RR1 } \\
<13 \mathrm{~ms}\end{array}$ & $\begin{array}{l}\text { R RR2 } \\
<41 \mathrm{~ms}\end{array}$ & $\begin{array}{l}\text { R LR2 } \\
<44 \mathrm{~ms}\end{array}$ & $\begin{array}{c}\text { RRR2-RLR2 } \\
<[5] \mathrm{ms}\end{array}$ & $\begin{array}{l}\text { LR1-RR1 } \\
<[1.2] \mathrm{ms}\end{array}$ & $\begin{array}{c}\text { LLR2-RLR2 } \\
<[7] \mathrm{ms}\end{array}$ & $\begin{array}{c}\text { LRR2-RRR2 } \\
<[7] \mathrm{ms}\end{array}$ \\
\hline $\mathrm{AA} 01$ & 12,60 & 34,40 & 29,80 & 4,60 & 13,00 & 27,20 & 29,20 & $-2,00$ & $-0,40$ & 5,20 & 2,60 \\
\hline $\mathrm{AA} 02$ & 13,00 & 30,80 & 34,60 & $-3,80$ & 11,60 & 43,20 & 42,60 & 0,60 & 1,40 & $-11,80$ & $-8,60$ \\
\hline AA04 & 14,20 & 30,00 & 26,60 & 3,40 & 13,40 & 38,60 & 0,00 & 38,60 & 0,80 & 30,00 & $-12,00$ \\
\hline AA06 & 13,00 & 41,00 & 0,00 & 41,00 & 13,00 & 64,00 & 48,00 & 16,00 & 0,00 & $-7,00$ & $-64,00$ \\
\hline AA07 & 13,80 & 26,20 & 24,20 & 2,00 & 14,00 & 25,20 & 33,20 & $-8,00$ & $-0,20$ & $-7,00$ & $-1,00$ \\
\hline $\mathrm{AA} 12$ & 13,40 & 33,60 & 36,60 & $-3,00$ & 12,60 & 43,60 & 38,60 & 5,00 & 0,80 & $-5,00$ & $-7,00$ \\
\hline AA13 & 12,40 & 31,00 & 0,00 & 31,00 & 13,00 & 41,00 & 51,60 & $-10,60$ & $-0,60$ & $-20,60$ & $-41,00$ \\
\hline AA15 & 13,00 & 47,00 & 31,60 & 15,40 & 12,20 & 30,80 & 0,00 & 30,80 & 0,80 & 47,00 & 0,80 \\
\hline AA18 & 13,80 & 45,00 & 41,60 & 3,40 & 13,80 & 55,00 & 0,00 & 55,00 & 0,00 & 45,00 & $-13,40$ \\
\hline AA19 & 14,00 & 45,80 & 0,00 & 45,80 & 13,40 & 43,80 & 45,40 & $-1,60$ & 0,60 & 0,40 & $-43,80$ \\
\hline $\mathrm{AA} 22$ & 13,40 & 33,60 & 34,60 & $-1,00$ & 13,20 & 32,20 & 36,20 & $-4,00$ & 0,20 & $-2,60$ & 2,40 \\
\hline AA23 & 13,40 & 38,00 & 0,00 & 38,00 & 13,60 & 34,00 & 30,00 & 4,00 & $-0,20$ & 8,00 & $-34,00$ \\
\hline AA25 & 13,60 & 0,00 & 0,00 & - & 11,40 & 36,80 & 49,90 & $-13,10$ & 2,20 & $-49,90$ & $-36,80$ \\
\hline AA26 & 11,00 & 30,00 & 31,60 & $-1,60$ & 13,80 & 59,60 & 63,20 & $-3,60$ & $-2,80$ & $-33,20$ & $-28,00$ \\
\hline AA27 & 12,40 & 30,40 & 37,80 & $-7,40$ & 13,20 & 34,00 & 39,00 & $-5,00$ & $-0,80$ & $-8,60$ & 3,80 \\
\hline AA28 & 13,40 & 40,20 & 39,60 & 0,60 & 12,80 & 30,60 & 35,60 & $-5,00$ & 0,60 & 4,60 & 9,00 \\
\hline AA34 & 13,00 & 33,20 & 37,60 & $-4,40$ & 12,20 & 37,20 & 31,20 & 6,00 & 0,80 & 2,00 & 0,40 \\
\hline AA40 & 13,40 & 38,40 & 0,00 & 38,40 & 13,20 & 0,00 & 0,00 & - & 0,20 & 38,40 & - \\
\hline AA48 & 12,00 & 34,80 & 39,80 & $-5,00$ & 13,40 & 33,40 & 35,40 & $-2,00$ & $-1,40$ & $-0,60$ & 6,40 \\
\hline AA49 & 12,80 & 24,00 & 29,20 & $-5,20$ & 13,00 & 36,80 & 0,00 & 36,80 & $-0,20$ & 24,00 & $-7,60$ \\
\hline AA53 & 12,00 & 0,00 & 0,00 & - & 13,80 & 0,00 & 0,00 & - & $-1,80$ & - & - \\
\hline AA55 & 13,80 & 40,00 & 43,40 & $-3,40$ & 12,60 & 35,60 & 31,60 & 4,00 & 1,20 & 8,40 & 7,80 \\
\hline AA58 & 14,00 & 0,00 & 0,00 & - & 14,00 & 0,00 & 0,00 & - & 0,00 & - & - \\
\hline AA59 & 11,40 & 33,80 & 34,60 & $-0,80$ & 13,20 & 29,60 & 28,80 & 0,80 & $-1,80$ & 5,00 & 5,00 \\
\hline AA60 & 12,60 & 42,00 & 30,00 & 12,00 & 13,20 & 49,60 & 47,00 & 2,60 & $-0,60$ & $-5,00$ & $-19,60$ \\
\hline
\end{tabular}

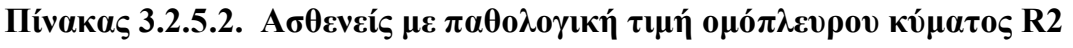

\begin{tabular}{|c|c|c|c|c|c|c|c|c|c|c|c|}
\hline $\begin{array}{c}\mathbf{A} / \mathbf{A} \\
\mathbf{K} \rho เ \tau .\end{array}$ & $\begin{array}{c}\text { LR1 } \\
<13 \mathrm{~ms}\end{array}$ & $\begin{array}{l}\text { L LR2 } \\
<41 \mathrm{~ms}\end{array}$ & $\begin{array}{l}\text { L RR2 } \\
<44 \mathrm{~ms}\end{array}$ & $\begin{array}{c}\text { LLR2-LRR2 } \\
<[5] \mathrm{ms}\end{array}$ & $\mid \begin{array}{c}\text { RR1 } \\
<13 \mathrm{~ms}\end{array}$ & $\begin{array}{l}\text { R RR2 } \\
<41 \mathrm{~ms}\end{array}$ & $\begin{array}{l}\text { R LR2 } \\
<44 \mathrm{~ms}\end{array}$ & $\begin{array}{c}\text { RRR2-RLR2 } \\
<[5] \mathrm{ms}\end{array}$ & $\begin{array}{l}\text { LR1-RR1 } \\
<[1.2] \mathrm{ms}\end{array}$ & $\begin{array}{l}\text { LLR2-RLR2 } \\
\quad<[7] \mathrm{ms}\end{array}$ & $\begin{array}{c}\text { LRR2-RRR2 } \\
<[7] \mathrm{ms}\end{array}$ \\
\hline $\mathrm{AA} 02$ & 13,00 & 30,80 & 34,60 & $-3,80$ & 11,60 & 43,20 & 42,60 & 0,60 & 1,40 & $-11,80$ & $-8,60$ \\
\hline $\mathrm{AA} 03$ & 12,20 & 27,40 & 60,40 & $-33,00$ & 10,00 & 0,00 & 23,80 & $-23,80$ & 2,20 & 3,60 & 60,40 \\
\hline AA06 & 13,00 & 41,00 & 0,00 & 41,00 & 13,00 & 64,00 & 48,00 & 16,00 & 0,00 & $-7,00$ & $-64,00$ \\
\hline AA09 & 10,60 & 0,00 & 0,00 & - & 11,20 & 0,00 & 0,00 & - & $-0,60$ & - & - \\
\hline AA10 & 12,40 & 44,20 & 0,00 & 44,20 & 10,80 & 40,60 & 46,60 & $-6,00$ & 1,60 & $-2,40$ & $-40,60$ \\
\hline $\mathrm{AA} 12$ & 13,40 & 33,60 & 36,60 & $-3,00$ & 12,60 & 43,60 & 38,60 & 5,00 & 0,80 & $-5,00$ & $-7,00$ \\
\hline $\mathrm{AA} 13$ & 12,40 & 31,00 & 0,00 & 31,00 & 13,00 & 41,00 & 51,60 & $-10,60$ & $-0,60$ & $-20,60$ & $-41,00$ \\
\hline AA15 & 13,00 & 47,00 & 31,60 & 15,40 & 12,20 & 30,80 & 0,00 & 30,80 & 0,80 & 47,00 & 0,80 \\
\hline AA18 & 13,80 & 45,00 & 41,60 & 3,40 & 13,80 & 55,00 & 0,00 & 55,00 & 0,00 & 45,00 & $-13,40$ \\
\hline AA19 & 14,00 & 45,80 & 0,00 & 45,80 & 13,40 & 43,80 & 45,40 & $-1,60$ & 0,60 & 0,40 & $-43,80$ \\
\hline $\mathrm{AA} 20$ & 11,00 & 45,60 & 0,00 & 45,60 & 9,80 & 38,00 & 49,60 & $-11,60$ & 1,20 & $-4,00$ & $-38,00$ \\
\hline AA21 & 12,20 & 0,00 & 28,60 & $-28,60$ & 12,00 & 41,00 & 0,00 & 41,00 & 0,20 & - & $-12,40$ \\
\hline AA23 & 13,40 & 38,00 & 0,00 & 38,00 & 13,60 & 34,00 & 30,00 & 4,00 & $-0,20$ & 8,00 & $-34,00$ \\
\hline AA25 & 13,60 & 0,00 & 0,00 & - & 11,40 & 36,80 & 49,90 & $-13,10$ & 2,20 & $-49,90$ & $-36,80$ \\
\hline AA26 & 11,00 & 30,00 & 31,60 & $-1,60$ & 13,80 & 59,60 & 63,20 & $-3,60$ & $-2,80$ & $-33,20$ & $-28,00$ \\
\hline AA29 & 12,20 & 43,00 & 58,20 & $-15,20$ & 12,40 & 40,20 & 55,80 & $-15,60$ & $-0,20$ & $-12,80$ & 18,00 \\
\hline AA35 & 12,80 & 0,00 & 0,00 & - & 11,00 & 46,40 & 53,80 & $-7,40$ & 1,80 & $-53,80$ & $-46,40$ \\
\hline AA38 & 12,60 & 0,00 & 71,20 & $-71,20$ & 9,80 & 39,80 & 0,00 & 39,80 & 2,80 & - & 31,40 \\
\hline AA39 & 12,20 & 43,40 & 0,00 & 43,40 & 12,40 & 41,20 & $\mathbf{0 , 0 0}$ & 41,20 & $-0,20$ & 43,40 & $-41,20$ \\
\hline AA40 & 13,40 & 38,40 & 0,00 & 38,40 & 13,20 & 0,00 & $\mathbf{0 , 0 0}$ & - & 0,20 & 38,40 & - \\
\hline AA43 & 11,80 & 29,60 & $\mathbf{0 , 0 0}$ & 29,60 & 12,00 & 31,80 & 37,00 & $-5,20$ & $-0,20$ & $-7,40$ & $-31,80$ \\
\hline AA44 & 12,40 & 50,00 & 41,20 & 8,80 & 12,00 & 48,20 & 40,60 & 7,60 & 0,40 & 9,40 & $-7,00$ \\
\hline AA46 & 12,00 & 48,40 & 62,80 & $-14,40$ & 12,20 & 46,40 & 41,00 & 5,40 & $-0,20$ & 7,40 & 16,40 \\
\hline AA49 & 12,80 & 24,00 & 29,20 & $-5,20$ & 13,00 & 36,80 & 0,00 & 36,80 & $-0,20$ & 24,00 & $-7,60$ \\
\hline AA53 & 12,00 & 0,00 & 0,00 & - & 13,80 & 0,00 & 0,00 & - & $-1,80$ & - & - \\
\hline AA56 & 13,00 & 39,80 & 48,00 & $-8,20$ & 12,80 & 38,80 & 39,60 & $-0,80$ & 0,20 & 0,20 & 9,20 \\
\hline AA58 & 14,00 & 0,00 & 0,00 & - & 14,00 & 0,00 & 0,00 & - & 0,00 & - & - \\
\hline AA60 & 12,60 & 42,00 & 30,00 & 12,00 & 13,20 & 49,60 & 47,00 & 2,60 & $-0,60$ & $-5,00$ & $-19,60$ \\
\hline AA61 & 12,60 & 39,60 & 44,60 & $-5,00$ & 10,80 & 31,00 & 28,80 & 2,20 & 1,80 & 10,80 & 13,60 \\
\hline AA65 & 11,20 & 40,40 & 0,00 & 40,40 & 12,00 & 47,40 & 0,00 & 47,40 & $-0,80$ & 40,40 & $-47,40$ \\
\hline
\end{tabular}




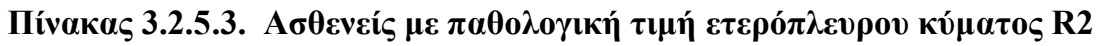

\begin{tabular}{|c|c|c|c|c|c|c|c|c|c|c|c|}
\hline $\begin{array}{r}\mathbf{A} / \mathbf{A} \\
\mathbf{K} \boldsymbol{\rho} \iota \tau .\end{array}$ & $\begin{array}{c}\text { LR1 } \\
<13 \mathrm{~ms}\end{array}$ & $\begin{array}{l}\text { L LR2 } \\
<41 \mathrm{~ms}\end{array}$ & $\begin{array}{l}\text { L RR2 } \\
<44 \mathrm{~ms}\end{array}$ & $\begin{array}{c}\text { LLR2-LRR2 } \\
<[5] \mathrm{ms}\end{array}$ & $\begin{array}{c}\text { RR1 } \\
<13 \mathrm{~ms}\end{array}$ & $\begin{array}{l}\text { R RR2 } \\
<41 \mathrm{~ms}\end{array}$ & $\begin{array}{l}\text { R LR2 } \\
<44 \mathrm{~ms}\end{array}$ & $\begin{array}{c}\text { RRR2-RLR2 } \\
<[5] \mathrm{ms}\end{array}$ & $\begin{array}{l}\text { LR1-RR1 } \\
<[1.2] \mathrm{ms}\end{array}$ & $\begin{array}{c}\text { LLR2-RLR2 } \\
<[7] \mathrm{ms}\end{array}$ & $\begin{array}{c}\text { LRR2-RRR } \\
<[7] \mathrm{ms}\end{array}$ \\
\hline $\mathrm{AA} 03$ & 12,20 & 27,40 & 60,40 & $-33,00$ & 10,00 & 0,00 & 23,80 & $-23,80$ & 2,20 & 3,60 & 60,40 \\
\hline AA04 & 14,20 & 30,00 & 26,60 & 3,40 & 13,40 & 38,60 & 0,00 & 38,60 & 0,80 & 30,00 & $-12,00$ \\
\hline AA06 & 13,00 & 41,00 & 0,00 & 41,00 & 13,00 & 64,00 & 48,00 & 16,00 & 0,00 & $-7,00$ & $-64,00$ \\
\hline AA09 & 10,60 & 0,00 & 0,00 & - & 11,20 & 0,00 & 0,00 & - & $-0,60$ & - & - \\
\hline AA10 & 12,40 & 44,20 & 0,00 & 44,20 & 10,80 & 40,60 & 46,60 & $-6,00$ & 1,60 & $-2,40$ & $-40,60$ \\
\hline AA11 & 11,20 & 34,60 & 29,00 & 5,60 & 11,40 & 32,00 & 49,00 & $-17,00$ & $-0,20$ & $-14,40$ & $-3,00$ \\
\hline AA13 & 12,40 & 31,00 & 0,00 & 31,00 & 13,00 & 41,00 & 51,60 & $-10,60$ & $-0,60$ & $-20,60$ & $-41,00$ \\
\hline AA14 & 11,60 & 29,40 & 0,00 & 29,40 & 12,20 & 31,80 & 30,40 & 1,40 & $-0,60$ & $-1,00$ & $-31,80$ \\
\hline AA15 & 13,00 & 47,00 & 31,60 & 15,40 & 12,20 & 30,80 & 0,00 & 30,80 & 0,80 & 47,00 & 0,80 \\
\hline AA16 & 11,40 & 36,20 & 30,00 & 6,20 & 11,80 & 38,60 & 0,00 & 38,60 & $-0,40$ & 36,20 & $-8,60$ \\
\hline AA18 & 13,80 & 45,00 & 41,60 & 3,40 & 13,80 & 55,00 & 0,00 & 55,00 & 0,00 & 45,00 & $-13,40$ \\
\hline AA19 & 14,00 & 45,80 & 0,00 & 45,80 & 13,40 & 43,80 & 45,40 & $-1,60$ & 0,60 & 0,40 & $-43,80$ \\
\hline AA20 & 11,00 & 45,60 & 0,00 & 45,60 & 9,80 & 38,00 & 49,60 & $-11,60$ & 1,20 & $-4,00$ & $-38,00$ \\
\hline $\mathrm{AA} 21$ & 12,20 & 0,00 & 28,60 & $-28,60$ & 12,00 & 41,00 & 0,00 & 41,00 & 0,20 & - & $-12,40$ \\
\hline AA23 & 13,40 & 38,00 & 0,00 & 38,00 & 13,60 & 34,00 & 30,00 & 4,00 & $-0,20$ & 8,00 & $-34,00$ \\
\hline AA25 & 13,60 & 0,00 & 0,00 & - & 11,40 & 36,80 & 49,90 & $-13,10$ & 2,20 & $-49,90$ & $-36,80$ \\
\hline AA26 & 11,00 & 30,00 & 31,60 & $-1,60$ & 13,80 & 59,60 & 63,20 & $-3,60$ & $-2,80$ & $-33,20$ & $-28,00$ \\
\hline AA29 & 12,20 & 43,00 & 58,20 & $-15,20$ & 12,40 & 40,20 & 55,80 & $-15,60$ & $-0,20$ & $-12,80$ & 18,00 \\
\hline AA 32 & 12,00 & 37,20 & 38,20 & $-1,00$ & 12,80 & 36,80 & 48,20 & $-11,40$ & $-0,80$ & $-11,00$ & 1,40 \\
\hline AA35 & 12,80 & 0,00 & 0,00 & - & 11,00 & 46,40 & 53,80 & $-7,40$ & 1,80 & $-53,80$ & $-46,40$ \\
\hline AA38 & 12,60 & 0,00 & 71,20 & $-71,20$ & 9,80 & 39,80 & 0,00 & 39,80 & 2,80 & - & 31,40 \\
\hline AA39 & 12,20 & 43,40 & 0,00 & 43,40 & 12,40 & 41,20 & 0,00 & 41,20 & $-0,20$ & 43,40 & $-41,20$ \\
\hline AA40 & 13,40 & 38,40 & 0,00 & 38,40 & 13,20 & 0,00 & 0,00 & - & 0,20 & 38,40 & - \\
\hline AA43 & 11,80 & 29,60 & 0,00 & 29,60 & 12,00 & 31,80 & 37,00 & $-5,20$ & $-0,20$ & $-7,40$ & $-31,80$ \\
\hline AA46 & 12,00 & 48,40 & 62,80 & $-14,40$ & 12,20 & 46,40 & 41,00 & 5,40 & $-0,20$ & 7,40 & 16,40 \\
\hline AA49 & 12,80 & 24,00 & 29,20 & $-5,20$ & 13,00 & 36,80 & 0,00 & 36,80 & $-0,20$ & 24,00 & $-7,60$ \\
\hline AA53 & 12,00 & 0,00 & 0,00 & - & 13,80 & 0,00 & 0,00 & - & $-1,80$ & - & - \\
\hline AA56 & 13,00 & 39,80 & 48,00 & $-8,20$ & 12,80 & 38,80 & 39,60 & $-0,80$ & 0,20 & 0,20 & 9,20 \\
\hline AA58 & 14,00 & 0,00 & 0,00 & - & 14,00 & $\mathbf{0 , 0 0}$ & 0,00 & - & $\mathbf{0 , 0 0}$ & - & - \\
\hline AA60 & 12,60 & 42,00 & 30,00 & 12,00 & 13,20 & 49,60 & 47,00 & 2,60 & $-0,60$ & $-5,00$ & $-19,60$ \\
\hline AA61 & 12,60 & 39,60 & 44,60 & $-5,00$ & 10,80 & 31,00 & 28,80 & 2,20 & 1,80 & 10,80 & 13,60 \\
\hline AA62 & 12,80 & 40,40 & 39,40 & 1,00 & 12,00 & 39,80 & 63,60 & $-23,80$ & 0,80 & $-23,20$ & $-0,40$ \\
\hline AA65 & 11,20 & 40,40 & 0,00 & 40,40 & 12,00 & 47,40 & 0,00 & 47,40 & $-0,80$ & 40,40 & $-47,40$ \\
\hline
\end{tabular}

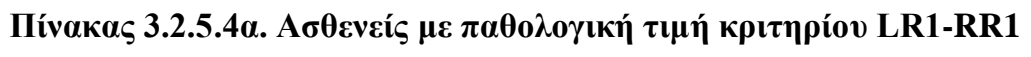

\begin{tabular}{|c|c|c|c|c|c|c|c|c|c|c|c|}
\hline 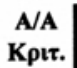 & $\begin{array}{c}\text { LR1 } \\
<13 \mathrm{~ms}\end{array}$ & $\begin{array}{l}\text { L LR2 } \\
<41 \mathrm{~ms}\end{array}$ & $\begin{array}{l}\text { L RR2 } \\
<44 \mathrm{~ms}\end{array}$ & $\begin{array}{c}\text { LLR2-LRR2 } \\
<[5] \mathrm{ms}\end{array}$ & $\begin{array}{c}\mathbf{R R 1} \\
<13 \mathrm{~ms}\end{array}$ & $\begin{array}{l}\text { R RR2 } \\
<41 \mathrm{~ms}\end{array}$ & $\begin{array}{l}\text { R LR2 } \\
<44 \mathrm{~ms}\end{array}$ & $\begin{array}{c}\text { RRR2-RLR2 } \\
<[5] \mathrm{ms}\end{array}$ & $\begin{array}{l}\text { LR1-RR1 } \\
<[1.2] \mathrm{ms}\end{array}$ & $\begin{array}{c}\text { LLR2-RLR2 } \\
<[7] \mathrm{ms}\end{array}$ & $\begin{array}{c}\text { LRR2-RRR2 } \\
<[7] \mathrm{ms}\end{array}$ \\
\hline $\mathrm{AA} 02$ & 13,00 & 30,80 & 34,60 & $-3,80$ & 11,60 & 43,20 & 42,60 & 0,60 & 1,40 & $-11,80$ & $-8,60$ \\
\hline $\mathrm{AA} 03$ & 12,20 & 27,40 & 60,40 & $-33,00$ & 10,00 & 0,00 & 23,80 & $-23,80$ & 2,20 & 3,60 & 60,40 \\
\hline AA10 & 12,40 & 44,20 & 0,00 & 44,20 & 10,80 & 40,60 & 46,60 & $-6,00$ & 1,60 & $-2,40$ & $-40,60$ \\
\hline $\mathrm{AA} 17$ & 11,40 & 37,00 & 32,00 & 5,00 & 9,20 & 33,20 & 37,60 & $-4,40$ & 2,20 & $-0,60$ & $-1,20$ \\
\hline $\mathrm{AA} 20$ & 11,00 & 45,60 & 0,00 & 45,60 & 9,80 & 38,00 & 49,60 & $-11,60$ & 1,20 & $-4,00$ & $-38,00$ \\
\hline AA25 & 13,60 & 0,00 & 0,00 & 0,00 & 11,40 & 36,80 & 49,90 & $-13,10$ & 2,20 & $-49,90$ & $-36,80$ \\
\hline AA26 & 11,00 & 30,00 & 31,60 & $-1,60$ & 13,80 & 59,60 & 63,20 & $-3,60$ & $-2,80$ & $-33,20$ & $-28,00$ \\
\hline $\mathrm{AA} 33$ & 12,00 & 31,20 & 33,20 & $-2,00$ & 10,20 & 33,80 & 33,20 & 0,60 & 1,80 & $-2,00$ & $-0,60$ \\
\hline AA35 & 12,80 & 0,00 & 0,00 & 0,00 & 11,00 & 46,40 & 53,80 & $-7,40$ & 1,80 & $-53,80$ & $-46,40$ \\
\hline AA38 & 12,60 & 0,00 & 71,20 & $-71,20$ & 9,80 & 39,80 & 0,00 & 39,80 & 2,80 & 0,00 & 31,40 \\
\hline AA48 & 12,00 & 34,80 & 39,80 & $-5,00$ & 13,40 & 33,40 & 35,40 & $-2,00$ & $-1,40$ & $-0,60$ & 6,40 \\
\hline AA53 & 12,00 & 0,00 & 0,00 & 0,00 & 13,80 & 0,00 & 0,00 & 0,00 & $-1,80$ & 0,00 & 0,00 \\
\hline AA54 & 12,80 & 34,40 & 32,60 & 1,80 & 9,80 & 30,80 & 30,40 & 0,40 & 3,00 & 4,00 & 1,80 \\
\hline AA55 & 13,80 & 40,00 & 43,40 & $-3,40$ & 12,60 & 35,60 & 31,60 & 4,00 & 1,20 & 8,40 & 7,80 \\
\hline AA57 & 10,80 & 38,20 & 36,60 & 1,60 & 12,40 & 34,80 & 30,80 & 4,00 & $-1,60$ & 7,40 & 1,80 \\
\hline AA59 & 11,40 & 33,80 & 34,60 & $-0,80$ & 13,20 & 29,60 & 28,80 & 0,80 & $-1,80$ & 5,00 & 5,00 \\
\hline AA61 & 12,60 & 39,60 & 44,60 & $-5,00$ & 10,80 & 31,00 & 28,80 & 2,20 & 1,80 & 10,80 & 13,60 \\
\hline AA63 & 10,40 & 33,20 & 27,60 & 5,60 & 12,20 & 31,80 & 33,40 & $-1,60$ & $-1,80$ & $-0,20$ & $-4,20$ \\
\hline AA64 & 11,20 & 39,40 & 36,40 & 3,00 & 12,60 & 38,60 & 40,20 & $-1,60$ & $-1,40$ & $-0,80$ & $-2,20$ \\
\hline
\end{tabular}

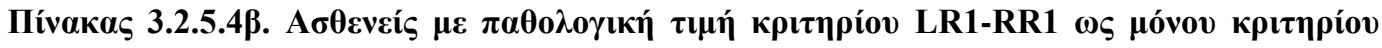

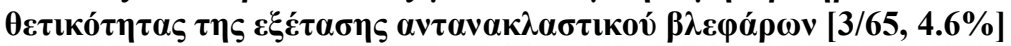

\begin{tabular}{|c|c|c|c|c|c|c|c|c|c|c|c|}
\hline $\begin{array}{c}\mathbf{A} / \mathbf{A} \\
\mathbf{K} \rho \mathbf{\tau} .\end{array}$ & $\mid \begin{array}{c}\text { LR1 } \\
<13 \mathrm{~ms}\end{array}$ & $\begin{array}{l}\text { L LR2 } \\
<41 \mathrm{~ms}\end{array}$ & $\begin{array}{l}\text { L RR2 } \\
<44 \mathrm{~ms}\end{array}$ & $\begin{array}{c}\text { LLR2-LRR2 } \\
<[5] \mathrm{msec}\end{array}$ & $\mid \begin{array}{c}\text { RR1 } \\
<13 \mathrm{~ms}\end{array}$ & $\begin{array}{l}\text { R RR2 } \\
<41 \mathrm{~ms}\end{array}$ & $\begin{array}{l}\text { R LR2 } \\
<44 \mathrm{~ms}\end{array}$ & $\begin{array}{c}\text { RRR2-RLR2 } \\
<[5] \mathrm{msec}\end{array}$ & \begin{tabular}{|l} 
LR1-RR1 \\
$<[1.2] \mathrm{ms}$
\end{tabular} & $\begin{array}{c}\text { LLR2-RLR2 } \\
<[7] \mathrm{ms}\end{array}$ & $\begin{array}{c}\text { LRR2-RRR } \\
\quad<[7] \mathrm{ms}\end{array}$ \\
\hline A33 & 12,00 & 31,20 & 33,20 & $-2,00$ & 10,20 & 33,80 & 33,20 & 0,60 & 1,80 & $-2,00$ & $-0,60$ \\
\hline A54 & 12,80 & 34,40 & 32,60 & 1,80 & 9,80 & 30,80 & 30,40 & 0,40 & 3,00 & 4,00 & 1,80 \\
\hline AA6 & 11,20 & 39,40 & 36,40 & 3,00 & 12,60 & 38,60 & 40,20 & $-1,60$ & $-1,40$ & $-0,80$ & $-2,20$ \\
\hline
\end{tabular}




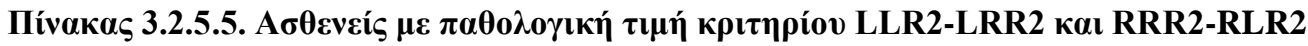

\begin{tabular}{|c|c|c|c|c|c|c|c|c|c|c|c|}
\hline $\begin{array}{c}\mathbf{A} / \mathbf{A} \\
\mathbf{K} \rho \iota \tau\end{array}$ & $\begin{array}{c}\text { LR1 } \\
<13 \mathrm{~ms}\end{array}$ & $\begin{array}{l}\text { L LR2 } \\
<41 \mathrm{~ms}\end{array}$ & $\begin{array}{l}\text { L RR2 } \\
<44 \mathrm{~ms}\end{array}$ & $\begin{array}{c}\text { LLR2-LRR2 } \\
<[5] \mathrm{ms}\end{array}$ & $\begin{array}{c}\text { RR1 } \\
<13 \mathrm{~ms}\end{array}$ & $\begin{array}{l}\text { R RR2 } \\
<41 \mathrm{~ms}\end{array}$ & $\begin{array}{l}\text { R LR2 } \\
<44 \mathrm{~ms}\end{array}$ & $\begin{array}{c}\text { RRR2-RLR2 } \\
<\text { [5] ms }\end{array}$ & $\begin{array}{l}\text { LR1-RR1 } \\
<[1.2] \mathrm{ms}\end{array}$ & $\begin{array}{c}\text { LLR2-RLR2 } \\
<[7] \mathrm{ms}\end{array}$ & $\begin{array}{c}\text { LRR2-RRR2 } \\
\quad<[7] \mathrm{ms}\end{array}$ \\
\hline $\mathrm{AA} 03$ & 12,20 & 27,40 & 60,40 & $-33,00$ & 10,00 & 0,00 & 23,80 & $-23,80$ & 2,20 & 3,60 & 60,40 \\
\hline $\mathrm{AA} 04$ & 14,20 & 30,00 & 26,60 & 3,40 & 13,40 & 38,60 & 0,00 & 38,60 & 0,80 & 30,00 & $-12,00$ \\
\hline AA06 & 13,00 & 41,00 & 0,00 & 41,00 & 13,00 & 64,00 & 48,00 & 16,00 & 0,00 & $-7,00$ & $-64,00$ \\
\hline AA07 & 13,80 & 26,20 & 24,20 & 2,00 & 14,00 & 25,20 & 33,20 & $-8,00$ & $-0,20$ & $-7,00$ & $-1,00$ \\
\hline AA08 & 11,60 & 29,40 & 31,40 & $-2,00$ & 12,00 & 39,80 & 28,80 & 11,00 & $-0,40$ & 0,60 & $-8,40$ \\
\hline AA09 & 10,60 & 0,00 & 0,00 & - & 11,20 & 0,00 & 0,00 & - & $-0,60$ & - & - \\
\hline AA 10 & 12,40 & 44,20 & 0,00 & 44,20 & 10,80 & 40,60 & 46,60 & $-6,00$ & 1,60 & $-2,40$ & $-40,60$ \\
\hline AA11 & 11,20 & 34,60 & 29,00 & 5,60 & 11,40 & 32,00 & 49,00 & $-17,00$ & $-0,20$ & $-14,40$ & $-3,00$ \\
\hline AA12 & 13,40 & 33,60 & 36,60 & $-3,00$ & 12,60 & 43,60 & 38,60 & 5,00 & 0,80 & $-5,00$ & $-7,00$ \\
\hline AA13 & 12,40 & 31,00 & 0,00 & 31,00 & 13,00 & 41,00 & 51,60 & $-10,60$ & $-0,60$ & $-20,60$ & $-41,00$ \\
\hline AA14 & 11,60 & 29,40 & 0,00 & 29,40 & 12,20 & 31,80 & 30,40 & 1,40 & $-0,60$ & $-1,00$ & $-31,80$ \\
\hline AA15 & 13,00 & 47,00 & 31,60 & 15,40 & 12,20 & 30,80 & 0,00 & 30,80 & 0,80 & 47,00 & 0,80 \\
\hline AA16 & 11,40 & 36,20 & 30,00 & 6,20 & 11,80 & 38,60 & 0,00 & 38,60 & $-0,40$ & 36,20 & $-8,60$ \\
\hline AA17 & 11,40 & 37,00 & 32,00 & 5,00 & 9,20 & 33,20 & 37,60 & $-4,40$ & 2,20 & $-0,60$ & $-1,20$ \\
\hline AA 18 & 13,80 & 45,00 & 41,60 & 3,40 & 13,80 & 55,00 & 0,00 & 55,00 & 0,00 & 45,00 & $-13,40$ \\
\hline AA19 & 14,00 & 45,80 & 0,00 & 45,80 & 13,40 & 43,80 & 45,40 & $-1,60$ & 0,60 & 0,40 & $-43,80$ \\
\hline AA20 & 11,00 & 45,60 & 0,00 & 45,60 & 9,80 & 38,00 & 49,60 & $-11,60$ & 1,20 & $-4,00$ & $-38,00$ \\
\hline AA21 & 12,20 & 0,00 & 28,60 & $-28,60$ & 12,00 & 41,00 & 0,00 & 41,00 & 0,20 & - & $-12,40$ \\
\hline AA23 & 13,40 & 38,00 & 0,00 & 38,00 & 13,60 & 34,00 & 30,00 & 4,00 & $-0,20$ & 8,00 & $-34,00$ \\
\hline AA24 & 10,25 & 26,25 & 20,95 & 5,30 & 10,20 & 19,90 & 20,00 & $-0,10$ & 0,05 & 6,25 & 1,05 \\
\hline AA25 & 13,60 & 0,00 & 0,00 & - & 11,40 & 36,80 & 49,90 & $-13,10$ & 2,20 & $-49,90$ & $-36,80$ \\
\hline AA27 & 12,40 & 30,40 & 37,80 & $-7,40$ & 13,20 & 34,00 & 39,00 & $-5,00$ & $-0,80$ & $-8,60$ & 3,80 \\
\hline AA28 & 13,40 & 40,20 & 39,60 & 0,60 & 12,80 & 30,60 & 35,60 & $-5,00$ & 0,60 & 4,60 & 9,00 \\
\hline AA29 & 12,20 & 43,00 & 58,20 & $-15,20$ & 12,40 & 40,20 & 55,80 & $-15,60$ & $-0,20$ & $-12,80$ & 18,00 \\
\hline AA 30 & 11,40 & 30,00 & 36,60 & $-6,60$ & 11,20 & 33,80 & 35,60 & $-1,80$ & 0,20 & $-5,60$ & 2,80 \\
\hline AA32 & 12,00 & 37,20 & 38,20 & $-1,00$ & 12,80 & 36,80 & 48,20 & $-11,40$ & $-0,80$ & $-11,00$ & 1,40 \\
\hline AA34 & 13,00 & 33,20 & 37,60 & $-4,40$ & 12,20 & 37,20 & 31,20 & 6,00 & 0,80 & 2,00 & 0,40 \\
\hline AA35 & 12,80 & 0,00 & 0,00 & - & 11,00 & 46,40 & 53,80 & $-7,40$ & 1,80 & $-53,80$ & $-46,40$ \\
\hline AA38 & 12,60 & 0,00 & 71,20 & $-71,20$ & 9,80 & 39,80 & 0,00 & 39,80 & 2,80 & - & 31,40 \\
\hline AA39 & 12,20 & 43,40 & 0,00 & 43,40 & 12,40 & 41,20 & 0,00 & 41,20 & $-0,20$ & 43,40 & $-41,20$ \\
\hline AA40 & 13,40 & 38,40 & 0,00 & 38,40 & 13,20 & 0,00 & 0,00 & - & 0,20 & 38,40 & - \\
\hline AA41 & 12,20 & 31,40 & 39,40 & $-8,00$ & 12,40 & 38,00 & 35,40 & 2,60 & $-0,20$ & $-4,00$ & 1,40 \\
\hline AA43 & 11,80 & 29,60 & 0,00 & 29,60 & 12,00 & 31,80 & 37,00 & $-5,20$ & $-0,20$ & $-7,40$ & $-31,80$ \\
\hline AA44 & 12,40 & 50,00 & 41,20 & 8,80 & 12,00 & 48,20 & 40,60 & 7,60 & 0,40 & 9,40 & $-7,00$ \\
\hline AA46 & 12,00 & 48,40 & 62,80 & $-14,40$ & 12,20 & 46,40 & 41,00 & 5,40 & $-0,20$ & 7,40 & 16,40 \\
\hline AA47 & 11,40 & 36,80 & 38,40 & $-1,60$ & 11,80 & 38,40 & 30,20 & 8,20 & $-0,40$ & 6,60 & 0,00 \\
\hline AA48 & 12,00 & 34,80 & 39,80 & $-5,00$ & 13,40 & 33,40 & 35,40 & $-2,00$ & $-1,40$ & $-0,60$ & 6,40 \\
\hline AA49 & 12,80 & 24,00 & 29,20 & $-5,20$ & 13,00 & 36,80 & 0,00 & 36,80 & $-0,20$ & 24,00 & $-7,60$ \\
\hline AA50 & 11,20 & 37,00 & 28,20 & 8,80 & 11,60 & 30,60 & 43,40 & $-12,80$ & $-0,40$ & $-6,40$ & $-2,40$ \\
\hline AA52 & 12,40 & 28,80 & 39,80 & $-11,00$ & 12,60 & 32,20 & 30,40 & 1,80 & $-0,20$ & $-1,60$ & 7,60 \\
\hline AA53 & 12,00 & 0,00 & 0,00 & - & 13,80 & 0,00 & 0,00 & - & $-1,80$ & - & - \\
\hline AA56 & 13,00 & 39,80 & 48,00 & $-8,20$ & 12,80 & 38,80 & 39,60 & $-0,80$ & 0,20 & 0,20 & 9,20 \\
\hline AA58 & 14,00 & 0,00 & 0,00 & - & 14,00 & 0,00 & 0,00 & - & 0,00 & - & - \\
\hline AA60 & 12,60 & 42,00 & 30,00 & 12,00 & 13,20 & 49,60 & 47,00 & 2,60 & $-0,60$ & $-5,00$ & $-19,60$ \\
\hline AA61 & 12,60 & 39,60 & 44,60 & $-5,00$ & 10,80 & 31,00 & 28,80 & 2,20 & 1,80 & 10,80 & 13,60 \\
\hline AA62 & 12,80 & 40,40 & 39,40 & 1,00 & 12,00 & 39,80 & 63,60 & $-23,80$ & 0,80 & $-23,20$ & $-0,40$ \\
\hline AA63 & 10,40 & 33,20 & 27,60 & 5,60 & 12,20 & 31,80 & 33,40 & $-1,60$ & $-1,80$ & $-0,20$ & $-4,20$ \\
\hline AA65 & 11,20 & 40,40 & 0,00 & 40,40 & 12,00 & 47,40 & 0,00 & 47,40 & $-0,80$ & 40,40 & $-47,40$ \\
\hline
\end{tabular}




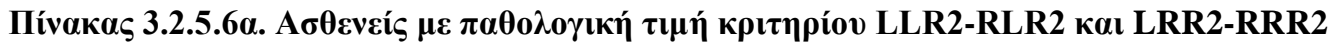

\begin{tabular}{|c|c|c|c|c|c|c|c|c|c|c|c|}
\hline $\begin{array}{c}\mathbf{A} / \mathbf{A} \\
\mathbf{K} \rho \iota \tau\end{array}$ & $\begin{array}{c}\text { LR1 } \\
<13 \mathrm{~ms}\end{array}$ & $\begin{array}{l}\text { L LR2 } \\
<41 \mathrm{~ms}\end{array}$ & $\begin{array}{l}\text { L RR2 } \\
<44 \mathrm{~ms}\end{array}$ & $\begin{array}{c}\text { LLR2-LRR2 } \\
\quad<[5] \mathrm{ms}\end{array}$ & $\begin{array}{c}\mathrm{RR1} \\
<13 \mathrm{~ms}\end{array}$ & $\begin{array}{l}\text { R RR2 } \\
<41 \mathrm{~ms}\end{array}$ & $\begin{array}{l}\text { R LR2 } \\
<44 \mathrm{~ms}\end{array}$ & $\begin{array}{c}\text { RRR2-RLR2 } \\
<[5] \mathrm{ms}\end{array}$ & $\begin{array}{l}\text { LR1-RR1 } \\
<|1.2| \mathrm{ms}\end{array}$ & $\begin{array}{c}\text { LLR2-RLR2 } \\
<[7] \mathrm{ms}\end{array}$ & $\begin{array}{c}\text { LRR2-RRR2 } \\
<[7] \mathrm{ms}\end{array}$ \\
\hline $\mathrm{AA} 02$ & 13,00 & 30,80 & 34,60 & $-3,80$ & 11,60 & 43,20 & 42,60 & 0,60 & 1,40 & $-11,80$ & $-8,60$ \\
\hline $\mathrm{AA} 03$ & 12,20 & 27,40 & 60,40 & $-33,00$ & 10,00 & 0,00 & 23,80 & $-23,80$ & 2,20 & 3,60 & 60,40 \\
\hline AA04 & 14,20 & 30,00 & 26,60 & 3,40 & 13,40 & 38,60 & 0,00 & 38,60 & 0,80 & 30,00 & $-12,00$ \\
\hline AA06 & 13,00 & 41,00 & 0,00 & 41,00 & 13,00 & 64,00 & 48,00 & 16,00 & 0,00 & $-7,00$ & $-64,00$ \\
\hline AA07 & 13,80 & 26,20 & 24,20 & 2,00 & 14,00 & 25,20 & 33,20 & $-8,00$ & $-0,20$ & $-7,00$ & $-1,00$ \\
\hline AA08 & 11,60 & 29,40 & 31,40 & $-2,00$ & 12,00 & 39,80 & 28,80 & 11,00 & $-0,40$ & 0,60 & $-8,40$ \\
\hline AA09 & 10,60 & 0,00 & 0,00 & - & 11,20 & 0,00 & 0,00 & - & $-0,60$ & - & - \\
\hline AA 10 & 12,40 & 44,20 & 0,00 & 44,20 & 10,80 & 40,60 & 46,60 & $-6,00$ & 1,60 & $-2,40$ & $-40,60$ \\
\hline AAl1 & 11,20 & 34,60 & 29,00 & 5,60 & 11,40 & 32,00 & 49,00 & $-17,00$ & $-0,20$ & $-14,40$ & $-3,00$ \\
\hline $\mathrm{AA} 12$ & 13,40 & 33,60 & 36,60 & $-3,00$ & 12,60 & 43,60 & 38,60 & 5,00 & 0,80 & $-5,00$ & $-7,00$ \\
\hline $\mathrm{AA} 13$ & 12,40 & 31,00 & 0,00 & 31,00 & 13,00 & 41,00 & 51,60 & $-10,60$ & $-0,60$ & $-20,60$ & $-41,00$ \\
\hline AA 14 & 11,60 & 29,40 & 0,00 & 29,40 & 12,20 & 31,80 & 30,40 & 1,40 & $-0,60$ & $-1,00$ & $-31,80$ \\
\hline AA 15 & 13,00 & 47,00 & 31,60 & 15,40 & 12,20 & 30,80 & 0,00 & 30,80 & 0,80 & 47,00 & 0,80 \\
\hline AA 16 & 11,40 & 36,20 & 30,00 & 6,20 & 11,80 & 38,60 & 0,00 & 38,60 & $-0,40$ & 36,20 & $-8,60$ \\
\hline AA 18 & 13,80 & 45,00 & 41,60 & 3,40 & 13,80 & 55,00 & 0,00 & 55,00 & 0,00 & 45,00 & $-13,40$ \\
\hline AA19 & 14,00 & 45,80 & 0,00 & 45,80 & 13,40 & 43,80 & 45,40 & $-1,60$ & 0,60 & 0,40 & $-43,80$ \\
\hline AA20 & 11,00 & 45,60 & 0,00 & 45,60 & 9,80 & 38,00 & 49,60 & $-11,60$ & 1,20 & $-4,00$ & $-38,00$ \\
\hline AA21 & 12,20 & 0,00 & 28,60 & $-28,60$ & 12,00 & 41,00 & 0,00 & 41,00 & 0,20 & - & $-12,40$ \\
\hline AA23 & 13,40 & 38,00 & 0,00 & 38,00 & 13,60 & 34,00 & 30,00 & 4,00 & $-0,20$ & 8,00 & $-34,00$ \\
\hline AA25 & 13,60 & 0,00 & 0,00 & - & 11,40 & 36,80 & 49,90 & $-13,10$ & 2,20 & $-49,90$ & $-36,80$ \\
\hline AA26 & 11,00 & 30,00 & 31,60 & $-1,60$ & 13,80 & 59,60 & 63,20 & $-3,60$ & $-2,80$ & $-33,20$ & $-28,00$ \\
\hline AA27 & 12,40 & 30,40 & 37,80 & $-7,40$ & 13,20 & 34,00 & 39,00 & $-5,00$ & $-0,80$ & $-8,60$ & 3,80 \\
\hline AA28 & 13,40 & 40,20 & 39,60 & 0,60 & 12,80 & 30,60 & 35,60 & $-5,00$ & 0,60 & 4,60 & 9,00 \\
\hline AA29 & 12,20 & 43,00 & 58,20 & $-15,20$ & 12,40 & 40,20 & 55,80 & $-15,60$ & $-0,20$ & $-12,80$ & 18,00 \\
\hline AA31 & 9,20 & 37,80 & 42,40 & $-4,60$ & 10,20 & 26,80 & 30,40 & $-3,60$ & $-1,00$ & 7,40 & 15,60 \\
\hline AA 32 & 12,00 & 37,20 & 38,20 & $-1,00$ & 12,80 & 36,80 & 48,20 & $-11,40$ & $-0,80$ & $-11,00$ & 1,40 \\
\hline AA35 & 12,80 & 0,00 & 0,00 & - & 11,00 & 46,40 & 53,80 & $-7,40$ & 1,80 & $-53,80$ & $-46,40$ \\
\hline AA38 & 12,60 & 0,00 & 71,20 & $-71,20$ & 9,80 & 39,80 & 0,00 & 39,80 & 2,80 & - & 31,40 \\
\hline AA39 & 12,20 & 43,40 & 0,00 & 43,40 & 12,40 & 41,20 & 0,00 & 41,20 & $-0,20$ & 43,40 & $-41,20$ \\
\hline AA40 & 13,40 & 38,40 & 0,00 & 38,40 & 13,20 & 0,00 & 0,00 & - & 0,20 & 38,40 & - \\
\hline AA43 & 11,80 & 29,60 & 0,00 & 29,60 & 12,00 & 31,80 & 37,00 & $-5,20$ & $-0,20$ & $-7,40$ & $-31,80$ \\
\hline AA44 & 12,40 & 50,00 & 41,20 & 8,80 & 12,00 & 48,20 & 40,60 & 7,60 & 0,40 & 9,40 & $-7,00$ \\
\hline AA46 & 12,00 & 48,40 & 62,80 & $-14,40$ & 12,20 & 46,40 & 41,00 & 5,40 & $-0,20$ & 7,40 & 16,40 \\
\hline AA47 & 11,40 & 36,80 & 38,40 & $-1,60$ & 11,80 & 38,40 & 30,20 & 8,20 & $-0,40$ & 6,60 & 0,00 \\
\hline AA48 & 12,00 & 34,80 & 39,80 & $-5,00$ & 13,40 & 33,40 & 35,40 & $-2,00$ & $-1,40$ & $-0,60$ & 6,40 \\
\hline AA49 & 12,80 & 24,00 & 29,20 & $-5,20$ & 13,00 & 36,80 & 0,00 & 36,80 & $-0,20$ & 24,00 & $-7,60$ \\
\hline AA52 & 12,40 & 28,80 & 39,80 & $-11,00$ & 12,60 & 32,20 & 30,40 & 1,80 & $-0,20$ & $-1,60$ & 7,60 \\
\hline AA53 & 12,00 & $\mathbf{0 , 0 0}$ & 0,00 & - & 13,80 & 0,00 & 0,00 & - & $-1,80$ & - & - \\
\hline AA55 & 13,80 & 40,00 & 43,40 & $-3,40$ & 12,60 & 35,60 & 31,60 & 4,00 & 1,20 & 8,40 & 7,80 \\
\hline AA56 & 13,00 & 39,80 & 48,00 & $-8,20$ & 12,80 & 38,80 & 39,60 & $-0,80$ & 0,20 & 0,20 & 9,20 \\
\hline AA57 & 10,80 & 38,20 & 36,60 & 1,60 & 12,40 & 34,80 & 30,80 & 4,00 & $-1,60$ & 7,40 & 1,80 \\
\hline AA58 & 14,00 & 0,00 & 0,00 & - & 14,00 & 0,00 & 0,00 & - & 0,00 & - & - \\
\hline AA 60 & 12,60 & 42,00 & 30,00 & 12,00 & 13,20 & 49,60 & 47,00 & 2,60 & $-0,60$ & $-5,00$ & $-19,60$ \\
\hline AA61 & 12,60 & 39,60 & 44,60 & $-5,00$ & 10,80 & 31,00 & 28,80 & 2,20 & 1,80 & 10,80 & 13,60 \\
\hline AA62 & 12,80 & 40,40 & 39,40 & 1,00 & 12,00 & 39,80 & 63,60 & $-23,80$ & 0,80 & $-23,20$ & $-0,40$ \\
\hline AA65 & 11,20 & 40,40 & 0,00 & 40,40 & 12,00 & 47,40 & 0,00 & 47,40 & $-0,80$ & 40,40 & $-47,40$ \\
\hline
\end{tabular}

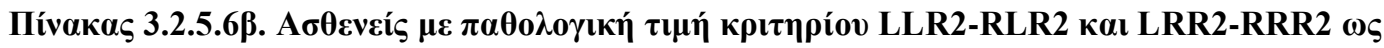

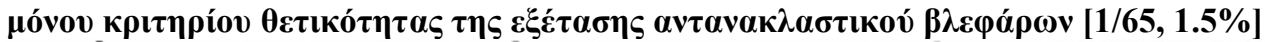

\begin{tabular}{|c|c|c|c|c|c|c|c|c|c|c|c|}
\hline $\begin{array}{r}\mathbf{A} / \mathbf{A} \\
\mathbf{K} \rho \iota \tau .\end{array}$ & $\begin{array}{c}\text { LR1 } \\
<13 \mathrm{~ms}\end{array}$ & $\begin{array}{l}\text { L LR2 } \\
<41 \mathrm{~ms}\end{array}$ & $\begin{array}{l}\text { L RR2 } \\
<44 \mathrm{~ms}\end{array}$ & $\begin{array}{c}\text { LLR2-LRR2 } \\
\quad<|5| \mathrm{ms}\end{array}$ & $\begin{array}{c}\text { RR1 } \\
<13 \mathrm{~ms}\end{array}$ & $\begin{array}{l}\text { R RR2 } \\
<41 \mathrm{~ms}\end{array}$ & $\begin{array}{l}\text { R LR2 } \\
<44 \mathrm{~ms}\end{array}$ & $\begin{array}{c}\text { RRR2-RLR2 } \\
\quad<|5| \mathrm{ms}\end{array}$ & $\begin{array}{l}\text { LR1-RR1 } \\
<|1.2| \mathrm{ms}\end{array}$ & $\begin{array}{c}\text { LLR2-RLR2 } \\
<[7] \mathrm{ms}\end{array}$ & $\begin{array}{c}\text { LRR2-RRR2 } \\
<|7| \mathrm{ms}\end{array}$ \\
\hline AA52 & 12,40 & 28,80 & 39,80 & $-11,00$ & 12,60 & 32,20 & 30,40 & 1,80 & $-0,20$ & $-1,60$ & 7,60 \\
\hline
\end{tabular}




\section{5. АПОТЕ $\Lambda$ $\Sigma$ MATA ME $\Lambda$ ETH $\Sigma-\Sigma Y N O \Lambda I K A$}

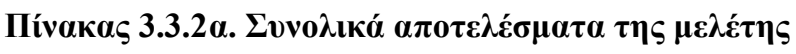

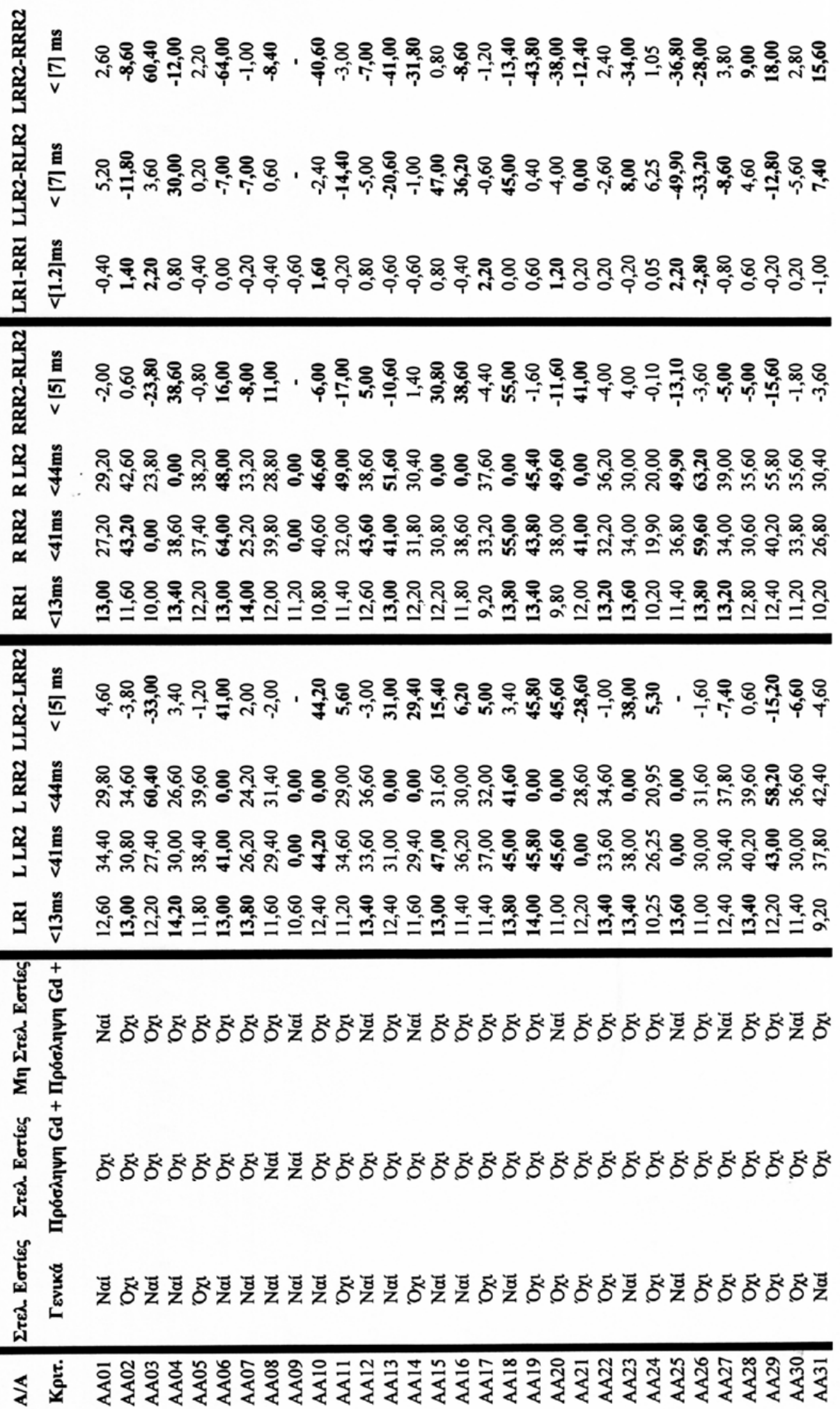




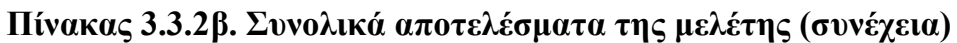

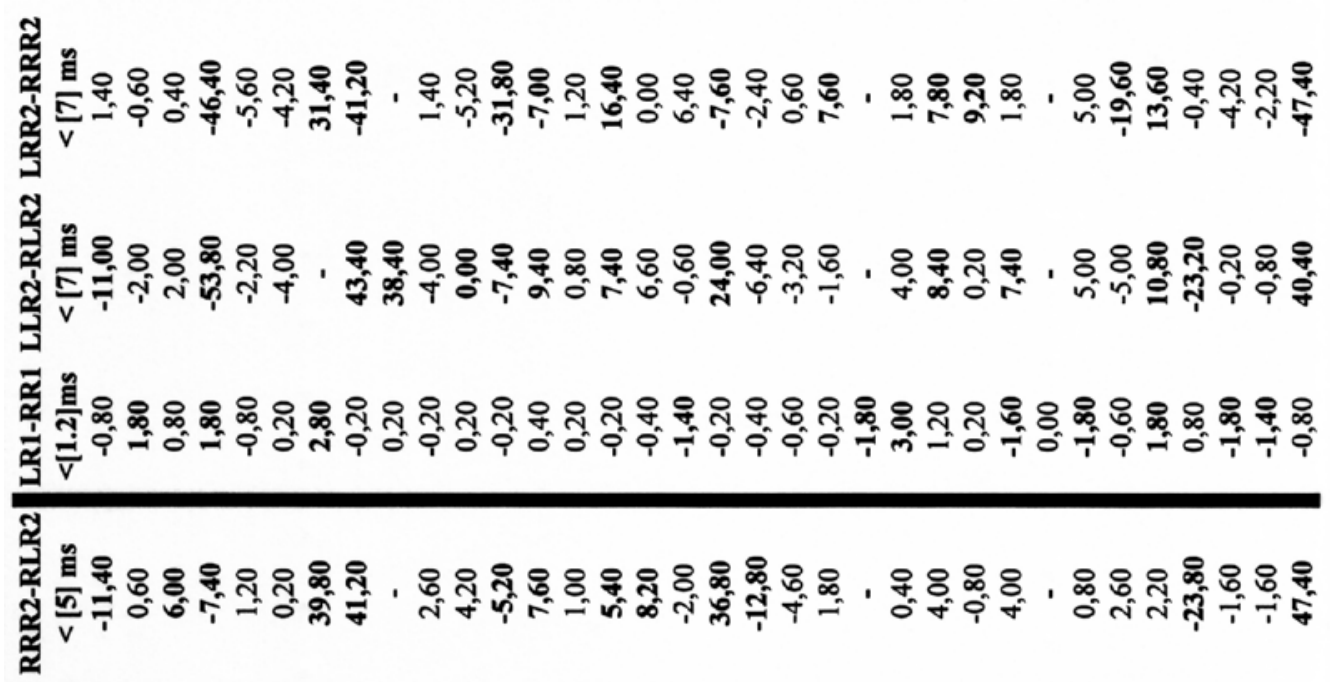

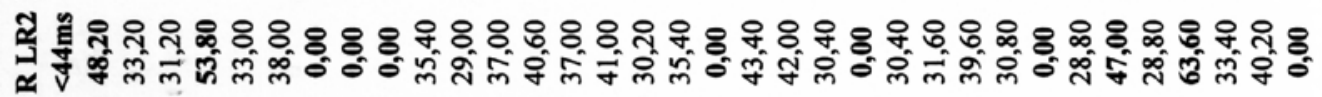

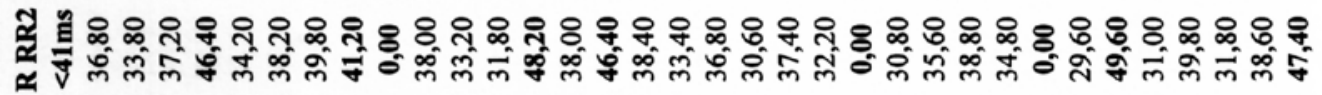

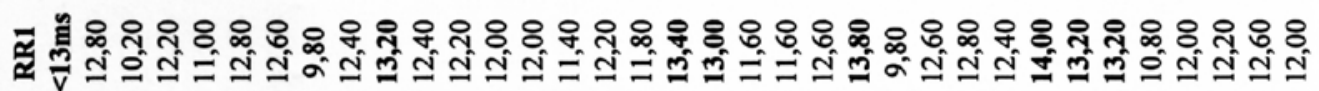

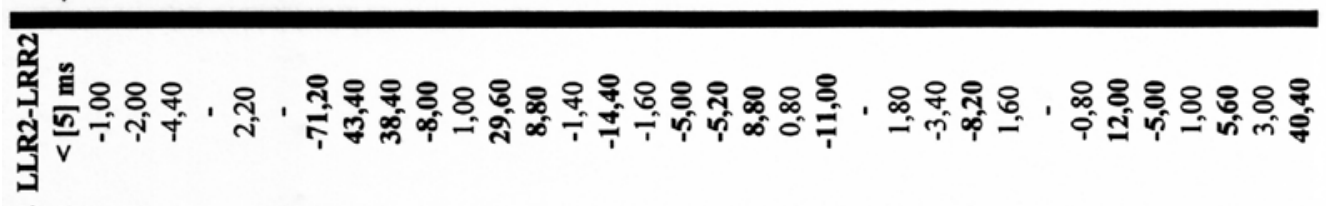

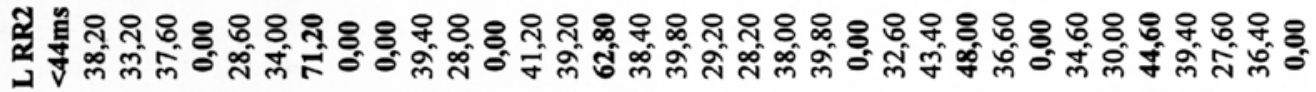

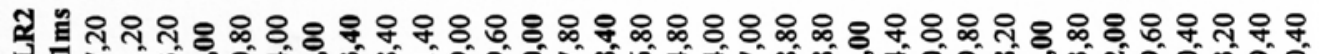

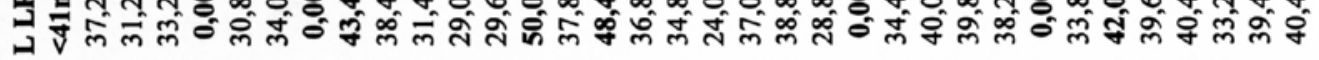

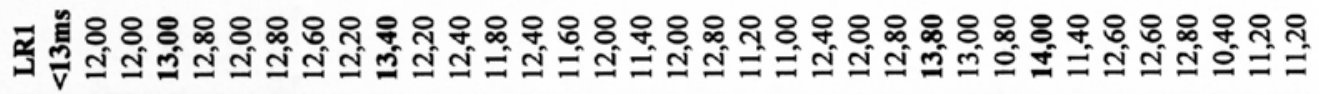

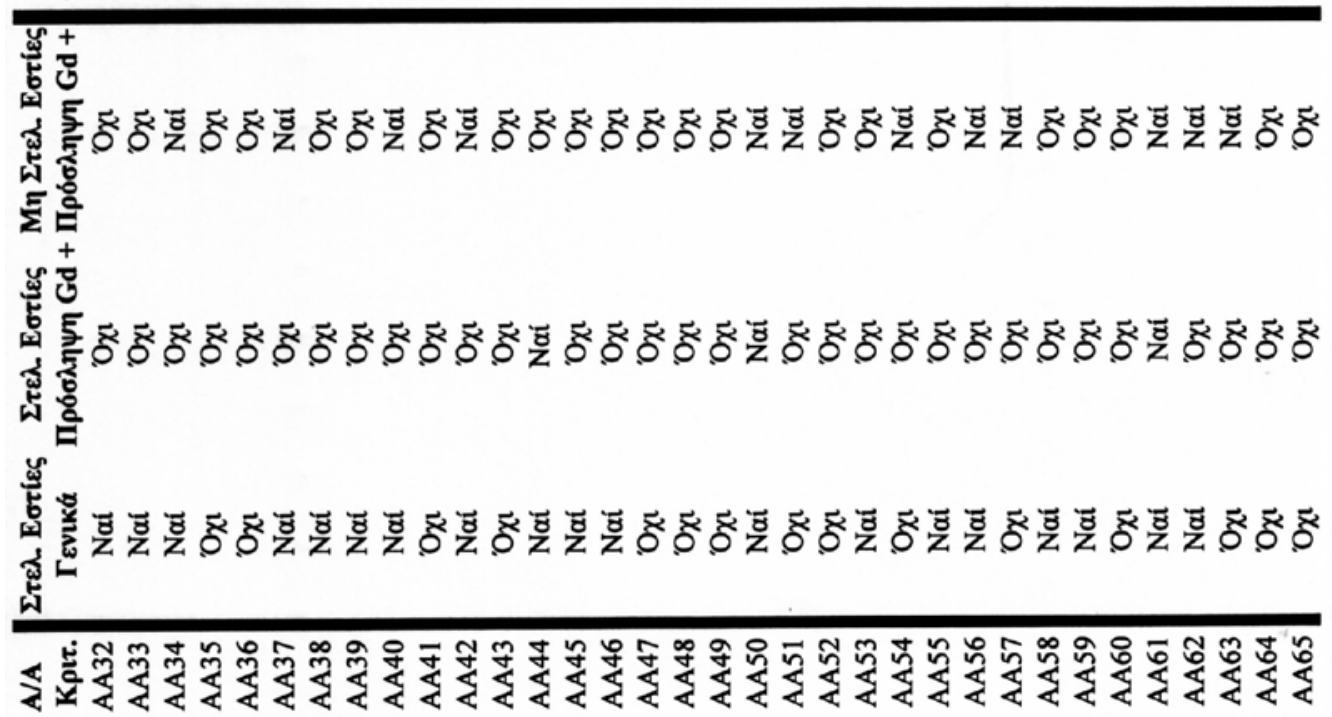




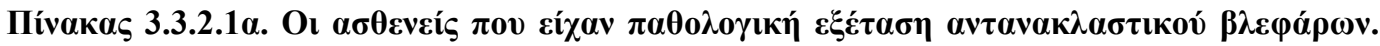

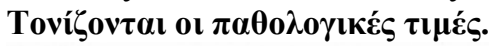

\begin{tabular}{|c|c|c|c|c|c|c|c|c|c|c|c|}
\hline $\mathbf{A} / \mathbf{A}$ & LR1 & L LR2 & L RR2 & LLR2-LRR2 & RR1 & R RR2 & R LR2 & RRR2-RLR2 & LR1-RR1 & LLR2-RLR2 & LRR2-RRR2 \\
\hline Kрıг. & $<13 \mathrm{~ms}$ & $<41 \mathrm{~ms}$ & $<44 \mathrm{~ms}$ & $<|5| \mathrm{ms}$ & $<13 \mathrm{~ms}$ & $<41 \mathrm{~ms}$ & $<44 \mathrm{~ms}$ & $<\mid 5] \mathrm{ms}$ & $<[1.2] \mathrm{ms}$ & $<|7| \mathrm{ms}$ & $<|7| \mathrm{ms}$ \\
\hline $\mathrm{AA} 01$ & 12,60 & 34,40 & 29,80 & 4,60 & 13,00 & 27,20 & 29,20 & $-2,00$ & $-0,40$ & 5,20 & 2,60 \\
\hline $\mathrm{AA} 02$ & 13,00 & 30,80 & 34,60 & $-3,80$ & 11,60 & 43,20 & 42,60 & 0,60 & 1,40 & $-11,80$ & $-8,60$ \\
\hline $\mathrm{AA} 03$ & 12,20 & 27,40 & 60,40 & $-33,00$ & 10,00 & 0,00 & 23,80 & $-23,80$ & 2,20 & 3,60 & 60,40 \\
\hline AA04 & 14,20 & 30,00 & 26,60 & 3,40 & 13,40 & 38,60 & 0,00 & 38,60 & 0,80 & 30,00 & $-12,00$ \\
\hline AA06 & 13,00 & 41,00 & 0,00 & 41,00 & 13,00 & 64,00 & 48,00 & 16,00 & 0,00 & $-7,00$ & $-64,00$ \\
\hline AA07 & 13,80 & 26,20 & 24,20 & 2,00 & 14,00 & 25,20 & 33,20 & $-8,00$ & $-0,20$ & $-7,00$ & $-1,00$ \\
\hline AA08 & 11,60 & 29,40 & 31,40 & $-2,00$ & 12,00 & 39,80 & 28,80 & 11,00 & $-0,40$ & 0,60 & $-8,40$ \\
\hline AA09 & 10,60 & 0,00 & 0,00 & - & 11,20 & 0,00 & 0,00 & - & $-0,60$ & - & - \\
\hline AA 10 & 12,40 & 44,20 & 0,00 & 44,20 & 10,80 & 40,60 & 46,60 & $-6,00$ & 1,60 & $-2,40$ & $-40,60$ \\
\hline AAl1 & 11,20 & 34,60 & 29,00 & 5,60 & 11,40 & 32,00 & 49,00 & $-17,00$ & $-0,20$ & $-14,40$ & $-3,00$ \\
\hline $\mathrm{AA} 12$ & 13,40 & 33,60 & 36,60 & $-3,00$ & 12,60 & 43,60 & 38,60 & 5,00 & 0,80 & $-5,00$ & $-7,00$ \\
\hline AA13 & 12,40 & 31,00 & 0,00 & 31,00 & 13,00 & 41,00 & 51,60 & $-10,60$ & $-0,60$ & $-20,60$ & $-41,00$ \\
\hline AA 14 & 11,60 & 29,40 & 0,00 & 29,40 & 12,20 & 31,80 & 30,40 & 1,40 & $-0,60$ & $-1,00$ & $-31,80$ \\
\hline AA15 & 13,00 & 47,00 & 31,60 & 15,40 & 12,20 & 30,80 & 0,00 & 30,80 & 0,80 & 47,00 & 0,80 \\
\hline AA 16 & 11,40 & 36,20 & 30,00 & 6,20 & 11,80 & 38,60 & 0,00 & 38,60 & $-0,40$ & 36,20 & $-8,60$ \\
\hline AA17 & 11,40 & 37,00 & 32,00 & 5,00 & 9,20 & 33,20 & 37,60 & $-4,40$ & 2,20 & $-0,60$ & $-1,20$ \\
\hline AA18 & 13,80 & 45,00 & 41,60 & 3,40 & 13,80 & 55,00 & 0,00 & 55,00 & 0,00 & 45,00 & $-13,40$ \\
\hline AA19 & 14,00 & 45,80 & 0,00 & 45,80 & 13,40 & 43,80 & 45,40 & $-1,60$ & 0,60 & 0,40 & $-43,80$ \\
\hline $\mathrm{AA} 20$ & 11,00 & 45,60 & 0,00 & 45,60 & 9,80 & 38,00 & 49,60 & $-11,60$ & 1,20 & $-4,00$ & $-38,00$ \\
\hline $\mathrm{AA} 21$ & 12,20 & 0,00 & 28,60 & $-28,60$ & 12,00 & 41,00 & 0,00 & 41,00 & 0,20 & 0,00 & $-12,40$ \\
\hline AA22 & 13,40 & 33,60 & 34,60 & $-1,00$ & 13,20 & 32,20 & 36,20 & $-4,00$ & 0,20 & $-2,60$ & 2,40 \\
\hline $\mathrm{AA} 23$ & 13,40 & 38,00 & 0,00 & 38,00 & 13,60 & 34,00 & 30,00 & 4,00 & $-0,20$ & 8,00 & $-34,00$ \\
\hline AA24 & 10,25 & 26,25 & 20,95 & 5,30 & 10,20 & 19,90 & 20,00 & $-0,10$ & 0,05 & 6,25 & 1,05 \\
\hline AA25 & 13,60 & 0,00 & 0,00 & - & 11,40 & 36,80 & 49,90 & $-13,10$ & 2,20 & $-49,90$ & $-36,80$ \\
\hline AA26 & 11,00 & 30,00 & 31,60 & $-1,60$ & 13,80 & 59,60 & 63,20 & $-3,60$ & $-2,80$ & $-33,20$ & $-28,00$ \\
\hline $\mathrm{AA} 27$ & 12,40 & 30,40 & 37,80 & $-7,40$ & 13,20 & 34,00 & 39,00 & $-5,00$ & $-0,80$ & $-8,60$ & 3,80 \\
\hline AA28 & 13,40 & 40,20 & 39,60 & 0,60 & 12,80 & 30,60 & 35,60 & $-5,00$ & 0,60 & 4,60 & 9,00 \\
\hline AA29 & 12,20 & 43,00 & 58,20 & $-15,20$ & 12,40 & 40,20 & 55,80 & $-15,60$ & $-0,20$ & $-12,80$ & 18,00 \\
\hline $\mathrm{AA} 30$ & 11,40 & 30,00 & 36,60 & $-6,60$ & 11,20 & 33,80 & 35,60 & $-1,80$ & 0,20 & $-5,60$ & 2,80 \\
\hline $\mathrm{AA} 31$ & 9,20 & 37,80 & 42,40 & $-4,60$ & 10,20 & 26,80 & 30,40 & $-3,60$ & $-1,00$ & 7,40 & 15,60 \\
\hline AA 32 & 12,00 & 37,20 & 38,20 & $-1,00$ & 12,80 & 36,80 & 48,20 & $-11,40$ & $-0,80$ & $-11,00$ & 1,40 \\
\hline AA 33 & 12,00 & 31,20 & 33,20 & $-2,00$ & 10,20 & 33,80 & 33,20 & 0,60 & 1,80 & $-2,00$ & $-0,60$ \\
\hline AA34 & 13,00 & 33,20 & 37,60 & $-4,40$ & 12,20 & 37,20 & 31,20 & 6,00 & 0,80 & 2,00 & 0,40 \\
\hline AA35 & 12,80 & 0,00 & 0,00 & - & 11,00 & 46,40 & 53,80 & $-7,40$ & 1,80 & $-53,80$ & $-46,40$ \\
\hline AA38 & 12,60 & 0,00 & 71,20 & $-71,20$ & 9,80 & 39,80 & 0,00 & 39,80 & 2,80 & - & 31,40 \\
\hline AA39 & 12,20 & 43,40 & 0,00 & 43,40 & 12,40 & 41,20 & 0,00 & 41,20 & $-0,20$ & 43,40 & $-41,20$ \\
\hline AA40 & 13,40 & 38,40 & 0,00 & 38,40 & 13,20 & 0,00 & 0,00 & - & 0,20 & 38,40 & - \\
\hline AA41 & 12,20 & 31,40 & 39,40 & $-8,00$ & 12,40 & 38,00 & 35,40 & 2,60 & $-0,20$ & $-4,00$ & 1,40 \\
\hline AA43 & 11,80 & 29,60 & 0,00 & 29,60 & 12,00 & 31,80 & 37,00 & $-5,20$ & $-0,20$ & $-7,40$ & $-31,80$ \\
\hline AA44 & 12,40 & 50,00 & 41,20 & 8,80 & 12,00 & 48,20 & 40,60 & 7,60 & 0,40 & 9,40 & $-7,00$ \\
\hline AA46 & 12,00 & 48,40 & 62,80 & $-14,40$ & 12,20 & 46,40 & 41,00 & 5,40 & $-0,20$ & 7,40 & 16,40 \\
\hline AA47 & 11,40 & 36,80 & 38,40 & $-1,60$ & 11,80 & 38,40 & 30,20 & 8,20 & $-0,40$ & 6,60 & 0,00 \\
\hline AA48 & 12,00 & 34,80 & 39,80 & $-5,00$ & 13,40 & 33,40 & 35,40 & $-2,00$ & $-1,40$ & $-0,60$ & 6,40 \\
\hline AA49 & 12,80 & 24,00 & 29,20 & $-5,20$ & 13,00 & 36,80 & 0,00 & 36,80 & $-0,20$ & 24,00 & $-7,60$ \\
\hline AA50 & 11,20 & 37,00 & 28,20 & 8,80 & 11,60 & 30,60 & 43,40 & $-12,80$ & $-0,40$ & $-6,40$ & $-2,40$ \\
\hline AA52 & 12,40 & 28,80 & 39,80 & $-11,00$ & 12,60 & 32,20 & 30,40 & 1,80 & $-0,20$ & $-1,60$ & 7,60 \\
\hline AA53 & 12,00 & 0,00 & 0,00 & - & 13,80 & 0,00 & 0,00 & - & $-1,80$ & - & - \\
\hline AA54 & 12,80 & 34,40 & 32,60 & 1,80 & 9,80 & 30,80 & 30,40 & 0,40 & 3,00 & 4,00 & 1,80 \\
\hline AA55 & 13,80 & 40,00 & 43,40 & $-3,40$ & 12,60 & 35,60 & 31,60 & 4,00 & 1,20 & 8,40 & 7,80 \\
\hline AA56 & 13,00 & 39,80 & 48,00 & $-8,20$ & 12,80 & 38,80 & 39,60 & $-0,80$ & 0,20 & 0,20 & 9,20 \\
\hline AA57 & 10,80 & 38,20 & 36,60 & 1,60 & 12,40 & 34,80 & 30,80 & 4,00 & $-1,60$ & 7,40 & 1,80 \\
\hline AA58 & 14,00 & 0,00 & 0,00 & - & 14,00 & 0,00 & 0,00 & - & 0,00 & - & - \\
\hline AA59 & 11,40 & 33,80 & 34,60 & $-0,80$ & 13,20 & 29,60 & 28,80 & 0,80 & $-1,80$ & 5,00 & 5,00 \\
\hline AA60 & 12,60 & 42,00 & 30,00 & 12,00 & 13,20 & 49,60 & 47,00 & 2,60 & $-0,60$ & $-5,00$ & $-19,60$ \\
\hline AA61 & 12,60 & 39,60 & 44,60 & $-5,00$ & 10,80 & 31,00 & 28,80 & 2,20 & 1,80 & 10,80 & 13,60 \\
\hline AA62 & 12,80 & 40,40 & 39,40 & 1,00 & 12,00 & 39,80 & 63,60 & $-23,80$ & 0,80 & $-23,20$ & $-0,40$ \\
\hline AA63 & 10,40 & 33,20 & 27,60 & 5,60 & 12,20 & 31,80 & 33,40 & $-1,60$ & $-1,80$ & $-0,20$ & $-4,20$ \\
\hline AA64 & 11,20 & 39,40 & 36,40 & 3,00 & 12,60 & 38,60 & 40,20 & $-1,60$ & $-1,40$ & $-0,80$ & $-2,20$ \\
\hline AA65 & 11,20 & 40,40 & 0,00 & 40,40 & 12,00 & 47,40 & 0,00 & 47,40 & $-0,80$ & 40,40 & $-47,40$ \\
\hline
\end{tabular}

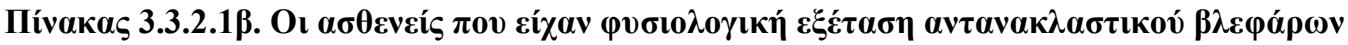

\begin{tabular}{|c|c|c|c|c|c|c|c|c|c|c|c|}
\hline $\begin{array}{c}\text { A/A } \\
\text { Kpı. }\end{array}$ & $\begin{array}{c}\text { LR1 } \\
<13 \mathrm{~ms}\end{array}$ & $\begin{array}{l}\text { L LR2 } \\
<41 \mathrm{~ms}\end{array}$ & $\begin{array}{l}\text { L RR2 } \\
<44 \mathrm{~ms}\end{array}$ & $\begin{array}{c}\text { LLR2-LRR2 } \\
<[5] \mathrm{ms}\end{array}$ & $\mid \begin{array}{c}\text { RR1 } \\
<13 \mathrm{~ms}\end{array}$ & $\begin{array}{l}\text { R RR2 } \\
<41 \mathrm{~ms}\end{array}$ & $\begin{array}{l}\text { R LR2 } \\
<44 \mathrm{~ms}\end{array}$ & $\begin{array}{c}\text { RRR2-RLR2 } \\
<[5] \mathrm{ms}\end{array}$ & $\begin{array}{l}\text { LR1-RR1 } \\
<[1.2] \mathrm{ms}\end{array}$ & $\begin{array}{c}\text { LLR2-RLR2 } \\
<[7] \mathrm{ms}\end{array}$ & $\begin{array}{c}\text { LRR2-RRR2 } \\
<[7] \mathrm{ms}\end{array}$ \\
\hline AA05 & 11,80 & 38,40 & 39,60 & $-1,20$ & 12,20 & 37,40 & 38,20 & $-0,80$ & $-0,40$ & 0,20 & 2,20 \\
\hline AA36 & 12,00 & 30,80 & 28,60 & 2,20 & 12,80 & 34,20 & 33,00 & 1,20 & $-0,80$ & $-2,20$ & $-5,60$ \\
\hline AA 37 & 12,80 & 34,00 & 34,00 & 0,00 & 12,60 & 38,20 & 38,00 & 0,20 & 0,20 & $-4,00$ & $-4,20$ \\
\hline $\mathrm{AA} 42$ & 12,40 & 29,00 & 28,00 & 1,00 & 12,20 & 33,20 & 29,00 & 4,20 & 0,20 & 0,00 & $-5,20$ \\
\hline $\mathrm{AA} 45$ & 11,60 & 37,80 & 39,20 & $-1,40$ & 11,40 & 38,00 & 37,00 & 1,00 & 0,20 & 0,80 & 1,20 \\
\hline AA51 & 11,00 & 38,80 & 38,00 & 0,80 & 11,60 & 37,40 & 42,00 & $-4,60$ & $-0,60$ & $-3,20$ & 0,60 \\
\hline
\end{tabular}




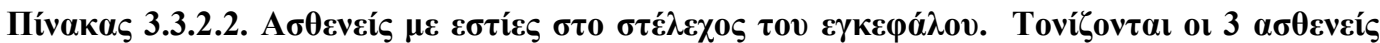

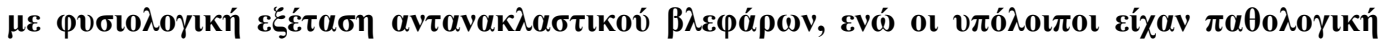

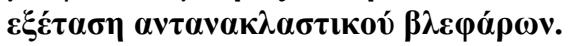

ชี

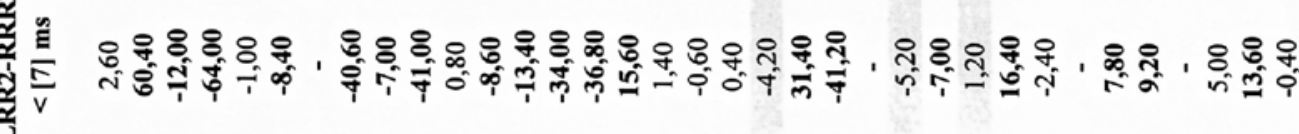
๘ี

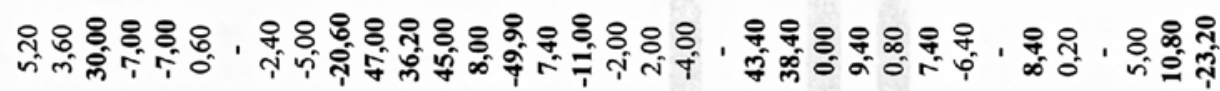

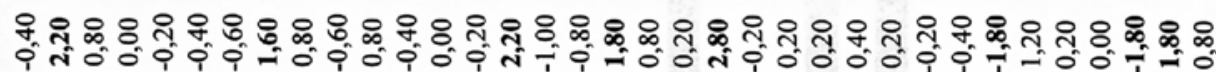

ฐ

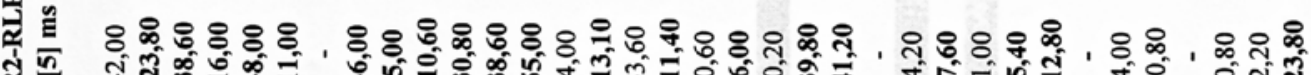

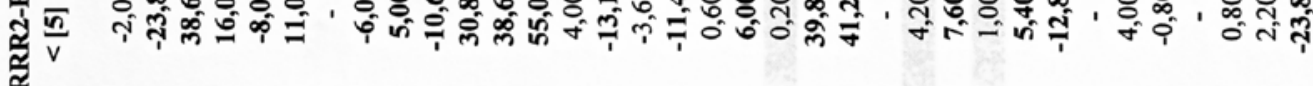

ฐ ฉ

‡

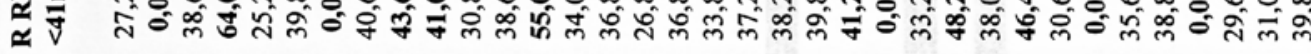

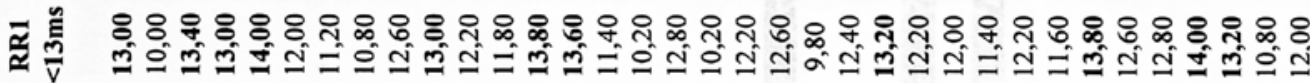

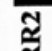

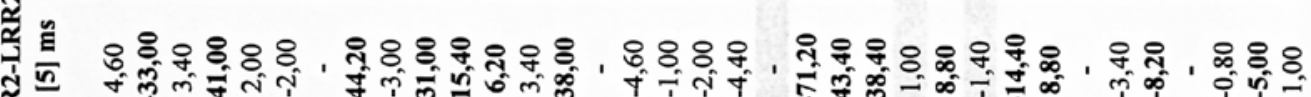

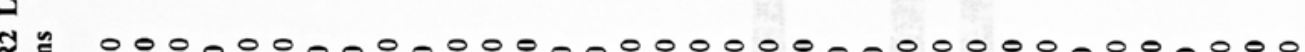

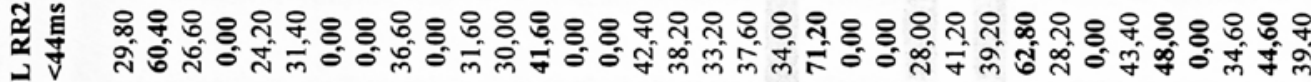
₹

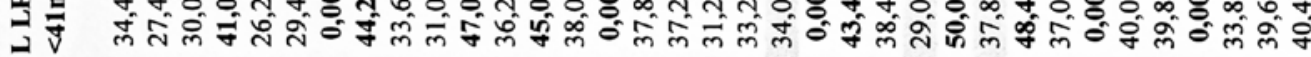

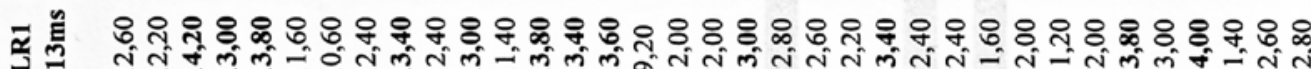
造

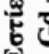

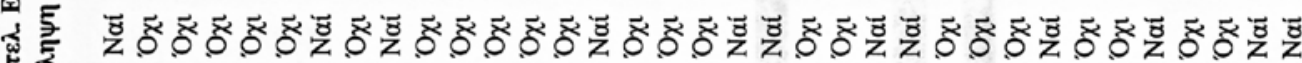
空宫 $+$ 苨

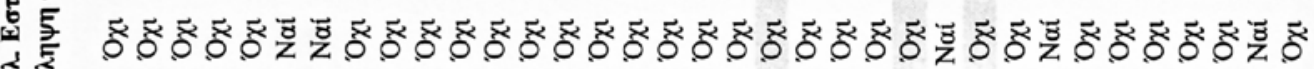
高

:

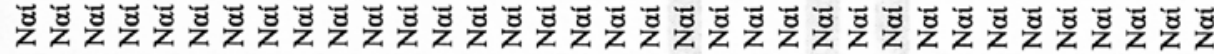

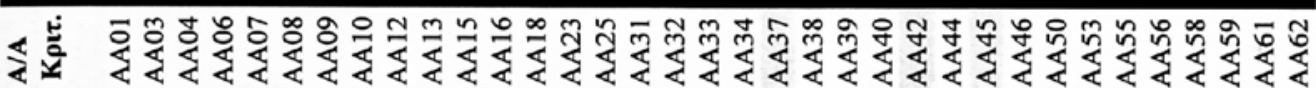

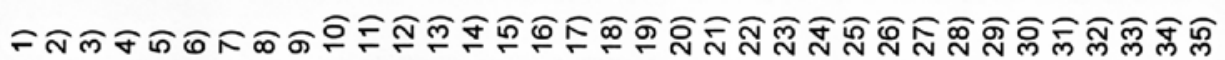




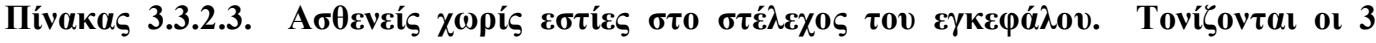

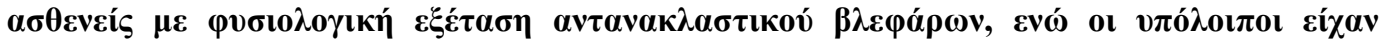

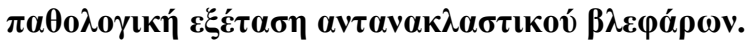

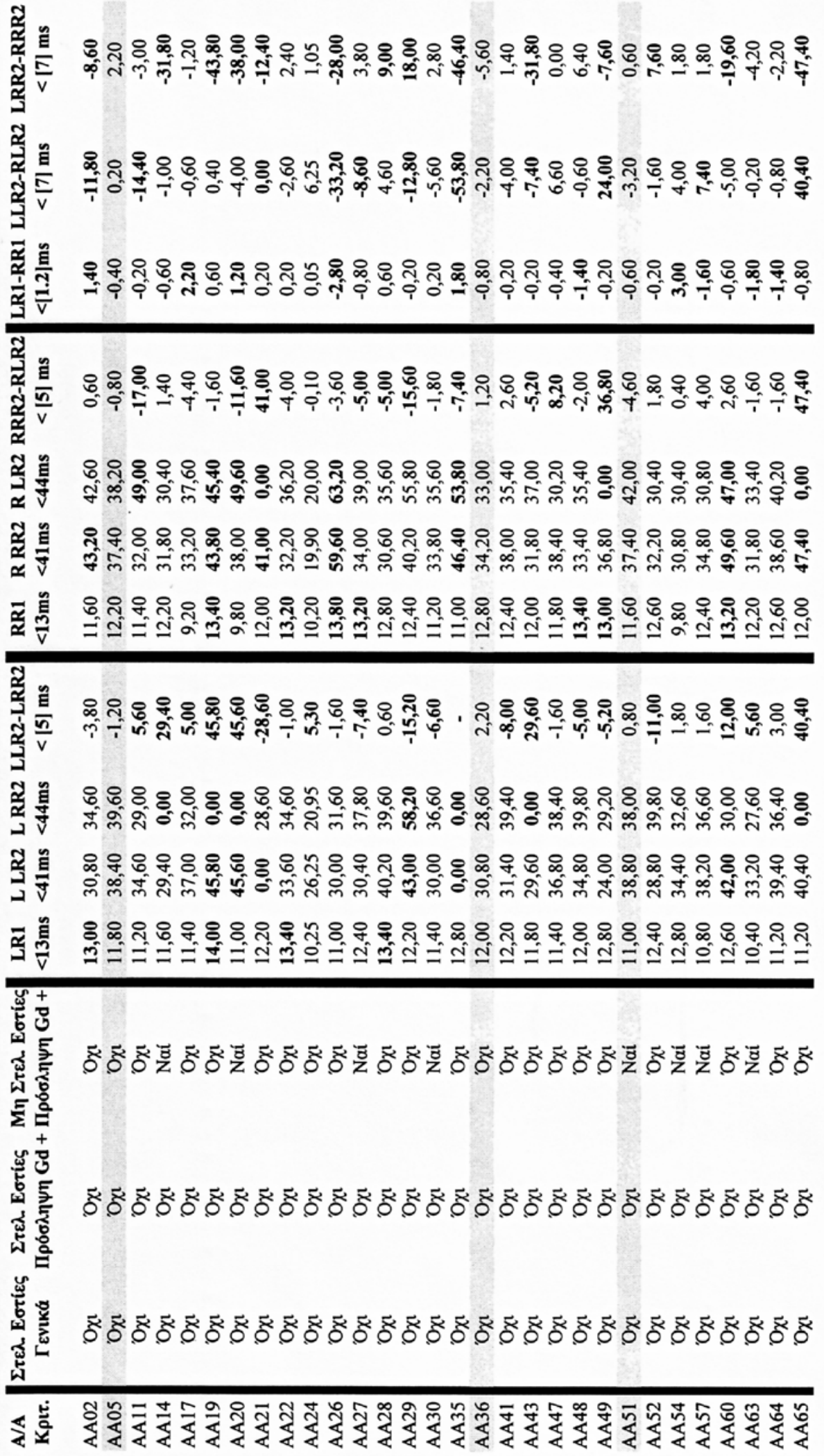

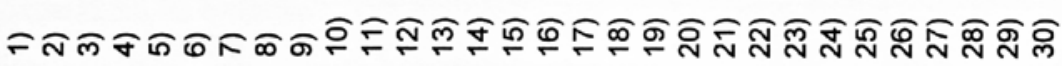




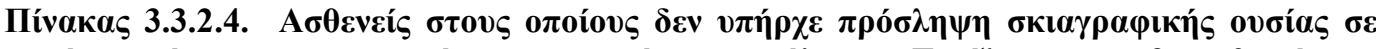

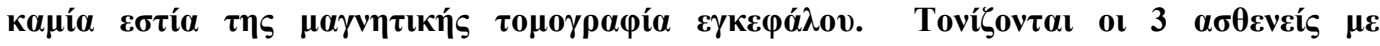

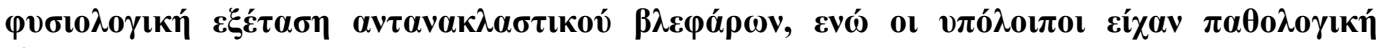

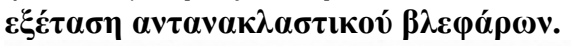

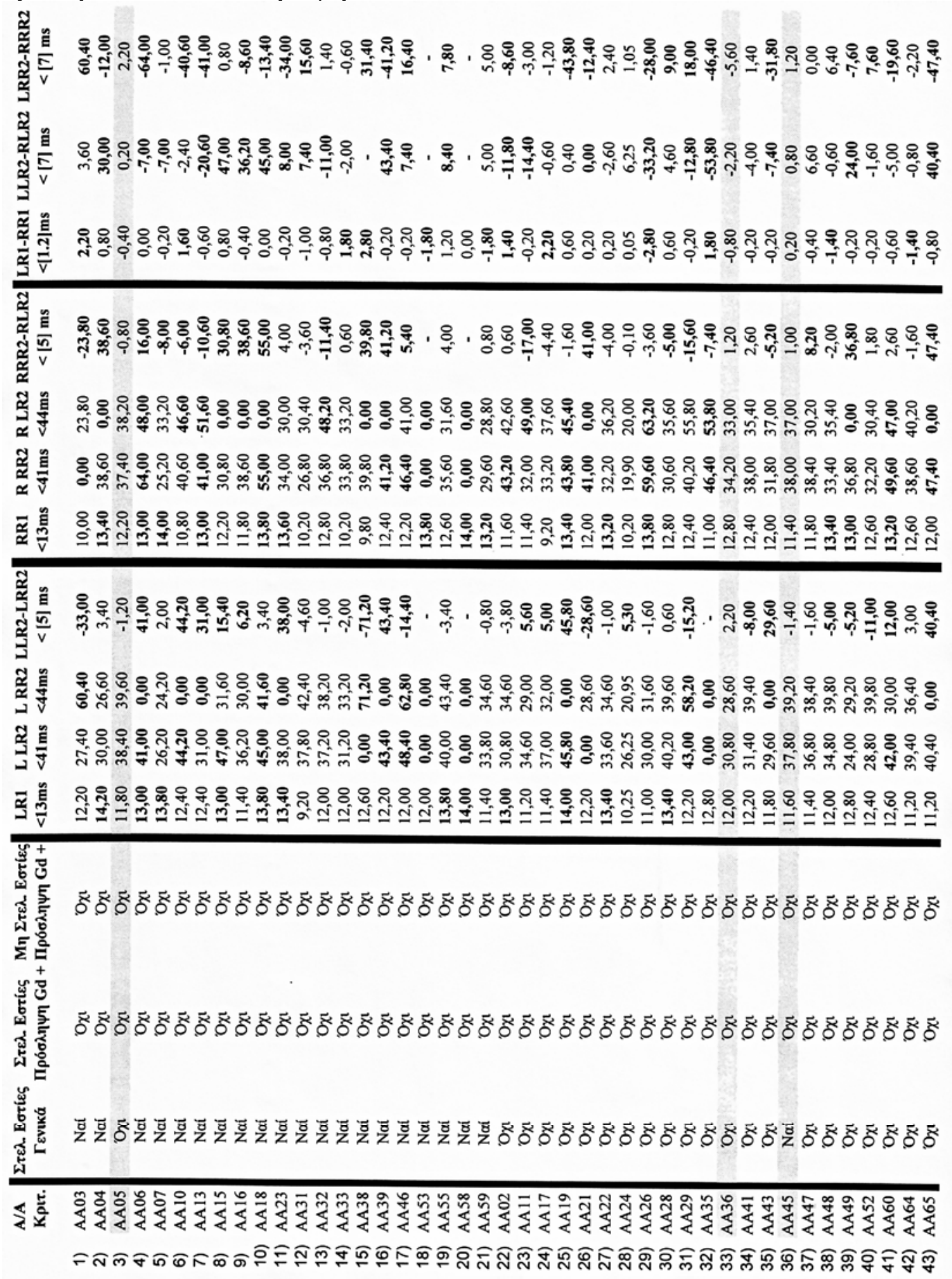




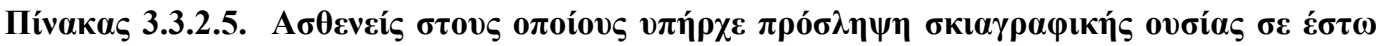

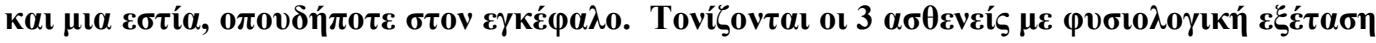

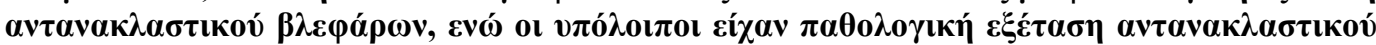
$\beta \lambda \varepsilon \varphi \alpha ́ \rho \omega v$.

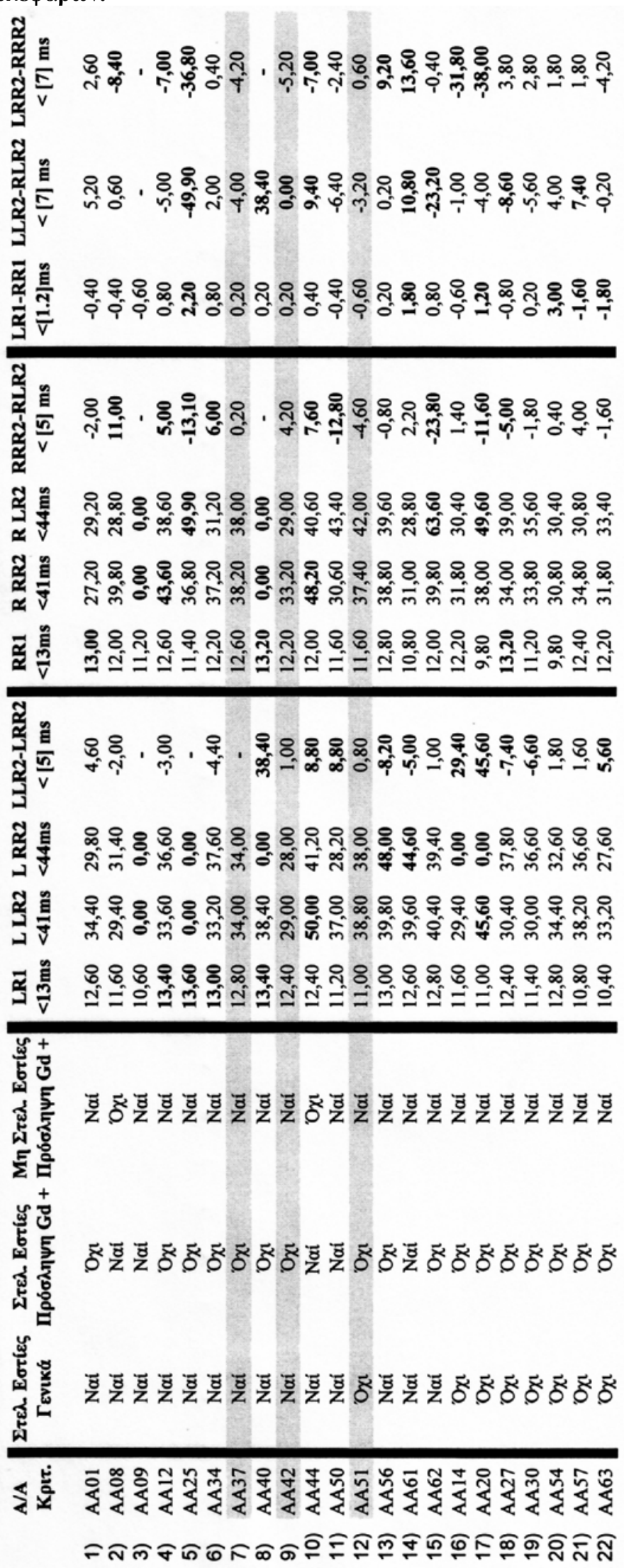




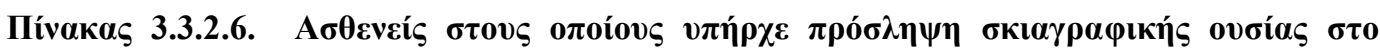

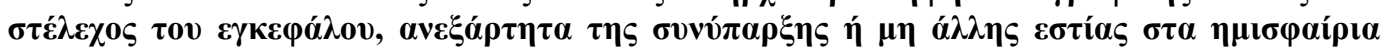

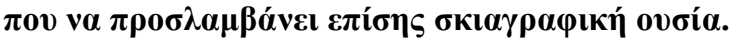

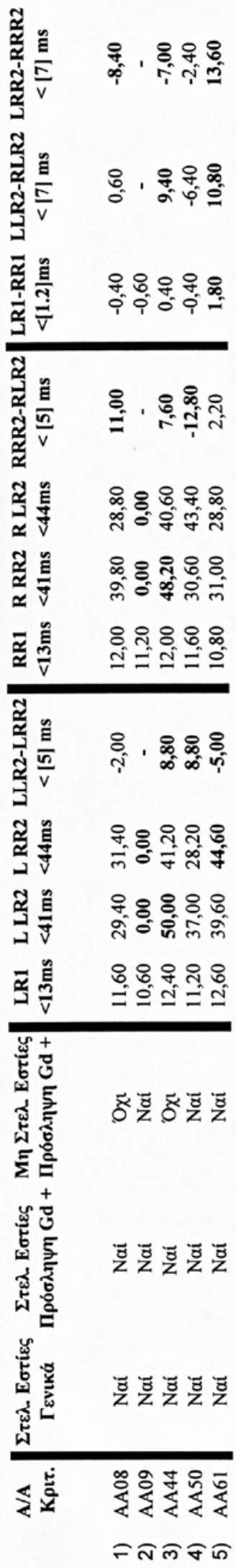




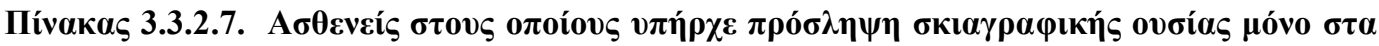

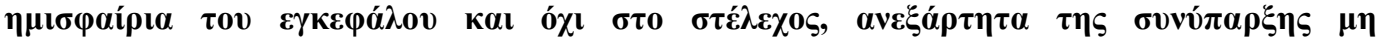

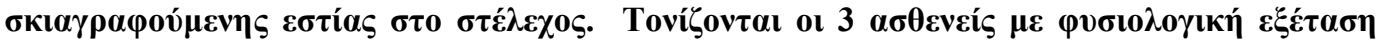

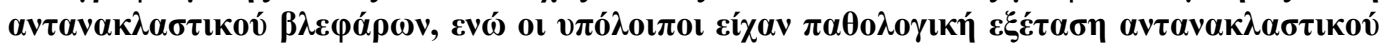

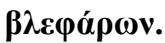

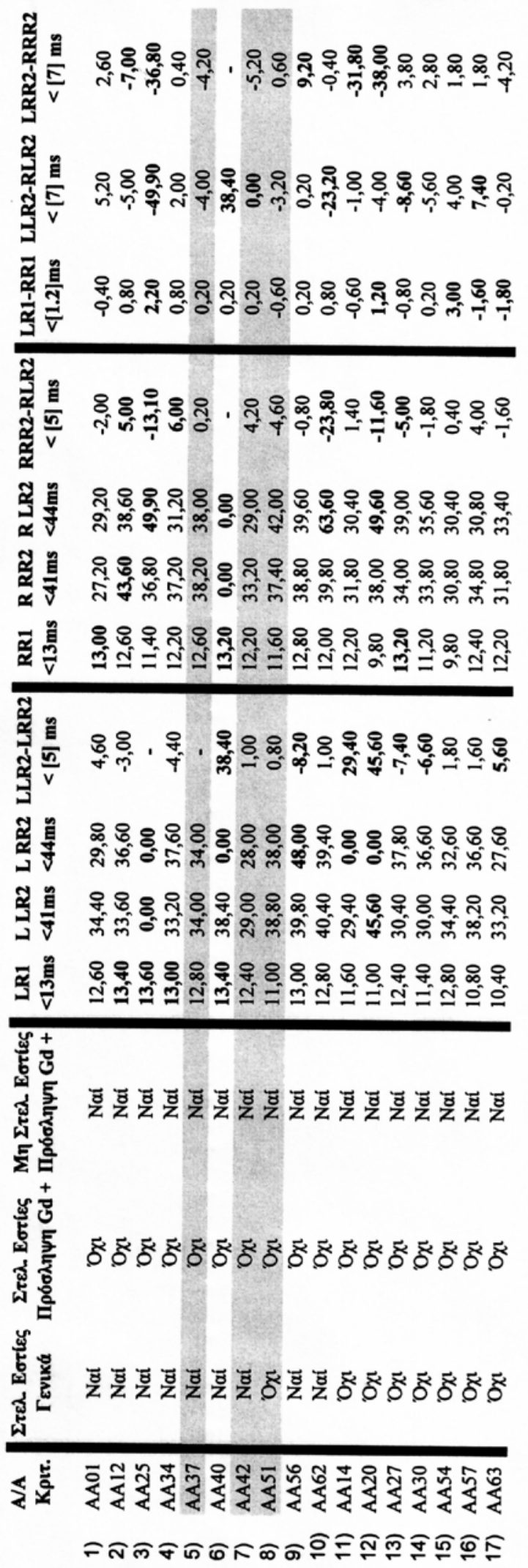




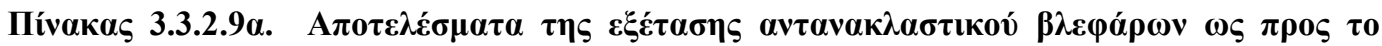

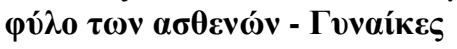

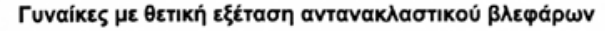

\begin{tabular}{|c|c|c|c|c|c|c|c|c|c|c|c|c|}
\hline & $\begin{array}{c}\text { A/A } \\
\text { Гvvaíkes }\end{array}$ & $\begin{array}{c}\text { LR1 } \\
<13 \mathrm{~ms}\end{array}$ & $\begin{array}{l}\text { L LR2 } \\
<41 \mathrm{~ms}\end{array}$ & $\begin{array}{l}\text { L RR2 } \\
<44 \mathrm{~ms}\end{array}$ & $\begin{array}{l}\text { LLR2-LRR2 } \\
<[5] \mathrm{msec}\end{array}$ & $\begin{array}{c}\text { RR1 } \\
<13 \mathrm{~ms}\end{array}$ & $\begin{array}{l}\text { R RR2 } \\
<41 \mathrm{~ms}\end{array}$ & $\begin{array}{l}\text { R LR2 } \\
<44 \mathrm{~ms}\end{array}$ & $\begin{array}{c}\text { RRR2-RLR2 } \\
<|5| \mathrm{msec}\end{array}$ & $\begin{array}{c}\text { LR1-RR1 } \\
<|1.2| \mathrm{msec}\end{array}$ & $\begin{array}{l}\text { LLR2-RLR2 } \\
<[7] \mathrm{msec}\end{array}$ & $\begin{array}{c}\text { LRR2-RRR2 } \\
<|7| \text { msec }\end{array}$ \\
\hline 1) & $\mathrm{AA} 01$ & 12,60 & 34,40 & 29,80 & 4,60 & 13,00 & 27,20 & 29,20 & $-2,00$ & $-0,40$ & 5,20 & 2,60 \\
\hline 2) & $\mathrm{AA} 02$ & 13,00 & 30,80 & 34,60 & $-3,80$ & 11,60 & 43,20 & 42,60 & 0,60 & 1,40 & $-11,80$ & $-8,60$ \\
\hline 3) & $\mathrm{AA} 03$ & 12,20 & 27,40 & 60,40 & $-33,00$ & 10,00 & 0,00 & 23,80 & $-23,80$ & 2,20 & 3,60 & 60,40 \\
\hline 4) & AA06 & 13,00 & 41,00 & 0,00 & 41,00 & 13,00 & 64,00 & 48,00 & 16,00 & 0,00 & $-7,00$ & $-64,00$ \\
\hline 5) & AA07 & 13,80 & 26,20 & 24,20 & 2,00 & 14,00 & 25,20 & 33,20 & $-8,00$ & $-0,20$ & $-7,00$ & $-1,00$ \\
\hline 6) & AA08 & 11,60 & 29,40 & 31,40 & $-2,00$ & 12,00 & 39,80 & 28,80 & 11,00 & $-0,40$ & 0,60 & $-8,40$ \\
\hline 7) & AA09 & 10,60 & 0,00 & 0,00 & 0,00 & 11,20 & 0,00 & 0,00 & 0,00 & $-0,60$ & 0,00 & 0,00 \\
\hline 8) & AAl1 & 11,20 & 34,60 & 29,00 & 5,60 & 11,40 & 32,00 & 49,00 & $-17,00$ & $-0,20$ & $-14,40$ & $-3,00$ \\
\hline 9) & $\mathrm{AA} 12$ & 13,40 & 33,60 & 36,60 & $-3,00$ & 12,60 & 43,60 & 38,60 & 5,00 & 0,80 & $-5,00$ & $-7,00$ \\
\hline 10) & $\mathrm{AA} 13$ & 12,40 & 31,00 & 0,00 & 31,00 & 13,00 & 41,00 & 51,60 & $-10,60$ & $-0,60$ & $-20,60$ & $-41,00$ \\
\hline 11) & AA14 & 11,60 & 29,40 & 0,00 & 29,40 & 12,20 & 31,80 & 30,40 & 1,40 & $-0,60$ & $-1,00$ & $-31,80$ \\
\hline 12) & AA 16 & 11,40 & 36,20 & 30,00 & 6,20 & 11,80 & 38,60 & 0,00 & 38,60 & $-0,40$ & 36,20 & $-8,60$ \\
\hline 13) & AA17 & 11,40 & 37,00 & 32,00 & 5,00 & 9,20 & 33,20 & 37,60 & $-4,40$ & 2,20 & $-0,60$ & $-1,20$ \\
\hline 14) & AA19 & 14,00 & 45,80 & 0,00 & 45,80 & 13,40 & 43,80 & 45,40 & $-1,60$ & 0,60 & 0,40 & $-43,80$ \\
\hline 15) & $\mathrm{AA} 20$ & 11,00 & 45,60 & 0,00 & 45,60 & 9,80 & 38,00 & 49,60 & $-11,60$ & 1,20 & $-4,00$ & $-38,00$ \\
\hline 16) & AA22 & 13,40 & 33,60 & 34,60 & $-1,00$ & 13,20 & 32,20 & 36,20 & $-4,00$ & 0,20 & $-2,60$ & 2,40 \\
\hline 17) & AA28 & 13,40 & 40,20 & 39,60 & 0,60 & 12,80 & 30,60 & 35,60 & $-5,00$ & 0,60 & 4,60 & 9,00 \\
\hline 18) & AA29 & 12,20 & 43,00 & 58,20 & $-15,20$ & 12,40 & 40,20 & 55,80 & $-15,60$ & $-0,20$ & $-12,80$ & 18,00 \\
\hline 19) & $\mathrm{AA} 30$ & 11,40 & 30,00 & 36,60 & $-6,60$ & 11,20 & 33,80 & 35,60 & $-1,80$ & 0,20 & $-5,60$ & 2,80 \\
\hline 20) & AA33 & 12,00 & 31,20 & 33,20 & $-2,00$ & 10,20 & 33,80 & 33,20 & 0,60 & 1,80 & $-2,00$ & $-0,60$ \\
\hline 21) & AA35 & 12,80 & 0,00 & 0,00 & 0,00 & 11,00 & 46,40 & 53,80 & $-7,40$ & 1,80 & $-53,80$ & $-46,40$ \\
\hline 22) & AA38 & 12,60 & 0,00 & 71,20 & $-71,20$ & 9,80 & 39,80 & 0,00 & 39,80 & 2,80 & 0,00 & 31,40 \\
\hline 23) & AA39 & 12,20 & 43,40 & 0,00 & 43,40 & 12,40 & 41,20 & 0,00 & 41,20 & $-0,20$ & 43,40 & $-41,20$ \\
\hline 24) & AA40 & 13,40 & 38,40 & 0,00 & 38,40 & 13,20 & 0,00 & 0,00 & 0,00 & 0,20 & 38,40 & 0,00 \\
\hline 25) & AA41 & 12,20 & 31,40 & 39,40 & $-8,00$ & 12,40 & 38,00 & 35,40 & 2,60 & $-0,20$ & $-4,00$ & 1,40 \\
\hline 26) & AA43 & 11,80 & 29,60 & 0,00 & 29,60 & 12,00 & 31,80 & 37,00 & $-5,20$ & $-0,20$ & $-7,40$ & $-31,80$ \\
\hline 27) & AA48 & 12,00 & 34,80 & 39,80 & $-5,00$ & 13,40 & 33,40 & 35,40 & $-2,00$ & $-1,40$ & $-0,60$ & 6,40 \\
\hline 28) & AA49 & 12,80 & 24,00 & 29,20 & $-5,20$ & 13,00 & 36,80 & 0,00 & 36,80 & $-0,20$ & 24,00 & $-7,60$ \\
\hline 29) & AA50 & 11,20 & 37,00 & 28,20 & 8,80 & 11,60 & 30,60 & 43,40 & $-12,80$ & $-0,40$ & $-6,40$ & $-2,40$ \\
\hline 30) & AA52 & 12,40 & 28,80 & 39,80 & $-11,00$ & 12,60 & 32,20 & 30,40 & 1,80 & $-0,20$ & $-1,60$ & 7,60 \\
\hline 31) & AA53 & 12,00 & 0,00 & 0,00 & 0,00 & 13,80 & 0,00 & 0,00 & 0,00 & $-1,80$ & 0,00 & 0,00 \\
\hline 32) & AA54 & 12,80 & 34,40 & 32,60 & 1,80 & 9,80 & 30,80 & 30,40 & 0,40 & 3,00 & 4,00 & 1,80 \\
\hline 33) & AA56 & 13,00 & 39,80 & 48,00 & $-8,20$ & 12,80 & 38,80 & 39,60 & $-0,80$ & 0,20 & 0,20 & 9,20 \\
\hline 34) & AA57 & 10,80 & 38,20 & 36,60 & 1,60 & 12,40 & 34,80 & 30,80 & 4,00 & $-1,60$ & 7,40 & 1,80 \\
\hline 35) & AA58 & 14,00 & 0,00 & 0,00 & 0,00 & 14,00 & 0,00 & 0,00 & 0,00 & 0,00 & 0,00 & 0,00 \\
\hline 36) & AA59 & 11,40 & 33,80 & 34,60 & $-0,80$ & 13,20 & 29,60 & 28,80 & 0,80 & $-1,80$ & 5,00 & 5,00 \\
\hline 37) & AA60 & 12,60 & 42,00 & 30,00 & 12,00 & 13,20 & 49,60 & 47,00 & 2,60 & $-0,60$ & $-5,00$ & $-19,60$ \\
\hline 38) & AA62 & 12,80 & 40,40 & 39,40 & 1,00 & 12,00 & 39,80 & 63,60 & $-23,80$ & 0,80 & $-23,20$ & $-0,40$ \\
\hline 39) & AA63 & 10,40 & 33,20 & 27,60 & 5,60 & 12,20 & 31,80 & 33,40 & $-1,60$ & $-1,80$ & $-0,20$ & $-4,20$ \\
\hline
\end{tabular}

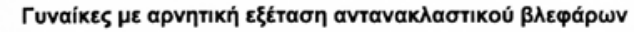

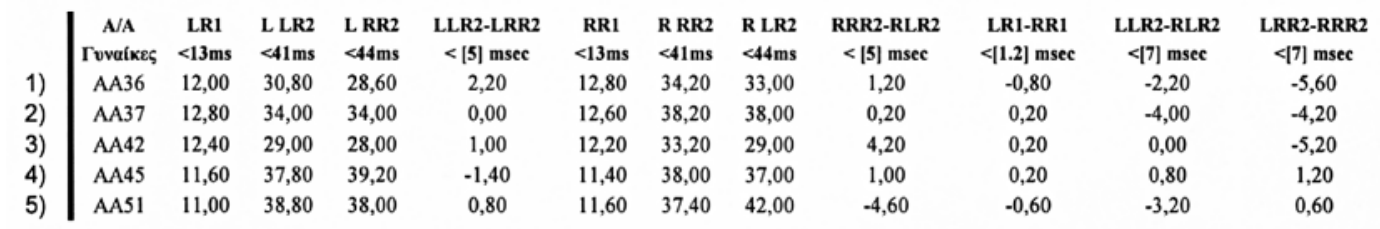




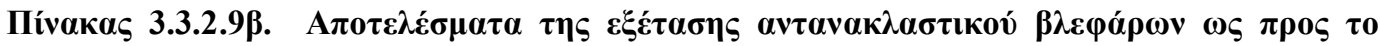

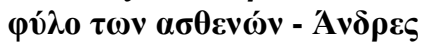

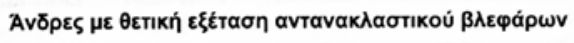

\begin{tabular}{|c|c|c|c|c|c|c|c|c|c|c|c|c|}
\hline & 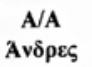 & $\begin{array}{c}\text { LR1 } \\
<13 \mathrm{~ms}\end{array}$ & $\begin{array}{l}\text { L LR2 } \\
<41 \mathrm{~ms}\end{array}$ & $\begin{array}{l}\text { L RR2 } \\
<44 \mathrm{~ms}\end{array}$ & $\begin{array}{c}\text { LLR2-LRR2 } \\
<[5] \mathrm{msec}\end{array}$ & $\begin{array}{c}\text { RR1 } \\
<13 \mathrm{~ms}\end{array}$ & $\begin{array}{l}\text { R RR2 } \\
<41 \mathrm{~ms}\end{array}$ & $\begin{array}{l}\text { R LR2 } \\
<44 \mathrm{~ms}\end{array}$ & $\begin{array}{c}\text { RRR2-RLR2 } \\
<|5| \text { msec }\end{array}$ & $\begin{array}{c}\text { LR1-RR1 } \\
<[1.2 \mid \text { msec }\end{array}$ & $\begin{array}{c}\text { LLR2-RLR2 } \\
<[7] \mathrm{msec}\end{array}$ & $<[7]$ msec \\
\hline 1) & AA04 & 14,20 & 30,00 & 26,60 & 3,40 & 13,40 & 38,60 & 0,00 & 38,60 & 0,80 & 30,00 & $-12,00$ \\
\hline 2) & AA10 & 12,40 & 44,20 & 0,00 & 44,20 & 10,80 & 40,60 & 46,60 & $-6,00$ & 1,60 & $-2,40$ & $-40,60$ \\
\hline 3 & AA15 & 13,00 & 47,00 & 31,60 & 15,40 & 12,20 & 30,80 & 0,00 & 30,80 & 0,80 & 47,00 & 0,80 \\
\hline 4) & AA18 & 13,80 & 45,00 & 41,60 & 3,40 & 13,80 & 55,00 & 0,00 & 55,00 & 0,00 & 45,00 & $-13,40$ \\
\hline 5) & AA21 & 12,20 & 0,00 & 28,60 & $-28,60$ & 12,00 & 41,00 & 0,00 & 41,00 & 0,20 & 0,00 & $-12,40$ \\
\hline 6) & AA23 & 13,40 & 38,00 & 0,00 & 38,00 & 13,60 & 34,00 & 30,00 & 4,00 & $-0,20$ & 8,00 & $-34,00$ \\
\hline 7) & AA24 & 10,25 & 26,25 & 20,95 & 5,30 & 10,20 & 19,90 & 20,00 & $-0,10$ & 0,05 & 6,25 & 1,05 \\
\hline 8) & AA25 & 13,60 & 0,00 & 0,00 & 0,00 & 11,40 & 36,80 & 49,90 & $-13,10$ & 2,20 & $-49,90$ & $-36,80$ \\
\hline & AA26 & 11,00 & 30,00 & 31,60 & $-1,60$ & 13,80 & 59,60 & 63,20 & $-3,60$ & $-2,80$ & $-33,20$ & $-28,00$ \\
\hline 0) & AA27 & 12,40 & 30,40 & 37,80 & $-7,40$ & 13,20 & 34,00 & 39,00 & $-5,00$ & $-0,80$ & $-8,60$ & 3,80 \\
\hline 1) & AA31 & 9,20 & 37,80 & 42,40 & $-4,60$ & 10,20 & 26,80 & 30,40 & $-3,60$ & $-1,00$ & 7,40 & 15,60 \\
\hline 2) & AA32 & 12,00 & 37,20 & 38,20 & $-1,00$ & 12,80 & 36,80 & 48,20 & $-11,40$ & $-0,80$ & $-11,00$ & 1,40 \\
\hline 3) & AA34 & 13,00 & 33,20 & 37,60 & $-4,40$ & 12,20 & 37,20 & 31,20 & 6,00 & 0,80 & 2,00 & 0,40 \\
\hline 4) & AA44 & 12,40 & 50,00 & 41,20 & 8,80 & 12,00 & 48,20 & 40,60 & 7,60 & 0,40 & 9,40 & $-7,00$ \\
\hline 15) & AA46 & 12,00 & 48,40 & 62,80 & $-14,40$ & 12,20 & 46,40 & 41,00 & 5,40 & $-0,20$ & 7,40 & 16,40 \\
\hline 6) & AA47 & 11,40 & 36,80 & 38,40 & $-1,60$ & 11,80 & 38,40 & 30,20 & 8,20 & $-0,40$ & 6,60 & 0,00 \\
\hline 17) & AA55 & 13,80 & 40,00 & 43,40 & $-3,40$ & 12,60 & 35,60 & 31,60 & 4,00 & 1,20 & 8,40 & 7,80 \\
\hline 18) & AA61 & 12,60 & 39,60 & 44,60 & $-5,00$ & 10,80 & 31,00 & 28,80 & 2,20 & 1,80 & 10,80 & 13,60 \\
\hline 19) & AA64 & 11,20 & 39,40 & 36,40 & 3,00 & 12,60 & 38,60 & 40,20 & $-1,60$ & $-1,40$ & $-0,80$ & $-2,20$ \\
\hline 20) & AA65 & 11,20 & 40,40 & 0,00 & 40,40 & 12,00 & 47,40 & 0,00 & 47,40 & $-0,80$ & 40,40 & $-47,40$ \\
\hline
\end{tabular}

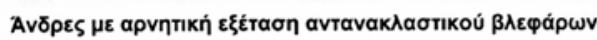

A/A LR1 L LR2 L RR2 LLR2-LRR2 RR1 R RR2 R LR2 RRR2-RLR2 LR1-RR1 LLR2-RLR2 LRR2-RRR2

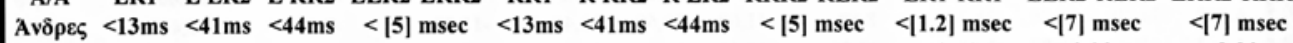

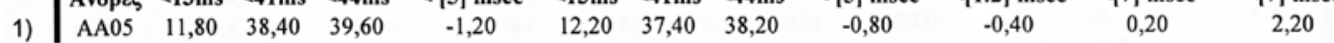




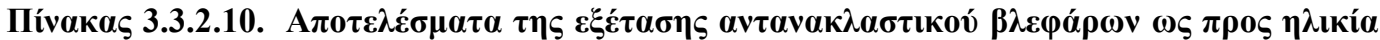

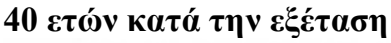

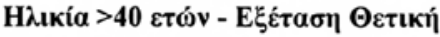

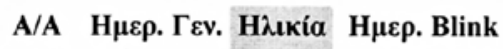

1) \begin{tabular}{lll|l} 
AA10 & $6 / 3 / 1936$ & 71 & $2 / 11 / 2006$
\end{tabular}

2) AA24 12/7/1938 $68 \quad 16 / 3 / 2006$

3) AA09 1/1/1947 $59 \quad 6 / 2 / 2006$

4) AA22 $18 / 2 / 1948 \quad 58 \quad 30 / 3 / 2006$

5) AA64 11/12/1953 53 27/9/2006

6) AA19 23/1/1955 $51 \quad 21 / 7 / 2006$

7) $\mathrm{AA} 15 \quad 3 / 1 / 1956 \quad 51 \quad 22 / 11 / 2006$

8) AA58 14/9/1956 50 $9 / 6 / 2006$

9) AA06 14/6/1957 $49 \quad 25 / 1 / 2007$

10) AA27 1/1/1958 $48 \quad 17 / 3 / 2006$

11) AA43 28/5/1958 $49 \quad 25 / 1 / 2007$

12) AA54 24/7/1959 $47 \quad 7 / 4 / 2006$

13) AA46 25/12/1959 $46 \quad 15 / 5 / 2006$

14) AA35 5/5/1960 $46 \quad 27 / 1 / 2006$

15) AA08 15/6/1960 $47 \quad 28 / 12 / 2006$

16) AA41 $12 / 11 / 1960 \quad 47 \quad 31 / 7 / 2007$

17) AA04 7/1/1961 $45 \quad 10 / 5 / 2006$

18) AA14 14/1/1961 $46 \quad 7 / 11 / 2006$

19) AA31 $18 / 4 / 1962 \quad 44 \quad 28 / 2 / 2006$

20) AA32 18/4/1962 $45 \quad 5 / 12 / 2006$

21) AA48 8/10/1963 $43 \quad 29 / 1 / 2007$

22) AA18 22/1/1963 $44 \quad 3 / 11 / 2006$

23) AA20 6/2/1965 $41 \quad 21 / 6 / 2006$

24) AA11 18/8/1965 $41 \quad 3 / 11 / 2006$

25) AA12 4/6/1966 $41 \quad 11 / 1 / 2007$

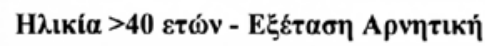

A/A H $\mu \varepsilon \rho . \Gamma \varepsilon v$. H人ıкí $\alpha$ H $\mu \varepsilon \rho$. Blink

1) AA05 14/12/1962 $44 \quad 28 / 12 / 2006$

2) AA36 7/10/1958 $47 \quad 24 / 2 / 2006$

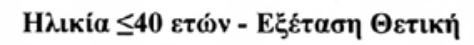

\begin{tabular}{|c|c|c|c|c|}
\hline & $\mathbf{A} / \mathbf{A}$ & 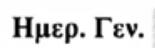 & 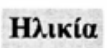 & 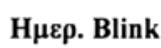 \\
\hline 1) & $\mathrm{AA} 02$ & $18 / 7 / 1966$ & 40 & $22 / 6 / 2006$ \\
\hline & $\mathrm{AA} 34$ & $2 / 10 / 1967$ & 39 & $3 / 8 / 2006$ \\
\hline & AA39 & $21 / 12 / 1967$ & 39 & $14 / 12 / 2006$ \\
\hline & AA29 & $4 / 2 / 1969$ & 37 & $29 / 3 / 2006$ \\
\hline & AA62 & $3 / 4 / 1970$ & 36 & $12 / 9 / 2006$ \\
\hline 6) & AA33 & $26 / 6 / 1970$ & 36 & $17 / 3 / 2006$ \\
\hline 7) & AA44 & 970 & 36 & $15 / 11 / 2006$ \\
\hline 8) & AA52 & $7 / 4 / 1971$ & 35 & $22 / 6 / 2006$ \\
\hline 9) & $\mathrm{AA} 03$ & $30 / 10 / 1971$ & 34 & $24 / 2 / 2006$ \\
\hline 10) & AA16 & $1 / 4 / 1972$ & 34 & $30 / 3 / 2006$ \\
\hline 11) & $\mathrm{AA} 21$ & $20 / 5 / 1972$ & 34 & $6 / 4 / 2006$ \\
\hline 12) & AA49 & $25 / 6$ & 34 & $28 / 2 / 2006$ \\
\hline 13) & AA28 & $11 / 8 / 1973$ & 33 & $12 / 1 / 2007$ \\
\hline 14) & AA60 & $26 / 12$ & 30 & 2006 \\
\hline 15) & $\mathrm{AA} 40$ & $8 / 1 /$ & 2 & $14 / 2$ \\
\hline 16) & AA57 & $7 / 2$ & 29 & 06 \\
\hline 17) & AA26 & $8 / 3 /$ & 29 & 2006 \\
\hline 18) & AA38 & $8 / 4$ & 29 & 12006 \\
\hline 19) & AA59 & $13 / 1$ & 29 & $0 / 5 / 2006$ \\
\hline 20) & AA63 & $17 / 10 / 1977$ & 29 & $30 / 10 / 2006$ \\
\hline 21) & AA47 & $8 / 12 / 1977$ & 29 & $16 / 11 / 2006$ \\
\hline 22) & AA01 & $15 / 12 / 1977$ & 29 & $6 / 10 / 2006$ \\
\hline 23) & AA55 & $17 / 4 / 1978$ & 28 & $30 / 3 / 2006$ \\
\hline 24) & AA 17 & $1 / 5 / 1978$ & 28 & $21 / 7 / 2006$ \\
\hline 25) & AA50 & $4 / 1 / 1$ & 27 & $4 / 7 / 2006$ \\
\hline 26) & AA65 & $1 / 10$ & 27 & 006 \\
\hline 27) & AA56 & $6 / 10$ & 26 & 2006 \\
\hline 28) & AA13 & $28 / 11 / 1980$ & 26 & $27 / 11 / 2006$ \\
\hline 29) & AA23 & $12 / 8 / 1981$ & 25 & $8 / 6 / 2006$ \\
\hline 30) & AA07 & $16 / 7 / 1982$ & 24 & $24 / 11 / 2006$ \\
\hline 31) & AA25 & 29/9/1986 & 20 & $31 / 3 / 2006$ \\
\hline 32) & AA53 & $7 / 3 / 1987$ & 19 & $27 / 3 / 2006$ \\
\hline 33) & AA61 & $3 / 8 / 1989$ & 17 & $12 / 4 / 2006$ \\
\hline 34) & AA30 & $4 / 10 / 1989$ & 17 & $26 / 9 / 2006$ \\
\hline
\end{tabular}

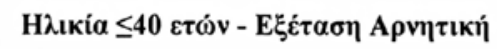

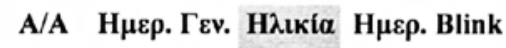

1) $\begin{array}{llll}\text { AA37 } & 23 / 12 / 1975 & 30 & 19 / 5 / 2006\end{array}$

2) AA42 7/3/1982 $24 \quad 22 / 6 / 2006$

3) AA45 18/8/1978 $28 \quad 12 / 5 / 2006$

4) AA51 22/10/1976 $30 \quad 21 / 7 / 2006$ 


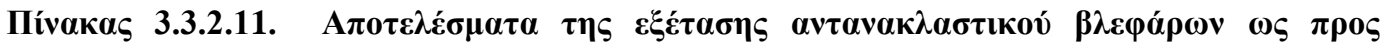

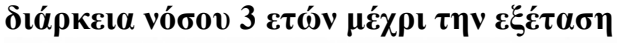

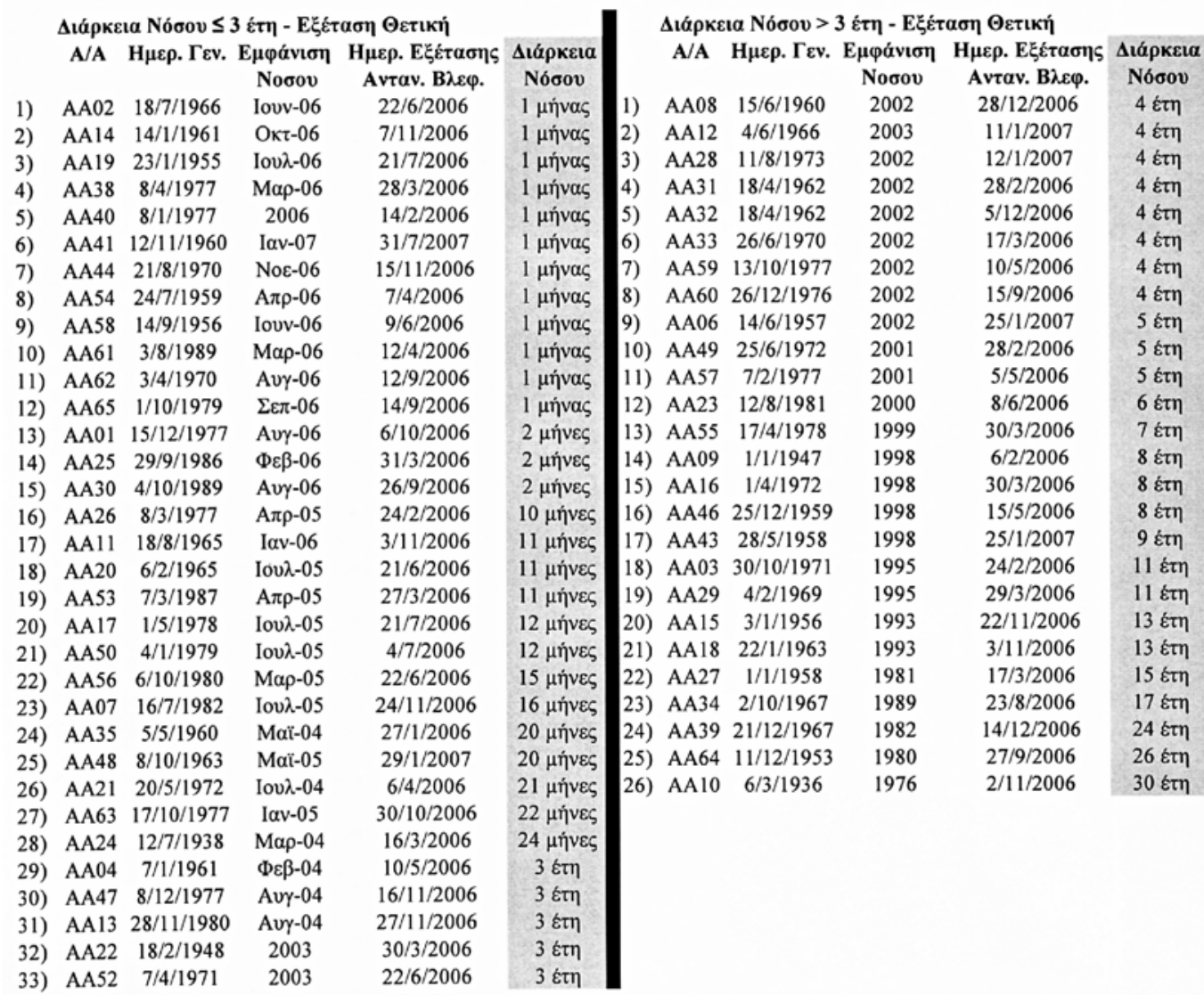

\begin{tabular}{|c|c|c|c|c|c|c|c|c|c|c|}
\hline \multicolumn{5}{|c|}{ 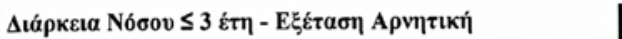 } & & \multicolumn{4}{|c|}{ 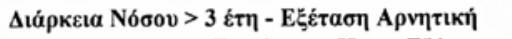 } & \multirow[b]{2}{*}{ 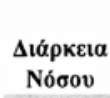 } \\
\hline $\mathbf{A} / \mathbf{A}$ & 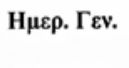 & 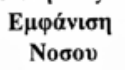 & 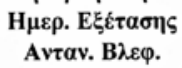 & 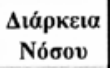 & & $\mathbf{A} / \mathbf{A}$ & 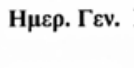 & 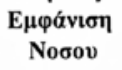 & 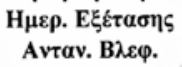 & \\
\hline A & & & $24 / 2 / 2006$ & & 1) & AA05 & 962 & 1998 & $28 / 12 / 2006$ & \\
\hline AA37 & $23 / 12 / 1975$ & Maï-06 & $19 / 5 / 2006$ & & & AA45 & $18 / 8 / 1978$ & 1998 & 006 & \\
\hline & $3 / 1982$ & Iovv-06 & $22 / 6 / 2006$ & $1 \mu \eta \dot{v a s}$ & & AA51 & $22 / 10 / 1976$ & 1997 & $21 / 7 / 2006$ & 9 हाп \\
\hline
\end{tabular}




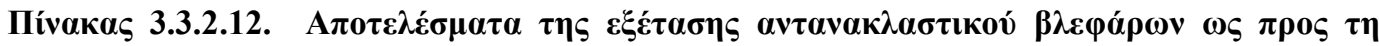

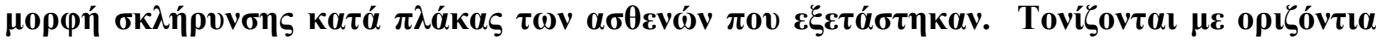

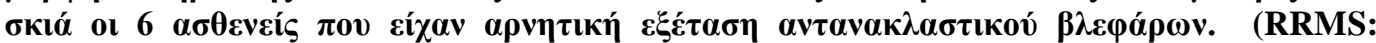

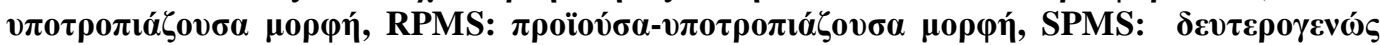
$\pi \rho 0 \ddot{0} 0$ $\sigma \alpha \mu \rho \rho \eta ์)$

\begin{tabular}{|c|c|c|c|c|c|c|c|c|c|c|c|c|c|}
\hline & $\mathbf{A} / \mathbf{A}$ & $\Phi 0 ́ \lambda_{0}$ & Нخıкía & 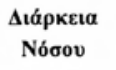 & 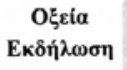 & $\begin{array}{l}\text { Морфі́ } \\
\text { Nóбov }\end{array}$ & & $\mathbf{A} / \mathbf{A}$ & Фúlo & H入ıкí $\alpha$ & 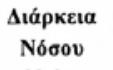 & 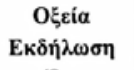 & 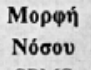 \\
\hline 1) & $\mathrm{AA} 01$ & $\Theta$ & 29 & $2 \mu$ ที่ & $O x_{1}$ & RRMS & 1) & $\mathrm{AA} 03$ & $\Theta$ & 34 & $11 \mathrm{Etm}$ & $O x_{1}$ & SPMS \\
\hline 2) & $\mathrm{AA} 12$ & $\Theta$ & 41 & 4 होग & $O x_{1}$ & RRMS & 2) & $\mathrm{AA} 05$ & A & 44 & 8 होग & $\mathrm{O}_{x 1}$ & SPMS \\
\hline 3) & $\mathrm{AA} 13$ & $\Theta$ & 26 & $2 \mathrm{\varepsilon tm}$ & $O_{x i}$ & RRMS & 3) & $\mathrm{AA} 10$ & A & 71 & $30 \mathrm{ktm}$ & $\mathrm{O} \times 1$ & SPMS \\
\hline 4) & AA25 & A & 20 & $2 \mu$ मांves & $\mathrm{O} x_{1}$ & RRMS & 4) & AA15 & A & 51 & 13 غ́זᄁ & $O x_{1}$ & SPMS \\
\hline 5) & AA28 & $\Theta$ & 33 & 4 ह́โๆ & $O x_{1}$ & RRMS & 5) & AA16 & $\Theta$ & 34 & $8 \mathrm{kt \tau}$ & $O_{x i}$ & SPMS \\
\hline 6) & $\mathrm{AA} 30$ & $\Theta$ & 17 & $2 \mu \eta ́ v \varepsilon \varepsilon$ & $O x_{1}$ & RRMS & 6) & AA18 & A & 44 & $13 \mathrm{~km}$ & $\mathrm{O} x 1$ & SPMS \\
\hline 7) & AA33 & $\Theta$ & 36 & $4 \mathrm{\varepsilon t \tau}$ & $O x_{1}$ & RRMS & 7) & AA23 & A & 25 & $6 \mathrm{Etm}$ & $O x_{1}$ & SPMS \\
\hline 8) & AA47 & A & 29 & $2 \mathrm{\varepsilon t \eta}$ & $\mathrm{O} \chi_{1}$ & RRMS & 8) & AA24 & A & 68 & $24 \mu \eta \dot{v \varepsilon \varsigma}$ & $\mathrm{O}_{x i}$ & SPMS \\
\hline 9) & AA51 & $\Theta$ & 30 & $9 \mathrm{\varepsilon tm}$ & $O x_{1}$ & RRMS & 9) & AA27 & A & 48 & 15 غ́โท & $O_{x 1}$ & SPMS \\
\hline 10) & AA52 & $\Theta$ & 35 & 3 हैtा & $\mathrm{O} \times 1$ & RRMS & 10) & AA29 & $\Theta$ & 37 & 11 होग & $\mathrm{O}_{\mathrm{X}}$ & SPMS \\
\hline 11) & $\mathrm{AA} 02$ & $\Theta$ & 40 & $1 \mu \eta ́ v a \varsigma$ & $\mathrm{Nal}$ & RRMS & 11) & AA34 & A & 39 & 17 ह́ता & $\mathrm{O} x_{1}$ & SPMS \\
\hline 12) & AA04 & A & 45 & $2 \mathrm{\varepsilon tm}$ & $\mathrm{Nat}$ & RRMS & 12) & AA39 & $\Theta$ & 39 & 24 होग & $O_{x_{1}}$ & SPMS \\
\hline 13) & AA06 & $\Theta$ & 49 & 5 Étᄁ & $\mathrm{Nal}$ & RRMS & 13) & AA64 & A & 53 & $26 \mathrm{Et \pi}$ & $\mathrm{O} x \mathrm{l}$ & SPMS \\
\hline 14) & AA07 & $\Theta$ & 24 & 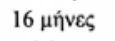 & $\mathrm{N} \alpha \mathrm{l}$ & RRMS & & & & & & & \\
\hline 15) & AA08 & $\Theta$ & 47 & 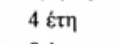 & $\mathrm{Nal}$ & RRMS & & & & & & & \\
\hline 16) & AA09 & $\Theta$ & 59 & $8 \dot{\varepsilon} \tau \eta$ & $\mathrm{Nal}$ & RRMS & & & & & & & \\
\hline 17) & AA14 & $\Theta$ & 46 & $1 \mu \eta ์$ vas & $\mathrm{N} \alpha 1$ & RRMS & & & & & & & \\
\hline 18) & AA17 & $\Theta$ & 28 & $12 \mu \eta \dot{v \varepsilon \varsigma}$ & $\mathrm{Nat}$ & RRMS & 1) & AA11 & $\Theta$ & 41 & $11 \mu \eta ́ v \varepsilon \varsigma$ & $\mathrm{Nal}$ & RPMS \\
\hline 19) & AA19 & $\Theta$ & 51 & $1 \mu \eta \dot{v} \vee \varsigma$ & $\mathrm{Nal}$ & RRMS & 2) & $\mathrm{AA} 21$ & A & 34 & $21 \mu \eta ీ v \varepsilon \varsigma$ & $\mathrm{N} \alpha \mathrm{t}$ & RPMS \\
\hline 20) & AA20 & $\Theta$ & 41 & $11 \mu$ ก́ves & $\mathrm{N} \alpha \mathbf{l}$ & RRMS & 3) & $\mathrm{AA} 31$ & A & 44 & 4 हाน & $\mathrm{Nal}$ & RPMS \\
\hline 21) & AA22 & $\Theta$ & 58 & $3 \mathrm{kt \eta}$ & $\mathrm{Nat}$ & RRMS & 4) & AA49 & $\Theta$ & 34 & 5 Étग & $\mathrm{Nal}$ & RPMS \\
\hline 22) & AA26 & A & 29 & $10 \mu \eta \dot{v \varepsilon \varsigma}$ & $\mathrm{N} \alpha \mathbf{l}$ & RRMS & 5) & AA54 & $\Theta$ & 47 & 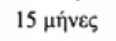 & $\mathrm{N} \alpha \mathrm{l}$ & RPMS \\
\hline 23) & AA35 & $\Theta$ & 46 & 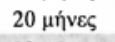 & $\mathrm{N} \alpha \mathbf{l}$ & RRMS & 6) & $\mathrm{AA} 32$ & A & 45 & 4 हाँ & $O_{x_{1}}$ & RPMS \\
\hline 24) & AA36 & $\Theta$ & 47 & $13 \mu \eta \dot{V} \in \varsigma$ & $\mathrm{Nat}$ & RRMS & 7) & AA55 & A & 28 & $7 \mathrm{Et \eta}$ & $O x_{1}$ & RPMS \\
\hline 25) & AA37 & $\Theta$ & 30 & 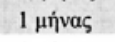 & $\mathrm{N} \alpha \mathrm{t}$ & RRMS & & & & & & & \\
\hline 26) & AA38 & $\Theta$ & 29 & $1 \mu \eta ́ v \alpha \varsigma$ & $\mathrm{N} \alpha 1$ & RRMS & & & & & & & \\
\hline 27) & AA40 & $\Theta$ & 29 & $1 \mu \eta \dot{v} v \alpha \zeta$ & $\mathrm{Nat}$ & RRMS & & & & & & & \\
\hline 28) & AA41 & $\Theta$ & 47 & $1 \mu \eta \dot{v} v \alpha \varsigma$ & $\mathrm{N} \alpha \mathrm{l}$ & RRMS & & & & & & & \\
\hline 29) & $\mathrm{AA} 42$ & $\Theta$ & 24 & $1 \mu \eta ́ v \alpha \varsigma$ & $\mathrm{Nal}$ & RRMS & & & & & & & \\
\hline 30) & AA43 & $\Theta$ & 49 & $9 \varepsilon \dot{z \eta}$ & $\mathrm{Nat}$ & RRMS & & & & & & & \\
\hline 31) & AA44 & A & 36 & $1 \mu \eta \dot{v a s}$ & $\mathrm{Nal}$ & RRMS & & & & & & & \\
\hline 32) & AA45 & $\Theta$ & 28 & $8 \varepsilon t \eta$ & $\mathrm{Nal}$ & RRMS & & & & & & & \\
\hline 33) & AA46 & A & 46 & $8 \varepsilon \dot{\varepsilon} \tau$ & $\mathrm{Nal}$ & RPMS & & & & & & & \\
\hline 34) & AA48 & $\Theta$ & 43 & $20 \mu \eta \mathfrak{v \varepsilon \varsigma}$ & $\mathrm{Nal}$ & RRMS & & & & & & & \\
\hline 35) & AA50 & $\Theta$ & 27 & $12 \mu \eta ́ v \varepsilon \varepsilon$ & $\mathrm{Nal}$ & RRMS & & & & & & & \\
\hline 36) & AA53 & $\Theta$ & 19 & $11 \mu \eta ́ v \varepsilon \varsigma$ & $\mathrm{N} \alpha \mathrm{t}$ & RRMS & & & & & & & \\
\hline 37) & AA56 & $\Theta$ & 26 & 1 Éо૬ & $\mathrm{N} \alpha \mathrm{l}$ & RRMS & & & & & & & \\
\hline 38) & AA57 & $\Theta$ & 29 & $5 \mathrm{ktm}$ & $\mathrm{Nal}$ & RRMS & & & & & & & \\
\hline 39) & AA58 & $\Theta$ & 50 & $1 \mu \eta \dot{v} v \alpha \varsigma$ & $\mathrm{N} \alpha \mathrm{l}$ & RRMS & & & & & & & \\
\hline 40) & AA59 & $\Theta$ & 29 & 4 हाग & $\mathrm{Nal}$ & RRMS & & & & & & & \\
\hline 41) & AA60 & $\Theta$ & 30 & $4 \mathrm{\varepsilon tᄁ}$ & $\mathrm{N} \alpha \mathrm{\iota}$ & RRMS & & & & & & & \\
\hline 42) & AA61 & A & 17 & $1 \mu$ ท́vas & $\mathrm{Nat}$ & RRMS & & & & & & & \\
\hline 43) & AA62 & $\Theta$ & 36 & 1 нйvas & $\mathrm{N} \alpha \mathrm{t}$ & RRMS & & & & & & & \\
\hline 44) & AA63 & $\Theta$ & 29 & 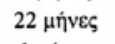 & $\mathrm{Nat}$ & RRMS & & & & & & & \\
\hline 45) & AA65 & A & 27 & $1 \mu \eta \dot{v a \varsigma}$ & $\mathrm{N} \alpha \mathbf{l}$ & RRMS & & & & & & & \\
\hline
\end{tabular}




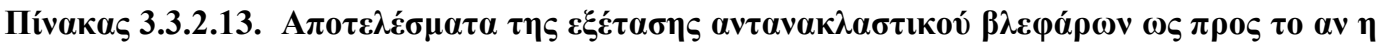

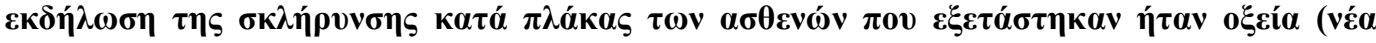

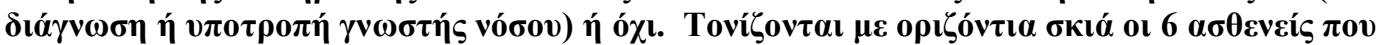

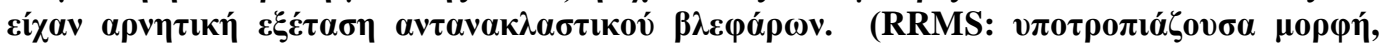

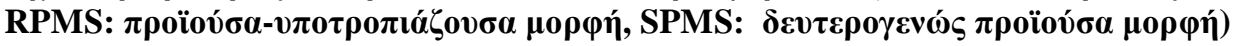

\begin{tabular}{|c|c|c|c|c|c|c|c|c|c|c|c|c|c|}
\hline & $\mathbf{A} / \mathbf{A}$ & Фv́خo & 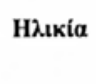 & 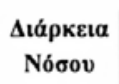 & 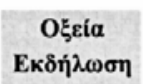 & 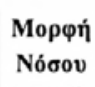 & & $\mathbf{A} / \mathbf{A}$ & Фủं & НАлккі $\alpha$ & 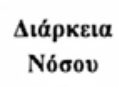 & 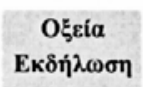 & 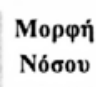 \\
\hline 1) & AA01 & $\Theta$ & 29 & $2 \mu \eta \dot{v} \varepsilon \varsigma$ & $O_{x 1}$ & RRMS & 1) & AA02 & $\Theta$ & 40 & $1 \mu \eta \dot{v} \times a \varsigma$ & $\mathrm{N} \alpha \mathbf{r}$ & RRMS \\
\hline 2) & AA12 & $\Theta$ & 41 & 4 होंग & $O_{x}$ & RRMS & 2) & AA04 & A & 45 & $2 \varepsilon \bar{\varepsilon}$ & $\mathrm{N} \alpha \mathrm{l}$ & RRMS \\
\hline 3) & AA13 & $\Theta$ & 26 & 2 દ́tᄁ & $\mathrm{O}_{x \mathrm{I}}$ & RRMS & 3) & AA06 & $\Theta$ & 49 & $5 \varepsilon \hat{\varepsilon ́ \eta}$ & $\mathrm{N} \alpha \mathbf{l}$ & RRMS \\
\hline 4) & AA25 & A & 20 & $2 \mu \eta ́ v \varepsilon \varsigma$ & $O x a$ & RRMS & 4) & AA07 & $\Theta$ & 24 & 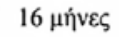 & $\mathrm{N} \alpha \iota$ & RRMS \\
\hline 5) & AA28 & $\Theta$ & 33 & 4 ह́tा & $O x 1$ & RRMS & 5) & AA08 & $\Theta$ & 47 & 4 ह่ात & $\mathrm{N} \alpha \mathbf{l}$ & RRMS \\
\hline 6) & $\mathrm{AA} 30$ & $\Theta$ & 17 & $2 \mu \eta \dot{v \varepsilon \varepsilon}$ & $O_{x 1}$ & RRMS & 6) & AA09 & $\Theta$ & 59 & $8 \varepsilon \dot{\varepsilon} \pi$ & $\mathrm{N} \alpha \mathbf{\imath}$ & RRM \\
\hline 7) & AA33 & $\Theta$ & 36 & $4 \dot{\varepsilon} \pi \eta$ & $O_{x 1}$ & RRMS & 7) & AA14 & $\Theta$ & 46 & $1 \mu \eta \dot{v} \alpha \varsigma$ & $\mathrm{Nal}$ & RRM \\
\hline 8) & AA47 & A & 29 & $2 \varepsilon \tilde{\xi}$ & $O x 1$ & RRMS & 8) & AA17 & $\Theta$ & 28 & $12 \mu \eta \dot{v} \vee \varsigma$ & $\mathrm{N} \alpha \mathbf{l}$ & RRM \\
\hline 9) & AA51 & $\Theta$ & 30 & $9 \mathrm{\varepsilon tm}$ & $O x_{1}$ & RRMS & 9) & AA19 & $\Theta$ & 51 & $1 \mu \eta \dot{v a \varsigma}$ & $\mathrm{N} \alpha \mathbf{l}$ & RRM \\
\hline \multirow[t]{2}{*}{ 10) } & AA52 & $\Theta$ & 35 & $3 \dot{\varepsilon ்}$ & 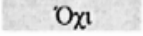 & RRMS & 10) & AA20 & $\Theta$ & 41 & $11 \mu \eta \dot{V E S}$ & $\mathrm{Nal}$ & RRM: \\
\hline & & & & & & & 11) & AA22 & $\Theta$ & 58 & 3 ह́ंग & $\mathrm{N} \alpha \mathbf{l}$ & RRM \\
\hline 11) & AA03 & $\Theta$ & 34 & 11 ह́ரП & $\mathrm{O} \times \mathrm{l}$ & SPMS & 12) & AA26 & A & 29 & $10 \mu \eta ́ v \varepsilon \varsigma$ & $\mathrm{N} \alpha \mathrm{t}$ & RRM \\
\hline 12) & AA05 & A & 44 & $8 \mathrm{ktm}$ & $O x_{1}$ & SPMS & 13) & AA35 & $\Theta$ & 46 & $20 \mu$ İVES & $\mathrm{Nat}$ & RRM \\
\hline 13) & AA10 & A & 71 & 30 ह่ரๆ & $\mathrm{O}_{x 1}$ & SPMS & 14) & $\mathrm{AA} 36$ & $\Theta$ & 47 & $13 \mu \eta \dot{v} \vee \varepsilon \varsigma$ & $N \alpha \imath$ & RRM \\
\hline 14) & AA15 & A & 51 & 13 Étᄁ & $O x_{1}$ & SPMS & 15) & AA37 & $\Theta$ & 30 & $1 \mu \eta \underline{v a s}$ & $\mathrm{Nal}$ & RRM \\
\hline 15) & AA16 & $\Theta$ & 34 & $8 \varepsilon \tilde{k}$ & $\mathrm{O}_{x 1}$ & SPMS & 16) & AA38 & $\Theta$ & 29 & $1 \mu \eta \underline{v a \zeta}$ & $\mathrm{N} \alpha \mathbf{t}$ & RRM \\
\hline 16) & AA18 & A & 44 & $13 \mathrm{kT \eta}$ & $O x$ & SPMS & 17) & $\mathrm{AA} 40$ & $\Theta$ & 29 & $1 \mu \eta \dot{v} \alpha{ }_{S}$ & $\mathrm{~N} \alpha \mathbf{1}$ & RRM \\
\hline 17) & AA23 & A & 25 & $6 \dot{\varepsilon} \tau \eta$ & $O x$ & SPMS & 18) & AA41 & $\Theta$ & 47 & $1 \mu \eta v^{\prime} \alpha \varsigma$ & $\mathrm{Nal}$ & RRM \\
\hline 18) & AA24 & A & 68 & $24 \mu \eta ́ v \varepsilon \varsigma$ & $O_{x_{1}}$ & SPMS & 19) & AA42 & $\Theta$ & 24 & $1 \mu \eta \dot{v}$ as & $\mathrm{N} \alpha \mathbf{l}$ & RRN \\
\hline 19) & AA27 & A & 48 & $15 \mathrm{kt \eta}$ & $O x_{1}$ & SPMS & 20) & AA43 & $\Theta$ & 49 & $9 \dot{\varepsilon} \tau \eta$ & $\mathrm{N} \alpha \mathbf{l}$ & RRM \\
\hline 20) & AA29 & $\Theta$ & 37 & 11 हाँा & $O x$ & SPMS & 21) & AA44 & A & 36 & $1 \mu \eta \dot{v} \alpha{ }^{\prime}$ & $\mathrm{N} \alpha \mathbf{l}$ & RRN \\
\hline 21) & AA34 & A & 39 & 17 غॉтך & $O x_{1}$ & SPMS & 22) & AA45 & $\Theta$ & 28 & $8 \mathrm{\varepsilon tm}$ & $\mathrm{N} \alpha$ & RRN \\
\hline 22) & AA39 & $\Theta$ & 39 & $24 \varepsilon \tau \eta$ & $\mathrm{O} x \mathrm{x}$ & SPMS & 23) & AA46 & A & 46 & $8 \varepsilon \pi \eta$ & $\mathrm{N} \alpha \mathrm{l}$ & RPM \\
\hline \multirow[t]{2}{*}{ 23) } & AA64 & A & 53 & 26 غॉๆ & $O x_{1}$ & SPMS & 24) & AA48 & $\Theta$ & 43 & $20 \mu \eta \dot{v} \nu \varepsilon \varsigma$ & $\mathrm{N} \alpha \mathrm{l}$ & RRM \\
\hline & & & & & & & 25) & AA50 & $\Theta$ & 27 & $12 \mu \eta \dot{v} \vee \varepsilon \varsigma$ & $\mathrm{N} \alpha \mathrm{I}$ & RRN \\
\hline 24) & AA32 & A & 45 & $4 \dot{\varepsilon} \pi \eta$ & $O x_{1}$ & RPMS & 26) & AA53 & $\Theta$ & 19 & $11 \mu$ İves & $\mathrm{Nat}$ & RRM \\
\hline \multirow[t]{14}{*}{ 25) } & AA55 & A & 28 & 7 Étᄁ & $\mathrm{O}_{\mathrm{x}}$ & RPMS & 27) & AA56 & $\Theta$ & 26 & 1 Éто૬ & $\mathrm{Nat}$ & RRMS \\
\hline & & & & & & & 28) & AA57 & $\Theta$ & 29 & $5 \varepsilon \mathrm{\varepsilon t \eta}$ & $\mathrm{N} \alpha \mathbf{l}$ & RRN \\
\hline & & & & & & & 29) & AA58 & $\Theta$ & 50 & $1 \mu \eta \dot{v} v \alpha \varsigma$ & $\mathrm{N} \alpha \mathbf{l}$ & RRN \\
\hline & & & & & & & 30) & AA59 & $\Theta$ & 29 & $4 \varepsilon \tilde{\xi} \eta$ & $\mathrm{N} \alpha \mathrm{t}$ & RRN \\
\hline & & & & & & & 31) & AA60 & $\Theta$ & 30 & $4 \varepsilon \dot{\varepsilon} \pi$ & $\mathrm{Nal}$ & RRN \\
\hline & & & & & & & 32) & AA61 & A & 17 & $1 \mu \eta \underline{v} \alpha \varsigma$ & $\mathrm{N \alpha l}$ & RRI \\
\hline & & & & & & & 33) & AA62 & $\Theta$ & 36 & $1 \mu \eta \dot{v} v \alpha \varsigma$ & $\mathrm{Nat}$ & RRA \\
\hline & & & & & & & 34) & AA63 & $\Theta$ & 29 & $22 \mu \eta ́ v \varepsilon \varsigma$ & $\mathrm{Nal}$ & RRN \\
\hline & & & & & & & 35) & AA65 & A & 27 & $1 \mu \eta v^{\prime} \mathrm{s}$ & $\mathrm{Nat}$ & RRN \\
\hline & & & & & & & 36) & AA11 & $\Theta$ & 41 & $11 \mu \eta \dot{v \varepsilon \varepsilon}$ & $\mathrm{Nat}$ & RPM \\
\hline & & & & & & & 37) & AA21 & A & 34 & $21 \mu \eta \mathfrak{v e \varsigma}$ & $\mathrm{N} \alpha$ & RPN \\
\hline & & & & & & & 38) & AA31 & A & 44 & $4 \varepsilon \dot{\varepsilon} t \eta$ & $\mathrm{Nal}$ & RPM \\
\hline & & & & & & & 39) & AA49 & $\Theta$ & 34 & $5 \dot{\varepsilon} \pi$ & $\mathrm{Nal}$ & RPN \\
\hline & & & & & & & 40) & AA54 & $\Theta$ & 47 & $15 \mu \eta \underline{v \varepsilon \varsigma}$ & $\mathrm{N} \alpha \mathrm{r}$ & RPN \\
\hline
\end{tabular}




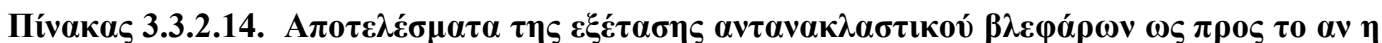

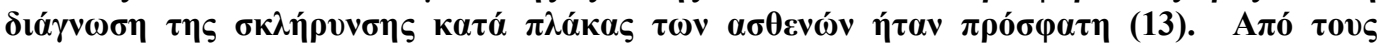

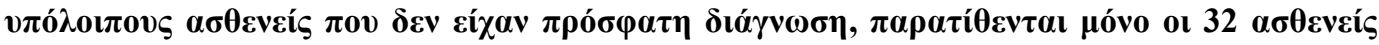

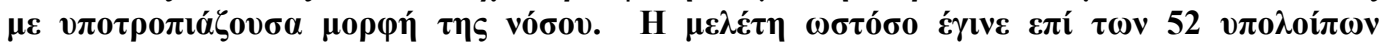

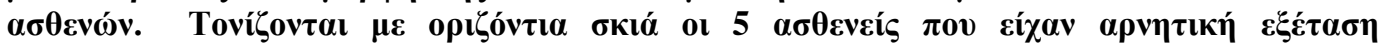

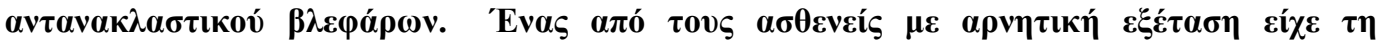

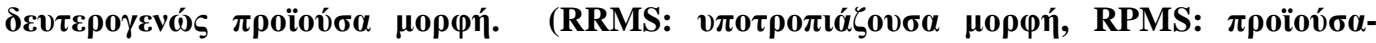

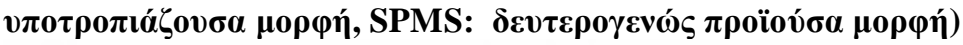

\begin{tabular}{|c|c|c|c|c|c|c|c|c|c|c|}
\hline & $\mathbf{A} / \mathbf{A}$ & Фט́ภo & 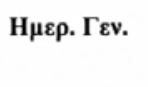 & 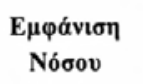 & H $\mu \varepsilon \rho$. Blink & Н入ıкі́а & 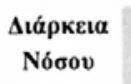 & $\begin{array}{c}\text { Néa } \\
\Delta \backslash \alpha \dot{\gamma} \gamma v \omega \sigma \eta\end{array}$ & 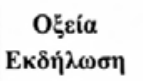 & 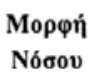 \\
\hline 1) & $\mathrm{AA} 02$ & $\Theta$ & 18/7/1966 & Iovv-06 & $22 / 6 / 2006$ & 40 & $1 \mu$ ท́ $v \alpha \varsigma$ & $\mathrm{N} \alpha \mathbf{\imath}$ & $\mathrm{N} \alpha \mathrm{l}$ & RRMS \\
\hline 2) & AA14 & $\Theta$ & $14 / 1 / 1961$ & Oкт-06 & $7 / 11 / 2006$ & 46 & $1 \mu \eta ́ v a \varsigma$ & $\mathrm{N} \alpha \mathrm{l}$ & $\mathrm{Nal}$ & RRMS \\
\hline 3) & AA19 & $\Theta$ & $23 / 1 / 1955$ & Iov $\lambda=06$ & $21 / 7 / 2006$ & 51 & $1 \mu \eta ́ v \alpha \varsigma$ & $\mathrm{Nal}$ & $\mathrm{Nal}$ & RRMS \\
\hline 4) & AA37 & $\Theta$ & $23 / 12 / 1975$ & Maï-06 & $19 / 5 / 2006$ & 30 & 1 ํำas & $\mathrm{N} \alpha \mathbf{l}$ & $\mathrm{Nal}$ & RRMS \\
\hline 5) & AA38 & $\Theta$ & $8 / 4 / 1977$ & M $\alpha \rho-06$ & $28 / 3 / 2006$ & 29 & $1 \mu \eta \dot{v a s}$ & $\mathrm{Nal}$ & $\mathrm{Nal}$ & RRMS \\
\hline 6) & AA40 & $\Theta$ & $8 / 1 / 1977$ & 2006 & $14 / 2 / 2006$ & 29 & 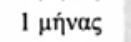 & $\mathrm{N} \alpha \mathbf{l}$ & $\mathrm{N} \alpha \mathrm{l}$ & RRMS \\
\hline 7) & AA41 & $\Theta$ & $12 / 11 / 1960$ & I $\alpha v-07$ & $31 / 7 / 2007$ & 47 & $1 \mu$ İvas & $\mathrm{N} \alpha \mathbf{l}$ & $\mathrm{N} \alpha \mathrm{l}$ & RRMS \\
\hline 8) & AA42 & $\Theta$ & $7 / 3 / 1982$ & Iovv-06 & $22 / 6 / 2006$ & 24 & $1 \mu \underline{\eta} v \alpha \varsigma$ & $\mathrm{N} \alpha \mathbf{l}$ & $\mathrm{N} \alpha \mathrm{l}$ & RRMS \\
\hline 9) & AA44 & A & $21 / 8 / 1970$ & No\&-06 & $15 / 11 / 2006$ & 36 & $1 \mu \eta \dot{v} \mathrm{v}$ & $\mathrm{N} \alpha \mathrm{l}$ & $\mathrm{N} \alpha \mathrm{l}$ & RRMS \\
\hline 10) & AA58 & $\Theta$ & $14 / 9 / 1956$ & Iouv-06 & 9/6/2006 & 50 & $1 \mu \eta \dot{v} \mathrm{v} \alpha$ & $\mathrm{N} \alpha \mathbf{l}$ & $\mathrm{N} \alpha 1$ & RRMS \\
\hline 11) & AA61 & A & $3 / 8 / 1989$ & M $\alpha \rho-06$ & $12 / 4 / 2006$ & 17 & 1 ні́vas & $\mathrm{Nal}$ & $\mathrm{Nal}$ & RRMS \\
\hline 12) & AA62 & $\Theta$ & $3 / 4 / 1970$ & Avy-06 & $12 / 9 / 2006$ & 36 & $1 \mu \eta \dot{v}$ va & $\mathrm{N} \alpha \mathbf{l}$ & $\mathrm{Nal}$ & RRMS \\
\hline 13) & AA65 & A & $1 / 10 / 1979$ & $\sum \varepsilon \pi-06$ & $14 / 9 / 2006$ & 27 & $1 \mu \eta \dot{v} \alpha \varsigma$ & $\mathrm{Nal}$ & $\mathrm{Nal}$ & RRMS \\
\hline 14) & AA04 & A & $7 / 1 / 1961$ & $\Phi \varepsilon \beta-04$ & $10 / 5 / 2006$ & 45 & 2 हाँ & $O x$ & $\mathrm{Nal}$ & RRMS \\
\hline 15) & AA06 & $\Theta$ & $14 / 6 / 1957$ & 2002 & $25 / 1 / 2007$ & 49 & $5 \varepsilon \pi$ & $\mathrm{O}_{x \mathrm{I}}$ & $\mathrm{N} \alpha \mathrm{l}$ & RRMS \\
\hline 16) & $\mathrm{AA} 07$ & $\Theta$ & $16 / 7 / 1982$ & Iov $\lambda-05$ & $24 / 11 / 2006$ & 24 & $16 \mu \eta \dot{v \varepsilon \varsigma}$ & $O x_{1}$ & $\mathrm{Nal}$ & RRMS \\
\hline 17) & AA08 & $\Theta$ & $15 / 6 / 1960$ & $\Delta \varepsilon \mathrm{k}-02$ & $28 / 12 / 2006$ & 47 & 4 ह́זा & $O x_{1}$ & $\mathrm{Nal}$ & RRMS \\
\hline 18) & AA09 & $\Theta$ & $1 / 1 / 1947$ & 1998 & $6 / 2 / 2006$ & 59 & 8 ह் & $O x 1$ & $\mathrm{~N} \alpha \mathrm{l}$ & RRMS \\
\hline 19) & AA17 & $\Theta$ & $1 / 5 / 1978$ & Iov $\lambda-05$ & $21 / 7 / 2006$ & 28 & 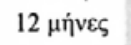 & $O x_{1}$ & $\mathrm{Nal}$ & RRMS \\
\hline 20) & AA20 & $\Theta$ & $6 / 2 / 1965$ & Iov $\lambda-05$ & $21 / 6 / 2006$ & 41 & $11 \mu \eta \dot{v \varepsilon \varsigma}$ & $O x i$ & $\mathrm{Nal}$ & RRMS \\
\hline 21) & AA22 & $\Theta$ & $18 / 2 / 1948$ & I $\alpha v-03$ & $30 / 3 / 2006$ & 58 & $3 \mathrm{Etm}$ & $\mathrm{OX}_{\mathrm{l}}$ & $\mathrm{N} \alpha \mathrm{l}$ & RRMS \\
\hline 22) & AA26 & A & $8 / 3 / 1977$ & A $\pi \rho-05$ & $24 / 2 / 2006$ & 29 & $10 \mu \eta \mathfrak{v \varepsilon \varsigma}$ & $O x$ & $\mathrm{~N} \alpha \mathrm{l}$ & RRMS \\
\hline 23) & AA35 & $\Theta$ & $5 / 5 / 1960$ & Maì-04 & $27 / 1 / 2006$ & 46 & $20 \mu \eta ́ v \varepsilon \varsigma$ & $O_{x l}$ & $\mathrm{Nal}$ & RRMS \\
\hline 24) & AA36 & $\Theta$ & $7 / 10 / 1958$ & 21-I $\alpha v$ & $24 / 2 / 2006$ & 47 & $13 \mu \eta ́ v \varepsilon \varsigma$ & $O x_{1}$ & $\mathrm{Nal}$ & RRMS \\
\hline 25) & $\mathrm{AA} 43$ & $\Theta$ & $28 / 5 / 1958$ & 1998 & $25 / 1 / 2007$ & 49 & $9 \varepsilon \pi \eta$ & $\mathrm{O} x_{1}$ & $\mathrm{Nal}$ & RRMS \\
\hline 26) & AA45 & $\Theta$ & $18 / 8 / 1978$ & 1998 & $12 / 5 / 2006$ & 28 & $8 \mathrm{\varepsilon tm}$ & $O x_{1}$ & $\mathrm{Nal}$ & RRMS \\
\hline 27) & AA46 & A & $25 / 12 / 1959$ & 1998 & $15 / 5 / 2006$ & 46 & $8 \dot{\varepsilon} \pi \eta$ & $O x_{1}$ & $\mathrm{Nal}$ & RPMS \\
\hline 28) & AA48 & $\Theta$ & $8 / 10 / 1963$ & Maï-05 & $29 / 1 / 2007$ & 43 & $20 \mu \eta \dot{v \varepsilon \varsigma}$ & $O_{x 1}$ & $\mathrm{~N} \alpha \mathrm{l}$ & RRMS \\
\hline 29) & AA50 & $\Theta$ & $4 / 1 / 1979$ & Iov $\lambda-05$ & $4 / 7 / 2006$ & 27 & $12 \mu \eta ் v \varepsilon \varsigma$ & $O x_{1}$ & $\mathrm{~N} \alpha \mathrm{l}$ & RRMS \\
\hline 30) & AA53 & $\Theta$ & $7 / 3 / 1987$ & $\mathrm{~A} \pi \rho-05$ & $27 / 3 / 2006$ & 19 & $11 \mu \eta \dot{v \varepsilon \varsigma}$ & $O_{x l}$ & $\mathrm{Nal}$ & RRMS \\
\hline 31) & AA56 & $\Theta$ & $6 / 10 / 1980$ & M $\alpha \rho-05$ & $22 / 6 / 2006$ & 26 & 1 غ́tos & $O x_{1}$ & $\mathrm{~N} \alpha \mathrm{l}$ & RRMS \\
\hline 32) & AA57 & $\Theta$ & $7 / 2 / 1977$ & 2001 & $5 / 5 / 2006$ & 29 & $5 \dot{\varepsilon} \tau \eta$ & $O_{x a}$ & $\mathrm{~N} \alpha \mathrm{l}$ & RRMS \\
\hline 33) & AA59 & $\Theta$ & $13 / 10 / 1977$ & 2002 & $10 / 5 / 2006$ & 29 & $4 \varepsilon \tilde{~ \varepsilon ॉ ~}$ & $O_{x 1}$ & $\mathrm{Nal}$ & RRMS \\
\hline 34) & AA60 & $\Theta$ & $26 / 12 / 1976$ & $\Sigma \varepsilon \pi-02$ & $15 / 9 / 2006$ & 30 & 4 Étᄁ & $O x_{1}$ & $\mathrm{Nal}$ & RRMS \\
\hline 35) & AA63 & $\Theta$ & $17 / 10 / 1977$ & I $\alpha v-05$ & $30 / 10 / 2006$ & 29 & $22 \mu \eta \dot{v \varepsilon \varepsilon \varsigma}$ & $0 x$ & $\mathrm{~N} \alpha \mathrm{l}$ & RRMS \\
\hline 36) & $\mathrm{AA} 01$ & $\Theta$ & $15 / 12 / 1977$ & Avy-06 & $6 / 10 / 2006$ & 29 & $2 \mu \eta \dot{v \varepsilon \zeta}$ & $O_{x 1}$ & $O x_{1}$ & RRMS \\
\hline 37) & $\mathrm{AA} 12$ & $\Theta$ & $4 / 6 / 1966$ & 2003 & $11 / 1 / 2007$ & 41 & $4 \dot{\varepsilon} \tau \eta$ & $O_{x_{1}}$ & $\mathrm{O}_{x_{1}}$ & RRMS \\
\hline 38) & AA13 & $\Theta$ & $28 / 11 / 1980$ & Avy-04 & $27 / 11 / 2006$ & 26 & $2 \varepsilon$ & $O_{x l}$ & $O_{x 1}$ & RRMS \\
\hline 39) & $\mathrm{AA} 25$ & A & 29/9/1986 & $\Phi \varepsilon \beta-06$ & $31 / 3 / 2006$ & 20 & $2 \mu \eta \dot{v \varepsilon \varsigma}$ & $\mathrm{O}_{x 1}$ & $O x_{1}$ & RRMS \\
\hline 40) & $\mathrm{AA} 28$ & $\Theta$ & $11 / 8 / 1973$ & Окт-02 & $12 / 1 / 2007$ & 33 & 4 होग & $O x_{1}$ & $O x_{1}$ & RRMS \\
\hline 41) & $\mathrm{AA} 30$ & $\Theta$ & $4 / 10 / 1989$ & Avy-06 & $26 / 9 / 2006$ & 17 & $2 \mu \eta ́ v \varepsilon \varepsilon$ & $\mathrm{O}_{x 1}$ & $\mathrm{O}_{x 1}$ & RRMS \\
\hline 42) & $\mathrm{AA} 33$ & $\Theta$ & $26 / 6 / 1970$ & $\Phi \varepsilon \beta-02$ & $17 / 3 / 2006$ & 36 & 4 ह่tก & $\mathrm{O}_{x 1}$ & $\mathrm{O}_{x_{1}}$ & RRMS \\
\hline 43) & $\mathrm{AA} 47$ & A & $8 / 12 / 1977$ & Avy-04 & $16 / 11 / 2006$ & 29 & $2 \mathrm{\varepsilon} \pi$ & $\mathrm{O}_{x 1}$ & $O x_{1}$ & RRMS \\
\hline 44) & AA51 & $\Theta$ & $22 / 10 / 1976$ & 1997 & $21 / 7 / 2006$ & 30 & $9 \varepsilon \dot{\varepsilon}$ & $O_{x 1}$ & $O_{x i}$ & RRMS \\
\hline 45) & AA52 & $\Theta$ & $7 / 4 / 1971$ & 2003 & $22 / 6 / 2006$ & 35 & $3 \varepsilon \dot{\varepsilon} \eta$ & $\mathrm{O}_{x 1}$ & $\mathrm{O}_{x_{1}}$ & RRMS \\
\hline
\end{tabular}

